\title{
On the History of Unified Field Theories. Part II. (ca. 1930 - ca. 1965)
}

\author{
Hubert F. M. Goenner \\ University of Göttingen \\ Institut für Theoretische Physik \\ Friedrich-Hund-Platz 1 \\ D-37077 Göttingen \\ Germany \\ email: goenner@theorie.physik.uni-goettingen.de \\ http://www.theorie.physik. uni-goettingen.de/ goenner
}

Accepted: 13 May 2014

Published: 23 June 2014

\begin{abstract}
The present review intends to provide an overall picture of the research concerning classical unified field theory, worldwide, in the decades between the mid-1930 and mid-1960. Main themes are the conceptual and methodical development of the field, the interaction among the scientists working in it, their opinions and interpretations. Next to the most prominent players, A. Einstein and E. Schrödinger, V. Hlavatý and the French groups around A. Lichnerowicz, M.-A. Tonnelat, and Y. Thiry are presented. It is shown that they have given contributions of comparable importance. The review also includes a few sections on the fringes of the central topic like Born-Infeld electromagnetic theory or scalar-tensor theory. Some comments on the structure and organization of research-groups are also made.
\end{abstract}

Keywords: Unified field theory, Differential geometry, History of science

This review is licensed under a Creative Commons Attribution-Non-Commercial 3.0 Germany License. http://creativecommons . org/licenses/by-nc/3.0/de/ 


\section{Imprint / Terms of Use}

Living Reviews in Relativity is a peer reviewed open access journal published by the Max Planck Institute for Gravitational Physics, Am Mühlenberg 1, 14476 Potsdam, Germany. ISSN 1433-8351.

This review is licensed under a Creative Commons Attribution-Non-Commercial 3.0 Germany License: http://creativecommons.org/licenses/by-nc/3.0/de/. Figures that have been previously published elsewhere may not be reproduced without consent of the original copyright holders.

Because a Living Reviews article can evolve over time, we recommend to cite the article as follows:

Hubert F. M. Goenner,

"On the History of Unified Field Theories. Part II. (ca. 1930-ca. 1965)",

Living Rev. Relativity, 17, (2014), 5. URL (accessed < date>):

http://www.livingreviews.org/lrr-2014-5

The date given as $<$ date $>$ then uniquely identifies the version of the article you are referring to.

\section{Article Revisions}

Living Reviews supports two ways of keeping its articles up-to-date:

Fast-track revision. A fast-track revision provides the author with the opportunity to add short notices of current research results, trends and developments, or important publications to the article. A fast-track revision is refereed by the responsible subject editor. If an article has undergone a fast-track revision, a summary of changes will be listed here.

Major update. A major update will include substantial changes and additions and is subject to full external refereeing. It is published with a new publication number.

For detailed documentation of an article's evolution, please refer to the history document of the article's online version at http://www. livingreviews.org/lrr-2014-5. 


\section{Contents}

$\begin{array}{llr}1 & \text { Introduction } & 7\end{array}$

2 Mathematical Preliminaries 12

2.1 Metrical structure ........................... 12

2.1 Affine structure . . . . . . . . . . . . . . . . . . . 14

2.1.2 Metric compatibility, non-metricity . . . . . . . . . . . . 16

2.2 Symmetries . . . . . . . . . . . . . . . . . . . . . . 19

2.2.1 Transformation with regard to a Lie group . . . . . . . . . . . . . 19

2.2 .2 Hermitian symmetry . . . . . . . . . . . . . . . . . . . . . . . . . . . . . . . . .

$2.2 .3 \lambda$-transformation . . . . . . . . . . . . . . . . . 20

2.3 Affine geometry . . . . . . . . . . . . . . . . . . . . . . . 20

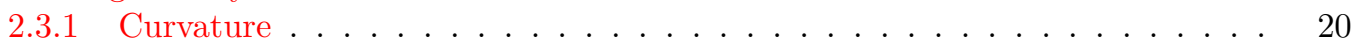

2.3.2 A list of "Ricci"-tensors . . . . . . . . . . . . . . . 22

2.3.3 Curvature and scalar densities . . . . . . . . . . . . . . . . . . . . . . . . . . . . .

2.3.4 Curvature and $\lambda$-transformation . . . . . . . . . . . . . . 24

2.4 Differential forms . . . . . . . . . . . . . . . . . . . . . . . . . 24

2.5 Classification of geometries . . . . . . . . . . . . . . . . . . . 25

2.5.1 Generalized Riemann-Cartan geometry . . . . . . . . . . . . . . . 25

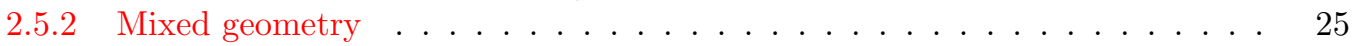

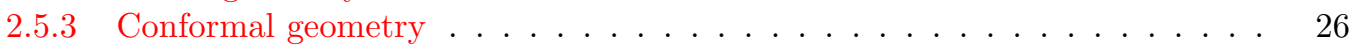

2.6 Number fields . . . . . . . . . . . . . . . . . . . . . . . 26

3 Interlude: Meanderings - UFT in the late 1930s and the 1940s 27

3.1 Projective and conformal relativity theory . . . . . . . . . . . . . . . 27

3.1 .1 Geometrical approach . . . . . . . . . . . . . . . . 28

3.1.2 Physical approach: Scalar-tensor theory . . . . . . . . . . . . . 30

3.2 Continued studies of Kaluza-Klein theory in Princeton, and elsewhere . . . . . . . 33

3.3 Non-local fields . . . . . . . . . . . . . . . . . . . . . . . . . . . . . . 35

3.3.1 Bi-vectors; generalized teleparallel geometry . . . . . . . . . . . . 35

3.3.2 From Born's principle of reciprocity to Yukawa's non-local field theory . . . 38

4 Unified Field Theory and Quantum Mechanics $\quad 40$

4.1 The impact of Schrödinger's and Dirac's equations . . . . . . . . . . . . . . . . . 40

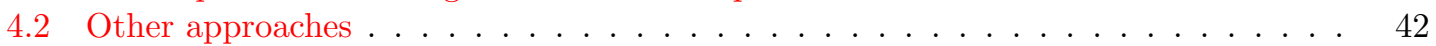

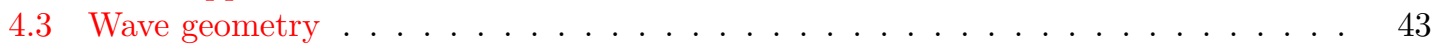

5 Born-Infeld Theory $\quad 45$

6 Affine Geometry: Schrödinger as an Ardent Player $\quad 48$

6.1 A unitary theory of physical fields . . . . . . . . . . . . . . . . . . . . . . . . . . . . . . .

6.1 Symmetric affine connection . . . . . . . . . . . . . . . . . 48

6.1.2 Application: Geomagnetic field . . . . . . . . . . . . . . 51

6.1 .3 Application: Point charge . . . . . . . . . . . . . . . . . . 52

6.2 Semi-symmetric connection . . . . . . . . . . . . . . . . 53

7 Mixed Geometry: Einstein's New Attempt $\quad \mathbf{5 7}$

7.1 Formal and physical motivation . . . . . . . . . . . . . . . . . 58

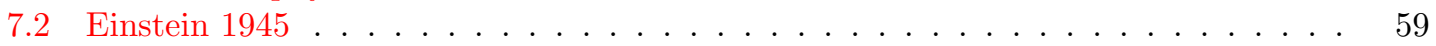

7.3 Einstein-Straus 1946 and the weak field equations . . . . . . . . . . . . 61 
8 Schrödinger II: Arbitrary Affine Connection $\quad \mathbf{6 5}$

8.1 Schrödinger's debacle . . . . . . . . . . . . . . . . . . . . . . . . . . . . . . . . . . . . . . . . .

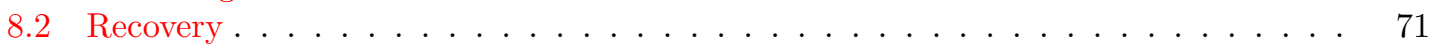

8.3 First exact solutions . . . . . . . . . . . . . . . . . . . 73

9 Einstein II: From 1948 on $\quad 75$

9.1 A period of undecidedness $(1949 / 50) \ldots \ldots \ldots \ldots$. . . . . . . . . . . . . . . . . . . . . . . . . 76

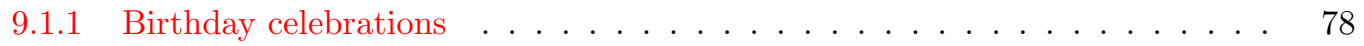

9.2 Einstein $1950 \ldots \ldots \ldots \ldots 79$

9.2.1 Alternative derivation of the field equations . . . . . . . . . . . . . . 79

9.2.2 A summary for a wider circle . . . . . . . . . . . . . . . . . . . . 80

9.2.3 Compatibility defined more precisely . . . . . . . . . . . . . . 83

9.2.4 An account for a general public . . . . . . . . . . . . . . . 85

9.3 Einstein $1953 \ldots \ldots \ldots$. . . . . . . . . . . . . . . . 87

9.3.1 Joint publications with B. Kaufman . . . . . . . . . . . . . . . 88

9.3 .2 Einstein's 74 th birthday $(1953) \ldots \ldots \ldots$. . . . . . . . . . . . . . . . . . . . 91

9.3.3 Critical views: variant field equation . . . . . . . . . . . . . . . . . . 91

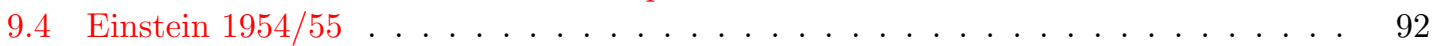

9.5 Reactions to Einstein-Kaufman . . . . . . . . . . . . . . . . . . 96

9.6 More exact solutions . . . . . . . . . . . . . . . . . . . . . . . 97

9.6.1 Spherically symmetric solutions . . . . . . . . . . . . . . . . . 97

9.6 .2 Other solutions . . . . . . . . . . . . . . . . . 99

9.7 Interpretative problems . . . . . . . . . . . . . . . . . . . 100

9.8 The role of additional symmetries . . . . . . . . . . . . . . . . . . . . 102

10 Einstein-Schrödinger Theory in Paris 104

10.1 Marie-Antoinette Tonnelat and Einstein's Unified Field Theory . . . . . . . . . . . 104

10.2 Tonnelat's research on UFT in $1946-1952 \ldots \ldots$. . . . . . . . . . . . . . 105

10.2.1 Summaries by Tonnelat of her work . . . . . . . . . . . . . . . . . . . . . . . . . . . . . . . . . . . . . . .

10.2 .2 Field equations . . . . . . . . . . . . . . . . . . . . . . . . . . . . . . . . . . . . . . . . . . . .

10.2.3 Removal of affine connection . . . . . . . . . . . . . . . . . . . . . . . . . . . . . . . . . . . . . . . . . .

10.3 Some further developments . . . . . . . . . . . . . . . . . . . . . . . . . . . . . . . . . . . . . . .

10.3.1 Identities, or matter and geometry . . . . . . . . . . . . . . . . . . . . . . . . . . . . . . . . . . . . . . .

10.3.2 Equations of motion . . . . . . . . . . . . . . . . 116

10.3.3 Tonnelat's extension of unified field theory . . . . . . . . . . . . . . 119

10.3.4 Conclusions drawn by M.-A. Tonnelat . . . . . . . . . . . . . . . . . . . 121

10.4 Further work on unified field theory around M.-A. Tonnelat . . . . . . . . . . . . . 122

10.4.1 Research by associates and doctoral students of M.-A. Tonnelat . . . . . . . 122

10.5 Research by and around André Lichnerowicz . . . . . . . . . . . . . . . . . . . . . . . . . . . . . . . . . . . . .

10.5.1 Existence of regular solutions? . . . . . . . . . . . . . . . . . . . . . . . . . . . . . . . . . . . . . . .

10.5.2 Initial value problem and discontinuities . . . . . . . . . . . . . . . . . . . . . . . . . . . . . . . . . . . . . . . . . .

10.5.3 Characteristic surfaces . . . . . . . . . . . . . . . . . 128

10.5.4 Some further work in UFT advised by A. Lichnerowicz . . . . . . . . . . . . 130

11 Higher-Dimensional Theories Generalizing Kaluza's 133

11.15 -dimensional theories: Jordan-Thiry theory . . . . . . . . . . . . . . . . 133

11.1.1 Scientists working at the IHP on the Jordan-Thiry unified field theory . . . 135

11.1.2 Scalar-tensor theory in the 1960s and beyond . . . . . . . . . . . . . 136

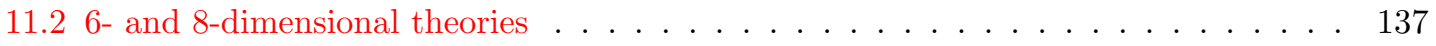

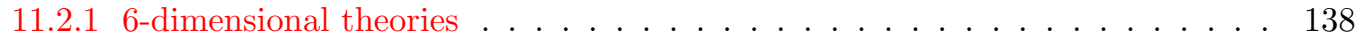


11.2.2 Eight dimensions and hypercomplex geometry . . . . . . . . . . . . . 140

12 Further Contributions from the United States 142

12.1 Eisenhart in Princeton . . . . . . . . . . . . . . . . . . . . . . . . . . . 142

12.2 Hlavatý at Indiana University . . . . . . . . . . . . . . . . . . . . . . . 144

12.3 Other contributions . . . . . . . . . . . . . . . . . . . . 147

13 Research in other English Speaking Countries 150

13.1 England and elsewhere . . . . . . . . . . . . . . . . . . . . . . 150

13.1.1 Unified field theory and classical spin . . . . . . . . . . . . . . 154

13.2 Australia . . . . . . . . . . . . . . . . . . . . . . . . . . . 157

13.3 India . . . . . . . . . . . . . . . . . . . . . . . . . . 158

14 Additional Contributions from Japan 160

15 Research in Italy $\quad \mathbf{1 6 1}$

15.1 Introduction . . . . . . . . . . . . . . . . . . . . . . . . 161

15.2 Approximative study of field equations . . . . . . . . . . . . . . . . 162

15.3 Equations of motion for point particles . . . . . . . . . . . . . . . 163

16 The Move Away from Einstein-Schrödinger Theory and UFT 165

16.1 Theories of gravitation and electricity in Minkowski space . . . . . . . . . . . . 166

16.2 Linear theory and quantization . . . . . . . . . . . . . . . . . . . . . 169

16.3 Linear theory and spin-1/2-particles . . . . . . . . . . . . . . . . . . 172

16.4 Quantization of Einstein-Schrödinger theory? . . . . . . . . . . . . . . . . 172

17 Alternative Geometries $\quad \mathbf{1 7 4}$

17.1 Lyra geometry . . . . . . . . . . . . . . . . . . . . . . . . . . 174

17.2 Finsler geometry and unified field theory . . . . . . . . . . . . . . 175

18 Mutual Influence and Interaction of Research Groups $\quad \mathbf{1 7 8}$

18.1 Sociology of science . . . . . . . . . . . . . . . . . . . . . . . 178

18.1.1 Princeton and UFT . . . . . . . . . . . . . . . . . . . 178

18.1.2 Mathematics and physics . . . . . . . . . . . . . . . 178

18.1 .3 Organization and funding . . . . . . . . . . . . . . . . . 179

18.2 After 1945: an international research effort . . . . . . . . . . . . . . . . . 179

18.2.1 The leading groups . . . . . . . . . . . . . . . . . . . . . . . . . 180

18.2.2 Geographical distribution of scientists . . . . . . . . . . . . . . . . . . 181

18.2.3 Ways of communications . . . . . . . . . . . . . . . . . . . . 182

18.2.4 International conferences and summer schools . . . . . . . . . . . . . . 185

19 On the Conceptual and Methodic Structure of Unified Field Theory $\quad \mathbf{1 8 7}$

19.1 General issues . . . . . . . . . . . . . . . . . . . . . . . . . . 187

19.1.1 What kind of unification? . . . . . . . . . . . . . . . . . 190

19.1.2 UFT and quantum theory . . . . . . . . . . . . . . . . . . . . . 191

19.1.3 A glimpse of today's status of unification . . . . . . . . . . . . . . . 194

19.2 Observations on psychological and philosophical positions . . . . . . . . . . 196

19.2.1 A psychological background to UFT? . . . . . . . . . . . . . . . . 196

19.2.2 Philosophical background . . . . . . . . . . . . . . . . . 198

20 Concluding Comment 201

$\begin{array}{ll}\text { References } & 202\end{array}$ 



\section{Introduction}

The dream of unifying all fundamental interactions in a single theory by one common representation still captures the mind of many a theoretical physicist. In the following, I will focus on the development of classical unified field theory (UFT) in the period from the mid-1930s to the mid1960s. One of the intentions then was to join the gravitational to the electromagnetic field, and, hopefully, to other fields (mesonic, ...) in "a single hyperfield, whose basis would be equivalent to that of the geometrical structure for the universe" ([376], p. 3). Einstein referred to his corresponding theories alternatively as the "generalized theory of gravitation", "(relativistic) theory of the non-symmetric (or asymmetric) field", and of "the theory of the total field". Schrödinger spoke of "unitary field theory"; this name was taken up later by Bergmann [24] or Takasu [598]. In Mme. Tonnelat's group, the name "théorie du champ unifié d'Einstein" (or d'Einstein-Schrödinger), or just "théorie unitaire (du champ)(d'Einstein)" was in use; Hlavatý called it "(Einstein) Unified (Field) Theory of Relativity". In other papers we read of "Einstein's Generalized Theory of Gravitation", "Einstein's equations of unified field", "theory of the non-symmetric field", "einheitliche Feldtheorie" etc. However, we should not forget that other types of unitary field theory were investigated during the period studied, among them Kaluza-Klein theory and its generalizations. In France, one of these ran under the name of "Jordan-Thiry" theory, cf. Sections 3.1.2 and 11.1.

Most important centers for research on unified field theory in the 1930s until the early 1950s were those around Albert Einstein in Princeton and Erwin Schrödinger in Dublin. Paris became a focus of UFT in the late 1940s up to the late 1960s, with a large group of students around both Mme. M.-A. Tonnelat in theoretical physics, and the mathematician A. Lichnerowicz. In comparison with the work of Einstein and Schrödinger, the contributions to UFT of the Paris groups have been neglected up to now by historians of physics although they helped to clarify consequences of the theory. These groups had a share both in the derivation of exact mathematical results and in contributing arguments for the eventual demise of UFT. The mathematician V. Hlavatý from Indiana University, Bloomington (USA), with one or two students, enriched the mathematically-oriented part of the UFT-community with his systematical studies in the 1950s. We will encounter many further researchers worldwide, especially sizeable groups in Italy, and in countries like Canada, England, India, and Japan. The time period is chosen such that Einstein's move from Berlin to Princeton approximately defines its beginning while its end falls into the 1960s which saw a revival of interest in general relativity theory [192], and the dying off of some still existing interest into classical unified field theory. Up to the 1940s, some hope was justified that the gravitational interaction might play an important role in the unification of the fundamental fields. With the growth of quantum field theory and developments in elementary particle physics, gravity became crowded out, however.

At the time, the mainstream in theoretical physics had shifted to quantum mechanics and its applications in many parts of physics and physical chemistry. Quantum field theory had been invented as a relevant tool for describing the quantum aspects of atoms, molecules and their interactions with P. Jordan, M. Born and W. Heisenberg having made first steps in 1926. Dirac had put forward his "second" quantization in 1927 which was then interpreted and generalized as field quantization by Jordan, Heisenberg, Klein, Pauli, and Wigner in 1927/28. Expert histories of quantum electrodynamics and its beginning have been presented ${ }^{1}$ by S. Schweber [562], O. Darrigol [109], and A. Pais [470]. Around the time when Einstein left Berlin, Heisenberg and others set up theories of the strong nuclear force. Fermi had introduced a theory of weak interactions in connection with beta-decay. Since 1932/33, besides electron, photon, and proton, three new particles, namely the neutron, positron and neutrino had come into play with the last two already having been found, empirically. Anyone doubting the existence of the neutron, had to give in after

1 Some of the relevant papers are reprinted in Miller's book [424] 
nuclear fission had been discovered and nuclear reactors been built. At the 1933 Solvay conference, L. de Broglie had proposed a neutrino theory of light, i.e., with the photon as a composite particle made up by two neutrinos [111, 112], and others like P. Jordan or G. Wentzel had followed suit $[314,315,687]$. For a while, this became a much debated subject in theoretical physics. Another great topic, experimentally, was the complicated physics of "cosmic rays" containing at least another new particle with a mass about 200 times that of the electron. It was called alternatively "heavy electron", "mesot(r)on", and "meson" and became mixed up with the particle mediating the nuclear force the name of which was "U-quantum", or "Yukon" after Yukawa's suggestion in 1934/35 concerning nuclear interactions. For the history cf. [63]. When the dust had settled around 1947, the "mesotron" became the muon and the pions were considered to be the carriers of the nuclear force (strong interaction). Since 1937, the muon had been identified in cosmic rays $[455,593]$. The charged pion which decays into a muon and a (anti-)neutrino via the weak interaction was detected in 1947, the uncharged one in 1950. In the 1940s, quantum electrodynamics was given a new kick by Feynman, Schwinger and Tomonaga. Up to the mid fifties, nuclear theory had evolved, the strong and weak nuclear forces were accepted with the neutrino observed only in 1957, after Einstein's death. Thus, the situation had greatly changed during the two decades since Einstein had started to get involved in unified field theory: in the 1920s only two fundamental interactions had been known, both long-range: the electromagnetic and the gravitational. Before 1926, neither non-relativistic quantum theory, nor relativistic quantum electrodynamics had been developed. In 1928, with Dirac's equation, "spin" had appeared as a new property of elementary particles. After a brief theoretical venture into spinors and the Dirac equation (cf. Section 7.3 of Part I and Section 4.1), against all of the evidence concerning new particles with half-integer spin and new fundamental interactions obtained in the meantime, Einstein continued to develop the idea of unifying only the electromagnetic and gravitational fields via pure geometry, cf. Section 7 below. His path was followed in much of the research done in classical UFT. Occasionally, as in Schrödinger's and Tonnelat's work, meson fields, treated as classical fields, were also included in the interpretation of geometric objects within the theory. The state of affairs was reflected, in 1950, in a note in the Scientific American describing Einstein's motivation for UFT as:

"to relate the physical phenomena in the submicroscopic world of the atom to those in the macroscopic world of universal space-time, to find a common principle explaining both electromagnetic forces and gravitational force [...]. In this inquiry Einstein has pursued a lonely course; most physicists have taken the apparently more promising road of quantum theory." ([564], p. 26)

In fact, the majority of the theoretical physicists working in field theory considered UFT of the Einstein-Schrödinger type as inadequate. Due to Einstein's earlier achievements, his fame and, possibly, due to his, Schrödinger's and de Broglie's reserve toward the statistical interpretation of quantum mechanics, classical or semi-classical approaches to field theory were favoured in their scientific research environments in theoretical physics. Convinced by the stature of these men, a rather small number of theoretical physicists devoted their scientific careers to classical unified field theory. Others wrote their $\mathrm{PhD}$ theses in the field and then quickly left it. A few mathematicians became attracted by the geometrical structures underlying the field (cf. [677], p. 30).

In their demands on UFT, Einstein and Schrödinger differed: while the first one never gave up his hope to find a substitute, or at least a needed foundation for quantum theory in his classical unified field theory, Schrödinger saw his theory as a strictly classical groundwork for an eventual alternative to quantum field theory or, as he expressed it himself, as "the classical analogue' of the true laws of Nature" ([551], p. 50). ${ }^{2}$ Einstein in particular followed his way towards UFT

\footnotetext{
2 In a different area, Schrödinger ventured to join quantum and gravitational theory in the picture of quantized eigen-vibrations of a spatially closed universe [543].
} 
unwaveringly in spite of failing success. Shortly before his death, he even reinterpreted his general relativity, the central concept of which had been the gravitational and inertial potentials encased in the (pseudo-)Riemannian metric tensor, through the lens of unified field theory:

"[...] the essential achievement of general relativity, namely to overcome 'rigid' space (i.e., the inertial frame), is only indirectly connected with the introduction of a Riemannian metric. The direct relevant conceptual element is the 'displacement field' $\left(\Gamma_{i k}^{l}\right)$, which expresses the infinitesimal displacement of vectors. It is this which replaces the parallelism of spatially arbitrarily separated vectors fixed by the inertial frame (i.e., the equality of corresponding components) by an infinitesimal operation. This makes it possible to construct tensors by differentiation and hence to dispense with the introduction of 'rigid' space (the inertial frame). In the face of this, it seems to be of secondary importance in some sense that some particular $\Gamma$-field can be deduced from a Riemannian metric [...]."3 (A. Einstein, 4 April 1955, letter to M. Pantaleo, in ([473], pp. XV-XVI); English translation taken from Hehl and Obuchov 2007 [244].)

To me, this is not a prophetic remark pointing to Abelian and non-Abelian gauge theories which turned out to play such a prominent role in theoretical physics, a little later. ${ }^{4}$ Einstein's gaze rather seems to have been directed backward to Levi-Civita, Weyl's paper of 1918 [688], and to Eddington. ${ }^{5}$ The Institute for Advanced Study must have presented a somewhat peculiar scenery at the end of the 1940s and early 50s: among the senior faculty in the physics section as were Oppenheimer, Placzek and Pais, Einstein remained isolated. That a "postdoc" like Freeman Dyson had succeeded in understanding and further developing the different approaches to quantum electrodynamics by Schwinger and Feynman put forward in 1948, seemingly left no mark on Einstein. Instead, he could win the interest and help of another Princeton postdoc at the time, Bruria Kaufman, for his continued work in UFT [587]. We may interpret a remark of Pauli as justifying Einstein's course:

"The quantization of fields turns out more and more to be a problem with thorns and horns, and by and by I get used to think that I will not live to see substantial progress for all these problems." ([493], p. 519)

In fact, for elementary particle theory, the 1950s and 1960s could be seen as "a time of frustration and confusion" ([686], p. 99). For weak interactions (four-fermion theory) renormalization did not work; for strong interactions no calculations at all were possible. W. Pauli was very skeptical toward the renormalization schemes developed: "[...] from my point of view, renormalization is a not yet understood palliative." (Letter to Heisenberg 29 September 1953 [491], p. 268.)

3 “[.. ]: die wesentliche Leistung der allgemeinen Relativitätstheorie, nämlich die Überwindung 'starren' Raumes, d.h. des Inertialsystems, ist nur indirekt mit der Einführung einer Riemann-Metrik verbunden. Das unmittelbar wesentliche begriffliche Element ist das die infinitesimale Verschiebung von Vektoren ausdrückende 'Verschiebungsfeld' $\left(\Gamma_{i k}^{l}\right)$. Dieses nämlich ersetzt den durch das Inertialsystem gesetzten Parallelismus räumlich beliebig getrennter Vektoren (nämlich Gleichheit entsprechender Komponenten) durch eine infinitesimale Operation. Dadurch wird die Bildung von Tensoren durch Differentiation ermöglicht und so die Einführung des 'starren' Raumes (Inertialsystem) entbehrlich gemacht. Demgegenüber erscheint es in gewissem Sinne von sekundärer Wichtigkeit, dass ein besonderes $\Gamma$-Feld sich aus der Existenz einer Riemann-Metrik deduzieren lässt."

${ }^{4}$ For the later development toward Poincaré gauge theory cf. [29].

${ }^{5}$ Einstein, through his interaction with Weyl, should have known Weyl's later paper with its then physically meaningful application of the gauge principle [692]. The paper by Yang \& Mills [712] did appear only shortly before his death. cf. also [464].

6 "Die Quantisierung der Felder erweist sich ja immer mehr als ein Problem mit Dornen und Hörnern, und allmählich gewöhne ich mich an den Gedanken, einen wirklichen Fortschritt bei all diesen Problemen nicht mehr zu erleben." 
About a month after Einstein's death, the mathematician A. Lichnerowicz had the following to say concerning his unified field theory:

"Einstein just has disappeared leaving us, in addition to many completed works, an enigmatic theory. The scientists look at it - like he himself did - with a mixture of distrust and hope, a theory which carries the imprint of a fundamental ambition of its creator." (cf. Lichnerowicz, preface of [632], p. VII.) ${ }^{7}$

In Bern, Switzerland, three months after Einstein's death, a "Jubilee Conference" took place commemorating fifty years of relativity since the publication of his famous 1905 paper on the electrodynamics of moving bodies. Unified field theory formed one of its topics, with 34 contributions by 32 scientists. In 1955, commemorative conferences were also held in other places as well which included brief reviews of UFT (e.g., by B. Finzi in Bari [203] and in Torino [203]). Two years later, among the 21 talks of the Chapel Hill Conference on "the role of gravitation in physics" published [119], only a single one dealt with the "Generalized Theory of Gravitation" [344]. Again five years later, after a conference on "Relativistic Theories of Gravitation", the astronomer George C. McVittie (1904-1988) could report to the Office of Naval Research which had payed for his attendance: "With the death of Einstein, the search for a unified theory of gravitation and electromagnetism has apparently faded into the background." (Quoted in [523], p. 211.) This certainly corresponded to the majority vote. At later conferences, regularly one contribution or two at most were devoted to UFT [302, 382]. From the mid-1960s onward or, more precisely, after the Festschrift for V. Hlavatý of 1966 [282], even this trickle of accepted contributions to UFT for meetings ran dry. "Alternative gravitational theories" became a more respectable, but still a minority theme. Not unexpectedly, some went on with their research on UFT in the spirit of Einstein, and some are carrying on until today. In particular, in the 1970s and 80s, interest in UFT shifted to India, Japan, and Australia; there, in particular, the search for and investigation of exact solutions of the field equations of the Einstein-Schrödinger unified field theory became fashionable. Nevertheless, Hlavatý's statement of 1958, although quite overdone as far as mathematics is concerned, continues to be acceptable:

"In the literature there are many approaches to the problem of the unified field theory.

Some of them strongly influenced the development of geometry, although none has received general recognition as a physical theory." ([269], preface, p. X.)

The work done in the major "groups" lead by Einstein, Schrödinger, Lichnerowicz, Tonnelat, and Hlavatý was published, at least partially, in monographs (Einstein: [150], Appendix II; [156], Appendix II); (Schrödinger: [557], Chapter XII); (Lichnerowicz: [371]); (Hlavatý: [269]), and, particularly, (Tonnelat: $[632,641,642]$ ). To my knowledge, the only textbook including the Einstein-Schrödinger non-symmetric theory has been written in the late 1960s by D. K. Sen [572]. The last monograph on the subject seems to have been published in 1982 by A. H. Klotz [334]. There exist a number of helpful review articles covering various stages of UFT like Bertotti [26], Bergia [19], Borzeszkowsi \& Treder [679], Cap [71], Hittmair [256], Kilmister and Stephenson [330, 331], Narlikar [453], Pinl [497], Rao [504], Sauer [528, 529], and Tonnelat ([645], Chapter 11), but no attempt at giving an overall picture beyond Goenner [228] seems to have surfaced. Vizgin's book ends with Einstein's research in the 1930s [678]. In 1957, V. Bargmann has given a clear four-page résumé of both the Einstein-Schrödinger and the Kaluza-Klein approaches to unified field theory [12]. ${ }^{8}$ In van Dongen's recent book, the epistemological and methodological positions

\footnotetext{
7 "Einstein vient de disparaître, nous laissant, à côté de tant de travaux achevés, une théorie énigmatique que les savants contemplent, comme lui-même, avec un mélange de méfiance et d'espoir, mais qui porte l'empreinte de l'ambition fondamentale de son créateur."

8 In the preface to Part I, the unconvincing chapter on UFT in the book by by Pais [469] was also included. The very brief "excursion into UFT" in a biography of W. Pauli ([194], pp. 260-273) is written specifically under the angle of Pauli's achievements and interests.
} 
of Einstein during his work on unified field theory are discussed [667].

The present review intends to provide a feeling for what went on in research concerning UFT at the time, worldwide. Its main themes are the conceptual and methodical development of the field, the interaction among the scientists working in it, and their opinions and interpretations. The review also includes a few sections on the fringes of the general approach. A weighty problem has been to embed the numerous technical details in a narrative readable to those historians of science lacking the mathematical tools which are required in many sections. In order to ease reading of chapters, separately, a minor number of repetitions was deemed helpful. Some sociological and philosophical questions coming up in connection with this review will be touched in Sections 18 and 19. These two chapters can be read also by those without any knowledge of the mathematical and physical background. Up to now, philosophers of science apparently have not written much on Einstein's unified field theory, with the exception of remarks following from a non-technical comparison of the field with general relativity. Speculation about the motivation of the central figures are omitted here if they cannot be extracted from some source.

The main groups involved in research on classical unified field theory will be presented here more or less in chronological order. The longest account is given of Einstein-Schrödinger theory. In the presentation of researchers we also follow geographical and language aspects due to publications in France being mostly in French, in Italy mostly in Italian, in Japan and India in English. ${ }^{9}$

We cannot embed the history of unified field theory into the external (political) history of the period considered; progress in UFT was both hindered by the second world war, Nazi- and communist regimes, and helped, after 1945, by an increasing cooperation among countries and the beginning globalization of communications. ${ }^{10}$

Part II of the "History of Unified Field Theory" is written such that it can be read independently from Part I. Some links to the earlier part [229] in Living Reviews in Relativity are provided.

\footnotetext{
9 After two fruitless attempts, with no answer received in one case and complete amnesia indicated in the other, I dropped the idea of consulting systematically all living contemporary witnesses about their past work. Some short biographies are provided, unsystematic, with the information taken from the internet and other available sources.

10 For a case study in mathematics (International Mathematical Union) cf. [356]. Political history is met, occasionally, in the biographies of scientists involved in research on UFT. Examples for interrupted or abruptly ended careers of little-known or unknown theoretical physicists are given; e.g., T. Hosokawa \& T. Iwatsuki (cf. Section 4.3), J. Podolanski (cf. Section 11.2.1) and H. Kremer (cf. Section 5).
} 


\section{Mathematical Preliminaries}

For the convenience of the reader, some of the mathematical formalism given in the first part of this review is repeated in a slightly extended form: It is complemented by further special material needed for an understanding of papers to be described.

\subsection{Metrical structure}

First, a definition of the distance $d s$ between two infinitesimally close points on a D-dimensional differential manifold $M_{D}$ is to be given, eventually corresponding to temporal and spatial distances in the external world. For $d s$, positivity, symmetry in the two points, and the validity of the triangle equation are needed. We assume $d s$ to be homogeneous of degree one in the coordinate differentials $d x^{i}$ connecting neighboring points. This condition is not very restrictive; it includes Finsler geometry [510, 199, 394, 4] to be briefly discussed in Section 17.2.

In the following, $d s$ is linked to a non-degenerate bilinear form $g(X, Y)$, called the first fundamental form; the corresponding quadratic form defines a tensor field, the metrical tensor, with $D^{2}$ components $g_{i j}$ such that

$$
d s=\sqrt{g_{i j} d x^{i} d x^{j}},
$$

where the neighboring points are labeled by $x^{i}$ and $x^{i}+d x^{i}$, respectively ${ }^{11}$. Besides the norm of a vector $|X|:=\sqrt{g_{i j} X^{i} X^{j}}$, the "angle" between directions $X, Y$ can be defined by help of the metric:

$$
\cos (\angle(X, Y)):=\frac{g_{i j} X^{i} Y^{j}}{|X||Y|} .
$$

From this we note that an antisymmetric part of the metrical's tensor does not influence distances and norms but angles.

We are used to $g$ being a symmetric tensor field, i.e., with $g_{i k}=g_{(i k)}$ with only $D(D+1) / 2$ components; in this case the metric is called Riemannian if its eigenvalues are positive (negative) definite and Lorentzian if its signature is $\pm(D-2)^{12}$. In this case, the norm is $|X|:=\sqrt{\left|g_{i j} X^{i} X^{j}\right|}$. In space-time, i.e., for $D=4$, the Lorentzian signature is needed for the definition of the light cone: $g_{i j} d x^{i} d x^{j}=0$. The paths of light signals through the cone's vertex are assumed to lie in this subspace. In unified field theory, the line element ("metric") $g_{i k}$ is an asymmetric tensor, in general. When of full rank, its inverse $g^{i k}$ is defined through ${ }^{13}$

$$
g_{m i} g^{m j}=\delta_{i}^{j}, \quad g_{i m} g^{j m}=\delta_{i}^{j} .
$$

In the following, the decomposition into symmetric and antisymmetric parts is denoted by ${ }^{14}$ :

$$
\begin{aligned}
g_{i k} & =h_{i k}+k_{i k}, \\
g^{i k} & =l^{i k}+m^{i k} .
\end{aligned}
$$

\footnotetext{
11 The second fundamental form comes into play when local isometric embedding is considered, i.e., when $M_{D}$ is taken as a submanifold of a larger space such that the metrical relationships are conserved. In the following, all geometrical objects are supposed to be differentiable as often as is needed.

12 Latin indices $i, j, k, \ldots$ run from 1 to $D$, or from 0 to $D-1$ to emphasize the single timelike direction. We are using symmetrization (anti-) brackets defined by $A_{(i j)}:=1 / 2\left(A_{i j}+A_{j i}\right)$ and $A_{[i j]}:=1 / 2\left(A_{i j}-A_{j i}\right)$, respectively.

13 Here, the Kronecker-symbol $\delta^{i}{ }_{k}$ with value +1 for $i=k$, and value 0 for $i \neq k$ is used. $\delta^{i}{ }_{k}$ keeps its components unchanged under arbitrary coordinate transformations.

14 Note the altered notation with regard to Eqs. (3) and (4) in Part I of this article, where the notation of [632] has been used. Here, we take over the notation of A. Lichnerowicz ([371], p. 255). The correspondences are $\gamma_{i k} \sim h_{i k}, \phi_{i k} \sim k_{i k}, h^{i k} \sim l^{i k}, f^{i k} \sim m^{i k}$ The inverses are defined with the same kernel letter. Also, in physical applications, special conditions for $h$ and $k$ might be needed in order to guarantee that $g$ is a Lorentz metric. Equation (3) reflects Hlavatý's notation, too.
} 
$h_{i k}$ and $l^{i k}$ have the same rank; also, $h_{i k}$ and $l^{i k}$ have the same signature [27]. Equation (2) looks quite innocuous. When working with the decompositions (3), (4) however, eight tensors are floating around: $h_{i k}$ and its inverse $h^{i k}$ (indices not raised!); $k_{i k}$ and its inverse $k^{i k} ; l^{i k} \neq h^{i k}$ and its inverse $l_{i k} \neq h_{i k}$, and finally $m^{i k} \neq k^{i k}$ and its inverse $m_{i k}$.

With the decomposition of the inverse $g^{j m}(4)$ and the definitions for the respective inverses

$$
h_{i j} h^{i k}=\delta_{j}^{k} ; k_{i j} k^{i k}=\delta_{j}^{k} ; l_{i j} l^{i k}=\delta_{j}^{k} ; m_{i j} m^{i k}=\delta_{j}^{k},
$$

the following relations can be obtained: ${ }^{15}$

$$
l^{i k}=l^{(i k)}=\frac{h}{g} h^{i k}+\frac{k}{g} k^{i m} k^{k n} h_{m n}
$$

and

$$
m^{i k}=m^{[i k]}=\frac{k}{g} k^{i k}+\frac{h}{g} h^{i m} h^{k n} k_{m n}
$$

where $g=: \operatorname{det}\left(g_{i k}\right) \neq 0, \quad k=: \operatorname{det}\left(k_{i k}\right) \neq 0, \quad h=: \operatorname{det}\left(h_{i k}\right) \neq 0$. We also note:

$$
g=h+k+\frac{h}{2} h^{k l} h^{m n} k_{k m} k_{l n}
$$

and

$$
g g^{i j}=h h^{i j}+k k^{i j}+h h^{i r} h^{j s} k_{r s}+k k^{i r} k^{j s} h_{r s} .
$$

Another useful relation is

$$
g^{2}=\frac{h}{l}
$$

with $l=\operatorname{det}\left(l_{i j}\right)$. From (9) we see that unlike in general relativity even invariants of order zero (in the derivatives) do exist: $\frac{k}{h}$, and $h^{k l} h^{m n} k_{k m} k_{l n}$; for the 24 invariants of the metric of order 1 in space-time cf. $[512,513,514]$.

Another consequence of the asymmetry of $g_{i k}$ is that the raising and lowering of indices with $g_{i k}$ now becomes more complicated. For vector components we must distinguish:

$$
\vec{y}_{k}:=g_{k j} y^{j}, \quad \overleftarrow{y}_{k}:=y^{j} g_{j k}
$$

where the dot as an upper index means that an originally upper index has been lowered. Similarly, for components of forms we have

$$
\vec{w}^{k}:=g^{k j} w_{j}, \quad \overleftarrow{w}^{k}:=w_{j} g^{j k}
$$

The dot as a lower index points to an originally lower index having been raised. In general, $\vec{y}_{k} \neq \overleftarrow{y}_{\dot{k}}, \vec{w}^{k} \neq \overleftarrow{w}^{k}$. Fortunately, the raising of indices with the asymmetric metric does not play a role in the following.

An easier possibility is to raise and lower indices by the symmetric part of $g_{j k}$, i.e., by $h_{j k}$ and its inverse $h^{i j}{ }^{16}$ In fact, this is often seen in the literature; cf. [269, 297, 298]. Thus, three new tensors (one symmetric, two skew) show up:

$$
\begin{array}{r}
\check{k}^{i j}:=h^{i s} h^{j t} k_{s t} \neq k^{i j}, \quad \check{l}_{i j}:=h_{i s} h_{j t} l^{s t} \neq l_{i j}, \\
\check{m}_{i j}:=h_{i s} h_{j t} m^{s t} \neq m_{i j}, \quad \check{h}^{i j}:=h^{i j} .
\end{array}
$$

${ }^{15}$ Cf. the table on p. 15 in [632], and Section 2.1.1 of Part I where further relevant historical references are given. E.g., Eq. (7) was also derived by Hlavatý [261], p. 110. (He denoted $m^{i k}$ by ${ }^{*} g^{[i k]}$.)

${ }^{16}$ Correspondingly, the simpler notation $y_{k}=h_{k r} y^{r}, \omega^{k}=h^{k r} \omega_{r}$ etc. is used. 
Hence, Ikeda instead of (9) wrote:

$$
g g^{i j}=h\left[h^{i j}\left(1+\frac{1}{2} k_{i j} \check{k}^{i j}\right)+\check{k}^{i j}-\check{k}^{i r} \check{k}_{s}^{j}+\frac{\rho}{2 h} \epsilon^{i j r s} k_{r s}\right],
$$

with $\rho:=\frac{1}{8} \epsilon^{i j l m} \check{k}_{i j} \check{k}_{l m}$. For a physical theory, the "metric" governing distances and angles must be a symmetric tensor. There are two obvious simple choices for such a metric in UFT, i.e., $h_{i k}$ and $l^{i k}$. For them, in order to be Lorentz metrics, $h<0\left(l:=\operatorname{det}\left(l^{i j}\right)<0\right)$ must hold. The light cones determined by $h_{i k}$ and by $l^{i k}$ are different, in general. For further choices for the metric cf. Section 9.7.

The tensor density formed from the metric is denoted here by

$$
\hat{g}^{i j}=\sqrt{-g} g^{i j}, \hat{g}_{i j}=(\sqrt{-g})^{-1} g_{i j} .
$$

The components of the flat metric (Minkowski-metric) in Cartesian coordinates is denoted by $\eta_{i k}$ :

$$
\eta_{i k}=\delta_{i}^{0} \delta_{k}^{0}-\delta_{i}^{1} \delta_{k}^{1}-\delta_{i}^{2} \delta_{k}^{2}-\delta_{i}^{3} \delta_{k}^{3}
$$

\subsubsection{Affine structure}

The second structure to be introduced is a linear connection (affine connection, affinity) L with $D^{3}$ components $L_{i j}{ }^{k}$; it is a geometrical object but not a tensor field and its components change inhomogeneously under local coordinate transformations. ${ }^{17}$ The connection is a device introduced for establishing a comparison of vectors in different points of the manifold. By its help, a tensorial derivative $\nabla$, called covariant derivative is constructed. For each vector field and each tangent vector it provides another unique vector field. On the components of vector fields $\mathrm{X}$ and linear forms $\omega$ it is defined by

$$
\stackrel{+}{\nabla}_{k} X^{i}=X_{\| k}^{i}:=\frac{\partial X^{i}}{\partial x^{k}}+L_{k j}^{i} X^{j}, \quad \stackrel{+}{\nabla}_{k} \omega_{i}:={\underset{+}{+} \omega_{i k}}^{i}=\frac{\partial \omega_{i}}{\partial x^{k}}-L_{k i}^{j} \omega_{j} .
$$

The expressions $\stackrel{+}{\nabla}_{k} X^{i}$ and $\frac{\partial X^{i}}{\partial x^{k}}$ are abbreviated by $X_{\| k}^{+}$and $X_{, k}^{i}=\partial_{k} X^{i}$. For a scalar $f$, covariant and partial derivative coincide: $\nabla_{i} f=\frac{\partial f}{\partial x_{i}} \equiv \partial_{i} f \equiv f_{, i}$. The antisymmetric part of the connection, i.e.,

$$
S_{i j}^{k}=L_{[i j]}^{k}
$$

is called torsion; it is a tensor field. The trace of the torsion tensor $S_{i}=: S_{i l}^{l}$ is called torsion vector or vector torsion; it connects to the two traces of the linear connection $L_{i}=: L_{i l}{ }^{l} ; \tilde{L}_{j}=: L_{l j}{ }^{l}$ as $S_{i}=1 / 2\left(L_{i}-\tilde{L}_{i}\right)$. Torsion is not just one of the many tensor fields to be constructed: it has a very clear meaning as a deformation of geometry. Two vectors transported parallelly along each other do not close up to form a parallelogram (cf. Eq. (22) below). The deficit is measured by torsion. The rotation $\stackrel{+}{\nabla}_{k} \omega_{i}-\stackrel{+}{\nabla}_{i} \omega_{k}$ of a 1 -form now depends on torsion $S_{k i}{ }^{r}$ :

$$
\stackrel{+}{\nabla}_{k} \omega_{i}-\stackrel{+}{\nabla}_{i} \omega_{k}=\frac{\partial \omega_{i}}{\partial x^{k}}-\frac{\partial \omega_{k}}{\partial x^{i}}-2 S_{k i}{ }^{r} \omega_{r}
$$

We have adopted the notational convention used by Schouten [537, 540, 683]. Eisenhart and others $[182,438]$ change the order of indices of the components of the connection:

$$
\bar{\nabla}_{k} X^{i}=X^{-}{ }_{\| k}:=\frac{\partial X^{i}}{\partial x^{k}}+L_{j k}^{i} X^{j}, \quad \bar{\nabla}_{k} \omega_{i}:=\omega_{-}^{i \| k}=\frac{\partial \omega_{i}}{\partial x^{k}}-L_{i k}{ }^{j} \omega_{j},
$$

17 Strictly, an affine connection is a connection in the frame bundle. In an arbitrary basis for differential forms (cotangent space), the connection can be represented by a 1-form. 
whence follows

$$
\bar{\nabla}_{k} \omega_{i}-\bar{\nabla}_{i} \omega_{k}=\frac{\partial \omega_{i}}{\partial x^{k}}-\frac{\partial \omega_{k}}{\partial x^{i}}+2 S_{k i}^{r} \omega_{r}
$$

As long as the connection is symmetric this does not make any difference because of

$$
\stackrel{+}{\nabla}_{k} X^{i}-\bar{\nabla}_{k} X^{i}=2 S_{[k j]}^{i} X^{j}=0
$$

For both kinds of derivatives we have:

$$
\stackrel{+}{\nabla}_{k}\left(v^{l} w_{l}\right)=\frac{\partial\left(v^{l} w_{l}\right)}{\partial x^{k}} ; \bar{\nabla}_{k}\left(v^{l} w_{l}\right)=\frac{\partial\left(v^{l} w_{l}\right)}{\partial x^{k}} .
$$

Both derivatives are used in versions of unified field theory by Einstein and others. ${ }^{18}$

A manifold provided with only a linear (affine) connection $\mathrm{L}$ is called affine space. From the point of view of group theory, the affine group (linear inhomogeneous coordinate transformations) plays a special rôle: with regard to it the connection transforms as a tensor; cf. Section 2.1.5 of Part I.

The covariant derivative with regard to the symmetrical part of the connection $L_{(k j)}^{l}=\Gamma_{j k}^{l}$ is denoted by $\stackrel{0}{\nabla}_{k}$ such that ${ }^{19}$

$$
\stackrel{0}{\nabla}_{k} X^{i}=X_{0}^{i} \underset{\| k}{\partial x^{k}}+\Gamma_{k j}^{i} X^{j}, \quad \stackrel{0}{\nabla}{ }_{k} \omega_{i}=\omega_{i \| k}=\frac{\partial \omega_{i}}{\partial x^{k}}-\Gamma_{k i}^{j} \omega_{j} .
$$

In fact, no other derivative is necessary if torsion is explicitly introduced, because of ${ }^{20}$

$$
\stackrel{+}{\nabla}_{k} X^{i}=\stackrel{0}{\nabla}{ }_{k} X^{i}+S_{k m}^{i} X^{m}, \quad \stackrel{+}{\nabla}_{k} \omega_{i}=\stackrel{0}{\nabla_{k}} \omega_{i}-S_{k i}{ }^{m} \omega_{m}
$$

In the following, $\Gamma_{i j}{ }^{k}$ always will denote a symmetric connection if not explicitly defined otherwise. To be noted is that: $\lambda_{[i, k]}=\stackrel{+}{\nabla}_{[k} \lambda_{i]}+\lambda_{s} S_{k i} s=\stackrel{0}{\nabla}_{[k} \lambda_{i]}$.

For a vector density of coordinate weight $z \hat{X}^{i}$, the covariant derivative contains one more term (cf. Section 2.1.5 of Part I):

$$
\stackrel{+}{\nabla}_{k} \hat{X}^{i}=\frac{\partial \hat{X}^{i}}{\partial x^{k}}+L_{k j}^{i} \hat{X}^{j}-z L_{k r}^{r} \hat{X}^{i}, \bar{\nabla}_{k} \hat{X}^{i}=\frac{\partial X^{i}}{\partial x^{k}}+L_{j k}^{i} \hat{X}^{j}-z L_{r k}^{r} \hat{X}^{i}
$$

The metric density of Eq. (13) has coordinate weight $z=1 .^{21}$ For the concept of gauge weight cf. (491) of Section 13.2.

\footnotetext{
18 In the literature, other notations and conventions are used. Tonnelat [632] writes $A_{k ; j}=: A_{k, j}-L_{k j}^{l} A_{l}$, and $A_{k ; j}=: A_{k, j}-L_{j k}^{l} A_{l}$. Thus like Einstein's notation, the + and - covariant derivatives are interchanged as compared to the notation used here. This was taken over by many, e.g., by Todeschini [609].

19 Here, we altered the notation of the covariant derivative with respect to a symmetric connection $\nabla_{k} X^{i}=$ $X_{\| k}^{i}$ introduced in (14) by shifting the number 0 from the index $i$, i.e., ${ }_{0}^{i} \| k$, to the sign of the derivation, i.e., $i \| k$. This simplifies notation, in particular if the same covariant derivative is applied to all indices of a tensor: $g_{i k \| l}$ in place of $g_{i k \| l}$.

20 This point of view was stressed by the mathematician J. A. Schouten; cf. Section 18.1.2.

21 Schouten's conventions are used here [540].
} 
A smooth vector field $\mathrm{Y}$ is said to be parallelly transported along a parametrized curve $\lambda(u)$ with tangent vector $\mathrm{X}$ iff for its components $Y_{\| k}^{i} X^{k}(u)=0$ holds along the curve. A curve is called an autoparallel if its tangent vector is parallelly transported along it in each point: ${ }^{22}$

$$
X_{\| k}^{i} X^{k}(u)=\sigma(u) X^{i}
$$

By a particular choice of the curve's parameter, $\sigma=0$ may be reached. Some authors use a parameter-invariant condition for auto-parallels: $X^{l} X^{i}{ }_{\| k} X^{k}(u)-X^{i} X^{l}{ }_{\| k} X^{k}(u)=0$; cf. [284].

A transformation mapping auto-parallels to auto-parallels is given by:

$$
L_{i k}^{j} \rightarrow L_{i k}^{j}+\delta^{j}{ }_{(i} \omega_{k)} .
$$

The equivalence class of auto-parallels defined by (23) defines a projective structure on $M_{D}$ [691], [690]. The particular set of connections

$$
{ }_{(p)} L_{i j}{ }^{k}=: L_{i j}{ }^{k}-\frac{2}{D+1} \delta_{(i}^{k} L_{j)}
$$

with $L_{j}=: L_{j m}^{m}$ is mapped into itself by the transformation (23), cf. [608].

In Section 2.2.3, we shall find the set of transformations $L_{i k}^{j} \rightarrow L_{i k}^{j}+\delta_{i}^{j} \frac{\partial \omega}{\partial x^{k}}$ playing a role in versions of Einstein's unified field theory.

From the connection $L_{i j}{ }^{k}$ further connections may be constructed by adding an arbitrary tensor field $T$ to it or to its symmetrized part:

$$
\begin{array}{r}
\bar{L}_{i j}{ }^{k}=L_{i j}{ }^{k}+T_{i j}{ }^{k}, \\
\overline{\bar{L}}_{i j}{ }^{k}=L_{(i j)}{ }^{k}+T_{i j}{ }^{k}=\Gamma_{i j}{ }^{k}+\tilde{T}_{i j} .
\end{array}
$$

By special choice of $\mathrm{T}$ or $\tilde{T}$ we can regain all connections used in work on unified field theories. One case is given by Schrödinger's "star"-connection:

$$
{ }^{*} L_{i j}{ }^{k}=L_{i j}{ }^{k}+\frac{2}{3} \delta_{i}^{k} S_{j},
$$

for which ${ }^{*} L_{i k}{ }^{k}={ }^{*} L_{k i}{ }^{k}$ or ${ }^{*} S_{i}=0$. The star connection thus shares the vanishing of the torsion vector with a symmetric connection. Further examples will be encountered in later sections; cf. (382) of Section 10.3.3.

\subsubsection{Metric compatibility, non-metricity}

We now assume that in affine space also a metric tensor exists. In the case of a symmetric connection the condition for metric compatibility reads:

$$
{ }^{\Gamma} \nabla_{k} g_{i j}=g_{i j, k}-g_{r j} \Gamma_{k i}^{r}-g_{i r} \Gamma_{k j}^{r}=0 .
$$

In Riemannian geometry this condition guaranties that lengths and angles are preserved under parallel transport. The corresponding torsionless connection ${ }^{23}$ is given by:

$$
\Gamma_{i j}^{k}=\left\{\begin{array}{l}
k \\
i j
\end{array}\right\}=\frac{1}{2} g^{k s}\left(\partial_{j} g_{s i}+\partial_{i} g_{s j}-\partial_{s} g_{i j}\right) .
$$

22 Many authors replace "auto-parallel" by "geodesic". We will reserve the name geodesic for curves of extreme length; cf. Riemannian geometry.

23 It sometime is named after the Italian mathematician T. Levi-Civita. 
In place of (28), for a non-symmetric connection the following equation was introduced by Einstein (and J. M. Thomas) (note the position of the indices!) ${ }^{24}$

$$
0=g_{i k-l} \| l=: g_{i k, l}-g_{r k} L_{i l}^{r}-g_{i r} L_{l k}^{r}
$$

As we have seen in Section 2.1.1, this amounts to the simultaneous use of two connections: $L_{i j}^{+}{ }^{k}=$ : $L_{(i j)}^{k}+S_{i j}{ }^{k}=L_{i j}{ }^{k}$ and $\bar{L}_{i j}{ }^{k}=: L_{(i j)}^{k}-S_{i j}{ }^{k}=L_{j i}{ }^{k}{ }^{25}$ We will name (30) "compatibility equation" although it has lost its geometrical meaning within Riemannian geometry. ${ }^{26}$ In terms of the covariant derivative with regard to the symmetric part of the connection, (30) reduces to

$$
0=g_{++-} \| \underset{0}{+g_{i k \| l}}-2 S_{(i|l| k)}+2 k_{r[i} S_{k] l}^{r} .
$$

In the 2nd term on the r.h.s., the upper index has been lowered with the symmetric part of the metric, i.e., with $h_{i j}$. After splitting the metric into its irreducible parts, we obtain ${ }^{27}$

$$
0=g_{+-\underline{k} \| l}=: h_{i k \| l}+k_{i k \| l}-2 S_{(i|l| k)}+2 k_{r[i} S_{k] l}^{r},
$$

or (cf. [632], p. 39, Eqs. (S1), (A1)):

$$
h_{i k \| l}+2 h_{r(i} S_{k) l}^{r}=0, \quad k_{i k \| l}+2 k_{r[i} S_{k] l}^{r}=0 .
$$

Eq. (32) plays an important role for the solution of the task to express the connection $L$ by the metric and its first partial derivatives. (cf. Section 10.2.3.)

In place of (30), equivalently, the \pm -derivative of the tensor density $\hat{g}^{i k}$ can be made to vanish:

$$
\hat{g}^{i k}{ }_{\| l}=\hat{g}_{, l}^{i k}+\hat{g}^{s k} L_{s l}^{i}+\hat{g}^{i s} L_{l s}^{k}-\hat{g}^{i k} L_{(l s)}^{s}=0 .
$$

From (30) or (33), the connection $L$ may in principle be determined as a functional of the metric tensor, its first derivatives, and of torsion. ${ }^{28}$ After multiplication with $\nu_{s}$, (33) can be rewritten as $\vec{\nabla}_{i} \vec{\nu}^{k}=g^{k s} \tilde{\nabla}_{i} \nu_{s}$, where $\tilde{\nabla}$ is formed with the Hermitian conjugate connection (cf. Section 2.2.2) $[396] .{ }^{29}$

\section{Remark:}

\footnotetext{
${ }^{24}$ In the notation used in Section 2.1.2 this is $g_{-\underset{+}{k} \| l}=0$.

${ }^{25}$ Santaló later called the expression "mixed covariant derivative" [524].

${ }^{26}$ M.-A. Tonnelat used the expression "equations de liaison" [641], p. 298 while B. Bertotti called (30) "the Christoffel relation" [25].

27 The corresponding Eq. (1) of [682], p. 382 is incorrect while its Eqs. (2), (3) correspond to the equations in (32).

28 Also, the relation $g^{r i}\left(g_{+} j \| l-\stackrel{L}{\nabla}_{l} g_{r j}\right)+2 L_{[k j]}^{l}=0$ trivially following from (30) was given a mathematical interpretation by F. Maurer-Tison [397]. M. Pastori suggested imaging torsion by parallelly propagating two vectors along each other on a two-sided (2-dimensional) surface with $L_{[k j]}^{l}$ on one side, and $L_{[j k]}^{l}$ on the other side ([485], p. 109/10.)

${ }^{29}$ In the derivative on the r.h.s., the minus-sign has been put underneath the nabla-sign in order of avoiding confusion with the tilde-sign above it.
} 
Although used often in research on UFT, the \pm -notation is clumsy and ambiguous. We apply the \pm -differentiation to (2), and obtain: $\left(g_{m i} g^{m j}\right)_{\| l}=g_{+-} \underset{+}{\| l} g^{m j}+g_{m i} g^{{ }^{+}-}{ }_{\| l}=\left(\delta_{i}^{j}\right)_{\| l}$. While the 1.h.s. of the last equation is well defined and must vanish by definition, the r.h.s. is ambiguous and does not vanish: in both cases $\delta_{\underline{i}}^{+}=-S_{i l}^{j} \neq 0, \delta_{i_{+}}^{{ }^{j}} \| l=S_{i l}^{j} \neq 0$. Einstein had noted this when

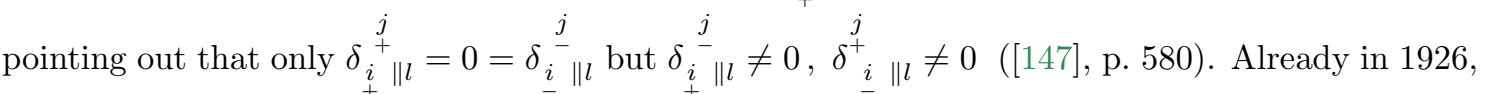
J. M. Thomas had seen the ambiguity of $\left(A_{i} B_{j}\right)_{\| l}$ and defined a procedure for keeping valid the product rule for derivatives [607]. Obviously, $\stackrel{0}{\nabla}_{k} \delta_{i}^{j}=0$.

A clearer presentation of (30) is given in Koszul-notation:

$$
\stackrel{ \pm}{\nabla}_{Z} g(X, Y):=Z g(X, Y)-g\left(\stackrel{+}{\nabla}_{Z} X, Y\right)-g\left(X, \bar{\nabla}_{Z} Y\right)
$$

The 1.h.s. of (34) is the non-metricity tensor, a straightforward generalization from Riemannian geometry:

$$
\stackrel{ \pm}{Q}(Z, X, Y):=\stackrel{ \pm}{\nabla}_{Z} g(X, Y)=Z^{l} X^{i} Y^{k} g_{i k-l}=-Z^{l} X^{i} Y^{k} \stackrel{ \pm}{Q_{l i k}} .
$$

(34) shows explicitly the occurrence of two connections; it also makes clear the multitude of choices for the non-metricity tensor and metric-compatibility. In principle, Einstein could have also used:

$$
\begin{gathered}
\stackrel{+}{\nabla}_{Z} g(X, Y):=Z g(X, Y)-g\left(\stackrel{+}{\nabla}_{Z} X, Y\right)-g\left(X, \stackrel{+}{\nabla}_{Z} Y\right), \\
\bar{\nabla}_{Z} g(X, Y):=Z g(X, Y)-g\left(\bar{\nabla}_{Z} X, Y\right)-g\left(X, \bar{\nabla}_{Z} Y\right), \\
\stackrel{00}{\nabla}_{Z} g(X, Y):=Z g(X, Y)-g\left(\stackrel{0}{\nabla}_{Z} X, Y\right)-g\left(X, \stackrel{0}{\nabla}_{Z} Y\right) .
\end{gathered}
$$

and further combinations of the 0 - and \pm -derivatives. His adoption of (30) follows from a symmetry demanded (Hermitian or transposition symmetry); cf. Section 2.2.2.

An attempt for keeping a property of the covariant derivative in Riemannian geometry, i.e., preservation of the inner product under parallel transport, has been made by J. Hély [249]. He

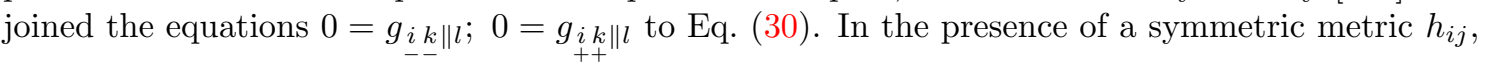
in place of Eqs. (25), (26) a decomposition

$$
L_{i j}{ }^{k}=\left\{\begin{array}{l}
k \\
i j
\end{array}\right)_{h}+u_{i j}{ }^{k}
$$

with arbitrary $u_{i j}{ }^{k}$ can be made. ${ }^{30}$ Hély's additional condition leads to a totally antisymmetric $u_{i j}{ }^{k}$.

We will encounter another object and its derivatives, the totally antisymmetric tensor:

$$
\epsilon_{i j k l}:=\sqrt{-g} \eta_{i j k l}, \quad \epsilon^{i j k l}:=(1 / \sqrt{-g}) \eta^{i j k l},
$$

${ }^{30}$ In Riemannian geometry, the decomposition holds:

$$
L_{i k}^{j}=\left\{{ }_{i k}^{j}\right\}_{g}+S_{i k}^{j}+S_{. i k}^{j}-S_{k \cdot i}^{j}+\frac{1}{2}\left(Q_{i k}^{j}-Q_{. i k}^{j}+Q_{k . i}^{j}\right),
$$

with torsion $S$ and non-metricity $Q$ ([540], p. 132). 
where $\eta^{i j k l}$ is the totally antisymmetric tensor density containing the entries $0, \pm 1$ according to whether two indices are equal, or all indices forming an even or odd permutation. For certain derivatives and connections, the object can be covariantly constant [473, 484]:

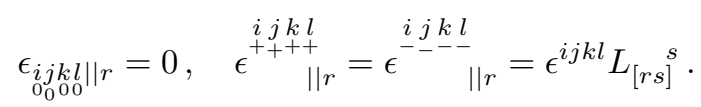

\subsection{Symmetries}

\subsubsection{Transformation with regard to a Lie group}

In Riemannian geometry, a "symmetry" of the metric with regard to a $C^{\infty}$-generator $X=\xi^{a} \frac{\partial}{\partial x^{a}}$ of a Lie algebra (corresponding, locally, to a Lie-group)

$$
\left[X_{(i)}, X_{(j)}\right]=c_{i j}{ }^{l} X_{(l)},
$$

is defined by

$$
\mathcal{L}_{\xi} g_{a b}=0=g_{a b, c} \xi^{c}+g_{c b} \xi_{, a}^{c}+g_{a c} \xi_{, b}^{c} .
$$

The vector field $\xi$ is named a Killing vector; its components generate the infinitesimal symmetry transformation: $x^{i} \rightarrow x^{i^{\prime}}=x^{i}+\xi^{i}$. Equation (43) may be expressed in a different form:

$$
\mathcal{L}_{\xi} g_{a b}=2 \stackrel{g}{\nabla}_{(a} \xi_{b)}=0 .
$$

In (44), $\stackrel{g}{\nabla}$ is the covariant derivative with respect to the metric $g_{a b}$ [Levi-Civita connection; cf. (29)]. A conformal Killing vector $\eta$ satisfies the equation:

$$
\mathcal{L}_{\eta} g_{a b}=f\left(x^{l}\right) g_{a b} .
$$

\subsubsection{Hermitian symmetry}

This is a generalization (a weakening) of the symmetrization of a real symmetric metric and connection: ${ }^{31}$ Hermitian "conjugate" metric and connection are introduced for a complex metric and connection by

$$
\tilde{g}_{i k}:=g_{k i} ; \tilde{L}_{i j}{ }^{k}:=L_{j i}{ }^{k} .
$$

In terms of the real tensors $h_{i k}, k_{i k}, L_{i j}{ }^{k}, S_{i j}{ }^{k}$, i.e., of $g_{i k}=h_{i k}+i k_{i k}, L_{i j}{ }^{k}=\Gamma_{i j}{ }^{k}+i S_{i j}{ }^{k}$ obviously $\tilde{g}_{i k}=\bar{g}_{i k}, \tilde{L}_{i j}{ }^{k}=\bar{L}_{i j}{ }^{k}$ holds, if the symmetry of $h_{i k}$ and the skew-symmetry of $k_{i k}$ are taken into account. For a real linear form $\omega_{i}:\left(\stackrel{+}{\nabla}_{k} \omega_{i}\right)^{\sim}=\bar{\nabla}_{i} \omega_{k}$. Hermitian symmetry then means that for both, metric and connection, $\tilde{g}_{i k}=g_{i k}, \tilde{L}_{i j}{ }^{k}:=L_{i j}{ }^{k}$ is valid. For the determinant $g$ of a metric with Hermitian symmetry, the relation $g=\bar{g}$ holds.

The property "Hermitian" (or "self-conjugate") can be generalized for any pair of adjacent indices of any tensor (cf. [149], p. 122):

$$
\tilde{A}_{\ldots i k \ldots}\left(g_{r s}\right):=A_{\ldots k i \ldots}\left(g_{s r}\right) .
$$

$\tilde{A}_{i j}$ is called the (Hermitian) conjugate tensor. A tensor possesses Hermitian symmetry if $\tilde{A}_{\ldots i k \ldots}\left(g_{r s}\right)=$ $A_{\ldots i k \ldots}\left(g_{r s}\right)$. Einstein calls a tensor anti-Hermitian if

$$
\tilde{A}_{\ldots i k \ldots}\left(g_{r s}\right):=A_{\ldots k i \ldots}\left(g_{s r}\right)=-A_{\ldots i k \ldots}\left(g_{r s}\right) .
$$

\footnotetext{
${ }^{31}$ In this section, the bar denotes complex conjugation.
} 
As an example for an anti-Hermitian vector we may take vector torsion $L_{i}=L_{[i l]}^{l}$ with $\tilde{L}_{i}=-L_{i}$. The compatibility equation (30) is Hermitian symmetric; this is the reason why Einstein chose it. For real fields, transposition symmetry replaces Hermitian symmetry.

$$
\tilde{g}_{i j}:=g_{j i} \stackrel{!}{=} g_{i j}, \tilde{L}_{i j}{ }^{k}:=L_{j i}{ }^{k} \stackrel{!}{=} L_{i j}{ }^{k},
$$

with $\tilde{A}_{i j}=A_{j i}$.

In place of (47), M.-A. Tonnelat used

$$
\tilde{A}_{\ldots i k \ldots}\left(L_{r s}^{t}\right):=A_{\ldots k i \ldots}\left(\tilde{L}_{r s}^{t}\right)
$$

as the definition of a Hermitian quantity [627]. As an application we find $\tilde{g}_{i k-}\left\|l=g_{\underline{i}++}\right\| l$ and $\hat{\hat{g}}^{i k-}{ }_{\| l}=\hat{g}^{-i k}{ }_{\mid l l}$.

\subsection{3 $\lambda$-transformation}

In (23) of Section 2.1.1, we noted that transformations of a symmetric connection $\Gamma_{i j}{ }^{k}$ which preserve auto-parallels are given by:

$$
{ }^{\prime} \Gamma_{i j}{ }^{k}=\Gamma_{i j}{ }^{k}+\lambda_{i} \delta_{j}^{k}+\lambda_{j} \delta_{i}^{k},
$$

where $\lambda_{i}$ is a real 1-form field. They were named projective by Schouten ([537], p. 287). In later versions of his UFT, Einstein introduced a "symmetry"-transformation called $\lambda$-transformation [156]:

$$
{ }^{\prime} \Gamma_{i j}{ }^{k}=\Gamma_{i j}{ }^{k}+\lambda_{j} \delta_{i}^{k} .
$$

Einstein named the combination of the "group" of general coordinate transformations and $\lambda$ transformations the "extended" group $U$. For an application cf. Section 9.3.1. After gauge- (YangMills-) theory had become fashionable, $\lambda$-transformations with $\lambda_{i}=\partial_{i} \lambda$ were also interpreted as gauge-transformations [702, 23]. According to him the parts of the connection irreducible with regard to diffeomorphisms are "mixed" by (52), apparently because both will then contain the 1 -form $\lambda_{i}$. Under (52) the torsion vector transforms like ' $S_{k}=S_{k}-\frac{3}{2} \lambda_{i}$, i.e., it can be made to vanish by a proper choice of $\lambda$.

The compatibility equation (30) is not conserved under $\lambda$-transformations because of $g_{i k \| l} \rightarrow$ $g_{i k \| l}-2 g_{i(k} \lambda_{l)}$. The same holds for the projective transformations (51), cf. ([430], p. 84). No generally accepted physical interpretation of the $\lambda$-transformations is known.

\subsection{Affine geometry}

We will speak of affine geometry in particular if only an affine connection exists on the 4-manifold, not a metric. Thus the concept of curvature is defined.

\subsubsection{Curvature}

In contrast to Section 2.1.3 of Part I, the two curvature tensors appearing there in Eqs. $(\mathrm{I}, 22)$ and $(\mathrm{I}, 23)$ will now be denoted by the \pm -sign written beneath a letter:

$$
\begin{aligned}
& K_{+}^{i}{ }_{j k l}=\partial_{k} L_{l j}{ }^{i}-\partial_{l} L_{k j}{ }^{i}+L_{k m}{ }^{i} L_{l j}{ }^{m}-L_{l m}{ }^{i} L_{k j}{ }^{m}, \\
& K^{i}{ }_{j k l}=\partial_{k} L_{j l}{ }^{i}-\partial_{l} L_{j k}{ }^{i}+L_{m k}{ }^{i} L_{j l}{ }^{m}-L_{m l}{ }^{i} L_{j k}{ }^{m} .
\end{aligned}
$$


Otherwise, this "minus"-sign and the sign for complex conjugation could be mixed up.

Trivially, for the index pair $j, k, \underline{-}_{j k l}^{i} \neq{\underset{+}{\tilde{K}}}_{j k l}^{i}$. The curvature tensors (53), (54) are skewsymmetric only in the second pair of indices. A tensor corresponding to the Ricci-tensor in Riemannian geometry is given by

$$
\underset{+}{K_{j k}}:=\underset{+}{K_{j k l}^{l}}=\partial_{k} L_{l j}{ }^{l}-\partial_{l} L_{k j}{ }^{l}+L_{k m}{ }^{l} L_{l j}{ }^{m}-L_{l m}{ }^{l} L_{k j}{ }^{m} .
$$

On the other hand,

$$
\underline{K}_{j k}:=\underline{-}_{j k l}^{l}=\partial_{k} L_{j l}^{l}-\partial_{l} L_{j k}^{l}+L_{m k}^{l} L_{j l}^{m}-L_{m l}^{l} L_{j k}^{m}
$$

Note that the Ricci tensors as defined by (55) or (56) need not be symmetric even if the connection is symmetric, and also that $\underline{-}_{j k} \neq \tilde{K}_{+}$jk when $\tilde{K}$ denotes the Hermitian (transposition) conjugate. Thus, in general

$$
\underline{K}_{[j k]}:=\partial_{[k} S_{j]}+\bar{\nabla}_{l} S_{k j}^{l}
$$

If the curvature tensor for the symmetric part of the connection is introduced by:

$$
\underset{0}{K_{j k l}^{i}}=\partial_{k} \Gamma_{l j}^{i}-\partial_{l} \Gamma_{k j}^{i}+\Gamma_{k m}^{i} \Gamma_{l j}^{m}-\Gamma_{l m}^{i} \Gamma_{k j}^{m}
$$

then

$$
\underline{K}^{i}{ }_{j k l}(L)=\underset{0}{K_{j k l}^{i}}(\Gamma)+S_{j l \| k}^{i}-S_{j k \| l}^{i}+S_{m k}^{i} S_{j l}^{m}-S_{m l}^{i} S_{j k}^{m} .
$$

The corresponding expression for the Ricci-tensor is:

$$
\underset{0}{K_{j k}}:=\underset{0}{K_{j k l}^{l}}=\partial_{k} \Gamma_{l j}^{l}-\partial_{l} \Gamma_{k j}^{l}+\Gamma_{k m}^{l} \Gamma_{l j}^{m}-\Gamma_{l m}^{l} \Gamma_{k j}^{m},
$$

whence follows:

$$
\underset{0}{K_{[j k]}}:=\partial_{[k} \Gamma_{j]}
$$

with $\Gamma_{k}=\Gamma_{k l}^{l}$. Also, the relations hold (for (63) cf. [549], Eq. (2,12), p. 278)):

$$
\begin{aligned}
& \underset{+}{K_{j k}}=\underset{0}{K_{j k}}+S_{j k \| l}^{l}-\underset{0}{l} S_{0}^{\| k}-S_{j k}^{m} S_{m}-S_{j l}^{m} S_{k m}^{l}, \\
& K_{-}{ }_{j k}=\underset{0}{K_{j k}}-S_{j k \| l}^{l}+\underset{0}{S_{0}^{\| k}}-S_{j k}^{m} S_{m}-S_{j l}{ }^{m} S_{k m}^{l} .
\end{aligned}
$$

A consequence of (62), (63) is:

$$
\underset{+}{K_{j k}}-\underset{-}{K_{j k}}=-2 S_{j \underset{0}{\| k}}+2 S_{j k}^{l} \underset{0}{l}, \underset{+}{K_{j k}}+\underset{-}{K_{j k}}=\underset{0}{2 K_{j k}}+2 S_{k m}{ }^{l} S_{l j}^{m}-2 S_{j k}^{m} S_{m} .
$$

Another trace of the curvature tensor exists, the so-called homothetic curvature ${ }^{32}$ :

$$
\underset{+}{V_{k l}}=\underset{+}{K_{j k l}^{j}}=\partial_{k} L_{l j}^{j}-\partial_{l} L_{k j}^{j}
$$

Likewise,

$$
\underline{-}_{k l}=\underline{-}_{j k l}^{j}=\partial_{k} L_{j l}^{j}-\partial_{l} L_{j k}^{j},
$$

\footnotetext{
32 In French also "courbure segmentaire".
} 
such that $\underline{-}_{k l}-V_{+} k l=2 \partial_{l} S_{k}-2 \partial_{k} S_{l}$. For the curvature tensor, the identities hold:

$$
\begin{array}{r}
\underline{K}^{i}{ }_{\{j k l\}}-2 \underline{-}_{\{j} S_{k l\}}{ }^{i}+4 S_{\{j k}{ }^{r} S_{l\} r}{ }^{i}=0, \\
\left.\underline{\nabla}_{\{k} \underline{K}^{i}|j| l m\right\} \\
{ }_{-j}+2 \underline{K}_{j r\{k}^{i} S_{l m\}}{ }^{r}=0 .
\end{array}
$$

where the bracket $\{\ldots\}$ denotes cyclic permutation while the index $|j|$ does not take part.

Equation (68) generalizes Bianchi's identity. Contraction on $i, j$ leads to:

$$
V_{j k}+2 K_{[j k]}=2 \nabla_{l} S_{j k}^{l}+4 S_{j k}^{r} S_{r}+4 \nabla_{[j} S_{k]}
$$

or for a symmetric connection (cf. Section 2.1.3.1 of Part I, Eq. (38)):

$$
V_{j k}+2 K_{[j k]}=0
$$

These identities are used either to build field equations without use of a variational principle, or for the identification of physical observables; cf. Section 9.7.

Finally, two curvature scalars can be formed:

$$
\underset{+}{K}=g^{i j} \underset{+}{K_{i j}}, \underset{-}{K}=g^{i j} \underline{-}_{i j}
$$

For a symmetric connection, an additional identity named after O. Veblen holds:

$$
\underset{0}{K_{j k l, m}^{i}}+\underset{0}{K^{i} l j m, k}+\underset{0}{K_{m l k, j}^{i}}+\underset{0}{K_{k m j, l}^{i}}=0
$$

The integrability condition for (30) is ([399], p. 225), [51]:

$$
g^{r i} R_{r l m}^{k}+g^{k r} R_{r l m}^{i}=0
$$

For a complete decomposition of the curvature tensor (53) into irreducible parts with regard to the permutation group further objects are needed, as e.g., $\epsilon^{a j k l}{ }_{+}^{K_{j k l}^{b}}=2 \epsilon^{a j k l} \partial_{[k} S_{l] j}^{b}$; cf. [348].

\subsubsection{A list of "Ricci"-tensors}

In many approaches to the field equations of UFT, a generalization of the Ricci scalar serves as a Lagrangian. Thus, the choice of the appropriate "Ricci" tensor plays a distinct role. As exemplified by Eq. (64), besides $\underset{+}{K_{j k}}$ and $\underline{-}_{j k}$ there exist many possibilities for building 2-rank tensors which could form a substitute for the unique Ricci-tensor of Riemannian geometry. In ([150], p. 142), Einstein gives a list of 4 tensors following from a "single contraction of the curvature tensor". Santalò derived an 8-parameter set of "Ricci"-type tensors constructed by help of $\underset{-}{K_{i k}}, S_{i k \| l}^{l}, \underline{-}_{i k}(\Gamma), S_{i \| k}, \quad S_{k \| i}, S_{i k}{ }^{m} S_{m}, S_{i} S_{k}, S_{i m}{ }^{l} S_{k l}{ }^{m}$ ([524], p. 345). He discusses seven of them used by Einstein, Tonnelat, and Winogradzki. ${ }^{33}$ The following collection contains a few examples of the objects used as a Ricci-tensor in variational principles/field equations of UFT besides $K_{i k}$ and $K_{i k}$ of the previous section. ${ }^{34}$ They all differ in terms built from torsion. Among

33 Santaló's covariant derivative denoted here with the star is just the regular covariant derivative with regard to

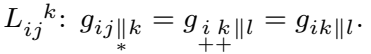

34 In the literature, notations differ from those given here, e.g., Winogradzki used $R_{j k}=-K_{j k}, \tilde{R}_{j k}=-K_{j k}$ [703]. Lichnerowicz had $P_{j k}=-K_{j k}$ and $P_{i k}^{*}=: E_{i k}$ etc. We try to indicate notational variations when necessary. 
them are:

$$
\begin{aligned}
& \stackrel{\mathrm{Her}}{K}_{i k}=-\frac{1}{2}\left(\underline{K}_{i k}+\underline{\tilde{K}}_{i k}\right)=P_{i k}
\end{aligned}
$$

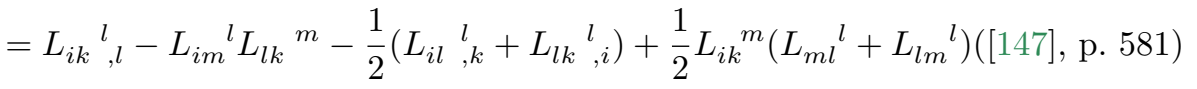

$$
\begin{aligned}
& =\frac{1}{2}\left(\underset{0}{K_{i k}}+\underset{0}{\tilde{K}_{i k}}\right)+\underset{\substack{i k \| l \\
\text { l }}}{l}+S_{i m}^{l} S_{k l}^{m} \\
& P_{i k}^{*}=L_{i k, l}{ }^{l}{ }^{2}-L_{i m}{ }^{l} L_{l k}{ }^{m}-\frac{1}{2}\left(L_{(i l), k}{ }^{l}, L_{(l k), i}{ }^{l}\right)+\frac{1}{2} L_{i k}{ }^{m}\left(L_{m l}{ }^{l}+L_{l m}{ }^{l}\right)([150], \text { p. 142) } \\
& =\stackrel{\mathrm{Her}}{K}_{i k}+S_{[i, k]}([371], \text { p. } 247-248)(76 \\
& { }^{(1)} R_{i k}=-K_{-} i k+\frac{2}{3}\left(\partial_{i} S_{k}-\partial_{k} S_{i}\right)([632], \text { p. } 129) \text {; } \\
& { }^{(2)} R_{i k}=\partial_{l} L_{i k}{ }^{l}-\partial_{k} L_{(i l)}{ }^{l}+L_{i k}{ }^{l} L_{(l m)}^{m}-L_{i m}{ }^{l} L_{l k}{ }^{m}+\frac{1}{3}\left(\partial_{i} S_{k}-\partial_{k} S_{i}\right)-\frac{1}{3} S_{i} S_{k}([632], \text { p. 129) } \\
& =-\underset{0}{K_{i k}}-\frac{2}{3} S_{\substack{[i \| k] \\
0}}+S_{i m}^{l} S_{k l}^{m}+\frac{1}{3} S_{i k}^{m} S_{m}-\frac{1}{3} S_{i} S_{k} \\
& { }^{(3)} R_{i k}={ }^{(2)} R_{i k}-\frac{1}{2} V_{+}{ },([632], \text { p. } 129) \text {; } \\
& U_{i k}=\stackrel{\text { Her }}{K}_{i k}-\frac{1}{3}\left[S_{i, k}-S_{k, i}+S_{i} S_{k}\right] \\
& =\underset{0}{K_{i k}}-S_{\substack{i k \| l \\
0}}^{l}+S_{i m}{ }^{l} S_{k l}{ }^{m}-\frac{2}{3} S_{[i \| k]}-\frac{2}{3} S_{i k}{ }^{m} S_{m}-\frac{1}{3} S_{i} S_{k},([151], \text { p. } 137 ; \\
& R_{i k}^{*}=-\underline{K}_{i k}+\stackrel{+}{\nabla}_{k} S_{i}=L_{i k}{ }^{l}, l-L_{i m}{ }^{l} L_{l k}{ }^{m}-L_{(i l), k}{ }^{l}+\frac{1}{2} L_{i k}{ }^{m}\left(L_{m l}{ }^{l}+L_{l m}{ }^{l}\right)([156], \text { p. 144) } \\
& R_{i k}^{* *}=R_{i k}^{*}-\left[(\log (\sqrt{-g}))_{, i}\right]_{\|_{-}}([156], \text { p. 144) }
\end{aligned}
$$

Further examples for Ricci-tensors are given in (475), (476) of Section 13.1.

One of the puzzles remaining in Einstein's research on UFT is his optimism in the search for a preferred Ricci-tensor although he had known, already in 1931, that presence of torsion makes the problem ambiguous, at best. At that time, he had found a totality of four possible field equations within his teleparallelism theory [176]. As the preceding list shows, now a 6-parameter object could be formed. The additional symmetries without physical support suggested by Einstein did not help. Possibly, he was too much influenced by the quasi-uniqueness of his field equations for the gravitational field.

\subsubsection{Curvature and scalar densities}

From the expressions $(73)$ to $(81)$ we can form scalar densities of the type: $\hat{g}^{i k} \stackrel{\text { Her }}{K}_{i k}$ to $\hat{g}^{i k} U_{i k}$ etc. As the preceding formulas show, it would be sufficient to just pick $\hat{g}^{i k} K_{i k}$ and add scalar densities built from homothetic curvature, torsion and its first derivatives in order to form a most general Lagrangian. As will be discussed in Section 19.1.1, this would draw criticism to the extent that such a theory does not qualify as a unified field theory in a stronger sense. 


\subsubsection{Curvature and $\lambda$-transformation}

The effect of a $\lambda$-transformation (52) on the curvature tensor $K_{-}^{i}{ }_{j k l}$ is:

$$
\underline{-}_{j k l}^{i} \rightarrow \underline{-}_{j k l}^{i}+2 \partial_{[k} \lambda_{l]} \delta_{j}^{i}
$$

In case the curvature tensor ${\underset{+}{i k l}}_{j k l}$ is used, instead of (52) we must take the form for the $\lambda$ transformation: ${ }^{35}$

$$
{ }^{\prime} \Gamma_{i j}{ }^{k}=\Gamma_{i j}{ }^{k}+\lambda_{i} \delta_{j}{ }^{k}
$$

Then

$$
\underset{+}{K_{j k l}^{i}} \rightarrow \underset{+}{K_{j k l}^{i}}+2 \partial_{[k} \lambda_{l]} \delta_{j}^{i}
$$

also holds. Application of (52) to ${\underset{+}{+}}_{j k l}^{i}$, or (85) to $\underline{-}_{j k l}^{i}$ results in many more terms in $\lambda_{k}$ on the r.h.s. For the contracted curvatures a $\lambda$-transformation leads to (cf. also [430]):

$$
\underset{-}{K_{j k}} \rightarrow \underset{-}{K_{j k}}-2 \partial_{[k} \lambda_{j]}, \underset{+}{V_{j k}} \rightarrow \underset{+}{V_{j k}}+2 \partial_{[j} \lambda_{k]}, \quad \underline{-}_{j k} \rightarrow \underline{-}_{j k}+8 \partial_{[j} \lambda_{k]} .
$$

If $\lambda_{i}=\partial_{i} \lambda$, the curvature tensors and their traces are invariant with regard to the $\lambda$-transformations of Eq. (52). Occasionally, ' $\Gamma_{i j}{ }^{k}=\Gamma_{i j}{ }^{k}+\left(\partial_{i} \lambda\right) \delta_{j}{ }^{k}$ is interpreted as a gravitational gauge transformation.

\subsection{Differential forms}

In this section, we repeat and slightly extend the material of Section 2.1.4, Part I, concerning Cartan's one-form formalism in order to make understandable part of the literature. Cartan introduced one-forms $\theta^{\hat{a}}(\hat{a}=1, \ldots, 4)$ by $\theta^{\hat{a}}:=h_{l}^{\hat{a}} d x^{l}$. The reciprocal basis in tangent space is given by $e_{\hat{\jmath}}=h_{\hat{\jmath}}^{l} \frac{\partial}{\partial x^{l}}$. Thus, $\theta^{\hat{a}}\left(e_{\hat{\jmath}}\right)=\delta_{\hat{\jmath}}^{\hat{a}}$. An antisymmetric, distributive and associative product, the external or "wedge" $(\wedge)$-product is defined for differential forms. Likewise, an external derivative $d$ can be introduced. ${ }^{36}$ The metric (e.g., of space-time) is given by $\eta_{\hat{\imath} \hat{k}} \theta^{\hat{\imath}} \otimes \theta^{\hat{k}}$, or $g_{l m}=\eta_{\hat{\imath} \hat{k}} h_{l}^{\hat{i}} h_{m}^{\hat{k}}$. The covariant derivative of a tangent vector with bein-components $X^{\hat{k}}$ is defined via Cartan's first structure equations,

$$
\Theta^{i}:=D \theta^{\hat{\imath}}=d \theta^{\hat{\imath}}+\omega^{\hat{\imath}} \wedge \theta^{\hat{l}},
$$

where $\omega_{\hat{k}}^{\hat{\imath}}$ is the connection-1-form, and $\Theta^{\hat{\imath}}$ is the torsion-2-form, $\Theta^{\hat{\imath}}=-S_{\hat{l} \hat{m}}{ }^{\hat{\imath}} \theta^{\hat{l}} \wedge \theta^{\hat{m}}$. We have $\omega_{\hat{\imath} \hat{k}}=-\omega_{\hat{k} \hat{\imath}}$. The link to the components $L_{[i j]}^{k}$ of the affine connection is given by $\omega_{\hat{k}}^{\hat{\imath}}=$ $h_{l}^{\hat{\imath}} h_{\hat{k}}^{m} L_{\hat{r} m}^{l} \theta^{\hat{r} 37}$. The covariant derivative of a tangent vector with bein-components $X^{\hat{k}}$ then is

$$
D X^{\hat{k}}:=d X^{\hat{k}}+\omega^{\hat{k}} X^{\hat{l}}
$$

By further external derivation on $\Theta$ we arrive at the second structure relation of Cartan,

$$
D \Theta^{\hat{k}}=\Omega_{\hat{l}}^{\hat{k}} \wedge \theta^{\hat{l}} .
$$

35 J. Winogradzki calls (85) a "त̃-transformation" ([703], p. 442).

36 The external derivative $d$ of linear forms $\omega, \mu$ satisfies the following rules:

(1) $d(a \omega+b \mu)=a d \omega+b d \mu$,

(2) $d(\omega \wedge \mu)=d \omega \wedge \mu-\omega \wedge d \mu$,

(3) $d d \omega=0$.

37 For an asymmetric connection, this corresponds to the + derivative. 
In Eq. (90) the curvature-2-form $\Omega_{\hat{l}}^{\hat{k}}=\frac{1}{2} R_{\hat{l} \hat{m} \hat{n}}^{\hat{k}} \theta^{\hat{m}} \wedge \theta^{\hat{n}}$ appears, which is given by

$$
\Omega_{\hat{l}}^{\hat{k}}=d \omega_{\hat{l}}^{\hat{k}}+\omega_{\hat{l}}^{\hat{k}} \wedge \omega_{\hat{l}}^{\hat{k}}
$$

$\Omega^{\hat{k}}$ is the homothetic curvature.

${ }^{k} \mathrm{~A}$ p-form in $\mathrm{n}$-dimensional space is defined by

$$
\omega=\omega_{\hat{i}_{1} \hat{i}_{2} \ldots \hat{i}_{p}} d x^{\hat{i}_{1}} \wedge d x^{\hat{i}_{2}} \wedge \cdots \wedge d x^{\hat{i}_{p}}
$$

and, by help of the so-called Hodge ${ }^{*}$-operator, is related to an (n-p)-form $)^{38}$

$$
\stackrel{*}{\omega}:=\frac{1}{(n-p) !} \epsilon_{\hat{k}_{1} \hat{k}_{2} \ldots \hat{k}_{n-p}}^{\hat{i}_{1} \hat{i}_{2} \ldots \hat{i}_{p}} \omega_{\hat{i}_{1} \hat{i}_{2} \ldots \hat{i}_{p}} d x^{\hat{k}_{1}} \wedge d x^{\hat{k}_{2}} \wedge \cdots \wedge d x^{\hat{k}_{n-p}} .
$$

\subsection{Classification of geometries}

A differentiable manifold with an affine structure is called affine geometry. If both, a (possibly non-symmetric) "metric" and an affine structure, are present we name the geometry "mixed". A subcase, i.e., metric-affine geometry demands for a symmetric metric. When interpreted just as a gravitational theory, it sometimes is called MAG. A further subdivision derives from the nonmetricity tensor being zero or $\neq 0$. Riemann-Cartan geometry is the special case of metric-affine geometry with vanishing non-metricity tensor and non-vanishing torsion. Weyl's geometry had non-vanishing non-metricity tensor but vanishing torsion. In Sections 2.1.3 and 4.1.1 of Part I, these geometries were described in greater detail.

\subsubsection{Generalized Riemann-Cartan geometry}

For the geometrization of the long-range fields, various geometric frameworks have been chosen. Spaces with a connection depending solely on a metric as in Riemannian geometry rarely have been considered in UFT. One example is given by Hattori's connection, in which both the symmetric and the skew part of the asymmetric metric enter the connection ${ }^{39}$ [240]:

$$
\begin{aligned}
{ }^{H} L_{i j}{ }^{k} & =1 / 2 h^{k l}\left(g_{l i, j}+g_{j l, i}-g_{j i, l}\right) \\
& =\left\{\begin{array}{l}
k \\
i j
\end{array}\right\}_{h}+1 / 2 h^{k l}\left(k_{l i, j}+k_{j l, i}+k_{i j, l}\right),
\end{aligned}
$$

where $h^{k l}$ is the inverse of $h_{k l}=g_{(k l)}$. As described in Section 6.2 of Part I, its physical content is dubious. As the torsion tensor does not vanish, in general, i.e.,

$$
{ }^{H} S_{i j}{ }^{k}=h^{k l}\left(k_{l[i, j]}+1 / 2 k_{i j, l}\right)
$$

this geometry could be classified as generalized Riemann-Cartan geometry.

\subsubsection{Mixed geometry}

Now, further scalars and scalar densities may be constructed, among them curvature scalars (Ricciscalars):

$$
\begin{aligned}
& \underset{+}{K}:=g^{j k} \underline{+}_{j k}=l^{j k}{ }_{+}^{K_{(j k)}}+m^{j k}{ }_{+}^{K_{[j k]}}, \\
& \underline{K}:=g^{j k} \underline{K}_{j k}:=l^{j k} \underline{K}_{(j k)}+m^{j k} \underline{K}_{[j k]} .
\end{aligned}
$$

\footnotetext{
38 Indices are moved with the Minkowski metric $\eta$, except for the totally antisymmetric $\epsilon_{\alpha \beta \gamma \delta}$. Here, both $\epsilon_{0123}=1$ and $\epsilon^{0123}=1$ hold, hence $\epsilon^{\alpha \beta \gamma \delta} \neq \eta^{\alpha \kappa} \eta^{\beta \lambda} \eta^{\gamma \mu} \eta^{\delta \nu} \epsilon_{\kappa \lambda \mu \nu}$; moving of indices would lead to $\epsilon^{0123}=-1$.

${ }^{39}$ Unlike in (92), Hattori used $h_{\lambda \mu}$ to denote the asymmetric metric and $g_{\alpha \beta}$ for its symmetric part.
} 
Here, $l^{j k}$ and $m^{j k}$ come from the decomposition into irreducible parts of the inverse of the nonsymmetric metric $g_{j k}$. Both parts on the r.h.s. could be taken as a Lagrangian, separately. The inverse $h^{j k}$ of the symmetric part, i.e., of $g_{(j k)}=h_{j k}$ could be used as well to build a scalar: $h^{j k} K_{+}(j k)$. Mixed geometry is the one richest in geometrical objects to be constructed from the asymmetric metric and the asymmetric connection. What at first may have appeared as an advantage, turned out to become an 'embarras de richesses': defining relations among geometric objects and physical observables abound; cf. Section 9.7 .

Whenever a symmetric tensor appears which is independent of the connection and of full rank, it can play the role of a metric. The geometry then may be considered to be a Riemannian geometry with additional geometric objects: torsion tensor, non-metricity tensor, skew-symmetric part of the "metric" etc. These might be related to physical observables. Therefore, it is moot to believe that two theories are different solely on the basis of the criterion that they can be interpreted either in a background of Riemannian or mixed geometry. However, by a reduction of the more general geometries to a mere Riemannian one plus some additional geometric objects the very spirit of UFT as understood by Einstein would become deformed; UFT explicitly looks for fundamental geometric objects representing the various physical fields to be described.

\subsubsection{Conformal geometry}

This is an "angle preserving" geometry: in place of a metric $g_{i j}\left(x^{m}\right)$ a whole equivalence class $\gamma_{i j}\left(x^{l}\right)=\rho^{2}\left(x^{n}\right) g_{i j}\left(x^{m}\right)$ with a function $\rho\left(x^{n}\right)$ obtains. Geometrical objects of interest are those invariant with regard to the transformation: $g_{i j}\left(x^{m}\right) \rightarrow \bar{g}_{i j}=\rho^{2}\left(x^{n}\right) g_{i j}\left(x^{m}\right)$. One such object is Weyl's conformal curvature tensor:

$$
C_{j k l}^{i}:=\underset{+}{K_{j k l}^{i}}-\frac{1}{n-2}\left(\delta_{k}^{i} K_{+}{ }_{j l}-\delta_{l}^{i} \underset{+}{K_{j k}}+g_{j l}{\underset{+}{K}{ }_{k}}_{i}-g_{j k}{\underset{+}{K_{l}}}_{i}^{i}\right)+\frac{1}{(n-1)(n-2)} \underset{+}{K}\left(\delta_{l}^{i} g_{j k}-\delta_{k}^{i} g_{j l}\right),
$$

where $n$ is the dimension of the manifold ( $n=4$ : space-time). $C_{j k l}^{i}$ is trace-free. For $n>$ $3, C_{j k l}^{i}=0$ is a necessary and sufficient condition that the space is conformally flat, i.e., $\gamma_{i j}\left(x^{l}\right)=$ $\rho^{2}\left(x^{n}\right) \eta_{i j}\left(x^{m}\right)([191]$, p. 92$)$.

If $\xi^{k}$ is a Killing vector field for $g_{i j}$, then $\xi^{k}$ is a conformal Killing vector field for $\bar{g}_{i j}$; cf. Eq. (45) in Section 2.1.2.

A particular sub-case of conformal geometry is "similarity geometry", for which the restricted group of transformations acts $g_{i j}\left(x^{m}\right) \rightarrow \gamma_{i j}\left(x^{l}\right)=k^{2} g_{i j}\left(x^{m}\right)$, with a constant $k$, cf. Section 3.1.

\subsection{Number fields}

In Section 2.3 of Part I, the possibility of choosing number fields different from the real numbers for the field variables was stated. Such field variables then would act in a manifold with real coordinates. A more deeply going change is the move to an underlying manifold with coordinates taken from another number field, e.g., complex spaces. The complex number field was most often used in connection with unified field theory in both roles. cf. A. Einstein, (complex space, Section 7.2), J. Moffat, (complex field on real space, Section 13) and A. Crumeyrolle, (hypercomplex manifold, Section 11.2.2).

As hypercomplex numbers are less well known, we briefly introduce them here. Let $z=$ $x+\epsilon y, x, y$ real and consider the algebra with two elements $I, \epsilon$, where $I$ is the unit element and $\epsilon^{2}=I$. $z$ is called a hypercomplex number. A function $f(z)=P(x, y)+\epsilon Q(x, y)$ will be differentiable in $z$ if

$$
\frac{\partial P}{\partial x}=\frac{\partial Q}{\partial y}, \quad \frac{\partial P}{\partial y}=\frac{\partial Q}{\partial x}
$$


The product of two identical real manifolds of dimension $\mathrm{n}$ can be made into a manifold with hypercomplex structure.

\section{Interlude: Meanderings - UFT in the late 1930s and the 1940s}

Prior to a discussion of the main research groups concerned with Einstein-Schrödinger theories, some approaches using the ideas of Kaluza and Klein for a unified field theory, or aspiring to bind together quantum theory and gravitation are discussed.

\subsection{Projective and conformal relativity theory}

Projective relativity theory had been invented expressly in order to avoid the fifth dimension of Kaluza-Klein theory. In Sections 6.3.2 and 7.2.4 of Part I, Pauli \& Solomon's paper was described. Also, in Section 6.3.2 of Part I, we briefly have discussed what O. Veblen \& B. Hoffmann called "projective relativity" [671], and the relationship to the Einstein-Mayer theory. Veblen \& Hoffmann had introduced projective tensors with components $T_{\sigma \ldots \tau}^{\alpha \ldots \beta}=\exp \left(N x^{0}\right) f_{\sigma \ldots \tau}^{\alpha \ldots \beta}\left(x^{1}, x^{2}, x^{3}, x^{4}\right)$ where $x^{1}, \ldots x^{4}$ are coordinates of space-time, $x^{0}$ is an additional parameter (a gauge variable) and $N$ a constant named "index". ${ }^{40} \quad x^{0}$ transforms as $x^{\prime 0}=x^{0}+\log \rho\left(x^{\alpha}\right)$. The auxiliary 5dimensional space appearing has no physical significance. A projective symmetric metric $G_{\alpha \beta}$ of index $2 N$ was given by $G_{\alpha \beta}=\Phi^{2} \gamma_{\alpha \beta}$ where $\Phi$ is an arbitrary projective scalar of index $N$. In addition, a third symmetric tensor $g_{\alpha \beta}=\gamma_{\alpha \beta}-\phi_{\alpha} \phi_{\beta}$, the gravitational metric, appeared. Here, $\phi_{\alpha}:=\gamma_{\alpha 0}$ is a projective vector. Likewise, the Levi-Civita connections $\Pi_{\alpha \beta}^{\delta}, \Gamma_{\alpha \beta}^{\delta}$ with $\Pi_{\alpha \beta}^{\delta}\left(G_{\sigma \tau}\right)=\Gamma_{\alpha \beta}^{\delta}\left(\gamma_{\sigma \tau}\right)+N\left(\delta_{\alpha}^{\delta} \Phi_{\beta}+\delta_{\beta}^{\delta} \Phi_{\alpha}-\gamma_{\alpha \beta} \Phi^{\delta}\right)$ with $\Phi_{\alpha}=\frac{1}{N} \frac{\partial \log \Phi}{\partial x^{\alpha}}$ and $\Phi^{\delta}=\gamma^{\delta \sigma} \Phi_{\sigma}$ were used. For arbitrary index $N$, the field equations were derived from the curvature scalar $P$ calculated from the connection $\Pi_{\alpha \beta}{ }_{\delta}^{\delta}\left(G_{\sigma \tau}\right)$. One equation could be written in the form of a wave equation:

$$
\frac{1}{\sqrt{-g}} \frac{\partial}{\partial x^{\sigma}}\left(\sqrt{-g} \gamma^{\sigma \tau} \frac{\partial \psi}{\partial x^{\tau}}\right)+\frac{5}{27}\left(R-3 N^{2}\right) \psi=0,
$$

where $R$ is the curvature scalar calculated from $g_{i j}$. Veblen \& Hoffmann concluded that: "The use of projective tensors and projective geometry in relativity theory therefore seems to make it possible to bring wave mechanics into the relativity scheme" ([671], abstract). How Planck's constant might be brought in, is left in the dark.

During the 1940s, meson physics became fashionable. Of course, the overwhelming amount of this research happened in connection with nuclear and elementary particle theory, outside of UFT, but sometimes also in classical field theory. Cf. the papers by F. J. Belinfante on the meson field, in which he used the undor-formalism ${ }^{41}[16,15]$. In his doctoral thesis of 1941, "Projective theory of meson fields and electromagnetic properties of atomic nuclei" suggested by L. Rosenfeld, Abraham Pais in Utrecht kept away from UFT and calculated the projective energy momentum tensor of an arbitrary field. Although citing the paper of Veblen and Hoffmann, in projective theory he followed the formalism of Pauli; in his application to the Dirac spinor-field, he used Belinfantes undors [466]. After this paper, he examined which of Kemmer's five types of meson fields were "in accordance with the requirements of projective relativity" ([467], p. 268).

\footnotetext{
${ }^{40} x^{0}$ can be interpreted as parametrizing a curve in a 5 -dimensional space; $\alpha, \ldots, \tau$ run through $0,1,2,3,4 ; i, j$ from 1 to 4 . The component $T_{00}$ of any projective tensor $T_{\alpha \beta}$ is a projective scalar.

41 "Undors of rank N" are quantities with $4^{N}$ components (in space-time) transforming like the products of N 4-component Dirac-spinors.
} 
It is unsurprising that B. Hoffmann in Princeton also applied the projective formalism to a theory intended to unify the gravitational and vector meson fields [278]. The meson field $\theta_{\alpha}$ was defined by Hoffmann via: $\theta_{\alpha}=\phi_{\alpha}-\Phi_{\alpha}$ with $\phi_{\alpha}=\gamma_{0 \alpha}$ and $\Phi_{\alpha}$ given above. Its space-time components $\theta_{j}$ form an affine vector from which the vector meson field tensor $\theta_{i j}=\frac{1}{2}\left(\frac{\partial \theta_{i}}{\partial x^{j}}-\frac{\partial \theta_{j}}{\partial x^{i}}\right)$ follows. The theory again contained three Riemannian curvature tensors (scalars). By skipping all calculations, we arrive at the affine form of Hoffmann's field equations

$$
\begin{aligned}
R^{i j}-\frac{1}{2} g^{i j}\left(R+12 N^{2}\right)+\frac{1}{2}\left(g^{r s} \theta_{r}^{i} \theta^{j}{ }_{s}+\frac{1}{4} g^{i j} \theta_{r}^{s} \theta^{r}{ }_{s}\right)-12 N^{2}\left(\theta^{i} \theta^{j}-\frac{1}{2} g^{i j} \theta^{s} \theta_{s}\right) & =0, \\
\theta^{i j}{ }_{j}+12 N^{2} \theta^{i} & =0 .
\end{aligned}
$$

In Hoffmann's words: "except for the term $-6 N^{2} g^{i j}$, these are the classical (i.e. unquantized) field equations for a vector meson and gravitational field in the general theory of relativity" ([278], p. 464). We could name them as well "Einstein-meson" equations in analogy to "Einstein-Maxwell" equations: no unification of both field had been reached. Also, no scalar meson field and the electromagnetic field were present in the theory.

Hoffmann then looked for a "broader geometrical base" than projective geometry in order to include the electromagnetic field. He found it in conformal geometry, or rather in a special subcase, similarity geometry [279]. ${ }^{42}$ It turned out that a 6 -dimensional auxiliary space was needed. We shall denote the coordinates in this $R^{6}$ by $A, B=0,1,2,3,4,5$. The components of a similarity tensor are $S_{\sigma \ldots \tau}^{\alpha \ldots \beta}=\exp \left((p-q) N x^{0}\right) f_{\sigma \ldots \tau}^{\alpha \ldots \beta}\left(x^{\alpha}\right)$, where $p, q$ are the number of covariant and contravariant coordinate indices while $N$ again is named the index of the tensor. In place of the transformations in projective geometry, now

$$
x^{0} \rightarrow x^{0}+\frac{1}{N} \log k, x^{j} \rightarrow x^{\prime j}\left(x^{k}\right), x^{5} \rightarrow \frac{1}{k^{2}} x^{5}
$$

hold. A symmetric tensor $S_{A B}$ in $R^{6}$ was given the role of metric; the assumptions $S_{05}=0$, and $\frac{S_{55}}{S_{00}}$ independent of $x^{\alpha}$ reduced the number of free functions. The definitions $s_{A B}:=\frac{S_{A B}}{S_{00}}$ and $\gamma_{A B}:=s_{A B}-s_{A 5} s_{B 5} / s_{55}, \gamma_{05}=0, \gamma_{00}=1$ led back to the former vector meson field via $\gamma_{j 0}$ and to a vector in $R^{6} \psi_{A}:=\frac{s_{A 5}}{\sqrt{s_{55}}}$ with $\psi_{0}=0, \psi_{5}$ independent of $x^{\alpha}$ and containing the electromagnetic 4 -vector $\psi_{j}$. To abreviate the story, Hoffmann's final field equations in space-time were:

$$
\begin{aligned}
R^{i j}-\frac{1}{2} g^{i j} R+2\left(g^{r s} \psi_{r}^{i} \psi_{s}^{j}+\frac{1}{4} g^{i j} \psi_{r}^{s} \psi_{s}^{r}\right)+\frac{1}{2}\left(g^{r s} \theta_{r}^{i} \theta^{j}{ }_{s}+\frac{1}{4} g^{i j} \theta_{r}^{s} \theta^{r}{ }_{s}\right) & \\
-20 N^{2}\left(\theta^{i} \theta^{j}-\frac{1}{2} g^{i j} \theta^{s} \theta_{s}\right) & =0, \\
\theta^{i j}{ }_{j}+20 N^{2} \theta^{i} & =0, \\
\psi^{i r} & =0 .
\end{aligned}
$$

The last equation with $\psi_{i j}=\frac{1}{2}\left(\frac{\partial \psi_{i}}{\partial x^{j}}-\frac{\partial \psi_{j}}{\partial x^{i}}\right)$ reproduced Maxwell's equations. In a sequel to this paper, Hoffmann claimed to have derived "the correct trajectories of charged meson testparticles in a combined gravitational, electromagnetic, and vector meson field" ([280], p. 1045).

\subsubsection{Geometrical approach}

It was Pascual Jordan ${ }^{43}$ who in physics re-applied projective geometry (cf. Section 2.1.3.3 of Part I) by showing that the transformation group $G_{5}$ of the 4-potential $A_{k}$ in electrodynamics, composed

42 O. Veblen had previously prepared the mathematical formalism for B. Hoffmann [670].

43 Pascual Jordan (1902-1980) was the only pioneer of quantum (matrix-) mechanics and quantum field theory who was not awarded a Nobel Prize unlike M. Born, W. Heisenberg and P. A. M. Dirac. After having been an 
of the gauge transformations

$$
A_{k^{\prime}}=A_{k}+\partial_{k} \chi, \quad x^{i^{\prime}}=x^{i}
$$

and coordinate transformations

$$
A_{k^{\prime}}=A_{s} \frac{\partial x^{s}}{\partial x^{k^{\prime}}}, x^{i^{\prime}}=f^{i}\left(x^{0}, \ldots, x^{3}\right)(k, s, i=0,1, \ldots, 3)
$$

in space-time, is isomorphic to the group of homogeneous transformations in five variables $X^{0}, X^{1}, \ldots, X^{4}[316]$ :

$$
X^{\prime \alpha}=X^{\alpha} F^{\alpha}\left(\frac{X^{\prime} 0}{X^{\prime 4}}, \frac{X^{\prime} 1}{X^{\prime 4}}, \frac{X^{\prime 2}}{X^{\prime 4}}, \frac{X^{\prime} 3}{X^{\prime 4}}\right),
$$

(no summation over $\alpha$ on the r.h.s.). ${ }^{44}$ Equivalently, the new coordinates $X^{\prime} \alpha$ are homogeneous functions of degree 1 of the old $X^{\alpha}$ and transform like a vector:

$$
X^{\prime \alpha}=X^{\rho} \frac{\partial X^{\prime \alpha}}{\partial X^{\rho}} \quad(\alpha, \rho=0,1, \ldots, 4) .
$$

For the coordinates $x^{i}$ of space-time, alternatively we may write $x^{i}=f^{i}\left(X^{0}, \ldots, X^{4}\right)$ or $\frac{\partial x^{i}}{\partial X^{\rho}} X^{\rho}=$ 0 . Jordan defined projector-components $P_{\alpha_{1}, \ldots, \alpha_{m}}^{\beta_{1}, \ldots, \beta_{n}}$ to transform under (106) like tensor-components $T_{\alpha_{1}, \ldots, \alpha_{m}}^{\beta_{1}, \ldots, \beta_{n}}$ which are homogeneous functions of degree $(n-m)$ in the $X^{\rho}$. Thus, $X^{\rho}$ itself is a projector just as the Minkowski (Euclidean) metric $g_{\alpha \beta}$ of $V_{5}$ with the invariant:

$$
J=g_{\alpha \beta} X^{\alpha} X^{\beta}
$$

Jordan introduced also a covariant derivative in $V_{5}$. The formalism is described in papers and his book [317, 319, 320]; a detailed presentation is given by G. Ludwig [384]. More generally, if $V_{5}$ is provided with a non-flat metric $g_{r s}$, the curvature scalar plays a prominent role in the derivation of the field equations within projective relativity. Ludwig also introduced arbitrary matter fields. At first, his Lagrangian for a scalar matter field $\psi$ within projective geometry was [383]

$$
L=\frac{1}{2}\left[a(J) \psi_{, \nu} \psi_{, \mu} g^{\mu \nu}+b(J) \psi^{2}\right]
$$

but then became generalized to

$$
L=U(J)\left[\stackrel{5}{R}+W(J) \psi_{, \nu} \psi_{, \mu} g^{\mu \nu}+V(J)\right] .
$$

To obtain the Lagrangian for the metrical field, $\psi$ was replaced by $J$ ([384], p. 57):

$$
L=U(J)\left[\stackrel{5}{R}+W(J) J_{, \nu} J_{, \mu} g^{\mu \nu}+V(J)\right]
$$

With

$$
{ }^{(5)} R={ }^{(4)} R+\frac{1}{4} J F_{r s} F^{r s}+\frac{2}{\sqrt{J} \sqrt{-g}} \partial_{r}\left(\sqrt{-g} g^{r s} \frac{\partial \sqrt{J}}{\partial x_{s}}\right) \text {. }
$$

assistant of R. Courant and M. Born in Göttingen and lecturer in Hamburg, in 1929 he became professor in Rostock; in 1944 he succeeded M. v. Laue at the (now Humboldt) University of Berlin. Due to his intellectual support of the Nazi-movement, after the second world war he had to wait until 1953 before again becoming full professor at the university of Hamburg. Apart from theoretical physics, Jordan also contributed to mathematics (Jordan algebras) and, less successfully, to biology and geology.

${ }^{44}$ Jordan denoted the 5 th coordinate by $x^{0}$, the time coordinate of space-time by $x^{4}$. 
we arrive at:

$$
L=U(J)\left[\stackrel{4}{R}+\frac{1}{4} J F_{r s} F^{r s}+J^{-1} g^{r s} \stackrel{4}{\nabla}_{r} \partial_{s} J+\left(W(J)-\frac{1}{2} J^{-2}\right) J_{, \nu} J_{, \mu} g^{\mu \nu}+V(J)\right],
$$

where $U(J), V(J), W(J)$ are arbitrary functions. As can be seen from (110), the 5-dimensional curvature scalar used by Jordan and by Thiry (cf. the next Section 3.1.2) follows as the subcase $U(J)=J^{-1 / 2}, W(J)=V(J)=0$ of the general expression (111). Ludwig, at the time of writing the preface to his book, e.g., in May 1951, seemingly did not know of Thiry's paper of 1948 [604] nor of his $\mathrm{PhD}$ thesis published also in 1951: in his bibliography Thiry's name and paper are missing.

Pauli had browsed in Ludwig's book and now distanced himself from his own papers on projective relativity of 1933 discussed briefly in Section 7.2.4 of Part I. ${ }^{45}$ He felt deceived:

"The deception consists in the belief that by the projective form, i.e., the homogeneous coordinates, the shortcomings of Kaluza's formulation have been repaired, and that one has achieved something beyond Kaluza. At the time, in 1933, I did not know explicitly the transition from Kaluza to the projective form (as in [20]); it is too simple and banal to the effect that the factual contents of both equivalent formulations could be somehow different." (letter of W. P. to P. Jordan, [490], p. 735): ${ }^{46}$

\subsubsection{Physical approach: Scalar-tensor theory}

Toward the end of the second world war, Kaluza's five- dimensional theory and projective relativity emerged once again as vehicles for a new physical theory which, much later, came to be known as "scalar-tensor theory of gravitation." ${ }^{47}$ Cosmological considerations related to the origin of stars seem to have played the major role for the building of a theory by P. Jordan in which the gravitational constant $\kappa=\frac{8 \pi G}{c^{2}}$ is thought to be varying in (cosmological) time and thus replaced by a scalar function [316] $]^{48}$. The theory nicely fit with Dirac's "large number hypothesis" [122, 123]. The fifteenth field variable in Kaluza's theory was identified by Jordan with this function, or in projective relativity, with the scalar: $J:=g_{\alpha \beta} X^{\alpha} X^{\beta}$ by setting $J=\frac{2 \kappa}{c^{2}}(\alpha, \beta=0,1, \ldots, 4)$ [321]. In space-time, the field equations for the gravitational field $g_{i j}$, the electromagnetic 4-potential $A_{k}=g_{4 k}$, and the $g_{44}$-variable $\kappa$ were derived by Jordan and Müller ${ }^{49}$ to be:

$$
\begin{array}{r}
G_{i k}+\frac{\kappa}{c^{2}} F_{i}{ }^{s} F_{k s}=-\frac{1}{2 \kappa}\left(\nabla_{k} \nabla_{i} \kappa-\frac{1}{2 \kappa} \nabla_{i} \kappa \nabla_{k} \kappa\right), \\
\kappa \nabla_{s} F^{s j}=-\frac{3}{2} \nabla_{s} \kappa F^{s j}, \\
G=-\frac{\kappa}{2 c^{2}} F_{r s} F^{r s}+\frac{1}{2 \kappa} g^{r s} \nabla_{r} \kappa \nabla_{s} \kappa-\frac{1}{\kappa} g^{r s} \nabla_{r} \nabla_{s} \kappa .
\end{array}
$$

45 His paper with J. Solomon was mentioned in Section 6.3.2 of Part I.

46 Die Täuschung besteht darin, daß man meint, durch die projektive Form, d.h. die homogenen Koordinaten, die Mängel der Kaluzaschen Formulierung behoben und überhaupt über Kaluza hinaus irgend etwas geleistet zu haben. Der Übergang von Kaluza zur projektiven Form** (der mir damals - 1933 - nicht explizite bekannt war) ist aber zu einfach und zu banal, als daß der sachliche Inhalt beider äquivalenter Formulierungen irgendwie verschieden sein könnte. - **Siehe z.B. P. Bergmann [20]."

47 cf. [230], sections of which are used here.

48 Due to the warfare started by Germany and afflicting her heavily at the time, the first paper on the subject by Jordan, submitted to Zeitschrift für Physik 46 in 1944, has not appeared. Jordan referred to proof sheets which I have not seen.

49 Equations (11) \& (12) of [321] corresponding to (113) \& (114) here contain each a misprint. Both were corrected in $[318]$. 
Jordan \& Müller denoted the Ricci-tensor in space-time by $G_{i k}$. P. G. Bergmann, in a paper submitted in August 1946 but published only in January of 1948, reported that work on a theory with a fifteenth field variable had been going on in Princeton:

"Professor Einstein and the present author had worked on that same idea several years earlier, but had finally rejected it and not published the abortive event" ([21], p. 255).

It may be that at the time, they just did not have an idea for a physical interpretation like the one suggested by P. Jordan. Although there were reasons for studying the theory further, Bergmann pointed out that there is an "embarras de richesses" in the theory: too many constructive possibilities for a Lagrangian. Nonetheless, in his subsequent paper on "five-dimensional cosmology", P. Jordan first stuck to the simplest Lagrangian, i.e. to the Ricci scalar in five dimensions [318]. In this paper, Jordan also made a general comment on attempts within unitary field theory of the Einstein-Schrödinger-type to embed corpuscular matter into classical field theory (cf. chapter 6 with Section 6.1 .1 below):

"The problem of the structure of matter can only be attacked as a problem in quantum mechanics; nevertheless, investigations of the singularities of solutions of the field equations retain considerable importance in this framework. [...] the wave functions of matter must be taken into account. Whether this program can be carried through, and to which extent, in the sense of an extension of geometry (to which Schrödinger's ideas related to the meson field seem to provide an important beginning) is such a widespread question [... ]". ${ }^{50}$ ([318], p. 205).

Jordan's theory received wider attention after his and G. Ludwig's books had been published in the early 1950s [319, 384]. In a letter to Jordan mentioned, Pauli also questioned Jordan's taking the five-dimensional curvature scalar as his Lagrangian. Actually, already in the first edition of his book, Jordan had accepted Pauli's criticism and replaced (110) by [compare with (109)]:

$$
J\left({ }^{(4)} R-\frac{J}{2} F_{r s} F^{r s}-\zeta \frac{\nabla_{r} J \nabla^{r} J}{J^{2}}\right) .
$$

He thus severed his "extended theory of gravitation" from Kaluza's theory. He also displayed the Lagrangian ([319], p. 139):

$$
\kappa^{\eta}\left({ }^{(4)} R-\zeta \frac{\nabla_{r} \kappa \nabla^{r} \kappa}{\kappa^{2}}\right)
$$

but then set one of the two free parameters $\eta=1$. One of those responding to this book was M. Fierz in Basel [195]. Before publication, he had corresponded with W. Pauli, sent him first versions of the paper and eventually received Pauli's placet; cf. the letter of Pauli to Fierz of 2 June 1956 in [492], p. 578. In the second edition of his book, Jordan also commented on a difficulty of his theory pointed out by W. Pauli: instead of $g_{i k}$ equally well $\phi(x) g_{i k}$ with arbitrary function $\phi$ could serve as a metric. ${ }^{51}$ This conformal invariance of the theory is preserved in the case that an electromagnetic field forms the matter tensor. A problem for the interpretation of mathematical objects as physical variables results: by a suitable choice of the conformal factor $\phi$, a "constant"

\footnotetext{
50 "Das Problem der Materiestruktur kann nur als quantentheoretisches Problem angegriffen werde; die Untersuchungen der Singularitäten der Lösungen der Feldgleichungen behält jedoch in diesem Rahmen erhebliche Bedeutung. [...] daß die Wellenfunktionen der Materie mitberücksichtigt werden. Ob oder in welchem Umfang dieses Programm durchführbar ist im Sinne einer Erweiterung der Geometrie (wozu die Schrödingerschen Ideen betreffs des Mesonenfeldes bedeutungsvolle Ansätze zu geben scheinen), ist eine so weit ausgreifende Frage, [...]".

51 In the 1990s, $\phi(x) g_{i k}$ was called "Jordan conformal frame" [104], "Fierz conformal frame metric" or "JordanFierz metric" $[103,105]$ in distinction to the "Einstein conformal frame metric" $g_{i k}$.
} 
gravitational coupling function could be reached, again. In his paper, M. Fierz suggested to either couple Jordan's gravitational theory to point particles or to a (quantum-) Klein-Gordon field in order to remove the difficulty. Fierz also claimed that Jordan had overlooked a physical effect. According to him, a dielectricity "constant" of the vacuum could be introduced $\epsilon_{0}=\kappa^{1+\frac{1}{\eta}}=J^{1+\eta}$ from looking at Jordan's field equations. His Lagrangian corresponded to $\kappa\left(R-\zeta \frac{\kappa_{, r} \kappa^{, r}}{\kappa^{2}}\right)-\frac{\epsilon_{0}}{c^{2}} F_{r s} F^{r s}$ with $J^{\eta}=\kappa$. On the other hand, in the MKSA system of physical units, the fine-structure constant is $\alpha=\frac{e^{2}}{\hbar c \epsilon_{0}}$. Thus, the fine-structure constant would depend on $\kappa$.

"Assumed that $\kappa$ be variable in cosmic spaces, then this variability must show up in the redshift of light radiated from distant stars." ([195], p. 134) 52

Because this had not been observed, Fierz concluded that $\kappa=-1$. Both, Pauli and Fierz gave a low rating to Jordan's theory ${ }^{53}$

Neither Pauli nor Fierz seem to have known that the mathematician Willy Scherrer at the university of Bern had suggested scalar-tensor theory already in 1941 before P. Jordan, and without alluding to Kaluza or Pauli's projective formulation. ${ }^{54}$ Also, in 1949, Scherrer had suggested a more general Lagrangian [532]:

$$
\mathcal{L}=(R-2 \Lambda) \psi^{2}+4 \omega g^{r s} \frac{\partial \psi}{\partial x^{r}} \frac{\partial \psi}{\partial x^{s}} \sqrt{-g} .
$$

$\psi$ is considered to be a scalar matter field. He had advised a student, K. Fink, to work on the Lagrangian $\mathcal{L}=\left(R+2 \omega g^{r s} \frac{\partial \psi}{\partial x^{r}} \frac{\partial \psi}{\partial x^{s}}\right) \sqrt{-g}$ [198]. The ensuing field equations correspond to those following from Jordan's Lagrangian if his parameter $\eta=0$ ([319] p. 140). Exact solutions in the static, spherically symmetric case and for a homogeneous and isotropic cosmological model were published in 1951 almost simultaneously by Fink $(\eta=0)$ and Heckmann, Jordan \& Fricke $(\eta=1)$ [243].

In 1953, W. Scherrer asked Pauli to support another manuscript on unified field theory entitled "Grundlagen einer linearen Feldtheorie" for publication in Helvetica Physica Acta but apparently sent him only a reprint of a preliminary short note [534]. Pauli was loath to get involved and asked the editor of this journal, the very same M. Fierz, what the most appropriate answer to Scherrer could be. He also commented:

"Because according to my opinion all "unified field theories" are based on dubious ideas - in particular it is a typically suspect idea of the great masters Einstein and Schrödinger to add up the symmetric and antisymmetric parts of a tensor - I have to pose the question [...]." (W. Pauli to M. Fierz, 15 Dec. 1953) ([491], p. 390-391) ${ }^{55}$

Scherrer's paper eventually was published in Zeitschrift für Physik [535]. In fact, he proposed a unified field theory based on linear forms, not on a quadratic form such as it is used in general relativity or Einstein-Schrödinger UFT. His notation for differential forms and tangent vectors living in two reference systems is non-standard. As his most important achievement he regarded "the

\footnotetext{
52 "Nimmt man an, dass $\kappa$ in kosmischen Räumen variabel sei, so müsste sich diese Variabilität auf die Rotverschiebung des von fernen Sternen ausgestrahlten Lichtes auswirken."

53 See letters of W. P. to M. F. of 30. 9. 1955, p. 350 and 2. 3. 1956, p. 531; of M. F. to W. P. of 8/11. 3. 1956, p. 539 in [492].

54 The paper (in German) was not readily accessible outside the German speaking countries and carried the misleading title "Zur Theorie der Elementarteilchen" (About the theory of elementary particles) [532]. Scherrer had seen a paper by Jordan \& Müller [321] and had hastened to secure priority for himself [533].

55 "Da ich der Meinung bin, daß alle "einheitlichen Feldtheorien" auf Bierideen basiert sind - insbesondere ist es eine typische Bieridee der großen Herren Einstein und Schrödinger, den symmetrischen und den antisymmetrischen Teil eines Tensors zusammenzuaddieren - muß ich die Frage stellen [...]."
} 
absolutely invariant and at the same time locally exact conservation laws." In his correspondence with Fierz, Pauli expressed his lack of understanding: "What he means with this, I do not know, because all generally relativistic field theories abound with energy laws"([491], p. 403). H. T. Flint wrote a comment in which he claimed to have shown that Scherrer's theory is kin to Einstein's teleparallelism theory [213]. For studies of Kaluza's theory in Paris (Jordan-Thiry theory) cf. Section 11.1.

\title{
3.2 Continued studies of Kaluza-Klein theory in Princeton, and else- where
}

As described in Section 6.3 of Part I, since 1927 Einstein and again in 1931 Einstein and Mayer ${ }^{56}$, within a calculus using 5-component tensorial objects in space-time, had studied Kaluza's approach to a unification of gravitation and electromagnetism in a formal 5-dimensional space with Lorentzsignature. A decade later, Einstein returned to this topic in collaboration with his assistant Peter Bergmann $[167,166]$. The last two chapters of Bergmann's book on relativity theory are devoted to Kaluza's theory and its generalization ([20]. Einstein wrote a foreword, in which he did not comment on "Kaluza's unified field theory" as the theory is listed in the book's index. He admitted that general relativity "[...] has contributed little to atomic physics and our understanding of quantum phenomena." He hoped, however, that some of its features as were "general covariance of the laws of nature and their nonlinearity" could contribute to "overcome the difficulties encountered at present in the theory of atomic and nuclear processes" ([20] p. V). Two years before Bergmann's book appeared, Einstein already had made up his mind against the five-dimensional approach:

\begin{abstract}
"The striving for most possible simplicity of the foundations of the theory has prompted several attempts at joining the gravitational field and the electromagnetic field from a unitary, formal point of view. Here, in particular, the five-dimensional theory of Kaluza and Klein must be mentioned. Yet, after careful consideration of this possibility, I think it more proper to accept the mentioned lack of inner unity, because it seems to me that the embodiment of the hypotheses underlying the five-dimensional theory contains no less arbitrariness than the original theory." 57
\end{abstract}

Nonetheless, in their new approach, Einstein and Bergmann claimed to ascribe "physical reality to the fifth dimension whereas in Kaluza's theory this fifth dimension was introduced only in order to obtain new components of the metric tensor representing the electromagnetic field" ([167], p. 683). Using ideas of O. Klein, this five-dimensional space was seen by them essentially as a four-dimensional one with a small periodical strip or a tube in the additional spacelike dimension affixed. The 4-dimensional metric then is periodic in the additional coordinate $x^{4}{ }^{58}$ With the fifth dimension being compact, this lessened the need for a physical interpretation of its empirical meaning. Now, the authors partially removed Kaluza's 'cylinder condition' $g_{\alpha \beta, 4}=0$ (cf. Section 4.2 of Part I, Eq. (109)): they set $g_{i k, 4} \neq 0$, but assumed $g_{i 4,4}=0$ and $g_{44}=1$ : the electrodynamic 4 potential remains independent of $x^{4}$. Due to the restriction of the covariance group (cf. Section 4.2,

\footnotetext{
56 Section 6.3.2 of Part I

57 "Das Streben nach möglichster Einheitlichkeit der Grundlagen der Theorie hat verschiedene Versuche veranlasst, das Gravitationsfeld und das elektromagnetische Feld unter einen einheitlichen formalen Gesichtspunkt zu bringen. Hier ist besonders die fünfdimensionale Theorie von Kaluza und Klein zu nennen. Nach sorgfältiger Abwägung dieser Möglichkeit halte ich es doch für richtiger, den genannten Mangel an innerer Einheitlichkeit der ursprünglichen Theorie hinzunehmen, weil mir der Inbegriff der der fünfdimensionalen Theorie zugrundeliegenden Hypothesen nicht weniger Willkür zu enthalten scheint als die ursprüngliche Theorie." ([143], p. 335)

58 We always keep $x^{0}$ as the time coordinate. Like Kaluza, Einstein and Bergmann denoted the time coordinate by $x^{4}$ and the additional spacelike coordinate by $x^{0}$. Einstein's and Bergmann's claim with regard to Kaluza can be debated; in my reading of his paper, Kaluza as well took the five dimensional space as physical ("eine fünfdimensionale Welt"). The conflicting claim is repeated by Bergmann ([20], p. 268).
} 
Part I, Eq. (112)), in space-time many more possibilities for setting up a variational principle than the curvature scalar of 5-dimensional space exist: besides the 4-dimensional curvature scalar $R$, Einstein \& Bergmann list three further quadratic invariants: $A_{r s} A^{r s}, g_{, 4}^{r s} g_{r s, 4},\left(g^{r s} g_{r s, 4}\right)^{2}$ where $A_{r s}:=\partial_{s} A_{r}-\partial_{r} A_{s}$. The ensuing field equations for the fourteen variables $g_{i k}$ and $A_{k}$ contain two new free parameters besides the gravitational and cosmological constants. Scalar-tensor theory is excluded due to the restrictions introduced by the authors. Except for the addition of some new technical concepts (p-tensors, p-differentiation) and the inclusion of projective geometry, Bergmann's treatment of Kaluza's idea in his book did not advance the field.

The mathematicians K. Yano and G. Vranceanu showed that Einstein's and Bergmann's generalization may be treated as part of the non-holonomic UFT proposed by them [713,681]. Vranceanu considered space-time to be a "non-holonomic" totally geodesic hypersurface in a 5-dimensional space $V^{5}$, i.e., the hypersurface cannot be generated by the set of tangent spaces in each point. Besides the metric of space-time $d s^{2}=g_{a b} d x^{a} d x^{b},(a, b=1,2,3,4)$, a non-integrable differential form $d s^{5}=d x^{5}-\phi_{a} d x^{a}$ defining the hypersurface was introduced together with the additional assumption $\frac{\partial}{\partial x^{5}} \phi_{a}=0$. The path of a particle with charge $e$, mass $m_{0}$ and 5 -vector $v^{A},(A=1, \ldots, 5)$ was chosen to be a geodesic tangent to the non-holonomic hypersurface. Thus $d v^{5} \stackrel{!}{=} 0$, and Vranceanu then took $v^{5}=\frac{e}{m_{0}}$. The electromagnetic field was defined as $F_{a b}=\frac{1}{2}\left(\phi_{b, a}-\phi_{a, b}\right)$. Both Einstein's and Maxwell's equations followed, separately, with the energy-momentum tensor of matter as possible source of the gravitational field equations: "One can also assume that the energy tensor $T_{a b}$ be the sum of two tensors one of which is due to the electromagnetic field [... ]". ([681], p. 525). ${ }^{59}$ His interpretation of the null geodesics which turn out to be independent of the electromagnetic field is in the spirit of the time: "This amounts to suppose for light, or as well for the photon, that its charge be null and its mass $m_{0}$ be different from zero, a fact which is in accord with the hypothesis of Louis de Broglie (Une nouvelle conception de la lumière; Hermann, Paris 1934)." ([681], p. 524) ${ }^{60}$ More than a decade later, K. Yano and M. Ohgane generalized the non-holonomic UFT to arbitray dimension: $n$-dimensional space is a non-holonomic hypersurface of $(n+1)$-dimensional Riemannian space. It is shown that the theory "[...] seems to contain all the geometries appearing in the five-dimensional unified field theories proposed in the past and to suggest a natural generalization of the six-dimensional unified field theories proposed by B. Hoffmann, J. Podolanski, and one of the present authors" ([714], pp. 318, 325-326). They listed the theories by Kaluza-Klein, Veblen-Hoffmann, Einstein-Mayer, Schouten-Dantzig, Vranceanu and Yano; cf. also Sections 3.1 and 11.2.1.

B. Hoffmann derived the geodesic equations of a magnetic monopole in the framework of a 6-dimensional theory [277]; cf. Section 11.2.1. The one who really made progress, although unintentionally and unnoticed at the time, was O. Klein who extended Abelian gauge theory for a particular non-Abelian group, which almost corresponds to $\mathrm{SU}(2)$ gauge theory [333]. For a detailed discussion of Klein's contribution cf. [237].

Einstein unceasingly continued his work on the "total field" but was aware of inherent difficulties. In a letter to his friend H. Zangger in Zurich of 27 February 1938, he wrote:

"I still work as passionately even though most of my intellectual children, in a very young age, end in the graveyard of disappointed hopes". ([560], p. 552) ${ }^{61}$

\footnotetext{
59 "On peut aussi supposer que le tenseur d'énergie $T_{a b}$ soit la somme de deux tenseurs dont un dû au champ électromagnétique, [...]".

60 "Cela revient à supposer pour la lumière ou bien pour le photon, que la charge électrique soit nulle et que la masse pondérable $m_{0}$ soit différente de zéro, fait qui serait d'accord avec l'hypothèse de M. Louis de Broglie."

61 "Ich arbeite immer noch so passioniert, trotzdem meine geistigen Kinder sehr jung auf dem Friedhof der enttäuschten Hoffnungen enden".
} 
At first he was very much fascinated by the renewed approach to unified field theory by way of Kaluza's idea. We learn this from the letter of 8 August 1938 to his friend Besso:

"After twenty years of vain searching, this year now I have found a promising field theory which is a quite natural sequel to the relativistic gravitational theory. It is in line with Kaluza's idea about the essence of the electromagnetic field." ([163], p. 321 $)^{62}$

\subsection{Non-local fields}

\subsubsection{Bi-vectors; generalized teleparallel geometry}

In 1943, Einstein had come to the conclusion that the failure of "finding a unified theory of the physical field by some generalization of the relativistic theory of gravitation" seemed to require "a decisive modification of the fundamental concepts" ([165], p. 1). He wanted to keep the fourdimensional space-time continuum and the diffeomorphism group as the covariance group, but wished to replace the Riemannian metric by a generalized concept. Together with the assistant at the Institute for Advanced Studies, Valentine Bargmann ${ }^{63}$, he set out to develop a new scheme involving "bi-vector fields". Unlike the concept of a bi-vector used by Schouten in 1924 ([537], p. 17) and ever since in the literature, i.e., for the name of a special antisymmetric tensor, in the definition by Einstein and Bargmann the concept meant a tensor depending on the coordinates of two points in space-time, an object which would be called "bi-local" or "non-local", nowadays. The two points, alternatively, could be imagined to lie in the same manifold ("single space"), or in two different spaces ("double space"). In the latter case, the coordinate transformations for each point are independent.

In place of the Riemannian metric, a contravariant bi-vector $g^{k 1}$ is defined via

$$
g_{i j} g^{j k} g^{2^{1}}=\delta_{i}^{1}
$$

and, similarly,

$$
g^{21} g_{m_{12}}=\delta_{\frac{k}{2}}^{i}
$$

Here, the numbers refer to the two points, while the Latin indices denote the usual tensor indices. The coordinate transformation $x^{i} \rightarrow{ }^{*} x^{i}$ for a simple "bi-vector" $T^{i k}$ is given by:

$$
\stackrel{*}{T}^{21}=\frac{\partial^{* i}}{\partial x^{2}} \frac{\partial^{m}}{\partial x^{1}} T^{m} T^{m l}
$$

Already here a problem was mentioned in the paper: there exist too many covariant geometric objects available for deriving field equations. This is due to the possibility to form covariant quantities containing only first order derivatives like the tensorial quantity: $\gamma_{i j k}:=g_{i j, k}-g_{i k, j}$. In order to cut down on this wealth, a new operation called "rimming" was introduced which

\footnotetext{
62 "Nun habe ich in diesem Jahr nach zwanzig Jahren vergeblichen Suchens eine aussichtsreiche Feld-Theorie gefunden, die eine ganz natürliche Fortsetzung der relativistischen Gravitationstheorie ist. Sie liegt auf der Linie der Kaluza'schen Idee vom Wesen des elektrischen Feldes."

63 Valentine Bargmann (1908-1989) was born in Berlin and began his studies there. In 1933, he moved to Zurich and received his doctorate with G. Wentzel. After his emigration to the United States he became assistant of A. Einstein at the Institute for Advanced Study (PIAS) from 1937 to 1946 . From 1946 on he joined the faculty of Princeton University as a mathematician until retirement. Among his interests were the representation theory of $S L(2, R)$ and the foundation and applications of Hilbert space representations by holomorphic functions (Bargmann spaces) [332].
} 
correlated a new "bi-vector" $\stackrel{f}{i k}_{\alpha \beta}$ with $g_{\alpha \beta}$ by multiplying it from left and right by tensors of full $\operatorname{rank} \omega_{\alpha}^{\alpha}{ }_{\alpha}^{k}, \omega_{\beta}^{k}$ where each is taken from one of the two manifolds (now Greek indices refer to the two different points) ${ }^{64}$ :

$$
\stackrel{*}{g}_{\alpha \beta}^{i k}=\omega_{\alpha}^{\alpha} g_{\alpha \beta}^{m}{ }_{\alpha \beta}^{\prime} \omega_{k}^{n}
$$

All tensors $\stackrel{*}{g}$ obtained by rimming $g$ were considered as different representations of the same field. The rimming of a contravariant "bi-vector" was done similarly by multiplying from the left with $\sigma_{i}{ }_{\beta}^{k}$ and from the right with tensors $\sigma_{\alpha}^{\alpha}{ }^{k}$. In order that (118), (119) remain invariant under rimming, the relations $\underset{\alpha}{\sigma}=\underset{\alpha}{\omega^{-1},}{ }_{\alpha}^{\prime} \sigma=\left(\begin{array}{c}{ }^{\omega} \\ \alpha\end{array}\right)^{-1}$, must hold. A comparison of (120) and (121) shows that a coordinate transformation can be combined with a rimming operation "in such a way that the bivector components remain invariant - i.e., behave like scalars under the resultant transformation" ([165], p. 4).

As a possible field equation, the authors now introduced "tensorial four-point equations":

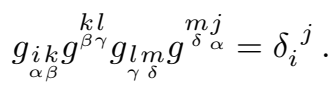

If $g_{i k}$ is treated as a matrix, it is easy to see that all higher-order tensorial equations $(6,8, \ldots-$ point) are dependent on the four-point equation. Next, the authors showed that by a special rimming operation $g_{i k}$ can be transformed into $\delta_{i k}$ such that the corresponding space-time is flat. The trace on $(\mathrm{i}, \mathrm{j})$ in $(122)$ leads to a scalar equation:

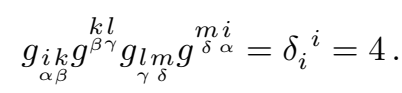

This equations apparently allows for non-flat solutions (cf. Eq. (13), p. 6 in [165]).

In another paragraph, the authors returned to the "single space"-version. Here, a symmetry condition is demanded: $g_{\alpha \beta}=g_{\beta \alpha}$. Now, the tensorial p-point equations admit the special case that the two points (coordinates $\alpha, \beta$ ) coincide. By a suitable rimming operation $g_{\alpha \alpha}=\eta_{i k}$ was reached where $\eta_{i k}$ is the matrix $\operatorname{diag}(1,-1,-1,-1)$. The rimming operations were performed with representations of the Lorentz group. Also, mixed bi-vectors $g_{\beta}^{i}{ }_{\beta}=\eta^{i l} g_{\alpha \beta}$ were introduced and a tensorial three-point equation had to be satisfied:

$$
g_{\underset{\beta}{\alpha}}^{i} g_{\underset{\gamma}{\beta}}^{k} g^{l} \underset{\alpha}{l}=\delta_{m}^{i}
$$

It characterizes flat space as well. After a discussion of complex rimming transformations, the theory was put into spinor form. Solutions were obtained of the relevant matrix equations, some of them due to the mathematician Carl L. Siegel (1896-1981), who stayed in Princeton at the time. Neither a link to physics nor a new UFT followed from this paper. The truly new feature of its approach was that the "metric" can join arbitrarily distant points, not just infinitesimally neighbouring ones. At this time, as in many other cases, Einstein expected the solution to physical problems from a solution to still unanswered mathematical questions.

${ }^{64}$ In order to avoid the same notation as for tensor densities, Einstein's $\hat{\omega}, \hat{\sigma}$ were replaced here by ' $\omega,{ }^{\prime} \sigma$ 
In a continuation of this paper, Einstein explicitly introduced the concept of connection: "I show that just as in the case of the infinitesimal theory this theory can be made very simple by separating the concepts and relations into those based exclusively on the affine connection and those where the affine connection is specialized by hypotheses on the structure of the field" ([146], p. 15). The mixed bi-vector $g_{\beta}^{i} \underset{\beta}{i}$ is interpreted as the (non-infinitesimal) affine connection because the relation

$$
A^{k}=g_{\alpha}^{\stackrel{k}{\beta}}{ }_{\alpha}^{i} A^{i}, \quad A_{\beta}=A_{\alpha} g_{{ }_{\beta}^{\alpha}}^{i}
$$

connects the points with coordinates $\alpha$ and $\beta{ }^{65}$ Two conditions are to be fulfilled: the displacement of a vector from $\alpha$ to $\beta$ and back does not change it, and "the scalar product of a covariant and a contravariant vector is invariant with respect to the affine connection". This led to

$$
g_{\alpha}^{i}{ }_{\alpha}^{i}=\delta_{k}^{i}, g_{k}^{i} \underset{\beta}{i} g_{j}^{k}{ }_{\alpha}^{k}=\delta_{j}^{i} .
$$

Taking into account the rimming operation, it is seen that (125) and (126) are invariant with respect to rimming. Again a tensorial three-point equation was written down which, in matrix form, reads as:

$$
\underset{\alpha \beta}{g} \underset{\beta \gamma}{g} \underset{\gamma \alpha}{g} g-1=0
$$

The consequences of (127) were the same as for the 4-point equation before: space-time is flat. To escape this conclusion, the trace of (127) could also be used "as a possible choice of a field law of a bi-vector field" ([146], p. 17). Furthermore, the symmetry of the metric could be replaced by the more general:

$$
g_{\alpha \beta}= \pm g_{\beta \alpha}
$$

In the remaining part of the paper, various possible cases were discussed and a new concept introduced: "volume invariance". For this, the rimming matrices were restricted to have determinant \pm 1 . Another field law then was proposed:

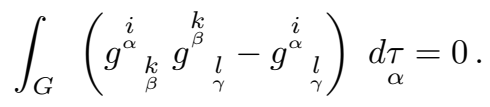

In a note in proof Einstein remarked that "W. Pauli and V. Bargmann have meanwhile succeeded in proving that (129), too, admits only "flat space" solutions"([146], p. 23). He slightly changed the equation into:

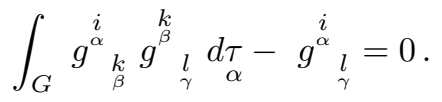

and ended the paper (and his publications on "bi-vectors") by stating: "At present, the author, in collaboration with W. Pauli, is trying to find out whether this equation has non-trivial solutions". Thus, besides a new mathematical scheme, and Einstein's method of "trial and error" for finding field equations, no progress in terms of unified field theory had been achieved.

The only physicist outside of Princeton who expressed an interest in this discovery of "a new form of geometrical connection of a continuum, the distant affine connection" was Schrödinger in Dublin. In his paper, he set out to

65 Under coordinate transformations, this new kind of "connection" transforms as the direct product of a vector at point $\beta$ and a 1-form at point $\alpha$. It thus is no affine (linear) connection in the sense of the one introduced in Section 2.1.1. In the bundle of linear frames, the mixed bi-vector operation would relate two fibers, but not lie in a section of the bundle. 
"[...] show how the new geometrical structure emerges, by generalization, from the one that was at the basis of Einstein's 'Distant Parallelism' (Fernparallelismus), and consisted in the natural union of an integrable (but in general non-symmetric) infinitesimal affine connection and a (in general not flat) Riemannian metric" ([550], p. 143).

He rewrote Einstein's mixed bi-vector with the help of the tetrads used in teleparallel geometry:

$$
g_{\beta}^{\alpha} \underset{\beta}{i}=\left(h_{\alpha}^{i}\right) \text { at } \beta\left(h_{k \alpha}\right) \text { at } \alpha
$$

Note that Schrödinger denoted the tetrad by $h_{a}^{\nu}$ with Latin indices $a, b, \ldots$ for the number of the leg, and Greek space-time indices such that $h_{\nu a} h_{b}^{\nu}=\delta_{a b}$. By comparing the parallel displacement of a 4-vector $\delta A^{\nu}=-\Delta_{\rho \sigma}^{\nu} A^{\rho} d x^{\sigma}$ with $A^{i}=g_{\alpha}^{i}{ }_{\alpha}^{i} A^{k}$ he arrived at his interpretation of $g_{k_{\alpha}^{\beta}}^{i}$ as a connection. As the relation between the affine connection - which he called "infinitesimal connection" - and the bi-vector connection, named "distant connection", he took:

$$
\Delta_{i j}{ }^{k}=\left\{\frac{\partial}{\partial x_{j}}\left(\begin{array}{c}
k \\
g_{\alpha}^{\beta} \\
\alpha
\end{array}\right)\right\}_{\alpha=\beta} .
$$

In the following paper with Friedrich Mautner, scholar at the Dublin Institute for Advanced Studies, who already had been acknowledged as coworker in the first publication, Schrödinger likened Einstein's three-point tensor in (127) to the curvature tensor: the three-point matrices of a distant affinity perform a transfer from the point $\alpha$ via $\rightarrow \beta \rightarrow \gamma$ back to the starting point $\alpha$. This is analogous to parallel transfer around a triangle formed by the points $\alpha, \beta, \gamma$; the change of a vector thus transported along the closed circuit is proportional to the curvature tensor, if it is skew symmetric also in the first pair of indices ([399], pp. 224-225). This analogy was carried on further; a formula corresponding to (59) of Section 2.3.1 was derived, with the symmetric affine connection replaced by the Christoffel symbols and torsion by contorsion, i.e., by $S_{k l i}+$ $S_{l i k}+S_{k i l}$. Both cases in (128) were treated and tentative field equations including a cosmological constant written down. The paper ended with the sentence: "Are these equations likely to give an appropriate description of physical fields?" As is known now, the answer to this question should have been "no". Thus, in this context, Schrödinger's papers also did not bring progress for UFT; nevertheless, they helped to make Einstein's papers more readable. The subject was also taken up by a Romanian mathematician, M. Haimovici (1906-1973), who instead introduced a space of point-couples, introduced axioms for defining a connection there, and established a relation to work by E. Cartan [239].

\subsubsection{From Born's principle of reciprocity to Yukawa's non-local field theory}

Much earlier, Max Born had followed a different if not entirely unrelated conceptual course: in 1938, he had introduced a "principle of reciprocity": "[ . . ] each general law on the $x$-space has an 'inverse image' in the $p$-space, in the first instance the laws of relativity" ([38], p. 327). In this note in Nature, Born added a Lorentzian metric $g_{a b}(p) d p^{a} d p^{b}$ in momentum space satisfying as well the corresponding Einstein field equations (cf. Section 4.2). Infeld in Princeton wanted to get some further information about this principle of reciprocity from Born, who was afraid that his idea be seized by the "terribly clever people over there". However, in his letter to Einstein of 11 April 1938, he described his joint work with Klaus Fuchs: to derive a "super-mechanics" with an 8-dimensional metric in phase space. A new fundamental ("natural") constant appeared leading to both an absolute length and an absolute momentum ([168], pp. 182-184). In a way, Born's formalism came near to Einstein's "double space" in his bi-vector theory. 
About a decade later, in 1947, H. Yukawa in an attempt to arrive at a theory of elementary particles, used exactly this "double space"-approach: "[...] the field in our case is not necessarily a function of $x_{\mu}$ alone, but may depend on $p_{\mu}$ also. [...] The generalized field can include not only the electromagnetic field and various types of meson fields but also the so-called pair-fields such as the meson pair field and the electron-neutrino field" ([716], p. 211-212). It seems that he saw his non-local field theory as introducing new degrees of freedom and leading a step away from the point-particle concept: a possibility for avoiding infinite self-energies. During his stay at the Princeton Institute for Advanced Study (PIAS) in 1948/49, Yukawa must have learned about Born's reciprocity principle and work around it [45, 46, 47], refered to it (cf. [718]) and embarked on his quantum-theory of non-local fields. In general, his theory now corresponded to the "single space" of Einstein and Bargmann: operators $U\left(X_{\mu}, r_{\mu}\right)$ depended on "two sets of real variables" (in the same space) $[719,720,721]$. According to Yukawa: " $X_{\mu}$ coincide with the ordinary coordinates $x_{\mu}$ in the limit of local field, so that the dependence on $X_{\mu}$ describes the asymptotic behavior and the dependence on $r_{\mu}$ characterizes the internal structure or inertial motion" ([722], p. 3 ). At this time, both Yukawa and Einstein lived in Princeton and got to know each other. It can reasonably be doubted, though, that they took note of each other's scientific work except superficially. In 1947, Yukawa possibly had not the least inkling of the Einstein-Bargmann paper of 1944. Of course, Bargmann in Princeton then could have told him. In the special issue of Review of Modern Physics for Einstein's 70th birthday in 1949, Max Born in Edinburgh dedicated an article about his reciprocity principle to Einstein:

"The theory of elementary particles which I propose in the following pages is based on the current conceptions of quantum mechanics and differs widely from the ideas which Einstein himself has developed in regard to this problem. [...] It can be interpreted as a rational generalization of his ("special") theory of relativity." ([40], p. 463)

Right next to Born's article, one by Yukawa was placed which dealt with meson theory. $\mathrm{He}$ made only a cryptic remark about his non-local field theory when expressing the then prevailing ignorance about elementary particle theory:

"Probably we need a broader background (such as the five-dimensional space or the quantized phase space) for field theory in order to cope with these problems, although it is premature to say anything definite in this connection." ([717], p.479)

He then gave references to his papers on non-local field theory. In a letter of 23 January 1949, Born informed Einstein also privately about his theory of elementary particles:

The laws of nature are invariant not only with regard to the relativistic transformations but also with regard to the substitutions $x^{\alpha} \rightarrow p_{\alpha}, p_{\alpha} \rightarrow-x^{\alpha}[\ldots]$. All amounts to replace your fundamental invariant $x^{\alpha} x_{\alpha}=R$ by the symmetrical quantity $S=R+P$ where $P=p^{\alpha} p_{\alpha} . S$ is an operator, the integer eigenvalues of which are the distances [...]." ([168], p. 242.) $)^{66}$

This story shows that a very loose kinship existed between the Einstein-Bargmann "bi-vector" method and Yukawa's non-local field theory with Born's reciprocity theory in the middle. Although some of those involved were in direct personal contact, no concrete evidence for a conscious transfer of ideas could be established.

66 "Die Naturgesetze sind invariant nicht nur gegen die relativistischen Transformationen, sondern auch gegen die Substitutionen $x^{\alpha} \rightarrow p_{\alpha}, p_{\alpha} \rightarrow-x^{\alpha}[\ldots]$. Es läuft darauf hinaus, dass statt Deiner Fundamental-Invariante $x^{\alpha} x_{\alpha}=R$ die symmetrische Grösse $S=R+P$ tritt, wo $P=p^{\alpha} p_{\alpha} . S$ ist ein Operator, dessen ganzzahlige Eigenwerte die Abstände [...] sind." 


\section{Unified Field Theory and Quantum Mechanics}

Obviously, Einstein did not trust an investigation like the experimental physicist Osborn's (19172003) trying to show by ideal measurements that the notion of curvature can be applied only "in the large" where "the domain of largeness is fundamentally determined by the momentum of the test particle with which the curvature is measured" - due to limitations from quantum mechanics [465]. Osborn's feeling obviously was shared by the majority of elementary particle physicists, in particular by F. Dyson:

"The classical field theory of Einstein - electromagnetic and gravitational together give us a satisfactory explanation of all large-scale physical phenomena. [...] But they fail completely to describe the behavior of individual atoms and particles. To understand the small-scale side of physics, physicists had to invent quantum mechanics and the idea of the quantum field." ([137], p. 60)

Nevertheless, there were other physicists like Einstein for whom no divide between classical and quantum field existed, in principle.

\subsection{The impact of Schrödinger's and Dirac's equations}

In the introduction to Section 7 of Part I, a summary has been given of how Einstein's hope that quantum mechanics could be included in a classical unified field theory was taken up by other researchers. A common motivation sprang from the concept of "matter wave" in the sense of a wave in configuration space as extracted from Schrödinger's and Dirac's equations. Henry Thomas Flint ${ }^{67}$ whom we briefly met in Section 7.1 of Part I, was one of those who wanted to incorporate quantum theory into a relativistic field theory for gravitation and electrodynamics. In Flint's imagination, the content of quantum mechanics was greatly condensed: it already would have been reproduced by the generation of a suitable relativistic wave equation for the wave function $\psi$ as a geometric object in an appropriate geometry. This might be taken as an unfortunate consequence of the successes of Schrödinger's wave theory. In the first paper of a series of three, Flint started with a 5 -dimensional curved space with metric $\gamma_{i j},(i, j=0,1, \ldots, 4)$ and an asymmetric connection:

$$
\Delta_{i j}^{k}=\Gamma_{i j}^{k}+T_{i j}^{k}, T_{i j}^{k}=S_{i j}^{k}-\Theta_{i j}^{k},
$$

where $\Gamma_{i j}^{k}(\gamma)$ is the Levi-Civita connection of the 5 -dimensional space, $\Delta_{[i j]}^{k}=S_{i j}^{k}$ the torsion tensor, and $\Theta_{i j}{ }^{k}$ an additional symmetric part built from torsion: $\Theta_{i j}{ }^{k}=S_{.}^{k}{ }_{(i j)}{ }^{\cdot}$ [204]. A scalar field $\psi$ was brought in through vector torsion:

$$
S_{i}=\partial_{i} \ln \psi
$$

and was interpreted as a matter-wave function. The metric is demanded to be covariantly constant with regard to $\Delta_{i j}{ }^{k}$, i.e., $\gamma_{i j ; l}=0$. In the next step, the Ricci-scalar of the 5-dimensional space is calculated. Due to (134), it contained the 5-dimensional wave operator. Up to here, an ensuing theory for the scalar field $\psi$ could be imagined; so far nothing points to quantum mechanics nor to particles. By using de Broglie's idea that the paths of massive and massless particles be given by geodesics in 5-dimensional space and O. Klein's relation between the 5th component of momentum and electric charge, Flint was led to equate the curvature scalar to a constant containing charge, mass and Planck's constant:

$$
\frac{1}{4} \gamma^{r s} R_{r s}(\Delta)=\frac{1}{\hbar^{2}}\left(m^{2} c^{2}+\frac{e^{2}}{\alpha^{2} \gamma_{55}}\right)
$$

67 Henry Thomas Flint (1890-1971) obtained a MSc from the University of Birmingham. After the first world war, he gained his DSc from the University of London while being a lecturer in physics at King's College London. Flint was appointed Professor of Physics at Bedford University of London in 1944 and stayed thereuntil 1956. 
Following Kaluza and Klein, $\alpha^{2} \gamma_{55}=\frac{16 \pi G}{c^{2}}$ was set with $G$ the Newtonian gravitational constant. The linear one-particle wave equation thus obtained contains torsion, curvature, the electromagnetic field $F_{a b},(a, b=0,1, \ldots, 3)$ and a classical spin tensor $A^{l m}$. It is:

$$
(\psi)^{-1} \gamma^{r s}\left(\frac{\partial^{2} \psi}{\partial x^{r} \partial x^{s}}-\Gamma_{r s}^{l} \partial_{l} \psi\right)-\frac{2 \pi i e}{h} F_{a b} A^{a b}+\frac{1}{4}\left(R(\gamma)-\frac{4 \pi G}{c^{2}} H\right)+\frac{4 \pi^{2}}{h^{2}}\left(m^{2} c^{2}-\frac{e^{2}}{c^{2}} \phi^{a} \phi_{a}\right)=0,
$$

where $R$ is the 4-dimensional curvature scalar, $H=F_{a b} F^{a b}$, and $\phi_{a}$ the electromagnetic 4-potential. The particle carries charge $e$ and mass $m$. While Flint stated that "[...] the generalized curvature, is determined by the mass and charge of the particle situated at the point where the curvature is measured" ([204], p. 420), the meaning of (136) by no means is as trivial as claimed. A solution $\psi$ determines part of torsion (cf. (134), but torsion is needed to solve (136); we could write $R=$ $R(\psi), \Gamma_{r s}^{l}=\Gamma_{r s}^{l}(\psi)$. Hence, (136) is a highly complicated equation.

As a preparation for the second paper in the series mentioned [205], a link to matrix theory as developed by Schrödinger was given through replacement of the metric "by more fundamental quantities", the 5 by 5 matrices $\alpha_{i}$ :

$$
\alpha_{i} \alpha_{j}+\alpha_{j} \alpha_{i}=2 \gamma_{i j}, \alpha_{i ; k}=T_{i k}{ }^{r} \alpha_{r},
$$

where the covariant derivative refers to the Levi-Civita connection of $\gamma_{i j}$. Both formulations, with and without matrices were said "to be in harmony". In this second paper, Dirac's equation is given the expression $\alpha^{s} \Pi_{s} \psi=0$ with $\Pi_{j}=p_{j}+\frac{e}{c} \phi_{j}, p_{j}=\frac{\hbar}{i} \frac{\partial}{\partial x^{j}}, \phi_{5}=0 . \frac{\partial}{\partial x^{5}}$ is replaced by $\frac{i m c}{\hbar}$. In place of (134) now

$$
S_{r} \alpha^{r} \psi=\alpha^{r} \frac{\partial \psi}{\partial x^{r}}
$$

is substituted. ${ }^{68}$ Dirac's equation then is generalized to $\alpha^{r} \frac{\partial \psi}{\partial x^{r}}+\frac{i}{\hbar} \Pi_{s} \alpha^{s} \psi=0$. The resulting wave equation of second order contains terms which could not be given a physical interpretation by Flint.

In the third paper of 1935 [206], Flint took up the idea of "matrix length" $d \sigma=\alpha_{j} d x^{j}$ Fock and Ivanenko had presented six years before without referring to them [215]. $\psi$ now is taken to be a column $\left(\psi_{1}, \psi_{2}, \ldots, \psi_{5}\right)^{t}$ and the matrix length of a vector $A^{k}$ defined to be $L:=A^{r} \alpha_{r} \psi$ such that $L^{2}=\left(\psi^{*} \psi\right) \gamma^{r s} A_{r} A_{s}$ with $\psi^{*}$ being the conjugate to $\psi$. Flint seemed undecided about how to interpret $\psi^{*} \psi$. On the one hand, he said that "[...] $\psi^{*} \psi$ has been interpreted as the density of matter" ([206], p. 439), on the other he apparently had taken note of the Kopenhagen interpretation of quantum mechanics (without sharing it) when writing:

"In connection with the equation of the electron path we have the suggestion that $\psi^{*} \psi$ respond to the certainty of finding the electron on the track" ([206], same page).

His conclusion, i.e., that quantum phenomena correspond to geometrical conceptions, and that the complete geometrical scheme includes quantum theory, gravitation, and electromagnetism could not hide that all he had achieved was to build a set of classical relativistic wave equations decorated with an $\hbar$. In a further paper of 1938, in the same spirit, Flint arrived at a geometrical "quantum law" built after the vanishing of the curvature scalar from which he obtained the Dirac equation in an external electrical field [207].

During the second world war, Flint refined his research without changing his basic assumption $[208,209,210]$, i.e., "that the fundamental equation of the quantum theory, which is the quantum equation for an electron in a gravitational and electromagnetic field, can be developed by an appeal to simple geometric ideas." His applications to "field theories of the electron, positron and meson" [211] and to "nuclear field theories" [212] follow the same line. No progress, either for

\footnotetext{
68 Raising and lowering of indices with $\gamma_{i j}, \gamma^{i j}$.
} 
the understanding of quantum mechanics nor for the construction of a unified field theory, can be discovered. Flint's work was not helped by contributions of others [6, 3]. After World War II, Flint continued his ideas with a collaborator [214]; in the meantime he had observed that Mimura also had introduced matrix length in 1935. As in a previous paper, he used the method by which Weyl had derived his first gauge theory combining gravitation and electromagnetism. Strangely enough, Weyl's later main success, the re-direction of his idea of gauging to quantum mechanics was not mentioned by Flint although he was up to show that "equations of the form of Dirac's equation can be regarded as gauge-equations" ([214], p. 260). Under parallel transport, the matrix length $L$ of a vector $A_{k}$ is assumed to change by $d L=\Theta R_{r} \alpha^{s} A_{s} \psi d x^{r}$, where $R_{r}$ is an operator (a matrix) corresponding to the 5 -vector $\phi_{k}$. Flint still was deeply entrenched in classical notions when approaching the explanation of the electron's rest mass: it should contain contributions from the electromagnetic and mesonic fields. The mathematician J. A. Schouten conjectured that "[t]he investigations of H. T. Flint are perhaps in some way connected with conformal meson theory [...]" ([539], p. 424).

That Flint was isolated from the physics mainstream may be concluded also from the fact that his papers are not cited in a standard presentation of relativistic wave-equations [84]. We dwelled on his research in order to illuminate the time lag in the absorption of new physics results among groups doing research, simultaneously. In this theme, we could have included the "tensor rear guard" (Fisher, Temple, etc.) who believed to be able to get around spinors.

\subsection{Other approaches}

We come back to a paper by M. Born which was referred to already in Section 3.3.2, but under a different perspective. In view of the problems of quantum field theory at the time with infinite selfenergy of the electron, the zero-point energies of radiation fields adding up to infinity etc., Max Born preferred to unify quantum theory and "the principle of general invariance", i.e., inertial fields rather than include the gravitational field. The uncertainty relations between coordinates and momenta served as a motivation for him to assume independent and unrelated metrics $g_{i j}\left(x^{l}\right)$ in configuration and $\gamma^{i j}\left(p^{n}\right)$ in momentum space [39]. As field equations in momentum space he postulated the Einstein field equations for a correspondingly calculated Ricci-tensor (as a function of momenta) $P^{k l}\left(p^{r}\right)$ :

$$
P^{k l}-\left(\frac{1}{2} P+\lambda^{\prime}\right) \gamma^{k l}=-\kappa^{\prime} T^{k l}
$$

The "nuclear constants" $\lambda^{\prime}, \kappa^{\prime}$ remained undetermined. Born was silent on the matter tensor. His applications of the formalism turned toward quantum electrodynamics, black body radiation and the kinetic theory of gases (of atoms). By choosing, in momentum space, the analogue to the Friedman cosmological solution with space sections of constant curvature, an upper limit $b$ for momentum ensued. The number of quantum states in volume elements $V$ of configuration space and in a volume element of momentum space turned out to be $g \frac{\pi^{2} V}{h^{3}} b$ and had many consequences e.g., for Planck's and Coulomb's laws and for nuclear structure. The parameter b determined all deviations from previous laws: the Coulomb law for two particles became $\frac{e_{1} e_{2}}{r_{12}^{2}} f\left(2 \frac{r_{12}}{\hbar} b\right)$ with the function $f(x)=\int_{0}^{x} J_{0}(y) d y$ and the Bessel function $J_{0}$; the Planck law for the energy density of black body radiation $u(\nu, T)=\frac{8 \pi h}{c^{3}} \frac{\nu^{3}}{\left(\exp \left(\frac{h \nu}{k T}-1\right)\left(1-(\nu \tau)^{2}\right)\right.}$ with $\tau=\frac{h}{b c}$. Born fixed $b$ such that the classical electron radius $r_{0}=\frac{\hbar}{b}=\frac{e^{2}}{m c^{2}}$. The paper's main result was a geometric foundation for the assumption of an upper limit for momentum - not a unification of quantum mechanics with anything else. Perhaps, Born had recycled an idea from his paper with Infeld, in which they had introduced an upper limit for the electrical field (cf. Section 5). 


\subsection{Wave geometry}

A group of theoreticians at the Physical Institute of Hiroshima University in Japan in the second half of the 1930s intensively developed a program for a unified field theory of a new type with the intention of combining gravitation and quantum theory. Members of the group were Yositaka Mimura, Tôyomon Hosokawa, Kakutarô Morinaga, Takasi Sibata, Toranosuke Iwatsuki, Hyôitirô Takeno, and also Kyosi Sakuma, M. Urabe, K. Itimaru. The research came to a deadly halt when the first atom bomb detonated over Hiroshima, with the hypo-center of the explosion lying 1.5 $\mathrm{km}$ away from the Research Institute for Theoretical Physics. ${ }^{69}$ After the second world war, some progress was made by the survivors. The theory became simplified and was summarized in two reports of the 1960s [427, 428].

In an introductory paper by Mimura, the new approach was termed "wave geometry" [425]. His intention was to abandon the then accepted assumption that space-geometry underlying microscopic phenomena (like in elementary particle physics), be the same as used for macroscopic physics. Schrödinger had argued in this sense and was cited by Mimura [541]. Einstein's original hope that space-time must not exist in the absence of matter, unfulfilled by general relativity, became revived on the level of "microscopic physics": "[... ] the microscopic space exists only when an elementary particle exists. In this sense, where there is no elementary particle, no 'geometry' exists" ([425], p. 101). Also "[...] according to our new theory, geometry in microscopic space differs radically from that of macroscopic [...]" ([425], p. 106). "70 "wave geometry" must not be considered as one specific theory but rather as the attempt for a theory expressing the claimed equivalence of geometry and physics.

The physical system, "the space-time-matter" manifold, was to be seen as a (quantum mechanical) state $\psi$, a 4-component (Dirac) spinor; "distance" in microscopic space became defined as an eigenvalue of a linear distance operator. In order to find this operator, by following Dirac, a principle of linearization was applied:

$$
d s \psi:=\gamma_{i} d x^{i} \psi
$$

with $\gamma_{i}=h_{i}^{a} \stackrel{\gamma}{a}_{a}$ where $h_{i}^{a}$ is an arbitrary tetrad (with the tetrad index $a=1,2,3,4$ ), and $\stackrel{0}{\gamma}_{i}$ denoting the Dirac matrices. If

$$
\gamma_{(i} \gamma_{j)}=g_{i j}
$$

is demanded, i.e.,

$$
\Sigma_{a} h_{i}^{a} h_{j}^{a}=g_{i j}
$$

the eigenvalues of the distance operator are $\pm \sqrt{g_{i j} d x^{i} d x^{j}}$. If Riemannian covariant differentiation is used, then

$$
\nabla_{i} \gamma_{j}:=\frac{\partial \gamma_{j}}{\partial x^{i}}-\left\{{ }_{i j}^{k}\right\} \gamma_{k}-\Gamma_{i} \gamma_{j}+\Gamma_{j} \gamma_{i}
$$

Here, $\Gamma$ denotes the spin connection. As the fundamental equation of the theory

$$
\nabla_{i} \psi=\Sigma_{i} \psi
$$

was written down where $\Sigma_{i}$ is an as yet undetermined 4 -vector with matrix entries. It was expected that (144) describe the gravitational, electromagnetic and the matter field "in unified form not discriminating macroscopic and microscopic phenomena" ([427], p. 11). In 1929, (140) had also been suggested by Fock and Ivanenko [215], a paper mentioned briefly in Section 7.2 of Part I. As we

69 "T. Iwatsuki and T. Hosokawa were killed, Y. Mimura (1898-1965) and T. Sibata were seriously wounded and K. Morinaga got heavily burned". All buildings and the library as well as the private homes of the researchers were destroyed. ([427], preface.)

70 Page numbers are taken from [428] wherein the paper has been reprinted. 
have seen, at around the same year 1935, H. T. Flint had set up a similar unified theory as Mimura [206]. The theory of Mimura and Takeno was to be applied to the universe, to local irregularities (galaxies) in the universe and to the atom. Only the Einstein cosmos and de Sitter space-time were allowed as cosmological metrics. For the atom, a solution in a space-time with metric $d s^{2}=$ $R^{2}\left(\left(d x^{1}\right)^{2}+\sin ^{2} x^{1}\left(d x^{2}\right)^{2}\right)+\frac{R^{2}}{\left(x^{3}\right)^{2}}\left(\left(d x^{3}\right)^{2}+\left(d x^{0}\right)^{2}\right)$ was obtained and a wave function "which can be identified with the Dirac level of the hydrogen atom if the arbitrary functions and constants in the equation are chosen suitably" ([427], p. 66). With a particular choice of $\Sigma_{i}$, (cf. [428], Eq. 4.15 on p. 4) the fundamental equation for $\psi$ was then determined to be the matrix equation:

$$
\left(\partial_{i}-\Gamma_{i}\right) \psi=\left(T_{i}^{5} \gamma_{5}-L_{i} I\right) \psi
$$

with $\Gamma_{i}:=\frac{1}{4}\left[h_{r}^{s} \partial_{i} h_{t}^{r}-\left\{\begin{array}{c}s \\ t i\end{array}\right\}_{g}\right] \gamma^{t} \gamma_{s} . T_{i}^{5}$ and $L_{i}$ are arbitrary vectors. In [428], a second fundamental equation was added:

$$
\gamma^{i}\left(\partial_{i}-\Gamma_{i}\right) \psi=\mu \psi
$$

with a scalar $\mu$. For complete integrability of (145), the Riemannian curvature tensor $K_{k l m}^{i}$ must vanish. Equation (145) reduces to $\partial_{i} \psi=0, g_{i j}=\eta_{i j}$ with the solution $\psi_{a}=$ const., $(a=$ $0,1, \ldots, 3)$. This being too restrictive, (145) was weakened to $\left(\partial_{i}-\Gamma_{i}\right) \psi=0$ for either $\psi_{0}=\psi_{3}=0$ or $\psi_{1}=\psi_{2}=0$ with the integrability conditions $\frac{\sqrt{g}}{2} \epsilon_{l m r s} K_{i j}{ }^{r s}= \pm K_{i j l m}$, respectively. T. Sibata gave a solution of this equation expressing self-duality for weak fields [576]. He also set out to show that Born-Infeld theory follows from his approach to wave geometry in the case of vacuum electrodynamics [577]. In this paper, the condition of complete integrability for his version of (144) read as $\frac{g}{4} \epsilon_{l m r s} \epsilon_{i j p q} K^{\text {pqrs }}= \pm K_{i j l m}$.

In 1938, T. Hosokawa even had extended wave geometry to Finsler geometry and applied to Milne's cosmological principle [287].

With its results obtained until 1945, wave geometry could not compete with quantum field theory. After the war, the vague hope was expressed that in a "supermicroscopic" space-time, elementary particle theory could be developed and that "the problem of internal space' of elementary particles may be interwoven with some 'hidden' relations to the structure of space-time." ([428], p. 41.) Clearly, the algebra of $\gamma$-matrices which is all what is behind the distance operator, was an insufficient substitute for the algebra of non-commuting observables in quantum field theory. 


\section{Born-Infeld Theory}

In 1934, M. Born and L. Infeld published a paper on "The Foundations of the New Field Theory" [42]. Its somewhat vague title hid a non-linear theory of the electromagnetic field using a nonsymmetric metric but denying a relationship with " "unitary' field due to Einstein, Weyl, Eddington, and others [...]". In fact, the original idea for the new theory originated in July 1933 while Born was still a member of the University of Göttingen but already on the move from Germany for vacations in South Tyrol to only return after World War II. Born's next publication, submitted in August 1933 without institutional address, dealt with the quantization of the electromagnetic field; in it the new Lagrangian was also shown [37]. In view of the problems with divergent terms in quantum (field) electrodynamics at the time, he set out to modify Maxwell's equations in such a way that an electron with finite radius $r_{0}$ could be described; its electric potential remained finite for $\frac{r}{r_{0}} \ll 1$ [36]. The Lagrangian for the new electrodynamics was $L=\frac{1}{a^{2}} \sqrt{1+a^{2}\left(H^{2}-E^{2}\right)}$ with the constant a of dimension $\frac{r_{0}^{2}}{e}$, where $e$ is the elementary electric charge and $r_{0}$ the electron radius. In the limit $a \rightarrow 0$ the Lagrangian of Maxwell's theory reappeared: $\frac{1}{a^{2}}+\frac{1}{2}\left(H^{2}-E^{2}\right)$. In the paper with Infeld, the Lagrangian is generalized in order to include the gravitational field:

$$
L=\sqrt{-\operatorname{det}\left(g_{(i j)}+f_{i j}\right)}-\sqrt{-\operatorname{det}\left(g_{(i j)}\right)}
$$

where $g_{(i j)}$ is the (Riemannian) metric and $f_{i j}=f_{[i j]}$ the electromagnetic field tensor; $g_{i j}=$ $g_{(i j)}+f_{i j}$, formally is an asymmetric metric. The Lagrangian (147) can be expressed by the two invariants of Maxwell's theory $F:=\frac{1}{2} f_{m r} f_{n s} g^{m n} g^{r s}$ and $G:=\frac{1}{4} \epsilon^{m n r s} f_{m n} f_{r s}==f^{* r s} f_{r s}$ as

$$
\mathcal{L}=\sqrt{-g} L=\sqrt{-g}\left(\sqrt{1+F-G^{2}}-1\right) .
$$

The new field equations become:

$$
\frac{\partial \sqrt{-g} f^{* i s}}{\partial x^{s}}=0, \frac{\partial \sqrt{-g} p^{i s}}{\partial x^{s}}=0
$$

with the definition $\sqrt{-g} p^{i k}:=\frac{\partial \mathcal{L}}{\partial f_{i k}}$. Insertion of $F=1 / b^{2}\left(\overrightarrow{\mathbf{B}}^{2}-\overrightarrow{\mathbf{E}}^{2}\right), \quad G=1 / b^{2} \overrightarrow{\mathbf{B}} \cdot \overrightarrow{\mathbf{E}}$ led to Maxwell's equations plus the relations between fields and inductions:

$$
\overrightarrow{\mathbf{H}}=b^{2} \frac{\partial L}{\partial \overrightarrow{\mathbf{B}}}=\frac{\overrightarrow{\mathbf{B}}-G \overrightarrow{\mathbf{E}}}{\sqrt{1+F-G^{2}}-1}, \overrightarrow{\mathbf{D}}=-b^{2} \frac{\partial L}{\partial \overrightarrow{\mathbf{E}}}=\frac{\overrightarrow{\mathbf{E}}+G \overrightarrow{\mathbf{B}}}{\sqrt{1+F-G^{2}}-1}
$$

"The quotient of the field strength expressed in the conventional units divided by the field strength in the natural units" was denoted by $b$ and named the "absolute field". As was well known, many asymmetric energy-momentum tensors for the electromagnetic field could be formulated. Years later, St. Mavridès took up this problem and derived identities for the symmetric Minkowski tensor, the fields and inductions, independent of whether the relations between fields and inductions were linear or more general [410]. Xinh Nguyen Xua then showed that with the relations (150), all the various energy-momentum tensors can be derived from one such symmetric tensor [711]. Born \& Infeld chose

$$
T_{k}^{l}=L \delta_{k}^{l}-\frac{f_{r k} f^{r l}-\delta_{k}^{l} G^{2}}{\sqrt{1+F^{2}-G^{2}}} .
$$

The static solution of the new equations for the potential of a point charge was determined to be

$$
\phi(r)=\frac{e}{r_{0}} \int_{r}^{\infty} \frac{d v}{\sqrt{1+v^{4}}}, r_{0}:=\sqrt{\frac{e}{b}} .
$$


It turned out that, from $E=m_{0} c^{2}:=\frac{b^{2}}{4 \pi} \int L d^{3} x, r_{0}$ could be calculated numerically via $2 \frac{e^{2}}{r_{0}}\left(\int_{0}^{\infty}(1-\right.$ $\left.\frac{x^{2}}{\sqrt{1+x^{4}}}\right)$ to take the value $r_{0}=1,2361 \frac{e^{2}}{m_{0} c^{2}}$ and thus $b$ could also be determined. According to Born and Infeld: "The new field theory can be considered as a revival of the old idea of the electromagnetic origin of mass" Also, the existence of an absolute field as a "natural unit for all field components and the upper limit for a purely electric field" ([42], p. 451) had been assumed.

Unsurprisingly, Pauli was unhappy with the paper by Born and Infeld as far as its inclusion of the gravitational field via $g_{(i j)}+f_{i j}$ was concerned. Instead, in his letter of 21 December 1933 to Max Born ([488], p. 241), he suggested to take as a Lagrangian density $\mathcal{L}=\sqrt{-g} F(P)$ with Kaluza's curvature scalar in 5 dimensions $P=R+\frac{k}{2 c^{2}} f_{r s} f^{r s}$. "In particular, it is possible to set $F(P)=\sqrt{1+\frac{1}{\text { const. }} P}$, and therefore your electrodynamics is compatible with the projective view on the electromagnetic and gravitational field." 71 But for Born, electrodynamics was in the focus. Three months after Pauli's criticism, he wrote to Einstein in connection with his paper with Infeld:

"Possibly, you will not agree, because I do not include gravitation. This is a rather basic point, where I have a different view as you in your papers on unitary field theory. Hopefully, I soon will be able to finalize my idea on gravitation" ([168], p. 167). ${ }^{72}$

Around the same time as Pauli, B. Hoffmann who had left Princeton for the University of Rochester, had had the same idea. It was couched in the language of projective theory on which he had worked with O. Veblen (cf. Section 6.3.2 of Part I) and on his own [275]. ${ }^{73}$ He suggested the Lagrangian $\mathcal{L}=(\sqrt{1+B}-1) \sqrt{-\gamma}$ where $\gamma_{i j}$ is the 5 -dimensional projective metric and $B$ the projective curvature scalar. Due to $B=R-g^{p r} g^{q s} f_{r q} f_{p s}$, his Lagrangian corresponds to Kaluza's. Born \& Infeld had remarked that in order to include gravitation in their theory, only Einstein's Lagrangian must be added to (148). Hoffmann now tried to obtain a static spherically symmetric solution for both theories with a non-vanishing electromagnetic field. In the augmented BornInfeld Lagrangian, the Minkowski metric could be used as a special case. According to Hoffmann this was no longer possible for his Lagrangian because "the electromagnetic field exerts a gravitational influence" ([275], p. 364). As he could not find a solution to his complicated field equations, the "degree of modification of the electrostatic potential by its own gravitational field" could not be determined.

In connection with the work of Euler and Kockel on the scattering of light by light under his guidance, W. Heisenberg wrote Pauli on 4 November 1934: "The terms to be added to the Lagrangian look like in the theory of Born and Infeld, but they are twenty times larger than those of Born and Infeld" ([488], p. 358). ${ }^{74}$ But Pauli had not changed his opinion; in connection with the scattering of light by light, he answered Heisenberg curtly: "I do not care about Born's theory" ([488], p. 372). Ten years later, in his letter to Einstein of 10 October 1944 Born assessed his theory with some reservation ([168], p. 212):

"[...] I always had a lot of understanding for your good Jewish physics, and much amusement with it; however, I myself have produced it only once: the non-linear elec-

71 "Insbesondere ist es also möglich, $F(P)=\sqrt{1+\frac{1}{\text { const. }} P}$ zu setzen, und daher ist Ihre Elektrodynamik mit der projektiven Auffassung von elektromagnetischem und Gravitationsfeld vereinbar."

72 "Du wirst vielleicht nicht einverstanden sein, weil ich die Gravitation nicht mitbehandele. Das ist ein prinzipieller Punkt, wo ich anderer Ansicht bin wie Du in Deinen Arbeiten über einheitliche Feldtheorie. Ich hoffe, bald dazu zu kommen, meine Idee über die Gravitation auszuarbeiten."

73 In this paper, he studied the relationship between the Einstein-Mayer unified field theory (cf. Section 6.4.3 of Part I) and projective relativity theory.

74 "Die Zusatzglieder zur Lagrangefunktion sehen ähnlich aus wie in der Theorie von Born und Infeld, sind aber etwa 20mal größer als jene bei Born und Infeld." 
trodynamics, and this is no particular success $[\ldots] . " 75$

Nevertheless, it had some influence on UFT; cf. Sections 6.1.3, 9.7, and 10.3.4.

Born and Infeld unsuccessfully tried to quantize their non-linear theory of the electromagnetic field by using the commutation rules of Heisenberg and Pauli for the field strenghts [43, 44]. They noticed that the theory could be presented differently according to whether the pairs $\vec{E}, \vec{B}$, or $\vec{D}, \vec{H} ; \vec{D}, \vec{B} ; \vec{E}, \vec{H}$ were chosen as independent variables. The authors took $\vec{D}, \vec{B}$ in order to avoid "formal difficulties". However, a perturbative approach by canonical quantization of either the field or the vector potential could not succeed because the interaction term in the Hamiltonian included higher powers of derivative terms. ${ }^{76}$

One who became attracted by the Born-Infeld theory was E. Schrödinger. He had come "across a further representation, which is so entirely different from all the aforementioned, and presents such curious analytical aspects, that I desired to have it communicated" ([542], p. 465). He used a pair $\mathcal{F}, \mathcal{G}$ of complex combinations of the 3 -vector fields $\vec{B}, \vec{E}, \vec{H}, \vec{D}$ such that $\mathcal{F}=\vec{B}-i \vec{D}, \mathcal{G}=\vec{E}+i \vec{H}$. The Lagrangian $\mathcal{L}$ was to be determined such that its partial derivatives with respect to $\mathcal{F}$ and $\mathcal{G}$ coincided with the complex conjugates: $\overline{\mathcal{F}}=\frac{\partial \mathcal{L}}{\partial \mathcal{G}}$ and $\overline{\mathcal{G}}=\frac{\partial \mathcal{L}}{\partial \mathcal{F}}$. The result is

$$
\mathcal{L}=\frac{\mathcal{F}^{2}-\mathcal{G}^{2}}{\mathcal{F} \cdot \mathcal{G}} .
$$

Born's constant $b$ was set equal to one. Schrödinger showed that his formulation was "entirely equivalent to Born's theory" and did not provide any further physical insight. Thus, Schrödinger's paper gave a witty formal comment on the Born-Infeld theory. Ironically, it had been financed by Imperial Chemical Industries, Limited.

S. Kichenassamy ${ }^{77}$ studied the subcase of an electromagnetic null field with matter tensor: $T_{i j}=A^{2} k_{i} k_{j}, \quad k_{i} k^{i}=0$ and showed that in this case the Born-Infeld theory leads to the same results as Maxwell's electrodynamics [328, 340].

\footnotetext{
75 “[...] Ich hatte immer viel Verständnis für Deine gute jüdische Physik und viel Vergnügen daran; aber selber gemacht habe ich es nur einmal: die nicht-lineare Elektrodynamik, und die ist doch kein besonderer Erfolg. [...]."

76 For a modern approach by stochastic quantization cf. [288].

77 The second paper was written together with his Brazilian student, Hugo F. Kremer, who did not finish his thesis in Paris. He nevertheless became director of the Physics Institute of the Federal University of Paraná during military dictatorship in Brazil which he seems to have supported. Kremer was shot to death in 1967 under obscure circumstances.
} 


\section{Affine Geometry: Schrödinger as an Ardent Player}

\subsection{A unitary theory of physical fields}

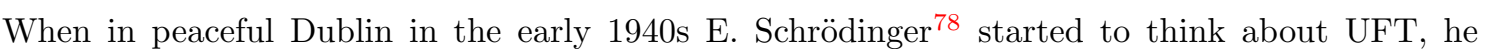
had in mind a theory which eventually would give a unitary description of the gravitational, electromagnetic and mesonic field. Mesons formed a fashionable subject of research at the time; they were thought to mediate nuclear interactions. They constituted the only other field of integral spin then known besides the gravitational and electromagnetic fields. Schrödinger had written about their matrix representations [546]. In his new paper, he deemed it "probable that the fields of the Dirac-type can also be accounted for. [...] It is pretty obvious that they must result from the self-dual and self-antidual constituents into which the anti-symmetric part of $R_{k l}$ can be split." ([545], p. 44, 57.) This is quickly constrained by another remark: "I do not mean that the new affine connection will be needed to account for the well-known Dirac fields". ([545], p. 58.) He followed the tradition of $\mathrm{H}$. Weyl and A. Eddington who had made the concept of affine connection play an essential role in their geometries - beside the metric or without any. He laid out his theory in close contact with Einstein's papers of 1923 on affine geometry (cf. Section 4.3.2 of Part I) and the nonlinear electrodynamics of M. Born \& L. Infeld [42] (cf. Section 5). On 10 May 1943, M. Born reported to Einstein about Schrödinger's work: “[...] He has taken up an old paper of yours, from 1923, and filled it with new life, developing a unified field theory for gravitation, electrodynamics and mesons, which seems promising to me. [...]" ([168], p. 194.) Einstein's answer, on 2 June 1943, was less than excited:

"Schrödinger was as kind as to write to me himself about his work. At the time I was quite enthusiastic about this way of thinking. Its weakness lies in the fact that its construction from the point of view of affine space is rather artificial and forced. Also, the link between skew symmetric curvature and the electromagnetic states of space leads to a linear relation between electrical fields and charge densities. [...]" ([168], p. 196.). ${ }^{79}$

As I suppose, the "At the time" refers to 1923. With "skew symmetric curvature", the antisymmetric part of the Ricci-tensor is meant. Schrödinger believed that Einstein had left affine theory because of "aesthetic displeasure" resulting from a mistake in his interpretation of the theory.

\subsubsection{Symmetric affine connection}

In his first papers on affine geometry, Schrödinger kept to a symmetric connection. ${ }^{80}$ There is thus no need to distinguish between $\stackrel{+}{\nabla}$ and $\bar{\nabla}$ in this context. Within purely affine theory there are fewer ways to form tensor densities than in metric-affine or mixed geometry. By contraction of the curvature tensor, second-rank tensors $K_{i j}$ and $V_{i j}$ are available (cf. Section 2.3.1) from which tensor densities of weight -1 (scalar densities) (cf. Section 2.1.5 of Part I) like $\sqrt{\operatorname{det}\left(K_{i j}\right)}$ or $\sqrt{\operatorname{det}\left(V_{i j}\right)}$ can be built. Such scalar densities are needed in order to set up a variational principle.

\footnotetext{
${ }^{78}$ Erwin Rudolf Josef Alexander Schrödinger (1887-1961), Austrian theoretical physicist. He was the creator of quantum wave mechanics with his famous "Schrödinger equation" suggested in 1926. Nobel prize in 1933. Professor at the Universities of Stuttgart, Breslau, Zürich, Berlin, Graz, and Wien. Professor at and director of the Dublin School of Theoretical Physics between 1940 and 1956.

79 "Schrödinger war so lieb, mir selbst über seine Arbeit zu schreiben. Ich war seinerzeit recht enthusiastisch bezüglich dieser Gedankenrichtung. Die Schwäche derselben liegt darin, daß die Konstruktion vom Standpunkt des affinen Raumes ziemlich künstlich und gezwungen ist. Auch führt die Verknüpfung der antisymmetrischen Krümmung mit den elektrischen Raumzuständen dazu, daß elektrische Felder mit Ladungsdichten linear verknüpft sind. [...]"

${ }^{80}$ O. Hittmair gave a brief review of Schrödinger's UFT in [256].
} 
In his paper, Schrödinger took as such a variational principle:

$$
\delta \int \mathcal{L}\left(K_{i j}\right) d \tau=0
$$

thus neglecting homothetic curvature as a further possible ingredient. ${ }^{81} K_{i j}(\Gamma)$ is the Ricci-tensor introduced in (55) or (56) due to $\Gamma$ being symmetric. Enthusiastically, he started at the point at which Einstein had given up and defined the symmetric and skew-symmetric quantities:

$$
\hat{g}^{i k}:=\frac{1}{2}\left(\frac{\partial \mathcal{L}}{\partial K_{i k}}+\frac{\partial \mathcal{L}}{\partial K_{k i}}\right), \quad \hat{f}^{i k}:=\frac{1}{2}\left(\frac{\partial \mathcal{L}}{\partial K_{i k}}-\frac{\partial \mathcal{L}}{\partial K_{k i}}\right) .
$$

The variation of (153) with respect to the components of the connection $\Gamma_{i j}{ }^{k}$ now can be written as:

$$
\hat{g}^{i k}{ }_{\| l}+\frac{1}{3} \delta_{l}^{k} \hat{f}_{\| s}^{i s}+\frac{1}{3} \delta_{l}^{i} \hat{f}_{\| s}^{k s}=0 .
$$

Note that (155) is formally the same equation which Einstein had found in his paper in 1923 when taking up Eddington's affine geometry [141]. A vector density $\hat{j}^{k}$ is introduced via

$$
\hat{j}^{k}:=\hat{f}^{i s}{ }_{\| s}=\frac{\partial \hat{f}^{i s}}{\partial x^{s}}
$$

with $\hat{j}^{k}$ being interpreted as the (electric) current density. By help of $\hat{g}^{i k}$ a (symmetric) metric tensor $g_{i k}$ is introduced by the usual relations:

$$
\hat{g}^{i k}=\sqrt{-\operatorname{det}\left(g_{l m}\right)} g^{i k}, g_{i s} g^{k s}=\delta_{i}^{k} \rightarrow g^{i k}=\sqrt{-\operatorname{det}\left(\hat{g}_{l m}\right)} \hat{g}^{i k} .
$$

(155) can be formally solved for the components of the symmetric connection to give:

$$
\Gamma_{i j}{ }^{k}=\left\{{ }_{i j}^{k}\right\}-\frac{1}{2} g_{i j} j^{k}+\frac{1}{6} \delta_{i}{ }^{k} j_{j}+\frac{1}{6} \delta_{j}^{k} j_{i}
$$

with $j_{k}=(-g)^{-\frac{1}{2}} g_{k s} \hat{j}^{s}, g=\operatorname{det}\left(g_{l m}\right) .{ }^{82}$ This expression is similar but unequal to the connection in Weyl's theory (cf. Section 4.1.1 of Part I, Eq. (100)). The intention is to express $\Gamma_{i j}{ }^{k}$ as a functional of the components of $K_{i k}$, insert the expression into (56) (for $L=\Gamma$ ), and finally solve for $K_{i k}$. What functions here as a metric tensor, is only an auxiliary quantity and depends on the connection (cf. (154), (157)).

In order to arrive at a consistent physical interpretation of his approach, Schrödinger introduced two variables conjugate to $\hat{g}^{i k}, \hat{f}^{i k}$ by:

$$
\gamma_{i k}:=K_{(i k)}, \phi_{i k}:=-K_{[i k]},
$$

and carried out what he called a contact transformation:

$$
\overline{\mathcal{L}}\left(\hat{g}^{l m}, \phi_{l m}\right)=\hat{g}^{l m} \gamma_{l m}-\mathcal{L} .
$$

From (160) we get:

$$
\gamma_{i k}=\frac{\partial \overline{\mathcal{L}}}{\partial \hat{g}^{i k}}, \quad \hat{f}^{i k}=\frac{\partial \overline{\mathcal{L}}}{\partial \phi_{i k}}
$$

\footnotetext{
${ }^{81} d \tau$ is the volume element.
}

82 Schrödinger's notation for $\hat{j}^{k}$ is $\hat{i}^{k}$. (158) is formal because, implicitly, in $g_{i j}$ through its dependency on $K_{i k}$ the connection $\Gamma$ still is present. Compare (158) to Einstein's equation (123) in Section 4.3.2 of Part I. We have not changed Schrödinger's kernel letter for the derived metric $g_{i j}$ in the hope that it will not be confused with the asymmetric metric in mixed geometry. 
With the new variables, Eqs. (155) and (156) may be brought into the form of the Einstein-Maxwell equations:

$$
\begin{array}{r}
-\left(G_{i k}-\frac{1}{2} g_{i k} G\right)=T_{i k}+\frac{1}{6}\left(j_{i} j_{k}-\frac{1}{2} g_{i k} j^{s} j_{s}\right), \\
\phi_{i k}=\frac{1}{6}\left(\frac{\partial j_{k}}{\partial x^{i}}-\frac{\partial j_{i}}{\partial x^{k}}\right), \\
\frac{\partial \hat{f}^{k s}}{\partial x^{s}}=\hat{j}^{k} .
\end{array}
$$

In (162), the (Riemannian) Ricci tensor $G_{i k}$ and Ricci scalar $G$ are formed from the auxiliary metric $g_{i k}$; the same holds for the tensor $T_{i k}:=-\left(\gamma_{i k}-\frac{1}{2} g_{i k} g^{r s} \gamma_{r s}\right)$. Of course, in the end, $g_{i k}$ and all quantities formed from it will have to be expresses by the affine connection $\Gamma$.

Schrödinger's assignment of mathematical quantities to physical observables is as follows:

$\phi_{i k}$ corresponds to the electromagnetic field tensor $(\overrightarrow{\mathbf{E}}, \overrightarrow{\mathbf{B}})$,

$\hat{f}^{i k}$ corresponds to its conjugate field quantity $(\overrightarrow{-\mathbf{D}}, \overrightarrow{\mathbf{H}})$,

$j^{k}$ corresponds to the electric 4-current density,

$T_{i k}$ corresponds to the "field-energy-tensor of the electromagnetic field".

We note from (163) that the electric current density is the negative of the electromagnetic 4-potential. The meson field is not yet included in the theory.

Up to here, Schrödinger did not specify the Lagrangian in (153). He then assumed:

$$
\overline{\mathcal{L}}=2 \alpha\left\{\sqrt{-\operatorname{det}\left(g_{i k}+\phi_{i k}\right)}-\sqrt{-\operatorname{det}\left(g_{i k}\right)}\right\},
$$

with a numerical constant $\alpha$ (his Eq. $(4,1)$ on p. 51). According to Schrödinger:

" $\mathcal{L}$ is essentially Born's Lagrangian, with $\phi_{k l}$ in place of his $(\overrightarrow{\mathbf{B}}, \overrightarrow{\mathbf{E}})[\ldots] \hat{f}^{i k}$ agrees in

form with Born's contravariant tensor-density $(\overrightarrow{\mathbf{H}}, \overrightarrow{-\mathbf{D}})[\ldots] . "([545]$, p. 52.)

This refers to the paper by Born and Infeld on a non-linear electrodynamics; ${ }^{83} \mathrm{cf}$. Section 5 . At the end of the paper, Schrödinger speculated about taking into account a cosmological constant, and about including a meson field of spin 1 described by a symmetrical rank 2 tensor $\psi_{i k}$ in a more complicated Lagrangian ${ }^{84}$ :

$$
\overline{\mathcal{L}}=2 \alpha\left\{\sqrt{-\operatorname{det}\left(g_{i k}+\phi_{i k}\right)}+\frac{\alpha^{\prime}}{\alpha} \sqrt{-\operatorname{det}\left(g_{i k}+\phi_{i k}+\psi_{i k}\right)}-\sqrt{-\operatorname{det}\left(g_{i k}\right)}\right\} .
$$

As field equations, he obtained the following system:

$$
\begin{array}{r}
G_{i k}=\frac{\alpha}{w}\left(\phi_{i}{ }^{r} \phi_{k r}+g_{i k}(w-1)-\frac{1}{6} j_{i} j_{k}\right), \\
\phi_{i k}=\frac{1}{6}\left(\frac{\partial j_{k}}{\partial x^{i}}-\frac{\partial j_{i}}{\partial x^{k}}\right), \\
\frac{1}{\sqrt{-g}} \frac{\partial}{\partial x^{r}}\left[\frac{\sqrt{-g}}{w}\left(\phi^{k r}-I_{2} \phi^{* k r}\right)\right]=g^{k r} j_{k}, \quad w=\sqrt{1+\frac{1}{2} \phi^{r s} \phi_{r s}-\left(I_{2}\right)^{2}} .
\end{array}
$$

Here, $\phi^{* i k}:=\frac{1}{2} \frac{1}{\sqrt{-g}} \epsilon^{i k r s} \phi_{r s}, I_{2}:=\frac{1}{4} \phi^{* r s} \phi_{r s}$ as in [545], p. 51, Eq. (4,3). For a physical interpretation, Schrödinger re-defined all quantities $g_{i k}, A_{k}, \phi_{i k}, j_{k}$ by multiplying them with constants having physical dimensions. This is to be kept in mind when his papers in which applications were discussed, are compared with this basic publication.

${ }^{83}$ Born and Schrödinger had worked together on absolute constants in field theory, and the uncertainty principle $[48,49]$

${ }^{84}$ This cannot occur in a representation of $\mathrm{SU}(2)$ where spin 1 is related to a skew symmetric tensor. 


\subsubsection{Application: Geomagnetic field}

Schrödinger quickly tried to draw empirically testable consequences from his theory. At first he neglected gravity in his UFT and obtained the equations "for not excessively strong electromagnetic fields":

$$
\begin{aligned}
& \vec{H}=\overrightarrow{\operatorname{rot}} \vec{A}, \quad \vec{E}=-\vec{\nabla} V-\frac{\partial \vec{A}}{\partial t}, \\
& \overrightarrow{\operatorname{rot}} \vec{H}-\frac{\partial \vec{E}}{\partial t}=-\mu^{2} \vec{A}, \quad \operatorname{div} \vec{E}=-\mu^{2} V,
\end{aligned}
$$

in which the electric current 4-density is replaced by the 4-potential; cf. (163). The equations then were applied to the permanent magnetic field of the Earth and the Sun [544]. Deviations from the dipole field as described by Maxwell's theory are predicted by (171). Schrödinger's careful comparison with available data did not show a contradiction between theory and observation, but remained inconclusive. This was confirmed in a paper with the Reverend J. McConnel ${ }^{85}$ [419] in which they investigated a possible (shielding) influence of the earth's altered magnetic field on cosmic rays (as in the aurora).

After the second world war, the later Nobel-prize winner Maynard S. Blackett (1897-1974) suggested an empirical formula relating magnetic moment $M$ and angular momentum $L$ of large bodies like the Earth, the Sun, and the stars:

$$
M=\beta \frac{\sqrt{G}}{2 c} L,
$$

with $G$ Newton's gravitational constant, $c$ the velocity of light in vacuum, and $\beta$ a numerical constant near $\frac{1}{4}$ [28]. The charge of the bodies was unimportant; the hypothetical effect seemed to depend only on their rotation. Blackett's idea raised some interest among experimental physicists and workers in UFT eager to get a testable result. One of them, the Portuguese theoretical physicist Antonio Gião, derived a formula generalizing (172) from his own unified field theory [225, 226]:

$$
M=2 \frac{\xi}{\chi} \frac{m_{0}}{2 e} G L,
$$

where $e, m_{0}$ are charge and rest mass of the electron, $\xi$ a constant, and $\chi$ the "average curvature of space-time".

Blackett conjectured "that a satisfactory explanation of (172) will not be found except within the structure of a unified field theory" [28]. M. J. Nye is vague on this point: "What he had in mind was something like Einstein asymmetry or inequality in positive and negative charges." ([460], p. 105.) Schrödinger seconded Blackett; however, he pointed out that it was "not a very simple thing" to explain the magnetic field generated by a rotating body by his affine theory. "At least a general comprehension of the structure of matter" was a necessary prerequisite ([554], p. 216). The theoretical physicist A. Papapetrou who had worked with Schrödinger joined Blackett in Manchester between 1948 and 1952. We may assume that the experimental physicist Blackett knew of Schrödinger's papers on the earth's magnetism within the framework of UFT and wished to use Papapetrou's expertise in the field. The conceptional link between Blackett's idea and UFT is that in this theory the gravitational field is expected to generate an electromagnetic field whereas, in general relativity, the electromagnetic field had been a source of the gravitational field.

\footnotetext{
85 J. McConnel (1915-1999), since 1968 senior professor at DIAS, 1969 to 1972 Director of the School of Theoretical Physics there.
} 
Theoreticians outside the circle of those working on unified field theory were not so much attracted by Blackett's idea. One of them was Pauli who, in a letter to P. Jordan of July 13, 1948, wrote:

"As concerns Blackett's new material on the magnetism of the earth and stars, I have the following difficulty: In case it is an effect of acceleration the dependency of the angular velocity must be different; in the case of an effect resulting from velocity, a translatory movement ought to also generate a magnetic field. Special relativity then requires that the matter at rest possesses an electric field as well. [...] I do not know how to escape from this dilemma." 86 ([489], p. 543)

Three weeks earlier, in a letter to Leon Rosenfeld, he had added that he "found it very strange that Blackett wrote articles on this problem without even mentioning this simple and important old conclusion." ([489], p. 539) This time, Pauli was not as convincing as usual: Blackett had been aware of the conclusions and discussed them amply in his early paper ([28], p. 664).

In 1949, the Royal Astronomical Society of England held a "Geophysical Discussion" on "Rotation and Terrestrial Magnetism" [519]. Here, Blackett tried to avoid Pauli's criticism by retaining his formula in differential form:

$$
d \underline{-} \sim G^{\frac{1}{2}} d m[\underline{\omega} \times \underset{-}{R}] \times \frac{r}{r^{3}} .
$$

For a translation $\underset{-}{\omega}=0$, and no effect obtains. T. Gold questioned Blackett's formula as being dependent on the inertial system and asked for a radial dependence of the angular velocity $\omega$. A. Papapetrou claimed that Blackett's postulate "could be reconciled with the relativistic invariance requirements of Maxwell's equations" and showed this in a publication containing Eq. (174), if only forcedly so: he needed a bi-metric gravitational theory to prove it [476]. In the end, the empirical data taken from the earth did not support Blackett's hypothesis and thus also were not backing UFT in its various forms; cf. ([19], p. 295).

\subsubsection{Application: Point charge}

A second application pertains to the field of an electrical point charge at rest [548]. Schrödinger introduced two "universal constants" which both appear in the equations for the electric field. The first is his "natural unit" of the electromagnetic field strength $b:=\frac{e}{r_{0}^{2}}$ called Born's constant by him, where $e$ is the elementary charge and $r_{0}:=\frac{e^{2}}{m_{0} c^{2}} \frac{2}{3} \int_{0}^{\infty} \frac{d x}{\sqrt{1+x^{4}}}$ the electron radius (mass $m_{0}$ of the electron). The second is the reciprocal length introduced in a previous publication $f:=\frac{b \sqrt{2 G}}{c^{2}}$ with Newton's gravitational constant $G$ and the velocity of light $c$. Interestingly, the affine connection has been removed from the field equations; they are written as generalized

86 "Betreffend Blackett's neues Material zum Erd- und Gestirnsmagnetismus [... ] habe ich folgende Schwierigkeit: handelt es sich um einen Beschleunigungseffekt, so sollte die Abhängigkeit von der Winkelgeschwindigkeit eine andere sein; ist es aber ein Geschwindigkeitseffekt, dann muss auch eine translatorische Bewegung ein Magnetfeld erzeugen. Nach der speziellen Relativitätstheorie muß dann aber die ruhende Masse ein entsprechendes elektrisches Feld haben. [...] Ich weiss keinen Ausweg aus diesem Dilemma. [...]" 
Einstein-Maxwell equations as in Born-Infeld theory ${ }^{87}$ (cf. Section 5):

$$
\begin{array}{r}
G_{i k}=f^{2}\left(\frac{c^{2}}{b^{2} w} \phi_{i}^{r} \phi_{k r}-g_{i k} \frac{w-1}{w}-\frac{\mu^{2} c^{2}}{b^{2}} A_{i} A_{k}\right), \\
\phi_{i k}=\frac{\partial A_{k}}{\partial x^{i}}-\frac{\partial A_{i}}{\partial x^{k}} \\
\frac{1}{\sqrt{-g}} \frac{\partial}{\partial x^{r}}\left[\frac{\sqrt{-g}}{w}\left(\phi^{k r}-\frac{c^{2}}{b^{2}} I_{2} \phi^{* k r}\right)\right]=\mu^{2} A^{k}, w=\sqrt{1+\frac{1}{2} \frac{c^{2}}{b^{2}} \phi^{r s} \phi_{r s}-\frac{c^{4}}{b^{4}}\left(I_{2}\right)^{2}} .
\end{array}
$$

If only a static electric field is present, $\phi^{* i k}=0$ and thus $I_{2}=\phi^{* i k} \phi_{i k}=0$.

An ansatz for an uncharged static, spherically symmetric line element is made like the one for Schwarzschild's solution in general relativity, i.e.,

$$
d s^{2}=\exp (\nu) d t^{2}-\exp (\lambda) d r^{2}-r^{2}(d \theta)^{2}-r^{2} \sin ^{2} \theta(d \phi)^{2} .
$$

The solution obtained was:

$$
\exp (\nu)=\exp (-\lambda)=1-f^{2}\left(r_{0}\right)^{2} \frac{1}{x} \int_{0}^{x} d x\left(\sqrt{1+x^{4}}-x^{2}\right)
$$

with $x:=\frac{r}{r_{0}}$. The integral is steadily decreasing from $x=0$ to $x \rightarrow \infty$, where it tends to zero. Like the Schwarzschild solution, (178) with (179) has a singularity of the Ricci scalar at $x=0=r$. For $r \rightarrow \infty$ the Schwarzschild (external) solution is reached. According to Schrödinger, "[...] we have here, for the first time, the model of a point source whose gravitational field is accounted for by its electric field energy. The singularity itself contributes nothing" ([548], p. 232).

Two weeks later, Schrödinger put out another paper in which he wrote down 16 "conservation identities" following from the fact that his Lagrangian is a scalar density and depends only on the 16 components of the Ricci tensor. He also compared his generalization of general relativity with Weyl's theory gauging the metric (cf. [689]), and also with Eddington's purely affine theory ([140], chapter 7, part 2). From (158) it is clear that Schrödinger's theory is not gauge-invariant. ${ }^{88} \mathrm{He}$ ascribed this weakness to the missing of a third fundamental field in the theory, the meson field. According to Schrödinger the absence of the meson field was due to his restraint to a symmetric connection. Eddington's theory with his general affine connection would house all the structures necessary to include the third field. It should take fifteen months until Schrödinger decided that he had achieved the union of all three fields.

\subsection{Semi-symmetric connection}

Schrödinger's next paper on UFT continued this line of thought: in order to be able to include the mesonic field he dropped the symmetry-condition on the affine connection ([549], p. 275). This brings homothetic $V_{i k}$ curvature into the game (cf. Section 2.3.1, Eq. (65)). Although covariant differentiation was introduced through $\bar{\nabla}_{k} X^{i}$ and $\bar{\nabla}_{k} \omega_{i}$, in the sequel Schrödinger split the connection according to $L_{i j}{ }^{k}=\Gamma_{i j}{ }^{k}+S_{i j}{ }^{k}$ and used the covariant derivative $\stackrel{0}{\nabla}$ (cf. Section 2.1.2) with regard to the symmetric part $\Gamma$ of the connection. ${ }^{89}$ In his first attempt, Schrödinger restricted

\footnotetext{
87 The seeming differences in the constants appearing in comparison with (167) - (169) result from a re-definition of the fields by Schrödinger.

88 The gauge transformations would have the form $g_{i k} \rightarrow \lambda g_{i k}, j_{k} \rightarrow j_{k}+a_{0}(\ln (\lambda))_{, k}$.

89 In Schrödinger's notation the homothetic curvature $\underline{V}_{i k}$ is denoted by $S_{i k}$. Furthermore, torsion $U_{i j}{ }^{k}$ corresponds to $S_{i j}{ }^{k}$. He denoted vector torsion by $V_{i}$.
} 
torsion to non-vanishing vector torsion by assuming:

$$
S_{i j}{ }^{k}=\delta_{i}^{k} Y_{j}-\delta_{j}^{k} Y_{i}
$$

with arbitrary 1-form $Y=Y_{i} d x^{i}$. Perhaps this is the reason why he speaks of "weakly asymmetric affinity" without giving a precise definition. Schouten called this type of connection "semisymmetric" (cf. Section 5 of Part I, Eq. (132)). Obviously, vector torsion $S_{i}=-3 Y_{i}$. The two contractions of the curvature tensor $K_{-}^{i}{ }_{j k l}$, i.e., $K_{-}$and $V_{-}$ik are brought into the form [cf. (63)]:

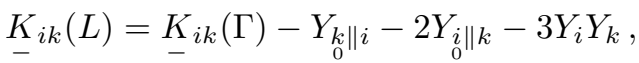

$$
\begin{aligned}
& \underline{V}_{i k}(L)=\underline{V}_{i k}(\Gamma)-3 Y_{i \| k}+3 Y_{0_{0} \| i} \text {. }
\end{aligned}
$$

Here, ${ }_{0}^{i \| k}$ denotes covariant differentiation with respect to the symmetric connection $\Gamma$. Two linear combinations of these tensors are introduced:

$$
M_{i k}:=\frac{1}{4}\left(V_{-i k}+K_{-}[i k]\right), \quad P_{i k}:=\frac{3}{2} K_{-}{ }_{i k}-\frac{1}{2} K_{-} k i+\frac{1}{2} V_{-} .
$$

A calculation shows that:

$$
P_{i k}=\left(K_{i k}\right)_{\Gamma}+Y_{k \| i}-4 Y_{i \| k}-3 Y_{i} Y_{k}
$$

with $\left(K_{i k}\right)_{\Gamma}$ being the Ricci-tensor formed with the symmetrized connection, and

$$
M_{i k}=Y_{k \| i}-Y_{i \| k}=Y_{k, i}-Y_{i, k} .
$$

In the same way as in (154) two tensor densities are introduced:

$$
\hat{g}^{i k}:=\frac{1}{2}\left(\frac{\partial \mathcal{L}}{\partial P_{i k}}+\frac{\partial \mathcal{L}}{\partial P_{k i}}\right), \quad \hat{f}^{i k}:=\frac{1}{2}\left(\frac{\partial \mathcal{L}}{\partial P_{i k}}-\frac{\partial \mathcal{L}}{\partial P_{k i}}\right),
$$

and a third one according to

$$
\hat{m}^{i k}=\frac{\partial \mathcal{L}}{\partial M_{i k}} .
$$

Conjugated variables to $\hat{g}^{i k}, \hat{f}^{i k}$ are [cf. (159]:

$$
\gamma_{i k}:=P_{(i k)}, \quad \phi_{i k}:=P_{[i k]} .
$$

The Lagrangian is to depend only on $P_{i k}$ and $M^{i k}$. A (symmetric) metric tensor $g_{i k}$ is introduced as in (157). Variation of the Lagrangian with respect to $P_{i k}$ and $M^{i k}$ leads to field equations now containing terms from homothetic curvature:

$$
\hat{g}^{i k}{ }_{\| l}+\delta_{l}^{k}\left(\frac{1}{3} \hat{f}^{i s}{ }_{\| s}-\hat{g}^{i s} Y_{s}\right)+\delta_{l}^{i}\left(\frac{1}{3} \hat{f}^{k s}{ }_{\| s}-\hat{g}^{k s} Y_{s}\right)+3 \hat{g}^{i k} Y_{l}
$$

and to the same equation (156) as before, as well as to an additional equation:

$$
\hat{m}_{\| s}^{i s}=\frac{\partial \hat{m}^{i s}}{\partial x^{s}}=0 .
$$

According to Schrödinger, (185) and (190) "form a self-contained Maxwellian set". The formal solution for the symmetric part of the connection replacing (158) now becomes:

$$
\Gamma_{i j}{ }^{k}=\left\{{ }_{i j}^{k}\right\}-\frac{1}{2} g_{i j} j^{k}+\delta_{i}{ }^{k}\left(\frac{1}{6} j_{j}+Y_{j}\right)+\delta_{j}^{k}\left(\frac{1}{6} j_{i}+Y_{i}\right) .
$$


In this paper, Schrödinger changed the relation between mathematical objects and physical observables:

The variables $(j, \phi, \hat{f})$ related to the Ricci tensor correspond to the meson field; whereas $(Y, M, \hat{m})$ related to torsion describe the electromagnetic field.

His main argument was:

"Now the gravitational field and the mesonic field are actually, to all appearance, universally and jointly produced in the same places, viz. in the heavy nuclear particles. They have at any rate their principal seat in common, while there is absolutely no parallelism between electric charge and mass" ([549], p. 282).

In addition, Schrödinger referred to Einstein's remark concerning the possibility of exchanging the roles of the electromagnetic fields $\overrightarrow{\mathbf{E}}$ by $\overrightarrow{\mathbf{H}}$ and $\overrightarrow{-\mathbf{D}}$ by $\overrightarrow{-\mathbf{B}}$ ([142], p. 418). "Now a preliminary examination of the wholly non-symmetrical case gives me the impression that the exchange of rôles will very probably be imperative, [...]" ([549], p. 282).

As to the field equations, they still were considered as preliminary because: "the investigation of the fully non-symmetric case is imperative and may have surprises in store." ([549], p. 282.) The application of Weyl's gauge transformations $g_{i k} \rightarrow \lambda g_{i k}$ in combination with

$$
Y_{k} \rightarrow Y_{k}-\frac{1}{3 \lambda} \frac{\partial \lambda}{\partial x^{k}}, \quad j_{k} \rightarrow j_{k}-\frac{1}{\lambda} \frac{\partial \lambda}{\partial x^{k}}
$$

leaves invariant $\Gamma_{i j}{ }^{k}, \phi_{i k}$, and $M_{i k}$, but changes $\gamma_{i k}$ and destroys the vanishing of the divergence $\hat{j}_{s}^{s}$. Schrödinger thought it to be "imperative to distinguish between the potential $j_{k}$ and the current-and-charge $j_{k}$ the two coinciding only in the original gauge." ([549], p. 284.) He also claimed that only the gravitational and mesonic fields had an influence on the auto-parallels (cf. Section 2.1.1, Eq. (22)).

These first two papers of Schrödinger were published in the Proceedings of the Royal Irish Academy, a journal only very few people would have had a chance to read, particularly during World War II, although Ireland had stayed neutral. Schrödinger apparently believed that, by then, he had made enough progress in comparison with Eddington's and Einstein's publications. ${ }^{90}$ Hence, he wrote a summary in Nature for the wider physics community [547]. At the start, he very nicely laid out the conceptual and mathematical foundations of affine geometry and gave a brief historical account of its use within unified field theory. After supporting "the superiority of the affine point of view" he discussed the ambiguities in the relation between mathematical objects and physical observables. An argument most important to him came from the existence of

"a third field [...], of equally fundamental standing with gravitation and electromagnetism: the mesonic field responsible for nuclear binding. Today no field-theory which does not embrace at least this triad can be deemed satisfactory at all." ([549], p. 574.) $)^{91}$

He believed to have reached "a fully satisfactory unified description of gravitation, electromagnetism and a 6-vectorial meson." ([547], p. 575.) Schrödinger claimed a further advantage of his approach from the fact that he needed no "special choice of the Lagrangian" in order to make the connection between geometry and physics, and for deriving the field equations.

\footnotetext{
90 That Schrödinger was satisfied with his first paper may be seen in its abbreviation $\mathrm{G} U \mathrm{~T}$ by him when he referred to it; GUT means "good" in German. The same gimmick later reappeared in connection with "Grand Unified Field Theories" ([256], p. 168).

91 At the time the distinction between the roles of the spin $1 / 2 \mu$-mesons which are leptons and the baryonic $\pi$-mesons of spin 0 or integer value had not yet been fully understood. Schrödinger considered bosonic mesonsonly.
} 
As to quantum theory, Schrödinger included a disclaimer (in a footnote): "The present article does not touch on it and has therefore to ignore such features in the conventional description of physical fields as are concerned with their quantum character [...]." ([549], p. 574.)

In a letter to Einstein of 10 October 1944, in a remark about an essay of his about Eddington and Milne, M. Born made a bow to Einstein:

"My opinion is that you have the right to speculate, other people including myself have not. [...] Honestly, when average people want to procure laws of nature by pure thinking, only rubbish can result. Perhaps Schrödinger can do it. I would love to know what you think about his affine field theories. I find all of it beautiful and full of wit; but whether it is true? [...]" ([168], p. 212-213) ${ }^{92}$

92 "Ich meine aber, daß Du das Recht hast zu spekulieren, andere Leute aber nicht, auch ich nicht. [...] Ich meine ganz ehrlich, wenn Durchschnittsleute sich durch reines Denken Naturgesetze verschaffen wollen, so kommt nur Mist heraus. Schrödinger kann das vielleicht. Ich wüsste gern, was Du über seine affinen Feldtheorien denkst. Ich find alles schön und geistreich; aber ob es wahr ist? [...]" 


\section{Mixed Geometry: Einstein's New Attempt}

After his move to Princeton, Einstein followed quite a few interests different from his later work on UFT. In the second half of the 1930s he investigated equations of motion of point particles in the gravitational field in the framework of his general relativity theory (with N. Rosen L. Infeld, and B. Hoffmann), and the conceptual intricacies of quantum mechanics (with B. Podolsky and N. Rosen). As we have seen in Section 3.2, in 1938-1943 he had turned back to Kaluza's 5-dimensional UFT (with P. G. Bergmann, V. Bargmann, and in one paper with W. Pauli) to which theory he had given his attention previously, in 1926-1928; cf. Section 6.3 of Part I. After joint work with V. Bargmann on bi-vector (bilocal) fields in 1944 (cf. Section 3.3.1), he took up afresh his ideas on mixed geometry of 1925 . He then stayed within this geometrical approach to UFT until the end of his life.

In the period 1923-1933 Einstein had tried one geometry after the other for the construction of UFT, i.e., Eddington's affine, Cartan's tele-parallel, Kaluza's 5 dimensional Riemannian geometry, and finally mixed geometry, a blend of affine geometry and Foerster's (alias Bach's) idea of using a metric with a skew-symmetric part. Unlike this, after the second world war he stuck to one and the same geometry with asymmetric fundamental tensor and asymmetric affine connection. The problems dealt with by him then were technical at first: what fundamental variables to chose, what "natural" field equations to take, and how to derive these in a satisfactory manner. Next, would the equations chosen be able to provide a set of solutions large enough for physics? Would they admit exact solutions without singularities? In physics, his central interest was directed towards the possibilities for the interpretation of geometrical objects as physical observables. During his life, he believed that the corpus of UFT had not yet become mature enough to allow for a comparison with experiment/observation. His epistemological credo lead him to distrust the probability interpretation of quantum theory as a secure foundation of fundamental physical theory; for him quantum mechanics amounted to no more than a useful "model". His philosophical position may also have demotivated him to the extent that, already in the late 1930s, he had given up on learning the formalism of quantum field theory in order to be able to follow its further development. ${ }^{93}$ To see him acquire a working knowledge of quantum field theory as a beginner, after World War II, would have been asking too much in view of his age and state of health. That he did not take into account progress in nuclear and elementary particle physics reached in the two decades since he first had looked at mixed geometry, was a further factor isolating him from many of his well known colleagues in theoretical physics.

As we shall note in Section 10.5.1, in 1942-1944 Einstein's interest was also directed to the question to what extent singularities occur in the solutions of the field equations of general relativity and of Kaluza-Klein. It is during this time that he wrote to Hans Mühsam in spring of 1942 (as quoted by Seelig [570], p. 412):

"But in my work I am more fanatical than ever, and really hope to have solved my old problem of the unity of the physical field. Somehow, however, it is like with an airship with which we can sail through the clouds but not clearly see how to land in reality, i.e on the earth." 94

Einstein's first three papers on UFT via mixed geometry $([142,147,179])$ all employ the metric $g$ and the connection $L$ as independent variables - with altogether 80 available components in local

\footnotetext{
93 A. Pais reported that Einstein had asked V. Bargmann "for a private survey of quantum field theory, beginning with second quantization". After one month "Einstein's interest waned." [469], p. 463.

94 "Aber im Arbeiten bin ich fanatischer als je und habe wirklich Hoffnung, mein altes Problem von der Einheit des Physikalischen Feldes gelöst zu haben. Es ist aber wie bei einem Luftschiff, mit dem man zwar in den Wolken herumsegeln kann, nicht aber klar sieht, wie man damit in der Realität, das heißt auf der Erde landen soll."
} 
coordinates while just $6+10$ of them are needed for a description of the gravitational and electromagnetic fields. (The number of the inherent "degrees of freedom" is a more complicated affair.) A strategy followed by Einstein and others seems to have been to remove the superfluous 64 variables in the affine connection by expressing them by the components of the metric, its first derivatives, and the torsion tensor. Since the matter variables were to be included in the geometry, at least in the approach to UFT by Einstein, enough geometrical objects would have to be found in order to represent matter, e.g., 4 components of the electric 4-current, 4 components of the magnetic 4current, 5 components for an ideal fluid, more for the unspecified energy-momentum-tensor in total. In Einstein's approach, the symmetric part of the metric, $h_{i j}$, is assumed to correspond to inertial and gravitational fields while the antisymmetric part $k_{i j}$ houses the electromagnetic field. The matter variables then are related to derivatives of the metric and connection (cf. Section 10.3.1). The field equations would have to be derived from such a Lagrangian in such a way that general relativity and Maxwell's equations be contained in UFT as limiting cases. Unlike the situation in general relativity, in metric-affine geometry a two-parameter set of possible Lagrangians linear in the curvature tensor (with the cosmological constant still to be added) does exist if homothetic curvature in (65), (66) of Section 2.3.1 is included. Nevertheless, Einstein used a Lagrangian corresponding (more or less) to the curvature scalar in Riemannian geometry $\sqrt{-\operatorname{det}\left(g_{i k}\right)} g^{l m} K_{l m}(L)$ without further justification. ${ }^{95}$ Such field equations, the main alternatives of which came to be named strong and weak, were used to express the connection as a complicated functional of the metric and its derivatives and to determine the two parts, symmetric and skew-symmetric, of the metric. This was fully achieved not before the 1950s; cf. Section 10.1.

Interestingly, in his second paper of 1945 using mixed geometry, Einstein did not mention his first one of 1925. It seems unrealistic to assume that he had forgotten what he had done twenty years earlier. His papers had been published in the proceedings of the Prussian Academy of Science in Berlin. Possibly, he just did not want to refer to the Prussian Academy from which he had resigned in 1933, and then been thrown out. This is more convincing than anything else; he never ever mentioned his paper of 1925 in a publication after 1933 [312]. There is a small difference between Einstein's first paper using mixed geometry [142] and his second [147]: He now introduced complex-valued fields on real space-time in order to apply what he termed "Hermitian symmetry"; cf. (46). After Pauli had observed that the theory could be developed without the independent variables being complex, in his next (third) paper Einstein used "Hermitian symmetry" in a generalized meaning, i.e., as transposition invariance [179]; cf. Section 2.2.2.

\subsection{Formal and physical motivation}

Once he had chosen geometrical structures, as in mixed geometry, Einstein needed principles for constraining his field equations. What he had called "the principle of general relativity", i.e., the demand that all physical equations be covariant under arbitrary coordinate transformations ("general covariance"), became also one of the fundamentals of Einstein's further generalization of general relativity. There, the principle of covariance and the demand for differential equations of 2nd order (in the derivatives) for the field variables had led to a unique Lagrangian $\left(\frac{1}{2 \kappa} R+\Lambda\right) \sqrt{-g}$, with the cosmological constant $\Lambda$ being the only free parameter. In UFT, with mixed geometry describing space-time, the situation was less fortunate: From the curvature tensor, two independent scalar invariants could be formed. Moreover, if torsion was used as a separate constructive element offered by this particular geometry, the arbitrariness in the choice of a Lagrangian increased considerably. In principle, in place of the term with a single cosmological constant $g_{i j} \Lambda$, a further

\footnotetext{
95 It can only be stressed that the curvature tensor $K_{i j k}^{l}(L)$ of a general affine connection $L$ allows for two contractions, corresponding to an (asymmetric) Ricci tensor $K_{i k}=K^{l}{ }_{i k l}$ and to what is called "homothetic curvature" $V_{i k}=K_{l i k}^{l}$. Schrödinger had included it in a theory; later Tonnelat (cf. Section 10.2) and Sciama [565] would also use homothetic curvature.
} 
term with two constants could be added: $h_{i j} \Lambda^{\prime}$ and $k_{i j} \Lambda^{\prime \prime}$.

In his paper of 1945 Einstein gave two formal criteria as to when a theory could be called a "unified" field theory. The first was that "the field appear as a unified entity", i.e., that it must not be decomposable into irreducible parts. The second was that "neither the field equations nor the Hamiltonian function can be expressed as the sum of several invariant parts, but are formally united entities". He readily admitted that for the theory presented in the paper, the first criterion was not fulfilled ([147], p. 578). As remarked above, the equations of general relativity as well as Maxwell's equations ought to be contained within the field equations of UFT; by some sort of limiting process it should be possible to regain them. A further requirement was the inclusion of matter into geometry: some of the mathematical objects ought to be identified with physical quantities describing the material sources for the fields. With UFT being a field theory, the concept of "particle" had no place in it. Already in 1929, in his correspondence with Elie Cartan, Einstein had been firm on this point:

"Substance, in your sense means the existence of timelike lines of a special kind. This is the translation of the concept of particle to the case of a continuum. [...] the necessity of such translatibility, seems totally unreasonable as a theoretical demand. To realize the essential point of atomic thought on the level of a continuum theory, it is sufficient to have a field of high intensity in a spatially small region which, with respect to its "timelike" evolution, satisfies certain conservation laws [...]." ([116], p. 95.) $)^{96}$

Thus, the discussion of "equations of motion" would have to use the concept of thin timelike tubes, and integrals over their surface, or some other technique. Einstein kept adamantly to this negatory position concerning point particles as shown by the following quote from his paper with N. Rosen of 1935 [178]:

"[...] writers have occasionally noted the possibility that material particles might be considered as singularities of the field. This point of view, however, we cannot accept at all. For a singularity brings so much arbitrariness into the theory that it actually nullifies its laws."

As we will see below, an idea tried by Einstein for the reduction of constructive possibilities, amounted to the introduction of additional symmetries like invariance with respect to Hermitian (transposition-) substitution, and later, $\lambda$-transformations; cf. Sections 2.2.2 and 2.2.3. Further comments on these transformations are given in Section 9.8.

Interestingly, the limiting subcase in which the symmetric part of the (asymmetric) metric is assumed to be Minkowskian and which would have lead to a generalization of Maxwell's theory apparently has been studied rarely as an exact, if only heavily overdetermined theory; cf. however $[450,600,502]$.

\subsection{Einstein 1945}

As early as in 1942, in his attempts at unifying the gravitational and electromagnetic fields, Einstein had considered using both a complex valued tensor field and a 4-dimensional complex space as a new framework. About this, he reported to his friend M. Besso in August 1942 ([163], p. 367):

"What I now do will seem a bit crazy to you, and perhaps it is crazy. [...] I consider a space the 4 coordinates $x^{1}, \ldots x^{4}$ which are complex such that in fact it is an 8 dimensional space. To each coordinate $x^{i}$ belongs its complex conjugate $x^{i}$. [...] In

\footnotetext{
${ }^{96}$ Here, the English translation given in [116] has been taken over.
} 
place of the Riemannian metric another one of the form $g_{i k}$ obtains. We ask it to be real, i.e., $g_{i k}=\bar{g}_{k i}$ must hold (Hermitian metric). The $g_{i k}$ are analytical functions of the $x^{i}$, and $x^{i}$. [ ...]"97

He then asked for field equations and for complex coordinate transformations. "The problem is that there exist several equations fulfilling these conditions. However, I found out that that this difficulty goes away if attacked correctly, and that one can proceed almost as with Riemann" ([163], p. 367-368).

During the 3 years until he published his next paper in the framework of mixed geometry, Einstein had changed his mind: he stuck to real space-time and only took the field variables to be complex [147]. He was not the first to dabble in such a mathematical structure. More than a decade before, advised by A. Eddington, Hsin P. Soh ${ }^{98}$, during his stay at the Massachusetts Institute of Technology, had published a paper on a theory of gravitation and electromagnetism within complex four-dimensional Riemannian geometry with real coordinates. The real part of the metric "[...] is associated with mass (gravitation) and the imaginary part with charge (electromagnetism)" ([581], p. 299).

Einstein derived the field equations from the Lagrangian ${ }^{99}$

$$
\mathcal{H}=K_{i k}{ }^{*} \hat{g}^{i k}
$$

with $^{100}$

$$
K_{i k}^{*}=: \stackrel{\text { Her }}{K}_{i k}-X_{\underline{i} \| k}
$$

and

$$
X_{i}=\frac{\partial(\log \sqrt{-g})}{\partial x_{i}}-\frac{1}{2}\left(L_{i m}^{m}+L_{m i}^{m}\right) .
$$

Here, $\stackrel{\text { Her }}{K}$ ik is the Hermitian-symmetrized Ricci-tensor obtained from the curvature tensor (54) as: $\stackrel{\mathrm{Her}}{K}_{i k}=\frac{1}{2}\left(\underline{-}_{i m k}^{m}+\underline{-}^{m}{ }_{k m i}\right) \quad($ cf. (73) of Section 2.3.2):

$$
\stackrel{\mathrm{Her}}{K}_{i k}=L_{i k}{ }_{, l}^{l}-L_{i m}{ }^{l} L_{l k}{ }^{m}-\frac{1}{2}\left(L_{i l}{ }_{, k}{ }^{l}+L_{l k}{ }_{, i}\right)+\frac{1}{2} L_{i k}{ }^{m}\left(L_{m l}{ }^{l}+L_{l m}{ }^{l}\right),
$$

97 "Was ich nun mache, wird Dir etwas verrückt erscheinen, und ist es vielleicht auch.[...] Ich betrachte einen Raum, dessen vier Koordinaten $x^{1}, \ldots x^{4}$ komplex sind, sodass es eigentlich ein 8-dimensionaler Raum ist. Zu jeder Koordinate $x^{i}$ gehört also der konjugiert komplexe $x^{i}$. [...] Anstelle der Riemann-Metrik tritt eine solche von der Form $g_{i k}$. Diese soll reell sein, was verlangt, dass $g_{i k}=\bar{g}_{k i}$ ist (hermitische Metrik). Die $g_{i k}$ sind analytische

Funktionen der $x^{i}$ und $x^{i}$. [...]"

98 Hsin P. Soh (Shu Xingbei) (1905-1983), after an education at Chinese Universities, continued his physics and mathematics studies at the University of California in San Francisco, at Cambridge University (with Eddington), and then at the Massachusetts Institute of Technology (with D. J. Struik). He received the chair position in the Department of Mathematics of Jinan University (Shanghai) and became professor at Zhejiang University (Hangzhou). His most famous student is the Nobel Prize winner Tsung-Dao Lee. Soh was purged heavily as anti-revolutionary in 1958 and rehabilitated fully only in 1979.

99 Termed Hamiltonian by him.

100 In Einstein's notation $S_{i} \| k$ corresponds to $S_{i} \| k$ of (16). We have replaced his $S_{i}$ by $X_{i}$ because of our denotation of the torsion vector (cf. definition after (15) in Section 2.1.1). Here, Einstein's $R_{i k}{ }^{*} \simeq K_{i k}{ }^{*}$ and his $R_{i k} \simeq \stackrel{\text { Her }}{K}_{i k}$. Due to the fields now taking values in the complex numbers, the symbol $\tilde{A}$ for the Hermitian conjugate is replaced by the symbol $\bar{A}$ for complex conjugation. 
with $L$ being a connection with Hermitian symmetry. Note that $K_{i k}{ }^{*}$ is not Hermitian thus implying a non-Hermitian Lagrangian $\mathcal{H}$.

The quantities to be varied are $\hat{g}^{i k}, L_{i k}{ }^{l}$. From $\delta \mathcal{H}=-\hat{U}_{m}^{i k} \delta L_{i k}^{m}+G_{i k} \delta \hat{g}^{i k}$, Einstein then showed that the first equation of the field equations:

$$
\hat{U}_{m}^{i k}=0, \quad G_{i k}=0
$$

is equivalent to the compatibility equation (30) while the second may be rewritten as

$$
G_{i k}=K_{i k}^{*}-\frac{1}{2 \sqrt{-g}}\left(\hat{g}^{l m} L_{l}\right)_{, m} g_{i k} .
$$

The proofs are somewhat circular, however, because he assumed (30) beforehand. He also claimed that a proof could be given that the equation

$$
L_{i}=0=\frac{1}{2}\left(L_{i m}^{m}-L_{m i}^{m}\right),
$$

expressing the vanishing of vector torsion, could be added to the field equations (197). Its second Eq. (198) would then bear a striking formal resemblance to the field equations of general relativity. The set

$$
\begin{array}{r}
0=g_{i k \| l}:=g_{i k, l}-g_{r k} L_{i l}^{r}-g_{i r} L_{l k}{ }^{r}, \\
S_{j}(L):=L_{[i m]}^{m}=0, \\
K_{j k}(L)=0
\end{array}
$$

with a more general connection $L$ later was named the strong field equations.

Equation (200) replaced the covariant constancy of the metric in general relativity, although, in general, it does not preserve inner products of vectors propagated parallelly with the same connection. The problem was already touched in Section 2.1.2: how should we define $\left(g_{i k} A^{i} B^{k}\right)_{\| l}$ ?

M. Pastori has shown that ([485], p. 112):

$$
\left(g_{i k} A^{i} B^{k}\right)_{, s} d x^{s}=\left(g_{+k-l} A^{i} B^{k}+g_{i k} A_{\| l}^{+} B^{k}+g_{i k} A^{i} B_{\| l}^{-\underline{k}}\right) .
$$

With the help of (20) and (31), we can re-write (203) in the form:

$$
\left(g_{i k} A^{i} B^{k}\right)_{, s} d x^{s}=\left(g_{i k-} \| l A^{i} B^{k}+g_{i k} A_{\| l}^{i} B^{k}+g_{i k} A^{i} B_{\| l}^{\stackrel{k}{0}}\right) .
$$

Hence, besides the connection $L_{i j}{ }^{k}$ a second one $L_{(i j)}{ }^{k}$ enters.

\subsection{Einstein-Straus 1946 and the weak field equations}

It turned out that the result announced, i.e., $L_{i}=0$, could not be derived within the formalism given in the previous paper ([147]). Together with his assistant Ernst Straus ${ }^{101}$, Einstein wrote

101 Ernst Gabor Straus (1922-1983) had to leave his birthplace Munich in 1933 with his family. He obtained his doctorate in mathematics at Columbia University in New York (1948) with Albert Einstein as his second adviser. He became an assistant of Einstein at the Institute for Advanced Study from 1950-1953. He spent his later academic career at the University of California, Los Angeles. 
a follow-up in which the metric field did not need to be complex [179]. Equation (199) now is introduced by a Lagrangian multiplier $\hat{A}^{i}$. The new Lagrangian is given by:

$$
\mathcal{H}=P_{i k} \hat{g}^{i k}+\hat{A}^{i} L_{i}+b_{k} \hat{g}_{, m}^{[k m]},
$$

where $P_{i k}$ is the same quantity as $\stackrel{\mathrm{Her}}{K}_{i k}$ of [147]; cf. also Eq. (74) of Section 2.3.2. ${ }^{102}$ The variables to be varied independently are $\hat{g}^{i k}, L_{i k}{ }^{l}$, and the multipliers $\hat{A}^{i}, b_{k}$. After some calculation, the following field equations arose:

$$
\begin{aligned}
L_{i} & =0, \\
\hat{g}^{i k}{ }_{\| l} & =0, \\
P_{(i k)} & =0, \\
P_{[i k], l}+P_{[k l], i}+P_{[l i], k} & =0 .
\end{aligned}
$$

The last Eq. (208) is weaker than $P_{[i k]}=0$; therefore this system of equations is named the weak field equations of UFT. However, cf. Section 9.2.2 for a change in Einstein's wording. From the calculations involved, it can be seen that (206) is equivalent to (30), and that $\hat{A}^{i}$ vanishes. The second multiplier satisfies:

$$
b_{i, k}-b_{k, i}=2 P_{[i k]} .
$$

On first sight, according to Eqs. (206) and (208), either the skew-symmetric part of $\hat{g}^{i k}$, or the skew-symmetric part of $P_{i k}$ might be related to the electromagnetic field tensor. In the paper, homothetic curvature $V_{i k}$ is introduced but not included in the Lagrangian. It will vanish as a consequence of the field equations given

In linear approximation, the ansatz $g_{i j}=\eta_{i j}+\gamma_{i j}$ is used with $\gamma_{(i j)}$ related to the gravitational and $\gamma_{[i j]}$ to the electromagnetic field. For the skew-symmetric part $\gamma_{[i j]}$ of the metric, the field equations reduce to

$$
\begin{aligned}
\eta^{j k} \gamma_{[i j], k} & =0 \\
\eta^{m n} \partial_{m} \partial_{n}\left(\gamma_{[i k], l}+\gamma_{[k l], i}+\gamma_{[l i], k}\right) & =0 .
\end{aligned}
$$

The system (210), (211) is weaker than the corresponding Maxwell's equations in vacuum. According to the authors, this is no valid objection to the theory "since we do not know to which solutions of the linearized equations there correspond rigorous solutions which are regular in the entire space." Only such solutions are acceptable but: "Whether such (non-trivial) solutions exist is as yet unknown" ([179], p. 737).

Einstein and Straus then discussed whether (207) and (208) could be replaced by $P_{i k}=0$. By again looking at the linear approximation, they "get a dependence of the electric from the gravitational field which cannot be brought in accord with our physical knowledge [...]" ([179], p. 737).

In the long last paragraph of the paper, the authors derive necessary and sufficient (algebraic) conditions for $g_{i k}$ in order that the Eqs. (30) or (206) determine the connection (in terms of the metric) "uniquely and without singularities". If we set $I_{1}:=\operatorname{det}\left(h_{i k}\right) ; I_{2}:=\frac{1}{4} \epsilon^{i j k l} \epsilon^{a b c d} h_{i a} h_{j b} k_{k c} k_{l d}$, and $I_{3}:=\operatorname{det}\left(k_{i k}\right)$, then they are given by ${ }^{103}$ :

$$
\begin{aligned}
I_{1} & \neq 0, \\
g=I_{1}+I_{2}+I_{3} & \neq 0, \\
\left(I_{1}-I_{2}\right)^{2}+I_{3} & \neq 0 .
\end{aligned}
$$

102 Do not mix up Einstein's $P_{i k}$ with Schrödinger's [cf. (183)].

103 Einstein used the notation $s_{i j}\left(a_{i j}\right)$ in place of $h_{i j}\left(k_{i j}\right)$ of this article. 
The second equation in (212) is equivalent to (8) in Section 2.1. We will have to compare this result with those to be given in Section 10, and in Section 12.2.

Einstein wrote on 6 March 1947 to Schrödinger that he:

"really does not yet know, whether this new system of equations has anything to do with physics. What justly can be claimed only is that it represents a consequent generalization of the gravitational equations for empty space." 104

And four months later (16 July 1946), Einstein confessed to Schrödinger:

"As long as the $\Gamma$ cannot be expressed by the $g_{i k, l}$ in the simplest way, one cannot hope to solve exact problems. Due to the diligence and inventiveness of my assistant Straus, we will have reached this goal, soon." 105

Both quotes are taken from the annotations of K. von Meyenn in ([489], p. 383). In fall, Pauli who had returned to Zürich wrote to Einstein:

"Schrödinger told me something about you. But I do not know whether you still keep to the field equations which you investigated with Straus at the time of my departure from Princeton (end of February). My personal conviction remains - not the least because of the negative results of your own numerous tries - that classical field theory in whatever form is a completely sucked out lemon from which in no way can spring something new. But I myself do not yet see a path, which leads us further in the principal questions." ([489], p. 384)

Unimpressed, Einstein went on squeezing the lemon for the next nine years until his death. On 9 April 1947, he wrote to his friend from student days, Maurice Solovine (1875-1958):

"I labour very hard with my Herr Straus at the verification (or falsification) of my equations. However, we are far from overcoming the mathematical difficulties. It is hard work for which a true mathematician would not at all muster the courage." $([160], \text { p. } 84)^{106}$

And, as may be added, for which a genuine true mathematician possibly would not muster enough interest. After all, the task is the resolution of a system of linear equations, well-known in principle, but hard to control for 64 equations. Nevertheless, Einstein's assistant in Princeton, E. Straus, in dealing with the weak field equations, continued to work at the problem of solving (206) for the connection. He worked with tensor algebra and presented a formal solution (cf. Eq. (1.9), p. 416 of [592]). However, it was not only unwieldy but useless in practice. Yet, the mathematical difficulty Einstein blamed for the slow progress made, was "the integration of malicious non-linear equations" (letter to H. Zangger of 28 July 1947 in [560], p. 579).

How appropriate Pauli's remark was, is made clear by a contemporary paper on "non-symmetric gravity theories". Damour, Deser \& McCarthy show that the theories of Einstein and Einstein \& Straus (together with further geometrical theories) "violate standard physical requirements" such

\footnotetext{
104 daß er "durchaus noch nicht weiß, ob dieses neue Gleichungssystem irgend etwas mit Physik zu tun hat. Was man wohl mit Recht behaupten kann, ist nur, daß es eine folgerichtige Verallgemeinerung der Gravitationsgleichungen des leeren Raumes darstellt."

105 "Solange man die $\Gamma$ nicht in der einfachsten Weise aus den $g_{i k, l}$ ausdrücken kann, hat man keine Hoffnung, strenge Probleme lösen zu können. Dank der wirklich großen Geschicklichkeit und Ausdauer meines Assistenten Straus werden wir in kurzem so weit sein."

106 "Ich plage mich sehr mit der Verifizierung (oder Falsifizierung) meiner Gleichungen mit meinem Herrn Straus. Wir sind aber weit von einer Überwindung der mathematischen Schwierigkeiten. Es ist ein hartes Geschäft, zu dem ein richtiger Mathematiker überhaupt die Courage nicht aufbringen würde."
} 
as to be free of ghosts ${ }^{107}$ and with absence of algebraic inconsistencies [101, 102]. On the other hand, the authors showed that the following Lagrangian, closely related to an expansion in powers of $k_{i j}=g_{[i j]}$ of the Einstein-Straus Lagrangian, would be acceptable:

$$
\mathcal{L}^{I}=\sqrt{h}\left[R(h)-\frac{1}{12} H_{r s t} H^{r s t}-\frac{2}{3} k^{l m}\left(\partial_{l} S_{m}-\partial_{m} S_{l}\right)\right]
$$

with $H_{r s t}:=\partial_{r} k_{s t}+\partial_{t} k_{r s}+\partial_{s} k_{t r}$. Indices are moved with the symmetric part of the asymmetric metric [100].

Another link from Einstein's Hermitian theory to modern research leads to "massive gravity" theories, i.e., speculative theories describing an empirically unknown spin-2 particle (graviton) with mass $[76,255]$. However, it is not clear whether these theories are free of ghosts.

${ }^{107}$ A ghost is a particle with negative kinetic energy. 


\section{Schrödinger II: Arbitrary Affine Connection}

After an interruption of more than two years, in January 1946, the correspondence between Schrödinger and Einstein resumed; he sent Schrödinger two unpublished papers, among them his paper with E. Straus [179].

"I am sending them to nobody else, because you are the only person known to me who is not wearing blinkers in regard to the fundamental questions in our science. The attempt depends on [...] the introduction of a non-symmetric tensor as the only relevant field quantity [...]. Pauli stuck out his tongue at me when I told him about it." (quoted from [446], p. 424.)

In his subsequent letter of 3 March 1946, Einstein pointed to a technical weakness of his theory: "the non-symmetric tensor is not the most simple structure that is covariant with respect to the group, but decomposes into the independently transforming parts $g_{(i k)}$ and $g_{[i k]}$; the consequence of this is that one can obtain a nondescript number of systems of second-order equations." ([446], p. 424. $)^{108}$ In both of the preceding papers $([147,179])$, Einstein had not given a single reference to any other publication. Due to the the difficulties concerning transatlantic communication during the war years 1943 and 1944, Einstein possibly might not have seen Schrödinger's six papers from 1943 and 1944.

In April, Schrödinger had progressed with his research such that he could present its essence before the Irish Academy. In hindsight, he confessed to Einstein in a letter of 1 May 1946:

"One thing I do know is that my first work [P.R.I.A. 1943] was so imbecilic that it now is repellent to everyone, including you [...]. This first work was no advance over 'Einstein 1923', but pretended to be." ([446], p. 426.)

M.-A. Tonnelat from Paris visited Schrödinger in Dublin during 1946 and, perhaps, discussions with her had influenced him. Unlike what he had stated in his earlier paper when using a semimetric connection two years before [549], Schrödinger now thought that: "[...] only the general case, dealt with here, is completely satisfactory and gives new information" ([551], 41). The "general case" meant: a general affine connection, named $\Delta_{i k}^{l}$ by him. After splitting it up into a symmetric part, the trace-free part $W_{i k}{ }^{l}$ of the torsion tensor $S_{i k}{ }^{l}$ and vector torsion:

$$
\Delta_{i k}^{l}=\Gamma_{i k}^{l}+W_{i k}^{l}+2 \delta_{[i}^{l} Y_{k]},
$$

with $\Gamma_{i k}^{l}:=\Delta_{(i k)}{ }^{l}, Y_{i}:=\frac{1}{3} S_{i l}^{l}$, and $W_{i l}^{l}=0$. Instead of the contractions of the curvature tensor (181), (182) now

$$
\begin{aligned}
& \underline{K}_{i k}(L)=\underline{-}_{i k}(\Gamma)-Y_{k \| i}-2 Y_{0} \underset{0}{\| k}-3 Y_{i} Y_{k}-W_{i k \| r}^{r}+W_{i r}^{s} W_{s k}^{r}+3 Y_{s} W_{i k}^{s}, \\
& \underline{V}_{i k}(L)=\underline{V}_{i k}(\Gamma)-3 Y_{i, k}+3 Y_{k, i}
\end{aligned}
$$

obtain. Again, $\|_{0} k$ denotes covariant differentiation with respect to the symmetric connection $\Gamma$. The quantities $\Gamma, W$, and $Y$ are varied independently. That $W_{i k}^{l}$ is tracefree is taken into account by help of a multiplier term in the variational principle:

$$
\delta \int d \tau\left[\hat{\mathcal{L}}\left(\underline{-}_{-}{ }_{i k}(L), \underline{-}_{l m}(L)\right)+2 \hat{p}^{r} W_{r s}^{s}\right]=0 .
$$

108 There is an abridged version of Moore's book in which this quote cannot be found, i.e., in [447]. 
The Lagrangian density $\hat{\mathcal{L}}$ is demanded to be a functional of the two contractions (215), (216) of the affine curvature tensor. The field variables $\hat{g}^{i k}, \hat{f}^{i k}, \gamma_{i k}$, and $\phi_{i k}$ are introduced as they were in Section 6.1 .1 by (154) and (159). A decomposition of (215) then leads to:

$$
\begin{aligned}
& \gamma_{i k}:=\underline{-}_{(i k)}(L)=\underline{-}_{(i k)}(\Gamma)-3 Y_{(i \| k)}-3 Y_{i} Y_{k}+W_{i r}^{s} W_{s k}^{r}, \\
& \phi_{i k}:=\underline{K}_{[i k]}(L)=\frac{1}{2}\left[\frac{\partial \Gamma_{r i}^{r}}{\partial x^{k}}-\frac{\partial \Gamma_{r k}^{r}}{\partial x^{i}}\right]-Y_{[i, k]}-W_{i k \| r}^{r}+3 Y_{s} W_{i k}^{s} .
\end{aligned}
$$

The field equations following from the variational principle (217) with respect to variation of $\Gamma$ and $W$ are similar to (189):

$$
\begin{array}{r}
\hat{g}_{\| l}^{i k}+\delta_{l}^{k}\left[\frac{1}{3} \hat{f}^{i s}-\hat{g}^{i s} Y_{s}-\frac{2}{3} \hat{r}^{i}\right]+\delta_{l}^{i}\left[\frac{1}{3} \hat{f}^{k s}{ }_{\| s}-\hat{g}^{k s} Y_{s}-\frac{2}{3} \hat{r}^{k}\right]+3 \hat{g}^{i k} Y_{l}=0 \\
\hat{f}_{\| l}^{i k}-\delta_{l}^{k}\left(\frac{1}{3} \hat{f}_{\| s}^{i s}+\hat{f}^{i s} Y_{s}\right)+\delta_{l}^{i}\left(\frac{1}{3} \hat{f}_{\| s}^{k s}+\hat{f}^{k s} Y_{s}\right)+3 \hat{f}^{i k} Y_{l}+\hat{g}^{i s} W_{s l}^{k}-\hat{g}^{k s} W_{s l}^{i}=0 .
\end{array}
$$

The abbreviation, or rather definition of the vector density $\hat{j}^{k}$ is as before $\left(=\frac{\partial \hat{f}^{k s}}{\partial x^{s}}\right)$ while

$$
\hat{r}^{k}:=\hat{s}_{\| r}^{k r}=\hat{s}_{, r}^{k r}
$$

with $^{109} \hat{s}^{i k}:=\frac{\partial \mathcal{L}}{\partial V_{i k}}$. The variation with respect to $Y^{k}$, after some calculation, led to the simple relation between the current densities:

$$
\hat{r}^{k}=\frac{1}{4} \hat{j}^{k}
$$

By the field equations, the dynamics of three fields were to be determined, gravitational, electromagnetic, and mesonic field:

"Because the Lagrangian is left undetermined for the time being, each of the three fields will be represented by two "conjugate" tensorial entities in the field equations, gravitation by $\hat{g}$ and $\gamma$; the skew fields by $\hat{f}$ and $\phi$ and by $\hat{s}$ and $V$ respectively." ([551], 44.)

In order to arrive at equations better separated in the new fields, Schrödinger redefined the field variables by forming the linear combinations:

$$
\begin{gathered}
{ }^{\prime} \phi_{i k}:=\phi_{i k}+\frac{1}{4} V_{i k}, \quad \quad \hat{f}^{i k}=\hat{f}^{i k}, \\
{ }^{\prime} V_{i k}=V_{i k}, \quad{ }^{\prime} \hat{s}^{i k}=\hat{s}^{i k}-\frac{1}{4} \hat{f}^{i k} .
\end{gathered}
$$

The equations for the Maxwellian field ' $\hat{s}, V$ are claimed to then be "kept entirely aloof from the rest by the remarkable fact that the $Y$-vector drops out rigorously from all the other equations except the last eqn. (216)". The fields $\hat{f}$, ' $\phi$ with the current density $\hat{j}$ are related to the meson field.

As in his earlier papers, a metric was introduced by (157), i.e., via $\hat{g}^{i k}$. Schrödinger then calculated the expression for the symmetric part of the connection as in (191) but now only in first approximation in $\hat{f}, W$ :

$$
\Gamma_{i j}^{k}=\left\{\begin{array}{l}
k \\
i j
\end{array}\right\}-\frac{1}{4} g_{i j} j^{k}+\delta_{i}^{k}\left(\frac{1}{12} j_{j}+Y_{j}\right)+\delta_{j}^{k}\left(\frac{1}{12} j_{i}+Y_{i}\right)+\ldots
$$

109 Schrödinger introduced the pair $\hat{s}^{i k}, V_{i k}$ as being duals (conjugates) 
The trace-free part of torsion was given by:

$$
W_{i k}^{l}=-\frac{1}{2} g^{l s}\left(f_{k s ; i}+f_{s i ; k}-f_{i k ; s}\right)+\frac{2}{3} \delta_{[i}^{l} j_{k]}+\ldots
$$

and the field equations for the meson field, again in linear approximation, were:

$$
\phi_{i k}=-\frac{3}{8}\left(\frac{\partial j_{k}}{\partial x^{i}}-\frac{\partial j_{i}}{\partial x^{k}}\right)+\frac{1}{2}\left(f_{i ; k ; s}^{s}-f_{k ; i ; s}^{s}-g^{r s} f_{i k ; r ; s}+\ldots\right), \hat{j}^{k}=\frac{\partial \hat{f}^{k s}}{\partial x^{s}} .
$$

The covariant derivative is the one formed with the Christoffel-symbol (Levi-Civita connection) built from (the symmetric) $g_{i k}$. Schrödinger did interpret (228) as Proca equation ${ }^{110}$ for the meson, "except for the term which contains explicitly second derivatives". According to him, the additional term was taking into account a slight direct influence of gravitation on the meson field ([551], 47).

In the same approximation, Schrödinger also wrote down gravitational field equations looking like Einstein's except for the fact that on the side of the matter tensor a number of geometrical objects do appear. They are said to describe the interaction of gravitational and electromagnetic fields as well as of gravitational and mesonic fields. A cosmological term could also be present.

Schrödinger's conclusion was cautious:

"This encourages one to regard an affine connection of space-time as the competent geometrical interpretation (from the classical point of view) of the three physical tensor fields we know." ([551], p. 50)

He questioned, however, that the classical field laws would "be of much help in guessing the true quantum laws of the meson" if they were violently non-linear.

In his correspondence with Schrödinger, Einstein doubted that a theory using only the connection (i.e., without additional metric) be feasible. He reported about difficulties in his theory to solve for the connection as a function of the metric and its first derivative:

"We have squandered a lot of time on this thing, and the result looks like a gift from the devil's grandmother." ([446], p. 426.)

In another letter of 16 July 1946 to Schrödinger, Einstein did explain the progress achieved "thanks to the truly great skill and persistence of my assistant Straus", then published in [179]. He also commented on conceptual differences. Schrödinger used the wave model for the transport of electromagnetic energy while Einstein thought this to be "really false on account of quantum actualities" ([446], p. 427). Another of his correspondents, W. Pauli, also did not believe in purely affine theory. He wrote to him on the same day at which Schrödinger's paper finally had been issued, i.e., on 21 November 1946:

"I personally am completely convinced - contrary to you as it seems - that for physics nothing reasonable follows from the affine connection without metric. Palatini's theorem again slams the door. I also believe that each tensor, e.g., the contracted curvature tensor, immediately must be split into a symmetric and a skew part* (* In general: tensors into their irreducible symmetry classes), and to avoid every adding sign between them. What God did separate, humans must not join.) ([489], p. 401)"111

110 The Proca equation for a vector field $\psi^{k}$ with mass $\mathrm{m}$ in curved (Riemannian) space-time would be $g^{r s} \psi^{k} ; r ; s+$ $\left(\frac{m c}{\hbar}\right)^{2} \psi^{k}=0$. In view of (224), the mass term in (228) is $\phi_{i k}$. It is unclear to me which term(s) is (are) disturbing for Schrödinger.

111 "Ich persönlich bin - wie es scheint im Gegensatz zu Dir - völlig davon überzeugt, daß für die Physik aus dem affinen Zusammenhang ohne Metrik nichts Vernünftiges herauskommt. Das Theorem von Palatini schlägt diese Türe wieder zu. Im übrigen glaube ich, man soll sofort jeden Tensor, z.B. den verjüngten Krümmungstensor, in einen symmetrischen und schiefen Teil spalten* (* Allgemein: Tensoren in ihre irreduziblen Symmetrieklassen) und jedes Pluszeichen zwischen diesen Teilen vermeiden. (Was Gott getrennt hat, sollen die Menschen auch nicht zusammenfügen.)" 


\subsection{Schrödinger's debacle}

Schrödinger kept Einstein informed about his continuing work on UFT within affine geometry "[...] by reports at about fortnightly intervals" ([446], p. 429). He had read the papers of Einstein, and Einstein \& Straus [179, 147] from the previous two years. (Cf. Sections 7.2 and 7.3 above.) Now he again presented his newest development of the theory to the Royal Irish Academy on 27 January 1947. Believing that he had made a break-through, he had written to Einstein a day earlier:

"Today I can report on a real advance. [...] In brief, the situation is this. If in the affine theory, which I have developed in general form in recent years, one takes the special, the only reasonable Lagrange function, namely the square root of the determinant of the Einstein tensor, then one obtains something fabulously good." ([446], p. 430.)

Well, this might have tasted a bit stale to Einstein because he had used this same Lagrangian about twenty five years ago [141] and abandoned the theory, nonetheless! (cf. Section 4.3.2 of Part I.) To the exuberant Schrödinger a modest statement to the Academy would not do: the press was also invited. So, Schrödinger began:

"The nearer one approaches truth, the simpler things become. I have the honour of laying before you today the keystone of the Affine Field Theory and thereby the solution of a 30 year old problem: the competent generalization of Einstein's great theory of 1915. The solution was

$$
\begin{array}{r}
\delta \int \mathcal{L}=0 \\
\text { with } \mathcal{L}=\sqrt{-\operatorname{det}\left(R_{r s}\right)}, \\
R_{i k} \equiv-\underline{-K}_{i k}=\frac{\partial \Gamma_{i l}^{l}}{\partial x_{k}}-\frac{\partial \Gamma_{i k}^{l}}{\partial x_{l}}+\Gamma_{m k}^{l} \Gamma_{i l}^{m}-\Gamma_{m l}^{l} \Gamma_{i k}^{m} .
\end{array}
$$

where $\Gamma$ is the general affinity of 64 components. That is all. From these three lines my friends would reconstruct the theory, supposing the paper I am handing in got hopelessly lost, and I died on my way home." ([446], p. 430-432.)

In the paper submitted together with his presentation, the Lagrangian (229) was given a factor $\frac{2}{\lambda}$ with a real constant $\lambda \neq 0$ playing an important role ([552], p. 164). Schrödinger first played its occurrence down, unconvincingly though, by saying that it could be transformed to \pm 1 , but in his final field equations, the constant stood for an additional "cosmological" term. In his own words (in a note "added in proof"), his field equations "[...] include 'the cosmological term' without containing a cosmological constant." ([552], p. 171.)

The presentation to the Academy and the press did not contain the finer details. For this kind of public, he wrote down the field equations in the reduced form: ${ }^{112}$

$$
\frac{\partial R_{i k}}{\partial x^{l}}-R_{s k}^{*} \Gamma_{i l}^{s}-R_{i s}^{*} \Gamma_{l k}^{s}=0
$$

with his "star"-connection (cf. (27))

$$
{ }^{*} \Gamma_{i k}^{l}:=\Gamma_{i k}^{l}+\frac{1}{3} \delta_{i}^{l}\left(\Gamma_{k s}^{s}-\Gamma_{s k}^{s}\right),
$$

while the complete equations in his paper are:

$$
\frac{\partial\left[R_{i k}^{*}+\mathcal{F}_{i k}\right]}{\partial x^{l}}-\left[R_{s k}^{*}+\mathcal{F}_{s k}\right]{ }^{*} \Gamma_{i l}{ }^{s}-\left[R_{i s}^{*}+\mathcal{F}_{i s}\right]^{*} \Gamma_{l k} s=0,
$$

112 This equation reappears in Schrödinger's presentation in his book ([557], p. 114). 
where

$$
\mathcal{F}_{i k}:=\frac{2}{3}\left(\frac{\partial \Gamma_{k}}{\partial x^{i}}-\frac{\partial \Gamma_{i}}{\partial x^{k}}\right) \equiv-\frac{4}{3} S_{[i, k]},
$$

if vector torsion $S_{i}$ is used. Equation (233) expresses nothing but the vanishing of the \pm -derivative of $R_{i k}^{*}+\mathcal{F}_{i k} \equiv-K_{-i k}^{*}+\mathcal{F}_{i k} . \quad R_{i k}^{*}\left(K_{-}^{*}\right)$ is formed with the "star"-connection. The (asymmetric) metric again was defined as a variational derivative with respect to $R_{i k}$. By some manipulation of the formalism, Schrödinger was able to show that (233) is equivalent to the slightly generalized weak field equations of Einstein \& Straus (206) - (208) (cf. below, Section 7.3):

$$
\begin{aligned}
\hat{g}^{[i s]}{ }_{, s} & =0, \\
R_{(i k)}^{*}-\lambda g_{i k} & =0, \\
\left(R_{[i k]}^{*}-\lambda g_{[i k]}\right)_{, l}+\left(R_{[k l]}^{*}-\lambda g_{[k l]}\right)_{, i}+\left(R_{[l i]}^{*}-\lambda g_{[i i]}\right)_{, k} & =0 .
\end{aligned}
$$

Schrödinger was well aware of this:

"We now have to endorse the remarkable fact, that the actual content of equations $[(236)-(237) \ldots]$ differs from the theory presented in Einstein's two papers, quoted above, (i.e., $[147,179]$ ) only by formal $\lambda$-terms. ${ }^{113}$ His theory amounts to putting $\lambda=0$ in (236) - (237). There is a formal difference in that he, from the outset, regards all skew tensors as purely imaginary. [...]" ([552], p. 167.)

Equations (235) to (237) were also called the "para-form" of his field equations [526]. They may be seen as 18 equations for the 16 field variables $g_{i k}$. According to Schrödinger: "The surplus of 2 equations is vindicated by two trivial identities, one between the first members of (237), and one between those of (236)." (236) is not a definition, such as it was used by Eddington (cf. Section 4.3.1 of Part I) but derived from the Lagrangian (229), the definition in (157) and:

$$
\hat{g}^{i k}:=\frac{\partial \mathcal{L}}{\partial R_{i k}} .
$$

Schrödinger also confessed that "it may turn out that I have overrated the practical advantage of (233) over (235) - (237)."

The Irish Press caught the bait: "Twenty persons heard and saw history being made in the world of physics yesterday as they sat in the lecture hall of the Royal Irish Academy, Dublin, and heard Dr. Erwin Schrödinger. [...] It was later told me that the theory should express everything in field physics." ([446], p. 432.) The news spread quickly; the science editor of The New York Times sent Schrödinger's statement and a copy of his paper to Einstein, and asked him for a comment. In the text supplied by Einstein which became also widely distributed, he said:

"Schrödinger's latest effort [...] can be judged only on the basis of mathematicalformal qualities, but not from the point of view of 'truth' (i.e., agreement with the facts of experience). Even from this point of view I can see no special advantages over the theoretical possibilities known before, rather the opposite. [...] It seems undesirable to me to present such preliminary attempts to the public in any form." ([446], p. 432-433.)

Schrödinger must have had second thoughts about his going public; he tried to justify himself vis-à-vis Einstein - although he had not yet seen Einstein's rebuff. In his letter, he admitted to have indulged "in a little hot air [...] I blew myself up quite a bit. [...] This thing is being

113 See also $(266-268)$ in Section 9.2.2. 
done for the purpose of obtaining cheap and fraudulent publicity for a discredited administration." His excuse was that he had tried by this "commotion" to increase his salary and to bring the authorities to reach a decision whether his wife, as a widow, could get a pension or not ([446], p. 433). Einstein replied coolly and curtly:

"I was not correct in my objection to your Hamilton-function. But your theory does not really differ from mine, only in the presentation and in the 'cosmological term' which mine lacks. [...] Not your starting-point but your equations permit a transition to vanishing cosmological constant, then the content of your theory becomes identical with mine" ([446], p. 434),

and stopped writing to Schrödinger for the next three years. Pauli seemingly had followed the events from Switzerland and wrote to Schrödinger calmly on 9 February 1947:

"Many thanks for your interesting letter of 26. Jan. I would have liked to only respond to it after your first enthusiasm about the new field equations will have given place to a more sober judgment (perhaps the letter is written still too early). Of course, progress is made by your decision to take a specific Lagrangian; also, the mathematical side of your thoughts to me seems extraordinarily clear. Nevertheless, my reservations with regard to a non-irreducible object as a basis continue unabatedly. [...]" ([489], p. 415). ${ }^{114}$

He then expressed in more detail, why for him, only irreducible tensors are the variables to be used. He emphasized that he was not against the "logical possibility" of Schrödinger's field equations, but could not accept their "necessity and naturalness". According to Pauli, already before "the next few years", it would become clear whether these field equations "have something to do with physics, or not." In a letter to Sommerfeld of 31 October 1947, Pauli agreed with Sommerfeld's "negative opinion concerning Einstein's present physics" and supported them with much the same arguments as those given to Schrödinger ([489], p. 475).

As to the physical interpretation of the geometrical objects in his "ultimate" theory, Schrödinger associated the two skew-symmetric fields $\hat{g}^{[i k]}, \mathcal{F}_{i k}$ as contravariant density and covariant field tensor, so that (234), and (233), or (235) amount to (modified) Maxwell equations. The quantities $\left.g_{[i k]}, \sqrt{-g} g^{i r} g^{k s} \mathcal{F}_{r s}, \sqrt{-g} g^{r i} g^{s k} \mathcal{F}_{r s}, \frac{1}{2} \sqrt{-g}\left(g^{r i} g^{k s}-g^{r k} g^{i s}\right) \mathcal{F}_{r s}\right)$ were assumed to be linked to "electric charge, mesonic charge and matter".

"We must not forget, that we are here faced with a truly unitary theory, in which we have to expect all fields to coalesce into an inseparable union, almost as close as that of the electric and magnetic field entailed by Restricted Relativity." ([552], p. 169.)

The episode differs from Einstein's repeated claims to have found the final unified field theory, in the 1920s and 30s, in that Einstein did not have to call in the press, and in fact was clever enough not do so. However, his friends in the press were covering his work to the extent, that for each new publication he received the same public attention as Schrödinger in this single case - staged by himself. By his public reaction to Schrödinger, Einstein solidified his position as the opinion leader in research concerning UFT.

114 "Vielen Dank für Deinen interessanten Brief vom 26.I. Ich wollte gerne mit dessen Beantwortung warten, bis Deine erste Begeisterung über die neuen Feldgleichungen einer nüchterneren Beurteilung gewichen sein wird (vielleicht ist also dieser Brief immer noch zu früh geschrieben). Es ist natürlich ein Fortschritt, daß Du Dich für eine bestimmte Lagrangefunktion entschieden hast; auch scheint mir die mathematische Seite Deiner Überlegungen außerordentlich klar. Dagegen bestehen meine Bedenken gegen das Stützen auf ein nicht-irreduzibles Gebilde in unverminderter Weise fort. [...]" 


\subsection{Recovery}

Schrödinger must have been depressed after so much self-confidence! To a friend he had written:

"I have found the unitary field equations. They are based on primitive affine geometry, [...] Albert did the main job in 1923, but missed the goal by a hair's breadth. The result is fascinatingly beautiful. I could not sleep a fortnight without dreaming of it." $([256], \text { p. } 168)^{115}$

The report of L. Bass that: "After a farcical debacle [...], Schrödinger put away the material in a file labeled despondently 'Die Einstein Schweinerei' (the Einstein mess)” ([13], p. 120) describes only a momentary halt. After a pause of almost one year, Schrödinger continued his publishing in this topic, i.e., UFT. As a beginning, he surveyed the possibilities for the construction of UFT in a whole class of geometries descending "from the theory of gravitation in empty space by very natural and straightforward generalization without any further artifice." ([555], p. 205.) He distinguished between the three cases: metrical, affine and mixed geometry depending on whether only $g_{i k}, \Gamma_{i k}^{l}$, or both can be regarded as independent variables. As a Lagrangian he took $\hat{g}^{r s} R_{r s}$, with as before $R_{i k}=-\underline{K}_{i k}$. The further classification depended on additional symmetry conditions on the basic variable(s). He dismissed the case of a non-symmetric metric and symmetric connection "since there is no simple and natural clue" by which the Levi-Civita connection should be replaced. It was perhaps this remark which induced J. I. Horváth to suggest "a selection principle for the final theory in the case of the affine theories", to wit: the field equations must be invariant against changes of the affinities which preserve the parallelism [284]. He derived such transformations (changes) from the weakened condition for auto-parallels (cf. Section 2.1.1, after (22)) and arrived, without noting it, at Einstein's $\lambda$-transformations (52) introduced before.

In commenting Schrödinger's work on affine field theories, Pauli also contributed to UFT, if only in a letter to Pascual Jordan of 13 July 1948. Once more he criticized the use of reducible tensors by Schrödinger, notably of the Ricci tensor $K_{i k}$ instead of its symmetric $K_{(i k)}$ and skew symmetric $K_{[i k]}$ parts, separately. He then derived the "mathematically simplest scalar densities" as building elements of the Lagrangian. If a symmetric connection is used, they are given by ${ }^{116}$

$$
H_{0}=\sqrt{\operatorname{det} K_{(i k)}}, \quad H_{1}=\sqrt{K_{(i p)} K_{(j q)} K_{[k r]} K_{[l s]} \epsilon^{i j k l} \epsilon^{p q r s}}, \quad H_{2}=K_{[i j]} K_{[k l]} \epsilon^{i j k l} .
$$

Possible Lagrangians then would be

$$
\mathcal{L}=H_{0}\left\{1+f_{1}\left(\frac{H_{1}}{H_{0}}\right)+f_{2}\left(\frac{H_{2}}{H_{0}}\right)\right\}, \quad \mathcal{L}^{\prime}=H_{0} f\left(\frac{H_{1}}{H_{0}}, \frac{H_{2}}{H_{0}}\right),
$$

with arbitrary functions $f_{1}, f_{2}, f$. Pauli's conclusion was:

"Even if we try to specialize these functions by simplifying arguments, a lot of arbitrariness remains. The impression prevails that the basic geometrical concepts have nothing to do with physics. Einstein did express it like this: 'the action function then is obtained by leering at another sheet of paper lying next to it, and on which the formulae for another theory can be seen.' "' ([489], p. 541-542). ${ }^{117}$

115 Hittmair gave neither a date nor further information about the "friend".

116 The form of these invariants obviously must correspond to the three invariants on the r.h.s. of (8) in Section 2.1. 117 "Selbst wenn man diese Funktionen durch Einfachheitsgesichtspunkte zu spezialisieren sucht, bleibt noch viel Willkür übrig. Man hat den Eindruck, daß die zu Grund liegende geometrische Konzeption nichts mit Physik zu tun hat. Einstein hat das so ausgedrückt: 'man gewinnt dann eben die Wirkungsfunktion durch Schielen auf ein anderes Blatt Papier, das daneben liegt und auf welchem die Formeln einer anderen Theorie stehen.' " 
Schrödinger was still convinced of his approach to unified field theory. In a paper of 1951, he showed a pragmatic attitude: He set out to solve approximately the field equations with asymmetric metric and asymmetric connection ${ }^{118}$ :

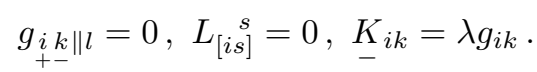

He argued that:

"[...] an assiduous application of such methods to weak fields is bound to tell us something on the interlacing of three things, gravitational field, electromagnetic field, and electric charges, all three of which spring from one basic conception. [...] One may hope that this will provide a better foundation to the quantum mechanical treatment of fields, which at present is based on a number of classical or pseudo-classical field theories of independent origin, cemented together by interaction terms'." ([558], p. 555.)

As a result he claimed that "a pure charge-free Maxwellian field of radiation is capable of producing a gravitational field which according to the old theory could only be produced by matter other than an electromagnetic field." By this, the non-vanishing of the trace of a correspondingly defined energy-momentum tensor is meant. He also offered three alternatives for an energy (pseudo) tensor which all vanish for a single plane wave. As to physical interpretations, $k_{i j}=g_{[i j]}$ is identified with the electromagnetic field with the space-space components standing for the electrical field. The magnetic 4-current vanishes in consequence of the field equations while the electrical 4 -current is added by hand and given by the expression $k_{i j, l}+k_{j l, i}+k_{l i, j}$. Despite Schrödinger's going beyond the linear approximation up to quadratic terms, the "[...] influence of both fields [i.e., gravitational and electromagnetic] on the motion of the charges and that of the gravitational field on the electromagnetic" was missing. Thus, the paper contained no new fundamental insights.

A favorable reaction came from a young Harvard mathematician R. L. Ingraham who was an assistant to Oswald Veblen in Princeton at the Institute for Advanced Studies in 1953. He set out to rewrite the field equations of Schrödinger's affine unified theory $[552,555]$ in "a more physically meaningful form" [305]. He assumed $h_{i j}$ to represent gravitation but found the direct link of the skew-symmetric part of $g_{i j}$ with the electromagnetic field as incorrect. By an elementary calculation presented a year later also by M.-A. Tonnelat (cf. [627] or the table on p. 15 of [632]), Eq. (33) is put into the form: ${ }^{119}$

$$
\left[\sqrt{\frac{h}{g}}\left(\sqrt{h} \check{k}^{r s}+\frac{1}{2} \frac{J_{2}}{\sqrt{h}} \epsilon^{r s p q} k_{p q}\right)\right]_{, s}=0
$$

with $J_{2}:=\frac{1}{8} \epsilon^{r s p q} k_{r s} k_{p q}$. This equation then is rewritten as one of the usual Maxwell equations (in a space with metric $h_{i j}$ ) with a complicated r.h.s. which then is made to vanish by the additional assumptions that $k_{i j}$ is a curl, $J_{2}=0$, and $\check{k}^{r s} k_{r s}=0$. Likewise, an additional condition was laid on the curvature tensor (cf. his Eq. (27a), p. 749) such that the field equation reduced to the Einstein vacuum equation with cosmological constant. That de Sitter space is a solution of Ingraham's equations with $k_{i j}$ representing some sort of plane wave, is unsurprising. Fortunately, this naive strategy of imposing additional conditions with the aim to obtain interpretable field equations, did not have many followers.

\footnotetext{
118 Here, he chose the notation $R_{i k} \sim+K_{i k}$.

119 For the notation see (13) in Section 2.1.
} 


\subsection{First exact solutions}

The first to derive several genuine exact, spherically symmetric and static (sss) solutions of Schrödinger's field equations with cosmological constant (235)-(237) in 1947 was his research assistant A. Papapetrou ${ }^{120}[475]$. His ansatz contained five unknown functions of the radial coordinate $r=x^{1}\left(x^{2}=\theta, x^{3}=\phi, x^{0}=c t\right): g_{00} \stackrel{*}{=} \gamma(r), g_{11} \stackrel{*}{=} \alpha(r), g_{22} \stackrel{*}{=} \beta(r), g_{33} \stackrel{*}{=} \beta \sin ^{2} \theta, g_{01}=$ $-g_{10} \stackrel{*}{=} w(r), g_{23}=-g_{32} \stackrel{*}{=} r^{2} v(r) \sin \theta$. In the paper, he treated the cases $v=0, w \neq 0$ and $v \neq 0, w=0, v$ real. After setting $\beta=r^{2}$ in the first case, integration of the field equations led to

$$
\begin{array}{r}
\gamma=\left(1+\frac{l^{4}}{r^{4}}\right)\left(1-\frac{2 m}{r}-\frac{\lambda}{3} r^{2}\right), \\
\alpha=\left(1-\frac{2 m}{r}-\frac{\lambda}{3} r^{2}\right)^{-1}, \\
w= \pm \frac{l^{2}}{r^{2}} .
\end{array}
$$

An assumption used was that asymptotically, i.e., for $r \rightarrow \infty, g_{(i k)} \rightarrow \eta_{i k}$. Thus, if $g_{(i j)}=h_{i j}$ is interpreted as the space-time metric (gravitational field tensor), $g_{[i j]}=k_{i j}$ as the electromagnetic field tensor, then a deviation from the Reissner-Nordström-de Sitter-solution of general relativity with $\gamma=(\alpha)^{-1}=\left(1-\frac{2 m}{r}+\frac{e^{2}}{r^{2}}-\frac{\lambda}{3} r^{2}\right)$ was obtained. Introducing the elementary electric charge by $l^{2}=e$, the source term for the point charge in (243) shows the wrong radial dependence, although from (243) we must conclude that a radial electromagnetic field is present. ${ }^{121}$ The solution does not describe the Coulomb field. At this point, this seems no serious objection to the theory, because other static spherically symmetric (SSS) solution might exist. However, cf. Section 9.6, where the most general sss solution is given.

In the second case, $v$ is replaced by $f=v r^{2}$ and only a particular solution with $\gamma=(\alpha)^{-1}=$ $\left(1-\frac{2 m}{r}-\frac{\lambda}{3} r^{2}\right), \beta=r^{2}, v=c$, with $c$ a constant was reached by Papapetrou. Thus, the radial electromagnetic field is constant. Moreover, this constant electric field does not influence the gravitational field. Papapetrou also discussed approximate solutions and concluded for them that $k_{i j}$ does not describe the electromagnetic field but the electromagnetic potential. This would rule out an interpretation of the solution in terms of an electric field. All these solutions would not have been acceptable to Einstein (in the sense of describing sources of electricity), because they were not free of singularities. Unlike for the gravitational field in general relativity or the electromagnetic field, for the "total" field in UFT singularities were no longer permitted:

"As I've said, one does't get away without singularities in the case of Maxwell's equations. But no reasonable person believes that Maxwell's equations can hold rigorously. They are, in suitable cases, first approximations for weak fields. It is now my belief that, for a serious and rigorous field theory, one must insist that the field be free of singularities everywhere." ([116], p. 93) ${ }^{122}$

We shall come back to the demand that exact solutions ought to be free of singularities in Sections 9.6.2 and 10.3.2.

\footnotetext{
120 Achilles Papapetrou (1907-1997) born in Northern Greece, had studied engineering in Athens. He received his $\mathrm{PhD}$ in theoretical physics 1935 at Technische Hochschule Stuttgart, Germany, under the supervision of Peter Paul Ewald. In 1946-1948 he became research fellow with Schrödinger at the Institute for Advanced Study in Dublin, later in Manchester (1948-1952) with P. M. S. Blackett; then professor at the Academy of Sciences, Berlin (1952-1961), and at the Institut Henri Poincaré, Paris 1960-1977.

121 This implies that $g_{4 i}, i=1,2,3$ denotes the electrical field. If, alternatively $\epsilon^{i j k l} g_{[i j]}$ is taken as the electromagnetic field tensor, then a magnetic field obtains.

122 "Wie schon gesagt, kommt man bei den Maxwell'schen Gleichungen nicht ohne Singularitäten aus. Aber kein vernünftiger Mensch glaubt, dass die Maxwell'schen Gleichungen streng gültig sein können. Sie sind günstigsten Falles erste Approximationen für schwache Felder." English translation taken from [116].
} 
For static metrics, Papapetrou was able to extend a result of Einstein [145], and Einstein \& W. Pauli [177] to the strong field equations of Einstein's UFT: non-singular static metrics of the strong field equations of UFT which would represent the (gravitational) field of a non-vanishing mass do not exist [474]. Note that this result depends on the identification of the symmetric part of the metric with the gravitational field (potential). A year later, a different proof was given by E. Straus for the weak field equations. In the same paper, Straus concluded: "There exists no static centrally symmetric solution of the field equations which is asymptotically flat and regular throughout" ([592], p. 420). For A. Lichnerowicz' contribution to the problem of Einstein \& Pauli cf. Section 10.5.1.

H. Takeno and two coworkers of the Hiroshima Institute for Theoretical physics also took up the search for exact solutions with spherical symmetry of Einstein's and Schrödinger's field equations [602]. Except for a different notation and an assumed time-dependency, the form of the metric was the same as Papapetrou's; it contained five free functions $A(r, t), B(r, t), C(r, t), f(r, t), h(r, t)$ (and the usual coordinates $x^{0}=t, x^{1}=r, x^{2}=\theta, x^{3}=\phi$ ):

$$
g_{a b}=\left(\begin{array}{cccc}
-A & 0 & 0 & f \\
0 & -B & h \sin \theta & 0 \\
0 & -h \sin \theta & -B \sin ^{2} \theta & 0 \\
-f & 0 & 0 & C
\end{array}\right) \text {, }
$$

Two types of solutions according to which $B^{2} \neq h^{2}, f \neq 0$ (type I), or $B^{2}=h^{2}, f=0$ (type II) were distinguished. And then, immediately, the time-dependence of the free functions was dropped. Assuming in addition $B=r^{2}, h=k r^{2}$, the authors derived the general solution of type II of Schrödinger's field equations (the weak equations with cosmological constant $\lambda$ ) to be:

$$
A^{-1}=1-\frac{2 m}{r}-\frac{\lambda}{3} r^{2}, B=r^{2}, C=a\left(1-\frac{2 m}{r}-\frac{\lambda}{3} r^{2}\right)\left(1+\frac{k_{1}}{r^{4}}\right), h=k r^{2}, f^{2}=\frac{a k_{1}}{r^{4}}
$$

where $a, k, k_{1}, m$ are integration constants. The solution generalized Papapetrou's two exact static solutions $\left(k_{1}=0, k \neq 0\right.$ and $\left.k_{1} \neq 0, k=0\right)$ by "combining" them. Apparently, at the time the authors did not know of Wyman's earlier paper, also containing solutions with $k \times k_{1} \neq 0$; see Section 9.6.1. The main conclusion drawn by Takeno et al. was that the fundamental equation (30) has no unique solution if $\operatorname{det}\left(g_{a b}\right)=2 \operatorname{det}\left(g_{(a b)}\right), \operatorname{det}\left(g_{[a b]}\right)=0$. This condition is consistent with (364) derived by M.-A. Tonnelat; cf. Section 10.2.3.

In his paper of 1951 discussed above in Section 8.2, Schrödinger expressed his disenchantment with regard to the search for exact solutions:

"One may hope that exact solutions, involving strong fields, will reveal the nature of the ultimate particles. I do not believe this, mainly because I do not believe the ultimate particles to be identifiable individuals that could be described in this fashion. Moreover, in the symmetric theory (i.e., in Einstein's theory of 1916) the exact solutions, involving strong fields, have disclosed the ingenuity of the mathematicians who discovered them, but nothing more. [...]" ([558], p. 3)

In view of the research done since, e.g., on black holes or cosmology, the last sentence possibly would not be upheld by him, today. 


\section{Einstein II: From 1948 on}

In the meantime, Einstein had gone on struggling with his field equations and, in a letter to M. Solovine of 25 November 1948, had become less optimistic ([160], p. 88):

"Scientifically, I am still lagging because of the same mathematical difficulties which make it impossible for me to affirm or contradict my more general relativistic field theory [...]. I will not be able to finish it [the work]; it will be forgotten and at a later time arguably must be re-discovered. It happened this way with so many problems." 123

In his correspondence with Max Born during the second half of the 1940s, Einstein clung to his refusal of the statistical interpretation of quantum mechanics. According to him, physics was to present reality in space and time without, as it appeared to him, ghostly interactions at a distance. In a letter of 3 March 1947, he related this to UFT:

"Indeed, I am not strongly convinced that this can be achieved with the theory of my continuous field although I have found for it an - until now - apparently reasonable possibility. Yet the calculatory difficulties are so great that I shall bite the dust until I myself have found an assured opinion of it. [...]" 124

In spite of such reservations, Einstein carried on unflagging with his research. In his next publication on UFT [148], he again took a complex (asymmetric) metric field. In order to justify this choice in comparison to Schrödinger's who "has based his affine theory [...] on real fields [...]", ${ }^{125}$ he presented the following argument: Just by multiplication and the use of a single complex vector $A_{i}$ a Hermitian tensor $A_{i} \bar{A}_{k}$ can be constructed. By adding four such terms, the Hermitian metric tensor $g_{i k}=\sum_{\kappa} \underset{\kappa}{c}{\underset{\kappa}{k}}_{i} \bar{A}_{\kappa}$ can be obtained. "A non-symmetric real tensor cannot be constructed from vectors in such close analogy" ([148], p. 39). Nevertheless, in Einstein's future papers, the complex metric was dropped.

The field equations were derived from the Lagrangian

$$
\mathcal{H}=P_{i k} \hat{g}^{i k},
$$

i.e., from the Lagrangian (205) without the multiplier terms. In order to again be able to gain the weak field equations, an additional assumption was made: the skew-symmetric part of the metric (density) $\hat{g}^{[i k]}$ be derived from a tensor "potential" $\hat{g}^{i k l}$ anti-symmetric in all indices. Thus, in the Lagrangian, $\hat{g}^{i k}$ is replaced by $\hat{g}^{i k}=\hat{g}^{(i k)}+\hat{g}_{, l}^{i k l}$. The motivation behind this trick is to obtain the compatibility equation (30) from $\delta \mathcal{H} / \delta \Gamma_{i k}^{m}=0$, indirectly. The skew-symmetric part of $\delta \mathcal{H} / \delta \Gamma_{i k}^{m}=0$ is formed and a trace taken in order to arrive at $\frac{1}{2} \hat{g}_{, l}^{[i k]}+\hat{g}^{(i k)} \Gamma_{[k s]}^{s}=0$. Introduction of $\hat{g}_{, l}^{i l}=\hat{g}_{, l s}^{i l s}$ then shows that $\Gamma_{i}=0$ holds. With the help of this equation, $\delta \mathcal{H} / \delta \Gamma_{i k}^{m}=0$ finally reduces to $(30)$.

123 "Wissenschaftlich bin ich immer noch gehemmt durch dieselben mathematischen Schwierigkeiten, die mir die Bestätigung oder Widerlegung meiner allgemeineren relativistischen Feldtheorie unmöglich machen [...]. Ich werde es nicht mehr fertig bringen; es wird vergessen werden und muss wohl später wieder entdeckt werden. So ist es ja schon mit so vielen Problemen gegangen."

124 "Allerdings bin ich nicht fest davon überzeugt, daß es mit der Theorie meines kontinuierlichen Feldes gemacht werden kann, obwohl ich hierfür eine bisher recht vernünftig erscheinende Möglichkeit gefunden habe. Die rechnerischen Schwierigkeiten sind jedoch so groß, daß ich ins Gras beißen werde, bevor ich selbst eine sichere Überzeugung hierüber erlangt habe [...]" [168], p. 215.

125 Einstein did not give a reference to one of Schrödinger's papers. 
The field equation following directly from independent variation with regard of $\Gamma_{i k}^{m}, \hat{g}^{(i k)}$ and $\hat{g}^{i k l}$ are: ${ }^{126}$

$$
\begin{aligned}
\hat{g}^{i k}{ }_{\| l} & =0, \\
P_{(i k)} & =0, \\
P_{[i k], l}+P_{[k l], i}+P_{[l i], k} & =0 .
\end{aligned}
$$

In addition, the equations hold:

$$
\hat{g}_{, k}^{[i k]}=0, \Gamma_{[k s]}^{s}=0 .
$$

As in [179], Einstein did not include homothetic curvature into the building of his Lagrangian with the same unconvincing argument: from his (special) field equations and (252) the vanishing of the homothetic curvature would follow.

In his paper, Einstein related mathematical objects to physical observables such that "the antisymmetric density $\hat{g}^{i k l}$ plays the role of an electromagnetic vector potential, the tensor $\hat{g}_{[i k], l}+$ $\hat{g}_{[k l], i}+\hat{g}_{[l i], k}$ the role of current density." More precisely, the dual object $j^{s} \sim \epsilon^{s i k l}\left(\hat{g}_{[i k], l}+\hat{g}_{[k l], i}+\right.$ $\left.\hat{g}_{[l i], k}\right)$ with vanishing divergence $j_{, s}^{s}=0$ is the (electric) current density ([148], p. 39).

Einstein summed up the paper for Pauli on one page or so and concluded: "The great difficulty lies in the fact that we do not have a method for deriving exact solutions free of singularities, which are the only ones of physical interest. The few things we were able to calculate strengthened my confidence in this theory." ([489], p. 518) ${ }^{127}$ In his answer three weeks later, Pauli was soft on "whether a mathematically unified combination of the electromagnetic and gravitational fields in a classical field theory is possible [...]", but adamant on its relation to quantum theory:

"[...] that I have another opinion than you on the question, mentioned in your letter, of the physical usability of singularity-free solutions of classical field equations. To me it deems that, even if such solutions do exist in a suitably chosen field theory, it would not be possible to relate them with the atomic facts in physics in the way you wish, ${ }_{128}$ namely in a way that avoids the statistical interpretation, in principle." ([489], p. 621.)

\subsection{A period of undecidedness (1949/50)}

With two sets of field equations at hand (the "strong" and "weak" versions), it cost some effort for Einstein to decide which of the two was the correct one. As will be seen in Section 9.2, early in 1949 he had found a new way of deriving the "weak" field equations, cf. [149]. ${ }^{129}$ In a letter of 16 August 1949 to his friend Besso, who had asked him to tell him about his generalized field equations, Einstein presented these "weak" equations and commented:

\footnotetext{
126 Einstein denoted the Hermitian Ricci tensor $P_{i k}=\stackrel{\text { Her }}{K}$ ik by $R_{i k}$.

127 "Die große Schwierigkeit liegt darin, daß man keine Methode hat, singlaritätenfreie strenge Lösungen abzuleiten, die ja allein physikalisch interessant sind. Das Wenige, was wir aber haben ausrechnen können, hat mein Vertrauen in diese Theorie gestärkt." (1. April 1948)

128 "[...] daß ich die in Ihrem Brief erwähnte Frage der physikalischen Brauchbarkeit von singularitätsfreien Lösungen von klassischen Feldgleichungen anders beurteile als Sie. Es scheint mir nämlich, daß selbst wenn solche Lösungen in einer passend gewählten Feldtheorie existieren, es nicht möglich wäre, sie mit den atomaren physikalischen Tatsachen in der von Ihnen gewünschten, eine statistische Deutung prinzipiell vermeidenden Weise in Beziehung zu setzen." (21. April 1948)

129 Submitted on 12 March 1949, but published only in 1950.
} 
"Now you will ask me: Did God tell this into your ear? Unfortunately, not. But the way of proceeding is: identities between the equations must exist such that they are compatible. [...] For their compatibility, i.e., that continuation from a [time-] slice is possible, there must be 6 identities. These identities are the means to find the equations. [...]" ([163], p. 410). ${ }^{130}$

Six weeks later, on 30 September 1949, Einstein had changed his mind: he now advocated the "strong" version (200) - (202) of Section 7.2.

"I recently found a very forceful derivation for this system; it shows that the equations follow from the generalized field as naturally as the gravitational equations from the postulate of the symmetric field $g_{(i k)}$. The examination of the theory still meets with almost unsurmountable mathematical difficulties [...]" ([163], p. 423). ${ }^{131}$

Consistent with Einstein's undecidedness are both, his presentation of UFT in Appendix II of the 3rd Princeton edition of The Meaning of Relativity [150], and another letter to Besso of 15 April 1950 [163]. In both, he had not yet come to a final conclusion as to which must be preferred, the "weak" or the "strong" equations. To Besso, he explained that the "weak" equations could be derived from a variational principle and thus are "compatible".

"On the other hand, one is pushed to the stronger system by formal considerations [...]. But the compatibility for this stronger system is problematic; i.e., at first one does not know whether the manifold of its solutions is sufficiently large. After many errors and efforts I have succeeded in proving this compatibility" ([163], p. 439). ${ }^{132}$

At first, Einstein seems to have followed a strategy of directly counting equations, variables, and identities. However, early in 1952 he seems to have had a new idea: the $\lambda$-transformations. He wrote to Besso on 6 March 1952 that he had made "very decisive progress (a couple of weeks ago)." The field equations, hitherto not uniquely determined theoretically, now were known:

"Apart from [coordinate-] transformation invariance, invariance also is assumed for the transformations of the non-symmetric 'displacement field' $\Gamma_{i k}^{l}:\left(\Gamma_{i k}^{l}\right)^{*}=\Gamma_{i k}^{l}+\delta_{i}^{l} \lambda_{k}$, where $\lambda_{k}$ is an arbitrary vector. In this extended group, the old gravitational equations are no longer covariant [...]."([163], p. 465) $)^{133}$

We will come back to his final decision in Section 9.2.3.

\footnotetext{
130 "Du wirst nun fragen: Hat Dir dies Gott ins Ohr gesagt? Leider nein. Aber das Mittel ist: Es muss zwischen den Gleichungen Identitäten geben, derart, dass sie kompatibel sind. [.. ] Es muss also zwischen den Gleichungen 6 Identitäten geben (18-12), damit sie "kompatibel" (d.h. von einem Schnitt aus fortsetzbar) sind. Diese Identitäten sind das Mittel, um die Gleichungen aufzufinden. [...]"

131 "Ich habe neuerdings eine sehr zwingende Ableitung für dies System gefunden; sie zeigt, dass diese Gleichungen ebenso natürlich aus dem verallgemeinerten Feld folgen, wie die Gravitationsgleichungen aus der Setzung des symmetrischen Feldes $g_{(i k)}$. Die Prüfung der Theorie stösst aber immer noch auf schier unüberwindliche mathematische Schwierigkeiten."

132 "Andererseits wird man zu dem stärkeren System durch formale Überlegungen gedrängt [...]. Von diesem stärkeren System ist aber die Kompatibilität problematisch; d.h., man weiss zunächst nicht, ob die Mannigfaltigkeit seiner Lösungen hinreichend gross ist. Nach vielen Irrtümern und Anstrengungen ist es mir gelungen, diese Kompatibiltät zu beweisen."

133 "Abgesehen von der Transformations-Invarianz soll Invarianz auch bestehen für die Transformation des nicht symmetrischen 'Verschiebungsfeldes' $\Gamma_{i k}^{l}:\left(\Gamma_{i k}^{l}\right)^{*}=\left(\Gamma_{i k}^{l}+\delta_{i}^{l} \lambda_{k}\right.$ wobei $\lambda_{k}$ ein beliebiger Vektor ist. In dieser erweiterten Gruppe sind die alten Gravitationsgleichungen nicht mehr kovariant [...]."
} 


\subsubsection{Birthday celebrations}

Einstein's seventieth birthday was celebrated in Princeton with a seminar on "The Theory of Relativity in Contemporary Science", in which E. P. Wigner, H. Weyl, and the astronomers G. M. Clemence and H. P. Robertson lectured. UFT was left aside [183]. Weyl, in his lecture "Relativity Theory as a Stimulus in Mathematical Research", came near to it when he said:

"The temptation is great to mention here some of the endeavors that have been made to utilize these more general geometries for setting up unified field theories encompassing the electromagnetic field beside the gravitational one or even including not only the photons but also the electrons, nucleons, mesons, and whatnot. I shall not succumb to that temptation." ([693], p. 539.)

As it suited to a former assistant of Einstein, in his article celebrating his master's 70th birthday, Banesh Hoffmann found friendly if not altogether exuberant words even for Einstein's struggle with UFT [281]. For 25 years Einstein had devoted his main scientific work to the problem of the structure of matter and radiation. He tried to gain an insight:

"[...] by abstract reasoning from a few general assumptions. In this he is following the heroic method that proved so successful [...] in the theory of relativity. Unfortunately there are many possible approaches, and since each requires a year or more of intensive computation, progress has been heartbreakingly slow."

That Hoffmann himself was a little outside of mainstream physics can be seen from his remark that quantum theory, now dominating physics, "has developed a stature comparable to that of the theory of relativity." ([281], p. 54/55.) Hoffmann was also one of the contributors to the special number of Reviews in Modern Physics "in commemoration of the seventieth birthday of Albert Einstein" issued in September 1949. Possibly, the best remembered paper among the 38 articles is Gödel's "new type of cosmological solutions", with local rotation and closed timelike world lines, now just named "Gödel's solution" [227]. Only E. Straus wrote an article about UFT: "Some results in Einstein's unified field theory" [592]. The others, big names and lesser known contributors except for the mathematician J. A. Schouten, shunned this topic. Schouten's contribution surveyed classical meson theories in view of their making contact with the conformal group [539]. In connection with Yukawa's prediction of a meson and with Hoffmann's similarity geometry (cf. Section 3.1), he boldly stated: "[...] the conformal field theory failed to ask for a meson field, but the meson field came and asked for a conformal theory!" (ibid., p. 423.) Einstein's oldest son Hans Albert reported on "Hydrodynamic Forces on a Rough Wall" [180].

Belatedly, toward the end of 1949, some sort of a "Festschrift" for Einstein appeared with 25 contributions of well-known physicists and philosophers, among them six Nobel prize winners [536]. Most interesting is Einstein's own additional contribution, i.e., his "Autobiographical Notes", written already in 1946. He described his intentions in going beyond general relativity and essentially presented the content of his paper with E. Straus [179] containing the "weak field equations" of UFT. His impression was:

"that these equations constitute the most natural generalization of the equations of gravitation. The proof of their physical usefulness is a tremendously difficult task, inasmuch as mere approximations will not suffice. The question is: 'What are the everywhere regular solutions of these equations?'” ([153], p. 93-94.) $)^{134}$

134 "Ich glaube, dass diese Gleichungen die natürlichste Verallgemeinerung der Gravitationsgleichungen darstellen. Die Prüfung ihrer physikalischen Brauchbarkeit ist eine überaus schwierige Aufgabe, weil es mit Annäherungen nicht getan ist. Die Frage ist: 'Was für im ganzen Raume singularitätsfreie Lösungen dieser Gleichungen gibt es?'” 


\subsection{Einstein 1950}

\subsubsection{Alternative derivation of the field equations}

As we have seen, one of Einstein's main concerns was to find arguments for choosing a quasiunique system of field equations for UFT. His first paper of 1949 opened with a discussion, mostly from the point of view of mathematics, concerning the possibilities for the construction of UFT with a non-symmetric fundamental tensor. According to Einstein: "The main difficulty in this attempt lies in the fact that we can build many more covariant equations from a non-symmetric tensor than from a symmetric one. This is due to the fact that the symmetric part $g_{(i k)}$ and the antisymmetric part $g_{[i k]}$ are tensors independently" ([149], p. 120). As the fundamental tensor is no longer considered symmetrical, the symmetry of the connection (as in Riemannian geometry) must also be weakened. By help of the conjugate quantities of Section 2.2.2 (Hermitian, transposition symmetry), Einstein's constructive principle then is to ask "that conjugates should play equivalent roles in the field-equations." According to him, this necessitates the introduction of the particular form (30) for the compatibility condition. In fact, for the conjugate: ${ }^{135}$

$$
\tilde{g}_{i k \|}\left\|l=g_{+-} i\right\| l,
$$

while

$$
\tilde{g}_{\substack{i k \\++l}}=g_{\underline{-}-\underline{i} \| l} \text {. }
$$

Einstein seemingly was not satisfied with the derivation of the field equations from a variational principle in "both previous publications" ([147, 179]), because of the status of equations $(252)$. To obtain them, either Lagrangian multipliers or a restriction of the metric ("must be derivable from a tensor potential") had to be used. Now, he wanted to test the field equations by help of some sort of Bianchi-identity such as (cf. Section 2.3.1, Eq. (68), or Section 2.1.3 of Part I, Eq. (30)):

$$
\stackrel{+}{K}^{i}{ }_{j\{k l \| m\}}=2 \stackrel{+}{K}^{i}{ }_{r\{k l} S_{m\} j}{ }^{r} .
$$

After a lengthy calculation, he arrived at: ${ }^{136}$

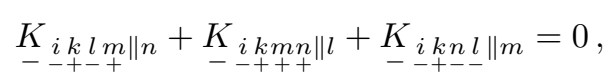

and by further trace-forming ${ }^{137}$

$$
g^{k l}\left[-K_{+-} \| m+\underset{++}{k m \| l}+\Sigma_{\underline{-}-\underline{m} \| k}\right]=0 .
$$

In Eq. (257), two contractions of $\underline{-}^{i}{ }_{j k l}$ were introduced: $-\underline{-}_{j k}:=\underline{-}^{l}{ }_{j k l}$ and $\Sigma_{m l}=-g_{m i} g^{j k} K_{-}^{i}{ }_{j k l}$. Neither $\Sigma_{m l}$ nor $\underline{K}_{j k}$ are Hermitian: We have $\underset{-}{-\tilde{K}_{l m}}=\Sigma_{m l}$. The anti-Hermitian part of $\underline{-}_{j k}$ is given by

$$
\underline{K}_{k l}-\underline{\tilde{K}}_{l k}=-\left(S_{l, k}+S_{k, l}\right)+L_{k l}^{s} S_{s} .
$$

If vector torsion is absent, i.e., $S_{k}=L_{[k s]}^{s}=0$, then $\underline{K}_{j k}$ becomes Hermitian, and $-K_{-} l m=\Sigma_{m l}$. Equation (257) then can be written as

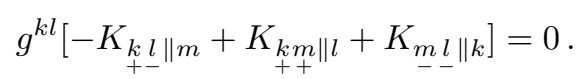

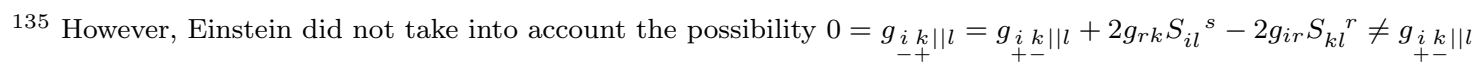
if torsion does not vanish.

136 Einstein used the curvature tensor $K_{j k l}^{i}=-\underline{-}_{j k l}^{i}$ (cf. (54)). The contraction $-K_{j k}$ is denoted $R_{j k}$ by him. 137 We replace Einstein's notation [149] $S_{i j}$ by $\Sigma_{i j}$ in order to avoid confusion with the torsion tensor.
} 
Therefore, Einstein demanded that the contribution of $\underline{-}_{[j k]}$ to the Eq. 259) be in general:

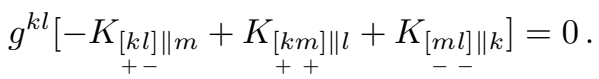

Schrödinger had derived (260) before by an easier method with the help of the Lie-derivative; cf. [556]. We will meet (260) again in Section 10.3.1. A split of (259) into symmetric and skew symmetric parts (inside the bracket) would give the equation:

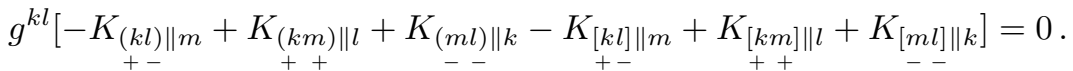

At best, as a sufficient condition, the vanishing of the symmetric and skew-symmetric parts separately could take place. Besides (260) the additional equation would hold:

$$
g^{k l}\left[-K_{+-}^{(k l) \| m}+\underset{++}{K_{(k m) \| l}}+K_{(-+} K_{(m l) \| k}\right]=0 .
$$

As we will see, by a further choice (cf. (265), this equation will be satisfied. Einstein first reformulated (260) into:

$$
g^{k l}\left[\underline{K}_{[k l], m}+\underline{-}_{[m k], l}+\underline{K}_{[l m], k}\right]=0,
$$

and then took

$$
\underline{-}_{[k l], m}+\underline{-}_{[l m], k}+\underline{-}_{[m k], l}=0
$$

as its solution and as part of the field equations. He then added as another field equation:

$$
\underline{K}_{(k l)}=0
$$

by which (262) is satisfied. Thus, in [149], with a new approach via identities for the curvature tensor and additional assumptions, Einstein had reached the weak field equations of his previous paper [148]. No physical interpretation of the mathematical objects appearing was given by him.

\subsubsection{A summary for a wider circle}

In Appendix II of the third Princeton edition of his book The Meaning of Relativity, Einstein gave an enlarged introduction on 30 pages into previous versions of his UFT. The book was announced with fanfare in the Scientific American [151]:

"[...] Einstein will set forth what some of his friends say is the long-sought unified field theory. The scientist himself has given no public hint of any such extraordinary development, but he is said to have told close associates at the Institute for Advanced Studies that he regards the new theory as his greatest achievement" ([564], p. 26).

It was Princeton University Press who had used Einstein's manuscript for this kind of advertising much to his distress; a page of it even "appeared on the front page of The New York Times under the heading 'New Einstein Theory Gives a Master Key to the Universe'." ([469], p. 350.) Einstein's comment to his friend M. Solovine, on 25 January 1950, was:

"Soon I will also send you the new edition of my little book with the appendix. A few weeks ago, it has caused a loud rustling noise in the newspaper sheets although nobody except the translator had really seen the thing. It's really drole: laurels in advance" ([160], p. 96). ${ }^{138}$

\footnotetext{
138 "Bald werde ich Ihnen auch die neue Auflage meines Büchleins übersenden mit dem Anhang, der vor ein paar Wochen in den Blättern ein starkes Rauschen erzeugt hat, obwohl niemand außer dem Übersetzer das Ding zu Gesicht bekommen hat. Es ist wahrhaft drollig: Lorbeeren auf Vorschuss".
} 
In the book, the translator is identified to have been Sonja Bargmann, the wife of Valentine, who also had translated other essays by Einstein.

In Appendix II, with the assumption that

(1) all equations remain unchanged with respect to simultaneous substitution of the $g_{i k}$ and $\Gamma_{i k}{ }^{l}$ by $\tilde{g}_{i k}$ and $\tilde{\Gamma}_{i k}^{l}$ (transposition invariance),

(2) all contractions of the curvature tensor (54) vanish,

(3) that (30) hold,

Einstein arrived at the field equations ${ }^{139}$ (30), (201), and (202):

$$
\begin{array}{r}
g_{i k \| l}:=g_{i k, l}-g_{r k} L_{i l}^{r}-g_{i r} L_{l k}^{r}=0, \\
S_{j}(L):=L_{[i m]}^{m}=0, \\
K_{j k}(L)=0 .
\end{array}
$$

In place of (266) the equivalent equations $g^{+-}{ }_{\| l}^{i k}=0$, or $\hat{g}^{i k}{ }_{\| l}^{+k}=0$ with $\hat{g}^{i k}=\sqrt{\operatorname{det}\left(g_{i k}\right)} g^{i k}$ can be used. Moreover, if in addition (267) is taken into account, then also

$$
\hat{g}_{, l}^{[i l]}=0
$$

is satisfied. This is due to a relation to be met again below [(cf. Eq. (276)]:

$$
\hat{g}^{[i l]}{ }_{\| l}^{+l}=\hat{g}_{, l}^{[i l]}-\hat{g}^{[i l]} S_{l} .
$$

([179], Eq. (3.4), p. 733.)

For Einstein, this choice ("System I") "is therefore the natural generalization of the gravitational equation" ([150], p. 144). A little later in the Appendix he qualified his statement as holding "from a formal mathematical point of view [...]" ([150], p. 150) because the manifold of solutions of "System I" might be too small for physical purposes. Moreover, "System I" could not be derived from a variational principle. He then set up such a variational principle ${ }^{140}$

$$
\mathcal{H}=P_{i k} \hat{g}^{i k}
$$

with the Hermitian Ricci tensor $P_{i k}$. As Einstein wanted to again get (267), he introduced another connection $\Gamma^{*}$ by $\Gamma_{i j}{ }^{k}=\Gamma_{i j}^{*}{ }^{k}-\frac{2}{3} \Gamma_{[i}^{*} \delta_{j]}^{k}$ which does not satisfy $\Gamma_{j}^{*}=\Gamma_{[i m]}^{*}=0$, such that just independent components could be varied. The result is his "System Ia":

$$
\begin{aligned}
\hat{g}^{i k}{ }_{\| l}^{+-}-\frac{1}{3}\left(\mathcal{M}^{i} \delta_{l}{ }^{k}-\mathcal{M}^{k} \delta_{l}{ }^{i}\right) & =0, \\
L_{i} & =0, \\
P_{i k} & =0,
\end{aligned}
$$

with $\mathcal{M}^{i}:=\hat{g}^{[i l]}{ }_{\| l}$. The following identity holds: ${ }^{141}$

\footnotetext{
139 Einstein wrote $\Gamma_{i j}{ }^{k}$ in place of $L_{i j}{ }^{k}$ used here.

${ }^{140}$ In ([150], p. 142), Einstein used the same symbol $R_{i k}$ (here called $P_{i k}$ ) for a different tensor equally denoted $R_{i k}$ in his previous paper [147], which he now named $A_{i k}$ ([150], p. 141), and which here is $P_{i k}^{*}$ (cf. Section 2.3.2, Eq. (75)). Worse, in the 4th Princeton edition of The Meaning of Relativity ([156], p. 140), the notation $R_{i k}$ now corresponded to $K_{-}$.

${ }^{141}$ In order to prove (276), use of
}

$$
\hat{g}^{i k}{ }_{\| l}^{+}=\hat{g}^{i k}, l+\hat{g}^{s k} L_{s l}{ }^{i}+\hat{g}^{i s} L_{l s}{ }^{k}-\hat{g}^{i k} L_{(l s)}^{s}
$$




$$
\mathcal{M}^{i} \equiv \hat{g}_{, l}^{[i l]}-\hat{g}^{[i l]} \Gamma_{l}
$$

In order to make vanish $\mathcal{M}^{i}$, with the help of a Lagrange multiplier $l_{i}$, he introduced the term $l_{i} \hat{g}_{, r}^{[i r}$ into the Lagrangian and arrived at 'System Ib', i.e., the weak field equations:

$$
\begin{aligned}
\hat{g}^{+k}{ }_{\| l} & =0, \\
L_{i} & =0, \\
P_{(i k)} & =0, \\
P_{[i k], l}+P_{[k l], i}+P_{[l i], k} & =0 .
\end{aligned}
$$

In all three systems, equations (249), (269), (272) are to be used for expressing the components of the (asymmetric) connection by the components of the (asymmetric) metric. The metric then is determined by the remaining equations for the Ricci tensor.

The only remarks concerning a relationship between mathematical objects and physical observables made by Einstein at the very end of Appendix II are:

(1) (269) shows that there is no magnetic current density present (no magnetic monopoles),

(2) the electric current density (or its dual vector density) is represented by the tensor $g_{[i k], l}+$ $\left.g_{[k l], i}+g_{[l i], k}\right)$.

In order to obtain these conclusions, a comparison to Maxwell's equations has been made (cf. (210) and (211) of Section 7.3). As for all of Einstein's papers in this class, $g_{(i k)}$ describes the gravito-inertial, and $g_{[i k]}$ the electromagnetic fields.

For the first time, Einstein acknowledged that he had seen Schrödinger's papers without giving a reference, though: "Schrödinger, too, has based his affine theory [...]". Max Born, in his review of Einstein's book, bluntly stated: "What we have before us might therefore be better described as a program than a theory." ([41], p. 751.) According to Born, Einstein "tries to find a theory of the classical type of such refined structure that it contains the essential features of atomic and quantum theory as consequences. There are at present few physicists who share this view." The review by W. H. McCrea reflected his own modesty. Although more cautious, he was very clear: ${ }^{142}$

"Nevertheless, what has been written here shows how much of the subsequent formulation appears to be entirely arbitrary and how little of it has received physical interpretation. It is clear that a tremendous amount of investigation is required before others than the eminent author himself are enabled to form an opinion of the significance of this work" ([420], p. 129).

The "eminent author" himself confessed in a letter to Max Born of 12 December 1951:

\footnotetext{
is necessary. Note the symmetrization in the last term of (275). For both $\hat{g}^{++} \|_{\| l}$ and $\hat{g}^{--}{ }_{\| l}^{[i k]}$ the identities contain further terms.

142 Note that the counting of editions is different in London and Princeton. Born's and McCrea's reviews refer to the 4th London edition which contained the famous Appendix II for the first time and was published in April 1950 (after a 3rd edition in 1946 with an appendix on cosmology corresponding to the appendix of the 2nd Princeton edition of 1946). Methuen's 5th edition came out in London in July 1951 [154], the 6th in 1956. Princeton's 3rd edition appeared also in 1950, containing Appendix II as well, the 4th edition in (March) 1953, the 5th in 1955. A further complication is that, in 1950 a further printing of the 3rd Princeton edition appeared [150] in which a mistake in Appendix II was corrected (cf. [490], p. 138, letter Pauli to Strauss; footnote 3 by K. von Meyenn). Although, on the back side of the title page in Methuen's 4th edition it is claimed: "This edition and the Princeton University Press third edition are identical" [151], Appendix II in the American and the British editions of 1950 are quite different! If translations into other languages with several editions by themselves are referred to by authors (like to the Italian translation, published by Einaudi), it becomes even harder to give a reliable reference.
} 
"Unfortunaletly, the examination of the theory is much too difficult for me. After all, a human being is only a poor wretch!" ([168], p. 258). ${ }^{143}$

Also, W. Pauli commented on this 3rd edition. A correspondent who inquired about "the prospects of using Einstein's new unified field theory as an alternative basis for quantum electrodynamics" obtained a demoralizing answer by him in a letter of 4 July 1950:

"Regarding Einstein's 'unified' field theory I am extremely skeptical. It seems not only arbitrary to add a symmetrical and an anti-symmetrical tensor together but there is also no reason why Einstein's system of equations should be compatible (the counting of identities between these equations given in the appendix of the new edition turned out to be incorrect). Certainly no work on similar lines will be done in Zürich." ([490], p. 137-138)

Einstein's former assistant and co-author Leopold Infeld sounded quite skeptical as well when he put the focus on equations of motion of charges to follow from "the new Einstein theory". By referring to the 3rd Princeton edition of The Meaning of Relativity he claimed that, in 1st approximation, "the equations of motion remain Newtonian and are uninfluenced by the electromagnetic field." But he offered immediate comfort by the possibility "that this negative result is no fault of Einstein's theory, but of the conventional interpretation by which it was derived" [303].

\subsubsection{Compatibility defined more precisely}

In a long paragraph ( $\S 7$ ) of Appendix II, of this 3rd Princeton edition, Einstein then asked about the definition of what he had termed "compatibility". This meant that "the manifold of solutions" of the different systems of field equations "is extensive enough to satisfy the requirements of a physical theory" ([150], p. 150), or put differently, the field equations should not be overdetermined. In view of the "System I"'s containing four more equations, i.e., 84, than the 80 unknowns, this might become a difficulty. Starting from the Cauchy problem, i.e., the time development of a solution off an initial hypersurface, he counted differential equations and the variables to be determined from them. ${ }^{144}$ To give an example for his method, he first dealt with general relativity and obtained the result that the general solution contains four free functions of three (spacelike) coordinates - "apart from the functions necessary for the determination of the coordinate system" ([150], p. 155). The corresponding results for "Systems Ia, (I)" according to him turned out to be: 16 , (6) arbitrary functions of three variables, respectively. In case "System I" should turn out to be too restrictive to be acceptable as a physical theory, Einstein then would opt for the "weak field equations" ("System Ib"). "However, it must be admitted that in this case the theory would be much less convincing than if system (I) can be preserved" ([150], p. 160).

This discussion calls back into memory the intensive correspondence Einstein had carried on between 1929 and 1932 with the French mathematician E. Cartan on an equivalent problem within the theory of teleparallelism, cf. Section 6.4.3 of Part I. At the time, he had asked whether his partial differential equations (PDEs) had a large enough set of solutions. Cartan had suggested an "index of generality" $s_{0}$ for first-order systems in involution which, essentially, gave the number of arbitrarily describable free data (functions of 3 spacelike variables) on an initial hypersurface $\left(t=t_{0}\right)$. He calculated such indices, for Maxwell's equations with currents to be $s_{0}=8$, and without $s_{0}=4$, for Einstein's vacuum field equations $s_{0}=4$, (in this case 4 free functions of 4 variables exist ${ }^{145}$ ), and of course, for Einstein's field equations in teleparallelism theory. Note that

143 "Die Prüfung der Theorie ist leider viel zu schwierig für mich. Der Mensch ist ja doch nur ein armes Luder!"

144 Today, we would say that he split the field equations into evolution- and constraint equations.

145 This meant that Cartan considered two solutions connected by a coordinate transformation as different while physicists identify them. This seems to imply that his index of generality is meaningless in physics. 
Maxwell's and Einstein's vacuum field equations according to Cartan exhibit the same degree of generality. It had taken Cartan a considerable effort of convincing Einstein of the meaningfulness of his calculations also for physics ([116], pp. 114, 147, 174). Already in the 3rd Princeton edition of The Meaning of Relativity, in Appendix II [150], Einstein tried to get to a conclusion concerning the compatibility of his equations by counting the independent degrees of freedom but made a mistake. As mentioned above, W. Pauli had noticed this and combined it with another statement of his rejection of the theory. Compatibility was shown later by A. Lichnerowicz [369] (cf. Section 10.5).

It is unknown whether Einstein remembered the discussion with Cartan or had heard of Pauli's remark, when he tackled the problem once more; in the 4th Princeton edition of his book The Meaning of Relativity, Appendix II [156] Cartan's name is not mentioned. In the meantime, Mme. Choquet-Bruhat, during her stay at Princeton in 1951 and 1952, had discussed the Cauch-problem with Einstein such that he might have received a new impulse from her. To make the newly introduced concept of "strength" of a system of PDE's more precise, he set out to count the number of free coefficients of each degree in a Taylor expansion of the field variables; if all these numbers are non-negative, he called the system of PDE's "absolutely compatible". He then carried out a calculation of the number of coefficients $\Omega_{n}$ remaining "free for arbitrary choice" for the free wave equation, Maxwell's vacuum equations, the Einstein vacuum equations, and particular field equations of UFT. Let us postpone the details and just list his results. ${ }^{146}$ For the wave equation $\Omega_{n}=\left(\begin{array}{c}n+3 \\ n\end{array}\right) \frac{6}{n}$. According to him "the factor $\frac{6}{n}$ gives the fraction of the number of coefficients (for the degree $n \gg 1$ ), which remain undetermined by the differential equation" ([156], p. 152). Similarly, he found for the Maxwell vacuum equations, $\Omega_{n}=\left(\begin{array}{c}n+3 \\ n\end{array}\right) \frac{12}{n}$. Einstein noted that by introducing the vector potential $A_{i}$, and taking into account the Lorentz gauge, i.e., by dealing with

$$
\eta^{r s} \partial_{r} \partial_{s} A_{i}=0, \quad \eta^{r s} \partial_{r} A_{s}=0
$$

the counting led to $\Omega_{n}=\left(\begin{array}{c}n+3 \\ n\end{array}\right) \frac{18}{n}$. He ascribed the increase in the number of freely selectable coefficients (loss of strength) to the gauge freedom for the vector potential. For the Einstein vacuum equations, he obtained $\Omega_{n}=\left(\begin{array}{c}n+3 \\ n\end{array}\right) \frac{15}{n}$. In applying his method to the "weak" field equations of Section 9.2.2, Einstein arrived at $\Omega_{n}=\left(\begin{array}{c}n+3 \\ n\end{array}\right) \frac{45}{n}$. In comparing this to the calculation for other field equations in UFT, he concluded that "the natural generalization of the gravitational equations in empty space" is given by the "weak" field equations ([156], p. 164).

Obviously, Einstein was not satisfied by his calculations concerning the "strength" of PDE's. In the 5th Princeton edition of his book The Meaning of Relativity, Appendix II [158], ${ }^{147}$ he again defined a system of PDE's as "absolutely compatible" if, in a Taylor expansion of the field variable $\Phi$, the number of free $n$-th order coefficients $\left.\frac{\partial^{n}}{\partial x^{1} \partial x^{2} \ldots \partial x^{n}} \Phi\right|_{P}$, at a point $P$ does not become negative. He then gave a name to the number of free coefficients calculated before: he called it "coefficient of freedom". The larger this coefficient is, the less acceptable to him is the system of PDE's. Let $p$ denote the number of field variables, $s$ the number of field equations of order $q$, and $w$ the number of identities among the field equations in the form of PDE's of order $q^{\prime}$. Then,

146 Einstein used a non-standard notation for combinatorics; we have transcribed it into symbols currently in use.

147 In the 5th Princeton and 6th London edition, now Appendix II is the same. 
writing $z$ in place of the previous $\Omega_{n}$, his formulas could be condensed into:

$$
\begin{array}{r}
z=p\left(\begin{array}{c}
n+3 \\
n
\end{array}\right)-[ \\
\left.=\left(\begin{array}{c}
n+3-q \\
n-q
\end{array}\right)-w\left(\begin{array}{c}
n+3-q^{\prime} \\
n-q^{\prime}
\end{array}\right)\right] \\
=\left(\begin{array}{c}
n+3 \\
n
\end{array}\right)\left\{a+\frac{z_{1}}{n}+\frac{z_{2}}{n^{2}}+\ldots\right\},
\end{array}
$$

with $a=p-s+w \stackrel{!}{\geq} 0$ required for absolute compatibility; $z_{1}=3\left(q s-q^{\prime} w\right)$ is the "coefficient of freedom." 148 Again, Einstein calculated $z_{1}$ for several examples, among them Maxwell's vacuum field equations in flat space-time:

$$
F_{, k}^{i k}=0,{ }^{*} F_{, k}^{i k}=0
$$

with the identities

$$
F_{, k i}^{i k}=0,{ }^{*} F_{, k i}^{i k}=0,
$$

Here $p=6, s=8, q=1, q^{\prime}=2, w=2$. In agreement with the result from the previous edition, the calculation led him to $a=0, z_{1}=12$. In contrast, it turned out that the "coefficient of freedom" for the gravitational vacuum field equations in general relativity, in the 2nd calculation became smaller, i.e., $z_{1}=12$ ([158], p. 139). Fortunately, now both equations have the same "index of generality" (Cartan) and the same "coefficient of freedom" (Einstein). Likewise, Einstein found $z_{1}=42$ instead of the previous $z_{1}=45$ for the "weak", and $z_{1}=48$ for a concurring system with transposition invariance such that he again adopted the "weak" one as before. (280) with its values for $a, z_{1}$ is not yet the correct formula. Such a formula was derived for involutive, quasi-linear systems of PDEs by a group of relativists around F. Hehl at the University of Cologne at the end of the 1980s ([595], Eqs. (2.9), (2.10), [596]). By their work, also the relation between the Cartan coefficient of generality and Einstein's coefficient of freedom has now been provided. According to M. Sué ([595], p. 398), it seems that Einstein's coefficient of freedom is better suited for a comparison of the systems investigated than Cartan's degré d'arbitraire. In mathematics, a whole subdiscipline has evolved dealing with the Cartan-Kähler theorem and the Cartan-Characters for systems of PDE's. Cf. [57].

The fact that Einstein had to correct himself in his calculations of the "coefficient of freedom" already may raise a feeling that there exists a considerable leeway in re-defining field variables, number and order of equations etc. Moreover, he did not prove the independence of the relative order of "strength" for two PDE's from mathematical manipulations affecting the form of the equations but not their physical content: remember (279), (282), (283). Regrettably, it is to be noted that Einstein's last attempt to gain a reliable mathematical criterion for singling out one among the many possible choices for the field equations in UFT remained unconvincing. ${ }^{149}$

\subsubsection{An account for a general public}

Following an invitation by the editors of Scientific American to report on his recent research, Einstein made it clear that he would not give

"[...] a detailed account of it before a group of readers interested in science. That should be done only with theories which have been adequately confirmed by experiment." ([152], p. 14.) ${ }^{150}$

148 If the system of field equations and identities consists of $s_{i}$ field equations of order $q_{i}$ and $w_{i}$ identities of order $q_{i}^{\prime}$, then $q s$ must be replaced by $\Sigma_{i} q_{i} s_{i}$ and $q^{\prime} w$ by $\Sigma_{i} q_{i}^{\prime} w_{i}$.

149 As has been shown from a modern point of view, the necessity for avoiding ghosts (negative energy solutions), algebraic inconsistencies, or other troubling features, made the strong system unacceptable [102].

150 Note the difference to our time where popular accounts of speculative theories abound (with some of them using 
He then talked about the epistemological basis of science, men's curiosity and passion for the understanding of nature before touching upon problems connected with a generalization of general relativity. Two questions were very important, though not yet fully answered: the uniqueness of the field equations and their "compatibility". He then sketched the three systems of field equations obtained, here denoted $E_{3}$ (System I), and $E_{1}, E_{2}$ (Systems Ia, Ib). He again stressed that $E_{3}$ (System I) "is the only really natural generalization of the equations of gravitation". However, it was not a compatible system as were the other two.

"The skeptic will say: 'It may well be true that this system of equations is reasonable from a logical standpoint. But this does not prove that it corresponds to nature.' You are right, dear skeptic. Experience alone can decide the truth. Yet we have achieved something if we have succeeded in formulating a meaningful and precise question." ([152], p. 17.)

Painstaking efforts and probably new mathematics would be required before the theory could be confronted with experiment. The article is illustrated by a drawing of Einstein's head by the American artist Ben Shahn ([152], p. 17).

There were not only skeptics but people like Dr. C. P. Johnson in the Chemistry Department of Harvard University who outrightly criticized "Dr. Albert Einstein's recent unified field theory" [311]. He pointed out that the theory permits a class of similarity solutions, i.e., with $g_{i k}\left(x^{l}\right)$ also $g_{i k}\left(k x^{l}\right)$ solves the field equations. For a system of two charged and one uncharged massive bodies he qualitatively constructed a contradiction with Coulomb's law. Einstein replied with a letter printed right after Johnson's by stating that if solutions depending upon a continuous parameter exist, "then the field equations must prevent the coexistence within one system of such elementary solutions pertaining to arbitrary values of their parameters." The underlying reason was that "for a system of field equations to be acceptable from a physical point of view, it has to account for the atomic structure of reality." This would entail that regions of space corresponding to a 'particle' have discrete masses and charges. The coexistence of similar solutions "in one and the same world" would make the theory unacceptable [161]. As we shall see, the situation of Einstein's UFT was worse: it did not lead to Coulomb's law - at least not in the lowest approximations. See Sections 9.3.3, 9.6, and Section 10.3.2.

Nevertheless, Einstein remained optimistic; in the same letter to Max Born, in which he had admitted his shortcoming vis-a-vis the complexity of his theory, he wrote:

"At long last, the generalization of gravitation from a formal point of view now is fully convincing and unambiguous - unless the Lord has chosen a totally different way which no one can imagine." 151

His Italian colleague Bruno Finzi was convinced that the final aim had been reached:

"[...] all physical laws laws of the macrocosm reduce to two geometrical identities

[...]. Therefore, the game is over, and the geometric model of the macrocosm has been constructed." ([200], p. 83) ${ }^{152}$

\footnotetext{
the multiverse concept), none of which rests on the least bit of empirical data. In fact, some theorists even propose to reconsider the need for empirical support of a physical theory rather than criticize such speculative theories. cf. the quote from Science magazine in ([707], p. 249).

151 "Die Verallgemeinerung der Gravitation ist nun endlich vom formalen Gesichtspunkt völlig überzeugend und eindeutig - wenn der Herrgott nicht einen total anderen Weg gewählt hat, von dem man sich keine Vorstellung machen kann." ([168], p. 258.)

152 “[...] tutte le leggi fisiche del macrocosmo si reducono poi a due identità geometriche [...]. E con ciò il gioco è fatto, e il modello geometrico del macrocosmo è costruito."
} 
However, at the end of his article, Finzi pointed out that it might be difficult to experimentally verify the theory, and thought it necessary to warn that even if such an empirical base had been established, this theory would have to be abandoned after new effects not covered by it were observed.

\subsection{Einstein 1953}

In the fourth edition of Einstein's The Meaning of Relativity, invariance with regard to $\lambda$-transformations was introduced as a new symmetry principle (cf. (52) of Section 2.2.3). Also transposition invariance is now claimed to be connected to the "indifference of the theory" (UFT) with respect "to the sign of electricity" ([156], p. 144). This interpretation rests on Einstein's identification of the electric current density with $g_{[i k], l}+g_{[k l], i}+g_{[l i], k}$. Einstein still grappled with the problem of how to set up a convincing system of field equations. As in the previous edition, he included (269) as an "a priori condition" in his variational principle by help of a (1-form)multiplier $\sigma_{i}$. However, he renounced using $\Gamma_{i}=0$. Without specification of the Lagrangian $\mathcal{H}$, from $\delta \int \mathcal{H} d \tau=\int\left(\hat{V}_{l}^{i k} \delta \Gamma_{i k}^{l}+W_{i k} \delta \hat{g}^{i k}\right) d \tau=0$ the field equations follow - without use of the multiplier-term - to be:

$$
\hat{V}_{l}^{i k}=0, \quad W_{i k}=0 ;
$$

- with use of the multiplier-term -

$$
\hat{V}_{l}^{i k}=0, \quad \hat{g}_{, l}^{[i l]}=0, \quad W_{i k}+\sigma_{[i, k]}=0 .
$$

Elimination of the multiplier $\sigma_{i}$ led to the equations (named "System II" by Einstein)

$$
\hat{V}_{l}^{i k}=0, \hat{g}_{, l}^{[i l]}=0, \quad W_{(i k)}=0, W_{[i k], l}+W_{[k l], i}+W_{[l i], k}=0 .
$$

A paragraph then was devoted to the choice of the proper Lagrangian. Einstein started from (196) and removed a divergence term in $\stackrel{\text { Her }}{K}_{i k} \hat{g}^{i k}$. After variation (with inclusion of the multiplier term) the ensuing field equations, Einstein's "system IIa", were:

$$
\begin{aligned}
& \hat{g}_{i k-1 \mid l}=0, \\
& \Gamma_{i}=0, \\
& \underline{K}_{(i k)}=0, \\
& \underline{K}_{[i k], l}+\underline{-}_{[k l], i}+\underline{-}_{[l i], k}=0,
\end{aligned}
$$

i.e., a version of the Einstein-Straus weak field equations. The road to the weak field equations (287) - (290) followed here still did not satisfy Einstein, because in it the skew-symmetric parts of both the metric and the connection could also be taken to be purely imaginary. In order to exclude this possibility and work with a real connection, he introduced $\lambda$-transformations and presented a further derivation of the field equations. He set up a variational principle invariant under the $\lambda$-transformation and arrived at the same system of field equations as before. The prize payed is the exclusion of a physical interpretation of the torsion tensor.

In a discussion covering twelve pages, Einstein again took up the question of "compatibility" from the previous edition and introduced the concept of the "strength" of a system of differential equations in order to bolster up his choice of field equation. A new principle applying to physical theories in general is put forward: "The system of equations is to be chosen so that the field quantities are determined as strongly as possible" ([156], p. 149). In Section 9.2.3, a detailed discussion of this new principle has been given such that we need not dwell on it. The paucity 
of physical input into Einstein's approach to UFT becomes obvious here. May it suffice to say that according to the new principle the weak field equations (277), (278) are called "stronger" than the strong field equations (268). However, this has lead to the misleading labeling of the system II as the "strong system" [704]. The relation of geometrical objects to physical observables remained unchanged when compared to the 3rd edition ([150]). Einstein saw a close relationship to Maxwell's theory only in the linear approximation where "the system decomposes into two sets of equations, one for the symmetric components of the field, and the other for the antisymmetric components.[...] In the rigorous theory this independence no longer holds." ([156], p. 147.)

Both, the concept of "strength" of a system of differential equations and the concluding $§ 5$ "General remarks concerning the concepts and methods of theoretical physics" point to Einstein's rather defensive position, possibly because of his feeling that the particular field equations of unified field theory for which he strove so hard, rested on flexible ground. This was due not only to the arbitrariness in picking a particular field equation from the many possibilities, but also to the failure of the theory to include a description of concepts forming an alternative to quantum theory. Einstein stuck to the classical field and rejected both de Broglie's "onde pilote", and Bohm's attempt away from the statistical interpretation of the wave function. At the very end of his Meaning of Relativity he explained himself in this way:

"[...] I see in the present situation no possible way other than a pure field theory, which then however has before it the gigantic task of deriving the atomic character of energy. [...] We are [...] separated by an as yet insurmountable barrier from the possibility of confronting the theory with experiment. Nevertheless, I consider it unjustified to assert, a priori, that such a theory is unable to cope with the atomic character of energy." ([156], p. 165.)

An indirect answer to this opinion was given by F. J. Dyson in an article on "field theory" in the Scientific American. He claimed "that there is an official and generally accepted theory of elementary particles, known as the 'quantum field theory'." According to him, while there still was disagreement about the finer details of the theory and its applications:

"The minority who reject the theory, although led by the great names of Albert Einstein and P. A. M. Dirac, do not yet have any workable alternative to put in its place." ([137], p. 57.)

Such kind of sober judgment did not bother The New York Times which carried an almost predictable headline: "Einstein Offers New Theory to Unify Law of the Cosmos." ([469], p. 350.)

Privately, in a letter to M. Solovine of 28 May 1953, Einstein seemed less assured. Referring to the appendix of this 4th edition of "The Meaning of Relativity", he said: "[...] Of course, it is the attempt at a theory of the total field; but I did not wish to give the thing such a demanding name. Because I do not know, whether there is physical truth in it. From the viewpoint of a deductive theory, it may be perfect (economy of independent concepts and hypotheses)." ([160], p. 96). ${ }^{153}$

\subsubsection{Joint publications with B. Kaufman}

In the Festschrift for Louis de Broglie on the occasion of his 60th birthday (15.8.1952) organized by M.-A. Tonnelat and A. George, Einstein again summarized his approach to UFT, now in an article

\footnotetext{
153 [...] "Es ist natürlich ein Versuch einer Theorie des Gesamtfeldes; aber ich wollte dem Ding keinen so anspruchsvollen Namen geben. Denn ich weiss ja nicht, ob physikalische Wahrheit darin steckt. Vom Standpunkt einer deduktiven Theorie ist es aber denkbar vollkommen (Sparsamkeit an unabhängigen Begriffen und Hypothesen)."
} 
with his assistant Bruria Kaufman ${ }^{154}$ [172]. In a separate note, as kind of a preface he presented his views on quantum theory, i.e., why he still was trying "[...] to solve the quantum riddle on another path or, to at least help for preparing such a solution." ([155], p. 4.) ${ }^{155}$ He expressed his well-known epistemological position that something like a "real state" of a physical system exists objectively, independent of any observation or measurement. A list of objections to the majority interpretation of quantum theory was given. At the end of the note, a link to UFT was provided:

"My endeavours to complete general relativity by a generalization of the gravitational equations owe their origin partially to the following conjecture: A reasonable general relativistic field theory could perhaps provide the key to a more perfect quantum theory. This is a modest hope, but in no way a creed." ([155], p. 14. $)^{156}$

As in the 4th edition of his book [156], the geometrical basics were laid out, and one more among the many derivations of the weak field equations of UFT given before was presented. At first, it looked weird, but in referring to a result of the "researches of E. Schrödinger" (without giving a reference, though) Einstein \& Kaufman took over Schrödinger's "star"-connection:

$$
{ }^{*} \Gamma_{i k}^{l}:=\Gamma_{i k}^{l}+\frac{2}{3} \delta_{[i}^{l} \Gamma_{k]}
$$

introduced in ([552], p. 165, Eq. (10)). For it, ${ }^{*} \Gamma_{k}=0$ holds which leads to simplifications. Under a $\lambda$-transformation the Ricci curvature is not invariant [cf. (87)]. In order to make the variational principle invariant, due to

$\delta \int d^{4} x \hat{g}^{i k} \underline{K}_{i k}(\Gamma)=\delta\left[\int d^{4} x \hat{g}^{i k} K_{-}\left({ }^{*} \Gamma\right)+2 \int d^{4} x \hat{g}_{, l}^{[i l]} \lambda_{i}\right]$, as an ad-hoc- (or as Einstein \& Kaufman called it, an a priori-) condition is needed:

$$
\hat{g}_{, l}^{[i l]}=0 .
$$

The further derivation of the field equations led to the known form of the weak field equations:

$$
\begin{aligned}
& g_{i k \|} \|_{*}=0, \\
& K_{*}(i k)=0, \\
& \underset{*}{K_{[}[k], l}+\underset{*}{K_{[k l], i}}+\underset{*}{K_{[l i], k}}=0 .
\end{aligned}
$$

Here, the covariant derivative refers to the connection ${ }^{*} \Gamma$ and $K_{*}(i k) \equiv K_{-}\left({ }_{i k}\left({ }^{*} \Gamma\right)\right.$.

In addition, a detailed argument was advanced for ruling out the strong field equations. It rests partially on their failure to guarantee the possibility to superpose weak fields. The method used is a weak-field expansion of the metric and the affine connection in a small parameter $\epsilon$ :

$$
g_{i k}=\epsilon \underset{1}{g_{i k}}+\epsilon_{2}^{2} g_{i k}+\ldots, \quad \Gamma_{i j}^{k}=\epsilon \Gamma_{1}{ }^{k}+\epsilon^{2} \Gamma_{2}{ }^{k}+\ldots
$$

154 Bruria Kaufman(-Harris) (1918-2010) received an MA from Hebrew University (Jerusalem) in 1938 and a PhD from Columbia University, New York in 1948. During the late 1940s she collaborated with Lars Onsager, and then from 1950 until the mid 1950s with Albert Einstein. Her own interests were, e.g., the application of spinor analysis to physical problems, and special functions seen from the angle of Lie algebra. Her 2nd marriage, in 1996, with Nobel prize winner W. Lamb ended in divorce.

155 “...] doch unablässig nach einem Wege gesucht habe, das Quantenrätsel auf einem anderen Weg zu lösen oder doch wenigstens eine Lösung vorbereiten zu helfen."

156 "Meine Bemühungen, die allgemeine Relativitätstheorie durch Verallgemeinerung der Gravitationsgleichungen zu vervollständigen, verdanken ihre Entstehung zum Teil der Vermutung, dass eine vernünftige allgemein relativistische Feldtheorie vielleicht den Schlüssel zu einer vollkommeneren Quantentheorie liefern könne. Dies ist eine bescheidene Hoffnung, aber durchaus keine Überzeugung." 
After expanding the field equations up to 2nd order, the authors came to the conclusion that the "strong equations" strongly constrain "the additivity of symmetric and antisymmetric weak fields. It seems that by this any usefulness of the 'strong system' is excluded from a physical point of view." ([172], p. 336.) ${ }^{157}$

In an appendix to the paper with title "Extension of the Relativistic Group" [172], Einstein combined the "group" of coordinate transformations with the $\lambda$-transformations to form a larger transformation group $U$. (cf. the letter to Besso mentioned in Section 9.1.) He then discussed the occurring geometric objects as representations of this larger group and concluded: "The importance of the extension of the transformation group to $U$ consists in a practically unique determination of the field equation." ([172], p. 341.) $)^{158}$

The next paper with Bruria Kaufman may be described as applied mathematics [173]. Einstein returned to the problem, already attacked in the paper with E. Straus, of solving (30) for the connection in terms of the metric and its derivatives. The authors first addressed the question: "What are necessary and sufficient conditions for constant signature of the asymmetric metric-field to hold everywhere in space-time?" At first, it was to be shown "that the symmetric part $g_{(i k)}$ of the tensor $g_{i k}$ is a Riemannian metric with constant signature". For a proof, the conditions $\operatorname{det}\left(g_{(i k)}\right) \neq 0$ and a further algebraic inequality were needed. In addition, the connection $\Gamma$, calculated from $\hat{g}_{i k \mid l l}=0$, had to be finite at any point and "algebraically determined". This is meant in the sense of interpreting $\hat{g}_{i k-} \| l=0$ as an inhomogeneous linear equation for the components of $\Gamma$. The situation was complicated by the existence of the algebraic invariants of the non-symmetric $g_{i k}$ as well as by the difficulty to solve for the connection as a functional of the metric tensor. Although not necessary for a solution of the field equations, according to the authors it is "of interest to give a closed expression for the $\Gamma$ as a function of the $g_{i k}$ and its first derivatives." This problem had been addressed before and partial results achieved by V. Hlavatý [258, 260], and S. N. Bose [52]. ${ }^{159}$ The papers by M.-A. Tonnelat published earlier and presenting a solution were not referred to at all [622, 623, 630, 629].

In the sequel, $g_{(i k)}$ is given a Lorentz signature. By using special coordinates in which $g_{i}^{k}=\rho_{i} \delta^{k}{ }_{i}$ (no summation on the index i), it can be seen that " $\rho_{1}=\frac{1}{\rho_{2}}$ is on the unit circle from which the point -1 has been excluded"; the other two roots $\rho_{3}=\frac{1}{\rho_{4}}$ are positive. It is shown in the paper that among the three algebraic invariants to be built from $g_{i}^{k}=g^{k s} g_{s i}$ only two are independent:

$$
S_{1}:=\sum_{s=1}^{4} \rho_{s}, S_{2}:=\sum_{r>s} \rho_{r} \rho_{s}
$$

As a "sufficient condition for regularity and unique algebraic determination of $\Gamma_{i k}{ }^{l}$ " the authors derive ([173], p. 237):

$$
S_{1} \neq 2 \neq S_{2}
$$

In a lengthy calculation filling six pages, a formal solution to the compatibility equation (30), seen as an algebraic equation for $\Gamma$ is then presented: "[...] it is cumbersome, and not of any practical utility for solving the differential equations" ([173], p. 238).

\footnotetext{
157 "Cette remarque montre à quel point les 'equations fortes' restreignent l'additivité des champs faibles symétrique et antisymétrique. Elle semble exclure d'un point de vue physique toute utilité du 'système fort'".

158 "La signification de l'extension du groupe de transformation en groupe $U$ consiste en ce que celui-ci détermine pratiquement les équations du champ d'une façon complète." The original English text of the paper was translated into French by Mme. M.-A. Tonnelat, the appendix by J. Winter.

159 The authors state that Hlavatý's papers "came to our attention after our own paper had been completed." ([173], p. 231, footnotes 1 \& 2.) Bose had submitted his paper to the Annals of Mathematics on Sept. 29 , 1952 and had sent it to Einstein; it appeared only in January 1954 - one issue prior to the paper by Einstein and Kaufman.
} 


\title{
9.3.2 Einstein's 74th birthday (1953)
}

Einstein agreed to let his 74th birthday be celebrated with a fund-raising event for the establishing of the Albert Einstein College of Medicine of Yeshiva University, New York. Roughly two weeks later, according to A. Pais The New York Times carried an article about Einstein's unified field theory on the front page [471]. It announced the appearance of the 4th Princeton edition of The Meaning of Relativity with its Appendix II, and reported Einstein as having stated that the previous version of 1950 of the theory had still contained one important difficulty. According to him: "[...] This last problem of the theory now finally has been solved in the past months." 160 Probably, this refers to Einstein's new way of calculating his "coefficient of freedom" introduced for mirroring the "strength" of partial differential equations. In a letter to Carl Seelig of 14 September 1953, Einstein tried to explain the differences between the 3rd and the 4th edition of The Meaning of Relativity:

\begin{abstract}
"A new theory often only gradually assumes a stable, definite form when later findings allow the making of a specific choice among the possibilities given a priori. This development is closed now in the sense that the form of the field laws is completely fixed. - The theory's mathematical consistence cannot be denied. Yet, the question about its physical foundation still is completely unsettled. This follows from the fact that comparison with experience is bound to the discovery of exact solutions of the field equations which seems impossible at the time being." ${ }^{61}$ ([570], p. 401-402)
\end{abstract}

\subsubsection{Critical views: variant field equation}

Already in 1950, Infeld had pointed to the fact that the equations of motion for particles following from Einstein's UFT (weak field equations), calculated in the same way as in general relativity, did not lead to the Lorentz equations of motion [304]. This result was confirmed by Callaway in 1953. Callaway identified the skew part of the fundamental tensor with the electromagnetic field and applied a quasistatic approximation built after the methods of Einstein and Infeld for deriving equations of motion for point singularities. He started from Einstein's weak field equations and showed that (208) could not influence the equations of motion. His conclusion was that he could reduce "Einstein's new unified field theory to something like Maxwell's equations in a sufficiently low approximation", but could not obtain the Lorentz equation for charged particles treated as singularities in an electromagnetic field [69].

In order to mend this defect, Kursunŏglu modified the Einstein-Straus weak field equations by beginning with the identity (257) and adding another identity formed from the the metric $g_{i j}=a_{i j}+i k_{i j}$ and its 1 st derivatives only [342, 343]: ${ }^{162}$

$$
g^{k l}\left[-g_{+-} l\left\|m+g_{++} m\right\| l+g_{\underline{m}-\underline{l}} \| k\right]=0 .
$$

\footnotetext{
160 This is a quotation and back-translation from the German edition of Pais' book ([472], p. 289).

161 "Eine neue Theorie nimmt eben oft nur allmählig eine feste definitive Form an, indem aufgrund späterer Erkenntnisse zwischen apriori sich bietenden Möglichkeiten eine ganz bestimmte Auswahl getroffen wird. Diese Entwicklung ist nun insofern abgeschlossen, als die Form der Feldgesetze völlig feststeht. - Die mathematische Folgerichtigkeit der Theorie lässt sich nicht bestreiten. Die Frage ihrer physikalischen Gültigkeit ist aber noch völlig ungeklärt. Es liegt dies daran, daß der Vergleich mit der Erfahrung an das Auffinden rechnerischer Lösungen der Feldgleichungen geknüpft ist, die sich einstweilen nicht gewinnen lassen."

${ }^{162}$ Kursunŏglu used a Hermitian metric $g_{i j}=a_{i j}+i \phi_{i j}$ and connection $\Gamma_{i j}{ }^{k}=\Gamma_{(i j)}^{k}+i \Gamma_{[i j]}^{k}$.
} 
Kursunŏglu's ensuing field equations were:

$$
\begin{aligned}
\hat{g}_{, l}^{[i l]} & =0, \\
K_{(i k)}+p^{2}\left(h_{i j}-b_{i j}\right) & =0, \\
\underline{L}_{[i k], l}+\underline{-}_{[k l], i}+\underline{-}_{[l i], k}+p^{2}\left(k_{[i k], l}+k_{[k l], i}+k_{[l i], k}\right) & =0,
\end{aligned}
$$

with $p$ real or imaginary, and ${ }^{163}$

$$
b^{i j}=\sqrt{\frac{l}{g}} l^{(i j)},
$$

where $b_{i j}$ is the inverse of $b^{i j} . I_{i k l}:=k_{[i k], l}+k_{[k l], i}+k_{[l i], k}$ is connected with the electrical 4-current density $J^{r}$ through $I_{i k l}:=\epsilon_{i k l r} J^{r}$.

In fact, as Bonnor then showed in the lowest approximation (linear in the gravitational, quadratic in the electromagnetic field), the static spherically symmetric solution contains only two arbitrary constants $e, m$ besides $p^{2}$ which can be identified with elementary charge and mass; they are separately selectable [33]. However, in place of the charge appearing in the solutions of the Einstein-Maxwell theory, now for $e^{2}$ the expression $e^{2} p^{2}$, and for $e, \frac{m e}{p^{2}}$ occurred in the same solution. The definition of mass seemed to be open, now. For vanishing electromagnetic field, the solution reduced to the solution for the gravitational field of general relativity.

In a discussion concerning the relation of matter and geometry, viz. matter as a "source" of geometry or as an intrinsic part of it, exemplified by the question of the validity of Mach's principle, J. Callaway tried to mediate between the point of view of A. Einstein with his unified field theory already incorporating matter, geometrically, and the standpoint of J. A. Wheeler who hoped for additional relations between matter and space-time fixing the matter tensor as in the case of the Einstein-Maxwell theory ([70], p. 779). Callaway concluded that "if the approach of field theory is accepted, it is necessary to construct a theory in which space-time and matter enter as equals." But he would not accept UFT as an alternative to quantum theory.

\subsection{Einstein 1954/55}

The last paper with B. Kaufman was submitted three months before, and appeared three months after Einstein's death [174]. In it, the authors followed yet two other methods for deriving the field equations of UFT. Although the demand for transposition-invariance was to play a considerable role in the setting up of the theory, in the end invariance under $\lambda$-transformations became the crucial factor. In the first approach $(\S 1)$, instead of the previously used connection $\Gamma$, Einstein and Kaufman introduced another one, $\Gamma^{*}$, containing four new variables (a 1-form) $\Lambda_{k}$ "supernumerary to the description of the field", and defined by:

$$
\Gamma_{i k}^{*}{ }^{l}=\Gamma_{i k}^{l}-\delta_{i}^{l} \Lambda_{k} .
$$

During variation of the Lagrangian, $\Gamma^{*}$ and $\Lambda_{k}$ were treated as independent variables; after the variation $\Lambda_{k}$ could be fixed arbitrarily ("normed"). This trick allowed that four variational field equations could replace four equations put in by hand as had been the equation $\Gamma_{k}=0$ in earlier approaches. The following relation resulted:

$$
\underset{-}{K}(\Gamma)_{i k}=\underset{-}{K}\left(\Gamma^{*}\right)_{i k}-2 \Lambda_{[i, k]},
$$

such that the Lagrangian could be written as

$$
\mathcal{H}(\Gamma)=\mathcal{H}\left(\Gamma^{*}\right)-2 \hat{g}^{r s} \Lambda_{[r, s]} .
$$

163 After use of Eq. (10). 
After the variation with respect to $\Gamma_{i k}^{*}{ }^{l}, \hat{g}^{i k}$, and $\Lambda_{k}$ the choice $\Lambda_{k}=\frac{2}{3} \Gamma_{k}$ was made leading to $\Gamma_{k}^{*}=0$. Although the wanted transposition-invariant field equations did come out, the authors were unhappy about the trick introduced. "The reason for our difficulties is that we require the field equations to be transposition-invariant, but we start out from a variational function which does not have that property. The question arises naturally whether we cannot find a form of the variational function which will itself be transposition-invariant, [...]." ([174], p. 131.) In order to obtain such a Lagrangian, they replaced the connection by a quantity $U_{i k}^{l}$ called "pseudo-tensor". It is transforming like a tensor only under linear coordinate transformations $(\S 2-\S 3)$ :

$$
U_{i k}^{l}:=\Gamma_{i k}^{l}-\Gamma_{i s}^{s} \delta_{k}^{l}
$$

From (307) we see that $U_{i k}^{l}$ does not transform like a connection. As a function of $U_{i k}^{l}$ the Ricci tensor:

$$
\underset{-}{K}(\Gamma(U))_{i k}=U_{i k, s}^{s}-U_{i s}^{r} U_{r k}^{s}+\frac{1}{3} U_{i r}^{r} U_{s k}^{s}
$$

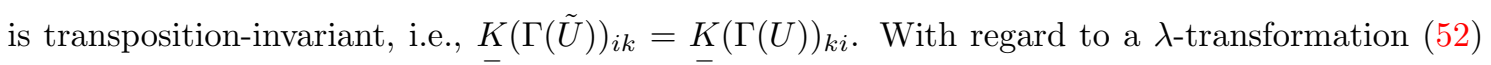
the "pseudo-tensor" $U$ transforms as:

$$
{ }^{\prime} U_{i k}^{l}=U_{i k}^{l}+2 \delta_{[i}^{l} \lambda_{k]}
$$

A short calculation shows that $K(\Gamma(U))_{i k}$ is invariant under (52).

As a Lagrangian, now $\mathcal{H}=\hat{g}^{i k} K(\Gamma(U))_{i k}$ was taken. Variation with respect to the variables $U_{i k}^{l}, \hat{g}^{i k}$, i.e.,

$$
\delta \mathcal{H}=\left(\hat{g}^{i k} \delta U_{i k}^{s}\right)_{, s}+\hat{N}_{s}^{i k} \delta U_{i k}^{s}+\underset{-}{K}(\Gamma(U))_{i k} \delta \hat{g}^{i k}
$$

led to the field equations:

$$
\begin{aligned}
& \hat{N}_{, s}^{i k} \equiv-\hat{g}_{, s}^{i k}-\hat{g}^{r k}\left(U_{r s}^{i}-\frac{1}{3} \delta_{s}^{i}\right) U_{r t}^{t}-\hat{g}^{i r}\left(U_{s r}^{k}-\frac{1}{3} \delta_{s}^{k} U_{t r}^{t}\right)=0, \\
& K(\Gamma(U))_{i k}=0
\end{aligned}
$$

with $K_{-}(\Gamma(U))_{i k}$ given by (308). Although the authors do not say it, Eqs. (311) and (312) are equivalent to the "weak" field equations (287)-(290). By inserting infinitesimal coordinate- and $\lambda$-transformations into (310), five identities called "Bianchi-identities" result. Modulo the field equations,

$$
\left(\hat{g}^{i k} \delta U_{i k}^{s}\right)_{, s}=0
$$

holds as well. With the help of special infinitesimal coordinate transformations and the Bianchiidentities a "conservation law for energy and momentum" is derived:

$$
\hat{T}_{i, s}^{s}=0
$$

where $\hat{T}_{i}^{k}:=\left(\hat{g}^{r s} U_{r s}^{k}\right)_{, i}$.

The results of this paper [174] were entered into the 5th Princeton edition of The Meaning of Relativity, Appendix II [158]. ${ }^{164}$ In "A note on the fifth edition" dated December 1954, Einstein wrote:

164 They are also reproduced in the 6th London (Methuen) edition [162]. 
"For I have succeeded - in part in collaboration with my assistant B. Kaufman - in simplifying the derivations as well as the form of the field equations. The whole theory becomes thereby more transparent, without changing its content" ([158], page before p. 1). ${ }^{165}$

From a letter to his friend Solovine in Paris of 27 February 1955, we note that Einstein was glad: "At least, yet another significant improvement of the general theory of the gravitational field (non-symmetric field theory) has been found. However, the thus simplified equations also cannot be examined by the facts because of mathematical difficulties". ([160], p. 138) ${ }^{166}$ In this edition of The Meaning of Relativity', he made a "remark on the physical interpretation". It amounted to as$\operatorname{sign} \hat{g}_{, s}^{[i s]}$ to the (vanishing) magnetic current density and $\frac{1}{2} \eta^{i k l m} g_{[i k], l}$ to the electric current density.

The paper with Kaufman ended with “Considerations of compatibility and 'strength' of the system of equations", a section reappearing as the beginning of Appendix II of the 5th Princeton edition. The $16+64$ variables $U_{i k}^{l}, \hat{g}^{i k}$ must satisfy the $16+64$ field equations (311), (312). The argument is put forward that due to $\lambda$-invariance (identification of connections with different $\lambda$ ) the $64 \Gamma$-variables were reduced to 63 plus an additional identity.

"In a system with no $\lambda$-invariance, there are $64 \Gamma$ and no counterbalancing identity. This is the deeper reason for the relative weakness of systems which lack $\lambda$-invariance. We hold to the principle that the stronger system has to be preferred to any weaker system, as long as there are no special reasons to the contrary." ([174], p. 137.)

However, it is to be noted that in the 5th Princeton edition the $\lambda$-transformation is reduced to $\lambda_{k}=\partial_{k} \lambda$ (Eq. (5) on p. 148). In a footnote, Appendix II of the 4 th Princeton edition of The Meaning of Relativity is given as a reference for the concept of "strength" of a system of differential equations (cf. Section 9.2.3). W. Pauli must have raised some critical questions with regard to the construction of the paper's Lagrangian from irreducible quantities. In her answer of 28 February 1956, B. Kaufman defended the joint work with Einstein by discussing an expression $\left(\alpha_{1} g^{i k}+\alpha_{2} g^{k i}\right) R_{i k}=\gamma^{i k} R_{i k}$ : "Now the point is here that $g^{i k}$ was introduced in our paper merely as a multiplying function such as to make, together with $R_{i k}$, a scalar. Hence $g^{i k}$ can just as well be this multiplier. The field equations we would get from this Lagrangian would be identical with the equations in our paper, except that they would be expressed in terms of $g^{i k}$." As to scalars quadratic in curvature she wrote: " [...] our paper does not claim that the system we give is $100 \%$ unique. In order to do that one would have to survey all possible additional tensors which could be used in the Lagrangian. We only considered the most 'reasonable' ones." ([492], pp. 526-527.)

Until 1955, more than a dozen people had joined the research on UFT and had published papers. Nevertheless, apart from a mentioning of H. Weyl's name (in connection with the derivation of the "Bianchi"-identities) no other author is referred to in the paper. B. Kaufman was well aware of this and would try to mend this lacuna in the same year, after Einstein had passed away.

At the "Jubilee Conference" in Bern in July 1955, ${ }^{167}$ based on her recent work with A. Einstein [174], B. Kaufman gave an account "[... of the logical steps through which one goes when trying to set up this generalization", i.e., of general relativity to the "theory of the non-symmetric field" ([322], p. 227). After she presented essential parts of the joint paper with Einstein, Kaufman

\footnotetext{
165 Einstein's original handwritten text (in German) can be read in the Einstein Archives Online, call nr. 2-14.00.

166 "Immerhin hat sich noch eine erhebliche Verbesserung der Verallgemeinerung der Theorie des Gravitationsfeldes gefunden (nichtsymmetrische Feldtheorie). Aber auch die so vereinfachten Gleichungen lassen sich wegen der mathematischen Schwierigkeiten noch nicht mit den Thatsachen prüfen."

167 Commemorating 50 years of what had been called "special relativity". B. Kaufman is listed there under the name of her first husband as "Harris-Kaufman".
} 
discussed its physical interpretation and some of the consequences of the theory. As in [148, 150], and [156], the electric current density is taken to be proportional to $g_{[i k], l}+g_{[k l], i}+g_{[l i], k}$. From this identification, transposition invariance receives its physical meaning as showing that "all equations of the theory shall be invariant under a change of the sign of electric charge" ([322], p. 229). With (252), i.e., $\hat{g}^{[i k]}{ }_{k}=0$, holding again in the theory, $\hat{g}^{i 4}$ (with $i=1,2,3$ ) is identified with the components of the magnetic field. In the linear approximation, the field equations decompose into the linear approximation of the gravitational field equations of general relativity and into the weaker form of Maxwell's equations already shown in (210), (211) of Section 7.3.

In the section "Results in the theory" of her paper, Kaufman tried to sum up what was known about the "theory of the non-symmetric field". Both in terms of the number of papers published until the beginning of 1955, and of researchers in UFT worldwide, she did poorly. She mentioned Schrödinger, Hlavatý, Lichnerowicz and M.-A. Tonnelat as well as one or two of their collaborators, and some work done in Canada and India. The many publications coming from Italian groups were neglected by her as well as contributions from Japan, the United States and elsewhere which she could have cited. Nevertheless, in comparison with Einstein's habit of non-citation, her references constituted a "wealth" of material. Of the few general results obtained, Lichnerowicz' treatment of the Cauchy initial value problem for the weak field equations of UFT and his proof that a unique solution exists seems to be the most important [369]. Unfortunately, his proof, within general relativity, that static, regular solutions behaving asymptotically like a Schwarzschild point particle (with positive mass) are locally Euclidean, could not be carried over to UFT. This was due to the complications caused by the field equations (311), (312). While (311) could be solved, in principle, for the $U_{i k}^{l}$ as functions of $\hat{g}^{i k}, \hat{g}_{, l}^{i k}$, its subsequent substitution into (312) led to equations too complicated to be solved - except in very special cases. In his summary of the conference, Pauli mocked Kaufman's report:

"We have seen how Einstein and Mrs. Kaufman struggled heroically [...], and how this fight has been led with the particular weapon of the $\lambda$-transformation. Certainly, all this is formally very correct; however, I was unable to make sense of the $\lambda$-transformations, either physically or geometrically." [486] ${ }^{168}$

The search for solutions of the weak field equations had begun already with exact spherically symmetric, static solution derived by a number of authors (cf. [475, 31, 32]; see Sections 8.3, and $9.6)$.

In a final section, B. Kaufman discussed two alternatives to what she now called "Einstein's theory" for the first time. The first is Schrödinger's purely affine version of the theory as presented in his book [557]. His field equations replacing (289), (290) were (cf. Section 8.1, Eq. (237):

$$
\begin{array}{r}
K_{(i k)}=\lambda g_{i k}, \\
\underline{-} K_{[i k], l}+\underline{-}_{[k l], i}+\underline{-}_{[l i], k}=\lambda\left(g_{[i k], l}+g_{[k l], i}+g_{[i], k}\right) .
\end{array}
$$

Here, $\lambda$ plays the role of cosmological constant. At first, in the affine theory, $g_{i k}$ is defined by the 1.h.s. of (315), but then this equations is read like an Einstein equation for the metric.

In a note added for the reprint in 1954 of his book, Schrödinger warned the readers of his chapter on UFT that he did not regard his unification of gravitation and electromagnetism:

168 "Wir haben dann gesehen, wie Einstein und Frau Kaufman einen heroischen Kampf gekämpft haben [...], und wie dieser Kampf mit der besonderen Waffe einer $\lambda$-Transformationen geführt worden ist. Das ist sicher in formaler Hinsicht alles richtig; aber ich habe weder einen physikalischen noch einen geometrischen Sinn dieser $\lambda$-Transformation sehen können." 
"[...] as anything like a well-established theory. It must be confessed that we have as yet no glimpse of how to represent electrodynamic interaction, say Coulomb's law. This is a serious desideratum. On the other hand we ought not to be disheartened by proofs, offered recently by L. Infeld, M. Ikeda and others, to the effect, that this theory cannot possibly account for the known facts about electrodynamic interaction. Some of these attempts are ingenious, but none of them is really conclusive." ([557], reprint 1954, p. 119.)

\subsection{Reactions to Einstein-Kaufman}

Schrödinger found the paper by Einstein and Kaufman in the Festschrift for L. de Broglie [172] "very important" and set out to draw some consequences. In particular, by using the approximationscheme of Einstein and Kaufman, he showed that "the electric current-four-vector is in general different from zero throughout the field" ([559], p. 13). In the strong field equations, $K_{[}[i k]=0$ led to the vanishing of the electric current density. Dropping the so-called cosmological term for convenience, Schrödinger now wrote Eq. (316) of his "weak" field equations in the form:

$$
\underline{K}_{[i k]}+X_{[i, k]}=0
$$

with a free vector-variable $X_{i} \cdot{ }^{169}$ Besides obtaining, in first approximation, Einstein's vacuum field equations of general relativity and one set of Maxwell's equations, he gave as the second set:

$$
\eta^{r s} \partial_{r} \partial_{s} g_{[i k]}-2 X_{[i, k]}=0
$$

From this he concluded that "the curl of the current is essentially the dual of the curl of $\Gamma_{i}$ " (his notation for $\left.X_{i}\right)$. Here, $g_{i k}=\eta_{i k}+g_{i k}+g_{2}+\ldots$

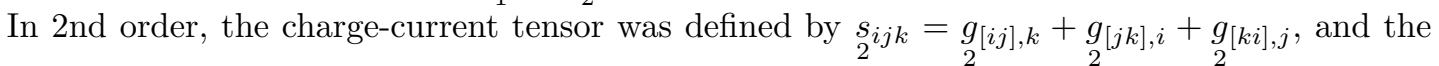
wave equation then $\eta^{r s} \partial_{r} \partial_{s} g_{2}[i k]=s_{2} i j k+\eta^{r m} \eta^{s n} g_{[r s]} B_{n m i k} . B_{n m i k}$ is a linear combination of the 2nd derivatives of $g_{i k}$. However, Schrödinger rejected this equation: "it is not invariant" ([559], p. 19). Since 1952 , Cornelius Lanczos had come to Dublin, first as a visiting, then as a senior professor, and, ultimately, as director at the Dublin Institute for Advanced Studies. In his paper, Schrödinger acknowledged "discussions with my friend professor Cornel Lanczos" ([559], p. 20).

In the Festschrift on the occasion of de Broglie's 60th anniversary, published only in 1953, C. F. von Weizsäcker expressed his opinion clearly that:

"[...] in the future, no reason exists for connecting the metric more closely to the electromagnetic field, and perhaps also to the meson field." ([680], p. 141.) $)^{170}$

One year later, consistent with this, and with Einstein's death "in April 1955, Schrödinger became quite depressed, for he was now convinced that his unified field theory was no longer tenable" ([446], p. 326). In any case, there is no further published research on UFT by him.

M. S. Mishra also studied Einstein's last publication written together with B. Kaufman [174] and solved (311) for the connection. He obtained M.-A. Tonnelat's result (364) of Section 10.2.3

\footnotetext{
169 Schrödinger's notation of the term $X_{[i, k]}$ is awkward: $\frac{4}{3} \Gamma_{[i, k]}$ with $\Gamma_{i}$ unrelated to his connection $\Gamma$.

170 “[... q q'il n'existe désormais aucune raison d'attacher plus intimement à la métrique précisément le champ électromagnétique et peut-être aussi le champ mésonique."
} 
[433]. Instead of beginning with (311) and (312) as Einstein and Kaufman had done, he then introduced "another set of field equations" by taking

$$
\begin{aligned}
\hat{N}_{, s}^{i k} \equiv-\hat{g}^{i k}{ }_{s}-\hat{g}^{r k}\left(U_{r s}^{i}-\frac{1}{3} \delta_{s}^{i}\right) U_{r t}^{t}-\hat{g}^{i r}\left(U_{s r}{ }^{k}-\frac{1}{3} \delta_{s}^{k} U_{t r}^{t}\right) & =0, \\
S_{i k} & =0,
\end{aligned}
$$

with the contracted curvature tensor which is transposition-symmetric $S_{i k}:=U_{i k, s}^{s}-U_{i s}^{t} U_{t k}^{s}+$ $\frac{1}{3} U_{i t}^{t} U_{r k}^{r}$. The solution to (319), (320) is given as:

$$
\Gamma_{l m}^{n}=\left\{\begin{array}{l}
n \\
l m
\end{array}\right\}+2 h^{n s}\left(k_{r(l} S_{m) s}{ }^{r}+\frac{1}{3} g_{s(l} S_{m)}\right)+S_{l m}{ }^{n},
$$

where $S_{l m}{ }^{n}$ is the torsion tensor, and $S_{m}(\Gamma)$ the torsion vector. Mishra then linearized the metric and showed the result to be equivalent to the linearized Einstein-Straus equations (cf. Section 7.3) In the same paper, Mishra suggested another set of field equations by starting from the transposed Ricci tensor and making it transposition invariant in the same manner as Einstein and Kaufman did in their case.

In a joint paper with M. L. Abrol, also directed to the Einstein-Kaufman version of Einstein's theory, Mishra claimed: "It is shown [...] that Infeld's method [cf. [304]] of approximation, to find the equations of motion of charged particles from the system of field equations, fails in this particular theory" [437]. This was due to some unknown terms in the 2nd and 3rd order of the approximation. After a modification of the field equations according to the method of Bonnor [cf. [34]], the Coulomb force appeared in 4th order.

\subsection{More exact solutions}

\subsubsection{Spherically symmetric solutions}

A hope for overcoming the difficulty of relating mathematical objects from UFT to physical observables was put into the extraction of exact solutions. In simple cases, these might allow a physical interpretation by which the relevant physical quantities then could be singled out. One most simple case with high symmetry is the static spherically symmetric (sss) field. Papapetrou's solution of Section 8.3 soon was generalized to the case $v \neq 0, w \neq 0$ by Wyman [709] which means that both, electric and magnetic fields, are now present. Wyman's three different solutions of the weak field equations contain one arbitrary function of the radial coordinate $r$, each. Wyman questioned the physical interpretation of $g_{(i j)}=h_{i j}$ and $g_{[i j]}=k_{i j}$ as standing for the gravitational field and the electromagnetic field, respectively. He built another expression:

$$
a_{i j}(g, L)=g_{(i j)}+q_{i} q_{j}, q_{i}=g_{i s} h^{s t} u_{t} / \sqrt{\left(1+\frac{1}{2} k_{m n} k^{m n}\right)},
$$

with $u_{i}=\frac{h_{i}}{\sqrt{h^{s t} h_{s} h_{t}}}$ and $h_{i}=h_{a b} k^{s a} S_{s i}{ }^{b}$. If $a_{i j}$ is chosen as a metric, then the unique solution of general relativity in this sss case, e.i., the Schwarzschild solution, results. Although Wyman's construction was very artificial, it clearly exemplified the unsurmountable impediment to UFT: the "embarras de richesses" in mathematical objects.

Wyman also questioned the boundary condition used at spacelike infinity: $\operatorname{limes}_{r \rightarrow \infty} g_{a b}=\eta_{a b}$, where $\eta_{a b}$ is the Minkowski metric. By looking at his (or Papapetrou's) sss solutions, he showed that different boundary condition could be set up leading to different solutions: $v r^{2} \rightarrow 0$ for $r \rightarrow \infty$ or $v \rightarrow 0$ for $r \rightarrow \infty$.

Apparently, this left no great impression; the search for sss solutions continued. As two sets of field equations were competing against each other, Einstein's (and Schrödinger's) weak and 
strong equations (with or without cosmological constant $\lambda$ ), we must distinguish the solutions suggested. In the case of sss fields, only one additional field equation, e.g., $R_{[23]}=0$, separates the strong from the weak equations. For the strong equations with $\lambda=0$, Bonnor [31] obtained the general exact solution in the cases for which either a magnetic or an electric field is present. He also generalized Papapetrou's solution for the weak equations to the case where the function $v$ is real or imaginary. The solutions describe spread out charges while the masses are banned into singularities. All solutions display an infinite set of "singular" surfaces between the radial coordinate $r=2 m$ and $r=\infty .{ }^{171}$ For some time, after a note by Bandyopadhyay [8], who claimed that for the strong equations $m e=0$ where $m, e$ are the parameters for mass and charge, ${ }^{172}$ a discussion took place whether isolated massless magnetic monopoles could exist. Since 1948, Papapetrou and Schrödinger had changed the assignment of components of $k_{i j}$ to the electric and magnetic fields; now $g_{[23]}, g_{[31]}, g_{[12]}$ stood for the electric field [479]. Ikeda, in a paper of 1955 , claimed to have shown "that a single magnetic pole cannot exist in the Einstein new theory, as in the Maxwell theory" ([298], p. 272). ${ }^{173}$ This result depended on Ikeda's identification of the electromagnetic field with

$$
F_{a b}=-\frac{1}{\sqrt{-g}}\left(\rho k_{a b}+\frac{h}{2} \epsilon_{a b r s} \check{k}^{r s}\right), \rho=\epsilon^{i j l m} k_{i j} k_{l m},
$$

where $h=\operatorname{det}\left(-h_{a b}\right)$. In 1960, Bandyopadhyay came back to the question and claimed that "the 'stronger' equations will not allow isolated magnetic poles with mass whereas the 'weaker' equations will allow the existence of such entities" ([10], p. 427). Bonnor's second paper of 1952 dealt with the strong equations in the case $v w \neq 0$. Again, the exact solutions described spread out charges of both signs with an infinity of singular surfaces. They were unphysical because they contained no parameter for the mass of the sources [32].

In her book, M.-A. Tonnelat discussed these solutions; her new contribution consisted in the calculation of the components of her connection $\Delta$ - Schrödinger's star connection (27) - and the Ricci $W(\Delta)$-tensor formed from it for the more general case of time-dependent spherically symmetric fields [629], ([632], p. 71, 73). By help of this calculation, her collaborator Stamatia Mavridès could present a general result: for $g_{[23]}=0, g_{[10]} \neq 0$ (as the non-vanishing components of $k_{i j}$ ) only static exact spherically symmetric solutions do exist [402]. Later in Italy, F. De Simoni published another generalization of Wyman's and Bonnor's solutions for the weak field equations; he used the Ricci tensor of Einstein and Straus (73) made Hermitian, i.e., $P_{i j}+\tilde{P}_{i j}$. His paper is not referred to in Tonnelat's book [114]. J. R. Vanstone mistakenly believed he had found timedependent spherically symmetric solutions, but the time dependency can be easily removed by a coordinate transformation [668]. Also B. R. Rao had calculated some, but not all components of the connection for the case of a time-dependent spherically symmetric field but had failed to find a time-dependent solution [502].

Unfortunately, all this work did not bring further insight into the physical nature of the sss solutions. The only physically "usable" solution remained Papapetrou's. He also proved the following theorem: "Spherically-symmetric solutions periodical in time of the "weak" field equations satisfying the boundary conditions $\hat{g}^{i k} \rightarrow \eta^{i k}$ for $r \rightarrow \infty$ are, in 1st approximation, identical to solutions of the "strong" field equations" [478].

\footnotetext{
${ }^{171}$ Note, however, that at the time, for Bonnor, $r=2 m$ was still a singularity, because he defined a singularity by one of the metrical components becoming 0 or $\infty$.

$172 \mathrm{e} \sim l^{2}$ in Papapetrou's solutions $(243-245)$.

173 "The Einstein new theory" is the one presented in the 3rd Princeton edition of The Meaning of Relativity.
} 


\subsubsection{Other solutions}

Still worse, in 1958 a sobering contribution from the Canadian mathematician Max Wyman ${ }^{174}$ and his German colleague Hans Zassenhaus ${ }^{175}$ cast doubt on any hope for a better understanding of the physical contents of UFT by a study of exact solutions. They investigated solutions of Einstein's non-symmetrical UFT with vanishing curvature tensor: in this case weak and strong field equations coincide. Unlike for flat space-time in general relativity, a large class of solutions resulted; a situation which, according to the authors, "[...] merely adds to the confusion and indicates that the form of the theory is far from complete". They went on: "However, as far as a satisfactory physical interpretation of such a theory goes, almost complete chaos seems to result." ([710], p. 228.) Strong words, indeed, but not without reason:

"[...] for a theory based on a non-symmetric tensor an infinity of tensors of all orders exist. The only hope to extract from this maze the proper mathematical expressions to use for physical quantities would thus have to be physical in nature. So far no such physical assumptions have been put forward" ([710], p. 229).

In the paper, seven distinct solutions were displayed. For one special case, i.e.,

$$
d s^{2}=h_{i j} d x^{i} d x^{j}=\left[1+\frac{1}{4}\left(x^{2}+y^{2}\right)\right] d t^{2}-d x^{2}-d y^{2}-d z^{2}, \quad F_{10}=-\frac{1}{2} x, \quad F_{20}=-\frac{1}{2} y,
$$

the standard interpretation $\left(F_{i j}=k_{i j}\right.$ electromagnetic field, $h_{i j}$ gravitational potential) "would attribute the electromagnetic field to a distribution of charge along the infinite planes $y= \pm x$ or along any of the equipotential $x^{2}-y^{2}=$ constant." For a weak gravitational field, the Newtonian potential would be $V=\frac{1}{8}\left(x^{2}+y^{2}\right)$ "corresponding to a distribution of mass of density $\frac{1}{2}$ inside an infinite cylinder". This is due to the approximated Newtonian equations of motion following from the geodesic equation for $h_{i j}$. Hence, for this solution, mass and charge are unrelated.

This result casts into doubt much of the work on exact solutions independently of any specific assignment of mathematical objects to physical variables. It vindicated Schrödinger's opinion that exact solutions were of useless for a better understanding of the particle-aspect of the theory; cf. the quotation at the end of Section 8.2. Nevertheless, the work of assembling a treasure of exact solutions continued. In 1954, it had still been supported by Kilmister \& Stephenson in this way: 'The true test of this theory [i.e., Einstein's weak field equations] as an adequate description of the physical world must await exact solutions of the field equations" [331].

Einstein did not live to see the results of Wyman \& Zassenhaus; now some of the non-singular exact solutions of the field equations of UFT he so much wished to have had, were at hand:

"The big difficulty [of UFT] lies in the lack of a method for deriving singularity-free exact solutions which alone are physically interesting. Yet the bit we have been able to calculate has strengthened my trust in this theory." (Einstein to Pauli, April 1, 1948 quoted from [489] $)^{176}$

How would he have dealt with the fact, unearthed in 1958, that such non-singular solutions not always offered a convincing physical interpretation, or even were unphysical?

\footnotetext{
174 Max Wyman (1916-1991) PhD at the California Institute of Technology. Since 1941 lecturer in mathematics and since 1956 full professor at the University of Alberta, Edmonton. President of this university 1969 - 1974.

175 Hans Julius Zassenhaus (1912-1991) PhD 1934 University of Hamburg with Hecke and Artin. Refused professorship at the University of Bonn in 1941. 1949-1959 professor at McGill University, Montreal, then at the University of Notre Dame, Indiana, and since 1964 at Ohio State University.

176 "Die große Schwierigkeit liegt darin, daß man keine Methode hat, singularitätsfreie strenge Lösungen abzuleiten, die ja allein physikalisch interessant sind. Das Wenige, was wir aber haben ausrechnen können, hat mein Vertrauen in diese Theorie gestärkt."
} 
The symmetry of so-called "1-dimensional" gravitational fields of general relativity, i.e., those for which the metric components depend on only a single coordinate, is high enough to try and solve for them field equations of UFT. In fact, already in 1951, Bandyopadhyay had found such a solution of the weak equations with $g_{[10]} \neq 0, g_{[23]}=0, g_{22}=g_{33}$ and had taken it as describing an infinite charged plate [7]. In 1953, E. Clauser presented another such 1-dimensional field as a solution of the weak equations with $g_{22} \neq g_{33}$ and saw it as representing a magnetostatic field [79]. B. R. Rao in 1959 generalized Bandyopadhyay's solution to the case $g_{[10]} \times g_{[23]} \neq 0$ without attempting to provide a physical interpretation [503].

Plane wave solutions of the weak and strong field equations of the form

$$
\begin{array}{r}
h_{i j} d x^{i} d x^{j}=\eta_{i j} d x^{i} d x^{j}-2\left(d x^{3}-d x^{0}\right)^{2} f\left(x^{1}, x^{2}, x^{3}-x^{0}\right), \\
k_{12}=k_{30}=0, \quad k_{31}=k_{10}=\sigma, \quad-k_{23}=k_{20}=\rho,
\end{array}
$$

with $\sigma, \rho$ functions of $x^{1}, x^{2}, x^{3}-x^{0}$ have been given by Takeno [601].

\subsection{Interpretative problems}

Already up to here, diverse assignments of geometrical objects to physical quantities (observables) were encountered. We now assemble the most common selections.

\section{a) Gravitational Field}

From the fact that the exact, statical, spherically symmetric solution of the weak field equations derived by A. Papapetrou did not coincide asymptotically, or far from the assumed location of the point source at $r=0$, i.e., for $r \rightarrow \infty$, with the corresponding solution of the Einstein-Maxwell equations (Reissner-Nordström) [475], a discussion of the relation between geometrical objects and physical observables arose. Perhaps the metric chosen to describe the gravitational potential ought not to be identified with $h_{i k}$ ! Let the inverse of $g_{i k}$ be given by

$$
g^{i k}=l^{(i k)}+m^{[i k]} .
$$

From a study of the initial value problem, A. Lichnerowicz suggested the use of the inverse $l_{i k}=l_{(i k)} \neq h_{i k}$ of $l^{i k}$ as the genuine metric ([371], p. 288). Schrödinger had already worked with it. A related suggestion made by several doctoral students of M.-A. Tonnelat (J. Hély, Pham Tan Hoang, M. Lenoir) was to use $a_{i j}$ with $a_{i s} a^{k s}=\delta_{i}^{k}$ and $a^{i j}=\sqrt{\frac{h}{g}} h^{i j}$ as metric [250, 271, 272], ([359], p. 92). In Section 9.6.1 we have seen that by another, if only very contrived definition of the metric, complete separation of the gravitational and electromagnetic fields could be achieved: the Schwarzschild solution could be made part of an exact solution of unified field theory [709]. The torsion tensor appeared in the definition of this metric.

\section{b) Electromagnetic Field and Charge Currents}

The same ambiguity arose for the description of the electromagnetic field: Einstein's specification that it be connected to $k_{i j}=g_{[i j]}$ was taken over by the majority ([147], p. 583). For dimensional reasons, this interpretation implies that a constant of dimension "length" will appear in the theory. In her discussion of two possibilities, St. Mavridès suggested $a_{i j}=\sqrt{\frac{g}{h}} h_{i j}$ for the metric and $s_{i j}=\sqrt{\frac{g}{h}} k_{i j}$ for the electromagnetic field [403, 400, 401, 404]. The first choice was supported by Pham Tan Hoang [271, 272]. Although making the electrical field regular at $r=0$ :

$$
E=\frac{e}{r_{0}^{2}} \frac{1}{\sqrt{1+\frac{r^{4}}{r_{0}}}},
$$


this choice did not fuse gravitational and electromagnetic fields any better. Mavridès' choice was guided by a comparison with the Born-Infeld non-linear electrodynamics ${ }^{177}$ [42], cf. also Section 5 . The fundamental invariants of the electromagnetic field now are given by $h_{p q} h_{r s} m^{p r} m^{q s}$ and $\frac{\sqrt{-l}}{\sqrt{m}}$ with $l=\operatorname{det}\left(l_{i j}\right), m=\operatorname{det}\left(m^{i j}\right)$ ([641], p. 345); cf. Section 5. Mme. Tonnelat, in her books, also discussed in detail how to relate observables as the gravitational and electromagnetic fields, the electric current density, or the energy-momentum tensor of matter to the geometric objects available in the theory ([632], Chapter VI; [636, 382]; cf. also Section 10.2.1). For the electromagnetic field tensor, four possibilities were claimed by her to be preferable: ${ }^{178} m_{i k} ; m^{i k} ; K_{[i k]} ; \epsilon^{i j k l} m_{k l}$. Tonnelat opted for $m_{i k}$, and also for the electric current density vector $J^{i}=\frac{1}{6 \sqrt{-h}} \epsilon^{i j k l}\left(m_{[i j], k}+\right.$ $\left.m_{[k i], j}+m_{[j k], i}\right)$. The field induction is defined via: $\hat{P}^{i k}=\frac{\partial \mathcal{L}}{\partial m_{i k}}$. Schrödinger had identified the electromagnetic field with the anti-symmetrical part $K_{[i k]}$ of the Ricci tensor [545, 549]; this suggestion was also made in $[138,141,5,93]$. On the other hand, Eq. (235) can be satisfied by $\hat{g}^{[i j]}=\frac{1}{2} \epsilon^{i j k l}\left(\partial_{k} A_{l}-\partial_{l} A_{k}\right)$ such that $F_{i j}:=\epsilon_{i j k l} \hat{g}^{[k l]}=\partial_{i} A_{j}-\partial_{j} A_{i}$ would naturally constitute the relationship to the electromagnetic field tensor $F_{i j}$ [203]. In this context, the electromagnetic induction would be $H^{i j} \sim \frac{1}{2} \epsilon^{i j k l} k_{k l}$, and alternatively, $\sim \epsilon^{i j k l} K_{k l}$ ([650], p. 370). We learned above in (323) of Section 9.6.1 that M. Ikeda used yet another definition of the electromagnetic field tensor.

If electrical currents are to be included, the following choices for the current density were considered by Einstein, by Straus, (cf. Sections 9.2.2 and 9.3), and by others [34, 637]:

$$
\begin{gathered}
j_{i j k}:=g_{\{[i j], k\}}, \\
j_{i j k}:=K_{\{}\{[i j], k\} .
\end{gathered}
$$

The second choice would either violate the weak field equations or forbid any non-zero current density. These alternatives are bound to the choice for the induction. Two possibilities were discussed by Mavridès:

$$
\begin{aligned}
j_{i j k} & :=k_{\{[i j], k\}}, \\
4 \pi j^{i} & :=\frac{1}{\sqrt{-h}} \partial_{l}\left(\sqrt{-h} h^{i r} h^{l s} k_{r s}\right) .
\end{aligned}
$$

In the 2nd case, Papapetrou's spherically symmetric static solutions would not make sense, physically [407]. ${ }^{179}$ Finzi proposed yet another expression: $j^{k}=\frac{1}{2} \epsilon^{k p q r} \stackrel{0}{\nabla}_{p} K_{q r}$ ([473], p. 288). Late in his life, Einstein gave $\hat{g}^{[i s]}$ the interpretation of magnetic current density [158].

An ambiguity always present is the assignment of the electric and magnetic fields to the components $k_{i 0}$ or $k_{a b}, a, b=1,2,3$, or vice versa in order to arrive at the correct Maxwell's equations.

Another object lending itself to identification with the electromagnetic field would be homothetic curvature encountered in Section 2.3.1, i.e., $V_{+} k l=\underset{+}{K_{j k l}^{j}}=\partial_{k} L_{l j}{ }^{j}-\partial_{l} L_{k j}{ }^{j}: L_{l j}{ }^{j}$ could then play the role of the vector potential. This choice has been made by Sciama, but with a complex curvature tensor $\stackrel{s}{K}_{j k l}^{j}$ [565]. In this case $\stackrel{s}{K}_{j k l}^{j}=\partial_{k} L_{[l j]}^{j}-\partial_{l} L_{[k j]}^{j}=2 S_{[j, k]}$. The vector potential thus is identified with the torsion vector.

177 As M.-A. Tonnelat did, Mme. Mavridès changed around her notation in some papers: for what is named here $l^{i j}, m^{i j}$, she first wrote $r^{i j}, g^{i j}$, i.e., $r^{i j}=g^{i j}+f^{i j}$, and, in her next paper, $g^{i j}, h^{i j}$; in the present paper, the full asymmetric metric is $\pi_{i j}=g_{i j}=a_{i j}+s_{i j}$ and its inverse $\pi^{i j}=g^{i j}=b^{i j}+t^{i j}$. In part of the tensors used, indices are moved with the metric; yet most tensors with upper and lower indices are just the inverses of each other. Also, in $[400,401]$, the electrical field became singular again at $r=0$.

178 Note that $m^{i k}=g^{[i k]}$ and $m_{i r} m^{k r}=\delta_{i}^{k}$; Tonnelat's notation is $f^{i k}=g^{[i k]}$.

179 In place of (332) Mme. Tonnelat wrote $j^{i}:=\partial_{l}\left(h^{i r} h^{l s} k_{r s}\right)$; cf. [641], p. 346. 
In a paper falling outside of the period of this review, H.-J. Treder ${ }^{180}$ suggested to also geometrize spinorial degrees of freedom by including them in the asymmetric metric; it took the form [651, 67]:

$$
g_{i j}=h_{i}^{A} h_{j}^{B} \eta_{A B}+\frac{1}{2} i C\left(\gamma_{i j}{ }^{\alpha \beta} \psi_{\alpha} \psi_{\beta}+\gamma_{i j}{ }^{\dot{\alpha} \dot{\beta}} \psi_{\dot{\alpha}} \psi_{\dot{\beta}}\right)
$$

where $h_{i}^{A}$ are tetrad components, $\psi_{\alpha}$ a 2-component Weyl-spinor, $C$ a constant (with dimension) and $\gamma_{i j}{ }^{\alpha \beta}=\sigma_{i}{ }_{\dot{\mu}} \sigma_{j}{ }^{\beta}{ }_{\dot{\nu}} \gamma^{\dot{\mu} \dot{\nu}} . \sigma_{i}{ }^{\alpha}{ }_{\dot{\mu}}$ are Pauli-matrices, $\gamma^{\dot{\mu} \dot{\nu}}$ corresponds to the antisymmetric $2 \times 2$ symbol $\epsilon^{\alpha \beta}$.

It is obvious that the assumed mapping of geometrical objects to physical variables had to remain highly ambiguous because the only arguments available were the consistency of the interpretation within unified field theory and the limit to the previous theories (Einstein-Maxwell theory, general relativity), thought to be necessarily encased in UFT. As we have seen, the hope of an eventual help from exact solutions had to be abandoned.

\section{c) Matter tensor}

In Einstein's understanding of UFT, the matter tensor for a continuous matter distribution should also become part of geometry. Again a precise attribution to geometrical objects could not be found. One way of approaching the problem was to reshape part of the field equations into the form of the old or a newly defined "Einstein tensor", and terms left over. These then were declared to constitute the "matter tensor". In her book, M.-A. Tonnelat discussed this problem in detail ([632], Chapter VII, A, pp. 109-117); cf. also Section 10.3.1. As late as 1963, Schrödinger could write:

"Thus it is as yet undecided what interpretation of the various tensors and densities is most likely to let the theory meet observed facts" ([557], reprinted 1963, p. 115).

\subsection{The role of additional symmetries}

The symmetries Einstein had introduced, i.e., transposition invariance and $\lambda$-transformations, played a major role in versions of UFT, but not in physics, in general. There are only a few papers with these symmetries as their topic. J. Winogradzki investigated the relationship of the results in Einstein's final approach to UFT (made together with B. Kaufman) to the theorems by Emmy Noether [704, 703]. She called invariance with regard to the group $U$ composed of coordinate- and $\lambda$-transformations which had been named the "extended" group by Einstein, $U$ - or $J$ - invariance:

$$
J\left(g_{i k}\right)=g_{i k}, J\left(L_{i j}{ }^{k}\right)=L_{i j}{ }^{k}+\Omega_{i k}{ }^{k},
$$

with $\Omega_{i j}{ }^{k}$ being independent of $g_{i j}$. As a necessary condition for the field equations following from a variational principle to be J-invariant, she derived $\Omega_{i j}{ }^{k}=\delta_{i}^{k} \lambda_{j}$. Thus, with the help of four axioms postulated by her, she arrived at the $\lambda$-transformations (52).

According to the 2 nd theorem by E. Noether, $\lambda$-invariance leads to four identities which were written out by Winogradzki for a Lagrangian density $\hat{A}$ (her "Hamiltonian"):

$$
\delta_{k}^{l} \frac{\delta \hat{A}}{\delta \Gamma_{k m}^{l}}-2 \hat{g}_{, s}^{[m s]}=0 .
$$

\footnotetext{
180 Hans-Jürgen Treder (1928-2006) was a theoretical physicist with an interest in general relativity, cosmology and astrophysics. He headed the Central Institute for Astrophysics of the German Academy of Sciences and became director of its Cosmic Physics department.
} 
Equation (334) relates $\hat{g}_{, s}^{[i s]}=0$ and the 16 field equations which are not invariant under $\lambda$ transformations.

P. G. Bergmann also discussed Einstein's $\lambda$-transformations, but just in the special form used in the 5th edition of The Meaning of Relativity, i.e., with $\lambda_{k}=\partial_{k} \lambda$. No wonder that he then concluded: "[...] the $\lambda$ transformation appears to be closely related in its conception to Weyl's original gauge transformation" ([23], p. 780). 


\section{Einstein-Schrödinger Theory in Paris}

Research on unified field theory in Paris centered around the mathematician A. Lichnerowicz, a student of Georges Darmois, and the theoretical physicist Marie-Antoinette Tonnelat. It followed two main lines: the affine or metric-affine approaches of Einstein and Schrödinger, and the 5dimensional unification originating with G. Nordström and Th. Kaluza. The latter theme was first studied in Paris by Y. Thiry, a former student of A. Lichnerowicz ("Jordan-Thiry-theory"), and by students of M.-A. Tonnelat; the first topic, "Einstein-Schrödinger theory", mainly by Tonnelat and her coworkers but no strict divide did exist. Between 1950 and the mid 1960s at least two dozen doctoral theses on topics in unitary field theory were advised by Lichnerowicz and/or by Tonnelat. Whereas the work of Tonnelat's students could be classified as applied mathematics, Lichnerowicz's interest, outside of pure mathematics, was directed to mathematical physics with its rigid proofs. This joined attack on unsolved questions and problematic features of classical unified field theory has made clear that (1) the theories under scrutiny were mathematically consistent, but (2) they could not be transformed into an acceptable part of physics.

\subsection{Marie-Antoinette Tonnelat and Einstein's Unified Field Theory}

We first make contact with Marie-Antoinette Tonnelat ${ }^{181}$ and her research group in the Institut Henri Poincaré. She had studied with Louis de Broglie. During the German occupation of Paris she continued to work with de Broglie and on her own in the field of (relativistic) "spin-particles", also under the influence of a gravitational field. She applied de Broglie's "méthode de fusion" to massive spin 1 particles (called photons) in order to arrive at particles with maximal spin 2: spin 2 corresponded to the graviton. The theory contained the graviton, three photons and two scalar particles (spin 0) all with non-vanishing mass. Each relevant field component satisfied the Klein Gordon equation. From there, she arrived at Maxwell's equations and the linearized version of the equation for Einstein spaces $R_{i j}=\lambda g_{i j}$. She carefully looked at the theory for a particle with spin 2 as a "unitary theory" and preferred to call it "a unitary formalism" ([616], p. 163, 164):

"[...] the theory of maximal spin 2 allows to show how a unitary theory presents itself, approximately, but in the framework of wave mechanics." 182

In this paper and in others in the early 1940s she also wrote down the standard commutation relations for the quantized spin-2 field [611, 616].

Many of her papers were published in the proceedings of the prestigious Academy of Sciences in Paris ${ }^{183}$ [613, 612, 614, 610, 611, 615, 616, 617]. The Academy's sessions had been interrupted for a mere three weeks due to the German occupation. According to its president:

\footnotetext{
181 Marie-Antoinette Tonnelat, née Baudot (1912-1980) first studied philosophy and then joined the group of theoretical physics around L. de Broglie at the Institut Henri Poincaré, in 1925. She wrote her PhD thesis with him on the "Theory of the photon in a Riemannian space" in 1939. The "second part" of the thesis was done under the supervision of Jean Perrin on "Artificial Radioactivity". It seems that she received her degree only in 1941. Since 1956 she became Professeur à la Faculté des Sciences of the University of Paris (Sorbonne); in this faculty she thus joined her teacher L. de Broglie. Mme. Tonnelat held a diploma in the history of science and, since 1949 regularly taught courses in this field as well. She also created an interdisciplinary seminar on the History of Sciences. Among her publications in this field, a book on the history of the relativity principle is to be noted [645]. In 1945 she received the prize "Pierson Perrin" and in 1970 the prize "Henri Poincaré" of the Academy of Sciences in Paris $[418,92]$. Tonnelat also published a volume of novellas.

182 “[...] la théorie de spin maximum 2 permet en outre de montrer comment peut se présenter d'une façon approchée, mais par la mécanique ondulatoire, une théorie unitaire."

183 The groups in Paris around de Broglie, Lichnerowicz and Tonnelat had the habit of publishing in the form of numerous 3-page communications in Comptes Rendus, a habit that still is cumbersome for historians of science even though Comptes Rendus have now been made accessible in the internet. After all, one first has to find unknown references in order to get a reasonable degree of completeness.
} 
"Despite the ordeal which oppresses the country, the Comptes Rendus attest that scientific research has not bent, and that the Academy of Sciences remains a focus of ardent and fruitful work. [...] Let us work." 184

Right after the war in 1946, like other young French scientists, Mme. Tonnelat apparently spent some time in Dublin with the group of E. Schrödinger. On the background of her previous work on a "unitary formalism" emerging from spin-2 particles, her interest in the unified field theories of Einstein and Schrödinger might have been brought forward during this stay with Schrödinger. Her scientific teacher, L. de Broglie, supported her research in UFT, although he himself stayed away from it. After briefly listing "innumerable attempts [...] to complete the general theory of relativity [...] and transform it into a "unified theory'", he went on to say:

"Einstein's efforts in this direction, ever characterized by the salient originality of his thought, will not be examined here. Despite their indisputable interest, they have not, to the best of our knowledge, attained any decisive success [...]. Moreover, the nature of the electromagnetic field is so intimately bound to the existence of quantum phenomena that any non-quantum unified theory is necessarily incomplete. These are problems of redoubtable complexity whose solution is still 'in the lap of the gods'" ([113], p. 121).

At the 8th Solvay Congress in 1948 in Brussels, Mme. Tonnelat presented a paper by L. de Broglie on the photon as composed of two neutrinos. Schrödinger asked a question afterwards about a tiny mass of the photon ([446], p. 444). During her work on unified field theory Tonnelat continued to study spin-particles, e.g., to regard a spin-1-particle as composed of two spin-1/2particles [625].

\subsection{Tonnelat's research on UFT in $1946-1952$}

As we will see, M.-A. Tonnelat made several attempts until she reached the final version of her unified field theory. In the warm-up, i.e., in her first paper on the subject, Tonnelat referred only to Schrödinger's theory [545, 549] and to Eyraud's thesis of 1926 (cf. Section 5 of Part I). By her theory, she intended to describe the gravitational, electromagnetic and mesonic fields [618]. The paper was reviewed by the American theoretical physicist A. H. Taub in Mathematical Reviews [0017205]:

"The author states without proof some formal consequences of a variational principle in which the action function is an unspecified function of a symmetric second order tensor and three (of which two are independent) anti-symmetric second order tensors. These four tensors are defined in terms of a general affine connection in a four dimensional space, and its derivatives. The connection is not assumed to be symmetric. The paper does not explain how the invariant element of volume entering into the action principle is defined."

By her, the torsion tensor $\Lambda_{i j}^{k}$ is defined through

$$
L_{i j}{ }^{k}=\Gamma_{i j}{ }^{k}+\frac{1}{2} \Lambda_{i j}{ }^{k}
$$

where $\Gamma_{i j}^{k}=\Gamma_{(i j)}^{k}$ and $\Lambda_{i j}{ }^{k}=\Lambda_{[i j]}^{k}$. Vector torsion is denoted by her as $\Lambda_{j}=\Lambda_{j k}^{k}$ and corresponds to our $2 S_{k}$. She introduced four tensors of rank two $K_{\mu \nu}, S_{\mu \nu}, T_{\mu \nu}, F_{\mu \nu}$ corresponding in our

184 "[...] Les Comptes rendus attestent que, malgré les épreuves qui accablent le pays, la recherche scientifique n'a pas fléchi et que l'Académie des Sciences demeure un foyer de travail ardent et fécond. [...] Travaillons. [...]" cf. Comptes Rendus, 212 1941, p. 19-21. 
notation (in the same order) to:

$$
\begin{array}{r}
\underline{K}_{(j k)}, \underline{-}_{[j k]}, \\
\partial_{k} L_{s j}^{s}-\partial_{j} L_{s k}^{s}, \partial_{k} S_{j}-\partial_{j} S_{k}
\end{array}
$$

with the torsion vector $S_{i} . T_{\mu \nu}$ corresponds to homothetic curvature $\underline{-}_{k l}=\underline{K}_{j k l}^{j}$ defined by (66) in Section 2.3.1. In the spirit of Schrödinger, a metric density is introduced by:

$$
\frac{1}{2} \hat{g}^{i k}:=\frac{\partial \mathcal{L}}{\partial \underline{-}_{(i k)}} .
$$

The Lagrangian is defined as a functional of these four tensors. By help of a "geometrically" defined matter tensor the resulting field equations are rewritten in the form of Einstein's equations.

In three following papers, Tonnelat had become acquainted with some of the past literature on the subject, and included references to Einstein [619,620, 621]. Her intention was to generalize this past work for an arbitrary affine connection in a more systematical way such as to "augment the interpretative possibilities of the theory." The same tensors as before (one symmetric, three antisymmetric) were introduced with a bewildering change of her own notation: $G_{\mu \nu}$ instead of $K_{\mu \nu}, F_{\mu \nu} \sim S_{\mu \nu}, S_{\mu \nu} \sim T_{\mu \nu}$, and $H_{\mu \nu} \sim F_{\mu \nu}$. Again, the Lagrangian is a functional of these four tensors and, possibly, the torsion vector. After dual (conjugate) field tensor densities were defined through:

$$
\bar{G}_{i k}=\frac{\partial \mathcal{L}}{\partial \underline{K}_{(i k)}}, \bar{F}_{i k}=\frac{\partial \mathcal{L}}{\partial F_{i k}}, \bar{S}_{i k}=\frac{\partial \mathcal{L}}{\partial S_{i k}}, \bar{H}_{i k}=\frac{\partial \mathcal{L}}{\partial H_{i k}},
$$

and a new quantity $\bar{R}^{i k}$ introduced by its symmetric and antisymmetric parts $\bar{R}^{i k}:=\bar{G}^{i k}-\bar{F}^{i k}$, the field equations could be written in the form:

$$
\stackrel{0}{\nabla}_{l} \bar{R}^{i k}=-\frac{1}{2} \bar{R}^{i k} S_{l}+\frac{1}{2}\left(\bar{R}^{i s} S_{l s}{ }^{k}-\bar{R}^{s k} S_{l s}{ }^{i}\right)+\frac{1}{3} \bar{R}^{i r} S_{r} \delta_{l}^{k}
$$

It is to be noted that this equation is purely formal: no definite expression for the Lagrangian had been chosen.

In the 2nd of the three notes, Tonnelat introduced a symmetric metric and split (340) into its symmetric and antisymmetric parts $(\mathrm{S})$ and $(\mathrm{A})$. According to her, the exact general solution to (A) was very difficult to produce; this is seen from the complicated expression in terms of quantities like the metric, its first derivative and torsion, known in principle. In the third note, the formalism is exemplified in second-order approximation and with additional restrictions, e.g., in de Sitter space. Proca's equation for the mesonic field emerges. In her next paper, (340) is rewritten in the form of a metric compatibility condition:

$$
\nabla_{l}^{\prime} r^{\stackrel{i j}{j}}=0
$$

where the covariant derivative $\nabla^{\prime}$ is defined with help of a new connection $L^{\prime} . r^{i k}$ is related to $\bar{R}^{i k}$ by $\bar{R}^{i k}=\sqrt{r} r^{i k}$ with $r:=\operatorname{det}\left(r_{i k}\right)$ and $r_{i k}$ the inverse of $r^{i k}{ }^{185}$ The decomposition

$\bar{R}^{i k}=\bar{G}^{i k}-\bar{F}^{i k}$ is also used. By a combination of symmetric and antisymmetric parts $r_{i k}=$ $\gamma_{i k}-\phi_{i k}$ two further tensors and their inverses are introduced, i.e., $\gamma_{i k}=\gamma_{(i k)}, \gamma^{i k}$ and $\phi_{i k}=$ $\phi_{[i k]}, \phi^{i k}$. Now

$L_{i j}^{\prime}{ }^{k}=L_{i j}{ }^{k}+\frac{1}{3} \delta_{i}^{k} \Lambda_{j}+\frac{1}{3}\left(\delta_{i}^{k} r_{l j}+\delta_{j}^{k} r_{i l}\right) s^{l}-r_{i j} s^{k}-\frac{1}{3} \delta_{j}^{k} r_{i l} f^{l}+\frac{1}{2} r_{i j}\left(f^{k}-f^{\bar{k}}\right)-\frac{1}{2}\left(\delta_{i}^{k} r_{j l}-\delta_{j}^{k} r_{i l}\right)\left(f^{l}+f^{\bar{l}}\right)$.

185 Thus, in the notation used here, $\bar{R}^{i k}$ should be written as $\hat{r}^{i k}$. 
Here, $f^{\bar{l}}=\gamma^{l m} \phi_{m n} f^{n}$, and $f^{n}=\frac{1}{\sqrt{-g}} \bar{F}^{n}=\stackrel{0}{\nabla}_{l} \bar{F}^{n l}$ with $\bar{F}^{n l}$ having been defined in (339). Apart from ingenious manipulations of a very general formalism, no physical progress with regard to Schrödinger's and Einstein's theories had been reached in this paper [622]. As Tonnelat herself confessed: "The physical interpretation of the thus formed tensors is far from being immediately clear." 186

Two summaries of her attempts with regard to affine field theory were presented by Tonnelat in 1951 and 1952 [627, 628]. It would be natural to discuss them together but M.-A. Tonnelat again complicated matters by an altered notation from one paper to the next. In 1951, for the first time, she introduced the transposed connection $\tilde{L}_{i j}{ }^{k}$ and thus worked with both $P_{j k}(L):=-K(L)_{j k}$ and $Q_{j k}(\tilde{L}):=-\underset{+}{K}(L)_{j k}$. Further basic tensors chosen were $S(L)_{j k}:=\partial_{k} L_{s j} s-\partial_{j} L_{s k}^{s}$ and $T(\tilde{L})_{j k}:=\partial_{k} L_{j s}^{s}-\partial_{j} L_{k s}^{s}$, and a fifth quantity $I_{j k}:=4 S_{j} S_{k}$ with $2 S_{k}$ corresponding to her $\Lambda_{k s}^{s}$. Having read the 2nd edition of Einstein's The Meaning of Relativity - as she claimed, ${ }^{187}$ she accepted Einstein's suggestion that only Hermitian quantities should be used as fundamental quantities in a Lagrangian. The following objects were introduced by her:

$$
\begin{aligned}
{ }^{(2)} R_{i k} & =P_{i k}(L)+P_{k i}(\tilde{L})-\frac{1}{4}\left(S(L)_{i k}+S(\tilde{L})_{k i}\right) \\
& =\partial_{l} L_{i k}{ }^{l}-\partial_{k} L_{(i l)}{ }^{l}+L_{i k}{ }^{l} L_{(l m)}^{m}-L_{i m}{ }^{l} L_{l k}{ }^{m}, \\
H_{i k} & =S(L)_{i k}+S(\tilde{L})_{k i}=2\left(\partial_{i} S_{k}-\partial_{k} S_{i}\right), \\
I_{j k} & :=4 S_{j} S_{k}, \\
L_{i k} & =P_{i k}(L)-P_{k i}(\tilde{L})-\frac{1}{2}\left(S(L)_{i k}-S(\tilde{L})_{k i}\right)=L_{i k}^{r} S_{r}-\partial_{i} S_{k}+\partial_{k} S_{i}, \\
K_{i k} & =-\frac{1}{2}\left(S(L)_{i k}-S(\tilde{L})_{k i}\right)=\partial_{k} L_{(i l)}^{l}-\partial_{i} L_{(k l)}^{l} .
\end{aligned}
$$

From these, Einstein had formed the linear combination:

$$
U_{i k}{ }^{(2)} R_{i k}-\frac{1}{6} H_{i k}-\frac{1}{12} I_{j k}
$$

which remains unchanged by a transformation of the connection: ${ }^{188}$

$$
L_{i j}{ }^{k} \rightarrow L_{i j}{ }^{k}+2 \delta_{[i}^{k} \phi_{j]}
$$

By choosing $\phi_{k}=-\frac{1}{3} S_{k}$, a new connection $\stackrel{*}{L}$ with vanishing torsion vector is obtained. Furthermore, $U(L)_{i k}=U(\stackrel{*}{L})_{i k}$. In place of the field densities (339), now the three quantities are used:

$$
\hat{R}_{i k}=\frac{\partial \mathcal{L}}{\partial U_{(i k)}}, \quad \hat{H}_{i k}=\frac{\partial \mathcal{L}}{\partial H_{i k}}, \quad \hat{I}_{i k}=\frac{\partial \mathcal{L}}{\partial I_{i k}},
$$

which are then manipulated similarly as in the previous paper. ${ }^{189}$ Eventually, Eq. (341) is obtained again, where the connection $L^{\prime}$ now is derived by beginning with $\stackrel{*}{L}$. The new Eq. (341) remains

186 "L'interprétation physique des tenseurs ainsi formés [...] est loin d'être immédiate." ([622], p. 184.)

187 [...]"un travail dont je viens de prendre connaissance [...]" ("[... ] a work of which I just learned"). The reference must be erroneous, because neither in the 2nd Princeton, nor in the 2nd London edition an appendix referring to UFT do appear. The content of her paper shows that she referred to the 4 th London edition of 1950 .

188 Note that this is neither a projective nor a $\lambda$-transformation. (cf. Section 2.2.3.)

189 In spite of the same notation, $\bar{R}_{i k}$ in Eq. (341) and the corresponding equation here, the objects must be kept apart. 
unchanged if the connection $L_{i j}^{\prime}{ }^{k}$ and $r_{i k}$ are replaced by the transposed objects. Up to here, a general formalism has been developed. In order to proceed, M.-A. Tonnelat then picked the same Lagrangian $\mathcal{L}$ as Schrödinger had in Eq. (229), i.e., $\mathcal{L}=\frac{2}{\lambda} \sqrt{-\operatorname{det}\left(R_{r s}\right)}$, yet based on her vectortorsion-free connection $\stackrel{*}{L}$. The field equations derived by her decompose into the fundamental ones (variation with regard to $U_{(i k)}$ ) and such of a "Maxwellian" type (variation with regard to $H_{i k}$ ). With the decomposition $r_{i k}=\gamma_{i k}-\phi_{i k}$ of the previous paper and the additional $r^{i k}=g^{i k}-f^{i k}$ together with the inverses of these four tensors, eight basic fields float around in the theory waiting for physical interpretation. Although still in a purely affine theory, the particular interpretation relating $r^{i k}$ or $r_{i k}$ with a metric could make the transition to mixed geometry [cf. Section 2.1 and Eqs. (3), (4)]). ${ }^{190}$ The field equations look very complicated such that an approximative handling seemed appropriate. For weak fields $\phi_{i k}$, i.e., up to 2 nd order, a Proca-type equation was obtained. For the approximated Ricci tensor, field equations of the form of Einstein's equations with cosmological constant and including the "metric" $r_{(i k)}=\gamma_{i k}$ were reached. The matter tensor is a complicated functional of the connections $L$ and $L^{\prime}$. Thus, formally, "matter" has been geometrized. Tonnelat did not give physical interpretations to the tensors appearing except for identifying $\bar{I}^{l}=\epsilon^{i j k l} I_{i j k}, I_{i j k}:=\partial_{i} \phi_{j k}+\partial_{j} \phi_{k i}+\partial_{k} \phi_{i j}$, or its dual $J^{k}=\frac{1}{6} \epsilon^{k l r s} I_{l r s}$ with the electric current density.

In a separate note, M.-A. Tonnelat discussed a possible relation $\frac{5 \lambda}{6}=\mu^{2}$ between the cosmological constant $\lambda$ appearing in the Proca equation obtained from her unified field theory in an approximation up to 2 nd order in $k_{i j}$ :

$$
\square k_{i j}=\frac{5 \lambda}{6} k_{i j}
$$

and the Proca equation for spin-1 or spin-2 particles of rest mass $\mu$ in a space of constant curvature derived by her [615]:

$$
\square k_{i j}=\mu^{2} k_{i j} .
$$

While she abstained from over-interpreting this relation in the sense of bringing both types of theories closer to each other, she still had some hope concerning the understanding of elementary particles:

"[...] the possibility remains of finding, thanks to the exact solution to the equation $0=g_{i k-} \| l$, a solution valid even in the case of strong fields, an explanation of the nature of the elementary particles. However, as Schrödinger very strongly emphasized, the realization of this hope remains quite problematical despite all efforts." ([626], p. 832) 191

In her subsequent paper [628], Tonnelat pledged to adopt "Einstein's notations". 192 Two connections were used, both free of vector torsion: ${ }^{(1)} \Delta_{i j}{ }^{k}:=L_{i j}{ }^{k}+\frac{2}{3} \delta_{[i}^{k} L_{j]}$ and ${ }^{(2)} \Delta_{i j}{ }^{k}:=$ $L_{i j}{ }^{k}+\frac{2}{3} \delta_{i}^{k} L_{j}\left(L_{j}=S_{j}\right)$. (1) $\Delta$ is the same as Schrödinger's "star"-connection; cf. Eq. (232) in Section 8.1 ${ }^{193}$ She then discussed at length which Ricci-tensor to take as basic variable, and

\footnotetext{
190 In mixed geometry, $\gamma_{i k}$ would correspond to our $h_{i k},-\phi_{i k}$ to $k_{i k}$. But as Tonnelat still worked in affine geometry, we continue to use her notation in this context and in this section.

191 " [...] il reste la possibilité de trouver, grâce à la solution exacte des équations $0=g_{i} k \| l$, solutions valable même dans le cas de champs très intenses, l'explication de la nature des particules élémentaires. Mais la réalisation de cet espoir, comme le souligne fort bien Schrödinger, demeure, malgré tous les efforts, assez problématique."

192 She now used $\Gamma_{\mu \nu}^{\rho}$ for the connection with $\Gamma_{\mu \nu}^{\rho}$ for the symmetric, $\Gamma_{\mu \nu}^{\rho}$ for the antisymmetric part. The torsion vector previously written as $\Lambda_{\mu}$ now is $2 \Gamma_{\mu}$. Nevertheless, the confusing relabeling of some of her previously defined objects continued: what had been $P_{\mu \nu}, R_{\mu \nu}, U_{\mu \nu}$ in [627] now had become $R_{\mu \nu}, U_{\mu \nu}, R_{\mu \nu}^{(3)}$, respectively, in [628].

193 Tonnelat, or Schrödinger never used the 1-parameter set of affine connections without torsion vector ${ }^{(a)} \Delta_{i j}{ }^{k}:=$ $L_{i j}{ }^{k}+2 a \delta_{(i}^{k} L_{j)}-\frac{2}{3} \delta_{j}^{k} L_{i}$ but just took special values $a=\frac{1}{3}$ or $a=\frac{2}{3}$.
} 
listed four possible expressions: $R_{\mu \nu}^{(0)} \simeq-_{-}^{K}(L)_{j k}, R_{\mu \nu}^{(1)} \simeq \underset{-}{K}\left({ }^{(1)} \Delta\right)_{j k}, R_{\mu \nu}^{(2)} \simeq \underset{-}{K}\left({ }^{(2)} \Delta\right)_{(j k)}$, and the Hermitian $R_{\mu \nu}^{(3)} \simeq \frac{1}{2}\left(\underline{K}_{-}\left({ }^{(2)} \Delta\right)_{(j k)}+\underline{K}_{-}\left({ }^{(2)}(\tilde{\Delta})_{(j k)}\right)\right.$. The formalism then is carried out with all $R_{\mu \nu}^{(A)}, A=0,1,2,3$. For the first time, $r_{i k}=\gamma_{i k}+\phi_{i k}$ is identified as the metric tensor. ${ }^{194}$ As before, it ensued from $\hat{R}^{i k}=\frac{\partial \mathcal{L}}{\partial R_{i k}}=\sqrt{-r_{s t}} r^{i k}$ and its reciprocal. The field equations following from variation with regard to the $R_{\mu \nu}^{(A)}$ looked like:

$$
\stackrel{L^{\prime}}{\nabla_{l}} \hat{R}^{i+-}=0
$$

where the connection $L_{i j}^{\prime}{ }^{k}$ in the covariant derivative is formed with the help of (1) $\Delta, r_{i k}, \gamma^{i k}, \phi_{l m}$, and $\partial_{l} \hat{F}^{l k}$. The same Lagrangian as before is used such that the field equations simply are:

$$
R_{i k}^{(A)},=\lambda r_{i k}, A=0,1,2,3
$$

For the further evaluation, for each of the four tensors $R_{i k}^{(A)}$ the corresponding connection as a functional of the fields must be inserted into (354). This leads to very complicated manipulations such that, again, Tonnelat decided to take the antisymmetric part $k_{i k}$ of $r_{i k}$ to be small of the order $\epsilon$, expand in $\epsilon$ and neglect all terms of the order $\epsilon^{3}$. The relation between the cosmological constant $\lambda$ expressed by the curvature radius of de Sitter space $\mathcal{R}$, i.e., $\lambda=-\frac{3}{\mathcal{R}}$ now is alternatively, $\mu^{2}=-\frac{2 \lambda}{3}$, or $\mu^{2}=-\frac{\lambda}{2}$.

A comparison of M.-A. Tonnelat's research with respect to Einstein's and Schrödinger's shows that, though first generalizing the class of possible Lagrangians enormously by including four tensor fields, in the end she went back to only one: she used Schrödinger's Lagrangian corresponding to Einstein's Lagrangian for general relativity. She avoided the additional equation within the field equations which demands that the torsion vector vanish by directly starting with a connection with zero torsion vector. Although this approach was new, ${ }^{195}$ most characteristic and important for her research seems to be that she directed her attention to "metric compatibility" in the sense of (200) of Section 7.2 and succeeded to "solve" it for the connection; cf. Section 10.2.3. She also showed that out of a purely affine theory, by proper definitions and interpretations, a theory within mixed geometry could be made. It was such a theory that she finally adopted.

\subsubsection{Summaries by Tonnelat of her work}

M.-A. Tonnelat summed up her research within Einstein-Schrödiner theory of almost a decade, published in many short notes in Comptes Rendus and in papers in other journals, in a monograph in 1955 [632]; it eventually became translated into English [642]. Unassumingly, she assessed the book as "[...] a collection of works with the sole aim of facilitating research on the subject" ([632], p. IX.) ${ }^{196}$ In the Mathematical Reviews [MR0076499], A. H. Taub not only gave a detailed description of its contents but also put the book into a larger perspective:

"In this book the author summarizes and discusses a great body of material on the Einstein and Schrödinger unified field theories. [...] The previous work of the author is collected and presented in a logical coherent fashion. The results obtained by other workers are also presented and compared. Thus, in this single volume containing an introduction and seven chapters one can obtain a well written complete and succinct account of the recent work in the field."

\footnotetext{
194 Note the change in sign in the decomposition of $r_{i k}$ with respect to [627].

195 It induced one of her students to speak of "a theory of type Einstein-Tonnelat [...]" ([56], p. 3).

196 "[... un recueil de travaux dont le but est uniquement de faciliter les recherches sur ce sujet".
} 
Tonnelat's associate J. Winogradzki, in her report on the book, gave a condensed list of the contents and found "that the major part of the work is devoted to the mathematical study of the field equations. The two last chapters deal with some physical content of the theory" [705]. Tonnelat clearly drew the line with regard to work by Lichnerowicz, e.g., the initial value problem. For her, the balance between the remaining problems of UFT and the results obtained was positive: "[...] Einstein's theory binds together the realization of a satisfying synthesis, originating from a very general principle, and the possibility of new provisions." ([632], p. 11) ${ }^{197}$ For her, UFT was a fruitful and important theory.

After another decade, in 1965, she published a second monograph on unified field theories reflecting now the development of research in all more prominent approaches to unified field theory. Her work and the results of her group concerning the Einstein-Schrödinger theory took up only one chapter [641]. A few of the doctoral theses she had advised were referred to. In its third part, the book aimed at presenting some connection between classical and quantum field theory. As a first approximation to an unknown nonlinear theory, Tonnelat's alternative theory of gravitation (linear gravity) investigated by her in the 1960s is included (cf. Section 16.1). In comparison with her first book, she had become even more modest:

"Whatever the future of the unitary theories might be, this book will have reached its objective, if it has somehow shown that the ties between electromagnetism and gravitation form a history of rebouncings the outcome of which is far from being written." $([641], \text { p. IX })^{198}$

In the following, some of the main aspects of her approach to UFT will be described.

\subsubsection{Field equations}

As she had moved from pure affine to mixed geometry during her research, M.-A. Tonnelat then started from the Lagrangian density $\hat{\mathcal{L}}=\hat{g}^{i j} R_{i j}$ where the reciprocal metric density $\hat{g}^{i j}$ is defined in (13), and $R_{i j}$ is one out of the list of possible Ricci-tensors. $\hat{\mathcal{L}}=\hat{\mathcal{L}}\left(\hat{g}^{i j}, L_{r s}{ }^{t}, \partial_{v} L_{r s}{ }^{t}\right)$. Variation with respect to $\hat{g}^{i j}$ and $L_{i j}{ }^{k}$ led her to the field equations. With $R_{i j} \stackrel{!}{=}-K_{i j}$ she arrived at:

$$
\begin{aligned}
\hat{g}_{\| l}^{i k} & =-\frac{2}{3} \delta_{l}^{k} \hat{g}^{i s} S_{s}+\hat{g}^{i k} S_{l}, \partial_{l} \hat{m}^{l k}=0, \\
K_{i j} & =0,
\end{aligned}
$$

where $\hat{m}^{i k}:=\sqrt{-\operatorname{det}\left(g_{s t}\right)} m^{l k}$ with $m^{i k}$ being the skew-symmetric part of the inverse metric, and $\left.S_{i}:=L_{[i j]}^{j}\right]^{199}$. After going over to the connection previously named ${ }^{(2)} \Delta_{i j}{ }^{k}$, i.e., Schrödinger's "star"-connection, Einstein's weak field equations followed from the variational principle in the form:

$$
\begin{array}{r}
D_{l} g^{i k}=0, \partial_{l} \hat{m}^{l k}=0, \\
\underline{K}_{(i j)}=0, \partial_{k} \underline{K}_{[i j]}+\partial_{j} \underline{K}_{[k i]}+\partial_{i} \underline{K}_{[j k]}=0 .
\end{array}
$$

\footnotetext{
197 “... [ la théorie d'Einstein unit la réalisation d'une synthèse satisfaisante, issue d'un principe très général, à des possibilités de prévisions nouvelles."

198 "Quel que soit l'avenir des théories unitaires, ce livre aura atteint son but s'il a montré, par quelque côté, que les rapports entre électromagnétisme et gravitation constituent une histoire à rebondissement dont le dénouement est loin d' être écrit."

199 Equation (356) corresponds to Tonnelat's Eq. I on p. 29 of [632]. The definition of the tensor density $\hat{m}^{i k}$ is given in her equation (1.52) on p. 19.
} 
where $D_{l}$ stands for the covariant derivative with respect to the new connection; $K_{i j}$ is the Ricci tensor $K\left({ }^{(2)} \Delta\right)_{i j}$ formed with ${ }^{(2)} \Delta$ and named $W_{i j}$ by Tonnelat (cf. [632], Eq. II, p. 31). If $S_{i}=0$ is added to Eqs. (355) and (356) Einstein's strong field equations obtain; they no longer follow from the variational principle. M. Lenoir used the fiber bundle of affine reference frames and the transformation groups implied by it to arrive at Tonnelat's field equations [358].

\subsubsection{Removal of affine connection}

A first objective was to use the equation $\hat{g}^{+\frac{i k}{+-}}{ }_{\| l}=0$ or, equivalently, (30) to express the affine connection $L_{i j}{ }^{k}=L_{i j}{ }^{k}\left(g_{r s} ; \partial_{k} g_{r s}\right)$ as a functional of the asymmetric metric $g_{i j}$ and its first derivatives in the same way as the Christoffel symbol had been expressed by the metric and its first derivatives. Now, the system comprises 64 linear equations for 64 variables $L_{i j}{ }^{k}$. As an already solved algebraic problem this might not create much interest for "pure" mathematicians: an inverse matrix must be found, if only a large one with functions as its elements. V. Hlavatý called for an "elementary algebraic device" to be invented. As a problem in applied mathematics, even in the computer age, it takes quite an effort to do this by computer algebra. The wish to obtain the solution in tensorial form aggravates matters. According to Hlavatý: "Finding such a device is by no means an easy task" ([269], p. 50).

In Riemann-Cartan theory, i.e., in a theory with symmetric metric and arbitrary linear connection, we obtain:

$$
g_{i j, k}+g_{i k, j}-g_{j k, i}-2 g_{j l} L_{[i k]}^{l}-2 g_{k l} L_{[j i]}^{l}-2 g_{i l} L_{(k j)}^{l}=0 .
$$

If $L_{[i k]}^{l} \neq 0$, the usual method of solving (359) for the Christoffel symbol as a functional of the metric and its first derivatives still works, ${ }^{200}$ but no longer for (30).

After E. Straus had not been able to present a manageable solution (cf. Section 7.3), M. A. Tonnelat invested a lot of work into the same methodical approach. In a series of steps involving many intermediate expressions which had to be replaced in the end, she obtained a solution. In her first attempt during the early 1950s [622, 623], summed up in her monographs [632, 642], the solution is achieved by first splitting $g_{\mu \nu}$ into its irreducible parts. If $u_{i j}{ }^{k}$ is defined similarly as in (39) but with a different $u_{i j}{ }^{k}$ by

$$
\tilde{L}_{i j}^{k}=\left\{{ }_{i j}^{k}\right\}_{h}+u_{i j}^{k}+S_{i j}^{k}
$$

the decomposition of (30) leads to

$$
\begin{array}{r}
h_{j l} u_{i k}{ }^{l}=\tilde{S}_{i j}{ }^{l} k_{l k}+\tilde{S}_{k j}{ }^{l} k_{l i}, \\
\left.h_{j l} \tilde{S}_{i k}{ }^{l}=\frac{1}{2} f_{i j k}+\stackrel{\{i j}{k}\right\}_{j} k_{i k}-\left(u_{i j}{ }^{l} k_{l k}-u_{k j}{ }^{l} k_{l i}\right),
\end{array}
$$

where ${ }^{201} \tilde{S}_{k j}^{l}$ is the torsion tensor of the connection $\tilde{L}_{i j}^{k}$, and $f_{i j k}=\partial_{i} k_{j l}+\partial_{j} k_{l i}+\partial_{l} k_{i j}$. We have met (361), (362) in principle already in Section 2.1.2. A. H. Taub had reviewed one of her papers on the subject from 1950 [MR0037634]: "These systems are then solved explicitly. Straus [592] has also solved this problem by another method after remarking that the method used in this paper is feasible." The main conclusion is that the symmetric part of the affine connection may

200 Although, in this case, the connection cannot be determined as a functional of the metric alone because of torsion.

201 Equations (361) and (362) correspond to Eqs. (3.17), (3.18) of ([632], p. 40); cf. also [454], p. 74, Eqs. (9), (10). Cf. as well Finzi's equations (44) and (45) in ([473], p. 283). His Eq. (45) contains an erroneous term. 
be expressed by its antisymmetric part from (361), while (362) then determines the antisymmetric part, in terms of "the fields" $\left\{\begin{array}{l}k \\ i j\end{array}\right\}_{h}, h_{i k}, k_{i k}$. In the next step thus $u_{i j}{ }^{k}$ is removed from (362). By a lengthy calculation, torsion is expressed by "the fields". Thus, the connection

$$
L_{i j}^{k}=\left\{\begin{array}{l}
k \\
i j
\end{array}\right\}+S_{i j}^{k}+2 h^{k s} S_{s(i}^{t} k_{j) t}
$$

is fully known. ${ }^{202}$ The procedure works if

$$
g\left(a^{2}+b^{2}\right) \neq 0
$$

where $a=: 2-\frac{g}{h}+\frac{6 k}{h}, b=: 2 \sqrt{\frac{k}{-h}}\left[3-\frac{g}{h}+\frac{k}{h}\right]$ and $g, h, k$ are the determinants of $g_{i k}, h_{i k}, k_{i k}$, respectively. $a$ and $b$ turn up during the elimination process in which $g \neq 0$ and $h \neq 0$ is always assumed. $g=2 h, k=0$ obviously leads to $a=b=0$ and thus leaves the solution indeterminate; this is a result also obtained in an independent calculation by H. Takeno and coworkers [602]. They acknowledged Tonnelat's solution in a note added in proof but found her condition $k \neq 0$ "too stringent". M.-A. Tonnelat dealt with the case $k=0$ in another paper and confirmed her general result, i.e., the condition $\frac{g}{h} \neq 2$ for a solution to exist [630]. Further careful investigations of possible subcases were made later by Hlavatý [269] and Mishra [434]; cf. Section 13.3.

A reproduction of Tonnelat's calculations would not bring further insight, the more so as lots of auxiliary symbols were introduced by her including indices with one and two strokes. M.A. Tonnelat has presented the method in detail not only in her books but also in an article [633], and in a talk given at the outstanding Relativity Jubilee Conference in 1955 in Bern ([631], p. 192197). She was keen on securing priority, i.e., for having found the solution already in 1949-1950. This seemed imperative to her because in the meantime V. Hlavatý [257, 259], and N. S. Bose $[52,50]$ had also published solutions of $g_{\underline{i}-+\| l}=0$ by other methods (for Hlavatý cf. Section 12.2). In fact, Hlavatý had reviewed Tonnelat's paper in Mathematical Reviews [MR0066128], in which she had shown that $\operatorname{det}\left(k_{i j}\right)=0$ did not affect her solution [630], and he added that "for the solution in the exceptional cases $\frac{g}{h}=0,2$ " one should consult a forthcoming paper of his [265]. While the limit $k_{i j} \rightarrow 0$ leads back to the well known results in general relativity, the other limit $h_{i j} \rightarrow \eta_{i j}$ seemingly has not been discussed intensively by Tonnelat.

Indeed, the whole procedure is drastically shortened and becomes very transparent if $h_{i j}=\eta_{i j}$ is assumed. In this context, apparently, no one did look at this particular case. N. N. Ghosh began with another simplified metric built like the general spherically symmetric metric, i.e., with only $h_{00}, h_{11}, h_{22}, h_{33} \neq 0$ and $k_{10}, k_{23} \neq 0$, but with all components being functions of the four coordinates $x^{0}, \ldots, x^{3}$ However, he managed to solve (30) for the connection only by adding 4 conditions for the first derivatives of $h_{i j}$ and $k_{i j}$ in an ad hoc manner [222]. S. N. Bose ${ }^{203}$ rewrote (30) into an inhomogeneous linear equation for tensorial objects $T_{i[j k]}, U_{i[j k]}$, i.e., $\operatorname{lh}\left(T_{i[j k]}\right)=U_{i[j k]}$ where $l h(T)$ is homogeneous and linear in $T$ [50]. Considered as matrix equation, its solution is $T=B U$. The matrix $\check{C}_{i}^{j}:=h^{j s} k_{s i}$, its eigenvalues and eigenvectors play an important role. Although the method is more transparent than Tonnelat's, the solution is just as implicitly given as hers.

Interestingly, at first Einstein seems to have had some doubts about her method of solution, because torsion expressed by the fields $h_{i j}, k_{i j}$, and their first derivatives depended on the choice of the object used as the (symmetric) metric and raised a question of compatibility: "Mr. A. Einstein has directed my attention to this difficulty." ([624], p. 2407). ${ }^{204}$ In the paper, M.-A. Tonnelat could disperse Einstein's reservations. In a letter to A. Einstein of 21 June 1951, L. de Broglie wrote:

202 Cf. Section 12.2, and also Hlavatý's derivation in ([260], p. 5).

203 This is Satyendra Nath Bose (1894-1974) of the Einstein-Bose statistics.

204 "M. A. Einstein a attiré mon attention sur cette question." 
"I am glad to learn that one of my former pupils, Mme. Tonnelat, who really is a remarkable person, has had contact with you with regard to her papers on the unitary theories, and that you have shown an interest in her results". ${ }^{205}$

P. G. Bergmann's report on the Jubilee Conference was noncommittal: "A. Tonnelat of the Sorbonne reported on some mathematical results she had obtained on this theory independently of Einstein and Kaufman." And a little later: "The papers by Kaufman and Tonnelat are too technical to be reported here." ([22], p. 493.)

In a later approach by M.-A. Tonnelat [636], the affine connection is expressed by the metric as above but without a decomposition of $g_{\mu \nu}$ - in a similar but very much more complicated way as in the case of the Levi-Civita connection (the Christoffel symbol). This second method does not work if $4 h+12 k=3 g$. An improvement of it was given by Dautcourt [110] who also showed that $4 h+12 k \neq 3 g$ does not guarantee a solution. V. Hlavatý used still another method to express the affine connection as a functional of the metric; cf. Section 12.2.

St. Mavridès applied Tonnelat's method in the case of $l_{i j}$ and $m_{i j}$, i.e., the inverses of $l^{i j}, m^{i j}$ in $g^{i j}$ being used as metric and electromagnetic field. ${ }^{206}$ As an existence-condition (364) appeared as well [406]. A plenitude of further work concerning this problem of how to express the affine connection by the asymmetric metric, its derivatives and torsion was done, with the uniqueness proof by Hlavatý \& Saenz among them [270]. It amounted to a mathematical discussion of all logically possible cases and subcases without furthering UFT as a physical theory; cf. Sections 12.2 and 13.3 .

As a functional of the metric, its first and second derivatives, the Ricci tensor becomes a rather complicated expression. To then find exact solutions of the remaining field equation in (340) is a difficult task. In a paper dealing with approximations of the field equations, M.-A. Tonnelat tried to show the superiority of her method by applying a scheme of approximations to her (weak) field equations [634]. However, the resulting equations of 4th order for weak electromagnetic fields $k_{i j}$ and of 1 st order for weak gravitational fields $h_{i j}$ are still as complicated as to not allow a physical interpretation. In fact, the solution of the problem to remove the connection from the field equations neither helped the search for exact solutions nor contributed to a convincing physical interpretation of the theory. Nonetheless, it was of crucial importance for the proofs given by A. Lichnerowicz that the initial value problem could be well posed in UFT.

In a later development, Eq. (200) had been made inhomogeneous:

$$
A_{i k l}=g_{i k-} \| l:=g_{i k, l}-g_{r k} L_{i l}^{r}-g_{i r} L_{l k}^{r} \neq 0 .
$$

According to Tonnelat, $A_{i k l}$ in (365) is a linear function of the first derivatives of the metric and an additional vector field with 2 free parameters; cf. Section 10.3.3, particularly Eq. (383). The simple expression J. Lévy [361] used, by starting from $\hat{g}^{i k}{ }_{\mid l}^{+l}$, was:

$$
A_{l}^{i k}=\hat{g}^{i k} S_{l}-\frac{2}{3} \delta_{l}^{k} \hat{g}^{i r} S_{r}
$$

leads back to the first equation of (355). As was shown in Section 9.6, the most promising approach seemed to investigate special cases (spherical or axial symmetry), or approximate solutions. This also was done by Tonnelat at the end of one of her papers of 1955 [631], and tried again, later, in a dissertation advised by her [59]. There, the components of the connection were calculated and compared with the results of Bonnor [32]. In fact, for high symmetry, the whole work of solving

205 This is a translation into English from the translation of the presumably French original into German by H. Sievers ([580], Appendix A.2.3).

${ }^{206}$ In the notation of Mme. Mavridès $l^{i j}, m^{i j}$ correspond to $h^{i j}, f^{i j}$. 
(30) is unneeded: the field equations were solved at the same time for both the the metric $g_{i j}$ and the connection; cf. [475].

A special application refers to Schrödinger's "star"-connection. In this case, for the skewsymmetric part:

$$
\left.{ }^{*} \Gamma_{[i j]}^{k}=k_{i j||}^{k}+\frac{1}{2} h^{k r} k_{\langle j i \|} \|_{0} r\right\rangle,
$$

where the bracket $\langle\ldots\rangle$ denotes cyclic permutation ([305], p. 745).

\section{Remark:}

It is interesting to confront Tonnelat's position with Schrödinger's. In his paper of 1951, he limited himself to an approximate solution of (30) by splitting the connection into its symmetrical and skew parts ([558], Eqs. $(1,4),(1,5))$ :

$$
\begin{array}{r}
L_{(i j)}^{k}=\left\{\begin{array}{l}
k \\
i j
\end{array}\right\}_{h}-h^{k r}\left(k_{j s} L_{[i r]}^{s}+k_{i s} L_{[j r]}^{s}\right)=\left\{\begin{array}{l}
k \\
i j
\end{array}\right\}_{h}-h^{k r}\left(k_{j s} S_{i r}{ }^{s}+k_{i s} S_{j r}{ }^{s}\right), \\
L_{[i j]}^{k}=S_{i j}{ }^{k}=\frac{1}{2} h^{k r}\left(k_{i r, j}+k_{r j, i}+k_{i j, r}\right)+h^{k r}\left(k_{j s} L_{(i r)}^{s}-k_{i s} L_{(j r)}^{s}\right),
\end{array}
$$

similarly as M.-A. Tonnelat had done in (361) and (362). Equations (368) and (369) are more convenient for the setting up of an approximation scheme. In Schrödinger's words: “ [...] from (369), if the $k_{i k}$ are small, the components of the tensor [torsion] are small of the same order. Hence, from (368) the symmetric affinity differs from the Christoffel-brackets only by quantities of the second order. Thus, using in (369) the Christoffel-brackets for $L_{(i j)}^{k}$ etc., one gets the tensor, with an error of the third order; and if this is used in (368), one gets $L_{(i j)}^{k}$ with an error of the fourth order." From this, "a series of ascending powers of the $k_{i k}$ " can be developed. Such an approximation up to a certain order, then can be inserted into the remaining field equations. ${ }^{207}$

\subsection{Some further developments}

From the mathematical point of view, the results of Tonnelat and Hlavatý may be interpreted as having simplified the study of the weak field equations to some degree. For physics, no new insights were gained. In order to make progress, topics like exact solutions, equations of motion of test particles, or the problem how to express continuously distributed "matter" had to be investigated. It is here that the conflict between the "dualistic" approach to UFT separating the fields and their sources, and the "purely geometric" one showed up clearly. In the latter, the (total) field itself defines its own sources.

\subsubsection{Identities, or matter and geometry}

As a consequence of the contracted Bianchi-identities, in general relativity the divergence of the Einstein tensor $G^{i j}:=R^{i j}-\frac{1}{2} R g^{i j}$ identically vanishes: $\nabla_{j} G^{i j}=0$. An implication of the field equations $G^{i j}=-\kappa T^{i j}$ then is the vanishing of the divergence of the so-called "matter" tensor or energy-momentum-stress tensor of "external" matter, a quantity without geometric significance. In general relativity, $T^{i j}$ is a functional of the metric, the matter variables, and eventually the connection through covariant derivatives according to the principle of minimal coupling. $\nabla_{j} T^{i j}=0$ is used to derive equations of motion for point particles or field equations for matter fields. In UFT, according to Einstein, no external matter is allowed to occur; matter variables are to be defined from

\footnotetext{
207 Schrödinger's paper had been submitted in December 1950, i.e., several months later than Tonnelat's first notes on the solution of (30) in Comptes Rendus of 9 January and 28 August 1950. No reference to her is given in his paper.
} 
within the geometry. ${ }^{208}$ Hence, a distinction between external and internal regions as exemplified by the corresponding Schwarzschild solutions in general relativity would be unnecessary. In the words of M.-A. Tonnelat:

"The immediate advantage of a unitary theory is that from the theory itself the form of the electromagnetic energy-momentum tensor and, perhaps, of the matter tensor can be extracted. The expression of this tensor then would be imposed by the very geometric principles, and not by conclusions from an alien theory as interesting as it might be." ([635], p. 6$)^{209}$

An example for an electromagnetic energy-momentum tensor built from geometric quantities is given in (422) of Section 10.5.4.

As we have seen in Section 9.2, Einstein derived an identity corresponding to the contracted Bianchi-identity; cf. (260). It may be rewritten in various forms such as $\partial_{l}\left[\underline{K}_{i k} \hat{g}^{l k}+\underline{-}_{k i} \hat{g}^{k l}-\right.$ $\left.\delta_{i}^{l} \underline{-}_{m n} \hat{g}^{m n}\right]=0$ [409], or

$$
\partial_{l}\left(\underline{K}_{(i k)} \hat{g}^{(l k)}\right)-\underline{-}_{(m n), i} \hat{g}^{(m n)}-\hat{g}^{[l m]} \underline{-}_{\{[l m], i\}}=0,
$$

where the brackets \{\} now denote cyclic permutation [81]. Prior to this, a direct derivation by use of the Lie derivative and the invariance of the Lagrangian had been presented by Schrödinger and Lichnerowicz [556, 368, 369]. Lichnerowicz gave the identity the form ([370], Eq. (6.2) or [371], p. 272-273):

$$
\partial_{s} \hat{N}_{k}^{s}-\frac{1}{2} K_{l m} \partial_{k} \hat{g}^{l m}=0,
$$

with $\hat{N}_{k}^{j}=\sqrt{-g} N_{k}^{j}:=\sqrt{-g}\left(H_{k}^{j}-\frac{1}{2} \delta_{k}^{j} H_{s}^{s}\right)$ and $2 H_{k}^{j}=-K_{k s} g^{j s}-K_{s k} g^{s j}$.

A reformulation led to

$$
\partial_{s} \hat{H}_{i}^{s}+\frac{1}{2} \hat{g}^{r s} \partial_{i} K_{-r s}=0,
$$

where the Ricci tensor $K_{-}$is derived from an arbitrary connection $L_{i j}{ }^{k}$. Expressed by the connection with vanishing vector torsion ${ }^{(2)} \Delta_{i j}{ }^{k}$, i.e., after the interchange of $L_{i j}{ }^{k}$ and ${ }^{(2)} \Delta_{i j}{ }^{k},(372)$ remains formally unchanged for $K_{-}=K_{-}\left({ }^{2}(2) \Delta_{i j}{ }^{k}\right)$. (372) can also be expressed as an ordinary divergence:

$$
\partial_{j}\left(\hat{T}_{k}^{j}+t_{k}^{j}\right)=0
$$

where $\hat{T}_{k}^{j}=\frac{1}{2}\left(\hat{T}_{k s} g^{j s}+\hat{T}_{s k} g^{s j}\right)$ and $\hat{T}_{i j}=\sqrt{-g} T_{i j}$ with $\underline{K}_{i j}-\frac{1}{2} g_{i j} K_{-}=\kappa T_{i j} \quad$ [555, 409], ([632], p. 110-113), ([641], p. 305-308). Expressions for a formal "matter tensor" and an "energy pseudotensor" $t_{k}^{j}$ may be read off here (Cf. Eq. (7.22) on p. 113 in [632]). The procedure is ambiguous, though, as is known from general relativity. In Mme. Tonnelat's group, (370) was named "identité de conservation" or "conditions de conservation", and exhibited in several forms. For a Lagrangian of the form $H_{r s}\left(L_{i j}{ }^{k}, \partial_{l} L_{i j}{ }^{k}\right) \hat{g}^{r s}+\hat{\mathcal{M}}$, where the scalar density $\hat{\mathcal{M}}$ is independent of the connection $L_{i j}{ }^{k}$, the "identities of conservation" can be given the form ([359], p. 89):

$$
\partial_{l} H_{i k} \hat{g}^{l k}+\partial_{l} H_{k i} \hat{g}^{k l}-\partial_{i} H_{r s} \hat{g}^{r s}+H_{r s} \partial_{i} \hat{g}^{r s}=0 .
$$

${ }^{208}$ In particular, this amounts to define a particle through a regular field concentration; Einstein did not describe a point particle by a singularity of the field.

209 "L'avantage immédiat d'une théorie unitaire est d'extraire de la théorie même la forme du tenseur d'impulsionénergie électromagnétique et peut-être du tenseur matériel. L'expression de ce tenseur serait alors imposée par les principes géométriques eux-mêmes et non par les conclusions d'une théorie étrangère, si intéressante soit-elle." 
For each solution of the corresponding field equations they are identically satisfied.

In the same spirit, (373) is often called "conservation law" although the quantity conserved need not correspond to a physical observable. An example of this is given by Kursunŏglu [343] who chose as his pseudo stress-energy-momentum tensor "beyond the shadow of doubt" to be:

$$
\mathcal{T}_{i}^{j}=\frac{1}{4 \pi p^{2}}\left\{\left(\hat{g}^{(s j)} \underline{-}_{(s i)}-\frac{1}{2} \delta_{i}^{j} \hat{g}^{(r s)} \underline{-}_{(r s)}\right)-\left(\hat{g}^{[s j]} \underline{-}_{[s i]}-\frac{1}{2} \delta_{i}^{j} \hat{g}^{[r s]} \underline{-}_{[r s]}\right)+\frac{1}{2}\left(\hat{g}^{r s}{ }_{, i} \mathcal{B}_{r s}^{j}-\delta_{i}^{j} \mathcal{B}\right)\right\},
$$

with $\mathcal{B}_{i j}^{k}=L_{i j}{ }^{k}-\delta_{j}^{k} L_{(i s)}{ }^{s}$, and $\mathcal{B}=\hat{g}^{i j} \mathcal{B}_{i j}, \mathcal{B}_{i j}=L_{i j}{ }^{s} L_{(s r)}^{r}-L_{i r}{ }^{s} L_{(j s)}{ }^{r} \cdot p$ is a constant defined in Section 9.3.3. Using the notations of Kursunŏglu, Clauser derived what he named contracted BianchiEinstein identity, i.e., (370): $\partial_{j} \hat{T}_{k}^{j}=0$ with $\left.\hat{T}_{k}^{j}=-2\left(\underline{K}_{(l k)} \hat{g}^{(l j)}\right)+\underline{K}_{[l k]} \hat{g}^{[l j]}\right)+\delta_{k}^{j}\left(\mathcal{B}+\underline{K}_{l m} \hat{g}^{l m}\right)-$ $\mathcal{B}_{l m}^{j} \hat{g}_{, k}^{l m}$. It is called a "strong" conservation law, because only Eqs. (250) and (251) of the weak field equation have been used, while for a "weak" conservation law all of the weak field equations would be needed [81]. $\hat{T}_{i}{ }^{j}$ is a tensor density only with regard to linear coordinate transformations.

Another approach for the introduction of the energy-momentum tensor of matter $T_{i k}$ is the following. First a symmetric metric must be be chosen, e.g., $h_{m n}$. Then in the symmetrical part of the Ricci tensor $K_{(i j)}(L)$ a term of the form of the Einstein tensor $G_{i k}\left(h_{m n}\right)$ is separated out. The field equations of UFT are then re-written as formal field equations of the type of Einstein's equations in general relativity plus terms left over. This remainder is identified as $T_{i k} \sim G_{i k}$. The method is applicable because mixed geometry can always be re-interpreted as Riemannian geometry with many extra fields (geometric objects). Its ambiguity lies in the choice of the Riemannian metric. Taking $h_{i j}$ as the metric, or the reciprocal of $\sqrt{\frac{l}{g}} l^{i j}$ or $\sqrt{\frac{g}{l}} l_{i j}$ like in [273, 390], or another of the many possible choices, makes a difference. By the formulation within a Riemannian geometry, the unifying strength of a more general geometry is given up, however. Also, according to a remark by M.-A. Tonnelat, the resulting equations $\nabla_{s} T^{i s}=0$ are satisfied identically if $K_{[i j]}(L)=0$ holds. Thus, the information about the gravitational field contained in the symmetric part of the field equation $K_{(i j)}(L)=0$ does not influence the equations of motions of matter following from $\nabla_{s} T^{i s}=0$ [637]. Related with this is the fact that the matter tensor "seems to vanish together with the electromagnetic field $g_{[i j]}$, or at least with a field the properties of which remind of the electromagnetic field" ([635], p. 7). ${ }^{210}$

In H.-J. Treder's access to a "matter" tensor in "the asymmetric field theory of Einstein", the subtraction was done not on the level of the Einstein tensor, but for the Lagrangian: from the Lagrangian density of UFT the Einstein Lagrangian was subtracted. An advantage is that the variational principle ensures the existence of an identity [649]. A disadvantage is that the Lagrangian density for the matter part depends not only on the metric but also on its derivatives of 1 st and 2nd order. For the metric Treder took the symmetric part $h_{i j}$ of the asymmetric fundamental tensor $g_{i j}$. His references went to Infeld and to Schrödinger's work, none to Tonnelat's. We conclude that Tonnelat's hope presented in the the first quotation above remained unfulfilled.

\subsubsection{Equations of motion}

Unlike in general relativity, in UFT, the equations of motion, in general, are no longer a direct consequence of the field equations. Ambiguities are bound to arise. ${ }^{211}$ A methodological concern was how to properly derive equations of motion for matter, in particular for point-particles, possibly charged and massive. Two problems arise: In principle, for a single non-interacting particle certain types of world lines could be defined as paths like the geodesics or auto-parallels in general relativity.

\footnotetext{
210 "Ce tenseur semble disparaître avec le champ électromagnétique $k_{m n}$, ou tout au moins avec un champ dont les propriétés rapellent celles du champ électromagnétique."

211 cf. Chapter VII B of Tonnelat's book of 1965 [641].
} 
But, what world lines should be investigated, the geodesics of $g_{(i j)}$, or of another object chosen as a representation of the gravitational potential (field)? The geodesics do not depend on $g_{[i j]}=k_{i j}$; in the usual interpretation of $k_{i j}$ as representing the electromagnetic field, its influence on such paths would be naught:

$$
\frac{d^{2} x^{i}}{d s^{2}}+\left\{{ }_{j k}^{i}\right\}_{h} \frac{d x^{j}}{d s} \frac{d x^{k}}{d s}=0 .
$$

Unlike this, in the equation for auto-parallels

$$
\frac{d^{2} x^{i}}{d s^{2}}+L^{i}{ }_{j k} \frac{d x^{j}}{d s} \frac{d x^{k}}{d s}=0
$$

the electromagnetic field would show up. Again, what kind of connection is to be used, with or without vector torsion ("star"-connection), axial torsion etc?

Secondly, in correspondence to the vacuum field equations of general relativity, the method of treating the motion of matter as motion of singular point particles as Einstein, Infeld \& Hoffmann had done with their approximation scheme in general relativity (EIH-method) [171, 170], would be in conceptual conflict with the spirit of UFT. Was it possible, here, to only consider the region outside of a world-tube around the moving body where the matter tensor $T_{i j}=0$ ? The alternative method of Fock with $T_{i j} \neq 0$ included the interior of the moving bodies as well. In order to avoid infinitely many degrees of freedom for extended bodies, some limit procedure had to be introduced. In the EIH-method, $\delta$-functions, i.e., distributions are used as matter sources, although Einstein's equations do not admit distributions as exact solutions. Nonetheless, many authors applied the EIH-method also in UFT. E. Clauser showed in great detail that the method is applicable there for charged particles [83]; cf. also Section 15. Pham Tan Hoang wrote his doctoral thesis by applying this "singularity-method" to unified field theory [273] (cf. Section 10.4.1). However, we have mentioned already in Section 9.3.3 that both, L. Infeld and J. Callaway were not even able to derive the results of Einstein-Maxwell theory.

With the singularity-method employed, it turned out to be non-trivial to reach the Lorentzforce, or even the Coulomb force in a "slow-motion-" and "weak-field-" approximation: $h_{i j}={ }^{(0)} h_{i j}+$ $\epsilon^{(1)} h_{i j}+\epsilon^{2}{ }^{(2)} h_{i j}+\ldots ; k_{i j}=\epsilon^{(1)} k_{i j}+\epsilon^{2}{ }^{(2)} k_{i j}+\ldots$, with only terms $\sim \frac{v}{c}$ being retained. In first approximation, only the motion of uncharged particles was described properly by the weak field equations if $h_{i j}$ is taken as the metric and $k_{i j}$ as the electromagnetic field [69]. This negative result remained valid up to 4th order in $\epsilon$ for $l^{i j}\left(l_{i j} \neq h_{i j}\right)$ chosen as the metric and $m^{i j}\left(m_{i j} \neq k_{i j}\right)$ as the electromagnetic field [271, 272]. Better results in which the Coulomb force could be made to appear were achieved by Treder and Clauser [650, 81, 82], and later by N. P. Chau, in a slightly changed theory, [77]; cf. also Section 15.1.

The electric potential is introduced via $g_{[m n]}^{(2)}=\epsilon_{m n s} \phi_{, s}^{(2)}$, where $n, m, s=1,2$, where $g_{[m n]}^{(2)}$ is the metric coefficient in the lowest order of an expansion. Here, the equations of motion for two electrical point-charges $\stackrel{1}{e}, \stackrel{2}{e}$ were following from 4 th-order equations for the electrostatic potential $\phi$ obeying

$$
\triangle \triangle \phi=0
$$

and leading to the solution $\phi=\frac{A}{r}+B+C r+D r^{2}$ instead of only the Coulomb potential. After the integration constants $B, D$ were argued away, the resulting equations of motion turned out to be, for the first point-charge:

$$
\stackrel{1}{m} \frac{d^{2} x}{d t^{2}}=-G_{N} \frac{\stackrel{1}{m} \stackrel{2}{m x}}{r_{12}^{3}}+(\stackrel{1}{A} C+\stackrel{2}{A} \stackrel{1}{C}) \frac{\stackrel{12}{e}) \frac{-}{r^{3}}}{{ }^{3}}-2 \stackrel{1}{C} \stackrel{2}{C} \frac{x}{r},
$$

where $\underline{x}:=x_{1}-x_{2}$ and $\underset{12}{r}:=\left|\underline{x}_{1}-\underline{x}_{2}\right|$; underlined letters denote 3 -vectors. The constants ${ }_{A}^{1}, \stackrel{2}{C}, \cdots+\ldots$ are referring to the electric potential of the two charges ([477], p. 197). However, 
in addition to the Coulomb force an unphysical force independent of distance also showed up. It could not be made to vanish without an accompanying loss of the Coulomb force. In order to remove this defect, the field equations had to be changed; cf. [77] and Section 10.4. In Section 9.3.3 we already encountered such an altered field equation.

In Section 13.1 we will present Bonnor's field equations which are leading, in lowest order, to Newton's equations for two charges $\stackrel{1}{e}, \stackrel{2}{e}$ :

$$
\stackrel{d^{2} x}{m} \frac{\stackrel{1}{m} \stackrel{2}{m x}}{d t^{2}}=-\frac{\stackrel{12}{e e x}}{r^{3}}+p^{2} q^{2} \frac{-}{r^{3}} .
$$

Here, $q$ is the ratio of $k_{i k}$ to the electromagnetic field strength, and $p$ a constant in an additional term in the Lagrangian. Bonnor ascribed the success due to this added term, quadratic in the skew-symmetric part of the metric $g_{i k}$, to the circumstance that "the corresponding terms [in the field equations] arising from the $k_{i k}$ refer not to potentials but to the electromagnetic field strengths. For this reason it is hardly to be expected that any minor modification of the tensor $R_{(i k)}$ will lead to the Coulomb force" ([33], p. 377). This is also obvious when Bonnor's term in the Lagrangian $p^{2} \hat{m}^{i k} \mathbf{k}_{\mathbf{i k}}$ is compared with the usual $F^{i k} F_{i k}$ in Maxwell's theory, because Bonnor is interpreting $k_{i k}$ not as an electromagnetic potential but as the electromagnetric field cf. [35].

A. Papapetrou, in a review concerning the problem of motion, pointed out that if Schrödinger's equations with a cosmological constant $\lambda$ are considered, i.e., Eqs. (236 and (237), then from $R_{\{[k l], i\}}-\lambda g_{\{[k l], i\}}=0$ the equation for the electrostatic potential follows [477]:

$$
\triangle(\triangle-2 \lambda) \phi=0 \text {. }
$$

For large distances, Coulomb's law then remained valid for an approximate solution $\phi \simeq e^{-2 \lambda r}\left[\frac{A}{r}+B+C r+D r^{2}\right]$ with vanishing $B, D$. The constant $C$ represents electric charge. A characteristic length $\frac{1}{\lambda}$ showed up. As Papapetrou wished to apply UFT to elementary particles like the electron, the problem was that $\frac{1}{\lambda}$ should be of the order of an electron radius and not be of cosmological significance. His general conclusion was:

"It appears impossible to come to a direct phenomenological use of this theory which would allow a satisfactory treatment of macroscopic problems. But this does not prove anything with regard to the applicability of the theory in the micro-physical domain." $([477], \text { p. 203. })^{212}$

A review by M. Lenoir [357] of progress made in the papers by Clauser [81], Treder [649] and Papapetrou [477] with regard to the Coulomb force, was reviewed itself by W. B. Bonnor in Mathematical Reviews [MR0119977].

For alternative field equations following from M.-A. Tonnelat's model cf. (383)-(385) in the next Section 10.3.3.

For continuous matter, a third approach within general relativity employed the vanishing of the covariant divergence of the matter tensor $T_{; l}^{i l}=0$. This assumed a convincing answer to the question of how to properly define a matter tensor within UFT as described in the previous Section 10.3.1. M.-A. Tonnelat, in particular, started from the form given to an identity by E. Schrödinger:

$$
\partial_{r}\left(\hat{g}^{s r} W_{s k}+\hat{g}^{r s} W_{k s}\right)-\hat{g}^{s r} \partial_{k} W_{s r}=0
$$

which is rewritten and re-interpreted within Riemannian geometry ([382], p. $207-209)$. The equations of motion are derived from volume-integration over equations of the form of (373) as will be seen in the next section.

212 "Il semble impossible de faire une utilisation phénoménologique directe de cette théorie qui permettrait un traitement satisfaisant des problèmes macroscopiques. Mais cela ne prouve rien à l'égard de l'applicabilité de la théorie dans le domaine microphysique." 


\subsubsection{Tonnelat's extension of unified field theory}

After having spent years of research with Einstein's Lagrangian $\hat{\mathcal{L}}=\hat{g}^{i j} R_{i j}$, M.-A. Tonnelat felt confident that this was not the way to proceed:

"Nevertheless, we are convinced that a modification of the generalization of the theory suggested by Einstein can lead, at least partially, to the goal Einstein himself had set. [...] if one wants to cling to the original form of this theory which has caused many hopes and initiated a flood of papers, he would not know how to achieve the objectives which had been proposed at first, within the strict scope of the theories' principles." ([302], p. $117-118)^{213}$

Hence she expanded her Lagrangian by including a normed 4-vector ([641], p. 353):

$$
\hat{\mathcal{L}}=\hat{g}^{i j} K_{i j}\left(L_{r s}^{t}, \Gamma_{p}\right)+\hat{A}^{p} S_{p}^{L}+\sigma\left(\hat{g}^{i j} \Gamma_{i} \Gamma_{j}-2 K^{2} \sqrt{-g}\right),
$$

where $L_{r s}^{t}$ is a connection without vector torsion $\left(\stackrel{L}{S}{ }_{i}=0\right) . \Gamma^{i}$ is the new 4 -vector (1-form), $\hat{A}^{p}, \sigma$ are Lagrangian multipliers, and $K$ is a constant not to be mixed up with the curvature scalar. For $K_{i j}$ the expression is chosen:

$$
K_{i j}=W_{i j}+\alpha\left(\partial_{i} L_{(j r)}^{r}-\partial_{j} L_{(i r)}^{r}\right)+2 q \partial_{[i} \Gamma_{j]}+p \Gamma_{i} \Gamma_{j}+2 \beta\left(\partial_{(i} \Gamma_{j)}-L_{i j}^{p} \Gamma_{p}\right) .
$$

$W_{i j}$ is the Ricci tensor formed with the connection $L_{r s}^{t}$. Note the four additional constant parameters $\alpha, \beta, p, q$ ! In a previous paper of $1960, \alpha=\beta=0$ had been assumed [637]. The ensuing field equations now were: ${ }^{214}$

$$
\begin{array}{r}
\hat{g}_{\| k}^{i j}{ }_{\| k}=-\frac{2}{3} \delta_{k}^{i} \partial_{s} \hat{g}^{[j s]}-\frac{2 \alpha}{3}\left(\delta_{k}^{i} \partial_{s} \hat{g}^{[j s]}+\delta_{k}^{j} \partial_{s} \hat{g}^{[i s]}\right)-2 \beta \hat{g}^{i j} \Gamma_{k}+\frac{2 \beta}{3}\left(\delta_{k}^{i} \hat{g}^{s j}+\delta_{k}^{j} \hat{g}^{i s}\right) \Gamma_{s}, \\
\left\{q+\frac{5 \beta}{3}(1+2 \alpha)\right\} \partial_{s} \hat{g}^{[i s]}=\left(\frac{4 \beta^{2}}{3}-p-\sigma\right) \hat{g}^{(i s)} \Gamma_{s}, \\
\omega_{i j}:=K_{i j}+\sigma\left(\Gamma_{i} \Gamma_{j}-K^{2} g_{i j}\right)-\theta_{i j}=0 .
\end{array}
$$

The choice $\alpha=\beta=\sigma=p=0 ; q=-1(q \neq 0)$ leads back to Einstein's strong field equations for a connection without torsion vector. Thus, M.-A. Tonnelat always assumed $\frac{4 \beta^{2}}{3}-p-\sigma \neq 0$. The subcase $\alpha=\beta=0$ first has been studied by doctoral students of Tonnelat in [361], and $[53,56]$. For this case, (383) leaves $\Gamma_{i}$ undetermined.

If the Lagrangian density $\hat{\mathcal{L}}$ is augmented by a phenomenological matter-Lagrangian density $\hat{\mathcal{L}}_{\text {mat }}$, then through $\Theta_{i j}:=-\frac{1}{\sqrt{-g}} \frac{\delta \mathcal{L}_{\text {mat }}}{\delta g^{i j}}=\theta_{i j}-\frac{1}{2} g_{i j} g^{r s} \theta_{r s}$ a phenomenological matter-tensor can be described.

However, it is possible to relinquish the phenomenological matter tensor by using the method of reformulating mixed or metric-affine geometry as Riemannian geometry. In $K_{i j}$, a Riemannian part $\stackrel{R i e}{K}_{i j}(a)$ relative to an arbitrarily chosen (symmetric) metric $a_{k j}$ must be exhibited. Eq. (385) will then be replaced by

$$
\omega_{i j}-\frac{1}{2} a_{i j} a^{r s} \omega_{r s}=\stackrel{\text { Rie }}{K}_{i j}(a)-\frac{1}{2} a_{i j} a^{r s} \stackrel{\text { Rie }}{K}_{r s}(a)-\chi \stackrel{\text { Rie }}{T}_{i j}
$$

213 "Toutefois nous sommes persuadés qu'une modification de la généralisation proposée par Einstein peut acheminer, au moins partiellement, au but qu'Einstein lui-même s'était fixé. [...] si l'on veut s'en tenir à la forme originale de cette théorie qui a suscité beaucoup d'espoir et provoqué un grand débordement de travaux, on ne saurait atteindre, dans le cadre strict de ses principes, les objectifs que l'on s'était tout d'abord proposés."

214 There is a difference in the signs of $p$ and $\sigma$ between ([637], p. 2893) and ([641], p. 353). Also $\kappa$ became used in place of $K$. 
We need not go through all the works similar to the approach in the previous theory of M.A. Tonnelat, but will only list the following items ([641], p. 363):

- as a metric, the quantity $\sqrt{\frac{g}{h}} l_{i j}$ was chosen; ${ }^{215}$

- geometrically, $\stackrel{l}{\Gamma}^{i}:=l^{i s} \Gamma_{s}$ corresponds to vector torsion $S^{i}$; physically, according to (384), it is linked to the current $\hat{g}^{(i r)} \Gamma_{r}$ and proportional to the 4 -velocity $u^{k}$ of a particle;

- from the equations of motion, in linear approximation, an acceleration term $\simeq \Gamma^{s} \partial_{s} \Gamma^{i}$ and the Lorentz force showed up as well as further terms characterizing other forces of unknown significance.

The last result needs a comment:

1) As admitted by Tonnelat, it is obtained only after partial neglect of the variational principle: instead of $\omega_{[i j]}=0$ which follows from $(386) \omega_{[i j]} \neq 0$ must be required in order to give a meaning to an equation like ([382], (108), p. 217):

$$
\frac{1}{2} \sqrt{\frac{g}{h}} g^{[r s]} \omega_{\{[i r], s\}}+\omega_{[i r]} \stackrel{\text { Rie }}{\nabla}_{s} g^{[r s]}=0 .
$$

(387) follows from (386) after a longer calculation omitting terms $\sim \partial_{k}\left(\sigma K^{2}\right)$. The covariant derivative is formed with regard to the Levi-Civita connection for the metric $\sqrt{\frac{g}{h}} l_{i j}$. "Hence, in the extended version of the asymmetric theory like in the initial version, the equations of motion can make sense only if at least one of the expressions $\omega_{[i j]}$ or $\Theta_{[i j]}$ does not vanish." 216

2) As usual, the equations of motion of charged particles were derived in linear approximation in which the electromagnetic field $k_{i j}$ is taken to be small of first order. It was expressed by a vector potential $\phi_{k}$ and an axial potential vector through $\chi_{i j}=\sqrt{-l} \epsilon_{i j l m} \partial^{[l} \chi^{m]}$ with $l=\operatorname{det}\left(l_{i j}\right)$ such that:

$$
k_{i j}=g_{[i j]}=2 \partial_{[i} \phi_{j]}+\sqrt{-l} \epsilon_{i j l m} \partial^{[l} \chi^{m]} .
$$

In the end, a simplified version of her extended theory is considered with $\alpha=\beta=p=0, k=$ $\frac{\sigma}{q} \neq 0$. The action is

$$
\hat{\mathcal{L}}=\hat{g}^{i j} K_{i j}\left(L_{r s}{ }^{t}, \Gamma_{p}\right)+\hat{A}^{p} \stackrel{L}{S_{p}}+\sigma\left(\hat{g}^{i j} \Gamma_{i} \Gamma_{j}-2 K^{2} \sqrt{-g}\right),
$$

with

$$
K_{i j}=W_{i j}+2 q \partial_{[i} \Gamma_{j]} .
$$

In the static case, only $\chi_{123}=: \chi$ and $\phi_{0}=: \phi$ remain $^{217}$ and satisfy the equations $\triangle \triangle \chi=0$ and $\triangle \phi=k \Gamma$ with $\Gamma=\Gamma_{0}, k=\frac{p+\sigma-4 \beta^{2} / 3}{q+\frac{5 \beta(1+2 \alpha)}{3}}=\frac{\sigma}{q} \neq 0$.

\footnotetext{
215 Caution: what is called $l_{i j}$ and $h$ here is $h_{i j}$ and $\gamma$ in Tonnelat's notation.

216 "Ainsi dans la version élargie de la théorie asymétrique comme dans la version initiale, les équations du mouvement ne peuvent avoir un sense que si l'on suppose que l'une au moins des expressions $\omega_{[i j]}$ ou $\Theta_{[i j]}$ n'est pas nulle." ([382], (108), p. 218.)

217 The symbol $\chi$ used here by Tonnelat differs from the $\chi$ introduced by her in (386); also $\chi_{i j}=: \partial^{r} \chi_{r i j}$.
} 


\subsubsection{Conclusions drawn by M.-A. Tonnelat}

In M.-A. Tonnelat's understanding, Einstein's unified field theory shows a number of new perspectives:

(1) the dynamics of both the electromagnetic and the gravitational fields are modified such that there appears to be also an influence of the gravitational field on the electromagnetic one;

(2) As a nonlinear electrodynamics follows, new effects will appear - as, e.g., "a diffusion of light by light".

(3) The relation between field strengths and inductions is similar as in nonlinear Born-Infeld theory ([632], p. 10.).

She believed that her extension of Einstein's field equations had alleviated the way toward the equations of motion for charged particles.

Altogether, she seems to have been optimistic about the importance of the theory although she was aware of the fact that its range of validity was unknown, and many conceptional questions still remained unanswered. At the time, Mme. Tonnelat's opinion possibly was the same as the one ascribed by her to the celebrities: "One may find with Einstein and Schrödinger a mixture of a certain discourage and of great hopes for the subject." [632], p. 11.) ${ }^{218}$ Four years later, after progress made had been criticized as irreconcilable with the axioms of UFT, her attitude still persisted:

"Nevertheless, the discouraging results obtained from different directions never have definitely compromised the theory; it is the ambiguity of the possible interpretations (choice of metric, interpretation of the skew-symmetric fields, etc) which have set straight issues for the inveterate and totalitarian unifiers." [382], p. 200). ${ }^{219}$

The choice of words impregnated by ideology like "inveterate" and "totalitarian" speaks for itself.

As has been remarked before, W. Pauli had criticized unified field theory approached through metric-affine geometry: he demanded that the fundamental objects must be irreducible with regard to the permutation group and also referred to Weyl ([487], Anm. 23, p. 273). In this view, an admissible Lagrangian might be $\mathcal{L}=a \hat{g}^{(i k)} K_{(i k)}+b \hat{g}^{[i k]} K_{[i k]}$ rather than $\mathcal{L}=\hat{g}^{i k} K_{i k}$. By an even stricter application, Pauli's principle would also rule out this Lagrangian, cf. Section 19.1.1.

In 1971, M.-A. Tonnelat veered away from the "unitary spirit" by giving more philosophical comments:

"It would be childish to think that, for Einstein, the existence of the unified fields would resolve into an ontological criss-cross of torsions and curvatures. It would be likewise improper to reduce such schemes to a pure formalism without any relation to a universe the objectivity of which they propose to present. [...] The objective pursued by unitary physics presents itself not as a realization with well defined contours but as a possible direction. [...]" ([645], p. 396) $)^{220}$

218 "On peut trouver chez Einstein et chez Schrödinger le mélange d'un certain découragement et de grands espoirs à ce sujet."

219 "Toutefois, les résultats décourageants obtenus dans divers directions n'ont jamais compromis définitivement la théorie car l'ambiguité des interprétations possibles (choix de la métrique, interprétation des champs antisymétriques, etc.) a toujours ménagé des issues pour les unitaristes totalitaires et impénitents."

220 "Il serait enfantin de penser que l'existence de champs unifiés se résolvait, pour Einstein, dans un entrecroisement ontologique de torsions et de courbures. Il serait tout aussi inexact de réduire ces schémas à un pur formalisme, sans aucun rapport avec un univers dont ils se proposeraient de traduire l'objectivité. [...] L'objectif que poursuit une physique unitaire se présente donc, - non comme une réalisation au contour bien défini, - mais comme une direction possible. [...]" 


\subsection{Further work on unified field theory around M.-A. Tonnelat}

Much of the work initiated by M.-A. Tonnelat has been realized in doctoral theses, predominantly in the framework of Einstein-Schrödinger field theory. About a dozen will be discussed here. They are concerned with alternative formulations of the field equations, with the identities connected with them, with exact solutions, and with equations of motion for (charged) particles in different approximation schemes.

\subsubsection{Research by associates and doctoral students of M.-A. Tonnelat}

As to the associates of M.-A. Tonnelat, it is unknown to me how Stamatia Mavridès got into theoretical physics and the group around M.-A. Tonnelat. She had written her doctoral dissertation in 1953 outside of physics [405]. For 5 years, since 1954, she contributed, alone and with Mme. Tonnelat, to Einstein-Schrödinger unitary field theory in many different aspects. Some of her publications have already been encountered. In Section 9.7, her assignment of physical variables to geometrical objects was noted, in Section 10.2.3 her contribution to the removal of the connection. Moreover, her contribution to spherically symmetric exact solutions mentioned in Section 9.6.1 must be kept in mind. Mme. Mavridès also took part in the research on linear field theories; cf. Section 16.1. Since the 1970s, her research interests have turned to astrophysics and cosmology [417].

Judith Winogradzki ${ }^{221}$ was a student of L. de Broglie with a thesis on "the contribution to the theory of physical quantities attached to spin-1/2-particles" [701]. With the topic of her thesis, she easily could have come into contact also with M.-A. Tonnelat. Although she had shown an interest in affine spaces before writing her dissertation [700], the concept of spinors permeated her subsequent research even more than unitary field theory. As noted in Section 9.8, group theory and conservation laws in special relativistic field theories and general relativity were also dear to her [706]. As was mentioned, she determined the identities following from Noether's theorems if applied to the group $U$ ( $\lambda$-and coordinate transformations) and asserted Einstein's claim that invariance under the group $\mathrm{U}$ is able to determine the field equations ${ }^{222}$. Also, Einstein's $\lambda$-transformations as an "extension of the relativistic group" were investigated by her [702]. In another paper, she set out to determine a "gauge"-group, named J-transformations, satisfying:

$$
J\left(g_{i k}\right)=g_{i k}, J\left(L_{i k}^{m}\right)=L_{i k}^{m}+\Lambda_{i k}^{m},
$$

where $\Lambda_{i k}{ }^{m}$ does not depend on the connection L; transposition symmetry is also excluded from (390). A variational principle with Lagrangian density $\hat{g}^{r s} R_{r s}$ is considered where $R_{i k}$ can equal $K_{i k}$ or $K_{+}$. J. Winogradzki then proved that $\Lambda_{i k}{ }^{m}=\delta_{i}^{l} \lambda_{k}$, is necessary, e.g., Einstein's $\lambda$ transformations appear. The weak field equations of Einstein and Straus are the only ones admitted [703]. Since 1956, no papers on UFT by her seem to exist.

We now come to some of the doctoral theses. Jack Lévy's Lagrangian for unified field theory is a slight generalization of Tonnelat's:

$$
\hat{\mathcal{L}}=\left[\hat{g}^{i j} K_{i j}\left(\Gamma_{r s}^{t}\right)+2 m X_{[i, j]}\right]+2 \hat{A}^{p}\left(\Gamma_{p}-k_{p}\right)+\sigma\left(\hat{g}^{i j} X_{i} X_{j}-2 \kappa^{2} \sqrt{-g}\right),
$$

where $\Gamma_{k}$ is the torsion vector of the arbitrary (asymmetric) connection $\Gamma_{i j}^{k}$, and $k_{i}$ an arbitrary one-form which can be a function of the arbitrary vector field $X_{k}$. The third term is introduced

\footnotetext{
221 Judith Winogradzki née Winterberg (1916-2006). She eventually became Professor of Theoretical Physics at the University of Rouen.

222 J. Winogradzki named "strong system" (système fort) what usually is called "weak" field equations ([704], p. 74)
} 
"in order to apply the normalization condition to an arbitrary vector $X_{k}$ and not necessarily to the torsion vector $\Gamma_{i}$. This is done to avoid any identification or anticipated interpretation of the fields" ([361], p. 249) ${ }^{223}$ Note that in Lévy's notation $X_{i}$ corresponds to $S_{i}$. The field equations follow from variation with regard to $\hat{g}^{i j}, \Gamma_{r s}{ }^{t}, X_{k}$, and the Lagrange multipliers $\hat{A}^{p}, \sigma$. Among the (weak) field equations, (235) is replaced by $\hat{g}^{[i s]}{ }_{, s}=\frac{3}{2} \hat{A}^{i}$ which allows the introduction of a non-vanishing electrical current if $\hat{g}^{[i s]}$ is related to the electromagnetic field (induction). Furthermore, in the field equations, Lévy put either $k_{i}=0$, or $k_{i}=k X_{i}$. In the first case the torsion vector drops out, while $X_{k}$ comes in. Also $\left(m \hat{g}^{[i s]}\right)_{, s}=\sigma \hat{g}^{(i s)} X_{s}$ with $m \neq 0$. In the second, the torsion vector stays and is normed: $\hat{g}^{i j} \Gamma_{i} \Gamma_{j}=2 \frac{\kappa^{2}}{k^{2}} \sqrt{-g}$ ); furthermore $\hat{g}^{[i s]}{ }_{s}=\frac{\sigma / k}{m+2 k / 3} \hat{g}^{(i s)} \Gamma_{s}$ with $m \neq-\frac{2 k}{3}$. Lévy then showed that both versions are mathematically and physically equivalent. The impression here and in the contributions below is that mathematical tricks were played to better the consistency of the approach without an improvement in the physical understanding of the theory.

Pham Tan Hoang's dissertation dealt with equations of motion. First, an explication of the EIH-method for the derivation of such equations for point particles in general relativity, and an introduction into the basics of unified field theory were given [274]. A useful result by him is the following. A coordinate system defined by

$$
g^{r s} L_{r s}^{k}=0,
$$

for an affine connection $L_{i j}^{k}$ had been named "isothermal" by M.-A. Tonnelat. ${ }^{224}$ In general relativity, in linear approximation, (392) reduces to the coordinate system introduced for obtaining the wave equation. According to Pham Tan Hoang one can set up a coordinate system in UFT such that it is also isothermal with regard to the particular Riemannian metric $a^{i j}=\sqrt{\frac{g}{h}} l^{i j}$ if vector torsion is vanishing. This he ascribes to the relation ([272], p. 67):

$$
g^{r s} L_{r s}^{k}=\sqrt{\frac{h}{g}} a^{r s}\left\{\begin{array}{c}
k \\
r s
\end{array}\right\}_{a} .
$$

Then, Pham Tan Hoang applied the EIH-method to the weak field equations. When $a_{i j}=\sqrt{g / l} l_{i j}$ with $l=\operatorname{det}\left(l_{r s}\right)$ is taken as the metric and $q^{i j}=\sqrt{l / g} m^{i j}$ as electromagnetic field, he obtained the same negative result as J. Callaway [69]: in linear approximation only uncharged particles can be described properly ([274], p. 89). No cure for this failure was found. In the end, the author could only bemoan the ambiguity inherent in the basis of the theory - implying structural richness, on the other side. We shall see that higher approximations had to be calculated in order to get the Coulomb field and the Lorentz force; cf. Section 15.3. Although dependent on the identifications made, another difficulty pointed out by Pham Tan Hoang is the vanishing of the charge current with the vanishing of $g_{[i j]}$. Moreover, the identification of a geometric object corresponding to the energy-momentum tensor of matter could not be made unambiguously.

In her thesis, Liane Bouche-Valere hoped to find acceptable equations of motion for a charged particle by "a method analogous to the one which provides them in the interior electromagnetic case in general relativity." ([56], p. 2-3.) By this, we must understand a method working with "conservation conditions". Her Lagrangian was (up to notation):

$$
\hat{\mathcal{L}}=\hat{g}^{i j}\left[W_{i j}\left(L_{r s}^{t}\right)+2 q \partial_{[i} \Gamma_{j]}+p \Gamma_{i} \Gamma_{j}\right]+\hat{A}^{k} L_{k}+\sigma\left(\hat{g}^{i j} \Gamma_{i} \Gamma_{j}-2 \kappa^{2} \sqrt{-g}\right),
$$

223 “ $[\ldots]$ nous pourrons faire porter la condition de normalisation sur un vecteur quelconque $X_{k}$ et non plus nécessairement sur le vecteur de torsion $\Gamma_{i}$; ceci afin d'éviter toute identification ou interprétation anticipée des champs."

224 Isothermal coordinates had been studied before by F. Maurer-Tison [395]. Mathematicians use the notion "isothermal coordinates" differently, e.g., for coordinates in which a Riemannian space is explicitly seen to be conformally flat. 
where $L_{k}$ is the torsion-vector of the arbitrary (asymmetric) connection $L_{i j}^{k}, W_{i j}$ the Ricci tensor formed from this connection, and $\hat{A}^{p}, \sigma$ are Lagrangian multipliers. $\Gamma_{i}$ is a normed vector field $[53,54,55,56]$. With regard to Tonnelat's Lagrangian in Section 10.3.3, the terms with constants $\alpha$ and $\beta$ are omitted. L. Bouche demonstrated the existence of 4 characteristic cones, three of which are the same as in (421) in Section 10.5.4 below, the 4th was not determined explicitly by her; cf. however the result of Nguyen Phong-Chau in Section 10.4. Her application of approximations (up to the second one) to the equations of motion has shown that the Lorentz-force could no longer be obtained as soon as the antisymmetric part of the field equation was satisfied. Also, her study of spherically symmetric solutions of the new field equations, due to their complexity could not be carried to a successful end.

The aim of the thesis by Marcel Bray was to study exact spherically and axially symmetric solutions of the weak field equations (417) - (419) and to compare them with exact solutions in general relativity. ${ }^{225}$ For a possible physical interpretation he had to make a choice among differing identifications between mathematical objects and physical observables. For the metric, interpreted as describing the inertial-gravitational potentials, he investigated two choices: the metric suggested by Maurer-Tison, cf. (412) of Section 9.7, and $g_{i j}=\sqrt{h / g} l_{i j}$. Unfortunately, his hope that his research "perhaps could also provide some helpful guiding principles for the choice of the metric" (p. 1) did not materialize. No solutions of physical interest beyond those already known were displayed by him [59].

A further systematic study of the possible field equations of Einstein-Schrödinger UFT was done by Nguyen Phong-Chau in his thesis [77, 456]. He started from a transposition invariant expression for the Ricci tensor with 7 parameters (cf. Section 2.3.2, Eqs. (381) and (382)) originally proposed by M.-A. Tonnelat:

$$
K_{i j}=a R_{i j}+b \tilde{R}_{j i}+a^{\prime} R_{j i}+b^{\prime} \tilde{R}_{i j}+c P_{i j}+d \tilde{P}_{i j}+e \Gamma_{i} \Gamma_{j},
$$

where $R_{i j}, P_{i j}$ are the two contractions of the curvature tensor of the asymmetric, torsionless affine connection $\Gamma_{i j}{ }^{k}$, whereas $\tilde{R}_{i j}, \tilde{P}_{i j}$ belong to the transposed connection $\tilde{\Gamma}_{i j}{ }^{k}=\Gamma_{j i}{ }^{k}$. $\Gamma_{i}$ is an arbitrary vector field. ${ }^{226}$ He concluded that only two cases had to be considered: version A of Einstein-Schrödinger with $K_{i j}=W_{i j}+2 p \partial_{[i} \Gamma_{j]}$; and version B with $K_{i j}=W_{i j}+r Q_{i j}$. Here, $W_{i j}, Q_{i j}$ are the contractions of the curvature tensor belonging to a connection $L_{i j}{ }^{k}$ with zero vector torsion while $p \neq 0, r$ are free constants. Thus, in version B only 76 (instead of 80) unknown components have to be determined and, for $r=\frac{1}{2}$, transposition invariance holds. Nguyen Phong-Chau succeeded in determining explicitly for arbitrary $r$ the fourth characteristic which L. Bouche had announced to exist for case B, but had not been able to specify in the case $r=0$ (for the other three cf. (421) below in Section 10.5.4) :

$$
\left[(1+\epsilon) h_{i j}-l_{i j}\right] d x^{i} d x^{j}=0, \epsilon=-1+\frac{4}{3} r(r-1), \epsilon \neq 0
$$

It thus seems possible that further linear combinations of the 3 quantities used for the definition of the "lightcone", i.e., $h_{i j}, l_{i j}, n_{i j}$ may occur.

In the framework of non-linear electromagnetism which should follow from UFT, he suggested an interpretation different from Maxwell's theory: the electromagnetic potential ought to be described by a tensor potential identified with the antisymmetric part of the metric $k_{i j}$, not just a

\footnotetext{
225 M. Bray used the notation of [632]. His Ricci tensor is $-K_{j k}$.

${ }^{226}$ Thus, $R_{i j}, P_{i j}$ correspond to $K_{i j}, V_{i j}$ of Section 2.3.1 and $\Gamma_{i j}{ }^{k}$ to $L_{i j}{ }^{k}$. At the beginning of [456], no clear distinction between the torsion vector $L_{[i s]}^{s}$ and $\Gamma_{j}$ was made.
} 
4-vector. In first approximation $\square k_{i j}=0$. A consequence would be that elementary particles must be described differently; beyond mass and charge, an electron would obtain further characteristics incompatible with spherical symmetry ([456], p. 354).

Further dissertations dealing with the generalization of Kaluza-Klein theory and with linear theories of gravitation in Minkowski space are discussed in Sections 11.1.1 and 16.1, 16.2, respectively.

\subsection{Research by and around André Lichnerowicz}

Within the Institut Henri Poincaré, a lively interaction between theoretical physicists, mathematicians and natural philosophers took place which tried to grab some of the mysteries from "the lap of the gods". One of the Paris mathematicians sharing Mme. Tonnelat's interest in metric affine geometry was André Lichnerowicz ${ }^{227}$. He looked mainly at problems of interest for a mathematician. In gravitation - both in general relativity and the "non-symmetric theory" - questions concerning the integration of the systems of partial differential equations representing the field equations were investigated, be it identities for curvature, the Cauchy problem arising from field equations in affine spaces [368, 370], existence and uniqueness of solutions and their global properties, or the compatibility of the field equations of both general relativity and UFT. In his own words: "[...] I could attack what interested me - the global problems of relativity, the keys to a real understanding of the theory." ([379], p. 104.) This has also been subsumed in Lichnerowicz's contribution to the Chapel Hill Conference of 1957 on the role of gravitation re-published in 2011 ([120], 65-75). For scalar-tensor theory with its 15 th scalar variable $\phi$, it is to be noted that Lichnerowicz not only discussed $\phi \sim \kappa^{-1},(\kappa$ the gravitational "constant") as a possibility like Ludwig and Just [385] but accepted this relation right away ([371], p. 202).

\subsubsection{Existence of regular solutions?}

In Section 7.1, it was pointed out that Einstein thought it imperative to banish singular solutions from his theory of the total field. Therefore it was important to get some feeling for whether general relativity theory would allow non-singular solutions or not. Einstein and Pauli set out to prove theorems in this regard [177]. Their result was that the vacuum field equation $R_{i k}=0$ did not admit any non-singular static solution describing a field with non-vanishing mass. For distances tending to infinity, the asymptotic values of the Schwarzschild solution were assumed. The proof held for any dimension of space and thus included the theory by Kaluza and Klein. However, prior to Einstein and Pauli, Lichnerowicz had proven a theorem almost identical to theirs; he had shown the non-existence of non-trivial regular stationary, asymptotically Euclidean vacuum solutions with $^{228} g_{00}=1-\epsilon_{00}, \epsilon_{00}>0$ [363, 362, 364]; cf. also Section 8.3. In a letter of 4 September 1945 ([489], p. 309), a double of which he had sent to Einstein, Lichnerowicz pointed this out to Pauli. ${ }^{229}$ In his response of 21 September 1945, Pauli found the condition on $g_{00}$ unphysical: why should $g_{00}>1$ be impossible near infinity? After he had studied the paper of Einstein and Pauli in more detail, Lichnerowicz commented on it in a further letter to Pauli of 11 November 1945. There, he also confessed to be "a bit shocked" about the fact that Einstein and Pauli had only

\footnotetext{
227 André Lichnerowicz (1915 - 1998). He received his doctorate with Georges Darmois on general relativity in 1939. First maître de conférences at the University of Strasbourg (transfered to Clermont-Ferrand during the German occupation), then in Paris; since 1949 professor at the faculty of science of the university of Paris. From 1952 until retirement in 1986 he held a chair for mathematical physics at the Collège de France in Paris. Member of the French Academy of Sciences since 1963. 1966-1973 president of Ministerial Commission on the Teaching of Mathematics. 228 Lichnerowicz used the index 4 for the time coordinate.

229 Einstein already had written a paper preceding the one with Pauli and published it in an Argentinian journal (University of Tucuman) both in English and Spanish [145, 144]. For the circumstances of the publishing cf. [220].
} 
proven "non-existence" of regular solutions while he had shown that Euclidean space is the only regular solution ([489], p. 325-326). Pauli, in his answer of 15 November 1945, apparently had suggested a related problem. On 15 December 1945, Lichnerowicz wrote back that he had solved this problem, outlined the structure of the proof, suggested a joint publication in Comptes Rendus, and congratulated Pauli for receiving the Nobel prize ([489], p. 333-335). A co-authored paper did not appear but Lichnerowicz published a short note: "W. Pauli signaled me his interest in the possibility to avoid any auxiliary hypothesis: he thought that this could be reached by a synthesis of our respective methods. In fact, this has happened: an important problem in relativity theory has been solved" [367]. A further proof of the occurrence of singularities for static gravitational fields in general relativity was given by A. Lichnerowicz and Y. Fourès-Bruhat [380]. That Pauli was impressed by Lichnerowicz's theorem is shown by his detailed discussion of it in his special lectures on relativity in 1953 as reported in ([194], p. 389-390).

As already mentioned in Section 8.3, A. Papapetrou, working at the time in Dublin with Schrödinger,

extended the theorem of Einstein and Pauli to a non-symmetric metric, i.e., to UFT with the strong field equations $R_{i k}=0, g_{+-} k \| l=0, \hat{g}^{[i s]}{ }_{, s}=0[474]$.

\subsubsection{Initial value problem and discontinuities}

The field equations for "Einstein's unitary theory" were presented by A. Lichnerowicz in the form [370]:

$$
\begin{array}{r}
g_{i k \| l}:=g_{i k, l}-g_{r k} L_{i l}{ }^{r}-g_{i r} L_{l k}{ }^{r}=0, \\
\hat{g}^{[i s]}{ }_{, s}=0, \\
P_{i k}=\frac{4}{3} \partial_{[i} S_{k]},
\end{array}
$$

where $S_{k}$ is a covariant vector to be identified with the torsion vector and $L_{i k}^{j}$ an "a priori arbitrary" affine connection corresponding to Schrödinger's star-connection [cf. (291)]: $L_{i k}{ }^{j} \rightarrow{ }^{*} \Gamma_{i k}{ }^{l}:=$ $\Gamma_{i k}^{l}+\frac{2}{3} \delta_{i}^{l} \Gamma_{k} . P_{i k}$ is the Ricci-tensor $-K_{j k}$ formed from $L_{i k}^{j}$ defined by (56). Note that from (399) follows $\partial_{k} P_{[i j]}+\partial_{j} P_{[k i]}+\partial_{i} P_{[j k]}=0$, but the reasoning backward holds only locally (local existence of a potential) ([369], p. 500), ([371], 267). In Section 10.3.1, the form given to the generalized Bianchi-identities by Lichnerowicz is shown.

The local initial value problem is the following: Let be given on a spacelike hypersurface $S\left(x^{0}=0\right)$, or $f\left(x^{k}\right)=0$, of the manifold (space-time) the components of the non-symmetric tensor $g_{i j}$ of class $\left(C^{1}, C^{3}\right.$ piecewise) and an affine connection of class $\left(C^{0}, C^{2}\right.$ piecewise $) .{ }^{230}$ With (397) having been solved, i.e., the connection expressed by the metric and its 1st derivatives, the task now is: Determine in a neighborhood of $S\left(x^{0}=0\right)$ the tensor $g_{i j}$ and the torsion vector $S_{k}$ such that they satisfy (398), (399). In his main results up to 1954, Lichnerowicz had shown that the local initial value problem for real and analytical data on $S$ and with $g^{00} \neq 0$ has a unique solution [368, 370, 371], Part II, Chap. VI), [369]. The proof included a normalization-condition for the torsion vector: $\partial_{s}\left(g^{s t} S_{t} \sqrt{|g|}\right)=0$ which was also used when in 1955, during the Jubilee-conference in Bern, A. Lichnerowicz reviewed global problems and theorems "of the relativistic equations". He now considered part of the field equations given above:

$$
R_{a b}-\frac{2}{3}\left(\partial_{a} S_{b}-\partial_{b} S_{a}\right)=0, \partial_{s}\left(g^{[s a]} \sqrt{|g|}\right)=0
$$

\footnotetext{
230 This means that all quantities on the manifold are twice continuously differentiable, and that in a change of coordinates the second derivatives of the coordinates are $C^{2}$, piecewise.
} 
with the Ricci tensor (previously denoted $\left.P_{i k}\right) R_{a b}=R_{a b}(L)$, where $L_{a b}{ }^{c}$ is the torsion-free connection. He could show that (400) "presents the same local mathematical coherence as the system of equations of general relativity" 231 ([372], p. 182). The first notice in Comptes Rendus [368] had been reviewed by V. Hlavatý, and then a paper by him containing the proofs announced appeared in the journal of Hlavatý's home university [369]. For the Einstein-Maxwell field equation the requirement that the initial data be analytic had been weakened by Lichnerowicz' student, Mme. Fourès-Bruhat, who also gave the first proof of existence and uniqueness of the local Cauchy problem for Einstein's field equations [217]. Lichnerowicz conjectured that such a proof also could be achieved for unitary field theory. Between January and June 1954, a correspondence between Lichnerowicz and Einstein on this topic has taken place; on 11 May 1954 he sent Einstein his paper on the compatibility of the field equations of UFT [368]. ${ }^{232}$ W. Pauli was impressed by Lichnerowicz' lecture at the conference in Bern:

"I believe, the most important else we have heard, was the report by Lichnerowicz on the Cauchy initial value problem in the nonlinear field equations of general relativity. I attach great importance to the study of such problems, because I suppose that it also will play an essential role with field quantization." ${ }^{233}$ [486].

Following Hadamard, Lichnerowicz had displayed the discontinuities of the Riemannian curvature tensor denoted by $\left[R_{j, r s}^{i}\right]$ on a characteristic $f=0$ by beginning with ${ }^{234}\left[\partial_{r} L_{j s}^{i}\right]=u_{j s}^{i} l_{r}, l_{i}=$ $\partial_{i} f$ which led to $\left[R_{j, r s}^{i}\right]=l_{r} u_{j s}^{i}-l_{s} u_{j r}^{i}$. As a consequence,

$$
l_{k}\left[R_{j, r s}^{i}\right]+l_{s}\left[R_{j, k r}^{i}\right]+l_{r}\left[R_{j, s k}^{i}\right]=0,
$$

and

$$
l_{q}\left[R_{j, r s}^{q}\right]=0, l^{r}\left[R_{p, r s}^{i}\right]=0 .
$$

Although A. Lichnerowicz had done the decisive steps in the formulation of the Cauchy initial value problem, a study of the case where it cannot be solved uniquely, i.e., $g^{00}=0$, was in order.

In several short notes in Comptes Rendus [291, 293, 292, 295], S. I. Husain applied the methods of A. Lichnerowicz for an investigation of the discontinuities of the curvature and Ricci tensors in mixed geometry, first with Einstein's "strong" and then the "weak" field equations ${ }^{235}$. Independently of the field equations, for the discontinuities the expressions of Lichnerowicz hold:

$$
\left[R_{j, r s}^{i}\right]=l_{r} A_{j s}^{i}-l_{s} A_{j r}^{i},\left[\partial_{j} S_{k}\right]=l_{j} b_{k}, l_{i}=\partial_{i} f,
$$

where $A_{j s}^{i}, b_{k}$ are his discontinuity-parameters and $R_{j, r s}^{i}=K_{-}^{i}{ }_{j k l}\left(L_{p q}{ }^{r}\right)$. Thus, (401) reappeared for the more general curvature tensor. For the "strong" equations, Husain derived

$$
l_{q}\left[R_{j, r s}^{q}\right]=0, l^{p}\left[R_{p, r s}^{i}\right]=0,
$$

using $l^{k}:=g^{k l} l_{l}$ [291]; the 2nd equation is non-trivial because there is no skew symmetry in the first two indices of the curvature tensor. For the "weak" system, he obtained instead [293]:

$$
l_{q}\left[R_{j, r s}^{q}\right] l^{i}=l^{p}\left[R_{p, r s}^{i}\right] l_{j} .
$$

231 "présente la même cohérence mathématique locale que le système des équations de la relativité générale."

232 Call numbers at the Einstein Collected Papers are 16-317.00 to 16-325.00.

233 "Ich glaube, das Wichtigste, das wir sonst gehört haben, war das Referat von Lichnerowicz über das Cauchysche Anfangswertproblem in den nichtlinearen Gleichungen der allgemeinen Relativitätstheorie. Ich lege sehr großen Wert auf das Studium dieser Probleme, weil ich bestimmt meine, dass es auch bei der Feldquantisierung [...] eine wesentliche Rolle spielen wird."

234 For this study within general relativity cf. [374].

235 I have not been able to find out with whom his thesis of 1960 was written [294]. 
In the next paper, he switched to the definition $l^{k}:=g^{(k l)} l_{l}=l^{k l} l_{l}$ and arrived at

$$
\begin{gathered}
l^{s} D_{s} l_{i}=0, l_{i}\left[P_{j k l}^{m}\right]+l_{l}\left[P_{j i k}^{m}\right]+l_{k}\left[P_{j l i}^{m}\right]=0, \\
2 l^{s} D_{s}\left[P_{j i l}^{m}\right]+l_{l}\left[D_{s} P_{j i}^{m}\right]+l_{i}\left[D_{s} P_{j l}^{m} s\right] \simeq 0,
\end{gathered}
$$

where $D_{s}$ is the covariant derivative with regard to the (Riemann) connection defined by $l_{i j}$, and $P_{j k l}^{m}(L)$ the curvature tensor belonging to the connection ${ }^{236} L_{p q}{ }^{r}$. The congruence-sign in (407) means "up to terms in $\left[R_{j, r s}^{i}\right]$ ". In a further paper, Husain obtained even $l^{s} D_{s}\left[P^{m}{ }_{j i l}\right] \simeq 0$ and concluded that the wave front of radiation propagates "with the fundamental speed" [295].

\subsubsection{Characteristic surfaces}

The propagation of waves with their characteristic surfaces was of great interest also in EinsteinSchrödinger theory. A naive mathematical approach would take $g_{i j} d x^{i} d x^{j}=0$ as the defining relation for the characteristic surface. However, from the point of view of physics, what is the "lightcone" of the gravitational field? This obviously depends on the identification of the gravitational potential (field) with a geometric object of unitary field theory. As we have seen in Section 9.7, different identifications were made. The hypersurface $S\left(x^{0}=0\right)$ represented by $f\left(x^{0}, x^{1}, x^{2}, x^{3}\right)=0$ and tangent to the cone $g^{r s} \partial_{r} f \partial_{s} f=0$ is a wave surface of the metric field $g^{r s}$. We already noted that Lichnerowicz preferred the inverse of $l^{i j}=g^{(i j)}$ as the "gravitational tensor" and thus defined the light cone through

$$
l_{r s} d x^{r} d x^{s}=0
$$

([371], p. 288; [370]; [372]). Lichnerowicz was interested in the initial value problem and in wave surfaces, he also looked at gravitational shock waves, characterized by discontinuities in the connection. Here, the task is to rewrite the field equations in terms of tensor distributions. As an application, Lichnerowicz took relativistic hydrodynamics and magnetohydrodynamics. ${ }^{237} \mathrm{He}$ advised the thesis of Pham Mau Quan on relativistic hydrodynamics [501], in which the various characteristic surfaces were investigated across which discontinuities of mass density, pressure, fluid velocity and heat transport vector, or of their gradients will occur. Lichnerowicz then succeeded to again solve the initial value problem for magnetohydrodynamics [377]. In Jordan-Thiry theory interpreted as a UFT, Mme. F. Hennequin and R. Guy studied fluid dynamics in more detail; cf. Section 11.1.1.

In view of the many possibilities of identifying a geometric object with the gravitational field, it can be understood why V. Hlavatý [260] and E. Clauser [80] did not follow the approach by Lichnerowicz. They used the inverse of $h_{i j}=g_{(i j)} \neq l_{i j}$ for the definition of the wave fronts:

$$
h^{i j} \partial_{r} f \partial_{s} f=0 \text {. }
$$

As seen in Section 2.1, $h^{i j} \neq l^{i j}$. Clauser dealt with a special case of Hlavatý's classification with connection:

$$
\Gamma_{i j}^{k}=\left\{\begin{array}{l}
k \\
i j
\end{array}\right\}_{h}+\frac{1}{2} h^{k s}\left(\stackrel{h}{\nabla}_{i} g_{s j}+\stackrel{h}{\nabla}_{j} g_{i s}+\stackrel{h}{\nabla}_{s} g_{i j}\right)+2 p_{s}\left(g_{i j} h^{k s}+2 k_{.[i}^{s} \delta_{j]}^{k}+k_{\cdot[j}^{s} \delta_{i]}^{k}-\delta_{.(i}^{s} \delta_{j)}^{k}\right),
$$

where $p_{k}:=\frac{1}{6}\left(\log \frac{g}{h}\right), K_{i}:=-\frac{1}{2}\left(\frac{k}{h}\right)_{, i}, K_{i}-p_{s} L_{i}^{s} \stackrel{!}{=} 0, L_{k}^{i}:=\delta_{k}^{i} \frac{g+2 k-h}{h}-k_{.}^{i s} k_{s k} \cdot g, h, k$ are the determinants of the corresponding tensors. Indices are moved with $h_{i j}$; the original spot of an index is noted by a dot. Clauser then was able to show that for the system:

$$
R_{(i j)}=0, R_{[i j]}=0, \nabla_{k}\left(K_{i}-p_{s} L_{i}^{s}\right)=0
$$

236 As Husain is not clear on this point but uses the notations of Lichnerowicz, this is my assumption.

237 However, A. Lichnerowicz had worked on general relativistic hydrodynamics already since the early 1940s [365], [366]. Again here, for general relativistic fluids, Y. Choquet-Bruhat has proven existence theorems [78]. 
the initial value problem is well defined and the characteristic surfaces are given by (409).

An important achievement was reached by Françoise Maurer-Tison who continued the investigation of Lichnerowicz. She pointed out that the characteristic cone of the metric ("light cone"), locally, can be decomposed into two cones described by the metrics $l_{i j}$ and $n_{i j}$ with

$$
n^{i j}:=2 \frac{h}{g} h^{i j}-l^{i j}, n_{i s} n^{k s}=\delta_{i}^{k},
$$

from which

$$
n_{i j}=\frac{g}{2 h+2 k-g}\left(2 h_{i j}-l_{i j}\right)
$$

follows. This left the use of the quantity $n_{i j}$ as a further possibility for the metric; cf. [408], ([398], p. 243-244), ([641], p. 339-344). In principle as many "light cones" as different interpretations made for the gravitational field can be found. However, in the literature studied, we will meet four different light cones being discussed; cf. also (421) and (396).

When two further approaches to the discontinuities of curvature tensors within the framework of UFT were published in 1961 in Comptes Rendus, the respective authors did not take notice of each other. In the first half of the year, L. Mas and A. Montserrat presented their three papers on "wave fronts" in unified field theory, while in the second half J. Vaillant published on "discontinuities" of the curvature tensor in Einstein-Schrödinger theory. Both continued the work of Lichnerowicz and Maurer-Tison.

In their first paper [390], Mas and Montserrat used the "compatibility equation" (33) in the form:

$$
{ }_{L} \bar{\nabla}_{k} g^{i j}=2 S_{r k}^{j} g^{i r},
$$

using the asymmetric connection $L_{i j}^{k}$ and introduced two further connections $M_{i j}^{k}=L_{(i j)}^{k}$ and the Riemannian connection obtained from $l_{i j}$ named $\Gamma_{i j}^{k}$. They referred to Husain's doctoral thesis [294] and like him called $P_{j i k}^{m}$ the curvature tensor belonging to $L_{i j}^{k}$. (405) and the second equation of (406) were reproduced, and the equation for the discontinuities of the Ricci tensor $\left[P_{i j}\right]=0$ added. For the curvature tensor $R^{m}{ }_{j i k}$ belonging to $\Gamma_{i j}^{k}$, the results of Lichnerowicz (401) and the first equation of (402) as well as $\left[R_{i j}\right]=0$ were shown to hold also in the case of Einstein's weak field equations. In the second paper, the light cone was defined by $h_{i j} l^{i} l^{j}=0$ with $h_{i j}=g_{(i j)}$ and the corresponding discontinuities for curvature derived [444]. The third paper then brought an investigation including three different characteristic surfaces [445]. Let $\stackrel{1}{g}_{i j}:=l_{i j}, \stackrel{2}{g}_{i j}:=h_{i j}, \stackrel{3}{g_{i j}}:=$ $n_{i j}$ where $n_{i j}$ is defined by (412), (413). Then, on the characteristics $\Sigma_{(s)}, s=1,2,3$ defined by ${ }_{g}^{s} i l_{i} l_{j}=0$ or $g^{00}=0, h^{00}=0, n^{00}=0$, respectively, for the curvature tensors $\stackrel{s}{R}^{m}{ }_{j i k}$ belonging to the Riemannian connections calculated from $\stackrel{s}{g}_{i j}$, the following discontinuities obtained:

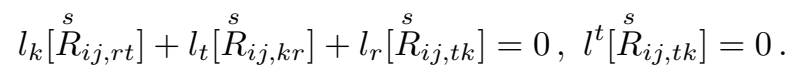

In his three notes in Comptes Rendus, Jean Vaillant took up as well the investigation of the discontinuities of the curvature tensor for the weak field equations [663, 664]. ${ }^{238}$ In 1964 he finished his $\mathrm{PhD}$ thesis on this and related subjects. ${ }^{239}$ For the discontinuities of the curvature tensor on a characteristic, J. Vaillant noted more precisely $\left[P_{i j k l}\right]=\frac{1}{2} K l_{i} l_{j} \eta^{p q r s} l_{p} k_{r s}\left(l_{k} h_{l q}-l_{l} h_{k q}\right)$. This expression satisfies $l_{i}\left[P_{j, r s}^{i}\right]=0$ and is consistent with (401) and (405). Vaillant also looked at the characteristics defined from (412) and by $h^{r s} l_{r} l_{s}=0$ of F. Maurer-Tison. For both, the discontinuities

$$
l_{i}\left[P_{m j k l}\right]+l_{l}\left[P_{m j i k}\right]+l_{k}\left[P_{m j l i}\right]=0, l^{r}\left[P_{i p, r s}\right]=0, l^{i}\left[P_{i p, r s}\right]=0
$$

238 In [663], p. 231, the field equations are marred by a misprint.

239 At the Collège de France with advisors Jean Leray and A. Lichnerowicz. 
were shown to hold. If $\gamma^{k}:=n^{k r} l_{r}$, then also

$$
\gamma^{p} \nabla_{p}\left[P_{j, r s}^{i}\right] \simeq 0, \gamma^{p} \nabla_{p}\left[\partial_{j} S_{k}\right] \simeq 0
$$

where the right hand sides contain linear combinations of $\left[P^{i}{ }_{j, r s}\right]$ and $\left[\nabla_{i} S_{j}\right]$, respectively. In his third paper [665], Vaillant concluded that the only surfaces on which discontinuities of the Ricci tensor can arise, are the characteristics $h^{r s} l_{r} l_{s}=0$ The results of S. I. Husain (cf. Section 10.5.2) were not mentioned by him.

\subsubsection{Some further work in UFT advised by A. Lichnerowicz}

The doctoral theses inspired by A. Lichnerowicz are about equally directed to Einstein-Schrödinger and Jordan-Thiry (Kaluza) theory. As interesting as the study of the Cauchy problem initiated by Lichnerowicz was, it also could not remove the ambiguities in the choice for the metric.

In her thesis "Aspects mathématiques de la théorie du champ unifié d'Einstein-Schrödinger", Françoise Maurer-Tison first wrote an introductory part on the geometrical background of unified field theory; she developed the concept of "coaffine connection", i.e., an infinitesimal connection on the fiber bundle of affine reference frames. In Part 2, Maurer-Tison investigated in detail the Cauchy initial value problem. The last Part 3 of her thesis is devoted to the "physical interpretation".

The "weak" field equations are written in the form: ${ }^{240}$

$$
\begin{aligned}
\partial_{\rho} g_{\lambda \mu}-L_{\lambda \rho}^{\sigma} g_{\sigma \mu}-L_{\rho \mu}^{\sigma} g_{\lambda \sigma} & =0, \\
\partial_{\rho}\left(g^{[\rho \beta]} \sqrt{-g}\right) & =0, \\
P_{\alpha \beta}-\frac{2}{3}\left(\partial_{\alpha} \Sigma_{\beta}-\partial_{\beta} \Sigma_{\alpha}\right) & =0,
\end{aligned}
$$

where $\Gamma_{\rho \mu}^{\sigma}$ is a linear connection with torsion-vector $\Sigma_{\beta}$ and $L_{\rho \mu}^{\sigma}$ a linear connection with vanishing torsion vector $\Sigma_{\beta}\left(=S_{\beta}\right)=0 . P_{\alpha \beta}(L)$ is the Ricci tensor [398].

For the Cauchy initial value problem, (418), (419) are rewritten into time-evolution equations

$$
\partial_{k}\left(g^{[k 0]} \sqrt{-g}\right)=0, P_{i 0}-\frac{2}{3}\left(\partial_{i} \Sigma_{0}-\partial_{0} \Sigma_{i}\right)=0(i, k=1,2,3),
$$

and constraint equations on the initial surface $x^{0}=0$ containing $\partial_{\rho}\left(g^{[\rho k]} \sqrt{-g}\right)=0$, and an equation for the Ricci tensor not written down here enclosing the metric, its first derivatives and the vector field $\Sigma_{i}$ ([398], p. 229). The existence of a solution is proven, and, in the case of analytic initial data, also its uniqueness. There exist three characteristic cones met before and defined by:

$$
\gamma_{i j} d x^{i} d x^{j}=0, l_{i j} d x^{i} d x^{j}=0, \quad h_{i j} d x^{i} d x^{j}=0,
$$

where $n^{i j}=\gamma^{i j}=\frac{2 h}{g} h^{i j}-l^{i j}$ [cf. (412) and (413)]. The first one (with $\gamma$ ) is declared to be the light cone, while the interpretation of the other two (time oriented) as wave fronts remains unclear (p. 241).

In Part 3, after a detailed calculation departing from a proper reformulation of the field equations and the "conservation equations", Maurer-Tison arrived at what she named energymomentum tensor of the electromagnetic field:

$$
\tau_{\lambda \mu}=P_{[\lambda \sigma]} m^{\rho \sigma} h_{\rho \mu}+P_{[\mu \sigma]} m^{\rho \sigma} h_{\lambda \rho}-\frac{1}{2} h_{\lambda \mu} P_{[\alpha \beta]} m^{\alpha \beta}
$$

${ }^{240}$ We have kept her notation, i.e., Greek indices run from 0 to 3, Latin indices from 1 to 3. 
Electromagnetic field $H_{\alpha \beta}$ and induction $K_{\alpha \beta}^{\prime}$ are identified as follows: $H_{\alpha \beta}=\frac{1}{2} \sqrt{-g} \epsilon_{\alpha \beta \gamma \delta} m^{\gamma \delta} ; K^{\prime \alpha \beta}$ $=-\frac{1}{2 \sqrt{-g}} \epsilon^{\alpha \beta \gamma \delta} \Delta k_{\gamma \delta}$. Here, $\underset{+-}{\Delta}$ is a generalized Laplacian (cf. her Eq. (40.4) on p. 255). Mme. Maurer-Tison did not only comment in technical terms on her impressive work; she also described the whole field very much to the point with accurate words:

"The unified field theory of Einstein-Schrödinger is attractive by its apparent simplicity and repellent by the finicky calculations it requires: it is a young theory with moderate baggage as long as it is investigated with rigour, but an immense load when the efforts are taken into account which have been tried to explore its possibilities" ([398], p. 187). ${ }^{241}$

The following doctoral thesis by Marcel Lenoir constitutes a link with the next Section 11.1. In it, he gave as his aim the introduction of a geometrical structure which permits the incorporation of Bonnor's supplementary term into the Lagrangian of UFT (cf. Section 13.1) resulting from the contraction of a suitable Ricci tensor ([359], p. 7). This is achieved by the introduction of space-time as a hypersurface of a 5 -dimensional space $V_{5}$ with metric tensor and asymmetric linear connection. The wanted supplementary terms follow from the curvature of $V_{5}$. Lenoir's approach to Bonnor's field equations is an alternative to (and perhaps more convincing) than the earlier derivation, in space-time, by F. de Simoni (cf. Section 15.1).

For a background, in the first two chapters of the thesis, the geometry of fiber bundles and of hypersurfaces was summed up. Lenoir stated the "weak" field equations in the form:

$$
\begin{array}{r}
\hat{g}^{\underline{i j}}{ }_{\| k}^{+}\left(=\partial_{k} \hat{g}^{i j}-\hat{g}^{i j} \Gamma_{k s}{ }^{s}+\hat{g}^{i s} \Gamma_{k s}{ }^{j}+\hat{g}^{s j} \Gamma_{s k}{ }^{i}\right)=\hat{g}^{i j} \Gamma_{k}-\frac{2}{3} \delta_{k}^{i} \hat{g}^{j s} \Gamma_{s}, \\
\partial_{s} \hat{g}^{[i s]}=0, \\
K_{i j}=0 .
\end{array}
$$

Following Kichenassamy, he distinguished UFT's of "type Einstein-Schrödinger" with $\partial_{s} \hat{g}^{[i s]}=0$ and of type "Einstein-Tonnelat" with $\partial_{s} \hat{g}^{[i s]}=\hat{F}^{i} \neq 0$ (cf. Section 10.3.3, Eq. (384)). From an effective Lagrangian conjured up from the curvature scalar in $V_{5}$, i.e.,

$$
\mathcal{L}=H_{i j}\left(\Gamma_{r s}^{t}, \partial_{u} \Gamma_{r s}^{t}\right)+\mathcal{B}\left(g_{r s}, \rho, A_{s}\right),
$$

Lenoir was able to derive extended field equations in space-time from which, by specialization, all three types of field equations emerged: Einstein-Schrödinger's, Einstein-Tonnelat's (cf. Section 10.3.3) and Bonnor's (pp. 61-71 of [359]). Lenoir also suggested alternatives for energymomentum tensors in order to obtain the equations of motion through the "conservation equations". He showed that identities will result as soon as all field equations are satisfied. In addition, a static, spherically symmetric solution of Bonnor's field equation was given and an extension of Birhoff's theorem obtained.

In the last chapter, Lenoir investigated whether Lichnerowicz's theorem on the non-existence of regular solutions could also be proven for his extended unitary theory but did not arrive at a conclusive result.

The doctoral thesis of another student of Lichnerowicz, Albert Crumeyrolle, contained two different topics [93]. In the larger part, research on the equations of motion of charged particles and on the energy-momentum tensor (corresponding to the "matter" tensor in UFT) was resumed in the framework of Einstein-Schrödinger theory. As to the equations of motion for charged

241 "La théorie du champ unifié d'Einstein-Schrödinger attire par son apparante simplicité et rebute par les calculations pénibles qu'elle nécessite; c'est une théorie jeune, dont le baggage est mince s'il est examiné avec rigueur, immense si l'on considère les efforts tentés pour explorer ses possibilités." 
particles, Pham Tan Hoang had simplified calculations by a more complete use of the isothermal condition (392) and by further improvements as mentioned in Section 10.4. Yet the negative result remained the same as the one already obtained by E. Clauser and H.-J. Treder, cf. Section 10.3.2. The same applies to Crumeyrolle's approximative calculation of the equations of motions in ([94], p. 390).

Because the energy-momentum tensor he constructed had to contain a metric field, Crumeyrolle investigated which of the three possibilities for the metric, i.e., $h_{i j}, l_{i j}$, and $\gamma_{i j}$ emerging from the Cauchy-problem (cf. (421) of Section 10.5.4) would be best for reaching the special relativistic energy-momentum tensor of the electromagnetic field. In fact, none was good enough. In first approximation, $h_{i j}$ fared best [94].

In the 2nd part of his thesis, an 8-dimensional auxiliary space was introduced in order to obtain more possibilities for field variables and (modified) field equations. ${ }^{242}$ A. Crumeyrolle provided this space $V_{8}$ with coordinates $x^{i}, x^{* i},(i=1,2,3,4)$ ("natural reference systems" in comparison with his adapted coordinates $z^{i}=x^{i}+\epsilon x^{* i}$; cf. Section 2.6); in $V_{8}$, he embedded space-time by $x^{* i}=0$. By use of the covariant derivative $\stackrel{+}{\nabla}$ in $V_{8}$, an affine connection with parts $\pi_{i}^{j}, \pi_{i *}^{j}, \pi_{i}^{j *}, \pi_{i *}^{j *}$ followed. In the special coordinate system, named "natural diagonal reference system", projection of the affine connection in $V_{8}$ into a 4-dimensional space led to both a connection $L_{i j}^{k}=L_{i * j}^{k *}=$ $L_{j * i *}^{k}=L_{j * i *}^{k *}=L_{i j *}^{k *}$, and a tensor $\Lambda_{i j}^{k}=L_{i j}^{k *}=L_{i * j}^{k}=L_{j i *}^{k}$ in space-time. Due to the increase in the number of field variables, he could derive two Ricci-tenors in this 4-dimensional space, identified with space-time:

$$
\begin{gathered}
\hat{R}_{j k}=-\partial_{k} L_{j l}^{l}+\partial_{l} L_{j k}^{l}-L_{m k}^{l} L_{j l}^{m}+L_{m l}{ }^{l} L_{j k}^{m}+L_{m * l}{ }^{l} L_{j k}{ }^{m *}-L_{m * k}{ }^{l} L_{j l}{ }^{m *}, \\
\hat{R}_{j * k}=-\partial_{k} L_{j * l}^{l}+\partial_{l} L_{j * k}^{l}-L_{m k}{ }^{l} L_{j * l}^{m}+L_{m l}{ }^{l} L_{j * k}^{m}+L_{m * l}{ }^{l} L_{j * k}^{m *}-L_{m * k}{ }^{l} L_{j l}{ }^{m *},
\end{gathered}
$$

and two tensorial objects:

$$
\begin{gathered}
\mathcal{P}_{j k}=-\partial_{k} L_{j l}{ }^{l}+\partial_{l} L_{j k}{ }^{l}-L_{m k}{ }^{l} L_{j l}{ }^{m}+L_{m l}{ }^{l} L_{j k}{ }^{m}+\Lambda_{l m}{ }^{l} \Lambda_{j k}{ }^{m}-\Lambda_{k m}{ }^{l} \Lambda_{j l}{ }^{m} \\
\overline{\mathcal{P}}_{j k}=-\partial_{k} \Lambda_{l j}{ }^{l}+\partial_{l} \Lambda_{k j}{ }^{l}-L_{m k}{ }^{l} \Lambda_{j l}{ }^{m}+L_{m l}{ }^{l} \Lambda_{k j}{ }^{m}+\Lambda_{l m}{ }^{l} L_{k j}{ }^{m}-\Lambda_{k m}{ }^{l} L_{l j}{ }^{m} .
\end{gathered}
$$

With these tensors, modified field equations which contained the "weak" Einstein equations including additional terms in $\mathcal{P}_{j k}$ then could be introduced ([96], p. 103-128). But a number of extra field equations had to be joined such as, still among others, $\Lambda_{[s j]}^{s}=0, d \overline{\mathcal{P}}_{[j k]}=0, \overline{\mathcal{P}}_{(j k)}=0$. cf. ([96], p. 126). Another approach by Crumeyrolle using a field of numbers different from the real numbers will be discussed in Section 11.2.2. In its Section XV ([97], pp. 126-130), it contains a new attempt at a unified field theory with a slightly changed formalism. As Crumeyrolle's aim was to regain the old Einstein-Schrödinger theory from a theory with additional field variables and field equations, his approach could not bring progress for an eventual physical interpretation of UFT.

${ }^{242}$ More general, the theory was carried through for spaces $V_{R}^{n}$ and $V_{R}^{2 n}$. 


\section{Higher-Dimensional Theories Generalizing Kaluza's}

\subsection{5-dimensional theories: Jordan-Thiry theory}

In January 1950, Yves Thiry ${ }^{243}$ submitted a thesis to the faculty of science of Paris University with the title "Mathematical study of the equations of a unitary theory with fifteen field variables". He exuberantly thanked his "master and friend Lichnerowicz" who obviously had initiated the work. Unfortunately, "Jordan and his school" had "obtained almost at the same time like us the equations which we will give in Chapter II. We had no knowledge about this except at a very late stage, and it is only recently that we could correspond with Jordan. He was so friendly as to send us his publications which we could not have procured otherwise." ${ }^{244}$ ([606], p. 6) In fact, it was A. Lichnerowicz, then at the university of Strasbourg, who had written to W. Pauli and asked for "Jordan's original paper" (cf. letter of W. Pauli to P. Jordan of 23. 3. 1948 in [489], p. 516). According to Pauli:

"Lichnerowicz is a pure mathematician who is occupied with the integration of Einstein's field equations. One of his students, Ives Thiry now has looked into the (not mutilated) Kaluza-theory (with $g_{55}$ ) and, so I believe, has simplified very much the calculational technique." 245

Since 1947/48, first together with A. Lichnérowicz, Thiry had published short notes about Kaluza's theory in Comptes Rendus [381, 604, 605]. On 19 January 1948, ${ }^{246}$ he sent one of his notes in Comptes Rendus to Albert Einstein [604]. In his publication preceding Lichnerowicz' letter to Pauli, Thiry had not yet given a physical interpretation of the scalar field [604]. Around that time, the interest in five dimensional relativity seems to have risen; we already have met P. G. Bergmann's paper of 1948 [21]. ${ }^{247}$ C. V. Jonsson, a student of O. Klein in Stockholm, also wrote a long paper about the theory's field equations and their linear approximation. He included the scalar field and dropped the cylinder condition. ${ }^{248}$ He then quantized the free field of this linear regime. [313]. Thiry's interest in Kaluza's theory was of mathematical nature:

"As to unitary field theories, it seems that their mathematical study has been quite neglected $[\ldots]$. We thought it useful to try a systematic mathematical study of a unitary field theory, and to find out whether such a theory is able to present the same coherence like general relativity." ([606], p. 3) ${ }^{249}$

Thiry's thesis laid out in three chapters the conceptual background of a 5-dimensional theory, the setting up and study of the field equations by help of Cartan's differential calculus, and results on

243 Yves (René) Thiry (1915-); studied physics in Strasbourg with A. Lichnerowicz, and since the early 1960s became professor for physics at the astrophysical institute of the University of Paris, then professor for celestial mechanics at the University Paris VI, and corresponding member of the Academy.

244 “[... ] obtenu à peu près en même temps que nous les équations que nous donnons au Chapitre II. Nous n'avons eu connaissance de ce fait que fort tard et ce n'est que récemment que nous avons pu correspondre avec Jordan, qui a eu l'amabilité de nous envoyer ses publications qu'il était alors impossible de se procurer autrement."

245 "Lichnerowicz ist ein reiner Mathematiker, der sich mit der Integration der Einsteinschen Feldgleichungen befasst. Einer seiner Schüler, Ives Thiry hat sich nun mit der (unverstümmelten) Kaluza-Theorie (mit $g_{55}$ ) beschäftigt und, glaube ich, die Rechentechnik sehr vereinfacht."

246 The call number of the Einstein Collected Papers (ECP) is 16-312.00.

247 Bergmann's paper appeared only on January 1, 1948 although it had been submitted on August 30, 1946. Thus he could not yet have reacted to Thiry's correspondence with Einstein.

248 I have not been able to verify that Jonsson's field equations for the case of the cylinder condition agree with Thiry's equations.

249 "Quant aux théories unitaires, il semble que leur étude mathématique ait été relativement negligée [... ] Il nous a paru utile de tenter une étude mathématique systématique d'une théorie unitaire et de voir si une telle théorie est susceptible de présenter la même cohérence que la théorie de la Relativité générale." 
regular solutions of the theory's field equations. Unlike in the approach by Einstein \& Bergmann (cf. Section 3.2), throughout, the cylinder condition $g_{\alpha \beta, 4}=0$ is upheld. Here, he used an argument from physics: no physical phenomena furnishes evidence for the existence of a fifth dimension ([606], p. 39). His access to 5-dimensional space made use of the fact that the equations of motion of charged particles are geodesics in Finsler geometry; for each value of $\frac{e}{m}$ (charge over mass) another Finsler space is needed with the metric:

$$
d s=\sqrt{g_{j k} d x^{j} d x^{k}}+\frac{e}{m} A_{l} d x^{l} .
$$

He then showed that a 5-dimensional Riemannian space could house all these geodesics. ${ }^{250}$

"The introduction of a fifth coordinate [...] thus shall justify itself by the fact that it imparts the role of geodesics to the trajectories of charged particles which they lost in space-time $[\ldots]^{\prime 251}$

In the third chapter, Thiry aimed at showing that his unitary field theory possessed the same mathematical coherence with regard to its global aspects as general relativity. By partially using methods developed by Lichnerowicz, he proved theorems on the global regularity of solutions such as: "A unitary field with normal asymptotic behavior (i.e., tending uniformly to Minkowski space at spatial infinity) cannot be regular everywhere." Thiry compared the proofs on the existence and regularity of solutions in O. Klein's version of Kaluza's theory and in his generalization and found them much simpler in his theory. These results are of a different nature than what Jordan had achieved; they are new and mathematically exact.

Yet Jordan was not in a hurry to read Thiry's thesis; he wrote to Pauli:

"By the way, in his thèse published in 1951, Thiry has studied systematically and extensively the theory with variable gravitational constant; [... I received it only after my book appeared and, at present, I have not read it very closely. It thus is not really clear to me whether it contains interesting novelties." ([490], p. 799/800) ${ }^{252}$

To Pauli, Thiry's global theorems might not have been "interesting novelties", because in his corresponding paper with Einstein on the non-regularity of solutions, the proof had been independent of the dimension of space [177]. Pauli, at first, also did not read Thiry's thèse, but responded arrogantly:

"The Thèses by Thiry are laying on my desk; however they are so appallingly thick (do not contain a reasonable abstract) such that it is so much simpler to not open the book and reflect about what must be inside." (W. Pauli to P. Jordan 8. 6. 1953, [491], p. 176$)^{253}$

Somewhat later, Pauli corrected himself and wrote to Jordan that in the preparation for a course "he nevertheless had read around in Thiry's Thèse" W. Pauli to P. Jordan 3 February 1954

\footnotetext{
250 In nuce, this idea can already be found in his paper with Lichnerowicz ([381], p. 531).

251 "L'introduction d'une cinquième coordonnée, [...] se justifiera donc par le fait qu'elle confière aux trajectories des particules électrisées le rôle de géodesiques qu'elles perdaient dans l'espace-temps[...]."

252 "Thiry hat ja übrigens in seiner 1951 veröffentlichten Thèse die Theorie mit variabler Gravitationskonstante systematisch und ausführlich studiert; [...] Ich habe es erst nach dem Erscheinen meines Buches von ihm bekommen und augenblicklich noch nicht sehr genau gelesen. Ich weiß also auch noch nicht recht, ob interessante Neuigkeiten darin stehen."

253 "Die Thèses von Thiry liegen auf meinem Tisch; sie sind aber so entsetzlich dick (haben auch keine vernünftige Zusammenfassung), daß es so viel einfacher ist, das Buch nicht aufzumachen und sich zu überlegen, was darinstehen muss." - Thiry's thesis comprises 122 pages.
} 
([491], p. 442). Note that neither of these two eminent theoretical physicists discussed Thiry's paper as regards its valuable content.

As to the field equations corresponding to (112) to (114), Thiry had calculated them in great detail with Cartan's repère mobile for both a Euclidean or Lorentz metric of $V_{5}$, and also with a 5-dimensional matter tensor of the form of dust $\rho u^{\alpha} u^{\beta}$. From the 15 th equation, he even had obtained "a new physical effect": uncharged dust-matter could generate an electromagnetic field [[606], p. 79, footnote (1)]. He linked this effect to Blackett's search for the magnetic field of a rotating body, described in Section 6.1.2. As to the interpretation of the fifteenth variable, the scalar field denoted by $V$ : for him $\chi_{T h}:=V^{2} G_{0}$ with $G_{0}=8 \pi G_{\text {Newton }} / c^{2}$ was the gravitational coupling factor ("facteur de gravitation") and put in front of the matter tensor [606], p. 72, 75, 77). In 1951, Y. Fourès-Bruhat proved existence and uniqueness theorems of "the unitary theory of Jordan-Thiry" [216, 218].

\subsubsection{Scientists working at the IHP on the Jordan-Thiry unified field theory}

Further investigation of the "5-dimensional unitary theory of Jordan-Thiry" as it was named by Lichnerowicz' and, later, by Thiry's students, resulted in several doctoral theses. In one such by Françoise Hennequin-Guyon, after approximation methods parametrized by $\frac{1}{c^{2}}$ had been developed by her for the equations of motion in general relativity, they then were applied to Jordan-Thiry theory. A metric conformally related to the metric induced from the 5-dimensional space $V_{5}$ was introduced such that the gravitational coupling function of the theory became a constant. The first approximation of a solution was then calculated [254]. Although stating that her interpretation via the conformal metric did agree with Jordan's view (p. 77 of her thesis), surprisingly she did not mention his book in her bibliography. Like Y. Thiry, in the general formalism F. Hennequin also considered the "interior" case with a matter tensor by postulating field equation in $V_{5}$

$$
S_{\alpha \beta}=\Theta_{\alpha \beta}, \alpha, \beta=0,1, \ldots, 4
$$

for "dust" (perfect fluid with zero pressure in 4 spatial dimensions): $\Theta_{\alpha \beta}=r \nu_{\alpha} \nu_{\beta}, \nu_{\alpha} \nu^{\alpha}=-1 . r>$ 0 later was called "pseudo-density", $\nu^{\alpha}$ is the 5 -velocity. After the projection of the formalism into space-time, the electromagnetic and the scalar field, named $\xi$, appeared. The conformal metric mentioned above is $\xi d s^{2}$ with $d s^{2}$ being the metric induced in space-time from $V_{5}$. Electromagnetic field $F_{i j}$ and induction $H_{i j}$ are assumed to be related by $H_{i j}=\xi^{3} F_{i j}$. Hennequin differed from Thiry by giving as ratio of charge and mass the expression $\frac{\mu}{\rho}=-c^{2} \frac{\beta h \sqrt{\xi}}{\sqrt{1+\frac{h^{2}}{\xi^{2}}}}, \kappa \rho=\frac{r}{\xi}\left(1+\frac{h^{2}}{\xi^{2}}\right)$ with $h=u_{0}$ being the timelike component of the 5 -velocity and $\beta$ a constant ([254], p. 53), while Thiry had had $\left(\frac{\mu}{\rho}\right)_{T h i}=\frac{\beta h}{\sqrt{1+\frac{h^{2}}{\xi^{2}}}}([606]$, p. 78). Her interpretation of the scalar field was also different from the one given by Lichnerowicz (cf. the end of Section 10.5).

In two subsequent theses of 1962 written by Robert Vallée and Pierre Pigeaud the defense of which was separated by only 4 months, Hennequin's research within 5-dimensional theory was continued and generalized to charged perfect fluids. The study of such fluids had been begun by Roland Guy ${ }^{254}$ who extended Lichnerowicz's results in relativistic hydrodynamics to "the formalism of the 5-dimensional unitary theories". His matter tensor included "pressure" $p$ : $\Theta_{\alpha \beta}=$ $r \nu_{\alpha} \nu_{\beta}-p \gamma_{\alpha \beta}, \gamma_{\alpha \beta}$ is the 5-dimensional Lorentz metric. His study of the congruences of the fluid's streamlines comprised those with rotation: a "tenseur de tourbillon" $\Omega_{\alpha \beta}=\partial_{\alpha}\left(F \nu_{\beta}\right)-\partial_{\beta}\left(F \nu_{\alpha}\right)$ was introduced by R. Guy which after projection to $V_{4}$ took the form:

$$
\Omega_{i j}=\frac{\mu}{\sqrt{\xi}}\left[\partial_{i}\left(F u_{j}\right)-\partial_{j}\left(F u_{i}\right)\right]+k \mu\left[\partial_{i}\left(F \phi_{j}\right)-\partial_{j}\left(F \phi_{i}\right)\right] i=0,1, \ldots, 3 .
$$

254 Roland Guy(1919-2006). A Swiss mathematical physicist who had written a doctoral thesis in Paris and later taught at the University of Montreal. His specialty besides differential geometry was the field of integral equations. 
Here, $\mu$ stands for electric charge, $\phi_{i}$ for the electromagnetic 4-potential, and $F=-\exp \int_{p_{0}}^{p} \frac{d p}{\rho+p}$ where $\rho$ is the mass density.

P. Pigeaud used two metrics, the "natural" one $\gamma$ following from the projection of $R_{5}$ to $R_{4}$, and another one conformal to it $\xi \gamma$. The first is employed for the calculation of the potentials (up to 2nd approximation), the 2nd for the study of the equations of motion. The reason is that for the "natural" metric uncharged test particles do not follow geodesics, yet for the conformal metric they do. At some point, Pigeaud had to make an ad hoc change of the energy-momentum tensor of matter (perfect fluid) not justified by empirical physics [495]. In a later development, Pigeaud did interpret the scalar fifteenth field variable as the field of a massive meson [387, 496]. The investigations within Jordan-Thiry theory were carried on by Aline Surin-Parlange to the case of perfect fluid matter with an equation of state $\rho=f(p)$ where $\rho, p$ are mass density and pressure, $\mathrm{f}$ is an arbitrary function. The Cauchy problem for this case was solved, the existence of hydrodynamical waves shown, and their propagation velocity determined. A. Surin compared both, the "singularity" method and the method using the vanishing of the divergence of the matter tensor in 1st order approximation: they gave the same results. Unlike F. Hennequin who had assumed for the metric components ${ }^{255}$ that $\gamma_{04}$ and $\gamma_{0 A}$ are of the same order $\frac{1}{c^{2}}$, A. Surin assumed $\gamma_{04}$ of order $\frac{1}{c^{2}}$, and $\gamma_{0 A}$ of the order $\frac{1}{c^{3}}$. Unfortunately, as in previous work, the resulting equation of motion for charged particles still were in conflict with the classical electrodynamic equations. Surin thus had to change her choice of metric to a conformal metric in space-time; she found that the equations of motion in first approximation are independent of the conformal metric. Nevertheless, she had to admit that this did not help: "These last results, particularly those concerning the first approximation to the equations of motion seem to necessitate a modification of the field equations." ${ }^{256}$ ([597], thèse , p. 126). A. Surin went on to calculate the 2nd approximation, an arduous task indeed, but which did not make the theory physically more acceptable. Moreover, in 2nd approximation, as in general relativity the results from both methods did not agree. Surin noted, that a modification of the field equations already had been suggested by H. Leutwyler [360], but did not comment on it. He had started from a variational principle mixing 5 - and 4-dimensional quantities:

$\delta \int d x^{1} \ldots d x^{4} R^{(5)} \sqrt{-g^{(4)}}=0$, where $R^{(5)}$ is the 5-dimensional curvature scalar expressible, due to the cylindricity condition, by quantities in space-time. He claimed to have removed the additional terms which precluded the derivation of the correct equations of motion. We will briefly present the further dissertation of R. Vallée below in Section 11.2.1. Huyen Dangvu also studied Jordan-Thiry theory and disagreed with its interpretation by Mariot \& Pigeaud as a theory describing massive mesonic particles ([106], p. 4309). According to him:

"It seems preferable to us to interpret $\phi$ as a scalar field without mass. Jordan-Thiry theory then will be a unitary theory of three massless fields: a scalar field, a vector field, and a 2-tensor field of spin 0, 1, 2, respectively." ${ }^{257}$

\subsubsection{Scalar-tensor theory in the 1960s and beyond}

Around Y. Thiry in Paris, the study of his theory continued with J. Hély producing static spherically symmetric solutions of the field equations [253, 252, 251]. They describe a point particle with charge and mass and include the Schwarzschild metric. Due to the different field equations, a

\footnotetext{
255 The index 0 denotes the time coordinate, index $A$ the 3 spatial coordinates of space-time, and the index 4 the additional space coordinate.

256 "Ces derniers résultats, surtout celui qui concerne la première approximation des équations de mouvement, semblent nécessiter une modification des équations du champ."

257 "Il nous semble donc préférable d'interpréter $\phi$ comme un champ scalaire sans masse. La théorie de JordanThiry sera alors une théorie unitaire de trois champs sans masse: un champ scalaire, un champ vectoriel et un champ de 2-tenseur, de spin respectivement $0,1,2 . "$
} 
direct comparison with the earlier solution by Heckmann et al. of Jordan's theory (cf. Section 3.1.2) seems not appropriate. However, H. Dangvu also contributed by looking at static and non-static spherically symmetric solutions to the Jordan-Thiry field equations in space-time. ${ }^{258}$ The solutions carry mass and some of them also electrical charge. Dangvu could compare them with those by Heckmann, Just, and Schücking in the Hamburg group around P. Jordan [108, 108].

From the full field (particle-) content of Kaluza-Klein theory, mainstream physics became interested most in the scalar field. In spite of the investigations within the framework of the ideas of Kaluza and Klein, and of Jordan's approach within projective relativity, attention to the scalar field evolved in complete separation from its origin in unitary field theory. Soon, scalar-tensor theories were understood strictly as alternative theories of gravitation. We comment briefly on the loss of this historical perspective the more so as current publications on scalar-tensor theory are more interested in the subsequent modern developments than in the historical record [219, 58]. In the Anglo-Saxon literature, scalar-tensor-theories run under the name of "Brans-Dicke theory", or, at best, Brans-Dicke-Jordan theory, i.e., two authors being late with regard to Jordan and Thiry are given most of the credit; cf. standard references like ([242], pp. 59, 64, 71, 77, 362); ([438], pp. 1068, 1070, 1098; ([698], pp. 125, 126, 341). ${ }^{259}$ True, the three groups of successive authors departed from different physical or mathematical ideas; Jordan from a varying gravitational constant as a hypothetical consequence of Dirac's large number hypothesis, Thiry from a mathematical study of Kaluza-Klein theory and its global aspects, and Brans \& Dicke from an implementation of their interpretation of Mach's principle. A fourth author, W. Scherrer, must be included who was the first of all, and who considered the scalar field as a matter field coupled to gravitation; vid. Section 3.1.2 and [230]. Yet, one should keep in mind that, together with Yang-Mills theories and, perhaps non-local field theory, scalar-tensor theories of gravitation may be also considered as one surviving offspring of unitary field theory. Hence a total loss of historical memory with regard to the origins of scalar-tensor theory appears unjustified. ${ }^{260}$

\subsection{6- and 8-dimensional theories}

Since Kaluza had proposed a 5-dimensional space as a framework for a unitary field theory of the gravitational and electromagnetic fields in 1919, both experimental elementary particle physics and quantum field theory had progressed. Despite the difficulties with divergencies, since the mid 1950s renormalization procedures had been stable enough to make quantum field theory acceptable and needed as a proper formalism for dealing with the known elementary particles. Nevertheless, in some approaches to unified field theory, it still was thought useful to investigate classical theory. Thus, in this context theories with new degrees of freedom for the new fields $(\pi$ - and $\mu$-mesons, neutrino) had to be constructed. We noticed that both in the Einstein-Schrödinger affine field theory and in the Jordan-Thiry extension of Kaluza's theory such attempts had been made. The increase in the dimension of space seemed to be a handy method to include additional fields. In Section 7.2.2 of Part I, papers of Rumer, Mandel, and Zaycoff concerning 6-dimensional space have been mentioned. None of them is referred to by the research described below. It involved theorists working independently in the USA, Great Britain, and France.

\footnotetext{
258 In scalar-tensor theory, Birkhoff's theorem no longer holds.

259 A reference to the 2nd edition of Jordan's book [320] which appeared almost a decade after his first suggestion of the theory most always is duly made but clouds the chronology. C. M. Will used the name "Jordan-Fierz-BransDicke theory (Brans-Dicke, for short)" ([699], Section 3.3). Cf. the discussion in Section 3.1.2.

260 A paper continuing the tradition and displaying an exact solution for "a simple variant of of projective relativity" is [341].
} 


\subsubsection{6-dimensional theories}

The first of these, B. Hoffmann in Princeton, wanted to describe particles with both electric charge $e$ and magnetic charge $\mu .{ }^{261}$ Because the paths of electrically charged particles could be described as geodesics of Kaluza's 5-dimensional space, he added another space-dimension. The demand now was that his particles with both kinds of charge follow geodesics in a 6-dimensional Riemannian (Lorentzian) space $R^{6}$. [277]. The coordinates in $R^{6}$ are denoted here by $A, B=0,1,2,3,4, \infty$, in $R^{5}$ by $\alpha, \beta, \cdots=0,1, \ldots, 4$, and in space-time $i, j,=1,2,3,4$. The metric $k_{A B}$ of $R^{6}$ contains, besides the metric $g_{i j}$ of space-time, two vector fields $k_{k 0}=\phi_{k}, k_{\infty j}=\psi_{k}$ and three scalar fields $k_{00}, k_{0 \infty}, k_{\infty, \infty}$. The scalar fields are disposed of immediately: $k_{00}=1$ (cylinder condition), $k_{0 \infty}=0, k_{\infty, \infty}=-1$, while $\phi_{k}$ is taken to be the electrical 4-potential: $\phi_{i j}=1 / 2\left(\frac{\partial \phi_{i}}{\partial x^{j}}-\frac{\partial \phi_{j}}{\partial x^{i}}\right)$ and $\psi_{k}$ the corresponding quantity following from the dual of the electrical field tensor $\psi^{i j}=\phi^{* i j}=$ $1 / 2(\sqrt{-g})^{-1} \epsilon^{i j r s} \psi_{r s}$.

The geodesic equation $\frac{d^{2} x^{A}}{d s^{2}}+K_{B C}^{A} \frac{d x^{B}}{d s} \frac{d x^{C}}{d s}=0$ with the Levi-Civita connection $K_{B C}^{A}$ is decomposed into 3 groups corresponding to $\frac{d^{2} x^{k}}{d s^{2}} \ldots, \frac{d^{2} x^{0}}{d s^{2}} \ldots, \frac{d^{2} x^{\infty}}{d s^{2}} \ldots$ From the last two equations follows $\phi_{C} \frac{d x^{C}}{d s}=$ const. $\stackrel{!}{=}-e / 2 m$ and $\psi_{C} \frac{d x^{C}}{d s}=$ const. $\stackrel{!}{=}-\mu / 2 m$ such that the projection of the geodesic equation of $R^{6}$ into space-time reads as: $\frac{d^{2} x^{k}}{d s^{2}}+\left\{\begin{array}{l}k \\ r s\end{array}\right\} \frac{d x^{r}}{d s} \frac{d x^{s}}{d s}-\frac{e}{m} \phi_{r}^{k} \frac{d x^{r}}{d s}-\psi_{r}^{k} \frac{\mu}{m} \frac{d x^{r}}{d s}=0$. In order that the geodesics are timelike curves, the condition $g^{m p} g^{n q}\left(\frac{\partial \phi_{p}}{\partial x^{q}}-\frac{\partial \phi_{q}}{\partial x^{p}}\right)=\frac{1}{2 \sqrt{-g}} \epsilon^{m n p q}\left(\frac{\partial \psi_{p}}{\partial x^{q}}-\right.$ $\left.\frac{\partial \psi_{q}}{\partial x^{p}}\right)$ must be fulfilled. It does as a consequence of the field equations derived from:

$$
\delta \int C \sqrt{-g} d x^{1} d x^{2} d x^{3} d x^{4}=0
$$

and which correspond merely to Maxwell's equations and to "the corresponding field equations of the projective theory" ( [277], p. 29). The curvature scalar of $R^{6}$ is $C=R-\phi_{s}^{r} \phi_{r}^{s}-\psi^{r}{ }_{s} \psi_{r}^{s}$ with $R$ being the curvature scalar of space-time. With topological questions not asked by him, Hoffmann's excursion into 6-dimensional space for describing the path of a magnetic monopole was entirely unnecessary. In fact, in his next paper he applied the method of Einstein and Mayer [175] presented in Section 6.3.2 of Part I to his particle with electric and magnetic charge and developed a theory in 4 dimensions [276].

In Manchester, where A. Papapetrou and P. M. S. Blackett worked on possible empirical consequences of UFT (cf. Section 6.1.2), while L. Rosenfeld wrote about the quantum theory of nuclear forces, a research fellow Julius Podolanski ${ }^{262}$ embarked on a unified field theory in six dimensions [498]. His motivation came from Dirac's equation, more precisely from the algebra of the $15 \gamma$-matrices, interpreted as representing the algebra of rotations in a 6-dimensional Riemannian space. His manifold $V_{6}$ is decomposed into a flat and geodesic 2-dimensional "sheet" generated by a spacelike and a timelike translation ${ }^{263}$, and a 4 -dimensional Lorentz space $V_{4}$ (identified with

\footnotetext{
261 In modern terminology, Hoffmann's particle is called dyon. His publication might have been a reaction to Dirac's paper on magnetic monopoles [121].

262 Julius Podolanski (1905-1955), born in Poland grew up in Germany (in what now is Thuringia) to where his parents had moved. He received his $\mathrm{PhD}$ at the university of Jena. After having been assistant there and then with W. Heisenberg in Leipzig, although a German citizen, due to his being of Jewish descent he could no longer work at a German university after 1933. Jobless at first, he then could join the publisher Akademische Verlagsgesellschaft, Leipzig and, when proofreading H. Kramers' article on Grundlagen der Quantenmechanik, he discovered errors and suggested improvements. Thus he got into contact with the impressed author. In 1937 he wrote letters to M. Born, H. Kramers and E. Schrödinger and presented them a manuscript on a new theory aimed at "replacing Dirac's theory of the electron." It unfortunately yielded two further particles, one spinless, the other uncharged with spin $1 / 2$. Kramers managed to get him a position as assistant at the University of Leiden. After hiding in Utrecht during the later years of the war, he afterwards obtained a position in Utrecht with L. Rosenfeld, and since 1948 joined him again in Manchester [517].

263 "Spacelike" and "timelike" refer to $V_{6}$, or to the indefinite 2-dimensional sheet space. Thus the signature of $V_{6}$ is \pm 4 .
} 
space-time), both normal to each other. Likewise, any 6-vector can be decomposed into a part in the 2-sheet and a 4-vector. A material point particle is assumed to follow a geodesic of $V_{6}$. From the connection of $V_{6}$, an induced connection in $V_{4}$ can be obtained. Invariance with regard to the translations is called "gauge"-invariance in $V_{4}$. This means that gauge-invariant quantities do not depend on the sheet-coordinates. It turned out that the metric of $V_{6}$, besides leading to the 4-dimensional metric $g_{i j}$, introduced two skew-symmetric tensors named fields of constraint. One of them is interpreted as the electromagnetic field. The interpretation of the second skew field is left open. The scalars in the theory (norms and inner product of the translations) are assumed constant. The field equations were derived from the curvature scalar of $V_{6}$ and obviously lead to Einstein's vacuum field equations in six dimensions; a matter tensor in $V_{6}$ is inserted by hand and restricted to charged pressureless matter. After a projection into $V_{4}$, it turned out that the currents and energy-momentum tensors of the two skew-symmetric fields had different signs: One field has a positive, the other a negative energy content. This was not seen as unphysical by the author; he commented: "So it seems that this theory may contribute to the problem of self-energy", an allusion to the divergence problem in quantum field theory ([498], p. 235), and to the fact that the energy density can become negative in quantum field theory. But he was disappointed that the 2nd skew field also had infinite reach: its quanta are massless. He tried to mend this by demanding that a particle follow a lightlike geodesic in $V_{6}$, but did not really succeed. His conclusion was: "A unitary field theory does not seem possible without introducing quantum theoretical concepts. This - classical - treatment therefore can only be incomplete" ([498], p. 258). Pauli noticed this paper; in the summer term 1953, he had given a course on general relativity including Kaluza's 5-dimensional theory. In a letter to Fierz of 3 July 1953 referring to this course we can read:

"In it, also Kaluza's 5th dimension did occur, and per se it is quite satisfying if now, in place of it, two additional dimensions with the 3-dimensional rotation group are introduced (this has already been suggested, if only formalistic, by e.g., Podolanski who has a 6-dimensional space)." ([491], p. 186-187.) ${ }^{264}$

Pauli's interest really went toward a paper of Pais who had suggested a 6-dimensional $\omega$-space consisting of space-time and an internal sphere-space affixed at any point [468]. From here, a direct line leads to Pauli's (unpublished) derivation of the non-Abelian $S U(2)$ gauge theory presented in letters to Pais. For a detailed history cf. O'Raifeartaigh's book ([463], Chapter 7). cf. also the section "A vision of gauge field theory" in [194], pp. 476-488.

When Josette Renaudie wrote her dissertation with A. Lichnerowicz and M.-A. Tonnelat on 6-dimensional classical unitary field theory in 1956, she also used elementary particles as her motivation [505, 506], ([507], p. 3; [508]). However, unlike Podolanski [498] and Yano \& Ogane [715], she worked in a 6-dimensional Lorentz space: 2 space-dimensions are added. While Yano \& Ogane employed a general 2-parameter isometry group and claimed to have the most general formalism, Renaudie admitted a general Abelian 2-dimensional isometry group, thus keeping 3 arbitrary scalar functions. Her two Killing vectors with regard to which the projection from 6dimensional $V_{6}$ along the trajectories of one Killing vector first to to $V_{5}$, and then with the 2nd Killing vector to space-time is performed, are spacelike. Again, an Einstein field equation $S_{A B}=$ $P_{A B},(A, B=0,1, \ldots, 5)$ is written down with the "matter tensor" $P_{A B}$ getting its interpretation backwards from the corresponding 4- and 5-dimensional quantities. The Einstein tensor now has 21 independent components and can be split into a rank 2 symmetric tensor, two 4 -vectors and 3 scalar functions. Renaudie gave two interpretations: (1) these variables stand for a hyperfield composed of gravitational, electromagnetic and mesonic fields (with the mesonic field a complex vector field),

264 "In dieser kam nämlich auch Kaluzas 5. Dimension vor und an sich ist es ganz befriedigend, wenn statt dieser nun zwei weitere Dimensionen mit der 3-dimensionalen Drehgruppe eingeführt werden (was auch, sehr formalistisch, bereits vorgeschlagen wurde, z. B. von Podolanski [...], der einen 6-dimensionalen Raum hat)." 
and (2) the field of a particle of maximal spin 1 in interaction with the gravitational field (p. 68/69). Note that in both interpretations the scalar fields remain unrelated to physical quantities. The terms in the field equations describing the interaction of the mesonic and electromagnetic fields are independent of the geometrical objects in space-time. The Cauchy initial value problem can be set up and solved properly. ${ }^{265}$

Five years later, another thesis advised by A. Lichnerowicz continued the work of J. Renaudie: R. Vallée investigated "The relativistic representation of charged perfect fluids" within 6- and 5-dimensional "space-times" [666]. According to its title, a focus lies in the material content, a perfect fluid, which also had been briefly dealt with by J. Renaudie ([506], cf. her Section 34). Vallée's ansatz for the matter tensor in six dimensions is taken over from space-time:

$$
\Theta_{I J}=(P+\Pi) W_{I} W_{J}-\Pi \Gamma_{I J},(I, J=0,1, \ldots, 5)
$$

where $\Gamma_{I J}$ is the metric in 6-dimensional space $V^{6}$ with Lorentz signature, $W_{I}$ with $W^{I} W^{J} \Gamma_{I J}=1$, the 6-velocity tangent to the streamlines, and $P>0, \Pi>0$ denote "density" and "pressure" ([666], p. 36). An equation of state $P+\Pi=f(\Pi)$ is assumed in order to get the corresponding relation in space-time. Projection into space-time (metric $g_{i j}$ ) via $V_{5}$ (metric $\gamma_{\alpha \beta}$ ) led to the matter tensor of a charged fluid of the form $\rho u_{i} u_{j}+\tau_{i j}^{\prime}$ with the electromagnetic energy-momentum tensor: $\tau_{i j}^{\prime}=\frac{1}{2}\left(H_{i}^{r} F_{j r}+F_{i}^{r} H_{j r}\right)-\frac{1}{4} g_{i j} H_{r s} F^{r s}$. Electromagnetic field $F_{i j}$ and induction field $H_{i j}$ were assumed to be related through $H_{i j}=\xi^{3} F_{i j}$ where $\xi^{2}=-\gamma_{00}$ stands for the additional scalar field in Jordan-Thiry theory. As with Renaudie, $V^{6}$ admits a 2-parameter Abelian group of global isometries. This is research extending the work of Renaudie to further classes of fluids; it fits to the doctoral theses described in Section 11.1.1 - in essence, they presented formal developments with restricted relevance to the envisaged physics of unitary field theory.

In 1963, Mariot \& Pigeaud again took up the 6-dimensional theory in a paper ${ }^{266}$ [388]. After the introduction of a conformal metric in $V_{4}, g_{i j}^{*}=\eta \xi g_{i j}, i, j=1,2,3,4$ with $\xi^{2}, \eta^{2}$ being the norms squared of the 2 Killing vectors from the additional dimensions, they studied the linear approximation of the theory with incoherent matter $m u_{i} u_{j}$. They were able to identify in $V_{4}$ matter tensors belonging to the electromagnetic field, to a neutral vector meson field and to both a massive neutral and a charged scalar meson field. Yet, some remaining terms were still not amenable to a physical interpretation.

\subsubsection{Eight dimensions and hypercomplex geometry}

As early as 1934, an eight-dimensional space with two time-dimensions was introduced in order to describe the gravitational field corresponding to an accelerated electromagnetic field. It then turned out that the two time coordinates were related by the eight geodesic equations such that, essentially, a 7-dimensional Lorentz space remained. Einstein's vacuum field equations in 8 dimensional space were assumed to hold [421, 422]. In view of the substantial input, the results, reached by approximate calculations only, were meagre: an approximate solution of the gravitational twobody problem; only static electric and constant magnetic fields could be described.

In the 1950s, the idea of using an 8-dimensional space as the stage for UFT seemingly arose by an extension of the mapping of connections in the same 4-dimensional space to a second copy of such a space. In Section 2.1.2 we have noted F. Maurer-Tison's interpretation of (30): The transport of a covariant vector $\nu_{i}$ with regard to the connection $\tilde{L}_{i j}{ }^{k}$ corresponds to the transport of the contravariant vector $\vec{\nu}^{k}=g^{k s} \nu_{s}$ with regard to $L_{i j}{ }^{k}$. This situation was turned into a geometry

\footnotetext{
265 Renaudie's papers were reviewed in Mathematical Reviews by K. Yano [MR0074981], who had published on the same subject, and by R. S. Mishra [MR0127958] who had not.

266 In the projection on a Lorentz space with 5 space dimensions, $x^{4}$ is the time coordinate.
} 
named semi-metric by Pierre-V. Grosjean ${ }^{267}$ in which two identical 4-dimensional spaces ("distinct universes") were introduced with the connection $L$ acting in one and its Hermitian conjugated connection $\tilde{L}$ in the other [235]. Inner products of vectors were allowed only if one vector is in the one space, the other in the second. His conclusion that the semi-metric geometry would be "the key to the unitary theory, in the same way as metric geometry was the key to general relativity" is more than overbearing. All that remains is a (physically empty) mathematical formalism the only consequence of which was to eventually motivate the scheme of applied mathematics to be discussed next.

As mentioned in Section 2.6, in close parallel to the complex numbers, a ring formed from $x+\epsilon y$ with real numbers $x, y$ and $\epsilon \times \epsilon=1$ can be introduced: the hypercomplex numbers. Albert Crumeyrolle ${ }^{268}$ built a unitary field theory of the Einstein-Schrödinger type on this number field $[96,97,98,95]$. In a generalization of the formalism noted in Section 10.5.4, he now introduced a 2ndimensional $C^{\infty}$-space $V^{2 n}$ and local charts around points by assigning n hypercomplex numbers $z^{\alpha}=x^{\alpha}+\epsilon x^{\alpha *}, \bar{z}=x^{\alpha}-\epsilon x^{\alpha *}, \alpha, \alpha *=1,2, \ldots, n$. In another basis, $z^{\alpha}=\frac{1}{2}\left(\xi^{\alpha}+\xi^{\alpha *}\right)+\epsilon \frac{1}{2}\left(\xi^{\alpha}-\right.$ $\left.\xi^{\alpha *}\right)$. The $x^{\alpha},\left(\xi^{\alpha}\right), x^{\alpha *},\left(\xi^{\alpha *}\right)$ transform among themselves. Again, a "natural adapted reference system" was introduced in which dual bases derive one from the other by the regular and diagonal $2 n \times 2 n$-matrix in $V^{2 n}$ :

$$
\left(\begin{array}{ll}
A & 0 \\
0 & \bar{A}
\end{array}\right)
$$

Here, $A, \bar{A}$ are $n \times n$-matrices with $A=\frac{\partial z^{\alpha^{\prime}}}{\partial z^{\lambda}}=\frac{\partial x^{\alpha^{\prime}}}{\partial x^{\lambda}}+\epsilon \frac{\partial x^{\alpha^{\prime}}}{\partial x^{\lambda *}}, \bar{A}=\frac{\partial z^{\alpha^{\prime}}}{\partial \bar{z}^{\lambda}}=\frac{\partial x^{\alpha^{\prime}}}{\partial x^{\lambda}}-\epsilon \frac{\partial x^{\alpha^{\prime}}}{\partial x^{\lambda *}}$. In $V^{2 n}$, locally a real symmetric covariant tensor could be introduced which decomposes in the coordinate system $\left(z^{\alpha}, z^{\alpha *}\right)$ into parts $\gamma_{\alpha \beta}, \gamma_{\alpha * \beta *}, \gamma_{\alpha \beta *}$. Likewise connections and special unitary connections were defined. In the end, a system generalizing the weak field equations of UFT resulted. For the physicist, and the more so for the historian of physics, the paper is a maze of mathematical structures. The author's statement that "a new field can be introduced the physical signification of which is not examined here but which could perhaps have something to do with the hypothesis of an inertial effect of the spin", remains unfounded ([97], p. 105).

267 Pierre-V. Grosjean (1912-2007), a Belgian mathematician, lived an uncommon life. After twenty years in the Congo as statistician for a mining business, meteorologist, and professor at a university there, he wrote his dissertation in Liège and then subsequently became a lecturer at the universities of Tunis, Caen and Rabat. He was elected full professor at the University of Mons, Belgium in 1968 where he stayed until retirement. He was also a writer with, among others, a book about his time in the Congo and three detective novels. He fought for the recognition of the Armenian genocide in Turkey after World War I.

268 Albert Crumeyrolle (1919-1992), after his work on unified field theory, gave important contributions to spinor structures and Clifford algebras. He was Professor at the University Paul Sabatier in Toulouse. 


\section{Further Contributions from the United States}

\subsection{Eisenhart in Princeton}

While Einstein pursued his research in UFT along the lines of mixed geometry at the Institute for Advanced Studies of Princeton, NJ, his colleague in the mathematics department of Princeton University, L. P. Eisenhart, kept quiet until the beginning of the 1950s. He had written a book on Non-Riemannian Geometry in 1927 [182], and since the twenties had had a long-standing interest in teleparallelism and UFT (cf. Section 6.4 of Part I and [181]). Being three years older than Einstein he had retired in 1945. Nevertheless, in the 1950s he wrote three further papers about UFT. He first introduced a non-symmetric metric $g_{i j}$ and a non-symmetric connection [184] in 1951. Unlike Einstein, whose papers he did not refer to, Eisenhart did not take the connection $L_{i j}{ }^{k}$ as an independent variable but built it entirely from the metric tensor and its first derivatives such as the Japanese physicist K. Hattori ${ }^{269}$ had done in the 1920s [240]:

$$
\begin{array}{r}
L_{i j}{ }^{k}=\Delta_{i j}{ }^{k}+a_{i j}{ }^{k}, \\
\Delta_{i j}{ }^{k}=\frac{1}{2} g^{(k l)}\left[g_{l i, j}+g_{j l, i}-g_{j i, l}\right],
\end{array}
$$

where $a_{i j}{ }^{k}$ is an arbitrary tensor. In fact, Eisenhart's $\Delta_{j i}{ }^{k}$ is exactly the same as Hattori's connection $g^{\rho \nu *}[\mu \lambda, \rho]([240]$, Eq. (1.6), p. 540). The tensor to be added was chosen by Eisenhart to be (indices are moved with the symmetric part of the metric):

$$
a_{i j k}=2 g_{[l k]} \Delta_{[i j]}^{l}-\frac{2}{3} \Delta_{[i j] k} .
$$

From (433) we notice that

$$
\Delta_{(i j)}^{k}=\left(\left\{\begin{array}{c}
k \\
i j
\end{array}\right)_{h}\right\}
$$

where the Christoffel symbol is formed with the symmetric part of the metric. The torsion tensor is given by:

$$
S_{i j}{ }^{k}=\Delta_{[i j]}^{k}+a_{[i j]}^{k}=\frac{1}{3} g^{(k l)} \Delta_{[i j] l}+2 g^{(k l)} g_{[s l]} \Delta_{[i j]}^{s} .
$$

Eisenhart used the curvature tensor $K_{-}^{i}{ }_{j k l}$ and its contraction $-K_{-}$jk After some manipulations, he obtained an equation for the Ricci tensor formed from $g_{(i j)}$ which is contained already in Hattori's paper (Eisenhart's Eq. (26)). ${ }^{270}$ Eisenhart's paper dealt only with differential geometry; no physical motivation or interpretation were given. This applies also to a subsequent publication in which, after formal manipulations, several expressions for possible curvature tensors and Einstein's Hermitian-symmetrized Ricci tensor (196) were derived [185]. The 3rd edition of Einstein's Meaning of Relativity [150] now was referred to.

Eisenhart's second attempt, after the death of Einstein, presented a new unified theory of gravitation and electromagnetism within metric-affine geometry [186, 187, 188], and [189]. Although in a different geometrical setting, eventually the theory formally led to the Einstein-Maxwell field equations in Riemannian geometry. The main difference to Einstein's approach was that Eisenhart kept the metric tensor $g_{i j}$ symmetric while embedding the electromagnetic field tensor $F_{i j}$ into the connection by an ad-hoc ansatz [186]:

$$
L_{i j}{ }^{k}=\left\{\begin{array}{l}
k \\
i j
\end{array}\right\}+F_{i j ; s} g^{k s} .
$$

\footnotetext{
269 Hattori's connection was already discussed in Section 2.5.1 and in Section 6.2. of Part I.

270 In all likelihood, Eisenhart did not know Hattori's paper. The substantial bibliography of his book [182] ends with 1927. Even Schouten's later extensive bibliography in [540] did not contain Hattori's paper.
} 
$F_{i j}$ is derived from a 4-potential and supposed to satisfy $F_{i}{ }_{; s}^{s}=0 .{ }^{271}$ Thus, vector torsion does vanish. In the first paper, Eisenhart's field equations were:

$$
R_{i j}-F_{i ; r}^{s} F_{j ; s}^{r}=0 \text {. }
$$

With his ansatz (437), the equation for the auto-parallels of the connection $L$ read as:

$$
\left(\frac{d x(s)^{i}}{d s}\right)_{\| l} \frac{d x(s)^{l}}{d s}=\text { const. } F_{m ; n}^{i} \frac{d x(s)^{m}}{d s} \frac{d x(s)^{n}}{d s} .
$$

These results were unphysical. Four months later, Eisenhart tried to find a remedy by postulating [187]:

$$
L_{i j}{ }^{k}=\left\{\begin{array}{l}
k \\
i j
\end{array}\right\}+F_{i j} \lambda^{k}
$$

with $\lambda^{k}$ satisfying

$$
\lambda_{i ; j}=\lambda_{i} \lambda_{l} F_{j}^{l}, \lambda_{i} \lambda^{i}=0 .
$$

All he arrived at was the field equation $R_{i j}=$ const. $\lambda_{i} \lambda_{j}$. A slight generalization of (441):

$$
\lambda_{i ; j}=\lambda_{i} \lambda_{l} F_{j}^{l}+F_{i j}, \lambda_{i} \lambda^{i}=1
$$

did not help much. Because he did not use a variational principle, Eisenhart always had to build his theories such that an identity in Riemannian geometry was guaranteed: the vanishing of the divergence of the Einstein tensor.

Again two months later, in his 3rd installment, Eisenhart finally arrived at the Einstein-Maxwell equation of general relativity [188]. This time, the ansatz (440) with (441) was changed substantially into:

$$
L_{i j}{ }^{k}=\left\{\begin{array}{l}
k \\
i j
\end{array}\right\}+g_{i j} \lambda^{k}, \lambda_{i ; j}=-\lambda_{i} \lambda_{j}-\left(F_{i l} F_{j}^{l}-\frac{1}{4} g_{i j} F_{r s} F^{r s}\right) .
$$

This equation is interesting because the energy-momentum tensor of the electromagnetic field is built directly into the connection (curvature). Eisenhart was aware that the Einstein-Maxwell equations did not represent the union of the gravitational and electromagnetic field aimed at. He quoted Einstein as saying that "A theory in which the gravitational field and electromagnetic field do not enter as logically distinct structures would be much preferable." ([188], p. 881.) This quotation was repeated in the last of this sequence of papers by Eisenhart, in which "the final result of my third paper" were derived "in a somewhat different manner." ([189], p. 333.)

In his last contribution to UFT, when he was 83 years old, Eisenhart returned to mixed geometry [190]. Starting from Einstein's condition (200) on the metric, he aimed at solving it for the connection as a function of the metric and its first derivatives. Unaware of the solutions given previously (cf. Section 10.2.3), he achieved this only with the help of the additional constraint for torsion:

$$
g_{[i r]} S_{j k}^{r}+g_{[j r]} S_{i k}^{r}=0 .
$$

From this, again the vanishing of vector torsion follows. In this special case, from (444) with the notation of (3), (4), Eisenhart's solution is given by:

$$
L_{(i j)}^{k}=\left\{\begin{array}{l}
k \\
i j
\end{array}\right\}_{h}, L_{[i j]}^{k}=S_{i j}^{k}=\frac{1}{2} m^{k r}\left(k_{r j, i}+k_{i r, j}+k_{j i, r}\right) .
$$

Seen in context, Eisenhart's papers on UFT from the 1950s did bring neither a new development in geometry nor an advance for physics. Cf. also the paper by Horvath [285] in Section 15.1.

271 The semicolon stands for the usual covariant derivative in Riemannian geometry. 


\subsection{Hlavatý at Indiana University}

Hlavaty $\dot{y}^{272}$ is the fourth of the main figures in UFT besides Einstein, Schrödinger, and Tonnelat. His research was published first in a sizeable number of articles in the Journal of Rational Mechanics and Analysis of Indiana University ${ }^{273}$ and in other journals; they were then transformed into a book [269]. According to its preface, his main intent was "to provide a detailed geometrical background for physical application of the theory". As he was very optimistic with regard to its relation to physics, he went on: "It so happens that the detailed investigation of Einstein's geometrical postulates opens an easy way to a physical interpretation" ([269], p. X). We have noticed in Section 9.7 that this possibly could not have been the case. In the preface of his book, Hlavatý became more explicit; his program was to encompass: (1) an investigation of the structure of the curvature and torsion imposed on space-time by the field equations, equations which he claimed to be "of a purely geometrical nature" without physical interpretation being "involved in them a priori". The two further points of his program, i.e., (2) an attempt to identify the gravitational field and the electromagnetic field by means of the field equations, and (3) an investigation of the physical consequences of his theory, were treated only in "an outline of the basic ideas" ([269], p. XVIII). In comparison with Einstein, Schrödinger and Tonnelat who followed their physical and mathematical intuition, Hlavatý's investigations were much more systematical and directed first to what could be proven by mathematics; whether a relation to physics could be established became secondary to him. Although mostly working and publishing alone, he corresponded with about 40 scientists working on UFT. He also was a frequent reviewer for Mathematical Reviews (cf. Section 18.1).

Hlavatý began by introducing a systematical classification of the non-symmetric metric $g_{i j}$ according to the non-vanishing eigenvalues of its skew-symmetric part $k_{i j}$ (remember $\left.\check{k}^{i j}=h^{i s} h^{i s} k_{s t}\right)$. He distinguished three classes:

$$
\begin{aligned}
& \text { class 1: } k=\operatorname{det}\left(k_{i j}\right) \neq 0 ; \\
& \text { class 2: } k=\operatorname{det}\left(k_{i j}\right)=0 \text { and } K^{2}:=\left(k_{i j} \check{k}^{i j}\right)^{2} \neq 0 ; \\
& \text { class 3: } k=\operatorname{det}\left(k_{i j}\right)=0 \text { and }\left(k_{i j} \check{k}^{i j}\right)^{2}=0 .
\end{aligned}
$$

In contrast to presentations in his articles [260, 261, 262, 263], in his book this simple algebraic problem is spread out on many pages ([269], 11-41). His student A. W. Sáenz simplified Hlavatý's proofs by looking at the algebraic structure of the electromagnetic field tensor [520].

Throughout his research, the symmetric part $g_{(i j)}=h_{i j}$ is used for raising and lowering indices. From Eq. (30) he concluded that there are metrics $g_{i j}$ for which this "metric compatibility" equation does not admit any solution", and cases in which (30) admits more than one solution [261]. According to him, the condition for uniqueness of the solution is $\operatorname{det}\left(g_{l m}\right) / \operatorname{det}\left(h_{r s}\right)=: g \neq 0$ if $\operatorname{det}\left(k_{l m}\right) / \operatorname{det}\left(h_{r s}\right):=k \neq 0$ (class 1$)$, and $\left.g(g-2)\right) \neq 0$ if $k=0$ (class 2,3$)$. The gravitational

\footnotetext{
272 Václav Hlavatý (1894-1969), mathematician, born in Czechoslovakia. PhD Charles University, Prague, 1922; post doctoral studies at universities in Holland, Rome, Paris and Oxford; professor of mathematics at Charles University, 1930-1948; visiting professor at Princeton University at the invitation of Albert Einstein, 1937-1938; a member of the Czech Socialist Party, entered politics in 1946; member of the Czech parliament in 1947; refused to sign Communist loyalty oath and left Czechoslovakia in 1948; taught one semester at the Sorbonne, 1948. In the fall of 1948 he accepted a professorship of mathematics at Indiana University. http://www.indiana.edu/ liblilly/ lilly/mss/index .php?p=hlavaty2

273 The Indiana University Mathematics Journal is a journal of mathematics published by Indiana University. Its first volume appeared in 1952, under the name Journal of Rational Mechanics and Analysis and was edited by V. Hlavatý and Clifford Truesdell. In 1957, Eberhard Hopf became editor; the journal name changed to the Journal of Mathematics and Mechanics, and Truesdell founded a separate successor journal, the Archive for Rational Mechanics and Analysis, now published by Springer-Verlag. The Journal of Mathematics and Mechanics later changed its name again to the present name.
} 
potential is identified with $g_{(i j)}=h_{i j}$ while the electromagnetic field is taken to be ${ }^{274}$ [261]:

$$
\begin{aligned}
f_{i j} & :=\frac{1}{\sqrt{g}}\left[\kappa \sqrt{|k|} k_{i j}-\frac{1}{2} \sqrt{|h|} \epsilon_{i j r s} \check{k}^{r s}\right] \\
f^{i j} & :=\frac{1}{\sqrt{g}}\left[\kappa \sqrt{|k|} \check{k}^{i j}-\frac{1}{2} \sqrt{|h|} \epsilon^{i j r s} k_{r s}\right]
\end{aligned}
$$

with $\kappa=-\operatorname{sgn}\left[\epsilon^{i j r s} k_{i j} k_{r s}\right]$. The above classification of $k_{i j}$ is thus valid also for the electromagnetic field. For classes 2 and 3, Hlavatý's definition of the electromagnetic field is a variant of one we have already met in Section 9.7.

His approach was more direct than Tonnelat's: mostly, he worked just with $g_{i j}, h_{i j}$ and the decomposition (363) met before:

$$
L_{i j}^{k}=\left\{\begin{array}{l}
k \\
i j
\end{array}\right\}+S_{i j}^{k}+U_{i j}^{k}
$$

where $U_{i j}{ }^{k}=2 h^{k r} S_{r(i}^{s} k_{j) s}$. The removal of the connection from (30) first gave torsion as a functional of the symmetric and skew symmetric parts of the metric via the linear equation:

$$
S_{p q r} X_{i j k}^{p q r}=K_{i j k}
$$

with $K_{i j k}:={\stackrel{\{\}_{h}}{\nabla}}_{k} k_{i j}+{\stackrel{\{\}_{h}}{\nabla}}_{j} k_{k i}+{\stackrel{\{\}_{h}}{\nabla}}_{i} k_{j k}$, and $X_{i j k}^{p q r}=X_{i j k}^{p q r}\left(\delta_{a}^{b}, k_{c t} h^{d t}\right)$ ([264], p. 320). After torsion is inserted into the decomposition of $L_{i j}{ }^{k}$ given before, the connection is known as a functional of $h_{i j}, k_{i j}$ and its first derivatives. Thus, after about 80 pages in his book including degenerate cases, in the end Mme. Tonnelat's calculations were only simplified a bit by Hlavatý and made more transparent, with some details added.

Hlavatý used the Ricci-tensors $K_{i j}$ and $P_{i j}^{*}$ (cf. (75) of Section 2.3.2), and proved that:

$$
\underline{K}_{i j}+P_{j i}^{*}=\stackrel{L}{\nabla}_{i} S_{j}
$$

He stressed that Einstein's weak field equations for UFT were of a purely geometrical nature with no physical interpretation needed. Equation (30) was written in the form [261]:

$$
\bar{\nabla}_{k} g_{i j}=2 S_{k j}{ }^{s} g_{i s}
$$

where the covariant derivative is defined by (16) in Section 2.1.1. The conditions on curvature are subsumed in

$$
K_{-}{ }_{i j}=\partial_{[i} X_{k]}
$$

with arbitrary $X_{k}$. For vanishing $X_{k}$, the strong field equations, for $X_{k} \neq 0$, the weak field equations are following.

According to Hlavatý, the first two classes cannot be handled simultaneously with the third class ([266], p. 421). This makes it more involved to read his papers, because the results proven by him must now be distinguished according to the special class of $k_{i j}$.

i) Fields of third class.

${ }^{274}$ In his monograph, a further sign-factor $\sigma$ is placed in front of the bracket [...] in (446) where $\sigma:=\operatorname{sgn}\left(\frac{\hat{e}}{|\hat{e}|}\right)$ with an arbitrary scalar density $\hat{e}$. Hlavatý denoted with $g, k$ what was named $\frac{g}{h}, \frac{k}{h}$ in Section 2.1. 
In the course of his investigations when he tried to interpret geometrical quantities in terms of physical variables, Hlavatý replaced the four equations $R_{\{[i k], j\}}=0$ following from (449) by four complicated looking equations:

$$
(G \check{Q})^{i r}{ }_{\| r}=2 U_{r s}^{i} \check{H}^{r s}+S_{r} G \check{Q}^{r i}+U_{r}\left(G \check{Q}^{r i}+2 H h^{r i}\right),
$$

where $\check{H}^{i j}$ and $H$ are the Ricci tensor and curvature scalar calculated from the symmetric part of the metric $h_{i j}$ (i.e., from the Levi-Civita connection). $G$ is a scalar function for which, for the 2 nd class $G=\frac{g}{h}$, and for the third class $G=\frac{g}{h}=1$ hold.

The tensor $Q_{i j}$ received its meaning from what Hlavatý called "the gravitational field equations", i.e., Einstein's equations with a geometrical energy-momentum tensor of matter:

$$
H_{i j}-\frac{1}{2} H h_{i j}=\frac{1}{2} T_{i j}
$$

where $T_{i j}:=v_{i} v_{j}+Q_{i j} . v_{i}$ describes both the charge density and the mass density of matter which thus are related. As charge density it is defined by: $v^{k} \sqrt{h}=\partial_{s}\left(f^{s k} \sqrt{h}\right)$, with $f^{i j}$ from (446); mass density by $v^{k} \sqrt{h}=\phi M \sqrt{h} u^{k}$ with a scalar function $\phi$ and the unit vector $u^{k} ; M$ is the mass of a particle ([269], p. 175-176). In Hlavatý's theory, Maxwell's equations were taken to be:

$$
\partial_{\{k} f_{i j\}}=0, v^{k} \sqrt{h}=\partial_{s}\left(f^{s k} \sqrt{h}\right) .
$$

Charge conservation was expressed by $\partial_{s}\left(v^{s} \sqrt{h}\right)=0$. As the first equation of (452) is equivalent to (449) plus $S_{i}=0$, we also have $v_{i}=-\frac{\sigma}{2} \epsilon_{p q r i} h^{p s}{\stackrel{\{\}_{h}}{\nabla}}_{s} \check{k}^{q r}$. Of course, the electromagnetic field in (446), for the third class, reduces to $f_{i j}=-\frac{1}{2} \sqrt{|h|} \epsilon_{i j l m} \check{k}^{l m}$.

For incoherent matter, $Q_{i j}=0$, and from $(451) \stackrel{\{\}_{h}}{\nabla}{ }_{s}\left(M u^{s} u^{k}\right)=0$. A somewhat disappointing consequence is that, in a manageable approximation, charged particles remain unaffected by the electromagnetic field: they move along geodesics in the gravitational field $h_{i j}$ ([264], p. 329; [269], p. 174, 187). Upon neglect of the cubic terms in $f_{i j}$, i.e., for $U_{i j} \simeq 0$, the equation of motion coincides with the geodesic equation:

$$
\frac{d^{2} x^{k}}{d \lambda^{2}}+\left\{\begin{array}{l}
k \\
i j
\end{array}\right\} \frac{d x^{i}}{d \lambda} \frac{d x^{j}}{d \lambda}+U_{i j}{ }^{k} \frac{d x^{i}}{d \lambda} u^{j}=0 .
$$

This is the more strange as Hlavatý claimed:

"In the unified theory the electromagnetic field is always present; hence we might look upon it as a primary field which [...] creates the gravitational field. However, there is at least one known electromagnetic field which does not create a gravitational field (i.e., the field of the plane wave in the electromagnetic theory of light)." ([266], p. 420.)

ii) Fields of class 1 and 2 .

Here, $G=\left(1+\frac{1}{2} f_{r s} \check{f}^{r s}+\frac{f}{h}\right), f=\operatorname{det}\left(f_{r s}\right)$, and two new quantities were introduced:

$$
F^{i j}=\sqrt{G} f^{i j}, \quad L_{i j}=-\phi \sqrt{\frac{f}{h}} F_{i j} .
$$

The skew-symmetric part of the asymmetric metric $g_{i j}$ now reads as $\sigma k_{i j}=\frac{1}{2} \epsilon_{i j k l} F^{k l}+L_{i j}$. Tangent vectors $w^{k}$ and $W^{k}$ were defined by

$$
w^{k} \sqrt{|h|}=\partial_{s}\left(f^{s k} \sqrt{|h|}\right), \quad W^{k} \sqrt{|h|}=\partial_{s}\left(F^{s k} \sqrt{|h|}\right)
$$


related by $W^{k}=\sqrt{G}\left(w^{k}+G_{s} f^{s k}\right)$ with $G_{s}=\frac{1}{2} \partial_{s} \ln G$. The gravitational and Maxwell equations (451) and (452) remain the same except for an exchange of $v_{i}$ by $w_{i}$ and a different complicated expression for $G Q_{i j}$ if $R_{\{[i k], j\}}=0$ is kept as a field equation; cf. [269], p. 204, Eq. (20.3a,b), p. 203 Eqs. (20.1), (20.2b). Hlavatý did present an exact spherically symmetric solution with $g_{00}=1, g_{11}=B\left(1-A r^{-4}\right), g_{22}=r^{2}, g_{33}=r^{2} \sin ^{2} \theta, A, B$ constants which is obtained from Papapetrou's solution (cf. Section 8.3) by setting there $m=\lambda=0$. The electromagnetic field in Hlavatý's solution is $f^{23}=\frac{\sqrt{A}}{r^{4} \sin ^{2} \theta}$. The gravitational function replacing the gravitational constant is $G=\left(1-A r^{-4}\right)^{-1}$. But in this case, according to Hlavatý "we are unable to derive the second set of Maxwell's equations from our field equations" ([269], p. 208). Therefore, as for classes 1, 2, this field equation again is replaced by (450). In consequence, for the motion of a particle Hlavatý obtained an improved result: A (massive) charged particle moving freely in the unified field $g_{i j}$ describes an auto-parallel of the unified connection $L_{i j}{ }^{k}$ ([269], p. 211). Thus, two of the three effects in the planetary system were the same as in general relativity; the third (Perihelion shift) in his theory depended on the electrical field of the sun. Hlavatý did not get as far as to clearly show the experimental physicist how this electric field enters the formula for the perihelion shift.

For paths of photons Eq. (453) still holds. If gravitation is neglected, i.e., $h_{i j}=\eta_{i j}$, Hlavatý found a discrepancy with the special relativistic explanation of the Michelson-Morley experiment. Although he referred to the judgment of Shankland et al. that Miller's result is erroneous [575], he concluded: "From the point of view of the unified field theory Miller's result, properly interpreted, is not necessarily at variance with the assumption of the constant velocity of light." ([266], p. 471).

Hlavatý's research will be appealing to some by its logical guideline concerning mathematical structures. His many special cases and set up "agreements" in proving results are somewhat bemusing for a physicist. An example is given by his publications dealing with the special case $h=0, g \neq 0$ when the symmetric part $h_{i j}$ of the metric $g_{i j}$ is degenerated [267, 268]. It is a purely mathematical exercise meant to fill a gap, but is without physical meaning. For the cases in which the theory could be applied to physical systems, in principle, Hlavatý was also forced to alter the original field equations in order to avoid objections against the unphysical results following from them. It is not unfair to conclude that he did not succeed in making a break-through in the sense of his physical interpretations being more convincing than those suggested by others.

The investigations of his doctoral student $\mathrm{R}$. Wrede ${ }^{275}$ were directed to the mathematical structure of the theory: He partially extended Hlavatýs theory to an n-dimensional space by adhering to the two principles: A.) The algebraic structure of the theory is imposed on the space by a general real tensor $g_{a b}$; B.) The differential geometrical structure is imposed on the space by the tensor $g_{a b}$ by means of a connection defined by (30). Hlavatý's third principle, i.e., the existence of the constraints $R_{a b}=\partial_{[a} X_{b]}, S_{a r}^{r}=L_{[a r]}^{r}=0$ with an arbitrary vector field $X_{a}$ is left out [708]. The paper solves (30) in $n$ dimensions for the various possible cases.

\subsection{Other contributions}

A theoretician of the younger generation and assistant at Princeton University, R. L. Arnowitt ${ }^{276}$ , tried to look at UFT from the point of view of the electromagnetic field forming a link between the description of microscopic charges by quantum field theory and macroscopic ray optics [5]. He introduced four postulates:

275 Robert C. Wrede $(1926$ - 2011) received his PhD in 1956 with Hlavatý. He became professor at San José State University, California, 1955-1994. Here, he concentrated on teaching and writing introductory mathematical textbooks. He was also active in university politics.

276 Richard L. Arnowitt (1928-) stayed at the Institute for Advanced Study in Princeton 1954-1956. Later Professor at Northeastern University in Boston and at Texas A\&M University in College Station, Texas. There, "Distinguished Professor Emeritus" (2007). He co-developed the ADM-formalism essential for recasting Einsteingravity into the Hamiltonian formalism. His publications include as diverse topics as the many body theory of liquid Helium and supergravity grand unification. 
(1) Any unified field theory should reduce to Einstein-Maxwell theory in a first approximation for weak electromagnetic fields.

(2) First-order corrections to the Coulomb field of the electron should not become appreciable for $r \geq 10^{-13} \mathrm{~cm}$.

(3) The affine connection has the form $\Gamma_{i j}{ }^{k}=C_{i j}{ }^{k}+\Gamma_{j} \delta_{i}^{k}$, where $\Gamma_{j}$ is related to the vector potential of the Maxwell field. Also $C_{i j}{ }^{k}=C_{j i}{ }^{k}$ is assumed.

(4) The Lagrangian must be invariant under the combined gauge transformation $\Gamma_{i j}{ }^{k} \rightarrow \Gamma_{i j}{ }^{k}+$ $\Lambda_{, j} \delta_{i}^{k}$ and (for the metric) $g_{i j} \rightarrow \exp [2 \Lambda(x)] g_{i j}$. The metric tensor is also symmetric.

The appearance of a microscopic length-parameter (and the cosmological constant) in his Lagrangian and the occurrence of two "metrical" tensors turned out to be a consequence of the postulates. The symmetrical first one $g_{i j}$ is supposed to "be measured by rods and clocks" and used to set up the Lagrangian; the second asymmetric one is derived from the Lagrangian: $\hat{g}^{\prime}{ }^{i j}:=\delta \mathcal{L} / \delta R_{i j}$. It is an auxiliary device for the introduction of the electromagnetic field. Arnowitt chose the Lagrangian:

$$
L=\int d^{4} x \mathcal{L}(x)=\int d^{4} x \sqrt{-g}\left[\alpha_{1} R_{i r} R_{j s} g^{i j} g^{r s}+\alpha_{2}\left(g^{r s} R_{r s}\right)^{2}\right]
$$

with two constants $\alpha_{1}, \alpha_{2}$ of dimension (length) ${ }^{2}$. The theory looks similar to Buchdahl's gaugeinvariant UFT published in the same year (cf. Section 13.1) but is different. Immediately,

$$
\hat{g}^{\prime(i j)}=\sqrt{-g}\left[\alpha_{1} R_{(r s)} g^{i r} g^{j s}+\alpha_{2} g^{i j} g^{r s} R_{(r s)}\right], \hat{g}^{\prime}[i j]=\alpha_{1} R_{[s r]} g^{i r} g^{j s} .
$$

In order to obtain the field equations, the quantities $C_{i j}{ }^{k}, \Gamma_{j}$ and $g^{i j}$ are to be varied. From the first two variations $\hat{g}_{; s}^{\prime[i s]}=0, \hat{g}_{; k}{ }_{; j}=0$ and $C_{i j}{ }^{k}=\frac{1}{2} h^{\prime(k s)}\left(h_{(i s), j}^{\prime}+h_{(j s), i}^{\prime}-h_{(i j), s}^{\prime}\right)$ resulted where $h^{\prime i j}$ was introduced by $\hat{g}^{\prime}(i j)=h^{i j} \sqrt{-h^{\prime}}$ and interpreted to be the "gravitational" metric tensor. The first of these equations was rewritten as sourceless Maxwell equation such that $g_{i j}$ is the "electromagnetic" metric tensor:

$$
\left[\sqrt{-g} g^{i r} g^{q s} f_{r s}\right]_{, q}=0
$$

with $f_{i j}=-a^{-\frac{1}{2}} R_{[i j]}$, and the constant a is determined later in a weak field approximation to be $a \simeq-\kappa^{2} \rho_{e} c^{2}$ with $\rho_{e} c^{2}=\frac{e^{2}}{r_{1}^{4}}, r_{1} \sim \frac{e^{2}}{m c^{2}}$ the classical electron radius, $m$ the electron mass, and $\kappa$ the gravitational constant in Einstein's equations.

After some manipulation, variation with respect to $g^{i j}$ led to:

$$
\alpha_{1}\left[g^{i p} R_{(k r)} g^{r s} R_{(p s)}-\frac{1}{4} \delta_{k}^{i} g^{q p} R_{(q r)} g^{r s} R_{(p s)}\right]+\alpha_{2}\left[g^{i p} R_{(k p)} g^{r s} R_{(r s)}-\frac{1}{4} \delta_{k}^{i}\left(g^{r s} R_{(r s)}\right)^{2}\right]=\alpha_{1} a T_{k}^{i}
$$

with

$$
T_{k}^{i}=-g^{i p} g^{r s} f_{k r} f_{p s}+\frac{1}{4} \delta_{k}^{i} g^{q p} f_{q r} g^{r s} f_{p s} .
$$

$T_{k}^{i}$ was interpreted as 'the electromagnetic stress-energy tensor". After linearization, (459) formally became Einstein's equations. Again, in a weak field approximation introduced by $g_{i j}=g_{(i j)}+h_{i j}$ with small $h_{i j}$, the free parameters $\alpha_{1}, \alpha_{2}$ were fixed to be $\alpha_{1} \simeq \kappa \rho_{e} c^{2}$ and $\left(\alpha_{2}\right)^{-1} \simeq 4 \lambda$ with the cosmological constant $\lambda$. Thus, $\alpha_{1}$ is the microscopic length parameter mentioned above. In linear approximation, the author also has obtained a static, spherically symmetric Schwarzschild-like solution with an event horizon and finite electrical field (and field energy) for $r \rightarrow 0$. 
A further contribution came from B. Kursunŏglu, whom we have met before in Section 9.3.3, now situated in Coral Gables, Florida. He continued to alter and study his variant of the EinsteinSchrödinger field equations [345]. In place of (300)-(302), he postulated the system: ${ }^{277}$

$$
\begin{aligned}
\hat{g}_{, l}^{[i l]} & =0, \\
\underline{-}_{(i k)}+p^{2}\left(h_{i j}-b_{i j}\right) & =0, \\
\underline{K}_{[i k]}+p^{2}\left(q k_{i k}-F_{i k}\right) & =0,
\end{aligned}
$$

where

$$
b_{i j}:=\frac{h_{i j}+k_{i r} \check{k}_{j}^{r}}{1+1 / 2 k_{r s} \check{k}^{r s}-\left(1 / 4{ }^{*} k^{r s} k_{r s}\right)^{2}}
$$

with

$$
{ }^{*} k^{r s}:=\frac{1}{2 \sqrt{-h}} \eta^{r s p q} k_{p q}
$$

The denominator is related to $g$.

The auxiliary field $F_{i j}$ satisfies the vacuum Maxwell equations.

${ }^{277}$ Kursunŏglu sets the skew part of the metric $k_{i j}=q^{-1} \phi_{i j} . q$ drops out everywhere except in his Eq. (463). 


\section{Research in other English Speaking Countries}

\subsection{England and elsewhere}

We have met the work of W. B. Bonnor of the University of Liverpool on UFT already before. After having investigated exact solutions of the "weak" and "strong" field equations, he set up his own by adding the term $p^{2} m^{i k} K_{i k}$ to Einstein's Lagrangian of UFT [34]. They are: ${ }^{278}$

$$
\begin{aligned}
g_{i k|| l} & =0, \\
S_{i-} & =0, \\
\stackrel{\operatorname{Her}}{K}_{(i k)}+p^{2} U_{(i j)} & =0, \\
\stackrel{\mathrm{Her}}{K}_{[i k], l}+\stackrel{\mathrm{Her}}{K}_{[k l], i}+\stackrel{\mathrm{Her}}{K}_{[l i], k}+p^{2}\left(U_{[i k], l}+U_{[k l], i}+U_{[l i], k}\right) & =0,
\end{aligned}
$$

with

$$
U_{i j}=g_{[i j]}-g^{[r s]} g_{i r} g_{j s}+\frac{1}{2} g^{[r s]} g_{r s} g_{i j}
$$

The assignment of $g_{(i j)}$ to the gravitational potentials and of $g_{[i j]}$ to the electromagnetic field was upheld while the electric current became defined as $J_{i j k}=g_{\{[i j], k\}}$.

A linearization $g_{i j}=\eta_{i j}+\gamma_{i j}$ of Bonnor's field equations up to the first order in $\gamma$ led to:

$$
\begin{aligned}
\gamma_{[i s], s} & =0, \gamma_{(i s), s k}+\gamma_{(k s), s i}-\gamma_{s s, k i}=0, \\
\gamma_{\{[i k], l\}, s s} & =-4 p^{2} \gamma_{\{[i k], l\}} .
\end{aligned}
$$

In first approximation the electric current is given by $J_{i j k}=\gamma_{\{[i j], k\}}$ such that the previous equation looked like $\partial^{s} \partial_{s} J_{i j k}=-4 p^{2} J_{i j k}$. For a spherically symmetric particle at rest with radial coordinate $r$, Bonnor obtained

$$
\frac{1}{r^{2}} \frac{d}{d r}\left(r^{2} \frac{d \rho}{d r}\right)=-4 p^{2} \rho
$$

where $\rho$ is the charge density. For $2 i p=a^{2}$ and $\rho \rightarrow 0$ for $r \rightarrow \infty$, the charge density will be restricted $^{279}$ to $\rho=$ const. $\frac{1}{r} e^{-a^{2} r}$. As M.-A. Tonnelat remarked, Bonnor's strategy was simply to add a term leading to Maxwell's energy-momentum-stress tensor ([634], p. 919). Abrol \& Mishra later re-wrote Bonnor's field equations with help of the connections defined in (51) and (52) of Section 2.2.3 [2].

In Trinity College, Cambridge, UK, in the mid-1950s research on UFT was carried out by John Moffat $^{280}$ as part of his doctoral thesis. It was based on a complex metric in (real) space-time: $g_{i j}:=s_{i j}+a_{i j}$ with real $s_{i j}$, imaginary $a_{i j}$, and $* s^{r i} s_{r k}=\delta_{k}^{i}$. Correspondingly, the symmetrical linear connection $\Gamma_{i j}{ }^{k}=* \Gamma_{i j}{ }^{k}+\hat{\Gamma}_{i j}{ }^{k}$ split into a real connection part $* \Gamma_{i j}{ }^{k}$ and an imaginary valued tensor $\hat{\Gamma}_{i j}{ }^{k}{ }^{281}$ His approach to UFT then differed considerably from Einstein's. In place

278 In fact, Bonnor used the Ricci tensor $P^{*}$ (cf. (75), but due to the field equations $\left(S_{i}=0\right)$ both tensors coincide.

279 Due to the approximation, the solution is not valid near the charge.

280 John Moffat (1932-) He obtained his PhD with Fred Hoyle and Abdus Salam. He has been a physics professor at the University of Toronto and also an adjunct Professor in physics at the University of Waterloo. He is a resident affiliate member of the Perimeter Institute for Theoretical Physics, Waterloo, Canada. His interests are alternative theories of gravitation, cosmology and (non-local) quantum field theory.

281 The notation is somewhat deceptive as the star is unrelated to complex conjugation; $* s^{i j} \simeq l^{i j}$, and his $* g_{i j} \simeq h_{i j}, \hat{g}_{i j} \simeq k_{i j}$. The hat-symbol has a different meaning from how it is used for tensor densities in this review. 
of (16) of Section 2.1.1 now

$$
\stackrel{\Gamma}{\nabla}_{k} X^{i}:=\frac{\partial X^{i}}{\partial x^{k}}+* \Gamma_{j k}^{i} X^{j}+\hat{\Gamma}_{j k}^{i} X^{j}=X_{; k}^{i}+\hat{\Gamma}_{j k}^{i} X^{j}
$$

where the semicolon indicates covariant derivation with regard to the real connection part. In place of (30) the compatibility condition

$$
g_{i j, k}-g_{r j} \Gamma_{i k}^{r}-g_{i r} \Gamma_{j k}^{r}=0
$$

was introduced. From this equation it turned out that the connection is formally akin to Hattori's (93), i.e., $\Gamma_{i j}^{s}\left[\delta_{s}^{k}+* s^{k r} a_{r s}\right]={ }^{H} L_{i k}{ }^{j}$ except that the imaginary $a_{i j}$ is entering on both sides; cf. Eqs. (8)-(10), p. 624 in [439]. It seems that Moffat did know neither Einstein's papers concerning UFT with a complex metric [147, 148] (see Section 7.2) nor Hattori's connection. This is unsurprising in view of his thesis advisors F. Hoyle and A. Salam which were not known as specialists in UFT.

With these complex valued mathematical objects, Moffat now built a "generalization of gravitation theory" [440] with the explicit purpose to find a theory yielding the correct equations of motion for charged particles (Lorentz force). ${ }^{282}$ Now, $g_{i j}:=* g_{i j}+\hat{g}_{i j}$ and $* s^{i r} * g_{r k}=\delta_{i}^{k}$. As a real Lagrangian, Moffat chose:

$$
\mathcal{L}=\sqrt{-g}\left[* g^{r s} * R_{r s}+\hat{g}^{r s} \hat{R}_{r s}\right]
$$

where presumably $\hat{g}^{r s}$ is defined by the decomposition of the inverse $* s^{i j}$ of $g_{i j}=* g_{r s}+\hat{g}_{r s}$ with $g_{i s} g^{k s}=\delta_{i}^{k}$, i.e., $g^{i j}=* s^{i j}+\hat{g}^{i j}$, although this relationship is not written down. His Ricci-tensors to be added to the list in Section 2.3.2 are the real and complex parts of $K_{j k}$ :

$$
\begin{gathered}
* R_{i j}:=-* \Gamma_{i j, r}^{r}+* \Gamma_{i r, j}^{r}+* \Gamma_{r j}{ }^{s} * \Gamma_{i s}^{r}-* \Gamma_{i j}^{r} * \Gamma_{r s}{ }^{s}+\hat{\Gamma}_{r j}{ }^{s} \hat{\Gamma}_{i s}^{r}-\hat{\Gamma}_{i j}^{r} \hat{\Gamma}_{r s}{ }^{s} \\
\hat{R}_{i j}:=-\hat{\Gamma}_{i j, r}^{r}+\hat{\Gamma}_{i r, j}^{r}+\hat{\Gamma}_{r j}{ }^{s} * \Gamma_{i s}^{r}-* \Gamma_{i j}^{r} \hat{\Gamma}_{r s}^{s}+* \Gamma_{r j}{ }^{s} \hat{\Gamma}_{i s}^{r}-\hat{\Gamma}_{i j}^{r} * \Gamma_{r s}^{s}
\end{gathered}
$$

W. Pauli's objection in its strict sense still applies in spite of the Lagrangian being a sum of irreducible terms.

For the field variables $\sqrt{-g} * g_{i j}, \sqrt{-g} \hat{g}_{i j}$, in empty space the field equations following from (474) are

$$
* R_{i j}=0, \hat{R}_{i j}=0 .
$$

Matter is introduced through the variation $\frac{\delta \mathcal{L}}{\delta g^{i j}}=-\frac{8 \pi}{c^{2}} \sqrt{-g} T_{i j}$ with "the complex-symmetric source term" $T_{i j}=G * T_{i j}+\hat{T}_{i j}, G$ the Newtonian gravitational constant. According to Moffat: "The real tensor $* T_{i j}$ represents the energy-momentum of matter, while $\hat{T}_{i j}$ is the charge-current distribution." A weak-field-approximation $g_{i j}=\eta_{i j}+h_{i j}+\gamma_{i j}$ with real $h_{i j}$ and imaginary $\gamma_{i j}$ is then carried through. In 1st approximation, the wave equation $\square \gamma_{i j}^{\prime}=16 \pi \hat{T}_{i j}$ resulted where $\gamma_{i j}^{\prime}:=\gamma_{i j}-\frac{1}{2} \delta_{i j} \gamma_{r r}$ and $\eta_{i j} \simeq-\delta_{i j}$. For slowly moving point particles and weak fields, MaxwellLorentz electrodynamics was reached. After an application of the EIH-approximation scheme up to the 6th approximation omitting cross tems between charge and mass, Moffat concluded: ${ }^{283}$

\footnotetext{
282 Some of the notation introduced previously [439] was changed by Moffat in [440]. The star referred to the real part, the hat to the imaginary part of an object. What had been the symbol for the covariant derivative with respect to $* \Gamma_{i j}{ }^{k}$ now denoted the partial derivative. Moffat made reading of his 2nd paper even more uncomfortable as he employed Einstein's commonly accepted notation for covariant derivatives $; i, ; i$ in a different sense: it now meant differentiation with respect to the real and imaginary parts of the connection.

283 The assumed use of a local geodesic coordinate system is highly dubious as such a system would change from one approximation to the next.
} 
"we have derived from the field equations the full Lorentz equations of motion with relativistic corrections for charged particles moving in weak and quasi-stationary electromagnetic fields." In a note added in proof he claimed that his method of winning the equations of motion was valid also for "quickly varying fields and fast moving particle" ([440], p. 487). In place of the ReissnerNordström metric, he obtained as a static centrally symmetric metric [441]:

$$
d s^{2}=\left(1-\frac{2 m}{r}\right) d t^{2}-\frac{\left(1-\frac{2 m}{r}\right)}{\left(1-\frac{2 m}{r}\right)^{2}+4 \frac{\epsilon^{2}}{r^{2}}}-r^{2} d \Omega^{2},
$$

where $\epsilon$ denotes the electric charge. Upon criticism by W. H. McCrea and W. B. Bonnor, Moffat included the "dipole procedure" of Einstein and Infeld in his derivation of the equations of motion [442]. R. P. Kerr found that the field equations (477), together with the boundary conditions at spacelike infinity, are not sufficient to determine the spherically symmetric solution. This holds even when four coordinate conditions are added [324].

In the 1950s, the difficulty with the infinities appearing in quantum field theory in calculations of higher order terms (perturbation theory) had been overcome by Feynman, Schwinger, Tomonoga and Dyson by renormalization schemes. Nevertheless, in 1952, Behram Kursunŏglu ${ }^{284}$ as a PhD student in Cambridge, UK, expressed the opinion,

"[...] that a correct and unified quantum theory of fields, without the need of the so-called renormalization of some physical constants, can be reached only through a complete classical field theory that does not exclude gravitational phenomena." ([343], p. 1396.)

So he looked for such a classical UFT and attempted to derive the "structure of the electron" from it. In Section 9.3.3 we already met Kursunŏglu's field equations, cf. (300)-(302), following from the Lagrangian:

$$
\mathcal{L}=\hat{g}^{r s} R_{r s}-2 p^{2}[\sqrt{-b}-\sqrt{-g}],
$$

where $b=\operatorname{det}\left(b_{k l}\right)$ and $b_{k l}$ is the inverse to the symmetric part $g^{(i j)}$ of the asymmetric metric $g_{i j}=a_{i j}+i q^{-1} \phi_{i j} .{ }^{285} \mathrm{I}$ assume that his Ricci tensor $R_{i j}$ is the same as the one used by Einstein in [149], i.e., $K_{j k}$.

By an approximation around Euclidean space $g_{i j}=-\delta_{i j}+h_{i j}+i \phi_{i j}$ which neglected the squares of $h_{i j}$, the cubes of $\phi_{i j}$, and interaction terms between $h_{i j}, \phi_{i j}$, Kursunŏglu obtained the following results:

$$
\begin{aligned}
& b_{i j}=-\delta_{i j}+h_{i j}-T_{i j}^{\prime}, \quad T_{i j}^{\prime}=\frac{1}{4} \delta_{i j} \Sigma_{r, s}\left(\phi_{r s} \phi_{r s}\right)-\Sigma_{r}\left(\phi_{i r} \phi_{j r}\right), \\
& \partial_{s} f_{s i}=J_{i}, \quad I_{l r s}=\Sigma_{t}\left(\epsilon_{l r s t} J_{t}\right), \quad f_{i j}=-\frac{1}{2} \Sigma_{k, l}\left(\epsilon_{i j k l} \phi_{k l}\right)=\partial_{i} A_{j}-\partial_{j} A_{i}, \\
& \frac{1}{2} \square h_{i j}+\frac{1}{2}\left[\delta_{i j} \Sigma_{r}\left(J_{r} J_{r}\right)-J_{i} J_{j}\right]+\Sigma_{r, s}\left(\partial_{r} \phi_{j s} \partial_{s} \phi_{i r}\right)+\partial_{i} \partial_{j}\left(\frac{1}{4} \Sigma_{r, s}\left(f_{r s} f_{r s}\right)\right) \\
& +\frac{1}{2}\left[\Sigma_{r}\left(f_{i r} J_{j r}\right)+\Sigma_{r}\left(f_{j r} J_{i r}\right)-\delta_{i j} \Sigma_{r, s}\left(f_{r s} J_{r s}\right)\right]=p^{2} T_{i j},
\end{aligned}
$$

284 Behram Kursunŏglu (1922 - 2003) graduated from the University of Edinburgh and received his doctoral degree in physics at the University of Cambridge. During the period of 1956-1958, he served as the dean of the Faculty of Nuclear Sciences and Technology at Middle East Technical University, Ankara. He held several teaching positions in the United States and, since 1958, a professorship at the University of Miami. In 1965, he was one of the co-founders of the Center for Theoretical Studies of the University of Miami, of which he became the first director.

${ }^{285} q^{-1} \phi_{i j}$ thus corresponds to $k_{i j}$ used here. 
where $J_{i j}=2 \partial_{[i} J_{j]}$, and $T_{i j}=-T_{i j}^{\prime}$. Both $\phi_{i j}$ and $f_{i j}$ are identified with the electromagnetic field:

$$
\left(\phi_{23}, \phi_{31}, \phi_{12} ; \phi_{41}, \phi_{42}, \phi_{43}\right)=\left(i E_{1}, i E_{2}, i E_{3} ; H_{1}, H_{2}, H_{3}\right) \text {. }
$$

By comparison of the equation for $h_{i j}$ with the Einstein field equations of general relativity, the relation $p^{2} q^{-2}=2 G / c^{4}$ with the gravitational constant $G$ ensued. Kursunŏglu then put the focus on the equation for the electrical current density derived from (302) after a lengthy calculation:

$$
\left(\square-\kappa^{2}\right) J_{k}=\kappa^{2} h J_{k}+w_{k}\left(h_{r s}, f_{l m}, J_{p}\right),
$$

where $w_{k}(\ldots)$ is a function describing the interaction terms; it consists of 11 products of $h$ with $f$ or $h$ or $J$ (and up to their 2 nd derivatives). The r.h.s. of (482) then was summarily replaced by

$$
j_{k}=\int_{-\infty}^{+\infty} d s e \delta^{4}(x-\xi) V_{k}(\xi)
$$

and "the electrostatic field due to an electron at rest" derived to be $|E|=\frac{e}{r^{2}}\left[1-e^{-\kappa r}-\kappa r e^{-\kappa r}\right]$. Kursunŏglu concluded that UFT "describes the charge density of an elementary particle as a short range field. It is not possible to measure the effects of an electron "radius" $\kappa^{-1}$ by having two electrons collide with an energy of the order of $m c^{2}$. Quantum theoretically the wavelength corresponding to this energy is $\frac{\hbar}{m c}$, which is much larger than $\kappa^{-1} . "$ ([343], p. 1375.)

As to the equations of motion, Kursunŏglu assumed that (30) is satisfied and, after some approximations, claimed to have obtained the Lorentz equation, in lowest order with an inertial mass $m_{0}=\frac{1}{2 c^{2}} \kappa e^{2}$ (cf. his equations (7.8)-(7.10)); thus according to him, inertial mass is of purely electromagnetic nature: no charge - no mass! Whether this result amounted to an advance, or to a regress toward the beginning of the 20th century is left open.

In a short note with G. Rickayzen, Kursunŏglu pointed out that the Born-Infeld non-linear electrodynamics followed from his "version of Einstein's generalized theory of gravitation" in the limit $p \rightarrow \infty$. In the note, a Lagrangian differing from (478) appeared:

$$
2 p^{2} \mathcal{L}=\hat{g}^{r s}\left(R_{r s}-i p^{2} B_{r s}\right)+2 p^{2}[\sqrt{-b}-\sqrt{-g}],
$$

where $B_{r s}=2 \partial_{[r} B_{s]}$ is an auxiliary field variable [509].

G. Stephenson from the University College in London altered Einstein's field equation as given in Appendix II of the 4th London edition of The Meaning of Relativity ${ }^{286}$ by replacing the constraint on vector torsion $S_{j}=0$ by $S_{r} S^{r}=a$ with constant $a$, and by introducing a vector-potential $A_{j}$ for the electromagnetic field tensor $k_{i j}$ [588]. His split of (30) for the symmetric part coincided with Tonnelat's (363), but differed for the skew-symmetric part from her (362) of Section 10.2.3. The missing term is involved in Stephenson's derivation of his result: Dirac's electrodynamics ${\left.\stackrel{\{}{V} \nabla_{s}^{k}\right\}}_{s} \breve{k}^{i s}=A^{i}$. Hence the validity of this result is unclear.

A year later, Stephenson delved deeper into affine UFT [589]. We quote from the review written by one of the opinion leaders, V. Hlavatý, for the Mathematical Reviews [MR0068357]:

"The Einstein paper contains three separate sections. In the first section the author expresses the symmetric part $\Lambda_{\lambda \mu}^{\nu}=\Gamma_{(\lambda \mu)}^{\nu}$ of the unified field connection $\Gamma_{\lambda \mu}^{\nu}$ by means of its skew symmetric part $S_{\lambda \mu}^{\nu}=\Gamma_{[\lambda \mu]}^{\nu}$ and vice versa. Then he identifies the electromagnetic field with $k_{\lambda \mu}=g_{[\lambda \mu]}$ and imposes on it the first set of Maxwell conditions

$$
\partial_{[\omega} k_{\mu \lambda]}=0 .
$$

286 For the various Princeton and London editions see the footnote in Section 9.2.2. 
The second set of Maxwell conditions is equivalent to the Einstein condition $S_{\lambda \alpha}^{\alpha}=0$. However, according to the author, there appears to be no definite reason for imposing the additional condition (1). In the second part the author considers Einstein's condition

$$
R_{[\mu \lambda]}=2 \partial_{[\mu} X_{\lambda]}
$$

coupled with

$$
R_{[\mu \lambda]}=-D_{\alpha} S_{\mu \lambda}^{\alpha}
$$

(where $D_{\alpha}$ denotes the covariant derivative with respect to $\Lambda_{\lambda \mu}^{\nu}$ ). Hence

$$
S_{\mu \lambda}^{\nu}=2 X_{[\mu} \delta_{\lambda]}^{\nu}+T_{\mu \lambda}^{\nu}
$$

where $T_{\mu \lambda}^{\nu}=T_{[\mu \lambda]}^{\nu}$ is a solution of

$$
D_{\alpha} T_{\mu \lambda}^{\alpha}=0
$$

Therefore $S_{\lambda \alpha}{ }^{\alpha}=0$ is equivalent to

$$
X_{\mu}=-\frac{1}{3} T_{\mu \alpha}{ }^{\alpha}
$$

and the field equations reduce to 16 equations (4) and $R_{(\mu \lambda)}=0$. In the third part the author considers all possibilities of defining $\Gamma_{\lambda \mu}^{\nu}$ by means of the derivatives of $g_{\lambda \mu}$ with all possible combinations of Einstein's signs $(++),(+-),(--)$. He concludes that in both cases (i.e. for real or complex $g_{\lambda \mu}$ ) only the $(+-)$ derivation leads to a connection $\Gamma_{\lambda \mu}^{\nu}$ without imposing severe restrictions on $g_{\lambda \mu}$."

\subsubsection{Unified field theory and classical spin}

Each of the three scientists described above introduced a new twist into UFT within the framework of - real or complex - mixed geometry in order to cure deficiencies of Einstein's theory (weak field equations). Astonishingly, D. Sciama ${ }^{287}$ at first applied the full machinery of metric affine geometry in order to merely describe the gravitational field. His main motive was "the possibility that our material system has intrinsic angular momentum or spin", and that to take this into account "can be done without using quantum theory" ([565], p. 74). The latter remark referred to the concept of a classical spin (point) particle characterized by mass and an antisymmetric "spin"tensor $s_{\alpha \beta}, \alpha, \beta=1,2,3$. Much earlier, Mathisson (1897-1940) [392, 391, 393], Weyssenhoff (1889-1972) [695, 694] and Costa de Beauregard (1911-2007) [87], had investigated this concept. For a historical note cf. [584]. Sciama did not give a reference to C. de Beauregard, who fifteen years earlier had pointed out that both sides of Einstein's field equations must become asymmetric if matter with spin degrees of freedom is generating the gravitational field. Thus an asymmetric Ricci tensor was needed. It also had been established that the deviation from geodesic motion of particles with charge or spin is determined by a direct coupling to curvature and the electromagnetic field $R_{j k l}^{i} \frac{d x^{j}}{d s} F^{k l}$ or, analogously, curvature and the classical spin tensor $R_{j k l}^{i} \frac{d x^{j}}{d s} s^{k l}$. The energymomentum tensor of a spin-fluid (Cosserat continuum), discussed in materials science, is skewsymmetric. What Sciama attempted was to geometrize the spin-tensor considered before just as another field in space-time.

\footnotetext{
287 Dennis William S. Sciama (1926-1999) was a British physicist who had earned his PhD in 1953 at Cambridge University with Paul Dirac. He taught at Cornell, King's College London, Harvard and the University of Texas at Austin, but mostly at Cambridge (1950s and 1960s) and the University of Oxford (1970s and early 1980s). In 1983, he became professor of Astrophysics at the International School of Advanced Studies (SISSA) in Trieste.
} 
Because he insisted on physical space as being described by Riemannian geometry, he had to cope with two geometries, the one with the full asymmetric metric $g_{i j}$, and space-time with metric $l_{i j}$ where $l_{r j} g^{(r k)}=\delta_{j}^{k}$, an attribution which we have seen before in the work of Lichnerowicz. This implied that spinless particles moved on geodesics of the metric $l_{i j}$, even if the gravitational field is generated by a massive spinning source, while spinning particles move on non-geodesic orbits determined by the non-symmetric connection. Sciama's field equations were:

$$
\begin{aligned}
g_{i k \|} \| l & =0, \\
+- & S_{i}=0, \\
K_{-}(L)-\frac{1}{2} g^{r s} K_{-r s}(L) g_{i k}+L_{i r, k}^{r}-L_{k r, i}^{r}-\frac{1}{2} g^{r s}\left(L_{r p, s}^{p}-L_{s q, r}^{q}\right) g_{i k} & =T_{i k},
\end{aligned}
$$

which, as he deemed, are "slightly different from the Einstein-Straus equations" ([565], p. 77). Conceptually, they are very different, because the matter tensor did not and in principle cannot enter the Einstein-Straus equations. Not very modestly, Sciama concluded that "further studies are required before one can decide whether the symmetric or the non-symmetric theory describes nature better." C. de Beauregard did not share Sciama's opinion concerning the motion of spinless particles; according to him, in linear approximation around flat space-time [89]:

$$
\frac{d u^{i}}{d s}=u_{l} \frac{d g^{[i l]}}{d s} .
$$

Perhaps, Sciama had convinced himself that mixed geometry was too rich in geometrical objects for the description of just one, the gravitational interaction. In any case, in his next five papers in which he pursued the relation between (classical) spin and geometry, he went into UFT proper [565]. He first dealt with the electromagnetic field which he identified with an expression looking like homothetic curvature: $V_{k l}=\stackrel{s}{K^{j}}{ }_{j k l}=\partial_{k} \Gamma_{l}-\partial_{l} \Gamma_{k}$. However, here $\Gamma_{k}:=L_{[k r]}^{r}=S_{k}$ In order to reach this result he had introduced a complex tetrad field ${ }^{288}$ and defined a complex curvature tensor $\stackrel{s}{K}^{i}{ }_{j k l}$ skew-symmetric in one pair of its indices and "skew-Hermitian" in the other. In analogy to Weyl's second attempt at gauge theory [692], he arrived at the trace of the tetrad-connection as his "gauge-potential" without naming it such. He also introduced a "principle of minimal coupling" as an equivalent to the "equivalence principle" of general relativity: matter must not directly couple to curvature in the Lagrangian of a theory. M.-A. Tonnelat and L. Bouche [646] then showed that Sciama's non-symmetric theory of the pure gravitational field [565] "implies that the streamlines of a perfect fluid $\left(T^{\mu \nu}=\rho v^{\mu} v^{\nu}\right)$ are geodesics of the Riemannian space with metric $g^{(i j)}$. These streamlines are not geodesics of the metric $g_{(i j)}$, but deviate from them by an amount which, in first approximation, agrees with a heuristic formula occurring in Costa de Beauregard's theory of the gravitational effects of spin [89]". ${ }^{289}$

In his next paper, Sciama described his endeavour of geometrizing classical spin within a general conceptual framework for unified field theory. His opening words made clear that he found it worthwhile to investigate UFT:

"The majority of physicists considers with some reserve unified field theory. In this article, my intention is to suggest that such a reserve is not justified. I will not

288 Not satisfied with the many names for a tetrad appearing in the literature, Sciama introduced a new one, namely eon which, however, did not catch on.

289 The sentence in quotes is taken from Sciama's review [MR0114629] in Mathematical Reviews of the paper of Tonnelat \& Bouche. 
explain or defend a particular theory but rather discuss the physical importance of non-Riemannian theories in general." ([566], p. 1.) ${ }^{290}$

Sciama's main new idea was that the holonomy group plays an important rôle with its subgroup, Weyl's $U(1)$, leading to electrodynamics, and another subgroup, the Lorentz group, leading to the spin connection. Although he gave the paper of Yang \& Mills [712] as a reference, he obviously did not know Utiyama's use of the Lorentz group as a "gauge group" for the gravitational field [661]. C. de Beauregard's reaction to Sciama's paper was immediate: he agreed with him as to the importance of embedding spin into geometry but did not like the two geometries introduced in [565]. He also suggested an experiment for measuring effects of (classical) spin in space-time [88].

In another paper of the same year, Sciama opted for a different identification of classical spin with geometrical structure: the skew-symmetric part of the connection no longer was solely connected with the electromagnetic field but with the spin angular moment of matter [567]. By introducing a field $\psi$ like a (classical) Dirac spinor, he defined the spin-flux as $S_{A B}{ }_{i}:=\frac{\partial \mathcal{L}}{\partial \psi_{, i}} \sigma_{A B} \psi$ where $\sigma_{A B}$ is a fitting representation of the Lorentz group. The indices $A, B=0,1,2,3$ are tetradindices ( real tetrad $e_{i}^{A}$ ), introduced by $g_{i j}=\eta_{A B} e_{i}^{A} e_{j}^{B}$. Seemingly, at that point Sciama had not known Cartan's calculus with differential forms and reproduced the calculation of tetrad connection and curvature tensor in a somewhat clumsy notation. The result of interest is:

$$
L_{[i j]}^{k}=S_{i j}^{k}+\delta_{[i}^{k} S_{j]}
$$

with $S_{i j}{ }^{k}=S_{A B}^{k} e_{i}^{A} e_{j}^{B}$. Use of a complex tetrad allowed him to define the electromagnetic field as before. At the time, he must have had an interaction with T. W. B. Kibble who's paper on "Lorentz Invariance and the Gravitational Field" introduced the Poincaré group as a gauge group ${ }^{291}$ [325]. Sciama's next paper did not introduce new ideas but presented his calculations and interpretation in further detail [568]. Two years later, when the ideas of Yang \& Mills and Utiyama finally had been accepted by the community as important for field theory, Sciama for the first time named his way of introducing the skew-symmetric part of the connection "the now fashionable "gauge trick" " ([569], p. 465, 466). His interpretation of UFT had changed entirely:

"We may note in passing that the result (7) [here Eq. (488)] suggests that unified field theories based on a non-symmetric connection have nothing to do with electromagnetism." ([569], p. 467)

C. de Beauregard had expressed this opinion three years earlier; moreover his doubts had been directed against the "unified theory of Einstein-Schrödinger-type" in total [90]. In the 1960s, the subject of classical spin and gravitation was taken up by F. W. Hehl [245] and developed into "Poincaré gauge theory" with his collaborators [246].

\footnotetext{
290 "La plupart des physiciens considèrent la théorie du champ unifié avec réserve. Mon intension, dans cet article, est de suggérer que cette réserve n'est pas justifiée. Je ne vais pas expliquer ou défendre une théorie particulière, mais plutôt discuter la signification physique des théories non Riemannienes en général."

291 Kibble received his doctorate in 1958 at Imperial College, London.
} 


\subsection{Australia}

H. A. Buchdahl ${ }^{292}$ in Tasmania, Australia, added a further definition for the electrical current, i.e., $\hat{j}^{k}=\hat{g}_{l, l}^{[k l]}$. Then, in linear approximation, from (211), the unacceptable restriction $\partial_{l} \partial^{l} j^{k}=0$ followed. In order to remedy this defect, Buchdahl suggested another set of field equations which, with an appropriate Lagrangian, did not imply any restriction on the thus defined electric current [64]:

$$
\begin{array}{r}
\hat{g}^{i k}{ }_{\| l}^{+}=0, g_{[i j]} \\
=\partial_{[k} A_{i]}, \hat{j}^{k}=\hat{g}_{, l}^{[k l]}, \\
\hat{B}^{(i j)}=0, \hat{B}_{, l}^{[i l]}=0,
\end{array}
$$

with $\hat{B}^{i j}=\delta \mathcal{L} / \delta g_{i j}, A_{k}$ an arbitrary vector. Unfortunately, from a linear approximation in which only the antisymmetric part of the metric is considered to be weak, an unacceptable result followed: "Consequently, if one wishes to maintain an unrestricted current vector is would seem that the introduction of a vector potential $A_{i}$ in the manner above must be abandoned." ([65], p. 1145.) With the asymmetric metric $g_{i j}$ having gauge weight +1 the determinant $g$ is of gauge weight +2 ( $+\frac{d}{2}$ for dimension $d$ of the manifold ).

Buchdahl then set out to build a gauge-invariant unified field theory by starting from Weyl space with symmetric metric $g_{i j}$ and linear connection $L_{i j}{ }^{k}=\left\{\begin{array}{l}k \\ i j\end{array}\right\}-\delta_{(i}^{k} k_{j)}+\frac{1}{2} g_{i j} k^{k}$. The gauge transformation is given by $g_{i j} \rightarrow \lambda g_{i j}, k_{i} \rightarrow k_{i}+\partial_{i}(\ln \lambda)$. Tensor densities now have both a coordinate weight $z$ [cf. (21) of Section 2.1.1], and a gauge weight $v$ defined via the covariant derivative by:

$$
\stackrel{+}{\nabla}_{k} \hat{X}^{i}=\frac{\partial \hat{X}^{i}}{\partial x^{k}}+L_{k r}^{i} \hat{X}^{r}-z L_{k r}^{r} \hat{X}^{i}-v k_{k} \hat{X}^{i}, \bar{\nabla}_{k} \hat{X}^{i}=\frac{\partial X^{i}}{\partial x^{k}}+L_{r k}^{i} \hat{X}^{r}-z L_{r k}^{r} \hat{X}^{i}-v k_{k} \hat{X}^{i}
$$

([65], p. 90). As a gauge-invariant curvature tensor and its contractions were used, the curvature scalar $R$ then is of gauge weight -1 . Consequently, a gauge-invariant Lagrangian density must contain terms quadratic in curvature like $\sqrt{-g} R^{2}$. Buchdahl used the gauge-invariant Hermitian Ricci tensor $-\stackrel{H}{K}_{i k}$ in Eq. (73) of Section 2.3.2, and the field equations [66]:

$$
\stackrel{\mathrm{Her}}{K}_{(i k)}=0, S_{i}=0 .
$$

Under scrutiny and by use of approximation methods and boundary conditions at (spatial) infinity, it turned out, according to H. A. Buchdahl, that these equations very likely did not have acceptable physical solutions ([66], p. 264). In view of the non-acceptance of Weyl's original gauge theory of the gravitational and electromagnetic fields, it is not surprising that Buchdahl's gauge-invariant UFT did not lead to much further research. One sequel was Mishra's paper [436] in which an exact solution in place of Buchdahl's approximate one for weak fields is claimed; closer inspection shows that it is only implicitly given (cf. Eq. (3.1), p. 84).

292 Hans A. Buchdahl (1919-2010), born in Mainz, Germany; sent to London in 1933 for higher education by his parents in view of the Nazi rule. After having obtained his degree at the London College of Science, in 1939, he was detained as a German National and deported to Australia in 1940. His abilities in mathematics were recognized soon and he became teaching assistant at the University of Tasmania in Hobart, part-time lecturer and research physicist. He received a doctorate there in 1948 and a DSc from Imperial College, London, in 1956. As a reader in Tasmania, he was called to become professor and head of the Department of Theoretical Physics at the Australian National University, in 1963 until retirement in 1984. His broad interests included geometrical optics, thermodynamics, theories of gravitation as well as tensor and spinor analysis. He wrote well received books in all of these fields. 


\subsection{India}

In a short note, the Indian theoretician G. Bandyopadhyay ${ }^{293}$ considered an affine theory using two variational principles such as Schrödinger [553] had sugested in 1946 [9]. Besides his Ricci tensor $R_{j k}$ corresponding to $K_{j k}$ of $(55)$ he used another one $\tilde{R}_{j k}$ turning out to be equal to: $\underline{K}_{j k}-2 \stackrel{+}{\nabla}_{j} S_{k}-V_{k j}$. The two Lagrangians used were $\left.\left.\mathcal{L}=\sqrt{\operatorname{det}\left(R_{j k}\right)}\right), \tilde{\mathcal{L}}=\sqrt{\operatorname{det}\left(\tilde{R}_{j k}\right)}\right)$. The resulting field equations were:

$$
\underline{K}_{i k-} \| l=0, \quad S_{i}=0 .
$$

A solution is given by

$$
K_{-}=\lambda g_{j k}, \quad g_{i k-} \| l=0 .
$$

R. S. Mishra ${ }^{294}$ refined Hlavatý's classification of the skew-symmetric part $k_{i j}$ by allowing all signatures ("indices of inertia") for the symmetric part $h_{i j}$ of the asymmetric metric and by splitting Hlavatý's third class into two [432]:

$$
\begin{aligned}
& \text { class 3: } k_{i s} \check{k}^{j s} \neq 0 \\
& \text { class 4: } k_{i s} \check{k}^{j s}=0, \quad k=0 .
\end{aligned}
$$

Here $k:=\frac{\operatorname{det}\left(k_{i j}\right)}{\operatorname{det}\left(h_{l m}\right)}$ and $K:=\frac{1}{4} k_{r s} \check{k}^{r s}$. He then studied the solutions of (30) for all classes and signatures and showed that for a Riemannian metric only the first two classes exist while for signature zero all four classes are possible. He also set out to show that the solution of M.A. Tonnelat (cf. Section 10.2.3) is valid only for the first class [434]. The conditions for Eqs. (30), or (448) to have a unique solution or to have at least one solution are derived and discussed in extenso in several further papers [431, 432, 346]. Tonnelat's conditions (364) are made more precise: $\frac{k}{h}>0, g\left(a^{2}+b^{2}\right) \neq 0: \frac{k}{h}<0, g \neq 0 ; k=0, g(g-2) \neq 0$. Except for re-deriving Tonnelat's result for class 1 (cf. [346], Eqs. (1.29)e, (1.30), p. 223), and polishing up Hlavatý's results by the inclusion of some degenerate subcases, e.g., for $(1+K)(1-3 K) \neq 0$ for $K^{2}=k$, no new mathematical ideas were introduced. Physics was not mentioned at all. Also, Mishra contradicted Kichenassamy's papers in which Tonnelat's results had been upheld contrary to criticism by Hlavatý [326, 327]. Like in Wrede's paper, Mishra considered the generalization of "the concepts of Einstein's unified field theory to n-dimensional space" as well and derived "some recurrence relations for different classes of $g_{\lambda \mu}$ " [435]. In another paper with S. K. Kaul, Mishra generalized Veblen's identities (71) of Section 2.3.1 to mixed geometry with asymmetric connection. The authors obtained 4 identities containing 8 terms each and with a mixture of \pm -derivatives [323]. I have seen no further application within UFT. From my point of view as a historian of physics, R. S. Mishra's papers are exemplary for estimable applied mathematics uncovering some of the structures of affine and/or mixed geometry without leading to further progress in the physical comprehension of unified field theory (cf. also [429, 296]).

The generation of exact solutions to the Einstein-Schrödinger theory became a fashionable topic in India since the mid 1960s. Following a suggestion of G. Bandyopadhyay, R. Sarkar assumed the

\footnotetext{
293 Gaganbihari Bandyopadhyay (? - ?). Formerly at Government College, Darjeeling, assistant professor of mathematics, Indian Institute of Technology, Kharakpur, and then Professor at the University of Calcutta in the Department of Applied Mathematics. Now retired.

294 Ratan Shanker Mishra (1918-?). Professor and Head of the Department of Mathematics at Gorakhpur and Allahabad from 1958-1963, and from 1963-1968. Head of the Department of Mathematics and Statistics at Banaras Hindu University in Varanasi since 1968. He has been a visiting professor in many countries, and worked and published with V. Hlavatý at Indiana University. His interests are well characterized by the title of his book Structures in a Differentiable Manifold (1978).
} 
asymmetric metric to have the form: ${ }^{295}$

$$
d s^{2}=H\left(d x^{0}\right)^{2}+2 I d x^{[1} d x^{0]}+\left(d x^{1}\right)^{2}+G\left(\left(d x^{2}\right)^{2}+\left(d x^{3}\right)^{2}\right)
$$

with $H, G$ and $I$ being functions of the single variable $x^{1}$ [526]. In Hlavatý's classification, the metric was of second class. In terms of physics, static, one-dimensional gravitational and electromagnetic fields were described. The particular set of solutions obtained consisted of metric components with algebraic functions of $\sinh \left(\frac{\sqrt{3 \lambda}}{2} x^{1}\right)$ and $\cosh \left(\frac{\sqrt{3 \lambda}}{2} x^{1}\right)$, and showed (coordinate?) singularities. As a physical interpretation, Sarkar offered the analogue to a Newtonian gravitating infinite plane. The limit $\lim _{\lambda \rightarrow 0}$ in the metric components led back to Bandyopadhyay's solution [7] referred to in Section 9.6.2 (with some printing errors removed by Sarkar):

$$
G=\left(k+\frac{3}{4} \sqrt{b} x^{1}\right)^{\frac{4}{3}}, \quad H=1 / b\left(k+\frac{3}{4} \sqrt{b} x^{1}\right)^{-\frac{2}{3}}\left[a-\frac{d^{2}}{\left(k+\frac{3}{4} \sqrt{b} x^{1}\right)^{\frac{8}{3}}}\right], \quad I=\frac{1}{\sqrt{b}} \frac{d}{\left(k+\frac{3}{4} \sqrt{b} x^{1}\right)^{\frac{5}{3}}}
$$

with constants $a, b, d, k$.

In a sequel [527], Sarkar used the asymmetric metric:

$$
d s^{2}=H\left(d x^{0}\right)^{2}+2 K d x^{[2} d x^{3]}+\left(d x^{1}\right)^{2}+G\left(\left(d x^{2}\right)^{2}+\left(d x^{3}\right)^{2}\right),
$$

where, again $G, H, K$ are functions of $x^{1}$. The solutions found are static and with coordinate singularities. To give just one metrical component:

$$
H=M^{-1}\left[C_{1} \exp (\mu x)+C_{2} \exp (-\mu x)\right]^{\frac{2}{3}} \times \exp \left[-\frac{2 L}{\mu \sqrt{C_{1} C_{2}}} \tan ^{-1} \sqrt{\frac{C_{1}}{C_{2}}} \exp (\mu x)\right]
$$

with $x=x^{1}, \mu=\sqrt{3 \lambda}, M, C_{1}, C_{2}$ constants. No physical interpretation was given.

In the same year 1965, H. Prasad and K. B. Lal engaged in finding cylindrically symmetric wave-solutions of the weak field equations (277), (278) with:

$$
h_{i j} d x^{i} d x^{j}=C\left[\left(d x^{0}\right)^{2}-\left(d x^{3}\right)^{2}\right]-A\left(d x^{1}\right)^{2}-B\left(d x^{2}\right)^{2}, k_{i j} d x^{i} d x^{j}=\left(\rho d x^{[1}+\sigma d x^{[2}\right)\left[d x^{3]}-d x^{0]}\right],
$$

where $A, B, C$ are functions of $x^{3}=z, x^{0}=t$, and $\rho, \sigma$ functions of $x^{3}-x^{0}=z-t$. The electromagnetic field is defined by $F_{i j}:=\frac{1}{2} \sqrt{-g} \epsilon_{i j r s} g^{r s}$. All 64 components of the connection were calculated, exactly. However, in order to determine the components of the Ricci-tensor, second and higher powers of $\rho, \sigma$ and their derivatives were omitted ("weak electromagnetic field"). Consequently, the solutions obtained, are only approximate. This holds also for solutions of the strong field equations (268) likewise considered. ${ }^{296}$ Sometimes, exact solutions were announced but given only implicitly, pending the solution of nonlinear 1st order algebraical or/and differential equations; for wave solutions cf. [347].

\footnotetext{
295 Here, $x^{0}$ is used instead of his $x^{4}$.

296 There are also three papers by N. N. Ghosh who tried products of functions depending on different coordinates for the components of the asymmetric metric in his attempt at solving the strong field equations [222, 223, 224]. Due to his awkward index notation and the many ad-hoc additional assumptions used, I could not find out what kind of new exact solutions he has found. A clearer presentation of his expressions for the symmetric and skew-symmetric parts of the metric would have helped; see also the brief remark on Gosh's 1st paper in Section 10.2.3.
} 


\section{Additional Contributions from Japan}

We already met Japanese theoreticians with their contributions to non-local field theory in Section 3.3.2, to wave geometry as presented in Section 4.3, and to many exact solutions in Section 9.6.1. The unfortunate T. Hosokawa showed, in the paper mentioned in Section 4.3, that "a group of motions of a Finsler space has at most 10 parameters" [287]. Related to the discussion about exact solutions is a paper by M. Ikeda on boundary conditions [299]. He took up Wyman's discussion of boundary conditions at spacelike infinity and tried to formulate such conditions covariantly. Thus, both spacelike infinity and the approach to it were to be defined properly. He expressed the (asymmetric) metric by referring it to an orthormal tetrad tetrad $g_{i j} \rightarrow a_{A B}=g_{i j} \xi_{A}^{i} \xi_{B}^{j}$, where $\xi_{A}^{i}$ are the tetrad vectors, orthonormalized with regard to $e_{A} \delta_{A B}, e_{A}= \pm 1$. The boundary condition then was $a_{A B} \rightarrow e_{A} \delta_{A B}$ for $\rho \rightarrow \infty$, where $\rho(P Q):=\int_{P}^{Q} \sqrt{\gamma_{i j} d u^{i} d u^{j}}$ and the integral is taken over a path from $P$ to $Q$ on a spacelike hypersurface $x^{k}=x^{k}\left(u^{1}, u^{2}, u^{3}, \sigma\right)$ with parameters $u^{i}$ and metric $\gamma$.

Much of the further research in UFT from Japan to be discussed, is concerned with structural features of the theory. For example, S. Abe and M. Ikeda generalized the concept of motions expressed by Killing's equations to a non-symmetric fundamental metric [1]. Although the Killing equations (43) remain formally the same, for the irreducible parts of $g_{a b}=h_{a b}+k_{a b}$, they read as:

$$
\mathcal{L}_{\xi} h_{a b}=2 \stackrel{h}{\nabla}_{(a} \check{\xi}_{b)}=0, \mathcal{L}_{\xi} k_{a b}=\xi^{c} \stackrel{h}{\nabla}_{c} k_{a b}+k_{b}^{c} \stackrel{h}{\nabla}_{a} \check{\xi}_{c}+k_{a}^{c} \stackrel{h}{\nabla}_{b} \check{\xi}_{c}=0 .
$$

In (497), $\check{\xi}_{c}=h_{c r} \xi^{r}$. All index-movements are done with $h_{a b}$. The authors derive integrability conditions for (497); it turns out that for space-time the maximal group of motions is a 6-parameter group. ${ }^{297}$

Possibly, in order to prepare a shorter way for solving (30), S. Abe and M. Ikeda engaged in a systematic study of the concomitants of a non-symmetric tensor $g_{a b}$, i.e., tensors which are functionals of $g_{a b}[301,300]$. A not unexpected result are theorems 7 and 10 in ([301], p. 66) showing that any concomitant which is a tensor of valence 2 can be expressed by $h_{a b}, k_{a b}, k_{a r} k_{b}^{r}, k_{a r} k_{s}^{r} k_{b}^{s}$ and scalar functions of $\frac{g}{h}, \frac{g}{k}$ as factors. In the second paper, pseudo-tensors (e.g., tensor densities) are considered.

A different mathematical interpretation of Hoffmann's meson field theory as a "unitary field theory" in the framework of what he called "sphere-geometry" was given by T. Takasu [598]. It is based on the re-interpretation of space-time as a 3-dimensional Laguerre geometry. The line element is re-written in the form

$$
d s^{2}=\left(d \xi^{1}\right)^{2}+\left(d \xi^{2}\right)^{2}+\left(d \xi^{3}\right)^{2}\left(\phi_{s}\left(x^{k}\right) d \xi^{s}+\phi_{4}\left(x^{k}\right) d t\right)^{2} .
$$

It can be viewed as "the common tangential segment of the oriented sphere with center $\xi^{k}$ and radius $\left.r=\int\left[\phi_{s} x^{k}\right) \frac{d \xi^{s}}{d t}+\phi_{4}\left(x^{k}\right)\right] d t \ldots "([599]$.

297 This is concordant with Maxwell's electrodynamics. 


\section{Research in Italy}

\subsection{Introduction}

In previous sections, we already have encountered several contributions from Italian researchers. The publication of the 3rd and 4th edition of Einstein's The Meaning of Relativity in an Italian translation in 1950 and $1953^{298}$ seems to have given a boost to research on UFT in Italy. Bruno Finzi ${ }^{299}$ went about a fresh derivation of the field equations (287) to (290). He started from the Lagrangian Einstein had used in the 4th Princeton edition of his book [156], i.e., $R_{i k}^{* *}$ [cf. Section 2.3.2, Eq. (83)]. However, he did not proceed by varying Einstein's Lagrangian with regard to the (asymmetric) metric but instead by varying its solenoidal and irrotational parts, separately [201]. While arriving at the correct result, his method is no less arbitrary than what Einstein had tried himself. As one of the major figures in research in the geometry of relativity and unified theories, Finzi became a favoured reviewer of UFT in Italy [473, 202, 203]. He made it very clear that the theory did not predict new empirical facts: "Until now, no prevision of verifiable new physical facts have emerged from this unified theory", ${ }^{300}$ but remained a firm believer in Einstein's unified theory:

"The charm of this theory lies in its generality, its simplicity, and, let's say it clearly, in its beauty, attributes which the utmost Einsteinian synthesis possesses, more than any other noted today." ([473], p. 306) $)^{301}$

In this spirit, contributions of mathematicians like I. Gasparini Cattaneo (1920-2011) [75], or A. Cossu (1922-2005) [85, 86], and more or less formal mathematical manipulations by Italian researchers played an important part in the work on UFT. The other leading elder figure Maria Pastori $^{302}$ and some of her students present a main example. Already in the 1930s, she had published on anisotropic and "conjugated" skew-symmetric tensors. Hattori's paper sparked her interest: she intended to reduce his two assumptions concerning skew-symmetric tensors to one [480, 482, 481]. Now in the 1950s, she studied the properties of the new tensorial objects appearing within UFT [483, 484, 485]. Elisa Brinis considered parallel transports conserving the scalar product of two vectors with a non-symmetric fundamental tensor [61].

B. Todeschini arrived at an inhomogeneous d'Alembert's equation in which the electromagnetic tensor coupled to torsion [609]. F. Graiff derived expressions for the commutation of the \pm derivatives; she also studied alternative forms for the electromagnetic tensor $\left(k_{i j}, R_{[i j]}\right.$, or its duals) in first and second approximation according to the scheme (499) given below [232, 233]. By adding a term to the Einstein-Straus Lagrangian depending only on the metric tensor, F. de Simoni showed how to trivially derive from a variational principle all the different systems of field equations of UFT including those suggested by Bonnor, or Kursunŏglu (cf. Section 13.1) [115]. Laura Gotusso generalized a theory suggested by Horváth with a Riemannian metric and

298 II significato della relatività, Torino: Einaudi (1950); 2nd ed. with appendix 2. Torino: Einaudi (1954).

299 Bruno Finzi (1899-1974). Graduation in 1919/20 in mathematics and industrial engineering at the University of Pavia. 1922 assistant of the mathematical physicist Umberto Cisotti at the Polytechnical Institute of Milano (Technical University). Professor of rational mechanics at the University of Milano in 1931 and later director of the mathematical Institute of the Politechnico. Contributions to classical fluid dynamics and aeronautics as well as to space-time-geometry. Established a sizeable school of reserchers in UFT.

300 "Nessuna previsione di nuovi fatti fisici accertabili ci è finora venuta da tale teorie unitaria"

301 "Il fascino di queste teorie sta nella loro generalità, nella loro semplicità, e, diciamolo pure, nella loro bellezza, caratteri questi che, più di ogni altra attualmente nota, possiede l'estrema sintesi einsteiniana."

302 Maria Pastori (1895-1975). After teaching at elementary school, she entered Scuola normale superiore di Pisa and graduated as number one. She continued teaching in middle school and in 1929 became regular assistant in mathematics at the University of Milano. With the exception of years at the University of Messina from 1934 to 1939, she spent her whole carrier at the university of Milano. Her contributions were mostly in tensor analysis and differential geometry but she also was interested in quantum mechanics and thermodynamics. 
the connection $L_{i k}^{l}=L_{(i k)}^{l}+F_{[i}^{l} j_{k]}$ where $j_{k}$ is the electrical current vector $j^{i}=\sigma \frac{d x^{i}}{d s}$. The torsion vector thus is proportional to the Lorenz force $S_{i}=\frac{1}{2} F_{i}{ }^{s} j_{s}$ and the autoparallels describe the motion of a charged point particle ${ }^{303}$ [285]. Gotusso generalized Horváth's theory by adding another tensor to the connection: $\stackrel{\text { Got }}{L}{ }_{i k}^{l}=L_{(i k)}{ }^{l}+F_{[i}{ }^{l} j_{k]}+U_{i k}{ }^{l}$ satisfying $U_{i k}{ }^{l} F^{i k}=0$. With regard to this connection $g_{\substack{i k \| l \\++}}=0$ [231]. The Ricci tensor belonging to the connection introduced was not calculated.

\subsection{Approximative study of field equations}

To P. Udeschini ${ }^{304}$ we owe investigations closer to physics. ${ }^{305}$ In a series of papers, he followed an approximative approach to the field equations of UFT by an expansion of the fundamental tensor starting from flat space:

$$
g_{i j}=\eta_{i j}+b_{i j}+c_{i j},
$$

where $b_{i j}, c_{i j}$ were assumed to be small of 1 st and 2 nd order. In linear approximation, the connection then read [652] as:

$$
\stackrel{1}{L}_{i j}^{k}=\frac{1}{2} \eta^{k r}\left(b_{r j, i}+b_{i r, j}-b_{j i, r}\right),
$$

whence follow the Eqs. (210) and (211) already obtained by Einstein and Straus. The field equations split into two groups related either to the gravitational potential $\left(b_{(i j)}\right)$ or to the electromagnetic field $F_{i j} \sim b_{[i j]}$. In the identification by Udeschini, i.e., $\epsilon^{i j r s} b_{[r s]}=: \psi F^{i j}$, where $\psi$ is a constant, the field equations then were:

$$
\square b_{(i j)}=0, \square b_{[i j]}=0, b_{[i r], s} \eta^{r s}=0 .
$$

The coordinate conditions $b_{(i r), s} \eta^{r s}-b_{r s, i} \eta^{r s}=0$ was used. In this approximation, the current density, $\hat{I}^{k}=\frac{1}{2} \epsilon^{k l m n} g_{m n, l}$ satisfies $\square \hat{I}^{k}=0$.

In 2nd approximation $([653,654,657]),(500)$ is replaced by:

$$
\stackrel{2}{L}_{i j}^{k}=\stackrel{1}{L}_{i j}^{k}+\frac{1}{2} \eta^{k r}\left(c_{r j, i}+c_{i r, j}-c_{j i, r}\right)-\eta^{k r}\left(b_{[s j]} \stackrel{1}{L}_{r i}{ }^{k}+b_{[i s]} \stackrel{1}{L}_{j r}{ }^{s}+b_{(r s)} \stackrel{1}{L}_{i j} s\right) .
$$

For $c_{i j}$, the field equations lead to an inhomogeneous wave equation $\square c_{(i j)}=B_{(i j)}$ with a lengthy expression for $B_{i j}$ built up from products of $b_{i j}$ and $\stackrel{1}{L}_{i j}{ }^{k}$ as well as squares of $\stackrel{1}{L}_{i j}{ }^{k}$. Further equations are $c_{[i r], s} \eta^{r s}=N_{i}$ and $\square \epsilon^{k l m n} c_{[m n], l}=\epsilon^{k l m n} B_{[m n], l}$, with $N_{i}$ being a sum of products of $b_{r s}$ and $b_{r s, t}$. For the electrical 4-current density, in 2nd approximation [658] $\square^{2} I^{k}=2 \epsilon^{k l m n} \eta^{r s} \eta^{p q} b_{[r m], p}\left(b_{(s n), q l}-b_{(n q), s l}\right)$. As a result, in the 2nd approximation the field equations for the gravitational and electromagnetic fields now intertwine. From his more general approach, Udeschini then reproduced the special case Schrödinger had treated in 1951 [558], i.e., $b_{(i j)}=0, c_{[i j]}=0$ : An electromagnetic field, small of first order, generates a gravitational field small of second order. The reciprocal case, i.e., a gravitational field small of first order cannot influence an electromagnetic field of second order [655].

\footnotetext{
303 A related kind of "unified" field theory was considered by Eisenhart; cf. (442) in Section 12.1.

304 Paolo Udeschini (1913 - 2006) Professor first at the University of Pavia (1950 - 1961); then professor for Rational Mechanics at the University of Milano.

305 We shall criticize him only in a particular case, e.g., when he defined the velocity of light by $c g_{00}$ where $c$ is the vacuum velocity of light and $g_{00}$ the time-time component of the metric. In second approximation, the velocity of light then is shown to obey a Poisson equation. Cf. [653], Section 4. Apparently, he did not know about early papers by Einstein, Nordström [457], and Ishiwara [306, 307].
} 
From this approximative approach, Udeschini calculated an additional term for the shift of the frequency $\nu$ of a spectral line by the unified field due to $g_{(00)}=b_{00}+c_{00}$ with $c_{00}=\psi^{2} \mu_{0}^{2} H^{2}$ and $H$ the polar magnetic field:

$$
\frac{\delta \nu}{\nu}=-\frac{1}{c^{2}} \delta U+\frac{1}{2} \psi^{2} \mu_{0}^{2}(\delta H)^{2} .
$$

$U$ is the Newtonian gravitational potential $[656,659,660]$. This result depends crucially on the interpretations for the gravitational and electromagnetic fields. If, in place of $g_{(i j)}$, the quantity $l_{i j}$ is chosen to describe the gravitational field (potential), then the 2nd term with the magnetic field drops out of (503) ([660], p. 446). Due to this and to further ambiguities, it makes no sense to test (503); at best, the constant $\psi$ eventually needed for other experiments could be determined.

L. Martuscelli studied the assignment of the electromagnetic tensor $F_{i j}$ to the quantity $R_{[i j]}$ [389]. In first approximation, $F_{i j}=\frac{1}{2} \square b_{[i j]}$, while in second approximation

$$
F_{i j}=\frac{1}{2} \square b_{[i j]}+\frac{1}{2} \square c_{[i j]}+A_{[i j]}
$$

with a lengthy expression for $A_{[i j]}$ again containing derivatives of products of $b_{i j}$ and $\stackrel{1}{L}_{i j}{ }^{k}$ and of products of $\stackrel{1}{L}_{i j}{ }^{k}$. A. Zanella wrote down a formal scheme of field equations which in any order $\mathrm{n}$ of the approximation looked the same as in the 2nd order field equations. The r.h.s. term in, e.g., $\square \stackrel{n}{c_{(i j)}}=\stackrel{n}{B}_{(i j)}$ contains combination of quantities obtained in all previous orders. Convergence was not shown [723].

\subsection{Equations of motion for point particles}

While Einstein refused to accept particles as singularities of the unified field, E. Clauser, P. Udeschini, and C. Venini in Italy followed Infeld (cf. Section 9.3.3) by assuming the field equations of UFT to hold only outside the sources of mass and charge treated as singularities:

"In the equations for the unified field, no energy-tensor has been introduced: only the external problem outside the sources of the unified field (masses and charges), assumed to be singularities, exists" ([659], p. 74). ${ }^{306}$

As mentioned in Section 10.3.2, Emilio Clauser (1917-1986) used the method of Einstein \& Infeld in order to derive equations of motion for point particles. In [81], he had obtained an integral formula for a 2-dimensional surface integral surrounding the singularities. With its help, Clauser was able to show that from Einstein's weak field equations for two or more "particles" all classical forces in gravitation and electromagnetism (Newton, Coulomb, and Lorentz) could be obtained [82]. In his interpretation, $g_{(i k)}$ stood for the gravitational, $g_{[i k]}$ for the electromagnetic field.

He expanded the fundamental tensor according to:

$$
\begin{aligned}
g_{00} & =1+\Sigma_{k=1} \lambda^{2 k} \alpha^{2 k} \stackrel{2 k}{g}_{00}, \\
g_{(m n)} & =-\delta_{m n}+\Sigma_{k=1} \lambda^{2 k} \alpha^{2 k} \stackrel{2 k}{g}(m n), \\
g_{[m n]} & =\Sigma_{k=1} \lambda^{2 k} \beta^{2 k}{\stackrel{k}{g_{[m n]}},} \\
g_{(0 m)} & =\Sigma_{k=1} \lambda^{2 k+1} \alpha^{2 k+1} \stackrel{2 k+1}{g}(0 m), \\
g_{[0 m]} & =\Sigma_{k=1} \lambda^{2 k+1} \beta^{2 k+1} \stackrel{2 k+1}{g}{ }_{[0 m]},
\end{aligned}
$$

306 "Nelle equazioni del campo unitario non viene introdotto alcun tensore energetico: esiste solo il problema unitario esterno alle sorgenti del campo (masse e cariche) assunta come singolarità [...]." 
where $m, n=1,2,3 ; \lambda \sim \frac{1}{c}$ with the vacuum-velocity of light $c .{ }^{307}$ In the $n$-th step of approximation, the field equations are:

$$
\stackrel{n}{R}_{(i k)}=0, \stackrel{n}{R}_{[i k], l}+\stackrel{n}{R}_{[k l], i}+\stackrel{n}{R}_{[l i], k}=0, \stackrel{n}{L}_{[i r]}^{r}=0 .
$$

In the symmetrical part of the fundamental tensor, only terms beginning with $\lambda^{4}$ contribute, in the skew-symmetric part terms from $\lambda^{3}$ on. The Newtonian and Coulomb forces exerted on on a "particle" from the others, appear in the terms $\sim \lambda^{4}$ together with a force independent of distance and not containing the masses of the "particles". After laborious calculations, the Lorentz force showed up in the terms $\sim \lambda^{6}$. This result is the very least one would have expected from UFT: to reproduce the effects of general relativity and of electrodynamics.

In a subsequent paper by Clauser, Einstein's weak system for the field equations of UFT was developed in every order into a recursive Maxwell-type system for six 3-vectors corresponding to electric and magnetic fields and intensities, and to electric and magnetic charge currents [83]. Quasi-stationarity for the fields was assumed.

C. Venini expanded the weak field equations by help of the formalism generated by Clauser and calculated the components of the fundamental tensor $g_{i k}$, or rather of $\gamma_{i k}:=\left(g_{(i k)}-\eta_{i k}-\right.$ $\frac{1}{2} \eta_{i k} \eta^{r s}\left(g_{(r s)}-\eta_{r s}\right)$, directly up to 2 nd approximation: $\stackrel{4}{\gamma}_{i k}, \stackrel{4}{\gamma}_{00}$, and $\stackrel{5}{\gamma}_{0 m}$ [672]. He applied it to calculate the inertial mass in 2nd approximation and obtained the corrections of special and general relativity; unfortunately the contribution of the electrostatic field energy came with a wrong numerical factor [673]. He also calculated the field of an electrical dipole in 2nd approximation [674]. Moreover, again by use of Clauser's equation of motion, Venini derived the perihelion precession for a charged point particle in the field of a second one. It depends on both the charges and masses of the particles. However, his formula is not developed as far as that it could have been used for an observational test [675]. In hindsight, it is astonishing how many exhausting calculations Clauser and Venini dedicated to determining the motion of point particles in UFT in view of the ambiguity in the interpretation and formulation of the theory.

$307 \lambda$ is introduced by $\tau=\lambda x^{0}$ for a hypersurface $\tau=$ const.; $c$ is measured in an inertial system. 


\section{The Move Away from Einstein-Schrödinger Theory and UFT}

Toward the end of the 1950s, we note tendencies to simplify the Einstein-Schrödinger theory with its asymmetric metric. Moreover, publications appear which keep mixed geometry but change the interpretation in the sense of a de-unification: now the geometry is to house solely alternative theories of the gravitational field.

Examples for the first class are Israel's and Trollope's paper ([308] and some of Moffat's papers [440, 441]. In a way, their approach to UFT was a backward move with its use of a geometry Einstein and Schrödinger had abandoned.

In view of the argument demanding irreducibility of the metric, Israel and Trollope returned to a symmetric metric but kept the non-symmetric connection:

"If, then, group-theoretical considerations are accepted as a basic guiding principle in the construction of a unified field theory, it will be logically most economical and satisfactory to retain the symmetry of the fundamental tensor $g_{i k}$, while admitting non-symmetrical $\Gamma_{i j}{ }^{k} . "([308]$, p. 778)

The Lagrangian was extended to contain terms quadratic in the curvature tensor as well:

$$
\left.\mathcal{L}=\sqrt{-g}\left(a \underline{\underline{K}}_{i j} g^{i j}+b \underset{-}{K}\right)^{2}+c \underline{\underline{K}}_{(i j)} \underline{K}_{(k l)} g^{i k} g^{j l}+d \underline{\underline{[}}_{[i j]}^{K_{[}} \underline{[k l]} g^{i k} g^{j l}\right),
$$

where $g^{i j}=g^{(i j)}$ and $K=K_{i j} g^{i j} ; a, b, \ldots$ arbitrary constants. The electromagnetic tensor is identified with $\underline{K}_{[i j]}$, and $L_{[i s]} \bar{s}=S_{i}$ "corresponds roughly to the 4-potential". The field equations, said to follow by varying $g^{i j}$ and $L_{[i j]}^{k}$ independently, are given by:

$$
\delta(\mathcal{L})=\hat{s}^{i j} \delta K_{(i j)}+\sqrt{-g} W_{r s} \delta g^{r s},
$$

with

$$
\begin{aligned}
\hat{s}^{i j} & =\sqrt{-g}\left[(a+2 b \underset{-}{K}) g^{i j}+2 c \underline{-}_{K^{[i j]}}+2 d \underline{-}^{(i j)}\right], \\
W_{i j} & =a\left(\underline{-}_{(i j)}-\frac{1}{2} \underset{-}{K} g_{i j}\right)+2 b \underset{-}{K}\left(\underline{K}_{(i j)}-\frac{1}{4} \underset{-}{K} g_{i j}\right)-2 c M_{i j}\left(\underline{K}_{[r s]}\right)-2 d M_{i j}\left(\underline{K}_{(r s)}\right) .
\end{aligned}
$$

$M_{i j}$ with $M_{i j} g^{i j}=0$ is the Maxwell energy-momentum-tensor calculated as if its argument were the electromagnetic field. For $b=c=d=0, W_{i j}$ reduces to the Einstein tensor. If $s_{i j}$ is defined by $s_{i r} \hat{s}^{j r}=\delta_{i}^{j} \sqrt{-\operatorname{det}\left(\hat{s}^{j r}\right)}$, an interpretation of $s_{i j}$ as the metric suggests itself. It corresponds to the definition of the metric by a variational derivative in the affine theories of Einstein and Schrödinger.

If $a \neq 0, b=0, c=0$ is assumed, and Schrödinger's star-connection (232) introduced, the field equations of Israel \& Trollope reduce to the system:

$$
\begin{gathered}
{\stackrel{\nabla}{\nabla_{s}}}^{*} \hat{s}^{i s}=0, S_{i}\left({ }^{*} L\right)=0, \hat{s}^{[i s]}{ }_{s}=0, \\
\underline{K}_{(i j)}=2 d M_{i j}\left(\underline{-}_{[r s]}\right), \underline{K}=0 .
\end{gathered}
$$

In the lowest order of an expansion $g_{i j}=\eta_{i j}+\epsilon \gamma_{i j}$, it turned out that the 3rd equation of (512) becomes one of Maxwell's equations, i.e., $\gamma^{i s}{ }_{, s}=0$, and the first equation of (513) reduces to $K_{(i j)}=0$. In an approximation up to the 4 th order, the Coulomb force and the equations of motion of charged particles in a combined gravitational and electromagnetic field were obtained. 


\subsection{Theories of gravitation and electricity in Minkowski space}

Despite her long-time work on the Einstein-Schrödinger-type unified field theory, M.-A. Tonnelat no longer seems to have put her sole trust in this approach: at the beginning of the 1960s, in her research group a new topic was pursued, the "Euclidean (Minkowskian) theory of gravitation and electricity", occasionally also named "theory of the graviton" [411]. In fact, she returned to the beginning of her research carrier: The idea of describing together quanta of spin 0, 1 and 2 in a single theory, like the one of Kaluza-Klein, about which she already had done research in the 1940s [616, 617] (cf. Section 10.1), seems to have been a primary motivation, cf. [638, 352]; in particular a direct analogy between vector and tensor theories as basis for a theory of gravitation. Other reasons certainly were the quest for an eventual quantization of the gravitational field and the difficulties with the definition of a covariant expression for energy, momentum and stresses of the gravitational field within general relativity [644]. Tonnelat also may have been influenced by the continuing work concerning a non-standard interpretation of quantum mechanics in the group around de Broglie. In the context of his suggestion to develop a quantum mechanics with non-linear equations, de Broglie wrote Einstein on 8 February 1954:

"Madame Tonnelat, whose papers on the unitary theories you know well, is interested with Mr. Vigier ${ }^{308}$ and myself in these aspects of the quantum problem, which of course are very difficult." 309

As mentioned by Tonnelat, the idea of developing a theory of gravitation with a scalar or vector potential in Minkowski space went back to the first decade of the 20th century ${ }^{310}$ [641]. At the same time, in 1961, when Tonnelat took up the topic again, W. Thirring investigated a theory in which gravitation is described by a tensor potential (symmetric tensor of rank 2) in Minkowski space. The allowed transformation group reduces to linear transformations, i.e., the Poincaré group. He showed that the Minkowski metric no longer is an observable and introduced a (pseudo-)Riemannian metric in order to make contact with physical measurement [603]. This was the situation Tonnelat and her coworkers had to deal with. In any case, her theory was not to be seen as a bi-metric theory like N. Rosen's [515, 516], re-discovered independently by M. Kohler [335, 336, 337], but as a theory with a metric, the Minkowski-metric, and a tensor field (potential) describing gravitation [638]. Seemingly, without knowing these approaches, Ph. DrozVincent suggested a bi-metric theory and called it "Euclidean approach to a metric" in order to describe a photon with non-vanishing mass [127]. In view of the difficulties coming with linear theories of gravitation, Tonnelat was not enthusiastic about her new endeavour ([641], p. 424):

"[...] a theory of this type is much less natural and, in particular, much less convincing than general relativity. It can only arrive at a more or less efficient formalism with regard to the quantification of the gravitational field." 311

A difficulty noted by previous writers was the ambiguity in choosing the Lagrangian for a tensor field. The most general Lagrangian for a massive spin-2 particle built from all possible invariants quadratic and homogeneous in the derivatives of the gravitational potential, can be obtained from a paper of Fierz and Pauli by replacing their scalar field $C$ with the trace of the gravitational

\footnotetext{
308 Jean-Pierre Vigier (1920-2004).

309 This again is a translation into English from the translation of the presumably French original into German by H. Sievers ([580], Appendix A.2.9).

310 Nordström's papers of 1912 to 1914 are referred to by her only in the references to Chapter XIII, p. 457. We add the paper by Einstein \& Fokker [169].

311 "[...] une théorie de ce genre est beaucoup moins naturelle et, partant, beaucoup moins convaincante que la relativité générale. Elle ne peut aboutir qu' à un formalisme plus ou moins efficace relatif au champ de gravitation quantifié."
} 
tensor potential: $C=\alpha \psi_{s}{ }^{s}, \alpha$ a proportionality-constant ([196], p. 216). ${ }^{312}$ Without the mass term, it then contained three free parameters $\alpha, a_{1}, a_{3}$. After fixing the constants in the Pauli-Fierz Lagrangian, Thirring considered:

$$
L=\frac{1}{2}\left[\psi_{p q, r} \psi^{p q, r}-2 \psi_{p q, r} \psi^{r q, p}+2 \psi_{p q}{ }^{p}{ }^{p} \psi_{r}^{r, q}-\psi_{r}^{r, q} \psi_{s, q}^{s}\right]-\frac{1}{2} M^{2}\left[\psi_{p q} \psi^{p q}-\psi_{r}^{r} \psi_{s}^{s}\right],
$$

where $M$ denotes a mass parameter.

Tonnelat began with a simpler Lagrangian: ${ }^{313}$

$$
\frac{1}{\sqrt{-g}} \mathcal{L}=\frac{1}{4}\left[\psi_{p q, r} \psi^{p q, r}-\psi_{r q, p} \psi^{p q, r}\right]-\frac{M^{2}}{2} \psi_{p q} \psi^{p q}
$$

where $\psi_{p q}$ is the gravitational potential. A more general Lagrangian than (515) written up in further papers by Tonnelat and Mavridès with constants $a, b, c$, and the matter tensor $M^{p q}$ [412, 640] corresponds to an alternative to the Pauli-Fierz Lagrangian which is not ghost-free: ${ }^{314}$

$$
\left.L=\frac{1}{4} \psi_{p q, r} \psi^{p q, r}+\frac{a}{2} \psi_{p r}{ }^{r} \psi_{, s}^{p s}+b \psi_{p q}^{, p} \psi_{r}^{r, q}+\frac{c}{2} \psi_{r}^{r, q} \psi_{s, q}^{s}\right]-\chi M^{p q} \psi_{p q} .
$$

The field equations of the most general case are easily written down. They are linear wave equations

$$
\square \psi_{p q}+\operatorname{lin}_{p q}\left(\partial_{r} \partial_{s} \psi_{m n}, a, b, c\right)=\chi T_{p q},
$$

with a tensor-valued linear function lin of its argument $\partial_{r} \partial_{s} \psi_{m n}$ also containing the free parameters. $T_{p q}\left(=M_{p q}\right)$ is the (symmetric) matter tensor for which, from the Lagrangian approach follows $\partial_{r} T^{k r}=0$. However, this would be unacceptable with regard to the conservation law for energy and momentum if matter and gravitational field are interacting; only the sum of the energy of matter and the energy of the tensor field $\psi_{m n}$ must be conserved:

$$
\partial_{r}\left(T^{k r}+t^{k r}\right)=0 .
$$

The so-called canonical energy-momentum tensor of the $\psi$-field is defined by

$$
t_{k}^{i}:=\frac{\partial \mathcal{L}}{\partial \psi_{r s, i}} \psi_{r s, k}-\delta_{k}^{i} \mathcal{L},
$$

and is nonlinear in the field variable $\psi$. For example, if in the general Lagrangian (516) $a=1, b=$ $-1, c=0$ are chosen, the canonical tensor describing the energy-momentum of the gravitational field is given by ([73], Eq. (1.3), p. 87) ${ }^{315}$ :

$$
t_{i k}=\frac{1}{2} \partial_{i} \psi_{r s} \partial_{k} \psi^{r s}-\frac{1}{4} \eta_{i k} \partial_{m} \psi_{r s} \partial^{m} \psi^{r s}-\frac{1}{4} \partial_{i} \psi \partial_{k} \psi+\frac{1}{8} \eta_{i k} \partial_{m} \psi \partial^{m} \psi,
$$

where $\psi:=\eta^{r s} \psi_{r s}$. As a consequence, (517) will have to be changed into

$$
\square \psi_{p q}+\operatorname{lin}_{p q}\left(\partial_{r} \partial_{s} \psi_{m n}, a, b, c\right)=\chi T_{p q}+t_{p q},
$$

312 Pauli and Fierz used a traceless symmetric tensor field as the gravitational potential.

313 In contrast to our identification of $A_{, k}=\partial_{k} A$, Tonnelat and Mavridès use the comma differently when denoting the gravitational field by the three-index-variable $\psi_{p q, r}:=\partial_{p} \psi_{q r}-\partial_{q} \psi_{p r}$. In her papers, the partial derivative is denoted strictly by $\partial_{k}$ or by $\nabla_{k}[412,640]$. Indices are raised and lowered with the Minkowski metric.

314 Note, that the 2nd term in Thirring's expression (514) is also contained in (516) due to Tonnelat's different notation. In another paper, even 4 free parameters were used: the first term in (516) obtained a free parameter of its own. Cf. [413]. All of Thirring's terms are contained in a Lagrangian given by S. Lederer and decorated by her with free parameters $\tau, \sigma, \gamma, \nu$ ([354], Eq. (III.23) p. 256). After the Lagrangian (516) is multiplied by 2 and compared with Lederer's, then her parameters $\sigma, \gamma, \nu$ correspond to $a, 2 b, c$, respectively.

315 A. Capella multiplied the Lagrangian by a factor $1 / 2$. 
which is a nonlinear equation. It is possible to find a new Lagrangian from which (521) can be derived. This process can be repeated ad infinitum. The result is Einstein's theory of gravitation as claimed in [238]. This was confirmed in 1968 by a different approach [118] and proved - with varying assumptions and degrees of mathematical rigidity - in several papers, notably [117] and [684]. In view of this situation, the program concerning linear theories of gravitation carried through by Tonnelat, her coworker S. Mavridès, and her PhD student S. Lederer could be of only very limited importance. This program, competing more or less against other linear theories of gravitation proposed, led to thorough investigations of the Lagrangian formalism and the various energy-momentum tensors (e.g., metric versus canonical). The (asymmetrical) canonical tensor does not contain the spin-degrees of freedom of the field; their inclusion leads to a symmetrical, so-called metrical energy-momentum tensor [17]. Which of the two energy-momentum-tensors was to be used in (521)? The answer arrived at was that the metrical energy-momentum-tensor tensor must be taken $[647,355,354] .{ }^{316}$

"In an Euclidean theory of the gravitational field, the motion of a test particle can be associated to conservation of mass and energy-momentum only if the latter is defined through the metrical tensor, not the canonical one" [647], p. 373). ${ }^{317}$

Because (518) is used to derive the equations of motion for particles or continua, this answer is important. In the papers referred to and in further ones, equations of motion of (test-) point particle without or within (perfect-fluid-)matter were studied. Thus, a link of the theory to observations in the planetary system was established [411, 413, 414]. In a paper summing up part of her research on Minkowskian gravity, S. Lederer also presented a section on perihelion advance, but which did not go beyond the results of Mme. Mavridès ([354], pp. 279-280). M.-A. Tonnelat also pointed to a way of making the electromagnetic field influence the propagation of gravitational waves by introducing an induction field $\hat{F}^{p q, r}$ for gravitation [639]. In the presence of matter, she defined the Lagrangian

$$
\mathcal{L}_{\text {mat }}=\frac{\sqrt{-\eta}}{4 \epsilon^{\prime}\left(\mu^{\prime}\right)^{2}} b^{p s} b^{q t} b^{r v} \psi_{p q, r} \psi_{s t, v}+\chi \psi^{p q} M_{p q}
$$

with

$$
b^{p q}:=\eta^{p q}+\left(\epsilon^{\prime} \mu^{\prime}-1\right) u^{p} u^{q}, \quad b_{p q}=\eta_{p q}-\frac{\epsilon^{\prime} \mu^{\prime}-1}{\epsilon^{\prime} \mu^{\prime}} u_{p} u_{q} .
$$

In $(523) u^{p}$ is the 4 -velocity of matter and $\epsilon^{\prime}, \mu^{\prime}$ constants corresponding now to a gravitational dielectric constant and gravitational magnetic permeability. The gravitational induction was $\hat{F}^{p q, r}=2 \frac{\partial \mathcal{L}_{\text {mat }}}{\partial \psi_{p q, r}}$ and the field equations became:

$$
\partial_{r} F^{r p, q}=\chi M^{p q}
$$

As M.-A. Tonnelat wrote:

"These, obviously formal, conclusions allow in principle to envisage the influence of an electromagnetic field on the propagation of the 'gravitational rays', i.e., a phenomenon inverse to the 2nd effect anticipated by general relativity" ([639], p. 227). ${ }^{318}$

\footnotetext{
316 There exists a paper in which, in (521), even a linear combination of both tensors is used [499].

317 "Dans une théorie euclidienne du champ de gravitation, le mouvement d'une particule d'épreuve ne peut donc être associé à la conservation de la masse et de l' impulsion-énergie qu'en définissant cette dernière au moyen du tenseur métrique, et non du tenseur canonique."

318 "Ces conclusions - évidemment formelles - permettraient en prévoir l'influence d'un champ électromagnétique sur la propagation de 'rayons de gravitation', c'est à dire un phénomène réciproque du deuxième effet prévu par la relativité générale."
} 
Tonnelat's doctoral student Huyen Dangvu worked formally closer to Rosen's bi-metric theory [107]. In the special relativistic action principle $\delta\left(-m c \int d s+\frac{1}{c} \int \mathcal{L}\right)=0$, he replaced the metric $\eta_{i j}$ by a metric $g_{i j}$ containing the gravitational field tensor $\phi_{i j}: g_{i j}=\eta_{i j}+\phi_{i j}$. This led to

$$
\delta_{g} I=0 ; I=-\mu_{0} c \int d s \sqrt{g_{i j} u^{i} u^{j}}+\frac{1}{c} \int d^{4} x \mathcal{L}\left(g_{i j}, \partial_{k} g_{i j}, \partial_{k} \partial_{l} g_{i j}\right),
$$

from which one group of field equations followed:

$$
L_{i j}=\mu_{0} c^{2} \frac{u^{i} u^{j}}{\sqrt{1+2 \psi_{r s} u^{r} u^{s}}},
$$

with $L_{i j}=\frac{1}{\sqrt{-g}}\left\{\frac{\partial \mathcal{L}}{\partial \phi_{i j}}-\partial_{r}\left(\frac{\partial \mathcal{L}}{\partial\left(\partial_{r} \phi_{i j}\right)}+\partial_{r} \partial_{s}\left(\frac{\partial \mathcal{L}}{\partial\left(\partial_{r} \partial_{s} \phi_{i j}\right)}\right)\right)\right\}$ and $u^{j}=\frac{d x^{j}}{d s}, g_{i j} u^{i} u^{j}=1$. The second group of field equations is adjoined ad hoc (in analogy with Maxwell's equations:

$$
g_{i r} \Delta_{j k}^{r}+g_{j r} \Delta_{i k}^{r}=2 \stackrel{g}{\nabla}_{k} \psi_{i j},
$$

where $\Delta_{i j}{ }^{k}=\frac{1}{2} g^{k s}\left(\partial_{i} g_{j s}+\partial_{j} g_{i s}-\partial_{s} g_{i j}\right)$. No further consequences were drawn from the field equations of this theory of gravitation in Minkowski space called "semi-Einstein theory of gravitation" after a paper of Painlevé of 1922, an era where such a name still may have been acceptable.

In the mid-1960s, S. Mavridès and M.-A. Tonnelat applied the linear theory of gravity in Minkowski space to the two-body problem and the eventual gravitational radiation sent out by it. Havas \& Goldberg [241] had derived as classical equation of motion for point particles with inertial mass $m_{A}, A=1,2, \ldots, n$ and 4 -velocity $u_{A}^{i}$ :

$$
m_{A} \eta_{i r} \dot{u}_{A}^{r}+\frac{11}{3} G m_{A}^{2} \eta_{i r}\left(\ddot{u}_{A}^{r}-u_{A}^{i} \eta_{l m} u_{A}^{l} \ddot{u}_{A}^{m}\right)=f_{i}\left(\partial_{m} g_{r s}^{\mathrm{ret}}\right),
$$

where $f_{i}$ is a functional of the derivatives of the retarded potential. The second term on the left hand side led to self-acceleration. In a calculation by S. Mavridès in the framework of a linear theory in Minkowski space with Lagrangian:

$$
L=\psi_{p q, r} \psi^{p q, r}+k \psi_{r}^{r, q} \psi_{s, q}^{s},
$$

the radiation-term was replaced by

$$
\frac{10+42 k}{3(1+k)} \frac{\chi c^{2}}{16 \pi} \kappa^{2} M_{A}^{2} \eta_{i r}\left(\ddot{u}_{A}^{r}-u_{A}^{i} \eta_{l m} u_{A}^{l} \ddot{u}_{A}^{m}\right),
$$

with $\chi, \kappa$ coupling constants and $k$ a numerical constant, $M_{A}$ is connected with gravitational mass [415]. No value of $k$ can satisfy the requirements of leading to the same radiation damping as in the linear approximation of general relativity and to the correct precession of Mercury's perihelion. By proper choice of $k$, a loss of energy in the two-body problem can be reached. Thus, in view of the then available approximation and regularization methods, no uncontested results could be obtained; cf. also [416]; [643], pp. 154-158; [644], pp. 86-90).

\subsection{Linear theory and quantization}

Together with the rapidly increasing number of particles, termed elementary, in the 1950s, an advancement of quantum field theories needed for each of the corresponding fundamental fields was imperative. No wonder then that the quantization of the gravitational field to which particles of spin 2 were assigned also received attention. Seen from another perespective: The occupation 
with attempts at quantizing the gravitational field in the framework of a theory in Minkowski space reflected clearly the external pressure felt by those busy with research in UFT. Until then, the rules of quantization had been successful only for linear theories (superposition principle). Thus, unitary field theory would have to be linearized and, perhaps, loose its geometrical background: in the resulting scheme gravitational and electromagnetic field become unrelated. The equations for each field can be taken as exact; cf. ([641], p. 372). For canonical quantization, a problem is that manifest Lorentz-invariance usually is destroyed due to the definition of the canonical variable adjoined to the field $\psi_{i j}$ :

$$
\Pi_{i j}:=\frac{\partial L}{\partial\left(\frac{\partial \psi_{i j}}{\partial t}\right)}, \Pi:=\frac{\partial L}{\partial\left(\frac{\partial \psi}{\partial t}\right)},
$$

where we referred to Tonnelat's Lagrangian (529) with $k=-\frac{1}{2}, \psi=\psi_{r}^{r}, t=\frac{1}{c} x^{0}$.

A. Lichnerowicz used the development of gravitational theories in Minkowski space during this period for devising a relativistic method of quantizing a tensor field $H_{\alpha \beta, \lambda \mu}(x)$ simulating the properties of the curvature tensor. ${ }^{319}$ In particular, the curvature tensor was assumed to describe a gravitational pure radiation field such that

$$
l^{\alpha} H_{\alpha \beta, \lambda \mu}=0, l_{\{\alpha} H_{\beta \gamma\}, \lambda \mu}=0, H_{\beta \mu}:=H_{\beta, \rho \mu}^{\rho}=0 .
$$

$l_{\alpha}$ is a null vector field tangent to the lightcone $l_{\alpha \beta} d x^{\alpha} d x^{\beta}=0$. Indices are moved with $l_{\alpha \beta}$ and $l^{\alpha \beta}$ where $l_{\alpha \rho} l^{\beta \rho}=\delta_{\alpha}^{\beta}$; cf. (4) and Section 10.5.3. Let $K_{\alpha \beta, \lambda \mu}(l)$ be the Fourier transform of $H_{\alpha \beta, \lambda \mu}(x)$ and build it up from plane waves:

$$
K_{\alpha \beta, \lambda \mu}(l)=\Sigma_{A, B} a(A, B, \vec{l})\left(l_{\alpha} n_{\beta}^{A}-l_{\beta} n_{\alpha}^{A}\right)\left(l_{\lambda} n_{\mu}^{B}-l_{\mu} n_{\lambda}^{B}\right)
$$

where $\mathbf{A}, \mathbf{B}=\mathbf{1}, \mathbf{2}$ and $n_{\alpha}^{A}$ are spacelike orthogonal and normed vectors in the 3 -space touching the lightcone along $l_{\alpha}$. The amplitudes $a(A, B, \vec{l})$ are then replaced by creation and annihilation operators satisfying the usual commutation relations [373], ([382], pp. 127-128). Lichnerowicz' method served as a model for his and M.-A. Tonnelat's group in Paris. We are interested in this formalism in connection with Kaluza-Klein theory as a special kind of UFT.

The transfer to Kaluza-Klein theory by Ph. Droz-Vincent was a straightforward application of Lichnerowicz' method: in place of (532):

$$
K_{\alpha \beta, \lambda \mu}(l)=\Sigma_{A, B} a(A, B, \vec{l})\left(l_{\alpha} n_{\beta}^{A}-l_{\beta} n_{\alpha}^{A}\right)\left(l_{\lambda} n_{\mu}^{B}-l_{\mu} n_{\lambda}^{B}\right)
$$

where now $\mathbf{A}, \mathbf{B}=\mathbf{0}, \mathbf{1}, \mathbf{2}$ and $x^{0}$ is the 4 th spacelike coordinate; the Greek indices are running from 0 to 4 . In the tensor $H_{\alpha \beta, \lambda \mu}(x)$ in $(1,4)$-space, through $H_{\alpha 0, \lambda \mu}(x)=\beta \partial_{\alpha} F_{\lambda \mu}, \beta$ a constant, also the electromagnetic field tensor $F_{\lambda \mu}$ is contained such that both, commutation relations for curvature and the electromagnetic field, could be obtained [129]. In a later paper, $\beta^{2}=2 \chi$ was set with $\chi$ being the coupling constant in Einstein's equations [133]. The commutation relations for the electromagnetic field $F_{i j}$ were ${ }^{320}$ :

$$
\left[F_{i j}(x), F_{l m}\left(x^{\prime}\right)\right]=\Sigma \eta_{l[i} \partial_{j] m} \mathcal{D}\left(x-x^{\prime}\right) .
$$

This would have to be compared to the Gupta-Bleuler formalism in quantum electrodynamics.

\footnotetext{
${ }^{319}$ His Greek indices run from 0 to 3 . We replace his symbol for cyclic permutation by $\{123\}$.

320 The Pauli-Jordan distribution $\mathcal{D}\left(x-x^{\prime}\right)$ is a particular solution of the homogeneous Klein-Gordon equation with the representation in wave-vector space $\mathcal{D}(x)=\frac{1}{2(2 \pi)^{3}} \int \frac{d^{3} k}{k_{0}}[\exp (-i \vec{x} \cdot \vec{k})-\exp (i \vec{x} \cdot \vec{k})]=-\frac{i}{(2 \pi)^{3}} \int d^{4} k\left[\theta\left(k^{0}\right)-\right.$ $\left.\theta\left(-k^{0}\right)\right] \delta\left(k_{r} k^{r}-m^{2}\right)\left[\exp \left(-i k_{r} x^{r}\right)\right.$.
} 
For linearized Jordan-Thiry theory, Droz-Vincent put [129, 134]:

$$
\sqrt{\gamma} \gamma^{\mu \nu}=\eta^{\mu \nu}-K \alpha^{\mu \nu} \quad \mu, \quad \nu=0,1, \ldots, 4
$$

for the metric density of $V^{5}$ and obtained the commutation relations:

$$
\left[\alpha_{\sigma \kappa}(x), \alpha_{\lambda \mu}\left(x^{\prime}\right)\right]=\beta^{2}\left(P_{\sigma \lambda} P_{\kappa \mu}+P_{\sigma \mu} P_{\kappa \lambda}\right) \mathcal{D}\left(x-x^{\prime}\right)
$$

with $P_{\sigma \lambda}=\eta_{\sigma \lambda}+\frac{1}{\epsilon^{2}} \frac{\partial^{2}}{\partial_{\sigma} \partial_{\lambda}}$ and the mass parameter $\epsilon^{2}$ introduced into the Klein-Gordon equation but not following from the field equations. In space-time, from (536):

$$
\sqrt{g} g^{i j}=\eta^{i j}-K \alpha^{i j}, \beta \phi_{i}=-K \alpha_{0 i}
$$

and

$$
\left[\phi_{i}(x), \phi_{j}\left(x^{\prime}\right)\right]=-K^{2} P_{i j} \mathcal{D}\left(x-x^{\prime}\right) .
$$

The relation to (534) is provided by $\beta \partial_{\mu} F_{i j}=K H_{\mu 0 i j}$. The tensor $H_{\alpha \beta \lambda \mu}=-\left(\partial^{2}{ }_{\sigma \lambda} \alpha_{\kappa \mu}-\right.$ $\partial^{2}{ }_{\sigma \mu} \alpha_{\kappa \lambda}+\partial^{2}{ }_{\kappa \mu} \alpha_{\sigma \lambda}-\partial^{2}{ }_{\sigma \lambda} \alpha_{\kappa \mu}$ ) corresponds to $H_{\alpha \beta, \lambda \mu}$ in (531).

S. Lederer studied linear gravitational theory also in the context of Kaluza-Klein-theory in five dimensions by introducing a symmetric tensor potential $\phi_{A B}, A, B=0,1, \ldots, 4$ comprising massive fields of spin 0,1 , and 2 ([353], pp. 381-283). For the quantization, she started from the linearization of the 5-dimensional metric in isothermal coordinates $\gamma_{A B}=\eta_{A B}+k_{A B}, \partial_{5} k_{A B}=0$, and the relation $k_{A B}=\phi_{A B}+\frac{a}{\mu^{2}} \partial_{A B} \Phi^{(0)}+b \eta_{A B} \Phi^{(0)}$, where $a, b$ are parameters with $1+a+3 b=0, \Phi^{(0)}=$ $\eta^{M N} \phi_{M N}$, and $\mu^{2} \neq 0$ is connected to the mass of the field. ${ }^{321}$ The $\phi_{A B}$ were expressed by creationand annihilation operators $K_{A B}(q), K_{C D}^{*}(q)$ and expanded in terms of an orthonormal tetrad $n_{S}^{(r)}$ with $\Sigma_{r} n_{A}^{(r)} n_{B}^{(r)}=\frac{1}{\mu^{2}} q_{A} q_{B}-\eta_{A B}$ tangential to the 4-dimensional surface $q^{R} q_{R}-\mu^{2}=0, q_{5}=0$, i.e., $K_{A B}=\Sigma_{r s} C(r, s, q) n_{A}^{(r)} n_{B}^{(s)}$. The $C(r, s, q)$ were assumed to be self-adjoined operators with commutation relations $\left[C^{*}(i j, q), C\left(l m, q^{\prime}\right)\right]=\left(\delta_{i m} \delta_{j l}+\delta_{j m} \delta_{i l}-\tilde{b} \delta_{i j} \delta_{l m}\right) \delta\left(q^{R} q_{R}-\mu^{2}\right) . \tilde{b}$ is a new numerical parameter. The commutation relations for the fields then were calculated to have the form:

$$
\left[\phi_{A B}(x), \phi_{C D}\left(x^{\prime}\right)\right]=\left(P_{A C} P_{B D}+P_{A D} P_{B C}-\tilde{b} P_{A B} P_{C D}\right) \mathcal{D}\left(x-x^{\prime}\right)
$$

with $P_{A B}=\eta_{A B}+\frac{1}{\mu^{2}} \frac{\partial^{2}}{\partial x^{A} \partial x^{B}}$ and the Pauli-Jordan distribution $\mathcal{D}\left(x-x^{\prime}\right)$. (539) translated into

$$
\begin{gathered}
{\left[k_{A B}(x), k_{C D}\left(x^{\prime}\right)\right]=} \\
\left(P_{A C} P_{B D}+P_{A D} P_{B C}-\frac{3-d}{6} P_{A B} P_{C D}-\frac{2}{9} d\left(\frac{3}{\mu^{4}} \frac{\partial^{4}}{\partial x^{A} \partial x^{B} \partial x^{C} \partial x^{D}}+\eta_{A B} \eta_{C D}\right) \mathcal{D}\left(x-x^{\prime}\right)(5\right.
\end{gathered}
$$

and is independent of a if $d=(2 \tilde{b}-1)(1+4 a)^{2}$ holds. The paper of S. Lederer discussed here in some detail is one midway in a series of contributions to the quantization of the linearized JordanThiry theory begun with the publications of C. Morette-Dewitt \& B. Dewitt, [448, 449], continued by Ph. Droz-Vincent [129, 128], ${ }^{322}$ and among others by A. Capella [72] and Cl. Roche [511].

These papers differ in their assumptions; e.g., Droz-Vincent worked with the traceless quantity $k_{A B}-\frac{1}{2} \eta_{A B} \eta^{M N} k_{M N}$; for $a=0, \tilde{b}=0$, and thus for $d=-1$ his results agree with those of S. Lederer. In his earlier paper, A. Capella had taken $\eta^{M N} k_{M N}=0$, and $\mu=0$. Claude Roche applied the methods of Ph. Droz-Vincent to the case of mass zero fields and quantized the gravitational and the electromagnetic fields simultaneously.

${ }^{321}$ Like her advisor Tonnelat, S. Lederer liked to change notation. In a previous paper $\Phi^{(0)} \rightarrow k, a \rightarrow b, b \rightarrow b-1 / 2$; cf. [351]. Caution is also required for a comparison of (540) below with the corresponding equation in ([351], p. 387). 322 The 3-page paper in Comptes Rendus should be, preferably, complemented by a reading of Droz-Vincent's thesis [128, 133]. 


\subsection{Linear theory and spin-1/2-particles}

With the progress in elementary particle theory, group theory became instrumental for the idea of unification. J.-M. Souriau was one of those whose research followed this line. His unitary field theory started with a relativity principle in 5-dimensional space the underlying group of which he called "the 5-dimensional Lorentz group" but essentially was a product of the 4-dimensional Poincaré group with the group $\mathrm{O}_{2}$ of 2-dimensional real orthogonal matrices. For its infinitesimal generator $A, \exp (2 \pi A)=1$ holds wherefrom he introduced the integer $n$ by $A^{2}=-n^{2}$. He interpreted $-n \leq 0$ as the electric charge of a particle and brought charge conjugation $\left(x^{5} \rightarrow-x^{5}\right)$ and antiparticles into his formalism [582]. Souriau also asked whether quantum electrodynamics could be treated in the framework of Thiry's theory, but for obvious reasons only looked at wave equations for spin-0 and spin- $1 / 2$ particles. As a result, he claimed to have shown the existence of two neutrinos of opposite chirality and maximum violation of parity in $\beta$-decay [583]. By comparing the (inhomogeneous) 5-dimensional wave equation for solutions of the form of the Fourier series $\phi=\Sigma_{n=-\infty}^{+\infty} \phi_{n}\left(x^{1}, \ldots, x^{4}\right) \exp \left(i n x^{5}\right)$ with the Klein-Gordon equation in an electromagnetic field, he obtained the spectrum of eigenvalues for charge $q$ and mass $m$ :

$$
q=n k \hbar, \quad m=\hbar \sqrt{\frac{n^{2}}{\xi^{2}}+a},
$$

where $k:=\frac{1}{\xi} \sqrt{\frac{\chi}{2 \pi}}, \chi=\frac{8 \pi G}{c^{2}}$ is the gravitational constant in Einstein's equations, and $a$ the "mass"term of the 5-dimensional wave equation, i.e., a free parameter. $\xi$ is the scalar field: $\xi^{2}=-g_{55}$. For $q=e, n=1, \xi$ is of the order of magnitude $\simeq 10^{-32} \mathrm{~cm}$. Souriau also rewrote Dirac's equation in flat space-time of five dimensions as an equation in quaternion space for 2 two-component neutrinos. His interpretation was that the electromagnetic interaction of fermions and bosons has a geometrical origin. The charge spectrum is the same as for spin-0 particles except that the constant $a$ in (541) is replaced by $-a^{2}$.

O. Costa de Beauregard applied the linear approximation of Souriau's theory for a field variable $H_{\alpha \beta}$ to describe the equations for a spin-1/2 particle coupled to the photon-graviton system. He obtained the equation $\partial_{\alpha} \partial^{\alpha} H_{5 k}=-2 i k_{5} \frac{\hbar \chi}{c} \bar{\phi} \gamma_{k} \phi(\alpha, \beta=1,2, \ldots, 5 ; k=1,2, \ldots, 4)$, where the wave function $\phi$ again depends on the coordinate $x^{5}$ via $\exp \left(k_{5} x^{5}\right)$; as before, $\chi$ is the coupling constant in Einstein's field equations. Comparison with electrodynamics led to the identification $k_{5}=\frac{e}{\hbar \sqrt{2 \chi}}$ with $e$ the electric charge. Costa de Beauregard also suggested an experimental test of the theory with macroscopic bodies [91].

\subsection{Quantization of Einstein-Schrödinger theory?}

Together with efforts at the quantization of the gravitational field as described by general relativity, also attempts at using Einstein-Schrödinger type unified theories instead began. Linearization around Minkowski space was an obvious possibility. But then the argument that the cosmological constant had appeared in some UFTs (Schrödinger) lead to an attempt at quantization in curved space-time. In the course of his research, A. Lichnerowicz developed a method of expanding the field equations around both a metric and a connection which are solutions of equations describing a fixed geometric backgrond [375]. Quantization then was applied to the quantities varied (semiclassical approximation). The theory was called "theory of the varied field" by Tonnelat [641], p. 441). ${ }^{323}$ Lichnerowicz determined the "commutators corresponding to vector meson and to an electromagnetic field (spin 1) on one hand and to a microscopic gravitational field (spin 2, mass 0 ) on the other hand [...] in terms of propagators" [378]. The linearization was obtained by looking at field equations for the varied metric and connection. Let $\Psi_{i j}:=\delta g_{i j}$ be such a variation

323 The program has been carried through for general relativity in her thesis by E. Blancheton [30]. 
of the metric $g_{i j}$ and $X_{i j}{ }^{k}:=\delta L_{i j}{ }^{k}$ a variation of the connection $L_{i j}{ }^{k}$. It is straightforward to show that the variation of the Ricci tensor is $\delta R_{i j}(L)=\nabla_{s} X_{i j}{ }^{s}-\nabla_{j} X_{i s}{ }^{s}=\left(-\delta K_{i j}(L)\right)$, where the covariant derivative is taken with regard to the connection formed from $g_{i j}$. Also, $2 X_{i j}{ }^{k}=$ $\nabla_{j} \Psi_{i}{ }^{k}+\nabla_{i} \Psi_{j}^{k}-\nabla^{k} \Psi_{j i}$. Ph. Droz-Vincent then looked at field equations for a connection with vanishing vector torsion and with Einstein's compatibility equation (30) varied, i.e., $\delta g_{i k-l} \| l=0$ :

$$
R_{i j}-\lambda g_{i j}=\frac{2}{3}\left(\partial_{i} \Gamma_{j}-\partial_{j} \Gamma_{i}\right)
$$

with cosmological constant $\lambda$ and arbitrary $\Gamma_{k}[131] .{ }^{324}$ The Riemannian metric which is varied solves $R_{i j}-\lambda g_{i j}=0$ (Einstein space). Droz-Vincent showed that the variation $\Psi_{i j}$ must satisfy the equations:

$$
\begin{array}{r}
\nabla^{r} \Psi_{[r j]}=0, \\
(\Delta+2 \lambda) \Psi_{(i j)}=\nabla_{i} k_{j}+\nabla_{j} k_{i}, \\
\left.(D+2 \lambda) \Psi_{[i j]}=\frac{8}{3} \partial_{[i} \delta \Gamma_{j}\right],
\end{array}
$$

where $D$ is a differential operator different from the Laplacian $\Delta$ for the Riemannian metric introduced by Lichnerowicz ([375], p. 28) such that $\nabla D \neq D \nabla . \quad k_{i}=k_{i}(\Psi)$ is defined by $k_{i}(\Psi)=\nabla^{r} \Psi_{r i}-\frac{1}{2} \nabla_{i} \Psi_{s}^{s}$ (indices moved with $g_{i j}$ ). Equation (543) follows from the vanishing of vector torsion.

Difficulties arose with the skew-symmetric part of the varied metric. Quantization must be performed such as to be compatible with this condition. The commutators sugested by Lichnerowicz were not compatible with (397). Droz-Vincent refrained from following up the scheme because:

"The endeavour to establish such a program is, to be sure, a bit premature in view of the missing secure physical interpretation of the objects to be quantized." 325

Ph. Droz-Vincent sketched how to write down Poisson brackets and commutation relations for the Einstein-Schrödinger theory also in the framework of the "theory of the varied field" ([130]. In general, the main obstacle for quantization is formed by the constraint equations, once the field equations are split into time-evolution equations and constraint equations. Droz-Vincent distinguished between proper and improper dynamical variables. The system $R_{i j}=0, D_{k} \hat{g}^{i j}=0$, where $D$ signifies covariant derivation with respect to the star connection (27), led to 5 constraint equations containing only proper variables arising from general covariance and $\lambda$-invariance. By destroying $\lambda$-invariance via a term $\hat{g}^{r s} \Gamma_{r} \Gamma_{s}$, one of the constraints can be eliminated. The Poisson brackets formed from these constraints were well defined but did not vanish. This was incompatible with the field equations. By introducing a non-dynamical timelike vector field and its first derivatives into the Lagrangian, Ph. Droz-Vincent could circumvent this problem. The physical interpretation was left open $[133,135]$. In a further paper, he succeeded in finding linear combinations of the constraints whose Poisson brackets are zero modulo the constraints themselves and thus acceptable for quantization [136].

\footnotetext{
324 Comparison with (399) lets enter the new terms with $\lambda$, and $\Gamma_{k}$ is the vector torsion of $\Gamma_{i j}{ }^{k}$ with $L_{i j}{ }^{k}=$ $\Gamma_{i j}^{k}+\frac{2}{3} \delta_{i}^{k} \Gamma_{j}$

325 "L'enterprise d'un tel programme est, cela va sans dire, quelque peu prématurée en l'absence d'une interprétation physique certaine des grandeurs à quantifier." ([133], p. 379.)
} 


\section{Alternative Geometries}

Although a linear theory of gravitation can be derived as a first approximation of EinsteinSchrödinger-type theories ([641], pp. 441-446), the results may be interpreted not only in UFT, but also in the framework of "alternative" theories of gravitation. Nevertheless, M.-A. Tonnelat remained defensive with respect to her linear theory:

" [...] this theory does not pursue the hidden aim of substituting general relativity but of exploring in a rather heuristic way some specifically tough and complex domains resulting from the adoption of the principles of a non-Euclidean theory [...]". ([382], p. 327$)^{326}$

We will now describe theories with a different geometrical background than affine or mixed geometry and its linearized versions.

\subsection{Lyra geometry}

In Lyra geometry, the notion of gauge transformation is different from its use in Weyl geometry. Coordinate and gauge transformations are given the same status: they are defined without a metric or a connection [386, 531]. A reference system now consists of two elements: Besides the usual coordinate transformations $x^{i} \rightarrow x^{i^{\prime}}=x^{i^{\prime}}\left(x^{1}, x^{2}, \ldots, x^{n}\right)$ a gauge transformation $x^{0^{\prime}}=f^{0}\left(x^{1}, x^{2}, \ldots, x^{n}, x^{0}\left(x^{1}, x^{2}, \ldots, x^{n}\right)\right)$ with gauge function $f$ is introduced. The subgroup of coordinate transformations is given by those transformations for which $f^{0}\left(x^{1}, x^{2}, \ldots, x^{n} ; x^{0}\right)=x^{0}$. A change of the reference system implies both coordinate and gauge transformations. Tangent vectors $\xi^{s}$ under a change of coordinates and gauge transform like:

$$
\xi^{i^{\prime}}=\lambda \frac{\partial x^{i^{\prime}}}{\partial x^{s}} \xi^{s}
$$

where $\lambda=\frac{x^{0^{\prime}}}{x^{0}}$ is the gauge factor (Lyra's "Eichverhältnis"); a basis of tangential space is given by $\frac{1}{x^{0}} \frac{\partial}{\partial x^{i}}$; a 1-form basis would be $x^{0} d x^{i}$. The metric then is introduced by $d s^{2}=g_{r s}\left(x^{0} d x^{r}\right)\left(x^{0} d x^{s}\right)$, and the asymmetric connection $\Gamma_{r s}^{i}-\frac{1}{2} \delta_{r}^{i} \phi_{s}$ is defined via

$$
d \xi^{i}=-\left(\Gamma_{r s}^{i}-\frac{1}{2} \delta_{r}^{i} \phi_{s}\right) \xi^{r}\left(x^{0} d x^{s}\right), \Gamma_{r s}^{i}=\Gamma_{s r}^{i}
$$

Here, similar to Weyl's theory, an arbitrary 1-form $\phi_{k}$ appears and the demand that the length of a transported vector be conserved leads to

$$
\Gamma_{i j}^{k}=\frac{1}{x^{0}}\left\{\begin{array}{l}
k \\
i j
\end{array}\right\}+\frac{1}{2}\left(\delta_{i}^{k} \phi_{j}+\delta_{j}^{k} \phi_{i}-g_{i j} g^{k r} \phi_{r}\right)
$$

with the Christoffel symbol calculated from $g_{i j}$. The curvature tensor is defined by

$$
-\underset{+}{K_{j k l}^{i}}=\frac{1}{\left(x^{0}\right)^{2}}\left[\partial_{k}\left(x^{0} \Gamma_{l j}{ }^{i}\right)-\partial_{l}\left(x^{0} \Gamma_{k j}^{i}\right)+x^{0} \Gamma_{k m}{ }^{i} x^{0} \Gamma_{l j}{ }^{m}-x^{0} \Gamma_{l m}{ }^{i} x^{0} \Gamma_{k j}{ }^{m}\right]
$$

Hence the curvature scalar becomes $K=\frac{R}{\left(x^{0}\right)^{2}}+\frac{3}{x^{0}} \phi_{; s}^{; s}+\frac{3}{2} \phi^{s} \phi_{s}+2 \stackrel{0}{\phi_{i}} \phi^{i}$ where the semicolon denotes covariant derivation with regard to $g_{i j}$, and $\stackrel{0}{\phi}_{i}=\frac{1}{x^{0}} \frac{\partial \log \left(x^{0}\right)^{2}}{\partial x^{i}} .327$ In the thesis of D. K. Sen, begun

326 “[...] cette théorie ne poursuit pas le but caché de se substituer à la Relativité Générale mais d'explorer de façon plutôt heuristique, quelques domaines tout spécialement coriaces et complexes dès que l'on adopte les principes d'une théorie non euclidienne [...]."

$327 R$ is the curvature scalar calculated from $g_{i j}$. 
with G. Lyra in Göttingen and finished in Paris with M.-A. Tonnelat, the field equations are derived from the Lagrangian $\mathcal{L}=\left(x^{0}\right)^{4} \sqrt{g} K$. In the gauge $x^{0}=1$, they are given by [571]:

$$
R_{i k}-\frac{1}{2} g_{i k} R+3 \phi_{i} \phi_{k}-\frac{3}{4} g_{i k} \phi^{s} \phi_{s}=-\kappa T_{i k} .
$$

Weyl's field equations in a special gauge are the same - apart from the cosmological term $\Lambda g_{i j}$. The problem with the non-integrability of length-transfer does not occur here. For further discussion of Lyra geometry cf. [572].

In relying on a weakened criterion for a theory to qualify as UFT suggested by Horváth (cf. Section 19.1.1), after he had added the Lagrangian for the electromagnetic field, Sen could interpret his theory as unitary. In later developments of the theory by him and his coworkers in the 1970s, it was interpreted just as an alternative theory of gravitation (scalar-tensor theory) [573, 574, 310]. In both editions of his book on scalar-tensor theory, Jordan mentioned Lyra's "modification of Riemannian geometry which is close to Weyl's geometry but different from it" ([319], p. 133; ([320], p. 154).

\subsection{Finsler geometry and unified field theory}

Already one year after Einstein's death, G. Stephenson expressed his view concerning UFT:

"The general feeling today is that in fact the non-symmetric theory is not the correct means for unifying the two fields." ${ }^{28}$

In pondering how general relativity could be generalized otherwise, he criticized the approach by Moffat [439] and suggested an earlier attempt by Stephenson \& Kilmister [591] starting from the line element [cf. (428)]:

$$
d s=\sqrt{g_{j k} d x^{j} d x^{k}}+A_{l} d x^{l},
$$

the geodesics of which correctly describe the Lorentz force.

Since Riemann's habilitation thesis, the possibility of more general line elements than those expressed by bilinear forms was in the air. One of Riemann's examples was "the fourth root of a quartic differential expression" (cf. Clifford's translation in [329], p. 113). Eddington had spoken of the space-time interval depending "on a general quartic function of the $d x$ 's" ([139], p. 11). Thus it was not unnatural that K. Tonooka from Japan looked at Finsler spaces with fundamental form $\sqrt[3]{a_{\alpha \beta \gamma} d x^{\alpha} d x^{\beta} d x^{\gamma}}[648]$, except that only $\left.\right|^{3} \sqrt{a_{\alpha \beta \gamma} d x^{\alpha} d x^{\beta} d x^{\gamma}} \mid$ is an acceptable distance. With (551), Stephenson went along the route to Finsler (or even a more general) geometry which had been followed by O. Varga [669], by Horváth [283] and by Horváth \& Moór, [286]. He mentioned the thesis of E. Schaffhauser-Graf [530] to be discussed below. In Finsler geometry, the line element is dependent on the direction of moving from the point with coordinates $x^{i}$ to the point with $x^{i}+d x^{i}$ :

$$
d s=\gamma_{j k}\left(x^{l}, \dot{x}^{m}\right) d x^{j} d x^{k}, \dot{x}^{m}=\frac{d x^{m}}{d u},
$$

with $u$ an arbitrary parameter. In the approach to Finsler geometry by Cartan [74], the starting point is the line element: ${ }^{329}$

$$
d s=L\left(x^{l}, \dot{x}^{m}\right) d u,
$$

with $L$ a homogeneous function of the velocity $\dot{x}^{m}$, and the Finsler metric defined by:

$$
\gamma_{j k}=\frac{1}{2} \frac{\partial^{2}\left(L^{2}\right)}{\partial \dot{x}^{j} \partial \dot{x}^{k}} .
$$

328 "Le sentiment général aujourd'hui est que la théorie non symétrique n' est pas en effet le moyen correct d'unifier les deux champs." ([590], p. 207.)

329 There is another road to Finsler space followed by Busemann [68]. 
Quantities $C_{i j}{ }^{k}$ and $L_{i j}{ }^{k}$ acting as connections are introduced through the change a tangent 4vector $X^{k}$ is experiencing:

$$
D X^{k}=d X^{k}+L_{s t}^{k} X^{s} d x^{t}+C_{s t}{ }^{k} X^{s} d \dot{x}^{s},
$$

where the totally symmetric object

$$
C_{i j k}=\gamma_{k s} C_{i j}{ }^{s}=\gamma_{k i, j}+\gamma_{k j, i}+\gamma_{i j, k}:=\frac{1}{4} \frac{\partial^{3}\left(L^{2}\right)}{\partial \dot{x}^{i} \dot{x}^{j} \dot{x}^{k}},
$$

transforms like a tensor. The asymmetric affine connection is defined by

$$
L_{i j}^{k}=\left\{{ }_{i j}^{k}\right\}_{(\gamma)}+\gamma^{k t}\left(C_{t i p} \frac{\partial G^{p}}{\partial \dot{x}^{j}}-C_{t j p} \frac{\partial G^{p}}{\partial \dot{x}^{i}}\right),
$$

with $\gamma_{i s} \gamma^{j s}=\delta_{i}^{j}$. The "geodesic coefficient" $G^{p}=\frac{1}{4} \gamma^{p r}\left[\frac{\partial^{2}\left(L^{2}\right)}{\partial \dot{x}^{r} \partial x^{s}} \dot{x^{s}}-\frac{\partial\left(L^{2}\right)}{\partial x^{r}}\right]$ is resulting from the Euler-Lagrange equation $\frac{d^{2} x^{k}}{d t^{2}}+2 G^{k}=0$ for $L$ reformulated with the Finsler metric. ${ }^{330}$ Equation $(555)$ can also be written as

$$
D X^{k}=d X^{k}+L_{s t}^{* k} X^{s} d x^{t},
$$

with Cartan's connection $L_{i j}^{*}{ }^{k}=L_{i j}{ }^{k}-\gamma^{k t} C_{t j p} G^{p}$.

Edith Schaffhauser-Graf at the University of Fribourg in Switzerland hoped that the various curvature and torsion tensors of Cartan's theory of Finsler spaces would offer enough geometrical structure such as to permit the building of a theory unifying electromagnetism and gravitation. She first introduced the object met before, also known as "Cartan" torsion:

$$
S_{i j k}=\frac{1}{2} L \frac{\partial \gamma_{i k}}{\partial \dot{x}^{j}}=\frac{1}{4} L \frac{\partial^{3}\left(L^{2}\right)}{\partial \dot{x}^{i} \dot{x}^{j} \dot{x}^{k}}=L C_{i j k} .
$$

and, by contraction with $\gamma^{k j}$, the "torsion" vector $S_{i}=\frac{1}{2} L \frac{\partial \ln |\gamma|}{\partial \dot{x}^{i}}$. With its help and its covariant derivative taken to be:

$$
S_{i \mid k}=\frac{\partial S_{i}}{\partial x^{k}}-\frac{\partial S_{i}}{\partial \dot{x}^{m}} \frac{\partial G^{m}}{\partial \dot{x}^{k}}-\left(\Gamma_{i k}^{m}-C_{i r}^{m} \Gamma_{s k}^{r} \dot{x}^{s}\right) S_{m},
$$

the electromagnetic field tensor is defined by: ${ }^{331}$

$$
F_{i j}:=S_{j \mid i}-S_{i \mid j} .
$$

The form of the covariant derivative (560) follows from the supplementary demand that the Finsler spaces considered do allow an absolute parallelism of the line elements. Schaffhauser-Graf used the curvature tensor 0. Varga had introduced in a "Finsler space with absolute parallelism of line elements" ([669], Eq. (37)), excluding terms from torsion. The main physical results of her approach are that a charged particle follows a "geodesic", i.e., a worldline the tangent vectors of which are parallel. The charge experiences the Lorentz force, yet this force, locally, can be transformed away like an inertial force. Also, charge conservation is guaranteed. For vanishing electromagnetic field, Einstein's gravitational theory follows. It seems to me that the prize paid, i.e., the introduction of Finsler geometry with its numerous geometric objects, was exorbitantly high.

Stephenson's paper on equations of motion [590], from which the quotation above is taken, was discussed at length by V. Hlavatý in Mathematical Reviews [MR0098611] reproduced below:

\footnotetext{
${ }^{330}$ In the present understanding of Finsler geometry in the framework of the tangent bundle, $C_{i j}{ }^{s}$ is one of the 5 non-vanishing torsion tensors ("Cartan torsion"). Three curvature tensors do exist. (cf. ([4], vol 2. p. 999-1000.) $G_{i}^{p}:=\frac{\partial G^{p}}{\partial \dot{x}^{i}}$ is named non-linear connection and usually denoted by $N_{i}^{p}$.

331 Schaffhauser-Graf used the symbol $P_{i j}$ in place of $F_{i j}$.
} 
The paper consists of three parts. In the first part, the author points out the difference between Einstein-Maxwell field equations of general relativity and Einstein's latest unified field equations. The first set yields the equations of motion in the form

$$
\frac{d^{2} x^{\nu}}{d s^{2}}+\left\{\begin{array}{c}
\nu \\
\lambda \mu
\end{array}\right\} \frac{d x^{\lambda}}{d s} \frac{d x^{\mu}}{d s}+F_{\mu}{ }^{\nu} \frac{d x^{\mu}}{d s}=0
$$

$\left(F_{\mu}^{\nu} d x^{\mu} / d s=\right.$ Lorentz' force vector $)$. Callaway's application of the EIH method to the second set does not yield any Lorentz force and therefore the motion of a charged particle and of an uncharged particle would be the same.

In the second part, the author discusses the attempt to describe unified field theory by means of a Finsler metric

$$
d s=A_{\lambda} d x^{\lambda}+\sqrt{g_{\lambda \mu} d x^{\lambda} d x^{\mu}},
$$

which leads by means of $\delta \int d s=0$ to (1) with $F_{\mu \lambda}=2 \partial_{[\lambda} A_{\mu]}$. However the tensor $R_{\mu \lambda}$ does not yield any appropriate scalar term which could be taken as Lagrangian. [...]

Remarks of the reviewer: 1) If $F_{\mu \lambda}$ is of the second or third class (which is always the case in Callaway's approximation) we could have $F_{\mu}^{\nu}\left(d x^{\mu} / d s\right)=0$ in (1) for $F_{\mu \lambda} \neq 0$. 2) The clue to Callaway's result is that four Einstein's equations $\partial_{\{\omega} R_{\mu \lambda\}}=0$ do not contribute anything to the equations of motion of the considered singularities. If one replaces these four differential equations of the third order by another set of four differential equations of the third order, then the kinematical description of the motion results in the form

$$
\frac{d^{2} x^{\nu}}{d q^{2}}+\Gamma_{\lambda}^{\nu}{ }_{\mu} \frac{d x^{\lambda}}{d q} \frac{d x^{\mu}}{d q}=0
$$

There is such a coordinate system for which the first approximation of (2) is the classical Newton gravitational law and the second approximation acquires the form (1). [...].

Stephenson dropped his plan to calculate the curvature scalar $R$ from (557) and (551), and to use it then as a Lagrangian for the field equations, because he saw no possibility to arrive at a term $R(g)+F_{s t} F^{s t}$ functioning as a Finslerian Lagrangian. His negative conclusion was: "It so seems that this particular generalization of Riemannian geometry is not able to lead to a correct implementation of the electromagnetic field." 332

332 "Il semble alors que cette généralisation particulière de la géometrie de Riemann n'est pas un procédé correct pour introduire le champ électromagnétique." ([590], p. 211.) 


\section{Mutual Influence and Interaction of Research Groups}

\subsection{Sociology of science}

\subsubsection{Princeton and UFT}

Einstein's unified field theory makes a good example for showing that the influence of a model scientist may be as important in driving research as ideas coming from physics or mathematics themselves. A realistic impression seems to be that most in the group of young workers busy with Einstein's UFT after the second world war were enticed by Einstein's fame and authority - perhaps mediated through the prestige of their professorial advisers. They went into the field despite its being disdained by mainstream-physicists. In view of the success of quantum field theory, it could hardly have been the methods and conceptions used in UFT that attracted them. In fact, many of those who wrote a doctoral thesis in the field dropped the subject quickly afterwards in favor of general relativity proper, or of some other field. It would be unfair to give too much importance to J. R. Oppenheimer's reckless rating of 1935 (unpublished at the time), i.e., "Princeton is a madhouse: its solipsistic luminaries shining in separate \& helpless desolation. Einstein is completely cuckoo" (Letter of J. R. Oppenheimer to his brother Frank from 11 January 1935 in [462], p. 190.) After all, much later during his time as director of the Institute for Advanced Study there, he supported people working on versions of UFT like R. L. Arnowitt who stayed at the Princeton Institute for Advanced Study from 1954 to 1956 and expressly thanked him in a paper ([5], p. 742). Nevertheless, while highly respected, Einstein and his theories lived there in splendid scientific isolation.

\subsubsection{Mathematics and physics}

Looking at the mutual "directions of influence" between mathematics and physics in the field described here, we may distinguish three fruitful exchanges. It is known that the mathematician Grossmann provided Einstein with the Ricci-calculus as a means of formulating General Relativity within Riemannian geometry. This very theory then radiated back into mathematics and helped to further study the previously introduced most general concept of affine connection (G. Hessenberg, T. Levi-Civita, E. Cartan, H. Weyl). Next, the transfer of this new geometrical concept into physics led to the unified field theories of Eddington, Einstein, and Schrödinger [138, 546, 548]. It seems to me that after this third interaction mathematics (geometry) no longer did profit from physical theory: the conceptual development of metric-affine geometry took place independently within mathematics (L. P. Eisenhart, O. Veblen, J. M. Thomas, T. Y. Thomas). Einstein's approach to a connection by way of the compatibility equation (30) was of very limited interest for mathematics. This was expressed most clearly by Schouten:

"[...] in the end there is nothing but an $X_{4}$ [a 4-dimensional manifold] with only two fields $g_{(i j)}$ and $g_{[i j]}$ and that [...] the differential concomitants of these fields are ordinary concomitants of $g_{(i j)}, g_{[i j]}$, the curvature tensor of the symmetric connection belonging to $g_{(i j)}$ and the covariant derivatives of these quantities with respect to this connection. [...] It may be useful to introduce this new connection in order to get some heuristic principles [...]. But nothing really new can ever arise from following this course." ([540], p. 184.)

Unified field theory has stimulated the fantasy of mathematicians such that they investigated conformal geometry [670] and projective geometry [538], or even ventured to apply odd geometries to geometrize physics. We have met some of them (Finsler, Sphere-, Lyra geometries). Certainly, mathematicians also both helped theoretical physicists to solve their equations and invented new equations for UFT. Unfortunately, to the exact solutions of such equations found, in most cases no 
physical meaning could be given. In physics, the next step in unification would be taken only in the 1960s through the joining of the weak and electromagnetic interactions in electroweak theory with gravitation being left aside, however. In mathematics, important developments leading to differential topology as well as to the theory of fiber bundles originated with E. Cartan among others - not with any of the theoretical physicist connected to the unified field theories of Einstein and Schrödinger. Gauge theory, seen as a development starting from Kaluza-Klein theory, and, more recently, string-theory (M-theory) then presented further examples for a fruitful interaction between physics and mathematics.

\subsubsection{Organization and funding}

Apart from the creative abilities of the individual scientist, knowledge production depends on the institutional organization of research and the ways of communication among researchers. Thus, within a review of the history of physics, beside the conceptional developments, questions pertaining to the sociology of science cannot easily be omitted.

During the period looked at, both, changes in the funding of scientific research, and in the institutional organization toward team work and less interaction with teaching, i.e., away from university research along Humboldt's idea of a close link between teaching and research, still were going on. In Germany, this development had begun with the establishment of the Kaiser Wilhelm Institutes (KWI, now Max Planck Institutes) since 1911, a combination of private and state funding, together with "Helmholtz-Gesellschaft", "Stifterverband", and most influential, the "Notgemeinschaft der deutschen Wissenschaft" predecessor of "Deutsche Forschungsgemeinschaft" since 1920 [676]. In France, where state-funding was the rule, the apparently not very successful "Caisse des recherches scientifiques" (1910-1934) was replaced by CNRS (Centre National de la Recherche Scientifique) only in 1939, right after the beginning of World War II. Of particular interest for the present review is the foundation of the Institut Henri Poincaré (IHP) in 1926 [579] which was built with private money but did not provide salaries. In the United States mostly private sponsors were involved; e.g., the Carnegie Corporation (1911), the Rockefeller Foundation (1913) and Einstein's "home" institution, the Princeton Institute for Advanced Study (PIAS) (since the 1930s). The Dublin Institute for Advanced Studies in Ireland (DIAS) was founded in 1935, but funded by the government (Department of Education). ${ }^{333}$ In Great Britain, after the Great War a mixture of state and private funding (University grants commission, Science Research Council) persisted, coordinated in part by the Royal Society. After the 2nd world war 35 Research Associations have sprung up.

Looking at four main figures in UFT, Einstein's and Hlavatý's research thus depended on private donors while Schrödinger in Dublin, Lichnerowicz, and Mme. Tonnelat in Paris were state-funded. Within this external framework, there existed micro-structures pertaining to the inner workings of the particular research groups.

\subsection{After 1945: an international research effort}

During the second world war, communications among the various scientists investigating UFT came to a halt - with the exception of the correspondence between nobel prize winners Einstein and Schrödinger. That the exchange between libraries then only slowly began to resume is clear from a letter of A. Lichnerowicz to W. Pauli of 6 October 1945: "In France, only one copy of Mathematical Review and one copy of the series Annals [of Mathematics] exist. Concerning the paper of Einstein published in 1941 at Tucuman, I know about it only through the report in

333 In France, an institute corresponding to the Princeton PIAS, i.e., l'Institut des Hautes Études Scientifiques (IHES) was established only in 1958 with the financial support of several grand enterprises. 
Mathematical Review; it never reached France" ([489], p. 317). ${ }^{334}$

From hindsight, after the second world war, research in classical unified field theory developed into a world-wide research effort. However, at the time it was no concerted action with regard to funding and organization; the only agreement among researchers consisted in the common use of scientific methods and concepts. As described in detail above in Sections 7 to 9, since the 1920s, it had been mainly Albert Einstein $(1879-1955)$ who had pursued research on UFT if we put aside H. Weyl and A. S. Eddington. Since his separation from Berlin, most important actors of the 1930s and 1940s were Einstein himself in Princeton, and somewhat later (from 1943 on) Erwin Schrödinger (1887-1961) in Dublin. After the war, in Paris the mathematician André Lichnerowicz (1915-1998) became interested in mathematical problems related with UFT, and a student of Louis de Broglie, and later professor Marie-Antoinette Tonnelat (1912-1980), built a sizable group working in the field. From the 1950s on Vavlav Hlavatý (1894-1969) in Bloomington/Indiana and his collaborators contributed prominently to the field. The Italian groups around Bruno Finzi (1899-1974) and Maria Pastori (1895-1975) were not as influential in the 1960s, perhaps because they wrote exclusively in Italian, published mostly in Italian journals and seemingly deemed networking less important although there were connections to France and the USA (cf. Sections 10 to $15)$.

\subsubsection{The leading groups}

The internal structure of the research "groups" differed greatly: in Princeton (as in Berlin), Einstein never had doctoral students but worked with post-docs like Peter Bergmann, Bannesh Hoffmann, Valentine Bargmann, Leopold Infeld, Bruria Kaufman, and Ernst Straus ${ }^{335}$. At the time, further people interested in unified field theory came to the Princeton Institute for Advanced Study (PIAS), e.g., Tullio Levi-Civita (1936), Vaclav Hlavatý (1937), Luis A. Santaló (1948-49) from Argentina, M. S. Vallarta from Mexico (1952), Wolfgang Pauli (1940 - 1946; 1949-50), Richard Lee Ingraham (1952-53), R. L. Arnowitt (1954-55), both from the USA, and D. W. Sciama (1954-55) from Great Britain. Schrödinger also worked with scientifically advanced people, mostly independent scholars at DIAS (J. McConnell, A. Papapetrou, O. H. Hittmair, L. Bass, F. Mautner, B. Bertotti). Unlike this, A. Lichnerowicz at the Collège de France and Mme. Tonnelat at the Institut Henri Poincaré (IHP) worked with many doctoral students. M.-A. Tonnelat had also two experienced collaborators whom she had not advised for her doctoral theses: Stamatia Mavridès [1954-57] and Judith Winogradzki [1954-59]. None of these scientists in Paris have been scholars at the Princeton Institute; possibly, they did not belong to the proper network.

The following students and young scientists wrote their PhD theses in M.-A. Tonnelat's group at the IHP or were interacting intensely with her on the Einstein-Schrödinger type of Unified Field Theories, and on linear theories of gravitation: Jacques Lévy - Thèse 1957; Pham Tan Hoang - Thèse 1957; Dipak K. Sen - Thèse, 1958; Jean Hély - Thèse 1959; Marcel Bray - Thèse 1960; Liane Bouche, née Valere - Thèse 1961; Huyen Dangvu - Thèse 1961; Mme. Aline Surin, née Parlange - Thèse 1963; Nguyên, Phong-Chau; - Thèse 1963; Philippe Droz-Vincent - Thèse 1963; ${ }^{336}$ Sylvie Lederer - Thèse 1964; Huyen Dangvu - Thèse 1966, Rudolphe Bkouche - Thèse 196?. S. Kichenassamy wrote his thesis with her on general relativity in 1958. That M.-A. Tonnelat advised doctoral students also on subjects outside of unified field theory is shown by the thesis of 1974 on classical renormalization by Th. Damour [99].

\footnotetext{
334 "Il n'existe en France qu'un exemplaire de la série Mathematical Review et un de la série Annals [of Mathematics]. [...] Quant au mémoire du professeur Einstein paru en 1941 à Tucuman, je ne le connais que par le referat des Mathematical Review et il n'est jamais parvenu en France."

335 We did not include Einstein's short-time mathematical assistants during 1949 and 1950 John George Kemeny (1926 - 1992) and Robert H. Kraichnan (1928-2008), both then post-docs.

$336 \mathrm{Ph}$. Droz-Vincent kindly has given my the correct date.
} 
Scientists and PhD students closer to A. Lichnerowicz besides Yvonne Bruhat / Fourès(-Bruhat) / Choquet-Bruhat - Thèse 1951, but unlike her working on Unified Field Theories, were: Yves Thiry - Thèse 1950; Pham Mau Quan - Thèse 1954; Josette Charles, née Renaudie - Thèse 1956; Françoise Maurer, née Tison - Thèse 1957; Françoise Hennequin, née Guyon - Thèse 1958; Pierre V. Grosjean - Thèse 1958; Robert Vallée - Thèse 1961; Albert Crumeyrolle - Thèse 1961; Marcel Lenoir - Thèse 1962; Jean Vaillant - Thèse 1964; ${ }^{337}$ Claude Roche - Thèse 1969; Alphonse Capella - Thèse 1972?. Eliane Blancheton wrote her thesis in general relativity in 1961.

Y. Thiry also supervised doctoral students. Among them is P. Pigeaud with a thesis on the application of approximations in Jordan-Thiry theory [495]. The thesis adviser for Monique SignorePoyet was Stamatia Mavridès (Thesis 1968). ${ }^{338}$

The relationship between M.-A. Tonnelat and A. Lichnerowicz must have been friendly and cooperative; he seems to have been the more influential in the faculty: having become a professor at the prestigious Collège de France while her application had not been successful ([92], p. 330). For the examination of Tonnelat's PhD-students, since 1960, Lichnerowicz was presiding the commission; she belonged to the two (or rarely three) examiners. Before Lichnerowicz, G. Darmois had been presiding several times. Tonnelat was backed by L. de Broglie; she became his successor as director of the "Centre de physique théorique" of Paris University (Sorbonne) in 1972.

From the Italian groups around Bruno Finzi and Maria Pastori (Milano) also a sizable number of doctoral degrees resulted. People working in UFT were: Paulo Udeschini (Pavia); Emilio Clauser (Milano); Elisa Brinis-Udeschini (Milano); Laura Gotusso (Milano); Bartolomeo Todeschini (Milano); Franco De Simoni (Pisa); Franca Graiff (Milano) [Student of M. Pastori]; Laura Martuscelli [Student of M. Pastori]; Angelo Zanella (Milano); Luigia Mistrangioli.

Of the seven doctoral students of V. Hlavatý, ${ }^{339}$ two were involved in work on UFT: Robert C. Wrede [PhD 1956] and Joseph Francis Schell [PhD 1957].

We notice the considerable number of female collaborators and PhD students both in France and Italy in comparison with all the other countries. In Germany, in particular, no woman scientist has worked in UFT in the period studied.

\subsubsection{Geographical distribution of scientists}

Research on UFT was done on all continents but essentially centered in Europe and in the United States of America. The contributing scientists came from more than 20 different countries. The largest number of researchers in UFT, between 1955 and 1956, worked in Paris. To a lesser extent work on UFT was done also in Asia, notably in Japan since the 1930s and in India since the 1950s. In the 1970s and 1980s, many papers on exact solutions of the Einstein-Schrödinger theory and alternatives were published by Indian scientists. In the early 1960s, V. Hlavatý had a coworker from India (R. S. Mishra). Of the six papers on UFT published by S. N. Bose in Calcutta from 1953-1955, five appeared in French journals, perhaps due to his contacts established during his previous stay in Paris in 1924/25.

Did those involved in UFT move from one place to another one? During the period considered, the mobility of scientists in Europe was seriously hampered by the Nazi-regime, the second world

337 A. Lichnerowicz was his 2nd advisor, Jean Leray the 1st one. Vaillant is professor emeritus at Universty P. Curie UPMC-Paris VI.

338 I have not found out by whom Roland Guy from Swizerland was advised in Paris. Guy (1919-2006) later became a mathematics professor in Canada. The same ignorance applies to the late Saiyid Izhar Husain (professor at Aligarh Muslim University, India). The thesis of P. V. Grosjean was handed in at the University of Liège "sous patronage Pr. Lichnerowicz, Collège de France". There were also those like Nguyen Xuan Xinh who, as a theoretician, wrote his thesis at the university of Paris in 1966 on Raman spectroscopy but then published an occasional paper concerning Born-Infeld theory. I assume that there must have been around many more master's degree students as among them, perhaps, Huguette Chevalier.

${ }^{339}$ Listed in the not always complete "Mathematics Genealogy Project" http://www .genealogy . math.ndsu.nodak. edu/. 
war and the ensuing occupation of Eastern Europe by the USSR ("cold war"). While doing their main work, the group leaders Einstein, Schrödinger, Lichnerowicz, and Tonnelat remained at the same place, respectively. After the war had ended, Tonnelat visited Schrödinger in Dublin. Because of the political situation, Hlavatý left his native Czechoslovakia, went to Paris as a guest professor at the Sorbonne (1948). He also spent some time in Princeton following an invitation by Einstein before obtaining a position at the University of Indiana. A. Papapetrou (1907-1997) who had obtained a doctoral degree at the Polytechnical University of Stuttgart, Germany, and had been professor in Athens, Greece, during 1940-1946, was the only major contributor to UFT who changed his positions several times. He first worked in Dublin until 1948, then at the University of Manchester until 1952 when he went to Berlin. There, he headed a group in general relativity at the Academy of Sciences of the GDR until 1962. From then on he stayed in Paris at the Institut Henri Poincaré until his retirement as a "Directeur de recherche" of the CNRS. Of course, there had always been exchanges between Paris and other centers, but they were not concerned with research on UFT. In 1946/47, the French mathematician Cecile Morette-[DeWitt] (1922-) originally affiliated with the Joliot-Curie group, spent a year at the Institute for Advanced Studies in Dublin. She did not work with Schrödinger on UFT but with P. H. Peng on mesons. Another well-known French mathematician, Yvonne Choquet-Bruhat, who had written her dissertation with A. Lichnerowicz, in 1951/52 was at PIAS.

John Archibald Wheeler must have spent some time in Paris in 1949; he worked on atomic and nuclear physics, though. In 1957 the quantum field theorist Arthur Wightman was a guest scientist at the University of Paris and in 1963/64 and 1968/69 at IHES (Institut des Hautes Études Scientifiques) near Paris. Stanley Deser was a Guggenheim fellow and guest professor at the Sorbonne (University of Paris) in 1966/67, and in 1971/72 as a Fulbright Fellow. Tonnelat and Mavridès visited the Pontifical Catholic University of Rio de Janeiro (PUC-Rio), Brazil, as guest professors in 1971. A. Lichnerowicz visited Princeton University in 1974, i.e., outside the period considered here.

\subsubsection{Ways of communications}

In 1923 to 1925, when research in classical unified field theory started, the only ways of communication among scientists apart from personal visits or encounters during the rare international conferences, were notes on paper in the form of personal letters by surface mail (Einstein and Pauli are famous for their postcards), and publications in scientific journals. Correspondence sometimes included manuscripts or proof sheets. Both, Einstein and Hlavatý left an enormous correspondence. ${ }^{340}$ The most notable change in available services during the period $1930-1960$ was the introduction of air mail and wireless services (world wide telex, since the 1930s) including radio broadcasts. $^{341}$ Radio broadcast as well as gramophone records (Einstein in Berlin!) were used mainly for educational purposes. In principle, telegrams also would have been available but they were unwieldy and too expensive for the communication of scientific content. To a lesser degree, the same may apply to the still costly telephone calls even within the same town. Paris:

The main meeting places seem to have been seminars of the various groups as there were in

Séminaire Janet (Séminaire de mécanique analytique et de mécanique céleste);

Séminaire de l'école normale supérieure;

Séminaire Théories physiques Institut Henri Poincaré [with invited talks by Einstein, Bonnor, Stephenson, Sciama, and others];

340 A systematic discussion of correspondence is outside my capability.

341 Although the first commercial tele-copying service was available already in 1865 between Paris and Lyon, its general use had to wait until the 70 s of the 20 th century. 


\section{Séminaire de Physique théorique (Séminaire Louis de Broglie).}

Séminaire de Physique mathématique du Collège de France (A. Lichnerowicz).

Séminaire sur la Mécanique quantique et les particules élémentaires (J. Winogradzki).

In London, a seminar on unified field theory existed at the University College (Imperial College) (ca. 1945 - ca. 1955) [G. Stephenson, C. Kilmister (1924-2010)], and continued at Kings College as a seminar on general relativity and cosmology initiated by the group around H. Bondi [F. Pirani, W. Bonnor, P. Higgs] (late 1950s-1977).

\section{Publications}

In total, about 150-170 scientists did take part in research on UFT between 1930 and 1965. If we distinguish three age cohorts according to year of birth, then in the group born until 1900 we find 27 people, from $1901-1920$ three more (30), and after 192118 persons. This is a biased preliminary survey among less than $50 \%$ of all involved, because the birth dates of the then doctoral students, of not so well known researchers, and of many scientists in India and Japan could not yet be obtained. Another grouping would be concerned with the differentiation between mathematicians and theoretical/mathematical physicists working in the field: roughly one third of the contributors to UFT were mathematicians.

Knowledge production in UFT is reflected by a yearly average of 18 papers published. ${ }^{342}$ The maximum of a 5-year-average in published papers per year at a peak of 40 papers occurred in the years just before and just after Einstein's death, i.e., in 1953-1957; the minimum with 4 papers in the period 1939-1943, i.e., during the years of the second world war. Looking at the absolute number of total yearly publications in UFT which appeared in Comptes Rendus de l'Académie des Sciences, Paris (French groups), and their percentage relative to papers on general relativity likewise published in Comptes Rendus, we conclude that the number of papers on UFT at most reached $20 \%$ of the papers on general relativity.

Do preferred journals exist in which researchers in the field of UFT published their research articles? Certainly, Einstein preferred the Annals of Mathematics, edited by Princeton University and, since 1933, co-edited by the Institute for Advanced Study. Likewise, Schrödinger used the Proceedings of the Royal Irish Academy and the Communications of the Dublin Institute for Advanced Studies, the organ of his home institution; M.-A. Tonnelat chose the Comptes Rendus de l'Academie des Sciences, Paris to which she had easy access through her former teacher L. de Broglie. ${ }^{343}$ Likewise, Lichnerowicz and the members of the French groups published first in Comptes Rendus. Finally, V. Hlavatý published mostly in a journal of his institution, Indiana University, i.e., in Journal of Rational Mechanics and Analysis. Thus, the main proponents of UFT did not have to submit their papers to an external refereeing process. However, all of the main figures did at some occasions; M.-A. Tonnelat and her coworkers also published in Journal de Physique et le Radium, Cahiers de Physique, and in Nuovo Cimento. ${ }^{344}$ V. Hlavatý sent his papers also to Italy (Rendiconti del Seminario Matematico dell'Università degli Studi di Padova, Rendiconti del Seminario Matematico, Università e Politecnico di Torino, Annali di Matematica), and to the Netherlands (Nieuw archief voor wiskunde), A. Lichnerowicz published in Rendiconti del Seminario Matemàtico e Fisico di Milano, etc. This remark is not meant as a criticism of quality but perhaps as an indication concerning the inaccessibility of many physics journals for

342 This is concluded from ca. 630 papers between $1930-1965$.

343 At the time, a note submitted to Comptes Rendus was accepted without external refereeing, but an academician had to present it.

344 Mme. Tonnelat as well as Mme. Winogradzki (for some time) were members of the editorial board of Cahiers de Physique. 
papers on UFT. ${ }^{345}$

As mentioned above, during the period considered (mid-1930s-mid-1960s) continuous communication almost exclusively happened via surface mail or, later, also by air mail. Thus, it is understandable that Schrödinger's papers, published during the war in the Proceedings of the Irish Academy, were not readily available in France, Italy, the USA, India or Japan. Schrödinger himself acknowledged having received information by correspondence with Einstein prior to publication of the Einstein-Straus-paper ([551], p. 44, footnote 3). Also, G. Bandyopadhyay in India learned of De Simoni's paper of 1954 only later (before 1963) through Bonnor ([11], p. 660). Of course, after the second world war, communication sped up. Nevertheless, Japanese and Indian researchers in the field of UFT seemingly did not read French journals because no references to Tonnelat are given in papers by Takeno and Ikeda [297, 298]. However, in a note added in proof, Takeno and his co-authors then in 1951 acknowledged having received information about a paper by Tonnelat of the year before [602]. Rao [504] included Tonnelat's book of 1955 and a paper by Mavridès of 1955 in his bibliography, but the way of listing shows that he might not have had them in his hands.

Although missing language skills did not forestall progress in the development of UFT, it might have been slowed down by it, in part. Knowledge of English and French was standard throughout Europe. Researchers in the United States apparently did not read articles in French. The large groups in Paris published mainly in French; the groups in Italy stuck to Italian such that the dissemination of their results occurred mostly via French authors (e.g., the books of M.A. Tonnelat), or through occasional visitors like Corben. The German language had virtually vanished for use in publications about UFT after the second world war.

\section{Co-authorships}

In order to obtain an impression of how people in the field interacted, the questions about coauthorships, inside and outside the various groups, may be asked. It was not to be expected that some of the group leaders, senior professors of the old style, would join to write a paper. This did not even occur between "colleagues" Marie-Antoinette Tonnelat and André Lichnerowicz in Paris if we leave aside the jointly edited proceedings of the Royaumont conference. As co-authors, we most often have the combination of group leader and co-worker/PhD student: Einstein \& Straus, Einstein \& Kaufman etc., Schrödinger \& Mautner, Schrödinger \& Papapetrou etc, M.-A. Tonnelat \& Liane Bouche, M.-A. Tonnelat \& Sylvie Lederer; A. Lichnerowicz \& Y. Thiry, A. Lichnerowicz \& Y. Fourès-Bruhat, Hlavatý \& Sáenz. Remarkably, Tonnelat did not publish in UFT jointly with her co-workers Judith Winogradzki (1916-2006) and Stamatia Mavridès. Winogradzki worked on UFT from 1954-1956 and then on spinors. Mavridès on UFT from 1954-1957, and also on Tonnelat's Euclidean theory of gravity from $1962-1964$. Thereafter, she went into cosmology and astrophysics.

All four leading figures summed up their research in books or, as was the case with Einstein and Schrödinger, in sections of their books on special and general relativity.

\section{Referee reports and citations}

It is interesting to find out who reviewed papers on UFT for the Mathematical Reviews. ${ }^{346}$ Principal group leaders like Einstein (but his co-workers as well), Schrödinger, and Tonnelat are not among them except for Hlavatý. In Paris, only Yvonne Fourès/Choquet-Bruhat and Mme. J. CharlesRenaudie wrote reports. I checked only 128 papers; they were reviewed by 14 people, half of which

\footnotetext{
345 Of course, A. Einstein and his correspondents, as well as scientists located in the United States (Callaway, Kursunŏglu) could publish on UFT in Physical Reviews.

346 The Mathematical Reviews were founded in 1940 as a counterpart to Zentralblatt in Nazi-Germany. For the development in reviewing cf. Siegmund-Schulze [578].
} 
were direct scientific contributors to UFT. Among the reviewers were six mathematicians. ${ }^{347}$ The theoretical physicist A. H. Taub is the one who wrote most reviews (31), followed by V. Hlavatý with 23 and M. Wyman with 20 reports. ${ }^{348}$ None of the papers by the Italian groups is in this sample. We note, however, that quite a few of such reviews are a mere description of the contents.

As to citations, the Italian group around Finzi, Pastori and Udeschini mostly referred to work inside the group. From the research done in other places, Princeton (Einstein, AE \& Straus) is quoted most often (23 times); next in frequency are Schrödinger's and Papapetrou's research in Dublin (quoted 11 times) and Hlavatý's papers (referred to 10 times). English authors (Bonnor, Stephenson) and French scientists (Lichnerowicz, Tonnelat) follow (4 times each). And vice-versa, by looking at the bibliographies in Tonnelat's books [632, 641], Italian authors stand for $7 \%$ of the entries, at best.

Citation of authors in Germany after the second world war (P. Jordan, E. Schücking, K. Just), occurred in research on scalar-tensor theory - as an outgrowth of Kaluza-Klein unitary theory mainly by the Paris group.

\subsubsection{International conferences and summer schools}

During the time-span considered, due to the external circumstances only a handful of international conferences took place at which the subject of gravitation and unified field theory could have been discussed. Most famous are the Volta Conference, in 1927 in Como, and the fifth Solvay Conference on Electrons and Photons in the same year in Brussels. The first conference, held at Lake Como, led to the public introduction of the uncertainty principle by Niels Bohr and Werner Heisenberg. In the 5th Solvay conference, the leading figures were Albert Einstein and Niels Bohr. Einstein, disenchanted with Heisenberg's "Uncertainty Principle", unceasingly fought the statistical interpretation of quantum mechanics. In both conferences, the discussion was not about UFT, specifically, but on classical theory versus quantum theory. The second Volta conference did not take place until 1932; its topic was "Europe". All further Volta conferences did not touch unified field theory. The same is true for the subsequent Solvay conferences: During the 11th Solvay Congress on "The structure and Evolution of the Universe" in 1958 the main topic discussed was "steady state" theory versus general relativistic cosmology; unified field theory remained untouched.

Since the 1960s, several international conferences on gravitation, as described by general relativity, were organized on a regular basis like the CNRS-colloquia and proceedings of the meetings by the International Society on Relativity and Gravitation. Three months after Einstein's death, a "Jubilee Conference" took place in Bern, Switzerland, commemorating fifty years of relativity since the publication of Einstein's famous 1905 paper on the electrodynamics of moving bodies. Two of its eight principal topics were aimed at Unified field theory; in fact, two plenary talks and three short communications concerning UFT out of the 36 spoken contributions by 32 scientists were then given. This conference was the starting one of a series organized by the "Committee on General Relativity and Gravitation", later to be absorbed by the "International Society on General Relativity and Gravitation". Follow-ups were GR-1: "Conference on the Role of Gravitation in Physics" in Chapel Hill, NC (Jan. 18-23, 1957), GR-2: Les théories relativistes de la gravitation. Colloques in Royaumont, France (21- 27 June 1959), GR-3: "Conférence internationale sur les théories relativistes de la gravitation" in Warszawa and Jablonna (25-31 July 1962), ${ }^{349}$ GR-4 "International Conference on Relativistic Theories of Gravitation" in London (1965), GR-5 "Conference on Gravity" in Tbilisi (USSR) (1968). During the conference in Chapel-Hill, only one paper on unified field theory was given (by Kursunŏglu), and, in a subsection of another one, by

347 Y. Choquet-Bruhat, V. Hlavatý, H. S. Ruse, J. A. Schouten, A. G. Walker, M. Wyman.

348 One report on a paper by G. Stephenson by Hlavatý has been reprinted in full in Section 17.2 .

349 Astonishingly, a conference on a conference, the "50-year-jubilee" of the Jablonna Conference has taken place in 2012, in Warsaw. 
Lichnerowicz, "the Cauchy problem for the asymmetric theory" was dealt with. M.-A. Tonnelat and Y. Thiry as well as Ph. Droz-Vincent reported about their research in the GR-2 proceedings. In the subsequent GR-3 conference UFT was represented only marginally. In 1958, L. de Broglie and M.-A. Tonnelat organized a meeting in Paris on "Actual problems in the theory of relativity". All but one of the articles were concerned with general relativity or astrophysics. By this, the move away from UFT is clearly seen [374]. Also, in April 1960, the first conference on the topic of gravitation in Japan, a "Symposium on Gravity", was held at the Research Institute for Fundamental Physics. Unified field theory was not discussed [662].

Summer schools sprang up like the Brandeis Summer Institute or the Summer Seminars on Applied Mathematics in Boulder and Ithaca, NY, since the 1960s. They reflected progress in the study of general relativity and relativistic astrophysics, but did not include any discussion of classical unified field theory. This can also be seen in the reports of a conference celebrating the 50th anniversary of general relativity in Berlin in 1965. J. A. Wheeler talked about "How is it today with Einstein's idea to comprehend everything as geometry?" His comment on Einstein's UFT was that none to Einstein's attempts had been successful, some even had led to unphysical results. The main thrust of his talk was an obvious effort to establish the Princeton school as the modern successor of Einstein's ideas (geons, wormholes, E-geometry) [697]. Thus, when communication in the form of conferences and summer schools finally was rapidly growing, since the mid 1960s, UFT was doomed: at best it came to be viewed as an old-fashioned pastime. ${ }^{350}$

The unfortunate parallelism C. Lanczos saw between the political changes caused by the dictatorships, "whether of the Russian, Italian or German variety", and the development of theoretical physics toward theories with high predictability catering to the needs of "industry and technology", after World War II, must be rejected. It was to serve a barely cloaked downgrading of quantum field theory in comparison with Einstein's "refined abstract thinking, armed with the mighty tools of advanced mathematics [...]" ([349], pp. 57-59).

\footnotetext{
350 Parts of the material of this section were used for a lecture at the university in Mainz (2010) and at a conference in Prague (2011), but have not appeared in print.
} 


\section{On the Conceptual and Methodic Structure of Unified Field Theory}

From his varied attempts at a unified field theory during the decades covered both in Part I and Part II of this review, it may be concluded that in this field of research Einstein never had or followed a program, in the strict sense, over a longer period of time. He seems to have been resistant to external influences as were, e.g., the fashionable attempts at an inclusion of the meson into all sorts of unitary field theory. Whether the last decade of his research pursuing structural investigations within mixed geometry and directed toward the establishment of field equations, as firmly grounded as his field equations of general relativity, is characterized by "enlightened perseverance" or "biased stubbornness" lies in the eye of the beholder. We shall now try to look from a more general point of view at the detailed and technical discussions given above.

\subsection{General issues}

Advantages of a theory unifying other theories are: (1) the conceptual structure of the unified theory will in general be richer, (2) its empirical content more inclusive, and (3) the limits of application of the sub-theories covered easier to determine ([221], p. 273, 276). On the first point UFT performs too well: The various forms of Einstein-Schrödinger unified field theory all provide us with too many mathematical objects as to allow a convincing selection of an unambiguous geometrical framework for a physical theory. To quote M.-A. Tonnelat:

"The multiplicity of structural elements brought into the game, the arbitrariness reigning over their interpretation, bring an unease into the theory which one cannot lightly make vanish in total." ([641], p. 299. $)^{351}$

In addition, particularly within mixed (or even metric-affine) geometry, the dynamics is highly arbitrary, i.e., possible field equations abound. Usually, the Lagrangian is built after the Lagrangian of general relativity, possibly because this theory was required to emerge from UFT in some limiting process. But L. A. Santalò has shown that the "weak" field equations can be reached from a Lagrange function linear in curvature and quadratic in torsion containing 5 free parameters ([524], Theorem 2, p. 350). ${ }^{352}$ There exists a 3-parameter Lagrangian which is transposition invariant and also leads to the "weak" system (p. 352). The same author has also proven that there are Lagrangians of the same class which are not invariant under $\lambda$-transformations (cf. Section 2.2.3) but still lead to the "weak" field equations (p. 351, Theorem 3). Thus, in spite of all symmetry- and plausibility arguments put forward, none of the field equations used by Einstein and Schrödinger acquired an equivalent position of uniqueness like the field equations of general relativity.

As to the 2nd and 3rd points, even if UFT had succeeded as a theory with a well-put particle concept, by the 1940s the newly discovered particles (neutron, mesons, neutrino) would have required another approach taking into account the quantum nature of these particles. Field quantization had been successfully developed for this purpose. Occasionally, the argument has been made that a unification of the two long-range fundamental forces within classical theory would have been enough to be asked for in "pre-quantum physics" ([234], p. 255). However, the end of pre-quantum physics must be set not later than 1925/26; in particular, the development of quantum electrodynamics had started already at the end of the 1920s; it is now part of the partial

351 "La multiplicité des éléments de structure mis en jeu, l'arbitraire qui préside à leur interprétation, introduisent dans la théorie un malaise qu'il est difficile de faire totalement disparaître."

352 In a subsequent paper the number of permitted free parameters in the Lagrangian became reduced to 4 [525]. 
unification achieved by the Glashow-Salam-Weinberg model (1967). Certainly, quantum field theory suffered from severe problem with infinities to be removed before the observables of the theory could provide numbers to be compared with measurements. At last, renormalization procedures did the job so well that an effect like the Lamb-shift could be calculated, with the inclusion of self-energy contributions, up to highest precision. ${ }^{353}$

Unlike this, the UFTs of the 1920s to the 1940s did not get to the stage where empirical tests could have been made. Actually, in later developments novel gravito-electromagnetic effects were derived from UFT; cf. Sections 6.1.2, 15.2. Unfortunately, they never led to observed results. Often, it is argued unconvincingly that this is due to the weakness of the gravitational field; for either a strong electrical or a strong gravitational field (neutron stars), measurable effects of the interaction of these fields could have been expected. In a way, UFT of the Einstein-Schrödinger type was as removed from an empirical basis then as quantum cosmology or string theory are at present. Pauli had been aware of this already in the 30s ([489], p. 789):

"It is odd how Einstein carries on physics nowadays. In effect, it is the method of a pure mathematician decreeing all from his desk who completely has lost contact with what physicists really do." 354

Ironically, when H. Weyl had suggested his generalization of Riemannian geometry by a purely mathematical argument, and then had used it to build a theory unifying gravitation and electromagnetism (cf. Section 4.1 of Part I), Einstein had refuted him for not having thought of the empirical consequences. Now, he followed the same course: He started from a mathematical structure and then aimed at turning it into a physical theory. There is a difference, though, because for his own theory Einstein was not able to derive testable consequences:

"The unified field theory now is self-contained. But its mathematical application is so difficult that I have not been capable to test it in some way in spite of all the efforts invested." (Letter to M. Solovine 12 February 1951, in [160], p. 106.) ${ }^{355}$

The speculative character of UFT was rendered yet more unattractive by its unsolved problems: how to describe matter, in particular the motion of charged particles. Doubts came up very early whether the Lorentz force could be extracted from the theory in the lowest steps of a non-trivial approximation. Instead of winning new results, many authors were content when they were able to reproduce effects already known from general relativity and Maxwell's theory; cf. Section 15.3. The missing empirical support was critically seen even within the community of workers in unified field theory:

"Unified theories do suggest to base the electromagnetic and gravitational fields on one and the same hyper-field - with the physical phenomena being explained by a geometrical structure imposed on space-time, independently from any phenomenological hypothesis. The ambition of such an explication in the spirit of Cartesian philosophy is recognized which, far from following the observational and experimental results step by step, pretends to anticipate them. The theory incorporates its actual provisions into a

\footnotetext{
353 We are aware of the fact that the mathematical difficulties in the description of interacting fields by quantum field theory are still waiting to be fully resolved.

354 "Es ist merkwürdig, wie Einstein jetzt Physik treibt. Es ist eigentlich die Methode eines alles vom Schreibtisch aus dekretierenden reinen Mathematikers, der den Kontakt zu dem, was die Physiker wirklich tun, vollkommen verloren hat."

355 "Die einheitliche Feldtheorie ist nun in sich abgeschlossen. Sie ist aber so schwer mathematisch anzuwenden, dass ich trotz aller aufgewandter Mühe nicht imstande bin, sie irgendwie zu prüfen."
} 
vast synthesis and furnishes them with a whole program of a posteriori verifications." ([94], p. 331) ${ }^{356}$

Due to his epistemological and methodical position, Einstein could not have cared less. With no empirical data around, he fitted the envisaged UFT to the various mathematical structural possibilities. As has been shown in great detail, originally, when struggling with a relativistic theory of gravitation, he had applied two methods: "induction from the empirical data" and "mathematical deduction" ([309], p. 500-501). In his later work, he confined himself to the second one by claiming that only mathematical simplicity and naturalness could lead to a fundamental theory reflecting unity. Intuition is played down by him in favour of quasi-axiomatic principles. This shift in Einstein's epistemology and methodology has been described in detail by J. D. Norton [458], D. Howard [290], and J. van Dongen [667]. However, we should not forget that both concepts, simplicity and naturalness, lack unambiguous mathematical or philosophical definitions.

In this context, Einstein's distinction between "constructive theories" and "theories of principle" may also be considered. The first ones are constructive, they "[...] attempt to build up a picture of the more complex phenomena out of the materials of a relatively simple formal scheme from which they start out [...]". The second important class called principle-theories "employ the analytic, not the synthetic, method. The elements which form their basis and starting-point are not hypothetically constructed but empirically discovered ones, general characteristics of natural processes, principles that give rise to mathematically formulated criteria which the separate processes or the theoretical representations of them have to satisfy." ([157], p. 228) According to Einstein, the theory of relativity belongs to the second class with its "logical perfection and security of the foundations". His unified field theory fits better to the description of a constructive theory.

The more delicate question why the unification of the fundamental forces must be sought by a geometrization of the fields, was rarely asked. In Weyl's approach, a pre-established harmony between mathematics and physics had been put forward as an argument. Y. Mimura and T. Hosokawa saw the "mission of physics" in looking for answers to the questions: "What is space-time in the world wherein physical phenomena occur?" and "By what laws are those physical phenomena regulated?" Their idea was that the properties of space-time are represented by physical laws themselves. "Thus theoretical physics becomes geometry. And that is why physical laws must be geometrized" ([426], p. 102). This circular remark of 1939 is less than convincing. Other paths could have been followed (and later were), e.g., one along a unifying (symmetry) group. ${ }^{357}$ A renowned scientist like P. A. M. Dirac shied away altogether from such a big sweep as unification is. He favoured an approximative approach to an eventually all encompassing theory: "One should not try to accomplish too much in one stage. One should separate the difficulties in physics one from another as far as possible, and then dispose of them one by one" ([125], quoted from [338], p. 373). On aesthetical grounds, Dirac came closer to Einstein:

"It seems to be one of the fundamental features of nature that fundamental physical laws are described in terms of a mathematical theory of great beauty and power, needing quite a high standard of mathematics for one to understand it. You may wonder: Why is nature constructed along these lines? One can only answer that our present

\footnotetext{
356 "Les théories unitaires se proposent de fondre en un même hyperchamp le champ électromagnétique et le champ de gravitation, les phénomènes physiques tirant leur explication d'une structure géométrique imposée à l'espacetemps en dehors de toute hypothèse phénoménologique - on réalise l'ambition d'une telle explication, bien dans l'esprit de la philosophie cartésienne, et qui loin de suivre pas à pas les résultat de l'observation et de l'experience, prétend les devancer, incorporant leurs apports actuels dans une vaste synthèse et leur fixant tout un programme de vérifications à posteriori."

357 In fact, in 1967, the idea was changed by the Japanese group: "We may say with confidence that gravitation should be geometrized, but some physical quantities might not be described as geometrical quantities." ([428], p. $40 / 41)$.
} 
knowledge seems to show that nature is so constructed. We simply have to accept it." $[124]$.

When Einstein geometrized gravitation, he had a good argument in the equality of inertial and gravitational mass. For electrodynamics and UFT, no such argument has been presented. In the 1950s, "charge-independence" as a property of strong interactions between baryons and mesons was discussed but not used for geometrization. ${ }^{358}$

\subsubsection{What kind of unification?}

According to Bargmann, the aim of UFT was: "(1) to deduce, at least in principle, all physical interactions from one law, (2) to modify the field equations in such a way that they would admit solutions corresponding to stable charged particles" ([12], p. 169). This general description of Einstein's eventual course for describing fundamental aspects of physical reality (nature) by one single theory can be complemented by further, more specific, details.

In Sections 8.1 and 10.3.4 we have mentioned Pauli's criticism with regard to the use of $g_{i j}=h_{(i j)}+k_{[i j]}$ in Einstein's and some of Schrödinger's unified field theories. As Mme. Tonnelat noted, this meant that the theory is unified only in "a weak sense" because the gravitational and electromagnetic fields are represented by different geometrical objects. Apart from the demand that the fundamental field quantities (metric, ...) must be irreducible with regard to the diffeomorphism group, Einstein had claimed symmetry with regard to $\lambda$-transformations, because these would mix the symmetric and skew-symmetric parts of the connection and thus counter criticism of the type Pauli had phrased. A further necessary condition for a unified field theory has been formulated: the Langrangian must not decompose into irreducible parts, i.e., it must not be expressed as a sum of several scalar densities but consist of a single "unified" term (cf. [308], p. 786). In principle, this was accepted also by Einstein as reported in Section 7.1. Sciama's unified field theory [567] forms an example ${ }^{359}$. A. Lichnerowicz called a theory "unitary in the strict sense"

"[...] if the exact field equations control an indecomposable hyper-field, and themselves cannot be fractionized into propagation equations of the gravitational and of the electromagnetic field but approximatively [...]." ([371], p. 152) ${ }^{360}$

In Kaluza-Klein theory the gravitational and electromagnetic fields are encased in one and the same geometrical object, the 5-dimensional metric. Just one term in the Lagrangian is needed. By M.-A. Tonnelat, such a theory has been called "unified in a strong sense" [627, 641], p. XVII. ${ }^{361}$ In his thesis on a generalization of Kaluza-Klein theory, Y. Thiry had defined a unitary theory for two fields by the requirements (1) that the two fields emanate from the same geometry, and (2) that they amalgamate into one hyper-field of which they represent nothing more than two different aspects ([606], p. 13). An example for a hyper-field taken from history would be the electromagnetic field tensor within special relativity.

Bargmann's second demand placed on UFT, the existence of stable solutions describing charged particles, remained unfulfilled within the Einstein-Schrödinger theories. With singularities of the fields being excluded, not even a satisfactory definition of a particle (beyond the concept of test

\footnotetext{
358 This far-fetched idea was followed up only in the late 1970s [452].

359 Horváth's weakening of this criterion in the sense that the Langrangian must not decompose into irreducible parts each of which has an independent geometrical meaning is contra-productive. By it, already Einstein-Maxwell theory could be interpreted as UFT [285].

360 "Une théorie sera unitaire au sens strict dans la mesure où les équations rigoureuses régissent un hyperchamp non décomposable et ne peuvent être fractionnées en équations de propagation du champ gravitationnel et du champ électromagnétique qu'approximativement, [...]"

361 cf. however Finzi for whom, in contrast, "la teoria affine è certamente più unitaria della teoria pentadimensionale." ("The affine theory certainly is more unitary than the five-dimensional theory".) ([203], p. 53)
} 
particle) could be given in such classical field theories. Einstein was very aware of this when he wrote to Besso ([163], p. 438): "E.g., a 'particle' in the strict sense of the word does not exist, because it does not fit to the program of representing reality by everywhere continuous, even analytical fields." ${ }^{362}$ Even today, a convincing definition of an interacting particle apparently does not exist. We have free fields describing particles and interaction terms introduced in the Lagrangian. Attempts at creating the concept of a single particle including its interaction with other particles have been attempted, unsuccessfully, e.g., one by G. Pétiau [494]. A precise definition of an interacting particle as a member of an ensemble of particles seems not outdated, but out of reach.

Methodological questions could be added. Why did Einstein, Schrödinger and most others working in unified field theory start with a metric and a connection as independent variables and then link them through a condition for the covariant derivative of the metric? To solve the latter condition for the connection has used up an immense amount of energy and time (plus printed paper) as we have seen before. Were they afraid of going one step back behind H. Weyl and other mathematicians who had recognized the independence of the concepts of metric and parallel transport? It might have been more direct to generalize, in a systematical investigation, the LeviCivita connection (Christoffel symbol) by building the connection as a functional of the symmetric and skew-symmetric parts of the metric and their first derivatives as Hattori and Eisenhart have done.

\subsubsection{UFT and quantum theory}

Einstein's position with regard to quantum mechanics, particularly his resistance to the statistical interpretation of it is well known, cf. [585, 586]. In this context, his abortive attempts at presenting contradictions within the Copenhagen interpretation of quantum mechanics during the 1927 Solvay conference in Brussels may be remembered. Ehrenfest's remark there is quoted by Heisenberg: "I' m ashamed of you, Einstein. You put yourself here just in the same position as your opponents in their futile attempts to refute your relativity theory" ([248], p. 107). A decade later, Einstein's comment on Bohr's riposte to the EPR-paper as reported by Léon Rosenfeld was that Bohr's position was logically possible, but: "so very contrary to my scientific instinct that I cannot forego the search for a more complete conception." ([518], p. 131). ${ }^{363}$ In his judgment on quantum mechanics, Einstein differed strongly from Pauli's who believed in the completeness of quantum mechanics:

"This generalization of the concept 'state' which involves a strict renouncement of a lawful description of the single, individual system for me seems to be necessary and, by the way also understandable, in view of the facts mentioned earlier. It is a consequence of the influence, unknown in principle, on the system being observed by the chosen measuring device. Due to this state of affairs, only as a consequence of this renouncement of a lawful description of an individual system seemingly is it possible to continue using the conception of 'closed system' and the closely related notion of space and time. In this sense, I deem the description of quantum mechanics to be complete." $([489], \text { p. } 520-521 .)^{364}$

\footnotetext{
362 "Es gibt z. B. nicht ein 'Teilchen' im strengen Sinne des Wortes, weil dies nicht zu dem Programm passt, die Realität durch überall kontinuierliche, ja sogar analytische Funktionen zu repräsentieren."

363 The retreat to his "scientific instinct" occurs also in other writings; cf. ([143], p. 342)

364 "Diese Verallgemeinerung des Begriffs 'Zustand', der einen grundsätzlichen Verzicht auf eine gesetzmäßige Beschreibung des Verhaltens des einzelnen individuellen Systems involviert, scheint mir angesichts der früher erwähnten Tatsachen notwendig und übrigens auch verständlich als Folge der prinzipiell unbekannten Beeinflussung des zu messenden Systems durch die als Beobachtungsmittel gewählte Meßanordnung. Bei dieser Sachlage scheint es nur infolge dieses Verzichtes auf eine gesetzmäßige Beschreibung des Einzelsystems möglich, überhaupt
} 
Therefore, Pauli questioned whether it was possible to unify the gravitational and electromagnetic fields in a classical field theory without taking note of "those facts in which the quantum of action plays an essential role". In fact, already at the time suggestions for a "unitary" field theory in the framework of quantum (field) theory were made: E. Stueckelberg saw electron, proton, neutron and the then only neutrino as states of one more fundamental elementary particle; the unitary field is a spinor with 16 components [594]. This was a step further than the neutrino theory of light (cf. Section 1). Heisenberg's later program of non-linear spinor theory as kind of a unified quantum field theory [247], although unsuccessful, belongs into this category. We are not surprised about Dirac's opposition: In a letter to Heisenberg of 6 March, 1967 he wrote:

"My main objection to your work is that I do not think your basic (non-linear field) equation has sufficient mathematical beauty to be a fundamental equation of physics. The correct equation, when it is discovered, will probably involve some new kind of mathematics and will excite great interest among the pure mathematicians, just like Einstein's theory of the gravitational field did (and still does). The existing mathematical formalism just seems to me inadequate." (Quoted from [339], p. 281.)

Perhaps, Dirac's foible for linear equations was behind this judgment. Much earlier, in 1942, after a colloquium in Dublin in which Eddington and Dirac had taken part, Schrödinger complained to Max Born:

"Your idea of getting their opinion on Born's theory is pathetic. ${ }^{365}$ That is a thing beyond their linear thoughts. All is linear, linear, - linear in the n'th power I would say if there was not a contradiction. Some great prophet may come ...' If everything were linear, nothing would influence nothing,' said Einstein once to me." ([446], p. 272.)

In fact, quantum mechanics and quantum field theory live very much on linearity (Hilbert space, linear operators). In principle, this does not forbid quantization of non-linear classical field theories like the non-linear electrodynamics of Born and Infeld (cf. Section 5). Already in 1933, in connection with this non-linear electrodynamics, Max Born confronted Einstein's opinion:

"For a long time Einstein had advocated the point of view that there must be a nonlinear field theory containing the quantum laws. We have here a non-linear field theory, but I do not believe that the quantization can be dispensed with. [...] I believe the following: every theory built up on classical foundations requires, for the completion of its assertions, an extension by initial and boundary conditions, satisfying only statistical laws. The quantum theory of the field provides this statistical completion [...] through an inner fusion of the statistical and causal laws." ([37], p. 434, 2nd footnote.)

Hence, Born's attempts at a theory compatible with both gravitation and quantum theory definitely left the framework of UFT [39]. It seems that M.-A. Tonnelat accepted Born's point of view.

A number of authors we have met before felt entitled to give general or very specific comments on the relationship between UFT and quantum theory. J. Callaway came to the conclusion that: "his [i.e., Einstein's UFT] theory will either be able to handle quantum phenomena or it will fail completely." ([70], p. 780.) Moffat \& Boal 1975 [443] just guessed: "It could be that the main significance of the $\lambda$-gauge transformations lies in the fact that it may influence the renormalizability

noch den Begriff 'abgeschlossenes System' und die mit ihm eng zusammenhängende gewöhnliche Vorstellung von Raum und Zeit in der Physik weiter zu verwenden. In diesem Sinne halte ich die Beschreibung der Quantenmechanik für vollständig."

365 I assume that this is a reference to the Born-Infeld theory. 
of the theory. [...]. It is possible that the unified field theory described here is renormalizable, because of its invariance under the extended gauge group of transformations." Within Jordan-Thiry theory as a "strongly unified" theory in the sense of M.-A. Tonnelat, the idea that its non-linearity would lead to elementary particles seemingly was given up. Moreover, in Sections 4.2, 4.3 and 16.2 we encountered two attempts, both also unsuccessful, toward a synthesis of classical field theory and quantum theory in the frameworks of wave geometry and 5-dimensional relativity. At the GR-2-Conference in Royaumont in 1959, Ph. Droz-Vincent spoke about the quantization of the theory's linear approximation; a mass term was introduced by hand. The theory then was interpreted as "a unitary theory of graviton-photon" ([132], p. 128).

A desperate argument, from the point of view of physics, in favour of UFT was advanced at the same conference by mathematician A. Lichnerowicz: there would be many good experiments in quantum theory, but no good (quantum field) theories. In UFT at least, we would have a theory with a definite mathematical meaning ([120], p. 149). For some like A. Proca, the hope in Einstein's genius overcame a sober assessment: "Convinced that every 'field' could be subjected to a theory of the type he had developed, he [Einstein] nurtured the 'modest hope' that such a theory possibly would bring forth the key to quantum theory. [500]"366

In fact, Einstein's claim that unified field theory would supersede quantum mechanics as a foundation for physics, could not be strenghtened by a recipe by which elementary particles were generated from classical field distributions. The concept of geon ("gravitational electromagnetic entity") was introduced by J. A. Wheeler in 1955 [696] in order to form a classical model for an elementary particle. It turned out to be a tinkering with the global topology of solutions. Although approximate solutions of Einstein's vacuum equations describing geons have been found (cf. [60]), they have not been proven to be stable entities. According to Wheeler

"A geon has exactly the property of being only an approximate solution; or rather, an accurate solution which is not fully stable with time - it leaks energy. Thus it is not in agreement with one's preconceived idea that there should be a particle-like solution that is fully stable; but aren't we being very brash if we say that the world isn't built that way? [...] Perhaps the stability of the particles we know is due to some intrinsic quantum character, which we cannot expect to show up before we have gone to the quantum level." ([120], p. 149)

Perhaps, this new concept led M.-A. Tonnelat once again to an optimistic comment ([635], p. 9):

"To the best of hypotheses, the unitary theories seem to explain, by classical methods, the formation of corpuscular structures out of the unified field. This attribution of particles to the field, postulated so energetically by Einstein, obviously is in a much too embryonic state to naturally explain the existence of different types of elementary particles." 367

Two years before, P. Bergmann's programmatic statement at the Chapel Hill conference, i.e.,

"The original motivation of unified field theory is get a theory of elementary particles, which includes electrons and not only hyper-fragments, and furthermore obviate the need for quantization which would result from the intrinsic non-linearity"

366 "Convaincu que tout 'champ' pouvait être justiciable d'une théorie du type de celles qu'il avait développées, il nourissait le 'modeste espoir' qu'une telle théorie livrerait peut-être aussi la clé de celle des quanta."

367 "Les théories unitaires semblent permettre, dans la meilleure hypothèse, d'expliquer par les méthodes classiques la formation des structures corpusculaires à partir du champ unifié. Cette réduction des particules au champ que postulait si énergiquement Einstein est évidemment dans un stade beaucoup trop embryonnaire pour expliquer avec naturel l'existence de différents types de particules élémentaires." 
had been instantly put into doubt by R. Feynman:

"Historically, when the unified field theory was first tackled, we had only gravitation, electrodynamics, and a few facts about quantization, [...]. In the meantime, the rest of physics has developed, but still no attempt starts out looking for the quantum effects. There is no clue that a unified field theory will give quantum effects." ([120], p. 149.)

Quantum mechanics, in particular as the measuring process is concerned, seemed not to have reached a generally accepted final interpretation. It looks as if Dirac wished to exploit this situation for making unitary field theory more respectable:

"And I think that it is quite likely that at some future time we may get an improved quantum mechanics in which there will be a return to determinism and which will, therefore, justify Einstein's point of view." ([126], p. 10.)

Dirac had in mind that application of the present quantum mechanics should not be pushed too far into domains of highest energy and smallest distances (p. 20). In view of the current brilliant empirical basis of quantum field theory, and the failure of all attempts to built a hidden-parameter theory, Dirac's remark is far from supporting Einstein's classical unified theory.

Einstein was well aware of the shortcomings of his "theory of the asymmetric field". The last paragraph of Appendix II in the 5th Princeton edition of The Meaning of Relativity reads as:

"One can give good reasons why reality cannot at all be represented by a continuous field. From the quantum phenomena it appears to follow with certainty that a finite system of finite energy can be completely described by a finite set of numbers (quantum numbers). This does not seem to be in accordance with a continuum theory, and must lead to an attempt to find a purely algebraic theory for the description of reality. But nobody knows how to obtain the basis of such a theory" [158].

His remark in a letter of 10 August 1954 to M. Besso led into the same direction: "Yet, by all means, I consider it as possible that physics cannot be founded on the field concept, i.e., on continuous structures. In this case, from my whole castle in the air, gravitational theory included, but also from the rest of contemporary physics nothing remains." 368

And, a fortnight before his death, he wrote that he did not want to dispense with "a complete real-description of the individual case" but also:

"On the other hand, it is to be admitted that the attempt to comprehend the undoubtedly atomistic and quantum-structure of reality on the basis of a consequential field theory encounters great challenges. By no means am I convinced that they can be overcome" 369 ([159], p. XVI )

\subsubsection{A glimpse of today's status of unification}

That unified field theory of the Einstein-Schrödinger type had become obsolete, was clear to theoretical physicists since the mid 1950s. In his introduction to the first conference on gravitation in Japan, in 1962, Nobel prize winner R. Utiyama wrote: "[...] it was no exaggeration to say that the old-fashioned mathematical investigation of Einstein's theory was not regarded as a field of

\footnotetext{
368 "Ich betrachte es aber als durchaus möglich, dass die Physik nicht auf den Feldbegriff gegründet werden kann, d.h. auf kontinuierliche Gebilde. Dann bleibt nichts von meinem ganzen Luftschloß inclusive Gravitationstheorie aber auch von der sonstigen zeitgenössischen Physik."

369 "Andererseits muss man zugeben, daß der Versuch, die unbezweifelbare atomistische und Quanten-Struktur der Realität auf dem Boden einer konsequenten Feld-Theorie zu begreifen auf große Schwierigkeiten stösst, von deren Überwindbarkeit ich keineswegs überzeugt bin".
} 
physics but rather a kind of mathematical play or a kind of metaphysics" ([662], p. 99). Einstein's former assistant P. G. Bergmann was less harsh: "Einstein spent the last five years of his life investigating this theory (the 'asymmetric' theory) without arriving at clear-cut answers. At the present time, all unified field theories must be considered speculative. But for a scientist who believes in the intrinsic unity of the physical universe, this speculative inquiry has an irresistible attraction" ([22], p. 492).

The idea of unifying all fundamental physical interactions in one common representation is as alive today as it was in Einstein's times. Its concrete realizations differ from UFT in important points: quantum fields are used, not classical ones, and all four fundamental interactions are taken into account - in principle. At first, Grand Unified Theories (GUTs) unifying only the electromagnetic, weak, and strong interactions were considered, e.g., with gauge group $S U(5)$. The breaking of this symmetry to the symmetry of the standard model of elementary particles $S U(3) \times S U(2) \times U(1)$ required the introduction of Higgs fields belonging to unattractive large representations of $S U(5)$ [561]. The concept of "spontaneous symmetry breaking" implying a dynamics exhibiting the full symmetry and a ground state with less symmetry is foreign to the Einstein-Schrödinger type of unitary theory. The GUTs studied have made predictions on the occurrence of new particles at a mass-scale (GUT-scale) outside the reach of present particle accelerators, and on the existence of topological defects such as cosmic strings, or domain walls. None were detected up to now. There was also a prediction on the decay of the proton by minimal $S U(5)$ which remained unsupported by subsequent measurements. Also, the simplest $S U(5)$-GUT does not bring together in one point the different energy-dependent couplings. This would be accomplished by a supersymmetric $S U(5)$-GUT.

Apparently, a main purpose of string theory has been to consistently unify all gauge interactions with gravity. However, "string phenomenology", i.e., the search for the standard model of elementary particles in (supersymmetric) string theory, has not been successful in the past 30 years. An optimistic assessment would be: "to obtain a connection between (string) theory and present (standard model) experiments is possible in principle but difficult in practice" ([451], p. 10).

The attempted inclusion of gravitation causes enormous conceptual and calculational difficulties which have not yet been overcome by candidates for "Theories of Everything" (TOEs), as are superstring theory, ${ }^{370}$ M-theory, ${ }^{371}$ brane-world scenarios ${ }^{372}$ etc. In the mid 1990 s, the 5 existing superstring theories in time plus a 10 -dimensional space ${ }^{373}$ via dualities have been shown to reduce, effectively, to one remaining theory. The extra dimensions are a means of allowing gravity to propagate into these dimensions while the other fundamental forces may be confined to fourdimensional spacetime. Problems caused by the number of additional spacelike dimensions required in modern unified theories are the unknown physics acting in them and the unacceptably large number of possibilities for space-time: the extra dimensions can be compactified in a giant number of different ways estimated to amount to $10^{500}$ (string theory landscape). A way out has been claimed by adherents of the multiverse-speculation: only a small number of the ground states are claimed to be "habitable". Thus, the fundamental constants of the universe would not be explained by physics but by some form of the anthropic principle. Up to now superstring- or M-theory have not been able to make explicit predictions about large distance physics. A recent presentation of string theory is given in [14].

\footnotetext{
370 Superstring theory means string theory with supersymmetry, a symmetry interchanging bosonic and fermionic particles.

${ }^{371} \mathrm{M}$-theory is an extension of string theory in an 11-dimensional Lorentz space which can be obtained as the strong coupling limit of type IIA string theory, or as a limit of the maximally-supersymmetric 11-dimensional supergravity. A complete description of M-theory is unknown.

372 Extension of the string concept. p-branes (-membranes) are p-dimensional spaces sweeping out a $(\mathrm{p}+1)$ dimensional "world" volume.

373 They are type I, types IIA, IIB and $S O(32)$ heterotic, $E_{8} \times E_{8}$ heterotic.
} 
In contrast to UFT, the modern theory of unified fields in the form of a set of rules and hopes purported by superstring theory has inspired greatly the development of some mathematical disciplines. Conceptually, non-Abelian gauge theory, supersymmetry and their geometrical realizations, as well as the renormalization group [461] now are part of the game. Even speculations about unification of such different objects as are elementary particles, microscopic black holes, and string states have been presented [522] - not to speak about even more speculative objects like black branes and blackfolds [193].

It seems that today's discussions divide theoretical physicists into two groups: those striving for a "Theory of Everything" 374 as the modern equivalent to UFT, and those believing that this is the kind of reductionism already disproved by present physical theory, in particular by manyparticle-phenomena [350]. In one of today's approaches, conceptual unification, i.e., the joinder of Heisenberg's uncertainty relations to gravitational theory (general relativity) and grand unification, has been set apart from the unification of spin-2-particles with all the other (elementary) particles, cf. [521]. . $^{375}$ The second point listed above could be expressed in more generality as: "One of the main goals of unified theory should be to explain the existence and calculate the properties of matter" ([236], p. 288). True, the present designs of TOEs have absorbed an immense amount of knowledge gained, theoretically and empirically, since the old days of classical UFT. Nevertheless, the elementary particle mass-spectrum is as unexplained today as it was then. In spite of the lauded Higgs-mechanism, the physical origin of mass is far from being understood. There seems to be a sizeable number of physicists with reserved attitude toward modern unified field theories.

\subsection{Observations on psychological and philosophical positions}

\subsubsection{A psychological background to UFT?}

According to guesswork propagated in the community, one of the reasons why both Albert Einstein and Erwin Schrödinger engaged in their enduring search for a unified field theory was that they hoped to repeat their grand successes: general relativity and its positive empirical test in 1919, and wave mechanics with its quick acceptance, in 1926. Schrödinger wrote to one of his lady lovers in January 1947 that he "had completely abandoned all hope of ever again making a really important contribution to science." But now, it looked as if he had been sent to Ireland by "the Old Gentleman" to live freely, without direct obligations, and follow his fancies. Which, of course, had brought him to the present final solution of how to set up unified field theory. His biographer W. Moore claimed that Schrödinger "was even thinking of the possibility of receiving a second Nobel prize" ([446] p. 434). In a way, world fame seems to cause addiction.

With Einstein, matters were more complicated. He certainly had no such wishes as Schrödinger; instead, he enjoyed using his fame as a propellant for making known his views, in other fields than in physics, in public. ${ }^{376}$ But, why would he begin anew with mixed geometry after roughly two decades? Did he want to not leave the territory to Schrödinger who had wandered into it since 1943 and had believed to be able to do better than Einstein? Einstein did not strive for a second Nobel prize, but was tied up by his philosophical thinking about reality and causality. It led him to believe that the epistemological basis of the ideas that had once lead to a splendid result, the

\footnotetext{
374 Another approach speaks of "Final Theory" as the collection of the final fundamental laws of nature; cf. [685]. Final is meant in the sense that no deeper explanation then will be needed for these laws.

375 Quoted from ms in the internet (introduction, p. 1) http://lss.fnal.gov/archive/other/fprint-93-25.pdf.

376 Among the many quotes ascribed to Einstein, I could find only one about fame, and it is right from the year he had become world-famous: "With fame I become more and more stupid, which of course is a very common phenomenon." (Ich werde nämlich mit der Berühmtheit immer dümmer, was ja eine ganz gewöhnliche Erscheinung ist.) Letter to H. Zangger of 24 December 1919.
} 
geometrization of the gravitational field within the framework of the continuum, by necessity must work again.

"The gravitational equations could be found only due to a purely formal principle (general covariance), i.e., due to faith in the imaginably greatest logical simplicity of the laws of nature. Because it was evident that the theory of gravitation was but a first step toward the discovery of field laws, it appeared to me that this logical way first must be thought to its end; only then one can hope to arrive at a solution to the quantum problem." Einstein to de Broglie 8 February 1954 ([580], Appendix A.2.8.) (777 $^{377}$

In the same letter, Einstein explained why he did not want to look like an "[...] ostrich permanently hiding his head in the relativistic sand in order to not have to face up to the evil quanta." 378 He thought this explanation could interest de Broglie "from a psychological point of view, [...]."

That successful physicists tend to come back to fruitful ideas in their previous work can be seen also in W. Heisenberg. In his case, the idea was to build theories just containing observables. His introduction of the S-matrix followed this objective he had applied when making the transition to quantum mechanics. Although valuable for scattering probabilities, and followed and extended by a number of well-known theoreticians, it has been criticized as having held up progress in elementary particle physics (Yang-Mills theory) [423].

At the very end of his life, Einstein was disappointed; he blamed "the physicists proper" for not understanding progress made by him. This is shown by his letter to Hans Mühsam of February 22, 1955:

"However, recently I decisively made progress. It refers to an improvement of the theory as far as its structure is concerned, but not with regard to finding solutions which could be examined through the [empirical] facts. [...] The matter is very plausible in itself and as perfect as to find increased interest among mathematicians. The physicists proper are rejecting it because they happened to maneuver themselves into a dead end - without noticing." 379

The reference to "the mathematicians" leads us back to Einstein's "logical-philosophical" thinking (letter to M. Solovine, 12 February 1951) in his later years as compared to physical argumentation. Fortunately, Einstein did not live long enough to be confronted with the deadly blow by Wyman \& Zassenhaus (Section 9.6.2) to his idea that knowledge of regular exact solutions would bring an advancement in the understanding of UFT.

From a point of view from outside the UFT-community, M. Fierz assessed the whole endeavor. In a letter to Pauli of October 9, 1951 he compared UFT with a particular tendency in psychology: "Likewise, the field-concept is analogous to the idea of milieu. Today, group-psychology is a big

\footnotetext{
377 "Die Gravitationsgleichungen waren nur auffindbar auf Grund eines rein formalen Prinzips (allgemeine Kovarianz), d.h. auf Grund des Vertrauens auf die denkbar größte logische Einfachheit der Naturgesetze. Da es klar war, dass die Gravitationstheorie nur einen ersten Schritt zur Auffindung der Feldgesetze darstellt, schien es mir, daß dieser logische Weg erst zu Ende gedacht werden muss, bevor man hoffen kann zu einer Lösung auch des Quantenproblems zu gelangen."

378 “[...] erscheinen wie der Wüsten-Vogel Strauss, der seinen Kopf dauernd in dem relativistischen Sand verbirgt, damit er die bösen Quanten nicht ins Auge sehen muss."

379 "[...] Allerdings habe ich neulich einen entscheidenden Fortschritt gemacht. Dieser bezieht sich auf eine Verbesserung der Theorie selber, was ihre Struktur anlangt, aber nicht auf das Auffinden von Lösungen, die an den Tatsachen geprüft werden könnten. [...] Die Sache ist aber in sich so plausibel und vollkommen, daß sie immer mehr Interesse bei den Mathematikern findet. Die eigentlichen Physiker dagegen verhalten sich ablehnend, weil sie sich in eine Sackgasse hineinmanövriert haben, ohne es zu merken." A photocopy of the letter is in the Einstein archive, call no. 38-450
} 
fashion in England and America. It is somewhat like Einstein's unitary field theory in the sense that the collective milieu is to explain everything such as the general field is to contain the whole of physics. Human beings are thus downgraded to herd animals - are made mechanical [...]." 380 ([490], p. 382.) His comparison seems to be a bit far-fetched, though. Perhaps, he felt that the field concept was no sufficient substitute for the notion of particle.

S. Schweber found a parallel between the manner of Einstein's theorizing and his views regarding world government and the organization to be established for preventing war ([563], p. 96).

\subsubsection{Philosophical background}

Often, mathematicians tend to be attracted by Platonic philosophy which assumes the existence of a world of ideas characterized by concepts like truth and beauty - with the possibility of only an approximative empirical approach to it. As H. Kragh pointed out in his biography of Dirac, since the 1930s Dirac supported the claim that, by following mathematical beauty, important advances in theory can be made ([339], p. 282). Kragh distinguished two aspects as to the function of such a "principle of mathematical beauty": it may serve as a recommendation for the process of theorybuilding, but it also may be used for a justification of a theory without strong empirical footing. In his Spencer Lecture of 1933, Einstein did not content himself with mathematical beauty:

"I am convinced that we can discover by means of purely mathematical constructions the concepts and the laws connecting them with each other, which furnish the key to the understanding of natural phenomena. Experience may suggest the appropriate mathematical concepts, but they most certainly cannot be deduced from it. Experience remains, of course, the sole criterion of physical utility of a mathematical construction. But the creative principle resides in mathematics. In a certain sense, therefore, I hold it true that pure thought can grasp reality, as the ancients dreamed." 381

Pauli found astonishing "[... ] Einstein's habit to call all those content with quantum mechanics 'positivists', 'empiricists', 'sensualists', 'phenomenalists', or the like." 382 In a personal note by him, we can read: "According to Einstein, proponents of quantum mechanics should say: 'the description is incomplete, yet its completion is meaningless because it has nothing to do with laws of nature.' Reduces to the question whether something about which no knowledge can be obtained exists. Einstein comments his own field theory "these laws are in Heaven, but not on Earth." 383 cf. ([491], 11 April 1953, p. 110).

After having worked on unified field theory since 1925, and having moved farther and farther away from experimental or observational evidence, Einstein needed such an epistemological and

\footnotetext{
380 "Der Feldbegriff hat auch eine Analogie zur Milieu-Vorstellung. Group-Psychology ist heute in England und Amerika große Mode. Das ist etwas wie die unitäre Feldtheorie Einsteins, indem das kollektive Milieu alles erklären soll, so wie das allgemeine Feld die ganze Physik enthalten soll. Der Mensch wird damit zum Herdentier degradiert - also unbewußt [...]."

381 English translation taken from ([339], p. 28.) cf. Einstein's original text ([164], p. 117): "Durch rein mathematische Konstruktion vermögen wir nach meiner Überzeugung diejenigen Begriffe und diejenige gesetzliche Verknüpfung zwischen ihnen zu finden, die den Schlüssel für das Verstehen der Naturerscheinungen liefern. Die brauchbaren mathematischen Begriffe können durch Erfahrung wohl nahegelegt, aber keinesfalls aus ihnen abgeleitet werden. Erfahrung bleibt natürlich das einzige Kriterium der Brauchbarkeit einer mathematischen Konstruktion für die Physik. Das eigentliche schöpferische Prinzip liegt aber in der Mathematik. In einem gewissen Sinne halte ich es also für wahr, daß dem reinen Denken das Erfassen des Wirklichen möglich sei, wie es die Alten geträumt haben."

382 "Um so erstaunlicher ist es mir, wenn Einstein die Gewohnheit hat, alle diejenigen, die mit der Quantenmechanik zufrieden sind, als 'Positivisten' 'Empiristen', 'Sensualisten', 'Phänomenalisten' oder sonstwie ähnlich zu titulieren."

383 "Einstein findet die Quantenmechaniker sollten sagen: 'die Beschreibung ist nicht vollständig, ihre Vervollständigung ist aber sinnlos, da sie nichts mit Naturgesetzen zu tun hat'. Läuft auf die Frage hinaus: ob etwas, über das man nichts wissen kann, existiert. Einstein sagt selbst über seine Feldtheorie, 'diese Gesetze sind im Himmel, aber nicht auf der Erde."'
} 
methodical justification for his research. He was convinced that there "is no logical path leading from the empirical material to the general principle which then supports logical deduction. [...] The further theory progresses, the clearer it becomes that the fundamental laws cannot be found inductively from the empirical facts (e.g., the gravitational field equations or the Schrödinger equation of quantum mechanics)." ([163], p. 468. $)^{384}$ Also, while Einstein ascribed a "creative principle" to mathematics, he just used this discipline as a quarry for the building of physical theories. After his decision for mixed geometry, a further creative influence of mathematics on his work can hardly be found. A mathematician who had followed the work in UFT by Einstein and others until 1945, stated bluntly:

"[...] the failure up to 1945 of the attempts at a unified theory might have been anticipated: each attempt was geometry and nothing more. The truism that, to get something empirically verifiable out of mathematics, something empirically known must be put into mathematics, appeared to have been overlooked" [18].

Now, many years later, we are permitted to extend Bell's date past 1945 until the end of the 1960s.

\section{What is reality?}

A central matter of dispute was Einstein's conception of reality. Philosophers of science have defined different categories of realism; to give as an example the one underlying the EPR-paper. Einstein's position then is classified accordingly; cf. [197], its review [62], and an interpretation of Einstein's understanding of locality and separability [289]. The following lines just reflect some aspects which have come up during this review.

W. Pauli formulated his opinion on the difference between Einstein and quantum physicists in a letter of 29 June 1953 to the Austrian philosopher F. Kröner (1989-1958): "It is due to the Einstein's restrictive philosophy' whereby an 'objective description of nature' is only such a description which demands potential validness without explicit reference to observation** ('detached observers')." ([491], p. 184-185. $)^{385}$ The annotation** reads as: "cf. also the final sentence of Einstein's 'The Meaning of Relativity', 4. Ed. 1953, Princeton University Press." The last two sentences in [156] have been quoted already toward the end of Section 9.3. In a letter to Heisenberg of 5 Juli 1954, Pauli explicated this:

"Essentially Einstein begins with a 'realistic metaphysics' (NB not with a deterministic one) assuring him a priori that observation cannot generate a state; e.g., if an observation leads to a ('quasi-sharp') position, then, in the 'objective-realistic description' of nature, even before the observation an 'element' would have been there which somehow 'corresponds' to the result of the observation. From it, Einstein infers his realistic dogma that in the 'objective-realistic description' the position of an electron ought to be determined 'quasi-sharply' always (in all states), i.e., up to quantities of ca. $10^{-13} \mathrm{~cm}$. Likewise, the position of the moon is determined independently of how we look at it. [...] The background of Einstein's realistic metaphysics is formed by

\footnotetext{
384 "Es gibt aber keinen logischen Weg, der vom empirischen Material zu dem allgemeinen Prizip führt, auf das sich dann die logische Deduktion stützt. [...] Je weiter die Theorie voranschreitet, desto deutlicher wird es, dass man die Grundgesetze nicht induktiv aus Erfahrungsthatsachen finden kann (z.B. die Feldgleichungen der Gravitationoder der Schrödinger-Gleichung der Quantenmechanik.)"

385 "Er liegt in der 'restriktiven Philosophie' Einsteins, wonach eine 'objektive Beschreibung' der Natur nach Einstein nur eine solche ist, welche potentielle Gültigkeit beansprucht, ohne auf eine Beobachtung explizite Bezug zu nehmen ('losgelöste Beobachter)'."'
} 
his belief that only it can ensure differentiation between the 'real' and what is merely imagined (dream, hallucination)." ([491], p. 706-707.) ${ }^{386}$

In the same direction went Pauli's letter to Max Born of 3 March 1954:

In conversations with Einstein, I have now seen that he takes exception to the essential premise of quantum mechanics that the "state" of a system is defined only by the specification of an experimental arrangement.[...] Einstein absolutely does not want to accept this. If one could measure precisely enough, this would be as true for macroscopic beadlets as for electrons. [...] But Einstein keeps the "philosophical" prejudice that (for macroscopic bodies) under all circumstances a "state" (said to be "real") can be "objectively" defined. This means without assigning an experimental set-up with the help of which the system (of macroscopic bodies) is investigated [...]. It appears to me that the discrepancy with Einstein may be reduced to this (his) assumption which I have called the notion or the "ideal" of the "detached observer". ${ }^{387}$ ([491], p. 509-510.)

In fact, for Einstein the quantum mechanical state function $\psi$ could not be interpreted as a "Realzustand": "The Realzustand cannot at all be described in present quantum theory but only (incomplete) knowledge with regard to a Realzustand. The 'orthodox' quantum theorists ban the concept of Realzustand in the first place (due to positivist considerations)". Einstein to Besso 10 August 1952 ([163], p. 483). ${ }^{388}$ This dispute might also be taken as an example for debaters using different categories: with Einstein arguing from ontology and Pauli methodologically. Modern experiments have vindicated Pauli's judgement about quantum physics and made obvious "the failure of Einstein's attempt to show the incompleteness of quantum theory." ([459], p. 182)

Until his death, Einstein insisted upon describing reality by a continuous field theory. In a letter of 16 October 1957 to Fierz, Pauli traced Einstein's attitude to an ancient philosophical dispute:

"I do not doubt that classical field physics pretty directly originates from the Stoa, in a continuous trend passing the ideas of the Renaissance and of the 17th century [...]. Insofar the synthesis of quantum theory and general relativity (and, generally, field quantization) is an unsolved problem, the old (ancient) conflict between atomists and the stoics continues." 389 ([493], p. 571)

\footnotetext{
386 "Einstein geht wesentlich von einer 'realistischen Metaphysik' aus (N.B. nicht von einer deterministischen), die ihn a priori vergewissert, daß die Beobachtung nicht einen Zustand erzeugen könne; wenn z.B. eine Beobachtung einen ('quasi-scharfen') Ort ergebe, so würde in der 'objektiven Real-Beschreibung' der Natur schon vor der Beobachtung ein 'Element' vorhanden sein, das diesem Beobachtungsresultat irgendwie 'entspreche'. Hieraus folgert Einstein dann sein realistisches Dogma, daß in der 'objektiven Real-Beschreibung' der Ort eines Elektrons immer (in allen Zuständen) 'quasi-scharf' (d.h. bis auf Größen von etwa $10^{-13} \mathrm{~cm}$ ) bestimmt sein müsse - ebenso wie der Ort des Mondes unabhängig davon, wie wir ihn anschauen, bestimmt sei. [...] Der Hintergrund von Einsteins realistischen Metaphysik ist der, daß er meint, nur diese könne eine Gewähr dafür bieten, 'das Wirkliche' von dem zu unterscheiden, 'was man sich blos einbildet' (Traum, Halluzination etc.)."

387 "Nun habe ich in Gesprächen mit Einstein gesehen,daß er Anstoß nimmt an der für die Quantenmechanik wesentlichen Voraussetzung, daß der "Zustand" eines Systems erst durch Angabe einer Versuchsanordnung definiert ist. [...] Davon will Einstein absolut nichts wissen. Wenn man genau genug messen könnte, wäre das natürlich für makroskopische Kügelchen genau so wahr wie für Elektronen. [...] Einstein aber hat das "philosophische" Vorurteil, daß sich (für makroskopische Körper) unter allen Umständen ein ("real" genannter) Zustand "objektiv" definieren lässt, d.h. ohne Angabe der Versuchsanordnung, mit deren Hilfe das System (der makroskopischen Körper) untersucht wird [...]. Es scheint mir, daß sich die Diskrepanz mit Einstein auf diese seine Voraussetzung reduzieren lässt, die ich die Idee oder das "Ideal" des "losgelösten Beobachters" genannt habe."

388 "Der Realzustand lässt sich in der gegenwärtigen Quantentheorie überhaupt nicht beschreiben, sondern nur ein (unvollständiges) Wissen inbezug auf einen Realzustand. Die 'orthodoxen' Quantentheoretiker verbieten überhaupt den Begriff des Realzustandes auf Grund positivistischer Erwägungen."

389 "Ich habe keinen Zweifel, daß die klassische Feldphysik ziemlich direkt von der Stoa "abstammt" in einer
} 


\section{Concluding Comment}

A leafing through the pages of this review of the history of classical unified field theory in the years between the mid 1930s and mid 1960s will suggest that some historians of physics, and almost all journalists writing about the evolution of classical unified field theories or "Theories of Everything" adjust their published views: their focus on Einstein's work in this field is not only excessive but unrealistic. This is due to at least two counts:

(1) Others like Schrödinger, Hlavatý and the totally neglected French groups (Tonnelat, Lichnerowicz) - not to point to the many further names given in this review - have contributed equal shares;

(2) In this field, Einstein has not produced novel ideas from which physics and mathematics could have benefited conceptually (cf. Section 18.1.2). Certainly, he was the most prominent player; it is fair to say that without the influence of the three eminent Nobel prize winners Einstein, Schrödinger and de Broglie (who backed M.-A. Tonnelat), the active period of research in classical UFT would have been shorter-lived, and its history much easier to write.

It remains a riddle why the signs pointing to a dead end of the Einstein-Schrödinger researchline of unified field theory, already highly visible before the 1950s, were overlooked or pushed aside by Einstein and others for so long: the flood of geometrical structures drowning a small number of physical concepts, the ambiguity in the dynamics for the "total field" (Lagrange density), the almost total lack of empirically testable output. Kaluza-Klein theory as the other type of unitary field theory fared much better. In combination with Weyl's second attempt toward a gauge principle, it paved the way to Yang-Mills theory able to describe the fundamental interactions with the exception of the gravitational. The final form of a generally accepted gauge theory of gravitation is still being discussed. Unfortunately, since the late 1930s, Einstein had written off Kaluza-Klein theory (cf. Section 3.2) and thus cannot be held in esteem for the successes of gauge theory.

In the wording of this review, the avowal, encountered occasionally, that Einstein's papers were read "sympathetically", will not be found. Like the papers of all other researchers, I tried to read his publications as neutrally as possible. While valuating research according to its place inside the body of unified field theory, I strove to judge past research from the physics at the time, not from the angle of the up-to-date state of the art. I am aware of the limits to this: the increase of knowledge since the period looked at, cannot be blanked out completely. Vice versa, no lesson from failed classical unified field theories is drawn here with regard to actual speculative theories with their claim to unify all four fundamental interactions. We just hope that current scientific practitioners are prepared to learn from the history of physics.

\section{Acknowledgements}

I am grateful to Thibault Damour, Institut des Hautes Études Scientifiques, Paris and member of the Academy of Sciences, Paris, for his helpful comments to Part I of this review, and for his advice in the final stages of writing Part II. My thanks go also to a referee whose thorough and thoughtful comments have helped to improve this article. It is my great pleasure to thank the staff of Living Reviews in Relativity, notably Mrs. Osswald and Mr. Schulz, for their friendly, generous and careful assistance.

kontinuierlichen, über die Ideen der Renaissance und des 17. Jahrhunderts führenden Entwicklung [... ]. Insoferne die Synthese von Quantentheorie und allgemeiner Relativitätstheorie (und Feldquantisierung überhaupt) ein offenes Problem ist, setzt sich der alte (antike) Konflikt zwischen den Atomisten und den Stoikern fort." 


\section{References}

[1] Abe, S. and Ikeda, M., "On groups of motions in space-time with a non-symmetric fundamental tensor $g_{\mu \nu}$. I", Tensor, New Ser., 10, 26-33 (1960). (Cited on page 160.)

[2] Abrol, M. L. and Mishra, R. S., "On the field equations of Bonnor's unified theory", Tensor, New Ser., 8, 14-20 (1958). (Cited on page 150.)

[3] Alfsen, E., "Schéma lagrangien de la théorie de l'électron de Flint", C. R. Hebd. Seanc. Acad. Sci., 232, 699-701 (1951). [ARK]. (Cited on page 42.)

[4] Antonelli, P. L., Handbook of Finsler Geometry, Vols. 1 and 2, (Kluwer, Boston, 2003). (Cited on pages 12 and 176.)

[5] Arnowitt, R. L., "Phenomenological Approach to a Unified Field Theory", Phys. Rev., 105, 735-742 (1957). [DOI]. (Cited on pages 101, 147, and 178.)

[6] Band, W., "On Flint's Five-Dimensional Theory of the Electron", Philos. Mag. (7), 29, 548-552 (1940). [DOI]. (Cited on page 42.)

[7] Bandyopadhyay, G., "Particular solutions of Einstein's recent unified field theories", Indian J. Phys., 25(5), 257-261 (1951). (Cited on pages 100 and 159.)

[8] Bandyopadhyay, G., "A strange feature of Einstein's most recent generalized field theory", Nature, 167, 648-649 (1951). [DOI]. (Cited on page 98.)

[9] Bandyopadhyay, G., "New Equation in the Affine Field Laws", Phys. Rev., 89, 1161 (1953). [DOI]. (Cited on page 158.)

[10] Bandyopadhyay, G., "General Static Spherically Symmetric solution of the 'Weaker' Equations of Einstein's Unified Theory", Sci. Cult., 25, 427-428 (1960). (Cited on page 98.)

[11] Bandyopadhyay, G., "A Theorem on Spherically Symmetric Solutions in Unified Theory", J. Math. Mech., 12, 655-662 (1963). (Cited on page 184.)

[12] Bargmann, V., "Relativity", Rev. Mod. Phys., 29, 161-174 (1957). [DOI]. (Cited on pages 10 and 190.)

[13] Bass, L., "Schrödinger: A Philosopher in Planck's Chair", Brit. J. Phil. Sci., 43(1), 111-127 (1992). [DOI]. (Cited on page 71.)

[14] Becker, K., Becker, M. and Schwarz, J. H., String Theory and M-Theory: A Modern Introduction, (Cambridge University Press, Cambridge; New York, 2007). (Cited on page 195.)

[15] Belinfante, F. J., "On the spin angular momentum of mesons", Physica, VI(9), 887-898 (1939). [DOI], [ADS]. (Cited on page 27.)

[16] Belinfante, F. J., "The undor equation of the meson field", Physica, VI(9), 870-886 (1939). [DOI]. (Cited on page 27.)

[17] Belinfante, F. J., "On the current and the density of the electric charge, the energy, the linear momentum and the angular momentum of arbitrary fields", Physica, 7, 449-474 (1940). [DOI]. (Cited on page 168.)

[18] Bell, E. T., The Development of Mathematics, (McGraw-Hill, New York, 1945), 2nd edition. [Google Books]. (Cited on page 199.) 
[19] Bergia, S., "Attempts at Unified Field Theories (1919-1955). Alleged Failure and Intrinsic Validation/Refutation Criteria", in Earman, J., Janssen, M. and Norton, J. D., eds., The Attraction of Gravitation: New Studies in the History of General Relativity, Third International Conference on the History of General Relativity at Johnstown, PA, U.S.A, 27-30 June 1991, Einstein Studies, 5, pp. 274-307, (Birkhäuser, Boston; Basel, 1993). (Cited on pages 10 and 52.)

[20] Bergmann, P. G., Introduction to the Theory of Relativity, (Prentice-Hall, Englewood Cliffs, NJ, 1942). [Google Books]. (Cited on pages 30 and 33.)

[21] Bergmann, P. G., "Unified Field Theory with Fifteen Field Variables", Ann. Math. (2), 49, 255-264 (1948). [DOI]. (Cited on pages 31 and 133.)

[22] Bergmann, P. G., "50 years of relativity", Science, 123(3195), 486-494 (1956). [DOI]. (Cited on pages 113 and 195.)

[23] Bergmann, P. G., "On Einstein's $\lambda$-Transformations", Phys. Rev., 103, 780-781 (1956). [DOI]. (Cited on pages 20 and 103.)

[24] Bergmann, P. G., "Unitary Field Theories", in De Sabbata, V. and Schmutzer, E., eds., Unified Field Theories of More Than 4 Dimensions: Including Exact Solutions, Proceedings of the International School of Cosmology and Gravitation held in Erice, Trapani, Sicily, 20 May-1 June 1982, pp. 1-10, (World Scientific, Singapore, 1983). (Cited on page 7.)

[25] Bertotti, B., "On the Relation Between Fundamental Tensor and Affinity in Unified Field Theory", Nuovo Cimento, 11, 358-365 (1954). [DOI]. (Cited on page 17.)

[26] Bertotti, B., "The Later Work of E. Schrödinger", Stud. Hist. Philos. Sci., 16, 83-100 (1985). [DOI]. (Cited on page 10.)

[27] Bkouche, R., "Sur la métrique asymétrique", C. R. Hebd. Seanc. Acad. Sci., 249, 2282-2284 (1959). [ARK]. (Cited on page 13.)

[28] Blackett, P. M. S., "The Magnetic Field of Massive Rotating Bodies", Nature, 159, 658-666 (1947). [DOI], [ADS]. (Cited on pages 51 and 52.)

[29] Blagojević, M. and Hehl, F. W., Gauge Theories of Gravitation: A Reader with Commentaries, (Imperial College Press, London, 2013). (Cited on page 9.)

[30] Blancheton, É., "Les équations aux variations de la relativité générale", Publ. Sci. Univ. Alger, Ser. A, 9, 31-117 (1962). Thèse de doctorat, 1961. (Cited on page 172.)

[31] Bonnor, W. B., "Static, spherically symmetric solutions in Einstein's unified field theory", Proc. $R$. Soc. London, Ser. A, 209, 353-368 (1951). [DOI]. (Cited on pages 95 and 98.)

[32] Bonnor, W. B., "The general static, spherically symmetric solution in Einstein's unified field theory", Proc. R. Soc. London, Ser. A, 210, 427-434 (1952). [DOI]. (Cited on pages 95, 98, and 113.)

[33] Bonnor, W. B., "Nonsymmetric Unified Field Theories", Phys. Rev., 92, 1067-1078 (1953). [DOI]. (Cited on pages 92 and 118.)

[34] Bonnor, W. B., "The equation of motion in the non-symmetric unified field theory", Proc. R. Soc. London, Ser. A, 226, 366-377 (1954). [DOI]. (Cited on pages 97, 101, and 150.)

[35] Bonnor, W. B., "Les équations du mouvement en théorie unitaire d'Einstein-Schrödinger", Ann. Inst. Henri Poincare, 15, 133-145 (1957). (Cited on page 118.)

[36] Born, M., "Modified Field Equations with a Finite Radius of the Electron", Nature, 132, 282 (1933). [DOI]. (Cited on page 45.) 
[37] Born, M., "On the Quantum Theory of the Electromagnetic Field", Proc. R. Soc. London, Ser. A, 143, 410-437 (1934). [DOI]. (Cited on pages 45 and 192.)

[38] Born, M., "Relativity and Quantum Theory", Nature, 141, 327-328 (1938). [DOI]. (Cited on page 38.)

[39] Born, M., "A Suggestion for Unifying Quantum Theory and Relativity", Proc. R. Soc. London, Ser. A, 165, 291-303 (1938). [DOI]. (Cited on pages 42 and 192.)

[40] Born, M., "Reciprocity Theory of Elementary Particles", Rev. Mod. Phys., 21, 463-473 (1949). [DOI]. (Cited on page 39.)

[41] Born, M., "Einstein and Relativity", Nature, 166, 751 (1950). [DOI]. Book review of The Meaning of Relativity, 4th edition, London: Methuen, 1950. (Cited on page 82.)

[42] Born, M. and Infeld, L., "Foundations of the New Field Theory", Proc. R. Soc. London, Ser. A, 144, 425-451 (1934). [DOI]. (Cited on pages 45, 46, 48, and 101.)

[43] Born, M. and Infeld, L., "On the Quantization of the New Field Theory I", Proc. R. Soc. London, Ser. A, 147, 522-546 (1934). (Cited on page 47.)

[44] Born, M. and Infeld, L., "On the Quantization of the New Field Theory II", Proc. R. Soc. London, Ser. A, 150, 141-166 (1935). [DOI]. (Cited on page 47.)

[45] Born, M. and Peng, H. W., "Quantum mechanics of fields. I. Pure fields", Proc. R. Soc. Edinburgh, 62, 40-57 (1944). (Cited on page 39.)

[46] Born, M. and Peng, H. W., "Quantum mechanics of fields. II. Statistics of pure fields", Proc. R. Soc. Edinburgh, 62, 92-102 (1944). (Cited on page 39.)

[47] Born, M. and Peng, H. W., "Quantum mechanics of fields. III. Electromagnetic field and electron field in interaction", Proc. R. Soc. Edinburgh, 62, 127-137 (1944). (Cited on page 39.)

[48] Born, M. and Schrödinger, E., "The absolute Field Constant in the New Field Theory", Nature, 135, 342 (1935). [DOI]. (Cited on page 50.)

[49] Born, M. and Schrödinger, E., "The Uncertainty Principle", Nature, 135, 261 (1935). (Cited on page 50.)

[50] Bose, S., "Solution d'une équation tensorielle invariante dans la théorie du champ unitaire", Bull. Soc. Math. Fr., 83, 81-88 (1955). Online version (accessed 20 June 2014): http://www .numdam.org/item?id=BSMF_1955_-83_-81_0. (Cited on page 112.)

[51] Bose, S. N., "Les identités de divergence dans la nouvelle théorie unitaire", C. R. Hebd. Seanc. Acad. Sci., 236, 1333-1335 (1953). [ARK]. (Cited on page 22.)

[52] Bose, S. N., "The Affine Connection in Einstein's New Unitary Field Theory", Ann. Math. (2), 59, 171-176 (1954). [DOI]. (Cited on pages 90 and 112.)

[53] Bouche, L., "Sur une autre forme des équations du champ dans une théorie unitaire du type EinsteinSchrödinger", C. R. Hebd. Seanc. Acad. Sci., 247, 2302-2304 (1958). [ARK]. (Cited on pages 119 and 124.)

[54] Bouche, L., "Les équations approchées du champ dans une théorie unitaire du type EinsteinSchrödinger", C. R. Hebd. Seanc. Acad. Sci., 249, 1321-1323 (1959). [ARK]. (Cited on page 124.)

[55] Bouche, L., "Identités de conservation dans une théorie du type Einstein-Schrödinger", C. R. Hebd. Seanc. Acad. Sci., 250, 3784-3785 (1960). [ARK]. (Cited on page 124.)

[56] Bouche, L., Une généralisation de la théorie du champ unifié relativiste d'Einstein-Schrödinger, Ph.D. thesis, (Faculté des Sciences de Paris, Paris, 1961). (Cited on pages 109, 119, 123, and 124.) 
[57] Boulier, F. and Neut, S., "Cartan's Characters and Stairs of Characteristic Sets", in Boztaş, S. and Shparlinski, I. E., eds., Applied Algebra, Algebraic Algorithms and Error-Correcting Codes, Proceedings of the 14th International Symposium, AAECC-14, Melbourne, Australia, November 26-30, 2001, Lecture Notes in Computer Science, 2227, pp. 363-372, (Springer, Berlin; New York, 2001). [DOI]. (Cited on page 85.)

[58] Brans, C. H., "The roots of scalar-tensor theory: an approximate history", arXiv, e-print, (2005). [arXiv:gr-qc/0506063]. (Cited on page 137.)

[59] Bray, M., Quelques solutions particulières en théorie du champ unifié, Ph.D. thesis, (Faculté des Sciences de Paris, Paris, 1960). (Cited on pages 113 and 124.)

[60] Brill, D. R. and Hartle, J. B., "Method of the Self-Consistent Field in General Relativity and its Application to the Gravitational Geon", Phys. Rev., 135, 271-278 (1964). [DOI]. (Cited on page 193.)

[61] Brinis, E., "Qualche illustrazione geometrica dello spazio unitario di Einstein", Rend. Ist. Lombardo Sci. Lett., Cl. Sci. Mat. Natur., III. Ser., 88, 531-538 (1955). (Cited on page 161.)

[62] Brown, H. R., "Book review: 'The Shaky Game - Einstein, Realism and the Quantum Theory", Am. J. Phys., 56(6), 571-573 (1988). (Cited on page 199.)

[63] Brown, L. M. and Rechenberg, H., "Quantum field theories, nuclear forces, and the cosmic rays (1934-1938)", Am. J. Phys., 59, 595-605 (1991). [DOI]. (Cited on page 8.)

[64] Buchdahl, H. A., "Variation of Integrals and the Field Equations in the Unitary Field Theory", Phys. Rev., 104, 1142-1145 (1956). [DOI]. (Cited on page 157.)

[65] Buchdahl, H. A., "Gauge-invariant generalization of field theories with asymmetric fundamental tensor", Quart. J. Math., 8, 89-96 (1957). [DOI]. (Cited on page 157.)

[66] Buchdahl, H. A., "Gauge-invariant generalization of field theories with asymmetric fundamental tensor (II)", Quart. J. Math., 9, 257-264 (1958). [DOI]. (Cited on page 157.)

[67] Buchner, K., "On Treder's Interpretation of Einstein's Unified Field Theory", Prog. Theor. Phys., 48(4), 1421-1422 (1972). (Cited on page 102.)

[68] Busemann, H., "The Geometry of Finsler Space", Bull. Am. Math. Soc., 56, 5-16 (1950). [DOI]. (Cited on page 175.)

[69] Callaway, J., "The equations of motion in Einstein's new unified field theory", Phys. Rev., 92, 1567-1570 (1953). [DOI]. (Cited on pages 91, 117, and 123.)

[70] Callaway, J., "Mach's principle and unified field theory", Phys. Rev., 96, 778-780 (1954). [DOI]. (Cited on pages 92 and 192.)

[71] Cap, F., "Über allgemeine Relativitätstheorie und einheitliche Feldtheorie", Acta Phys. Austriaca, 6, 135-156 (1952). (Cited on page 10.)

[72] Capella, A., "Sur la quantification du champ unitaire en théorie de Jordan-Thiry à l'approximation linéaire", C. R. Hebd. Seanc. Acad. Sci., 250, 2140-2142 (1960). [ARK]. (Cited on page 171.)

[73] Capella, A., "Théorie minkowskienne de la gravitation", C. R. Hebd. Seanc. Acad. Sci., 258, 87-89 (1964). [ARK]. (Cited on page 167.)

[74] Cartan, É., Les Espaces de Finsler, Actualités Scientifiques et Industrielles, 79, (Hermann, Paris, 1934). (Cited on page 175.)

[75] Cattaneo-Gasparini, I., "Sulle geodetiche di una $V_{n}$ relative a una connessione affine", Rend. Lincei, 22, 146-154 (1957). (Cited on page 161.) 
[76] Chamseddine, A. H. and Mukhanov, V., "Massive Hermitian Gravity", J. High Energy Phys., 2012(08), 036 (2012). [DOI], [arXiv:1205.5828]. (Cited on page 64.)

[77] Chau, N. P., "Le problème de mouvement en théorie du champ unifié asymétrique", C. R. Hebd. Seanc. Acad. Sci., 252, 4123-4125 (1961). [ARK]. (Cited on pages 117, 118, and 124.)

[78] Choquet-Bruhat, Y., "Theoremes d'existence en mécanique des fluides relativistes", Bull. Soc. Math. France, 6, 155-175 (1958). (Cited on page 128.)

[79] Clauser, E., "Una particolare soluzione delle equazione einsteiniane della relatività unitaria", Rend. Lincei, 15(3-4), 171-177 (1953). (Cited on page 100.)

[80] Clauser, E., "Sui fronti d'onda nella teoria unitaria Einsteiniana", Rend. Ist. Lombardo Sci. Lett., Cl. Sci. Mat. Natur., III. Ser., 87, 473-492 (1954). (Cited on page 128.)

[81] Clauser, E., "Movimento di particelle nel campo unitario einsteiniano", Rend. Lincei, Ser. VIII, 21, 408-416 (1956). (Cited on pages 115, 116, 117, 118, and 163.)

[82] Clauser, E., "Legge di moto nell'ultima teoria unitaria einsteiniana", Nuovo Cimento, 7, 764-788 (1958). [DOI]. (Cited on pages 117 and 163.)

[83] Clauser, E., "Condizioni di integrabilià e moto di particelle nell'ultima teoria unitaria einsteiniana", Rend. Lincei, 26, 498-505 (1959). (Cited on pages 117 and 164.)

[84] Corson, E. M., Introduction to Tensors, Spinors, and Relativistic Wave-Equations, (Blackie \& Son, London and Glasgow, 1953). (Cited on page 42.)

[85] Cossu, A., "Alcune osservazioni sul confronto tra connessioni affini e metriche riemanniane", Rend. Lincei, 14, 29-35 (1953). (Cited on page 161.)

[86] Cossu, A., "Alcune osservazioni sulle connessioni tensoriali", Rend. Mat. Appl. (5), 13, 373-390 (1955). (Cited on page 161.)

[87] Costa de Beauregard, O., "Sur la dynamique des milieux doués d'une densité de moment cinétique propre", C. R. Hebd. Seanc. Acad. Sci., 214, 904-906 (1942). [ARK]. (Cited on page 154.)

[88] Costa de Beauregard, O., "L'hypothèse de l'effet gravitationnel de spin", Cah. Phys., 12(99), 407-415 (1958). (Cited on page 156.)

[89] Costa de Beauregard, O., "Métrique asymétrique et représentation des changements d'axes locaux. Application á la théorie de l'effet gravitationnel de spin”, C. R. Hebd. Seanc. Acad. Sci., 250, 984-986 (1960). [ARK]. (Cited on page 155.)

[90] Costa de Beauregard, O., "L'effet gravitationnel de spin sur la particule de spin zéro", C. R. Hebd. Seanc. Acad. Sci., 253, 1761-1763 (1961). [ARK]. (Cited on page 156.)

[91] Costa de Beauregard, O., "Sur le terme en 55 de la théorie unitaire pentadimensionnelle", $C . R$. Hebd. Seanc. Acad. Sci., 255, 3383-3385 (1962). [ARK]. (Cited on page 172.)

[92] Costabel, P., "Marie-Antoinette Tonnelat (1912-1880)", Rev. Hist. Sci., 36(3-4), 329-331 (1983). (Cited on pages 104 and 181.)

[93] Crumeyrolle, A., Sur quelques interprétations physiques et théoriques des équations du champ unitaire d'Einstein-Schrödinger, Ph.D. thesis, (University of Paris, Paris, 1961). (Cited on pages 101 and 131.)

[94] Crumeyrolle, A., "Sur quelques interprétations physiques et théoriques des équations du champ unitaire d'Einstein-Schrödinger", Riv. Mat. Univ. Parma (2), 3(2), 331-391 (1962). (Cited on pages 132 and 189.) 
[95] Crumeyrolle, A., "Sur des variétés différentielles dont les coordonnées appartiennent à une extension quadratique du corps des réels et application à la théorie unitaire d'Einstein-Schrödinger", $C . R$. Hebd. Seanc. Acad. Sci., 256, 2121-2123 (1963). [ARK]. (Cited on page 141.)

[96] Crumeyrolle, A., "Sur quelques interprétations physiques et théoriques des équations du champ unitaire d'Einstein-Schrödinger. II", Riv. Mat. Univ. Parma (2), 5, 85-132 (1964). (Cited on pages 132 and 141.)

[97] Crumeyrolle, A., "Variétés différentiables à coordonnées hypercomplexes. Applications à une géométrisation et à une généralisation de la théorie d'Einstein-Schrödinger", Ann. Fac. Sci. Toulouse (4), 26, 105-137 (1964). Online version (accessed 20 June 2014): http://www.numdam.org/item?id=AFST_1962_4_26_-105_0. (Cited on pages 132 and 141.)

[98] Crumeyrolle, A., "Variétés hypercomplexes et théorie unitaire d'Einstein", in Atti del Settimo Congresso dell'Unione Matematica Italiana, Proceedings of the congress held in Genova, 30 September - 5 October 1963, pp. 383-384, (Edizioni Cremonese, Roma, 1965). (Cited on page 141.)

[99] Damour, T., "A new and consistent method for classical renormalization", Nuovo Cimento B, 26, 157-164 (1975). [DOI], [ADS]. (Cited on page 180.)

[100] Damour, T., "On some links between mathematical physics and physics in the context of general relativity", in Flato, M., Kerner, R. and Lichnerowicz, A., eds., Physics on Manifolds, Proceedings of the International Colloquium in honour of Yvonne Choquet-Bruhat, Paris, June 3-5, 1992, Mathematical Physics Studies, 15, pp. 59-65, (Kluwer, Dordrecht; Boston, 1994). (Cited on page 64.)

[101] Damour, T., Deser, S. and McCarthy, J., "Theoretical problems in nonsymmetric gravitational theory", Phys. Rev. D, 45, R3289-R3291 (1992). [DOI], [ADS]. (Cited on page 64.)

[102] Damour, T., Deser, S. and McCarthy, J., "Nonsymmetric gravity theories: Inconsistencies and a cure", Phys. Rev. D, 47, 1541-1556 (1993). [DOI]. (Cited on pages 64 and 85.)

[103] Damour, T. and Esposito-Farèse, G., "Tensor-multi-scalar theories of gravitation", Class. Quantum Grav., 9, 2093-2176 (1992). [DOI]. (Cited on page 31.)

[104] Damour, T., Gibbons, G. W. and Gundlach, C., "Dark matter, time-varying G, and a dilaton field", Phys. Rev. Lett., 64, 123-126 (1990). [DOI], [ADS]. (Cited on page 31.)

[105] Damour, T. and Nordtvedt, K., "General relativity as a cosmological attractor of tensor-scalar theories", Phys. Rev. Lett., 70, 2217-2219 (1993). [DOI]. (Cited on page 31.)

[106] Dăngvu, H., "Étude des équations du champ de Jordan-Thiry en repère naturel adapté", $C$. $R$. Hebd. Seanc. Acad. Sci., 260, 4307-4310 (1965). [ARK]. (Cited on page 136.)

[107] Dăngvu, H., "Contribution à une formulation Minkowskienne de la gravitation Einsteinienne par une méthode du type Rosen", Cah. Phys., 21(198), 53-99 (1967). (Cited on page 169.)

[108] Dăngvu, H., "Nonstatic Solutions with Spherical Symmetry for Jordan-Thiry field equations", $C$. R. Acad. Sci. Ser. A, 267, 61-64 (1968). (Cited on page 137.)

[109] Darrigol, O., "La genèse du concept de champ quantique", Ann. Phys. (Paris), 9, 433-501 (1984). (Cited on page 7.)

[110] Dautcourt, G., "Sur la solution de l'équation d'Einstein $g_{\mu_{\nu} ; \rho}=0$ ", C. R. Hebd. Seanc. Acad. Sci., 249, 2159-2161 (1959). [ARK]. (Cited on page 113.)

[111] de Broglie, L., "Sur la nature du photon", C. R. Hebd. Seanc. Acad. Sci., 198, 135-138 (1934). [ARK]. (Cited on page 8.)

[112] de Broglie, L., "La théorie du photon et la mécanique ondulatoire relativiste des systèmes", $C$. $R$. Hebd. Seanc. Acad. Sci., 203, 473-477 (1936). [ARK]. (Cited on page 8.) 
[113] de Broglie, L., "A general survey of the scientific work of Albert Einstein", in Schilpp, P., ed., Albert Einstein: Philosopher-Scientist, pp. 109-127, (Tudor, New York, 1951). (Cited on page 105.)

[114] De Simoni, F., "Le soluzioni generali della statica a simmetria sferica nell'ultima teoria unitaria di Einstein", Rend. Lincei, Ser. VIII, 16, 348-355 (1954). (Cited on page 98.)

[115] De Simoni, F., "Sulle equazioni di campo della teoria relativistica unitaria", Rend. Lincei, Ser. VIII, 18, 297-304 (1955). (Cited on page 161.)

[116] Debever, R., ed., Elie Cartan-Albert Einstein. Lettres sur le parallélisme absolu 1929-1932, (Académie Royale de Belgique and Princeton University Press, Bruxelles, 1979). (Cited on pages 59, 73, and 84.)

[117] Deser, S., "Self-interaction and gauge invariance", Gen. Relativ. Gravit., 1, 9-18 (1970). [DOI], [ADS], [arXiv:gr-qc/0411023]. (Cited on page 168.)

[118] Deser, S. and Laurent, B. E., "Gravitation without self-interaction", Ann. Phys. (N.Y.), 50, 76-101 (1968). [DOI]. (Cited on page 168.)

[119] DeWitt, B. S., ed., Papers from the Conference on the Role of Gravitation in Physics Held at the University of North Carolina, Chapel Hill, North Carolina, January 18-23, 1957, Proceedings from the Conference held at the University of North Carolina, Chapel Hill, North Carolina, January 18 -23, 1957, Rev. Mod. Phys., 29, (APS, College Park, MD, 1957). (Cited on page 10.)

[120] DeWitt, C. M. and Rickles, D., eds., The Role of Gravitation in Physics: Report from the 1957 Chapel Hill Conference, WADC Technical Report 57-216 from the Conference at Chapel Hill, North Carolina, January 18-23, 1957, Edition Open Accesss: Sources, 5, (Max Planck Research Library for the History and Development of Knowledge, Berlin, 2011). URL (accessed 11 February 2014): http://edition-open-access.de/sources/5/. (Cited on pages 125, 193, and 194.)

[121] Dirac, P. A. M., "Quantized singularities in the electromagnetic field", Proc. R. Soc. London, Ser. A, 133, 60-73 (1931). [DOI]. (Cited on page 138.)

[122] Dirac, P. A. M., "The Cosmological Constants", Nature, 139, 323 (1937). [DOI], [ADS]. (Cited on page 30.)

[123] Dirac, P. A. M., "A new basis for cosmology", Proc. R. Soc. London, Ser. A, 165, 199-208 (1938). [DOI], [ADS]. (Cited on page 30.)

[124] Dirac, P. A. M., "The Evolution of the Physicist's Picture of Nature", Sci. Am., 208(5), 45-53 (1963). [DOI]. (Cited on page 190.)

[125] Dirac, P. A. M., "Methods in Theoretical Physics", in Salam, A., ed., From a Life of Physics: Special Supplement of the IAEA Bulletin 1969, International Symposium on Contemporary Physics, Trieste, Italy, 1968, IAEA Bulletin, pp. 21-28, (International Atomic Energy Agency, Wien, 1969). also published in: From a Life of Physics, World Scientific, 1989, pp.19-30, ed. Salam, A. (Cited on page 189.)

[126] Dirac, P. A. M., Directions in Physics, (Wiley, New York, 1978). (Cited on page 194.)

[127] Droz-Vincent, P., "Généralisation des équations d'Einstein correspondant à l'hypothèse d'une masse non nulle pour le graviton", C. R. Hebd. Seanc. Acad. Sci., 249, 2290-2292 (1959). [ARK]. (Cited on page 166.)

[128] Droz-Vincent, P., Quantification du champ à l'approximation linéaire en théorie de Jordan-Thiry, Ph.D. thesis, (Université de Paris. Faculté des sciences, Paris, 1959). (Cited on page 171.)

[129] Droz-Vincent, P., "Une méthode de quantification en théorie unitaire pentadimensionnelle", $C . R$. Hebd. Seanc. Acad. Sci., 248, 1790-1792 (1959). [ARK]. (Cited on pages 170 and 171.) 
[130] Droz-Vincent, P., "Crochets de Poisson dans le cas d'un champ au lagrangien linéaire", C. R. Hebd. Seanc. Acad. Sci., 253, 2862-2864 (1961). [ARK]. (Cited on page 173.)

[131] Droz-Vincent, P., "Sur les équations du champ unitaire varié", C. R. Hebd. Seanc. Acad. Sci., 252, 3405-3407 (1961). [ARK]. (Cited on page 173.)

[132] Droz-Vincent, P., "Quantification de la théorie de Jordan-Thiry", in Lichnerowicz, A. and Tonnelat, M. A., eds., Les Théories Relativistes de la Gravitation, Actes du colloque tenu à Royaumont, les 21-27 Juin 1959, Colloques Internationaux du CNRS, 91, pp. 359-372, (Éditions du CNRS, Paris, 1962). (Cited on page 193.)

[133] Droz-Vincent, P., "Contribution á la quantification des théories relativistes de électromagnétisme et de la gravitation", Cah. Phys., 18(170), 369-405 (1964). (Cited on pages 170, 171, and 173.)

[134] Droz-Vincent, P., "Quantification du champ pentadimensionnel linéarisé", Cah. Phys., 18(161), 2537 (1964). (Cited on page 171.)

[135] Droz-Vincent, P., "Suppression des contraintes du champ de gravitation par l'introduction d'une source vectorielle", C. R. Hebd. Seanc. Acad. Sci., 259, 515-518 (1964). [ARK]. (Cited on page 173.)

[136] Droz-Vincent, P., "Poisson brackets of the constraints in unified field theory", Ann. Inst. Henri Poincare, 7(4), 319-331 (1967). (Cited on page 173.)

[137] Dyson, F. J., "Field Theory", Sci. Am., 188(4), 57-64 (1953). [DOI]. (Cited on pages 40 and 88.)

[138] Eddington, A. S., "A generalisation of Weyl's theory of the electromagnetic and gravitational fields", Proc. R. Soc. London, Ser. A, 99, 104-122 (1921). [DOI]. (Cited on pages 101 and 178.)

[139] Eddington, A. S., The Mathematical Theory of Relativity, (Cambridge University Press, Cambridge, 1923). (Cited on page 175.)

[140] Eddington, A. S., The mathematical theory of relativity, (Cambridge University Press, Cambridge, 1930), 2nd edition. (Cited on page 53.)

[141] Einstein, A., "Zur allgemeinen Relativitätstheorie", Sitzungsber. Preuss. Akad. Wiss., 1923(V), 3238 (1923). (Cited on pages 49, 68, and 101.)

[142] Einstein, A., "Einheitliche Feldtheorie von Gravitation und Elektrizität", Sitzungsber. Preuss. Akad. Wiss., 1925(XXII), 414-419 (1925). (Cited on pages 55, 57, and 58.)

[143] Einstein, A., "Physik und Realität", J. Franklin Inst., 221(3), 313-337 (1936). [DOI]. (Cited on pages 33 and 191.)

[144] Einstein, A., "Demonstración de la no existencia de campos gravitacionales sin singularidades de masa total no nula", Rev. Univ. Nac. Tucuman, Ser. A, 2, 5-10 (1941). (Cited on page 125.)

[145] Einstein, A., "Demonstration of the non-existence of gravitational fields with a non-vanishing total mass free of singularities", Rev. Univ. Nac. Tucuman, Ser. A, 2, 11-15 (1941). (Cited on pages 74 and 125.)

[146] Einstein, A., "Bivector Fields II", Ann. Math. (2), 45, 15-23 (1944). [DOI]. (Cited on page 37.)

[147] Einstein, A., "A Generalization of the Relativistic Theory of Gravitation", Ann. Math. (2), 46, 578-584 (1945). [DOI]. (Cited on pages 18, 23, 57, 58, 59, 60, 61, 62, 65, 68, 69, 79, 81, 100, and 151.)

[148] Einstein, A., "A generalized theory of gravitation", Rev. Mod. Phys., 20, 35-39 (1948). [DOI]. (Cited on pages $75,76,80,95$, and 151.)

[149] Einstein, A., "The Bianchi identities in the generalized theory of gravitation", Can. J. Math., 2, 120-128 (1950). [DOI]. (Cited on pages 19, 76, 79, 80, and 152.) 
[150] Einstein, A., The Meaning of Relativity, (Princeton University Press, Princeton, 1950), 3rd rev. edition. including 'The Generalized Theory of Gravitation'. (Cited on pages 10, 22, 23, 77, 81, 82, $83,84,88,95$, and 142.)

[151] Einstein, A., The Meaning of Relativity, (Methuen, London, 1950), 4th edition. with further appendix. (Cited on pages 23, 80, and 82.)

[152] Einstein, A., "On the Generalized Theory of Gravitation", Sci. Am., 182(April), 13-17 (1950). [DOI]. (Cited on pages 85 and 86.)

[153] Einstein, A., "Autobiographical Notes", in Albert Einstein: Philosopher-Scientist, The Library of Living Philosophers, VII, pp. 1-95, (Tudor Publishing, New York, 1951), 2nd rev. edition. ed. Schilpp, P. A. (Cited on page 78.)

[154] Einstein, A., The Meaning of Relativity, (Methuen, London, 1951), 5th rev. edition. including "The Generalized Theory of Gravitation". (Cited on page 82.)

[155] Einstein, A., "Einleitende Bemerkungen über Grundbegriffe", in Louis de Broglie: physicien et penseur, pp. 4-15, (Éditions Albin Michel, Paris, 1953). (Cited on page 89.)

[156] Einstein, A., The Meaning of Relativity, (Princeton University Press, Princeton, 1953), 4th rev. edition. including 'The Generalized Theory of Gravitation'. (Cited on pages 10, 20, 23, 81, 84, 87, $88,89,95,161$, and 199.)

[157] Einstein, A., Ideas and Opinions, (Crown Publishers, New York, 1954). (Cited on page 189.)

[158] Einstein, A., The Meaning of Relativity, (Princeton University Press, Princeton, 1955), 5th rev. edition. including 'The Generalized Theory of Gravitation'. (Cited on pages 84, 85, 93, 94, 101, and 194.)

[159] Einstein, A., "Prefazione di Albert Einstein", in Pantaleo, M., ed., Cinquant'anni di relatività 19051955, pp. XV-XVII, (Editrice Universitaria, Firenze, 1955). (Cited on page 194.)

[160] Einstein, A., Albert Einstein. Briefe an Maurice Solovine, (Gauthier-Villars, Paris, 1956). ed. Solovine, M. (Cited on pages 63, 75, 80, 88, 94, and 188.)

[161] Einstein, A., "A Comment on a Criticism of Unified Field Theory", Phys. Rev., 89, 321 (1956). (Cited on page 86.)

[162] Einstein, A., The Meaning of Relativity, (Methuen, London, 1956), 6th edition. Appendix II. (Cited on page 93.)

[163] Einstein, A., Correspondance avec Michele Besso 1903-1955, (Hermann, Paris, 1972). (Cited on pages $35,59,60,77,191,199$, and 200.)

[164] Einstein, A., Albert Einstein: Mein Weltbild, Ullstein Materialien, (Ullstein, Frankfurt, 1984). (Cited on page 198.)

[165] Einstein, A. and Bargmann, V., "Bivector Fields", Ann. Math. (2), 45, 1-14 (1944). [DOI]. (Cited on pages 35 and 36. )

[166] Einstein, A., Bargmann, V. and Bergmann, P. G., "On the Five-Dimensional Representation of Gravitation and Electricity", in Theodore von Kármán Anniversary Volume: Contributions to Applied Mechanics and Related Subjects by the Friends of Theodore Von Kármán on His Sixtieth Birthday, pp. 212-225, (Caltech, Pasadena, 1941). (Cited on page 33.)

[167] Einstein, A. and Bergmann, P., "On a Generalization of Kaluza's Theory of Electricity", Ann. Math. (2), 39, 683-701 (1938). [DOI]. (Cited on page 33.) 
[168] Einstein, A., Born, H. and Born, M., Briefwechsel: 1916-1955 kommentiert von Max Born; Geleitwort von Bertrand Russell; Vorwort von Werner Heisenberg, (Edition Erbrich, Frankfurt am Main, 1982). (Cited on pages 38, 39, 46, 48, 56, 75, 83, and 86.)

[169] Einstein, A. and Fokker, A. D., "Nordströmsche Gravitationstheorie vom Standpunkt des absoluten Differentialkalküls", Ann. Phys. (Leipzig), 44, 321-328 (1914). [DOI]. (Cited on page 166.)

[170] Einstein, A. and Infeld, L., "On motion of particles in general relativity", Can. J. Math., 1, 209-241 (1949). [DOI]. (Cited on page 117.)

[171] Einstein, A., Infeld, L. and Hoffmann, B., "The Gravitational Equations and the Problem of Motion", Ann. Math. (2), 39, 65-100 (1938). [DOI]. (Cited on page 117.)

[172] Einstein, A. and Kaufman, B., "Sur l'état actuel de la théorie générale de la gravitation", in Louis de Broglie: physicien et penseur, pp. 321-342, (Éditions Albin Michel, Paris, 1953). (Cited on pages 89, 90, and 96.)

[173] Einstein, A. and Kaufman, B., "Algebraic Properties of the Field in the Relativistic Theory of the Asymmetric Field", Ann. Math., 59, 230-244 (1954). [DOI]. (Cited on page 90.)

[174] Einstein, A. and Kaufman, B., "A New Form of the General Relativistic Field Equations", Ann. Math. (2), 62, 128-138 (1955). [DOI]. (Cited on pages 92, 93, 94, and 96.)

[175] Einstein, A. and Mayer, W., "Einheitliche Theorie von Gravitation und Elektrizität", Sitzungsber. Preuss. Akad. Wiss., 1931(XXV), 541-557 (1931). (Cited on page 138.)

[176] Einstein, A. and Mayer, W., "Systematische Untersuchung über kompatible Feldgleichungen, welche in einem Riemannschen Raume mit Fernparallelismus gesetzt werden können", Sitzungsber. Preuss. Akad. Wiss., 1931(XIII), 257-265 (1931). (Cited on page 23.)

[177] Einstein, A. and Pauli, W., "On the Non-Existence of Regular Stationary Solutions of Relativistic Field Equations", Ann. Math. (2), 44, 131-137 (1943). [DOI]. (Cited on pages 74, 125, and 134.)

[178] Einstein, A. and Rosen, N., "The particle problem in the general theory of relativity", Phys. Rev., 48, 73-77 (1935). [DOI]. (Cited on page 59.)

[179] Einstein, A. and Straus, E. G., "A Generalization of the Relativistic Theory of Gravitation, II", Ann. Math. (2), 47, 731-741 (1946). [DOI]. (Cited on pages 57, 58, 62, 65, 67, 68, 69, 76, 78, 79, and 81.)

[180] Einstein, H. A. and El-Samni, E.-S. A., "Hydrodynamic Forces on a Rough Wall", Rev. Mod. Phys., 21, 520-524 (1949). [DOI]. (Cited on page 78.)

[181] Eisenhart, L. P., "Einstein's recent theory of gravitation and electricity", Proc. Natl. Acad. Sci. USA, 12, 125-129 (1926). [DOI]. (Cited on page 142.)

[182] Eisenhart, L. P., Non-Riemannian Geometry, AMS Colloquium Publications, 8, (American Mathematical Society, Providence, RI, 1927). (Cited on pages 14 and 142.)

[183] Eisenhart, L. P., ed., The Theory of Relativity in Contemporary Science, Papers read at the Celebration of the Seventieth Birthday of Professor Albert Einstein in Princeton, March 19, 1949, Proc. Amer. Phil. Soc., 93, (American Philosophical Society, Philadelphia, 1949). [Google Books]. (Cited on page 78.)

[184] Eisenhart, L. P., "Generalized Riemann Spaces", Proc. Natl. Acad. Sci. USA, 37, 311-315 (1951). [DOI]. (Cited on page 142.)

[185] Eisenhart, L. P., "Generalized Riemann Spaces. II.", Proc. Natl. Acad. Sci. USA, 38, 505-508 (1952). [DOI]. (Cited on page 142.) 
[186] Eisenhart, L. P., "A Unified Theory of General Relativity and Electromagnetism", Proc. Natl. Acad. Sci. USA, 42, 249-251 (1956). (Cited on page 142.)

[187] Eisenhart, L. P., "A Unified Theory of General Relativity and Electromagnetism. II", Proc. Natl. Acad. Sci. USA, 42, 646-650 (1956). (Cited on pages 142 and 143.)

[188] Eisenhart, L. P., "A Unified Theory of General Relativity and Electromagnetism. III", Proc. Natl. Acad. Sci. USA, 42, 878-881 (1956). (Cited on pages 142 and 143.)

[189] Eisenhart, L. P., "A Unified Theory of General Relativity and Electromagnetism. IV", Proc. Natl. Acad. Sci. USA, 43, 333-336 (1957). [DOI]. (Cited on pages 142 and 143.)

[190] Eisenhart, L. P., "Generalized Spaces of General Relativity", Proc. Natl. Acad. Sci. USA, 45, 17591762 (1959). [DOI]. (Cited on page 143.)

[191] Eisenhart, L. P., Riemannian Geometry, (Princeton University Press, Princeton, NJ, 1964). 5th printing (1st printing 1926). (Cited on page 26.)

[192] Eisenstaedt, J., "The low water mark of general relativity, 1925 - 1955", in Howard, D. and Stachel, J., eds., Einstein and the History of General Relativity, Based on the proceedings of the 1986 Osgood Hill Conference, North Andover, Massachusetts, 8-11 May, Einstein Studies, 1, pp. 277-292, (Birkhäuser, Boston; Basel, 1989). (Cited on page 7.)

[193] Emparan, R., Harmark, T., Niarchos, V. and Obers, N. A., "Essentials of Blackfold Dynamics", J. High Energy Phys., 2010(03), 063 (2010). [DOI], [arXiv:0910.1601]. (Cited on page 196.)

[194] Enz, C. P., No Time to be Brief: A Scientific Biography of Wolfgang Pauli, (Oxford University Press, Oxford, 2002). [DOI]. (Cited on pages 10, 126, and 139.)

[195] Fierz, M., "On the physical interpretation of P. Jordan's extended theory of gravitation", Helv. Phys. Acta, 29, 128-134 (1956). (Cited on pages 31 and 32.)

[196] Fierz, M. and Pauli, W., "On relativistic wave equations for particles of arbitrary spin in an electromagnetic field", Proc. R. Soc. London, Ser. A, 173, 211-232 (1939). [DOI], [ADS]. (Cited on page 167.)

[197] Fine, A., The Shaky Game: Einstein, Realism and the Quantum Theory, (The University of Chicago Press, Chicago; London, 1986). (Cited on page 199.)

[198] Fink, K., "Metrisches Feld und skalares Materiefeld", Comment. Math. Helv., 25, 26-42 (1951). [DOI]. (Cited on page 32.)

[199] Finsler, P., Über Kurven und Flächen in allgemeinen Räumen, Lehrbücher und Monographien aus dem Gebiete der exakten Wissenschaften: Mathematische Reihe, 11, (Birkhäuser, Basel, 1951). Unveränderter Nachdruck der Dissertation von 1918. (Cited on page 12.)

[200] Finzi, B., "La récente théorie relativiste unitaire d'Einstein", Rend. Mat. Appl. (5), 11(1), 75-87 (1952). (Cited on page 86.)

[201] Finzi, B., "Su le equazioni di campo della teoria relativistica unitaria di Einstein", Rend. Lincei, 14, 581-588 (1953). (Cited on page 161.)

[202] Finzi, B., "Calcolo tensoriale: teorie relativistiche e teorie unitarie (Cinquantenario della relatività di Einstein 1905-1955)", Conf. Semin. Mat. Univ. Bari, 15, 42-51 (1955). (Cited on page 161.)

[203] Finzi, B., "Teorie relativistiche unitarie", in Atti del Quinto Congresso dell'Unione Matematica Italiana, Proceedings of the congress held in Pavia and Torino, 6-9 October 1955, pp. 35-56, (Edizioni Cremonese, Roma, 1956). (Cited on pages 10, 101, 161, and 190.) 
[204] Flint, H. T., "A Relativistic Basis of Quantum Theory", Proc. R. Soc. London, Ser. A, 144, 413-424 (1934). (Cited on pages 40 and 41.)

[205] Flint, H. T., "A Relativistic Basis of Quantum Theory II", Proc. R. Soc. London, Ser. A, 145, 645-656 (1934). (Cited on page 41.)

[206] Flint, H. T., "A Relativistic Basis of the Quantum Theory. III", Proc. R. Soc. London, Ser. A, 150, 421-441 (1935). [DOI]. (Cited on pages 41 and 44.)

[207] Flint, H. T., "The Analogy between the Photon and the Electron and the Derivation of the Quantum Equation", Proc. Phys. Soc., 50, 899-909 (1938). [DOI]. (Cited on page 41.)

[208] Flint, H. T., "The Theory of the Electric Charge and the Quantum Theory. - Part I", Philos. Mag. (7), 29, 330-343 (1940). [DOI]. (Cited on page 41.)

[209] Flint, H. T., "The Theory of the Electric Charge and the Quantum Theory. - Part II", Philos. Mag. (7), 29, 417-433 (1940). [DOI]. (Cited on page 41.)

[210] Flint, H. T., "The Theory of the Electric Charge and the Quantum Theory. - Part III", Philos. Mag. (7), 33, 369-383 (1942). [DOI]. (Cited on page 41.)

[211] Flint, H. T., "A Study of the Nature of the Field Theories of the Electron and Positron and of the Meson", Proc. R. Soc. London, Ser. A, 185, 14-34 (1944). (Cited on page 41.)

[212] Flint, H. T., "Quantum Equations and Nuclear Field Theories", Philos. Mag. (7), 36, 635-643 (1945). [DOI]. (Cited on page 41.)

[213] Flint, H. T., "Grundlagen zu einer linearen Feldtheorie", Z. Phys., 142, 401-405 (1955). [DOI]. (Cited on page 33.)

[214] Flint, H. T. and Williamson, E. M., "Die Beziehung der Quantentheorie zu den Theorien der Gravitation und des Elektromagnetismus und eine Anwendung auf die Theorie des Elektrons", Z. Phys., 135(3), 260-269 (1953). [DOI]. (Cited on page 42.)

[215] Fock, V. A. and Ivanenko, D. D., "Über eine mögliche geometrische Deutung der relativistischen Quantentheorie", Z. Phys., 54, 798-802 (1929). [DOI]. (Cited on pages 41 and 43.)

[216] Fourès-Bruhat, Y., "Théorèmes d'existence et d'unicité pour les équations de la théorie unitaire de Jordan-Thiry", C. R. Hebd. Seanc. Acad. Sci., 232, 1800-1802 (1951). [ARK]. (Cited on page 135.)

[217] Fourès-Bruhat, Y., "Théorèmes d'existence pour certains systèmes d'équations aux dérivées partielles non linéaires", Acta Math., 88, 141-225 (1952). [DOI]. (Cited on page 127.)

[218] Fourès-Bruhat, Y., "Le problème de Cauchy dans la théorie relativiste de l'électromagnetisme et dans la théorie unitaire de Jordan-Thiry", in Mercier, A. and Kervaire, M., eds., Fünfzig Jahre Relativitätstheorie - Cinquantenaire de la Théorie de la Relativité - Jubilee of Relativity Theory, Proceedings of the conference held in Bern, Switzerland, $11-16$ July 1955, Helv. Phys. Acta Suppl., 29, pp. 76-78, (Birkhäuser, Basel, 1956). [DOI]. (Cited on page 135.)

[219] Fujii, Y. and Maeda, K.-I., The Scalar-Tensor Theory of Gravitation, Cambridge Monographs on Mathematical Physics, (Cambridge University Press, Cambridge; New York, 2003). [Google Books]. (Cited on page 137.)

[220] Galvagno, M. and Giribet, G., "The particle problem in classical gravity: a historical note on 1941", Eur. J. Phys., 26, S97-S110 (2005). [DOI]. (Cited on page 125.)

[221] Geiger, G., "Intertheory relations from unified field theories", J. Gen. Philos. Sci., 22(2), 263-282 (1991). [DOI]. (Cited on page 187.) 
[222] Ghosh, N. N., "On the solution of $\Gamma$ 's for a type of Non-Symmetric Tensor Field $g_{\mu \nu}$ ", Prog. Theor. Phys., 13(6,), 587-593 (1955). [DOI]. (Cited on pages 112 and 159.)

[223] Ghosh, N. N., "On a solution of Field Equations in Einstein's Unified Field Theory. I", Prog. Theor. Phys., 16, 421-428 (1956). [DOI]. (Cited on page 159.)

[224] Ghosh, N. N., "On a solution of Field Equations in Einstein's Unified Field Theory. II", Prog. Theor. Phys., 17(2), 131-138 (1957). [DOI]. (Cited on page 159.)

[225] Gião, A., "Sur le magnétisme des masses en rotation", C. R. Hebd. Seanc. Acad. Sci., 224, 1813-1815 (1947). [ARK]. (Cited on page 51.)

[226] Gião, A., "Sur l'effet mécano-magnétique à l'intérieur des masses sphériques en rotation. Application au champ magnétique terrestre", C. R. Hebd. Seanc. Acad. Sci., 226, 645-647 (1948). [ARK]. (Cited on page 51.)

[227] Gödel, Kurt, "An Example of a New Type of Cosmological Solutions of Einstein's Field Equations of Gravitation", Rev. Mod. Phys., 21, 447-450 (1949). [DOI]. (Cited on page 78.)

[228] Goenner, H., "Unified Field Theories: From Eddington and Einstein Up To Now", in De Sabbata, V. and Karade, T. M., eds., Relativistic Astrophysics and Cosmology, Vol. 1, Proceedings of the Sir Arthur Eddington Centenary Symposium, Nagpur, India 1984, pp. 176-196, (World Scientific, Singapore, 1984). (Cited on page 10.)

[229] Goenner, H., "On the History of Unified Field Theories", Living Rev. Relativity, 7, lrr-2004-2 (2004). [DOI], [ADS]. URL (accessed 20 May 2014):

http://www.livingreviews.org/lrr-2004-2. (Cited on page 11.)

[230] Goenner, H., "Some remarks on the genesis of scalar-tensor theories", Gen. Relativ. Gravit., 44, 2077-2097 (2012). [DOI], [arXiv:1204.3455 [gr-qc]]. (Cited on pages 30 and 137.)

[231] Gotusso, L., "Contributi alla teoria relativistica de Horváth", Rend. Ist. Lombardo Accad. Sci. Lett., Sci. Mat. Fis. Chim. Geol., Ser. A, 93, 64-72 (1959). (Cited on page 162.)

[232] Graiff, F., "Formule di commutazione e trasporto ciclico nei recenti spazi di Einstein", Rend. Ist. Lombardo Sci. Lett., Cl. Sci. Mat. Natur., III. Ser., 87, 105-110 (1954). (Cited on page 161.)

[233] Graiff, F., "Sul tensore elletromagnetico in una recente teoria unitaria", Rend. Ist. Lombardo Sci. Lett., Cl. Sci. Mat. Natur., III. Ser., 88, 833-840 (1955). (Cited on page 161.)

[234] Graves, J. C., The Conceptual Foundation of Contemporary Relativity Theory, (MIT Press, Cambridge, MA; London, 1971). (Cited on page 187.)

[235] Grosjean, P.-V., "La géométrie 'semi-métrique' et la théorie unitaire d'Einstein-Schrödinger", $C . R$. Hebd. Seanc. Acad. Sci., 247, 1562-1565 (1958). [ARK]. (Cited on page 141.)

[236] Gross, D., "Einstein and the Quest for a Unified Theory", in Galison, P. L., Holton, G. and Schweber, S. S., eds., Einstein for the 21st Century: His Legacy in Science, Art, and Modern Culture, pp. 287297, (Princeton University Press, Princeton, NJ, 2008). (Cited on page 196.)

[237] Gross, D. J., "Oscar Klein and gauge theory", in Lindström, U., ed., The Oskar Klein Centenary, Proceedings of the Symposium, held 19-21 September 1994 in Stockholm, Sweden, p. 94, (World Scientific, Singapore; River Edge, NJ, 1995). [arXiv:hep-th/9411233]. (Cited on page 34.)

[238] Gupta, S. N., "Einstein's and Other Theories of Gravitation", Rev. Mod. Phys., 29, 334-336 (1957). [DOI]. (Cited on page 168.)

[239] Haimovici, M., "Sur les espaces d'Einstein à connexion affine", C. R. Hebd. Seanc. Acad. Sci., 224, 94-96 (1947). [ARK]. (Cited on page 38.) 
[240] Hattori, K., "Über eine formale Erweiterung der Relativitätstheorie und ihren Zusammenhang mit der Theorie der Elektrizität", Phys. Z., 29, 538-549 (1928). (Cited on pages 25 and 142.)

[241] Havas, P. and Goldberg, J. N., "Lorentz-invariant equations of motion of point masses in the general theory of relativity", Phys. Rev., 128, 398-414 (1962). [DOI]. (Cited on page 169.)

[242] Hawking, S. W. and Ellis, G. F. R., The Large Scale Structure of Space-Time, Cambridge Monographs on Mathematical Physics, (Cambridge University Press, Cambridge, 1973). [Google Books]. (Cited on page 137.)

[243] Heckmann, O., Jordan, P. and Fricke, W., "Zur erweiterten Gravitationstheorie. I", Z. Astrophys., 28, 113-149 (1951). (Cited on page 32.)

[244] Hehl, F. and Obukhov, Y. N., "Élie Cartan's torsion in geometry and in field theory, an essay", Ann. Fond. Louis de Broglie, 32(2-3), 157-194 (2007). Online version (accessed 20 June 2014): http://aflb.ensmp.fr/AFLB-322/aflb322m595.htm. (Cited on page 9.)

[245] Hehl, F. W. and Kröner, E., "Über den Spin in der allgemeinen Relativitätstheorie. Eine notwendige Verallgemeinerung der Einsteinschen Feldgleichungen", Z. Phys., 187, 478-489 (1965). [DOI]. (Cited on page 156.)

[246] Hehl, F. W., von der Heyde, P., Kerlick, G. D. and Nester, J. M., "General relativity with spin and torsion: Foundations and prospects", Rev. Mod. Phys., 48, 393-416 (1976). [DOI]. (Cited on page 156.)

[247] Heisenberg, W., "Quantization of non-linear wave equations", in Bellamy, E. H. and Moorhouse, R. G., eds., The 1954 Glasgow Conference on Nuclear \& Meson Physics, International Union of Pure and Applied Physics conference held in Glasgow, Scotland, 13-17 July 1954, pp. 293-295, (Pergamon Press, London, 1955). (Cited on page 192.)

[248] Heisenberg, W., "Quantum Theory and its Interpretation", in Rozental, S., ed., Niels Bohr: His life and work as seen by his friends and colleagues, pp. 94-108, (North-Holland, Amsterdam, 1967). (Cited on page 191.)

[249] Hély, J., "Sur une représentation du champ unitaire", C. R. Hebd. Seanc. Acad. Sci., 238, 1375-1377 (1954). [ARK]. (Cited on page 18.)

[250] Hély, J., "Sur la représentation du champ unitaire par un tenseur $g_{i k}$ non symétrique", C. R. Hebd. Seanc. Acad. Sci., 241, 645-647 (1955). [ARK]. (Cited on page 100.)

[251] Hély, J., "Modèles statiques à symétrie sphérique en Relativité générale et en théorie de KaluzaKlein", C. R. Hebd. Seanc. Acad. Sci., 256, 5291-5293 (1963). [ARK]. (Cited on page 136.)

[252] Hély, J., "Solutions statiques à symétrie sphérique des équations de Jordan-Thiry", C. R. Hebd. Seanc. Acad. Sci., 256, 2784-2785 (1963). [ARK]. (Cited on page 136.)

[253] Hély, J., "Solutions statiques des équations de Jordan-Thiry", C. R. Hebd. Seanc. Acad. Sci., 256, 1933-1934 (1963). [ARK]. (Cited on page 136.)

[254] Hennequin, F. G., Étude mathématique des approximations en relativité générale et en théorie unitaire de Jordan-Thiry, (Gauthier-Villars, Paris, 1958). Thèse de doctorat, Paris 1956. (Cited on page 135.)

[255] Hinterbichler, K., "Theoretical aspects of massive gravity", Rev. Mod. Phys., 84, 671-710 (2012). [DOI], [ADS], [arXiv:1105.3735 [hep-th]]. (Cited on page 64.)

[256] Hittmair, O., "Schrödinger's Unified Field Theory Seen 40 Years Later", in Kilmister, C. W., ed., Schrödinger: Centenary Celebration of a Polymath, pp. 165-175, (Cambridge University Press, Cambridge; New York, 1987). (Cited on pages 10, 48, 55, and 71.) 
[257] Hlavatý, V., "The Einstein Connection of the Unified Theory of Relativity", Proc. Natl. Acad. Sci. USA, 38, 415-419 (1952). [DOI]. (Cited on page 112.)

[258] Hlavatý, V., "The Elementary Basic Principles of the Unified Theory of Relativity. A", Indiana Univ. Math. J., 1(4), 539-562 (1952). [DOI]. (Cited on page 90.)

[259] Hlavatý, V., "The Schrödinger Final Affine Field Laws", Proc. Natl. Acad. Sci. USA, 38(12), 10521058 (1952). [DOI]. (Cited on page 112.)

[260] Hlavatý, V., "The Elementary Basic Principles of the Unified Theory of Relativity B", J. Rational Mech. Anal., 2(1), 1-52 (1953). (Cited on pages 90, 112, 128, and 144.)

[261] Hlavatý, V., "The Elementary Basic Principles of the Unified Theory of Relativity $C_{1}$ : Introduction", J. Rational Mech. Anal., 3, 103-146 (1954). (Cited on pages 13, 144, and 145.)

[262] Hlavatý, V., "The Elementary Basic Principles of the Unified Theory of Relativity $C_{2}$ : Applications I", J. Rational Mech. Anal., 3, 147-179 (1954). (Cited on page 144.)

[263] Hlavatý, V., "The Elementary Basic Principles of the Unified Theory of Relativity $C_{3}$ : Applications II", J. Rational Mech. Anal., 3, 645-689 (1954). (Cited on page 144.)

[264] Hlavatý, V., "Report on the recent Einstein unified field theory", Rend. Sem. Mat. Univ. Padova, 23, 316-332 (1954). Online version (accessed 12 February 2014): http://www.numdam.org/item?id=RSMUP_1954_-_23__316_0. (Cited on pages 145 and 146.)

[265] Hlavatý, V., "The Elementary Basic Principles of the Unified Theory of Relativity B3", J. Rational Mech. Anal., 4(5), 653-679 (1955). (Cited on page 112.)

[266] Hlavatý, V., "The Elementary Basic Principles of the Unified Theory of Relativity $C_{4}$. General case", J. Rational Mech. Anal., 5, 419-472 (1955). (Cited on pages 145, 146, and 147.)

[267] Hlavatý, V., "The Elementary Basic Principles of the Unified Theory of the Second Kind, I.", J. Math. Mech., 7, 323-354 (1958). (Cited on page 147.)

[268] Hlavatý, V., "The Elementary Basic Principles of the Unified Theory of the Second Kind, II.", J. Math. Mech., 7, 323-354, 833-866 (1958). (Cited on page 147.)

[269] Hlavatý, V., Geometry of Einstein's Unified Field Theory, (P. Noordhoff, Groningen, 1958). Online version (accessed 23 June 2014):

http://www .archive.org/details/geometryofeinste029248mbp. (Cited on pages 10, 13, 111, 112, 144, 146, and 147.)

[270] Hlavatý, V. and Sáenz, A. W., "Uniqueness theorems in the unified theory of relativity", J. Rational Mech. Anal., 2(3), 523-536 (1953). (Cited on page 113.)

[271] Hoang, P. T., "L'emploi de la métrique $h^{\mu \nu}$ et des champs $f^{\mu \nu}$ pour l'obtention des équations du mouvement", C. R. Hebd. Seanc. Acad. Sci., 241, 170-172 (1955). [ARK]. (Cited on pages 100 and 117.)

[272] Hoang, P. T., "Sur le choix de la métrique en théorie unitaire", C. R. Hebd. Seanc. Acad. Sci., 241, 1919-1921 (1955). [ARK]. (Cited on pages 100, 117, and 123.)

[273] Hoang, P. T., "Conditions de conservation pour le tenseur d'impulsion-énergie en théorie unitaire d'Einstein-Schrödinger", C. R. Hebd. Seanc. Acad. Sci., 243, 1600-1603 (1956). [ARK]. (Cited on pages 116 and 117.) 
[274] Hoang, P. T., La méthode des singularités pour les équations du mouvement en relativité générale et en théorie du champ unifié, Ph.D. thesis, (Université de Paris (1896-1968), Faculté des sciences, Paris, 1957). Online version (accessed 12 February 2014):

http://www.numdam.org/item?id=ASNSP_1958_3_12_4_425_0. Soc. Tipografica 'Oderisi', Gubbio, 1958. Also published in: Ann. Scuola Norm. Sup. Pisa Cl. Sci., Ser. 3, 12, 1958, 425-477 and 13, 1959, 13-75. (Cited on page 123.)

[275] Hoffmann, B., "On the New Field Theory", Proc. R. Soc. London, Ser. A, 148, 353-364 (1934). (Cited on page 46.)

[276] Hoffmann, B., "A Generalization of the Einstein-Mayer Field Theory", Quart. J. Math., 7, 32-42 (1936). [DOI]. (Cited on page 138.)

[277] Hoffmann, B., "A Generalization of the Kaluza-Klein Field Theory", Quart. J. Math., 7, 20-31 (1936). [DOI]. (Cited on pages 34 and 138.)

[278] Hoffmann, B., "The Vector Meson Field and Projective Relativity", Phys. Rev., 72, 458-465 (1947). [DOI]. (Cited on page 28.)

[279] Hoffmann, B., "The Gravitational, Electromagnetic, and Vector Meson Field and the Similarity Geometry", Phys. Rev., 73, 30-35 (1948). [DOI]. (Cited on page 28.)

[280] Hoffmann, B., "The Gravitational, Electromagnetic, and Vector Meson Fields and the Similarity Geometry", Phys. Rev., 73(9), 531-532 (1948). [DOI]. (Cited on page 28.)

[281] Hoffmann, B., "The influence of Albert Einstein", Sci. Am., 180, 53-55 (1949). [DOI]. (Cited on page 78.)

[282] Hoffmann, B., ed., Perspectives in Geometry and Relativity: Essays in honor of Václav Hlavatý, (Indiana University Press, Bloomington; London, 1966). (Cited on page 10.)

[283] Horváth, J. I., "A Geometrical Model for the Unified Theory of Physical Fields", Phys. Rev., 80, 901 (1950). [DOI]. (Cited on page 175.)

[284] Horváth, J. I., "Contribution to the Final Affine Field Law", Bull. Acad. Polon. Sci. Cl. III, 3, 151-155 (1955). (Cited on pages 16 and 71.)

[285] Horváth, J. I., "Contribution to the Unified Theory of Physical Fields", Nuovo Cimento, 4, 577-581 (1956). [DOI]. (Cited on pages 143, 162, and 190.)

[286] Horváth, J. I. and Moór, A., "Entwicklung einer einheitlichen Feldtheorie begründet auf die Finslersche Geometrie", Z. Phys., 131, 544-470 (1952). [DOI]. (Cited on page 175.)

[287] Hosokawa, T., "Finslerian Wave geometry and Milne's world-structure", J. Sci. Hiroshima Univ., Ser. A, 8, 249-270 (1938). (Cited on pages 44 and 160.)

[288] Hotta, H., Namiki, M. and Kanenaga, M., "Stochastic quantization of Born-Infeld field", arXiv, e-print, (2004). [arXiv:hep-th/0405136]. (Cited on page 47.)

[289] Howard, D., "Einstein on Locality and Separability", Stud. Hist. Philos. Sci., 16, 171-201 (1985). [DOI]. (Cited on page 199.)

[290] Howard, D., "Einstein and the Development of Twentieth-Century Philosophy of Science", in Janssen, M. and Lehner, C., eds., The Cambridge Companion to Einstein, Cambridge Companions to Philosophy, 11, pp. 354-376, (Cambridge University Press, New York, 2014). (Cited on page 189.)

[291] Husain, S. I., "Sur les discontinuités des tenseurs de courbure en théorie unitaire d'Einstein", C. R. Hebd. Seanc. Acad. Sci., 246, 3020-3022 (1958). [ARK]. (Cited on page 127.) 
[292] Husain, S. I., "Sur la propagation des discontinuités du tenseur de courbure en théorie unitaire d'Einstein", C. R. Hebd. Seanc. Acad. Sci., 248, 2965-2967 (1959). [ARK]. (Cited on page 127.)

[293] Husain, S. I., "Sur les discontinuités des tenseurs de courbure en théorie unitaire d'Einstein (systéme faible des équations du champ)", C. R. Hebd. Seanc. Acad. Sci., 248, 194-196 (1959). [ARK]. (Cited on page 127.)

[294] Husain, S. I., Etude des ondes et de la radiation totale en champ unitaire d'Einstein, Ph.D. thesis, (Faculté des Sciences de Paris, Paris, 1960). (Cited on pages 127 and 129.)

[295] Husain, S. I., "La radiation totale en théorie unitaire du champ d'Einstein", C. R. Hebd. Seanc. Acad. Sci., 250, 466-467 (1960). [ARK]. (Cited on pages 127 and 128.)

[296] Husain, S. I. and Mishra, R. S., "Projective change of affine connections in Einstein's unified field", Tensor, New Ser., 6, 26-31 (1956). (Cited on page 158.)

[297] Ikeda, M., "On Static Solutions of Einstein's Generalized Theory of Gravitation. I", Prog. Theor. Phys., 12, 17-30 (1954). [DOI]. (Cited on pages 13 and 184.)

[298] Ikeda, M., "On Static Solutions of Einstein's Generalized Theory of Gravitation. II", Prog. Theor. Phys., 13, 265-275 (1955). [DOI]. (Cited on pages 13, 98, and 184.)

[299] Ikeda, M., "On boundary conditions in the Non-Symmetric Unified Field Theory", Prog. Theor. Phys., 15, 1-11 (1956). [DOI]. (Cited on page 160.)

[300] Ikeda, M., "On tensorial concomitants of a non-symmetric tensor $g_{\mu \nu}$. II.", Tensor, New Ser., 7, 117-127 (1957). (Cited on page 160.)

[301] Ikeda, M. and Abe, S., "On tensorial concomitants of a non-symmetric tensor $g_{\mu \nu}$. I", Tensor, New Ser., 7, 59-69 (1957). (Cited on page 160.)

[302] Recent Developments in General Relativity: Dedicated to Leopold Infeld in Connection with his 60th Birthday, (Pergamon Press; PWN, New York; Warszawa, 1962). (Cited on pages 10 and 119.)

[303] Infeld, L., "The New Einstein Theory and the Equations of Motion", Nature, 166, 1075 (1950). [DOI]. (Cited on page 83.)

[304] Infeld, L., "The New Einstein Theory and the Equations of Motion", Acta Phys. Pol., 10, 284-293 (1950). (Cited on pages 91 and 97.)

[305] Ingraham, R. L., "Contributions to the Schrödinger Non-Symmetric Affine Unified Field Theory", Ann. Math. (2), 52, 743-752 (1950). [DOI]. (Cited on pages 72 and 114.)

[306] Ishiwara, J., "Grundlagen einer relativistischen elektromagnetischen Gravitationstheorie", Phys. Z., 15, 294-298 (1914). (Cited on page 162.)

[307] Ishiwara, J., "Grundlagen einer relativistischen elektromagnetischen Gravitationstheorie, II", Phys. Z., 15, 506-510 (1914). (Cited on page 162.)

[308] Israel, W. and Trollope, R., "New Possibilities for a Unified Field Theory", J. Math. Phys., 2, 777-786 (1961). [DOI]. (Cited on pages 165 and 190.)

[309] Janssen, M., Renn, J., Sauer, T., Norton, J. D. and Stachel, J., "A Commentary on the Notes on Gravity in the Zurich Notebook", in Renn, J., ed., The Genesis of General Relativity, Vol. 2: Einstein's Zurich Notebook: Commentary and Essays, Boston Studies in the Philosophy of Science, 250, pp. 541-714, (Springer, Dordrecht, 2007). [DOI]. (Cited on page 189.)

[310] Jeavons, J. S., McIntosh, C. B. G. and Sen, D. K., "A correction to the Sen and Dunn gravitational field equations", J. Math. Phys., 16, 320-321 (1975). [DOI]. (Cited on page 175.) 
[311] Johnson, C. P., "A Criticism of a Recent Unified Field Theory", Phys. Rev., 89, 320-321 (1956). (Cited on page 86.)

[312] Johnson, C. R., "Equivalence of Einstein's 1925 unified field theory and his relativistic theory of the nonsymmetric field", Am. J. Phys., 47, 425 (1979). [DOI]. (Cited on page 58.)

[313] Jonsson, C. V., "Studies on five-dimensional relativity theory", Ark. Fys., 3(8), 87-129 (1951). (Cited on page 133.)

[314] Jordan, P., "Zur Neutrinotheorie des Lichtes", Z. Phys., 93, 464-472 (1935). [DOI]. (Cited on page 8.)

[315] Jordan, P., "Beiträge zur Neutrinotheorie des Lichtes. I", Z. Phys., 102(3-4), 243-252 (1936). (Cited on page 8.)

[316] Jordan, P., "Zur projektiven Relativitätstheorie", Nachr. Akad. Wiss. Goettingen II, Math.-Phys. $K l .$, 1945, 39-41 (1945). (Cited on pages 29 and 30.)

[317] Jordan, P., "Erweiterung der projektiven Relativitätstheorie", Ann. Phys. (Leipzig), 1, 219-228 (1947). [DOI]. (Cited on page 29.)

[318] Jordan, P., "Fünfdimensionale Kosmologie", Astron. Nachr., 276, 193-208 (1948). [DOI]. (Cited on pages 30 and 31.)

[319] Jordan, P., Schwerkraft und Weltall. Grundlage der theoretischen Kosmologie, (Vieweg, Braunschweig, 1952). (Cited on pages 29, 31, 32, and 175.)

[320] Jordan, P., Schwerkraft und Weltall, Die Wissenschaft, 133, (Vieweg, Braunschweig, 1955), 2nd edition. (Cited on pages 29, 137, and 175.)

[321] Jordan, P. and Müller, C., "Field Equations with a Variable 'Constant' of Gravitation", Z. Naturforsch., 2a, 1-2 (1947). (Cited on pages 30 and 32.)

[322] Kaufman, B., "Mathematical Structure of the Non-Symmetric Field Theory", in Mercier, A. and Kervaire, M., eds., Fünfzig Jahre Relativitätstheorie - Cinquantenaire de la Théorie de la Relativité - Jubilee of Relativity Theory, Proceedings of the conference held in Bern, Switzerland, 11-16 July 1955, Helv. Phys. Acta Suppl., 29, pp. 227-238, (Birkhäuser, Basel, 1956). [DOI]. (Cited on pages 94 and 95.)

[323] Kaul, S. K. and Mishra, R. S., "Generalized Veblen's identities", Tensor, New Ser., 8, 159-164 (1958). (Cited on page 158.)

[324] Kerr, R. P., "On Spherically Symmetric Solutions in Moffat's Unified Field Theory", Nuovo Cimento, 8, 789-797 (1958). [DOI]. (Cited on page 152.)

[325] Kibble, T. W. B., "Lorentz Invariance and the Gravitational Field", J. Math. Phys., 2, 212-221 (1961). [DOI]. (Cited on page 156.)

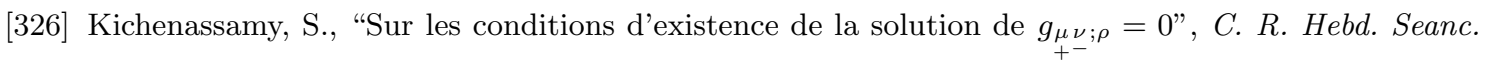
Acad. Sci., 244, 168-170 (1957). [ARK]. (Cited on page 158.)

[327] Kichenassamy, S., "Sur un cas particulier de la solution de $g_{\mu_{+} ; \rho}=0$ ", C. R. Hebd. Seanc. Acad. Sci., 244, 2007-2009 (1957). [ARK]. (Cited on page 158.)

[328] Kichenassamy, S., "Sur le champ électromagnétique singulier en théorie de Born-Infeld", C. R. Hebd. Seanc. Acad. Sci., 248, 3690-3692 (1959). [ARK]. (Cited on page 47.)

[329] Kilmister, C. W., General Theory of Relativiy: Selected Readings in Physics, (Pergamon Press, Oxford, 1973). (Cited on page 175.) 
[330] Kilmister, C. W. and Stephenson, G., "An Axiomatic Criticism of Unified Field Theories - I", Nuovo Cimento, 11, 91-105 (1954). [DOI]. (Cited on page 10.)

[331] Kilmister, C. W. and Stephenson, G., "An Axiomatic Criticism of Unified Field Theories - II", Nuovo Cimento, 11, 118-140 (1954). [DOI]. (Cited on pages 10 and 99.)

[332] Klauder, J. R., "Valentine Bargmann 1908-1989", in Biographical Memoirs, Vol. 76, pp. 36-49, (National Academy Press, Washington, DC, 1999). Online version (accessed 20 May 2014): http://www.nap.edu/openbook.php?record_id=6477. (Cited on page 35.)

[333] Klein, O., "On the Theory of Charged Fields", in New Theories in Physics, Conference organized in collaboration with the International Union of Physics and the Polish Intellectual Co-operation Committee, Warsaw, May $30-$ June 3, 1938, pp. 77-93, (International Institute of Intellectual Cooperation, Paris, 1939). (Cited on page 34.)

[334] Klotz, A. H., Macrophysics and Geometry: From Einstein's unified field theory to cosmology, (Cambridge University Press, Cambridge; New York, 1982). (Cited on page 10.)

[335] Kohler, M., "Die Formulierung der Erhaltungssätze der Energie und des Impulses in der allgemeinen Relativitätstheorie", Z. Phys., 131, 571-602 (1952). [DOI]. (Cited on page 166.)

[336] Kohler, M., "Die Formulierung der Erhaltungssätze der Energie und des Impulses in der allgemeinen Relativitätstheorie. II", Z. Phys., 134, 286-305 (1953). [DOI]. (Cited on page 166.)

[337] Kohler, M., "Zur Herleitung der Feldgleichungen in der allgemein-relativistischen Gravitationstheorie", Z. Phys., 134, 306-316 (1953). [DOI]. (Cited on page 166.)

[338] Kragh, H., "The Genesis of Dirac's Relativistic Theory of Electrons", Arch. Hist. Exact Sci., 24, 32-67 (1981). [DOI]. (Cited on page 189.)

[339] Kragh, H. S., Dirac: A Scientific Biography, (Cambridge University Press, Cambridge; New York, 1990). [Google Books]. (Cited on pages 192 and 198.)

[340] Kremer, H. and Kichenassamy, S., "Sur le champ électromagnétique singulier dans une théorie du type Born-Infeld", C. R. Hebd. Seanc. Acad. Sci., 250, 1192-1194 (1960). [ARK]. (Cited on page 47.)

[341] Kühnel, A. and Schmutzer, E., "Exakte Lösung der Feldgleichungen einer einfachen Variante der projektiven Relativitätstheorie für eine geladene Punktsingularität”, Ann. Phys. (Leipzig), 462(56), 243-250 (1961). [DOI]. (Cited on page 137.)

[342] Kurşunoğlu, B., "On Einstein's Unified Field Theory", Phys. Rev., 82, 289-290 (1951). [DOI]. (Cited on page 91.$)$

[343] Kurşunoğlu, B., "Gravitation and Electrodynamics", Phys. Rev., 88, 1369-1379 (1952). [DOI]. (Cited on pages 91, 116, 152, and 153.)

[344] Kurşunoğlu, B., "Correspondence in the Generalized Theory of Gravitation", Rev. Mod. Phys., 29, 412-416 (1957). [DOI]. (Cited on page 10.)

[345] Kurşunoğlu, B., "Relativity and Quantum Theory", Nuovo Cimento, 15, 729-756 (1960). [DOI]. (Cited on page 149.)

[346] Lal, K. B. and Mishra, R. S., "Einstein's connections. I: Degenerate cases of the first class", Tensor, New Ser., 10, 218-237 (1960). (Cited on page 158.)

[347] Lal, K. B. and Singh, S. P., "The wave solutions of non-symmetric unified field theories of Einstein, Bonnor and Schrödinger", Tensor, New Ser., 23, 369-375 (1972). (Cited on page 159.)

[348] Lamson, K. W., "On the Curvature Tensor of Einstein's Generalized Theory of Gravitation”, Can. J. Math., 5, 120-128 (1953). [DOI]. (Cited on page 22.) 
[349] Lanczos, C., "Albert Einstein and the Role of Theory in Contemporary Physics", American Scientist, 47(3), 41-59 (1959). (Cited on page 186.)

[350] Laughlin, R. B. and Pines, D., "The Theory of Everything", Proc. Natl. Acad. Sci. USA, 97, 28-31 (2000). [DOI]. (Cited on page 196.)

[351] Lederer, S., "Sur la quantification en théorie pentadimensionnelle (approximation linéaire)", C. R. Hebd. Seanc. Acad. Sci., 253, 386-388 (1961). [ARK]. (Cited on page 171.)

[352] Lederer, S., "Sur la représentation pentadimensionnelle des particules de spin 2, 1 et 0", C. R. Hebd. Seanc. Acad. Sci., 252, 3946-3948 (1961). [ARK]. (Cited on page 166.)

[353] Lederer, S., "Étude et quantification d'une théorie unitaire pentadimensionnelle", J. Phys. Radium, 23, 381-385 (1962). [DOI]. (Cited on page 171.)

[354] Lederer, S., "Le formalisme lagrangien et son application aux théories minkowskiennes de la gravitation et de l'électromagnétisme", Cah. Phys., 18, 233-284 (1964). (Cited on pages 167 and 168.)

[355] Lederer, S. and Tonnelat, M.-A., "Identités et lois du mouvement déduites d'un lagrangien. Application à la définition de l'impulsion-énergie dans une théorie euclidienne de la gravitation", Nuovo Cimento, 34, 883-900 (1964). [DOI]. (Cited on page 168.)

[356] Lehto, O., Mathematics Without Borders: A History of the International Mathematical Union, (Springer, Berlin; New York, 1998). (Cited on page 11.)

[357] Lenoir, M., "Les équations du mouvement en théorie du champ unifié", Cah. Phys., 14, 331-339 (1956). (Cited on page 118.)

[358] Lenoir, M., "Principe d'une théorie unitaire. Interprétation basée sur l'emploi d'un espace fibré", $C$. R. Hebd. Seanc. Acad. Sci., 248, 1944-1946 (1959). [ARK]. (Cited on page 111.)

[359] Lenoir, M., Sur quelques généralisations de la théorie unitaire d'Einstein-Schrödinger, Ph.D. thesis, (Faculté des Sciences de Paris, Paris, 1962). (Cited on pages 100, 115, and 131.)

[360] Leutwyler, H., "Sur une modification des théories pentadimensionnelles destinée à éviter certaines difficultés de la théorie de Jordan-Thiry", C. R. Hebd. Seanc. Acad. Sci., 251, 2292-2294 (1960). [ARK]. (Cited on page 136.)

[361] Levy, J., "Une version modifiée de la théorie du champ unifié d'Einstein", J. Phys. Radium, 20, 747-750 (1959). [DOI]. (Cited on pages 113, 119, and 123.)

[362] Lichnerowicz, A., "Espaces-temps extérieurs réguliers partout", C. R. Hebd. Seanc. Acad. Sci., 206, 313-316 (1938). [ARK]. (Cited on page 125.)

[363] Lichnerowicz, A., "Sur les singularités du $d s^{2}$ extérieur", C. R. Hebd. Seanc. Acad. Sci., 206, 157-159 (1938). [ARK]. (Cited on page 125.)

[364] Lichnerowicz, A., Sur certaines problèmes globaux relatifs au système des équations d'Einstein, Ph.D. thesis, (Faculté des Sciences de Paris, Paris, 1939). (Cited on page 125.)

[365] Lichnerowicz, A., "Sur des théorèmes d'unicité relatifs aux équations gravitationelles du cas intérieur", Bull. Sci. Math., 65, 54-72 (1941). (Cited on page 128.)

[366] Lichnerowicz, A., "Sur l'invariant intégral de l'hydrodynamique relativiste", Ann. Sci. Ec. Norm. Sup. (3), 58, 285-304 (1941). Online version (accessed 20 June 2014): http://www.numdam.org/item?id=ASENS_1941_3_58__285_0. (Cited on page 128.)

[367] Lichnerowicz, A., "Sur le charactère euclidien d'espaces-temps extérieurs statiques partout réguliers", C. R. Hebd. Seanc. Acad. Sci., 222, 432-434 (1946). [ARK]. (Cited on page 126.) 
[368] Lichnerowicz, A., "Compatibilité des équations de la théorie unitaire d'Einstein-Schrödinger", $C . R$. Hebd. Seanc. Acad. Sci., 237, 1383-1386 (1953). [ARK]. (Cited on pages 115, 125, 126, and 127.)

[369] Lichnerowicz, A., "Compatibilité des Équations de la Théorie Unitaire du Champ d'Einstein", J. Rational Mech. Anal., 3, 487-521 (1954). (Cited on pages 84, 95, 115, 126, and 127.)

[370] Lichnerowicz, A., "Étude des équations de la théorie unitaire d'Einstein", Rend. Sem. Mat. Fis. Milano, 25, 121-133 (1955). [DOI]. (Cited on pages 115, 125, 126, and 128.)

[371] Lichnerowicz, A., Théories relativistes de la gravitation et de l'électromagnétisme: Relativité générale et théories unitaires, (Masson, Paris, 1955). (Cited on pages 10, 12, 23, 100, 115, 125, 126, 128, and 190.)

[372] Lichnerowicz, A., "Problèmes généraux d'intégration des équations de la relativité", in Mercier, A. and Kervaire, M., eds., Fünfzig Jahre Relativitätstheorie - Cinquantenaire de la Théorie de la Relativité - Jubilee of Relativity Theory, Proceedings of the conference held in Bern, Switzerland, 11 - 16 July 1955, Helv. Phys. Acta Suppl., 29, pp. 176-191, (Birkhäuser, Basel, 1956). [DOI]. (Cited on pages 127 and 128.)

[373] Lichnerowicz, A., "Sur un procédé de quantification du champ de gravitation", C. R. Hebd. Seanc. Acad. Sci., 247, 433-436 (1958). [ARK]. (Cited on page 170.)

[374] Lichnerowicz, A., "Ondes Électromagnétiques et Ondes Gravitationnelles en Relativité Générale", in Costa de Beauregard, O., Dupeyrat, R. and de Broglie, L., eds., Problèmes actuels en théorie de la relativité, Réunion d'études et de mises au point, tenues sous la présidence de Louis de Broglie, p. 51, (Éditions de la Revue d'Optique théorique et instrumentale, Paris, 1959). (Cited on pages 127 and 186.)

[375] Lichnerowicz, A., "Propagateurs et commutateurs en relativité générale", Publ. Math. IHES, 10, 5-56 (1961). [DOI]. (Cited on pages 172 and 173.)

[376] Lichnerowicz, A., "Differential Geometry and Physical Theories", in Hoffmann, B., ed., Perspectives in Geometry and Relativity: Essays in honor of Václav Hlavatý, pp. 1-6, (Indiana University Press, Bloomington; London, 1966). (Cited on page 7.)

[377] Lichnerowicz, A., Relativistic Hydrodynamics and Magnetohydrodynamics: Lectures on the Existence of Solutions, Mathematical Physics Monograph Series, (W. A. Benjamin, New York, 1967). ed. Wightman, A. S. (Cited on page 128.)

[378] Lichnerowicz, A., "Relativity and Mathematical Physics", in Pentalo, M. and Finis, I., eds., Relativity, Quanta, and Cosmology in the Development of the Scientific Thought of Albert Einstein, Vol. 2, pp. 403-472, (Johnson, New York, 1979). (Cited on page 172.)

[379] Lichnerowicz, A., "Mathematics and General Relativity: A Recollection", in Eisenstaedt, J. and Kox, A. J., eds., Studies in the History of General Relativity, Proceedings of the 2nd Conference on the History of General Relativity, Luminy, Marseille, France, 6-9 September 1988, Einstein Studies, 3, pp. 103-108, (Birkhäuser, Boston; Basel, 1992). [Google Books]. (Cited on page 125.)

[380] Lichnerowicz, A. and Fourès-Bruhat, Y., "Théorème global sur les $d s^{2}$ extérieurs généraux d'Einstein", C. R. Hebd. Seanc. Acad. Sci., 226, 2119-2120 (1948). [ARK]. (Cited on page 126.)

[381] Lichnerowicz, A. and Thiry, Y., "Problèmes de calcul des variations liés à la dynamique classique et à la théorie unitaire du champs", C. R. Hebd. Seanc. Acad. Sci., 224, 529-531 (1947). [ARK]. (Cited on pages 133 and 134.)

[382] Lichnerowicz, A. and Tonnelat, M. A., eds., Les Théories Relativistes de la Gravitation, Actes du colloque tenu à Royaumont, les 21 - 27 Juin 1959, Colloques Internationaux du CNRS, 91, (Éditions du CNRS, Paris, 1962). (Cited on pages 10, 101, 118, 120, 121, 170, and 174.) 
[383] Ludwig, G., "Skalares Materiefeld in der projektiven Relativitätstheorie mit variabler Gravitationskonstante", Z. Naturforsch., 2a, 482-489 (1947). (Cited on page 29.)

[384] Ludwig, G., Fortschritte der projektiven Relativitätstheorie, Die Wissenschaft, 105, (Vieweg, Braunschweig, 1951). (Cited on pages 29 and 31.)

[385] Ludwig, G. and Just, K., "Zur Jordanschen Theorie der Materie-Entstehung", Z. Phys., 143, 472-476 (1955). [DOI]. (Cited on page 125.)

[386] Lyra, G., "Über eine Modifikation der Riemannschen Geometrie", Math. Z., 54, 52-64 (1951). [DOI]. (Cited on page 174.)

[387] Mariot, L. and Pigeaud, P., "Le champ mésonique en théorie hexadimensionnelle", C. R. Hebd. Seanc. Acad. Sci., 257, 2248-2251 (1963). [ARK]. (Cited on page 136.)

[388] Mariot, L. and Pigeaud, P., "Le champ mésonique scalaire en théorie de Jordan-Thiry", C. R. Hebd. Seanc. Acad. Sci., 257, 621-623 (1963). [ARK]. (Cited on page 140.)

[389] Martuscelli, L., "Sopra una possibile modificazione della teoria unitaria de Einstein", Rend. Ist. Lombardo Sci. Lett., Cl. Sci. Mat. Natur., III. Ser., 88, 607-615 (1955). (Cited on page 163.)

[390] Mas, L. and Montserrat, A., "Sur les fronts d'onde en théorie unitaire d'Einstein", C. R. Hebd. Seanc. Acad. Sci., 252, 382-384 (1961). [ARK]. (Cited on pages 116 and 129.)

[391] Mathisson, M., "Das zitternde Elektron und seine Dynamik", Acta Phys. Pol., 6, $218-227$ (1937). (Cited on page 154.)

[392] Mathisson, M., "Neue Mechanik materieller Systeme", Acta Phys. Pol., 6, 163-200 (1937). (Cited on page 154.)

[393] Mathisson, M., "Relativistic dynamics of a spinning magnetic particle", Proc. Cambridge Philos. Soc., 38, 40-60 (1942). [DOI]. (Cited on page 154.)

[394] Matsumoto, M., Foundations of Finsler Geometry and Special Finsler Spaces, (Kaiseisha Press, Otsu, Japan, 1986). (Cited on page 12.)

[395] Maurer-Tison, F., "Sur les coordonnées isothermes en théorie unitaire", C. R. Hebd. Seanc. Acad. Sci., 243, 1196-1198 (1956). [ARK]. (Cited on page 123.)

[396] Maurer-Tison, F., "Une interprétation géométrique des équations qui déterminent la connexion affine en fonction du tenseur fondamental en théorie unitaire du champ", C. R. Hebd. Seanc. Acad. Sci., 245, 995-998 (1957). [ARK]. (Cited on page 17.)

[397] Maurer-Tison, F., "L'espace fibré des corepères affines et son rôle fondamental en théorie unitaire d'Einstein-Schrödinger", C. R. Hebd. Seanc. Acad. Sci., 246, 240-243 (1958). [ARK]. (Cited on page 17.)

[398] Maurer-Tison, F., "Aspects mathématiques de la théorie unitaire du champ d'Einstein", Ann. Sci. Ec. Norm. Sup. (3), 76, 185-269 (1959). Online version (accessed 20 June 2014): http://www . numdam.org/item?id=ASENS_1959_3_76_3_185_0. (Cited on pages 129, 130, and 131.)

[399] Mautner, F. and Schrödinger, E., "Infinitesimal Affine Connections with Twofold Einstein-Bargmann Symmetry", Proc. R. Irish Acad. A, 50, 223-231 (1945). (Cited on pages 22 and 38.)

[400] Mavridès, S., "Courant et charge en théorie unitaire d'Einstein", C. R. Hebd. Seanc. Acad. Sci., 238, 1643-1644 (1954). [ARK]. (Cited on pages 100 and 101.)

[401] Mavridès, S., "Le choix de la métrique et du champ électromagnétique en théorie unitaire d'Einstein", C. R. Hebd. Seanc. Acad. Sci., 239, 637-640 (1954). [ARK]. (Cited on pages 100 and 101.) 
[402] Mavridès, S., "Solution non statique à symétrie sphérique des équations de la théorie unitaire d'Einstein", C. R. Hebd. Seanc. Acad. Sci., 239, 1597-1599 (1954). [ARK]. (Cited on page 98.)

[403] Mavridès, S., "Sur le choix de la métrique et du champ électromagnétique en théorie unitaire d'Einstein", C. R. Hebd. Seanc. Acad. Sci., 238, 1566-1568 (1954). [ARK]. (Cited on page 100.)

[404] Mavridès, S., "Choix de la métrique et du champ électromagnétique en théorie unitaire d'Einstein. Lien avec la théorie de Born-Infeld", J. Phys. Radium, 16, 482-488 (1955). [DOI]. (Cited on page 100.)

[405] Mavridès, S., Étude quantitative de l'isohémagglutination des hématies du groupe A, (Besancon: Jacques \& Demontrond, Paris, 1955). Thèse de doctorat, Paris 1953. (Cited on page 122.)

[406] Mavridès, S., "La solution générale des équations d'Einstein $g^{\mu_{+} \nu_{-} ;}=0$ ", C. R. Hebd. Seanc. Acad. Sci., 241, 173-174 (1955). [ARK]. (Cited on page 113.)

[407] Mavridès, S., "Sur une nouvelle définition du courant et de la charge en théorie unitaire d'Einstein", C. R. Hebd. Seanc. Acad. Sci., 240, 404-406 (1955). [ARK]. (Cited on page 101.)

[408] Mavridès, S., "Étude algébrique d'un tenseur métrique et d'un champ électromagnétique général en théorie unitaire d'Einstein-Schrödinger", C. R. Hebd. Seanc. Acad. Sci., 244, 1149-1151 (1957). [ARK]. (Cited on page 129.)

[409] Mavridès, S., "Identités de Bianchi et identités de conservation en théorie unitaire d'EinsteinSchrödinger", C. R. Hebd. Seanc. Acad. Sci., 244, 2482-2484 (1957). [ARK]. (Cited on page 115.)

[410] Mavridès, S., "Le tenseur d'impulsion-énergie électromagnétique en présence de matière chargée", C. R. Hebd. Seanc. Acad. Sci., 249, 637-639 (1959). [ARK]. (Cited on page 45.)

[411] Mavridès, S., "Mouvement d'une planète en théorie du graviton", C. R. Hebd. Seanc. Acad. Sci., 253, 2478-2480 (1961). [ARK]. (Cited on pages 166 and 168.)

[412] Mavridès, S., "Énergie gravitationelle en théorie euclidienne du champ de gravitation", C. R. Hebd. Seanc. Acad. Sci., 255, 2232-2234 (1962). [ARK]. (Cited on page 167.)

[413] Mavridès, S., "Vérifications expérimentales et théorie euclidienne du champ de gravitation", $C$. $R$. Hebd. Seanc. Acad. Sci., 257, 4139-4142 (1963). [ARK]. (Cited on pages 167 and 168.)

[414] Mavridès, S., "Trajectoires des satellites dans le champ de gravitation d'une sphère en rotation, en théorie minkowskienne linéaire", C. R. Hebd. Seanc. Acad. Sci., 258, 4655-4658 (1964). [ARK]. (Cited on page 168.)

[415] Mavridès, S., "Rayonnement d'une particule et loi du mouvement. Comparaison entre la Relativité générale et une méthode phénoménologique", C. R. Hebd. Seanc. Acad. Sci., 261, 3549-3552 (1965). [ARK]. (Cited on page 169.)

[416] Mavridès, S., "Gravitational Radiation and Renormalization of Mass in General Relativity and in a Lorenz-Invariant Theory", Nuovo Cimento, 45, 859-866 (1966). [DOI]. (Cited on page 169.)

[417] Mavridès, S., L'Univers Relativiste, (Masson, Paris, 1973). (Cited on page 122.)

[418] Mavridès, S., "Marie-Antoinette Tonnelat (1912-1980)", Ann. Inst. Henri Poincare A, 38, 1-6 (1983). Online version (accessed 14 May 2013):

http://www.numdam.org/item?id=AIHPA_1983__38_1_1_0. (Cited on page 104.)

[419] McConnell, J. and Schrödinger, E., "The Shielding Effect of Planetary Magnetic Fields", Proc. R. Irish Acad. A, 49, 259-273 (1944). (Cited on page 51.)

[420] McCrea, W. H., "The Meaning of Relativity", Math. Gaz., 35, 127-129 (1951). [DOI]. (Cited on page 82.) 
[421] Meksyn, D., "Unified Field Theory, Part I: Electromagnetic Field", Philos. Mag., 17, 99-112 (1934). (Cited on page 140.)

[422] Meksyn, D., "Unified Field Theory, Part II: Gravitation", Philos. Mag., 17, 476-482 (1934). (Cited on page 140.)

[423] Migdal, A. A., "Paradise Lost. Part One", personal homepage, Migdal, A. A. URL (accessed 11 February 2014):

http://alexandermigdal.com/prose/paradise1.shtml. (Cited on page 197.)

[424] Miller, A. I., Early Quantum Electrodynamics: A Source Book, (Cambridge University Press, Cambridge; New York, 1994). [Google Books]. (Cited on page 7.)

[425] Mimura, Y., "Relativistic Quantum Mechanics and Wave Geometry", J. Sci. Hiroshima Univ., Ser. A, 5, 99-106 (1935). (Cited on page 43.)

[426] Mimura, Y. and Hosokawa, T., "Space, time, and laws of nature", in Mimura, Y., Takeno, H., Sakuma, K. and Ueno, Y., eds., Wave Geometry II, Scientific Reports of the Research Institute for Theoretical Physics, 6, (Hiroshima University, Takehara, 1967). (Cited on page 189.)

[427] Mimura, Y. and Takeno, H., eds., Wave Geometry I, Scientific Reports of the Research Institute for Theoretical Physics, 2, (Hiroshima University, Takehara, 1962). (Cited on pages 43 and 44.)

[428] Mimura, Y., Takeno, H., Sakuma, K. and Ueno, Y., eds., Wave Geometry II, Scientific Reports of the Research Institute for Theoretical Physics, 6, (Hiroshima University, Takehara, 1967). (Cited on pages 43,44 , and 189.)

[429] Mishra, R. S., "Basic Principles of Unified Field Theory", Nuovo Cimento, 4, 907-916 (1956). [DOI]. (Cited on page 158.)

[430] Mishra, R. S., "The Field Equations of Einstein's and Schrödinger's Unified Theory", Tensor, New Ser., 6, 83-89 (1956). (Cited on pages 20 and 24.)

[431] Mishra, R. S., "Einstein's Connections, II. Nondegenerate Case", J. Math. Mech., 7(6), 867-892 (1958). (Cited on page 158.)

[432] Mishra, R. S., "Einstein's Connections: III - Degenerate Cases of Second Class", Nuovo Cimento, 10, 965-984 (1958). [DOI]. (Cited on page 158.)

[433] Mishra, R. S., "A Study of Einstein's Equations of Unified Field", Nuovo Cimento, 8, 632-642 (1958). [DOI]. (Cited on page 97.)

[434] Mishra, R. S., "Einstein's connections", Tensor, New Ser., 9, 8-43 (1959). (Cited on pages 112 and 158.)

[435] Mishra, R. S., " $n$-dimensional considerations of unified theory of relativity. Recurrence relations", Tensor, New Ser., 9, 217-225 (1959). (Cited on page 158.)

[436] Mishra, R. S., "Solutions of Gauge-invariant Generalization of Field Theories with Asymmetric Fundamental Tensor", Quart. J. Math., 14, 81-85 (1963). [DOI]. (Cited on page 157.)

[437] Mishra, R. S. and Abrol, M. L., "Equations of motion in unified field theory. I.", Tensor, New Ser., 10, 151-160 (1960). (Cited on page 97.)

[438] Misner, C. W., Thorne, K. S. and Wheeler, J. A., Gravitation, (W. H. Freeman, San Francisco, 1973). [ADS]. (Cited on pages 14 and 137.)

[439] Moffat, J., "Generalized Riemannian Spaces", Proc. Cambridge Philos. Soc., 52, 623-625 (1956). [DOI]. (Cited on pages 151 and 175.) 
[440] Moffat, J., "The foundations of a generalization of gravitation theory", Proc. Cambridge Philos. Soc., 53, 473-488 (1957). [DOI]. (Cited on pages 151, 152, and 165.)

[441] Moffat, J., "The Static Spherically Symmetric Solutions in a Unified Field Theory", Proc. Cambridge Philos. Soc., 53, 489-493 (1957). [DOI]. (Cited on pages 152 and 165.)

[442] Moffat, J., "On the Motion of Charged Particles in the Complex-Symmetric Unified Field Theory", Nuovo Cimento, 7, 107-109 (1958). [DOI]. (Cited on page 152.)

[443] Moffat, J. W. and Boal, D. H., "Solutions of the nonsymmetric unified field theory", Phys. Rev. D, 11, 1375-1382 (1975). [DOI]. (Cited on page 192.)

[444] Montserrat, A. and Mas, L., "Sur les fronts d'onde en théorie unitaire d'Einstein. Cas d'une charactéristique de Maurer-Tison", C. R. Hebd. Seanc. Acad. Sci., 252, 1110-1112 (1961). [ARK]. (Cited on page 129.)

[445] Montserrat, A. and Mas, L., "Sur les fronts d'onde en théorie unitaire d'Einstein. Étude générale", C. R. Hebd. Seanc. Acad. Sci., 252, 3751-3753 (1961). [ARK]. (Cited on page 129.)

[446] Moore, W., Schrödinger: Life and Thought, (Cambridge University Press, Cambridge; New York, 1990). [Google Books]. (Cited on pages 65, 67, 68, 69, 70, 96, 105, 192, and 196.)

[447] Moore, W., A Life of Erwin Schrödinger, (Cambridge University Press, Cambridge; New York, 1994). [Google Books]. (Cited on page 65.)

[448] Morette-DeWitt, C. and DeWitt, B. S., "Sur une théorie unitaire à cinq dimensions. I. Lagrangien", C. R. Hebd. Seanc. Acad. Sci., 241, 167-168 (1955). [ARK]. (Cited on page 171.)

[449] Morette-DeWitt, C. and DeWitt, B. S., "Sur une théorie unitaire à cinq dimensions. II. Hamiltonien. Relations de commutation", C. R. Hebd. Seanc. Acad. Sci., 241, 279-281 (1955). [ARK]. (Cited on page 171.)

[450] Motz, L., "Gauge Invariance and Classical Electrodynamics", Phys. Rev., 89, 60-66 (1953). [DOI]. (Cited on page 59.)

[451] Muñoz, C., "Desperately Seeking the Standard Model", in Sanz, V., Abel, S., Santiago, J. and Faraggi, A., eds., String Phenomenology 2003, Proceedings of the 2nd International Conference on String Phenomenology, Durham, UK, 4 July-4 August 2003, pp. 357-377, (World Scientific, Singapore, 2004). [DOI], [ADS], [arXiv:hep-ph/0312091]. (Cited on page 195.)

[452] Murphy, G. L., "A Unified Einstein-Yukawa Theory”, Prog. Theor. Phys., 58(5), 1622-1626 (1977). [DOI]. (Cited on page 190.)

[453] Narlikar, V. V., "From General Relativity to a Unified Field Theory", in Narlikar, V. V., ed., Proceedings of the Fortieth Indian Science Congress, Part II, Section 1: Mathematics, Lucknow, India 1953, pp. 1-20, (Indian Science Congress, Calcutta, 1954). Presidential Address. (Cited on page 10.)

[454] Narlikar, V. V. and Tiwari, R., "On Einstein's generalized theory of gravitation", Proc. Natl. Inst. Sci. India, 15, 73-79 (1949). (Cited on page 111.)

[455] Neddermeyer, S. H. and Anderson, C. D., "Note on the Nature of Cosmic-Ray Particles", Phys. Rev., 51, 884-886 (1937). [DOI], [ADS]. (Cited on page 8.)

[456] Nguyen, P.-C., "Contribution à l'étude des théories du champ unifié du type théorie d'EinsteinSchrödinger", Ann. Inst. Henri Poincare, 18(4), 303-357 (1964). (Cited on pages 124 and 125.)

[457] Norton, J. D., "Einstein, Nordström and the Early Demise of Scalar, Lorentz-Covariant Theories of Gravitation", Arch. Hist. Exact Sci., 45, 17-94 (1992). [DOI]. (Cited on page 162.) 
[458] Norton, J. D., "NNature is the Realisation of the Simplest Conceivable Mathematical Ideas': Einstein and the Canon of Mathematical Simplicity", Stud. Hist. Phil. Mod. Phys., 31, 135-170 (2000). [DOI]. (Cited on page 189.)

[459] Norton, J. D., "Little boxes: A simple implementation of the Greenberger, Horne, and Zeilinger result for spatial degrees of freedom", Am. J. Phys., 79, 182-188 (2011). [DOI]. (Cited on page 200.)

[460] Nye, M. J., Blackett: Physics, War, and Politics in the Twentieth Century, (Harvard University Press, Cambridge, 2004). (Cited on page 51.)

[461] O'Connor, D. J. and Stephens, C. R., "Geometry, the Renormalization Group and Gravity", in Hu, B. L., Ryan Jr, M. P. and Vishveshwara, C. V., eds., Directions in General Relativity, Vol. 1, Proceedings of the 1993 International Symposium, Maryland: Papers in honor of Charles Misner, pp. 272-287, (Cambridge University Press, Cambridge; New York, 1993). (Cited on page 196.)

[462] Oppenheimer, R., Robert Oppenheimer: Letters and Recollections, Stanford Nuclear Age Series, (Harvard University Press, Cambridge, MA; London, 1980). eds. Smith, A. K. and Weiner, C. (Cited on page 178.)

[463] O'Raifeartaigh, L., The Dawning of Gauge Theory, Princeton Series in Physics, (Princeton University Press, Princeton, NJ, 1997). [Google Books]. (Cited on page 139.)

[464] O'Raifeartaigh, L. and Straumann, N., "Gauge theory: Historical origins and some modern developments", Rev. Mod. Phys., 72, 1-23 (2000). [DOI]. (Cited on page 9.)

[465] Osborne, M. F. M., "Quantum theory restrictions on the General Theory of Relativity", Phys. Rev., 75, 1579-1584 (1949). [DOI]. (Cited on page 40.)

[466] Pais, A., "The Energy-momentum Tensor in Projective Relativity", Physica, 8, 1137-1160 (1941). [DOI]. (Cited on page 27.)

[467] Pais, A., "Meson fields in projective space", Physica, 9, 267-284 (1942). [DOI]. (Cited on page 27.)

[468] Pais, A., " $\omega$-space theory", in Imai, I., ed., Proceedings of the International Conference of Theoretical Physics 1953, Kyoto and Tokyo, September 1953, pp. 156-161, (Science Council of Japan, Tokyo, 1954). (Cited on page 139.)

[469] Pais, A., 'Subtle is the Lord ...': The Science and the Life of Albert Einstein, (Oxford University Press, Oxford; New York, 1982). [Google Books]. (Cited on pages 10, 57, 80, and 88.)

[470] Pais, A., Inward Bound: Of Matter and Forces in the Physical World, (Oxford University Press, Oxford; New York, 1986). (Cited on page 7.)

[471] Pais, A., Einstein lived here, (Oxford University Press, New York, 1994). (Cited on page 91.)

[472] Pais, A., Ich vertraue auf Intuition: Der andere Albert Einstein, (Spektrum Akademischer Verlag, Heidelberg, 1995). (Cited on page 91.)

[473] Pantaleo, M., ed., Cinquant'anni di relatività. Prefazione di Albert Einstein, (Editrice Universitaria, Firenze, 1955), 2nd edition. (Cited on pages 9, 19, 101, 111, and 161.)

[474] Papapetrou, A., "The question of non-singular solutions in the generalized theory of gravitation", Phys. Rev., 73, 1105-1108 (1948). [DOI]. (Cited on pages 74 and 126.)

[475] Papapetrou, A., "Static Spherically Symmetric Solutions in the Unitary Field Theory", Proc. R. Irish Acad. A, 52, 69-86 (1948). (Cited on pages 73, 95, 100, and 114.)

[476] Papapetrou, A., "A 4-dimensional Generalization of Wilson's Hypothesis", Philos. Mag., 41, 399-404 (1950). (Cited on page 52.) 
[477] Papapetrou, A., "Le problème du mouvement dans la Relativité générale et dans la théorie du champ unifié d'Einstein", Ann. Inst. Henri Poincare, 15, 173-203 (1957). Online version (accessed 19 May 2014):

http://www.numdam.org/item?id=AIHP_1957__15_3_173_0. (Cited on pages 117 and 118.)

[478] Papapetrou, A., "Un théorème en théorie du champ unifié à $g_{\mu \nu}$ non symétrique", C. R. Hebd. Seanc. Acad. Sci., 252, 2821-2823 (1961). [ARK]. (Cited on page 98.)

[479] Papapetrou, A. and Schrödinger, E., "The Point-Charge in the Non-symmetric Field Theory", $N a$ ture, 168, 40-41 (1951). [DOI]. (Cited on page 98.)

[480] Pastori, M., "Sui tensori emisotropi coniugati", Rend. Lincei, Ser. VI, 13, 109-114 (1931). (Cited on page 161.)

[481] Pastori, M., "Proprietà dei tensori emisimmetrici coniugati", Rend. Lincei, Ser. VI, 16, 311-316 (1932). (Cited on page 161.)

[482] Pastori, M., "Tensori emisimmetrici coniugati", Rend. Lincei, Ser. VI, 16, 216-220 (1932). (Cited on page 161.)

[483] Pastori, M., "Sull'ufficio del tensore fondamentale nell'ultima teoria di Einstein", Rend. Ist. Lombardo Sci. Lett., Cl. Sci. Mat. Natur., III. Ser., 84, 509-518 (1951). (Cited on page 161.)

[484] Pastori, M., "Sulle equazioni del campo elettromagnetico nell'ultima teoria di Einstein", Rend. Lincei, 12, 302-307 (1952). (Cited on pages 19 and 161.)

[485] Pastori, M., "Sullo spazio della recente teoria unitaria di Einstein", in Convegno Internazionale di Geometria Differenziale, Proceedings of the conference held in Venezia, Bologna, Pisa, Italy, 20 - 26 September 1953, pp. 107-113, (Edizioni Cremonese, Roma, 1954). (Cited on pages 17, 61, and 161.)

[486] Pauli, W., "Schlußwort durch den Präsidenten der Konferenz", in Mercier, A. and Kervaire, M., eds., Fünfzig Jahre Relativitätstheorie - Cinquantenaire de la Théorie de la Relativité - Jubilee of Relativity Theory, Proceedings of the conference held in Bern, Switzerland, 11-16 July 1955, Helv. Phys. Acta Suppl., 29, pp. 261-267, (Birkhäuser, Basel, 1956). Online version (accessed 13 February 2014):

http://retro.seals.ch/digbib/view?pid=hpa-001:1956:29::861. (Cited on pages 95 and 127.)

[487] Pauli, W., Relativitätstheorie, (P. Boringhieri, Torino, 1963). Reprint with annotations from the 1958 English edition. (Cited on page 121.)

[488] Pauli, W., Wissenschafticher Briefwechsel mit Bohr, Einstein, Heisenberg u.a., Band II: 1930 - 1939, Sources in the History of Mathematics and Physical Sciences, 6, (Springer, Berlin; New York, 1985). [DOI]. ed. von Meyenn, K. (Cited on page 46.)

[489] Pauli, W., Wolfgang Pauli. Wissenschaftlicher Briefwechsel mit Bohr, Einstein, Heisenberg u.a, Bd. III: 1940-1949, Sources in the History of Mathematics and Physical Sciences, 11, (Springer, Berlin; New York, 1993). [DOI]. ed. von Meyenn, K. (Cited on pages 52, 63, 67, 70, 71, 76, 99, 125, 126, 133, 180, 188, and 191.)

[490] Pauli, W., Wissenschaftlicher Briefwechsel mit Bohr, Einstein, Heisenberg u.a. Band IV, Teil I: 1950-1952, Sources in the History of Mathematics and Physical Sciences, 14, (Springer, Berlin; New York, 1996). [DOI]. ed. von Meyenn, K. (Cited on pages 30, 82, 83, 134, and 198.)

[491] Pauli, W., Wissenschaftlicher Briefwechsel mit Bohr, Einstein, Heisenberg u.a. Band IV, Teil II: 1953-1954, Sources in the History of Mathematics and Physical Sciences, 15, (Springer, Berlin; New York, 1999). [DOI]. ed. von Meyenn, K. (Cited on pages 9, 32, 33, 134, 135, 139, 198, 199, and 200.)

[492] Pauli, W., Wissenschaftlicher Briefwechsel mit Bohr, Einstein, Heisenberg u.a., Band IV, Teil III: 1955 - 1956, Sources in the History of Mathematics and Physical Sciences, 17, (Springer, Berlin; New York, 2001). [DOI]. von Meyenn, K. (Cited on pages 31, 32, and 94.) 
[493] Pauli, W., Wolfgang Pauli. Wissenschaftlicher Briefwechsel mit Bohr, Einstein, Heisenberg u.a, Bd. IV, Teil IV, A: 1957, Sources in the History of Mathematics and Physical Sciences, 18, (Springer, Berlin; New York, 2005). [DOI]. ed. von Meyenn, K. (Cited on pages 9 and 200.)

[494] Pétiau, G., "Recherches sur un modèle de représentation unifiée du système des particules élémentaires", Cah. Phys., 16(148), 491-516 (1962). (Cited on page 191.)

[495] Pigeaud, P., Contributions à l'étude des approximations en théorie unitaire pentadimensionnelle de Jordan-Thiry, Ph.D. thesis, (Faculté des Sciences de Paris, Paris, 1962). (Cited on pages 136 and 181.)

[496] Pigeaud, P., "Unified Fields in Pentadimensional Theory", Rep. Math. Phys., 14, 261-284 (1978). [DOI]. (Cited on page 136.)

[497] Pinl, M., "Begriff und Ziel der einheitlichen Feldtheorien", Math.-Phys. Semesterber., 4(3-4), 183-194 (1955). (Cited on page 10.)

[498] Podolanski, J., "Unified field theory in six dimensions", Proc. R. Soc. London, Ser. A, 201, 234-260 (1950). [DOI]. (Cited on pages 138 and 139.)

[499] Poyet, M., "Sur le choix d'un tenseur d'énergie-impulsion gravitationelle en théorie euclidienne de la gravitation", C. R. Hebd. Seanc. Acad. Sci., 256, 3594-3596 (1963). [ARK]. (Cited on page 168.)

[500] Proca, A., "Ce que nous devons à Einstein", in Proca, G. A., ed., Alexandre Proca, 1897-1955: Eeuvre scientifique publiée, p. 727, (G. A. Proca, Paris, 1988). Obituary Albert Einstein, Le Figaro Littéraire, 23 Avril 1955. (Cited on page 193.)

[501] Quan, P. M., Sur une théorie relativiste des fluides thermodynamiques, (Cooperativa Tipografica Azzoguidi, Bologna, 1955). Thèse de doctorat, Paris 21. 6. 1954. (Cited on page 128.)

[502] Rao, B. R., "The External Field of a Non-Static Centrally Symmetric Isolated System in the Unified Field Theory", Proc. Natl. Inst. Sci. India, 19, 723-738 (1953). (Cited on pages 59 and 98.)

[503] Rao, J. R., "Rigorous solution of Einstein's unified theory for a special type of symmetry", Acta Phys. Austriaca, 12, 251-261 (1959). (Cited on page 100.)

[504] Rao, J. R., "Unified theories of Einstein and Schrödinger", J. Math. Phys. Sci., 6, 381-418 (1972). (Cited on pages 10 and 184.)

[505] Renaudie, J., "Théories unitaires à six dimensions. Equations du champ", C. R. Hebd. Seanc. Acad. Sci., 240, 399-401 (1955). [ARK]. (Cited on page 139.)

[506] Renaudie, J., "Théories unitaires à six dimensions. Interprétation pour le champ mésoniqueélectromagnétique", C. R. Hebd. Seanc. Acad. Sci., 240, 2380-2382 (1955). [ARK]. (Cited on pages 139 and 140.)

[507] Renaudie, J., "Étude mathématique d'une théorie hexadimensionnelle du champ unifié", Bull Sci. Com. Trav. Hist. Sci., 1, 1-72 (1957). (Cited on page 139.)

[508] Renaudie, J., "Théorie hexadimensionnelle du champ de certaines particules", Sem. Janet, 2(2), 1-8 (1958-1959). Online version (accessed 11 February 2014): http://www.numdam.org/item?id=SJ_1958-1959__2__A2_0. (Cited on page 139.)

[509] Rickayzen, G. and Kurşunoğlu, B., "Unified Field Theory and Born-Infeld Electrodynamics", Phys. Rev., 89, 522-523 (1953). [DOI]. (Cited on page 153.)

[510] Riemann, B., Über die Hypothesen, die der Geometrie zu Grunde liegen, (Wissenschaftliche Buchgesellschaft, Darmstadt, 1959). Reprint. (Cited on page 12.) 
[511] Roche, C., "Sur la quantification du champ à l'approximation linéaire en théorie unitaire de JordanThiry", C. R. Hebd. Seanc. Acad. Sci., 250, 3128-3130 (1960). [ARK]. (Cited on page 171.)

[512] Romain, J., "Invariants différentiels d'un espace non riemannian", C. R. Hebd. Seanc. Acad. Sci., 244, 2777-2779 (1957). [ARK]. (Cited on page 13.)

[513] Romain, J., "Invariants différentiels d'un tenseur asymétrique du second ordre à quatre dimensions", C. R. Hebd. Seanc. Acad. Sci., 251, 1336-1338 (1960). [ARK]. (Cited on page 13.)

[514] Romain, J., "Invariants différentiels de connexion d'un espace non-riemannian", C. R. Hebd. Seanc. Acad. Sci., 254, 2928-2930 (1962). [ARK]. (Cited on page 13.)

[515] Rosen, N., "General Relativity and Flat Space. I", Phys. Rev., 57, 147-150 (1940). [DOI]. (Cited on page 166.)

[516] Rosen, N., "General Relativity and Flat Space. II", Phys. Rev., 57, 150-153 (1940). [DOI]. (Cited on page 166.)

[517] Rosenfeld, L., "Dr. Julius Podolanski”, Nature, 175, 795-796 (1955). (Cited on page 138.)

[518] Rosenfeld, L., "Niels Bohr in the Thirties", in Rozental, S., ed., Niels Bohr. His life and work as seen by his friends and colleagues, pp. 114-136, (North-Holland, Amsterdam, 1967). (Cited on page 191.)

[519] "Rotation and Terrestrial Magnetism", Observatory, 69, 100-105 (1949). [ADS]. (Cited on page 52.)

[520] Sáenz, A. W., "Elementare Herleitung der von Hlavatý angegebenen kanonischen Formen für den elektromagnetischen Tensor in der einheitlichen Feldtheorie", Z. Phys., 138, 489-498 (1954). [DOI]. (Cited on page 144.)

[521] Sánchez, N. G., "String Quantum Gravity", in Sánchez, N. and Zichichi, A., eds., String Quantum Gravity and Physics at the Planck Energy Scale, International worshop on theoretical physics (Second Chalonge Course), Erice, Sicily, $21-28$ June 1992, The Science and Culture Series, pp. 1-69, (World Scientific, Singapore; New Jersey, 1993). (Cited on page 196.)

[522] Sánchez, N. G., "Conceptual unification of elementary particles, black holes, quantum de Sitter and Anti de Sitter string states", Int. J. Mod. Phys. A, 19, 4173-4200 (2004). [DOI]. (Cited on page 196.)

[523] Sánchez-Ron, J. M., "George McVittie, The Uncompromising Empiricist", in Kox, A. J. and Eisenstaedt, J., eds., The Universe of General Relativity, Based on the Proceedings of the 6 th International Conference on the History of General Relativity, held in Amsterdam on June 26-29, 2002, Einstein Studies, 11, pp. 189-221, (Birkhäuser, Boston; Basel, 2005). [DOI]. (Cited on page 10.)

[524] Santaló, L. A., "On Einstein's Unified Field Theory", in Hoffmann, B., ed., Perspectives in Geometry and Relativity: Essays in honor of Václav Hlavatý, pp. 343-352, (Indiana University Press, Bloomington, IN, 1966). (Cited on pages 17, 22, and 187.)

[525] Santaló, L. A., "Unified field theories of Einstein's type deduced from a variational principle: Conservation laws", Tensor, New Ser., 25, 383-389 (1972). (Cited on page 187.)

[526] Sarkar, R., "Rigorous Static Solution Corresponding to a Particular Form of the Fundamental Tensor in Schrödinger Unified Field Theory", J. Math. Mech., 14, 183-193 (1965). (Cited on pages 69 and 159.)

[527] Sarkar, R., "On solutions of Schrödinger's unified field equations corresponding to a particular form of the fundamental non-symmetric tensor", Tensor, New Ser., 17, 227-237 (1966). Addendum: ibid. 19, 51-54 (1968). (Cited on page 159.)

[528] Sauer, T., "Field-equations in teleparalell space-time: Einstein's Fernparallelismus approach toward unified field theory", Historia Math., 33, 399-439 (2006). [DOI], [physics/0405142]. (Cited on page 10.) 
[529] Sauer, T., "Einstein's Unified Field Theory Program", in Janssen, M. and Lehner, C., eds., The Cambridge Companion to Einstein, Cambridge Companions to Philosophy, 9, pp. 281-304, (Cambridge University Press, New York, 2014). (Cited on page 10.)

[530] Schaffhauser-Graf, E., "Versuch einer 4-dimensionalen einheitlichen Feldtheorie der Gravitation und des Elektromagnetismus", J. Rational Mech. Anal., 2, 743-765 (1953). (Cited on page 175.)

[531] Scheibe, E., "Über einen verallgemeinerten affinen Zusammenhang", Math. Z., 57, 65-74 (1952). [DOI]. (Cited on page 174.)

[532] Scherrer, W., "Zur Theorie der Elementarteilchen", Verh. Schweiz. Naturf. Gesellsch., 121, 86-87 (1941). Online version (accessed 20 June 2014): http://retro.seals.ch/digbib/view?pid=sng-005:1941:121::94. (Cited on page 32.)

[533] Scherrer, W., "Über den Einfluss des metrischen Feldes auf ein skalares Materiefeld", Helv. Phys. Acta, 22, 537-551 (1949). [DOI]. (Cited on page 32.)

[534] Scherrer, W., "A propos des théories unitaires du champ", C. R. Hebd. Seanc. Acad. Sci., 237, 554-555 (1953). [ARK]. (Cited on page 32.)

[535] Scherrer, W., "Grundlagen zu einer linearen Feldtheorie", Z. Phys., 138, 16-34 (1954). (Cited on page 32.)

[536] Schilpp, P. A., ed., Albert Einstein: Philosopher-Scientist, The Library of Living Philosophers, VII, (Tudor, New York, 1949). (Cited on page 78.)

[537] Schouten, J. A., Der Ricci-Kalkül, (Springer, Berlin, 1924). (Cited on pages 14, 20, and 35.)

[538] Schouten, J. A., "La théorie projective de la relativité", Ann. Inst. Henri Poincare, 5(1), 51-88 (1935). (Cited on page 178.)

[539] Schouten, J. A., "On Meson Fields and Conformal Transformations", Rev. Mod. Phys., 21, 421-424 (1949). [DOI]. (Cited on pages 42 and 78.)

[540] Schouten, J. A., Ricci-Calculus: An Introduction to Tensor Analysis and Its Geometrical Applications, Die Grundlehren der Mathematischen Wissenschaften, X, (Springer, Berlin; Heidelberg, 1954), 2nd edition. [DOI]. (Cited on pages 14, 15, 18, 142, and 178.)

[541] Schrödinger, E., "Über die Unanwendbarkeit der Geometrie im Kleinen", Naturwissenschaften, 22, 518-520 (1934). [DOI]. (Cited on page 43.)

[542] Schrödinger, E., "Contributions to Born's New Theory of the Electromagnetic Field", Proc. R. Soc. London, Ser. A, 150, 465-477 (1937). (Cited on page 47.)

[543] Schrödinger, E., "Sur la théorie du monde d'Eddington", Nuovo Cimento, 15, 246-254 (1938). [DOI]. (Cited on page 8.)

[544] Schrödinger, E., "The Earth's and the Sun's Permanent Magnetic Fields in the Unitary Field Theory", Proc. R. Irish Acad. A, 49, 135-148 (1943). (Cited on page 51.)

[545] Schrödinger, E., "The General Unitary Theory of the Physical Fields", Proc. R. Irish Acad. A, 49, 43-56 (1943). (Cited on pages 48, 50, 101, and 105.)

[546] Schrödinger, E., "Systematics of Meson-Matrices", Proc. R. Irish Acad. A, 49, 29-42 (1943). (Cited on pages 48 and 178.)

[547] Schrödinger, E., "The Affine Connexion in Physical Field Theories", Nature, 153, 572-575 (1944). [DOI]. (Cited on page 55.)

[548] Schrödinger, E., "The Point Charge in the Unitary Field Theory", Proc. R. Irish Acad. A, 49, 225-235 (1944). (Cited on pages 52, 53, and 178.) 
[549] Schrödinger, E., "The Union of the three Fundamental Fields (Gravitation, Meson, Electromagnetism)", Proc. R. Irish Acad. A, 49, 275-287 (1944). (Cited on pages 21, 53, 55, 56, 65, 101, and 105.)

[550] Schrödinger, E., "On Distant Affine Connection", Proc. R. Irish Acad. A, 50, 143-154 (1945). (Cited on page 38.)

[551] Schrödinger, E., "The General Affine Field Laws", Proc. R. Irish Acad. A, 51, 41-50 (1946). (Cited on pages $8,65,66,67$, and 184.)

[552] Schrödinger, E., "The Final Affine Field Laws I.", Proc. R. Irish Acad. A, 51, 163-171 (1947). (Cited on pages $68,69,70,72$, and 89 .)

[553] Schrödinger, E., "The Relation Between Metric and Affinity", Proc. R. Irish Acad. A, 51, 147-150 (1947). (Cited on page 158.)

[554] Schrödinger, E., "The Final Affine Field Laws. II.", Proc. R. Irish Acad. A, 51, 205-216 (1948). (Cited on page 51.)

[555] Schrödinger, E., "The Final Affine Field Laws II.", Proc. R. Irish Acad. A, 51, 205-216 (1948). (Cited on pages 71,72 , and 115.)

[556] Schrödinger, E., "The Final Affine Field Laws III.", Proc. R. Irish Acad. A, 52, 1-9 (1948). (Cited on pages 80 and 115.)

[557] Schrödinger, E., Space-Time Structure, (Cambridge University Press, Cambridge; New York, 1950). [Google Books]. (Cited on pages 10, 68, 95, 96, and 102.)

[558] Schrödinger, E., "Studies in the Non-Symmetric Generalization of the Theory of Gravitation. I", Commun. Dublin Inst. Adv. Stud. A, 6, 551-579 (1951). (Cited on pages 72, 74, 114, and 162.)

[559] Schrödinger, E., "Electric Charge and Current Engendered by Combined Maxwell-Einstein Fields", Proc. R. Irish Acad. A, 56, 13-21 (1954). (Cited on page 96.)

[560] Schulmann, R., ed., Seelenverwandte: Der Briefwechsel zwischen Albert Einstein und Heinrich Zangger 1910-1947, (Verlag Neue Zürcher Zeitung, Zürich, 2012). (Cited on pages 34 and 63.)

[561] Schwarz, J. H., "Status of Superstring and M-Theory", Int. J. Mod. Phys. A, 25, 4703-4725 (2010). [DOI], [arXiv:0812.1372]. (Cited on page 195.)

[562] Schweber, S. S., QED and the men who made it: Dyson, Feynman, Schwinger, and Tomonaga, (Princeton University Press, Princeton, NJ, 1994). [Google Books]. (Cited on page 7.)

[563] Schweber, S. S., "Einstein and Nuclear Weapons", in Galison, P. L., Holton, G. and Schweber, S. S., eds., Einstein for the 21st Century: His Legacy in Science, Art, and Modern Culture, pp. 72-97, (Princeton University Press, Princeton, 2008). (Cited on page 198.)

[564] “A new Einstein theory?", Sci. Am., 182(January), 26 (1950). Editorial note. (Cited on pages 8 and 80.)

[565] Sciama, D. W., "On a non-symmetric theory of the pure gravitational field", Proc. Cambridge Philos. Soc., 54, 72-80 (1958). [DOI]. (Cited on pages 58, 101, 154, 155, and 156.)

[566] Sciama, D. W., "Les bases physiques de la théorie du champ unifié", Ann. Inst. Henri Poincare, 17, 1-11 (1961). (Cited on page 156.)

[567] Sciama, D. W., "On the Interpretation of the Einstein-Schrödinger Unified Field Theory", J. Math. Phys., 2, 472-477 (1961). [DOI]. (Cited on pages 156 and 190.) 
[568] Sciama, D. W., "On the analogy between charge and spin in general relativity", in Recent Developments in General Relativity: Dedicated to Leopold Infeld in Connection with his 60th Birthday, pp. 415-439, (Pergamon Press / PWN, New York; Warszawa, 1962). (Cited on page 156.)

[569] Sciama, D. W., "The Physical Structure of General Relativity", Rev. Mod. Phys., 36, 463-469 (1964). [DOI]. (Cited on page 156.)

[570] Seelig, C., Albert Einstein: Leben und Werk eines Genies unserer Zeit, (Europa Verlag, Zürich, 1960). (Cited on pages 57 and 91.)

[571] Sen, D. K., Sur une nouvelle théorie unitaire et un modèle statique cosmologique de l'univers basée sur la géométrie de Lyra, Ph.D. thesis, (Faculté des Sciences de Paris, Paris, 1958). (Cited on page 175.)

[572] Sen, D. K., Fields and/or Particles, (Academic Press, London; New York, 1968). (Cited on pages 10 and 175.)

[573] Sen, D. K. and Dunn, K. A., "A scalar-tensor theory of gravitation in a modified Riemannian manifold", J. Math. Phys., 12, 578-586 (1971). [DOI]. (Cited on page 175.)

[574] Sen, D. K. and Vanstone, J. R., "On Weyl and Lyra Manifolds", J. Math. Phys., 13, 990-993 (1972). [DOI]. (Cited on page 175.)

[575] Shankland, R. S., McCuskey, S. W., Leone, F. C. and Kuerti, G., "New Analysis of the Interferometer Observations of Dayton C. Miller", Rev. Mod. Phys., 27, 167-178 (1955). [DOI], [ADS]. (Cited on page 147.)

[576] Sibata, T., "A first approximate solution of the Morinaga's equation: $\frac{\sqrt{\Delta}}{2} \epsilon_{s t p q} K_{i m}{ }^{p q}=K_{l m s t}$ ", J. Sci. Hiroshima Univ., Ser. A, 5, 189-203 (1935). (Cited on page 44.)

[577] Sibata, T., "Wave geometry unifying Einstein's law of gravitation and Born's theory of electrodynamics", J. Sci. Hiroshima Univ., Ser. A, 8, 51-79 (1938). (Cited on page 44.)

[578] Siegmund-Schultze, R., "Scientific control in mathematical reviewing and German-U.S.-American relations between the two World Wars", Historia Math., 21(3), 306-329 (1994). [DOI]. (Cited on page 184.)

[579] Siegmund-Schultze, R., "The Institute Henri Poincaré and mathematics in France between the wars", Rev. Hist. Sci., 62(1), 247-283 (2009). [DOI]. (Cited on page 179.)

[580] Sievers, H., "Louis de Broglie und die Quantenmechanik", arXiv, e-print, (1998). [arXiv:physics/9807012]. (Cited on pages 113, 166, and 197.)

[581] Soh, H. P., "A Theory of Gravitation and Electricity", J. Math. Phys. (MIT), 12, 298-305 (1932). (Cited on page 60.)

[582] Souriau, J.-M., "Conséquences physiques d'une théorie unitaire", C. R. Hebd. Seanc. Acad. Sci., 248, 1478-1480 (1959). [ARK]. (Cited on page 172.)

[583] Souriau, J.-M., "Five-dimensional relativity", Nuovo Cimento, 30, 565-578 (1963). [DOI]. (Cited on page 172.)

[584] Średniawa, B., "Myron Mathisson's and Jan Weyssenhoff's Work on the Problem of Motion in General Relativity", in Eisenstaedt, J. and Kox, A. J., eds., Studies in the History of General Relativity, Proceedings of the 2nd Conference on the History of General Relativity, Luminy, Marseille, France, 6-9 September 1988, Einstein Studies, 3, pp. 400-406, (Birkhäuser, Boston; Basel, 1992). [Google Books]. (Cited on page 154.) 
[585] Stachel, J., "Einstein and the quantum: 50 years of struggle", in Colodny, R. G., ed., From Quarks to Quasars: Philosophical Problems of Modern Physics, Pittsburgh Series in Philosophy and History of Science, pp. 349-385, (Pittsburgh University Press, Pittsburgh, 1986). Reprinted in Stachel (2002), pp. 367-402. (Cited on page 191.)

[586] Stachel, J., "Einstein and Quantum Mechanics", in Einstein from ' $B$ ' to ' $Z$ ', Einstein Studies, 9, pp. 403-426, (Birkhäuser, Boston; Basel, 2002). (Cited on page 191.)

[587] Steinberger, J., "Early Particles", Annu. Rev. Nucl. Part. Sci., 47, xiii-xlii (1997). [DOI]. (Cited on page 9.)

[588] Stephenson, G., "Affine Field Structure of Gravitation and Electromagnetism", Nuovo Cimento, 10, 354-355 (1953). [DOI]. (Cited on page 153.)

[589] Stephenson, G., "Some Properties of Non-Symmetric Unified Field Theories", Nuovo Cimento, 12, 279-284 (1954). [DOI]. (Cited on page 153.)

[590] Stephenson, G., "La géometrie de Finsler et les théories du champs unifié", Ann. Inst. Henri Poincare, 15, 205-215 (1957). Online version (accessed 20 May 2014): http://www . numdam.org/item?id=AIHP_1957_-15_3_205_0. (Cited on pages 175, 176, and 177.)

[591] Stephenson, G. and Kilmister, C. W., "A Unified Field Theory of Gravitation and Electromagnetism", Nuovo Cimento, 10, 230-235 (1953). [DOI]. (Cited on page 175.)

[592] Straus, E. G., "Some Results in Einstein's Unified Field Theory", Rev. Mod. Phys., 21, 414-420 (1949). [DOI]. (Cited on pages 63, 74, 78, and 111.)

[593] Street, J. C. and Stevenson, E. C., "Penetrating Corpuscular Component of the Cosmic Radiation (Abstract 40)", Phys. Rev., 51, 1005 (1937). [DOI]. in: Minutes of the Washington Meeting, April 29, 30 and May 1, 1937. (Cited on page 8.)

[594] Stueckelberg, E. C. G., "Radioactive $\beta$-Decay and Nuclear Exchange Force as a Consequence of a Unitary Field Theory", Nature, 137, 1032 (1936). [DOI]. (Cited on page 192.)

[595] Sué, M., "Involutive systems of differential equations: Einstein's strength versus Cartan's degré d'arbitraire", J. Math. Phys., 32, 392-399 (1989). (Cited on page 85.)

[596] Sué, M. and Mielke, E. W., "Strength of the Poincaré gauge field equations in first order formalism", Phys. Lett. A, 139, 21-26 (1990). (Cited on page 85.)

[597] Surin, A., Étude du schéma fluide parfait et des équations de mouvement dans les théories pentadimensionnelles de Jordan-Thiry et de Kaluza-Klein, Mémorial des Sciences Mathématiques, 159, (Gauthier-Villars, Paris, 1965). Online version (accessed 13 February 2014):

http://www.numdam.org/item?id=MSM_1965_159__1_0. Thèse de doctorat, Paris 1963. (Cited on page 136.)

[598] Takasu, T., "Sphere-geometrical Unitary Field Theories", Compos. Math., 10, 95-116 (1952). Online version (accessed 19 June 2014):

http://www . numdam.org/item?id=CM_1952__10__95_0. (Cited on pages 7 and 160.)

[599] Takasu, T., "A combined field theory as a three-dimensional non-holonomic parabolic Lie geometry and its quantum mechanics", Yokohama Math. J., 1, 105-116 (1953). (Cited on page 160.)

[600] Takeno, H., "On some spherical wave solutions of non-symmetric unified field theories", Tensor, New Ser., 6, 90-103 (1956). (Cited on page 59.)

[601] Takeno, H., "Some plane wave-like solutions of non-symmetric unified field theory", Tensor, New Ser., 11, 263-269 (1961). (Cited on page 100.) 
[602] Takeno, H., Ikeda, M. and Abe, S., "On solutions of new field equations of Einstein and those of Schrödinger", Prog. Theor. Phys., 6, 837-848 (1951). [DOI]. (Cited on pages 74, 112, and 184.)

[603] Thirring, W. E., "An Alternative Approach to the Theory of Gravitation", Ann. Phys. (N.Y.), 16, 96-117 (1961). [DOI]. (Cited on page 166.)

[604] Thiry, Y., "Les équations de la théorie unitaire de Kaluza", C. R. Hebd. Seanc. Acad. Sci., 226, 216-218 (1948). [ARK]. (Cited on pages 30 and 133.)

[605] Thiry, Y., "Sur la régularité des champs gravitationels et électromagnétiques dans les théories unitaires", C. R. Hebd. Seanc. Acad. Sci., 226, 1881-1882 (1948). [ARK]. (Cited on page 133.)

[606] Thiry, Y., "Etude mathématique des équations d'une théorie unitaire à quinze variables de champ", J. Math. Pures Appl., 30, 275-396 (1951). (Cited on pages 133, 134, 135, and 190.)

[607] Thomas, J. M., "On various geometries giving a unified electric and gravitational theory", Proc. Natl. Acad. Sci. USA, 12, 187-191 (1926). [DOI]. (Cited on page 18.)

[608] Thomas, T. Y., "On the projective and equi-projective geometry of paths", Proc. Natl. Acad. Sci. USA, 11, 199-203 (1925). (Cited on page 16.)

[609] Todeschini, B., "Sul potenziale elettromagnetico nella teoria unitaria di Einstein", Rend. Lincei, Ser. VIII, 14, 495-500 (1953). (Cited on pages 15 and 161.)

[610] Tonnelat, M.-A., "Densité-flux et densité d'énergie dans la théorie du corpuscule de spin 2", $C$. $R$. Hebd. Seanc. Acad. Sci., 212, 384-389 (1941). [ARK]. (Cited on page 104.)

[611] Tonnelat, M.-A., "La seconde quantification dans la théorie du corpuscule de spin 2", C. R. Hebd. Seanc. Acad. Sci., 212, 430-434 (1941). [ARK]. (Cited on page 104.)

[612] Tonnelat, M.-A., "Sur la fusion de deux particules de spin 1", C. R. Hebd. Seanc. Acad. Sci., 212, 187-189 (1941). [ARK]. (Cited on page 104.)

[613] Tonnelat, M.-A., "Sur la théorie du photon dans un espace de Riemann", Ann. Phys. (Paris), 15, 144 (1941). (Cited on page 104.)

[614] Tonnelat, M.-A., "Sur les ondes planes de la particule de spin 2 (graviton)", C. R. Hebd. Seanc. Acad. Sci., 212, 263-266 (1941). [ARK]. (Cited on page 104.)

[615] Tonnelat, M.-A., "Sur une interprétation possible des grandeurs issues d'un tenseur symétrique dans la théorie de la particule de spin 2", C. R. Hebd. Seanc. Acad. Sci., 212, 687-689 (1941). [ARK]. (Cited on pages 104 and 108.)

[616] Tonnelat, M.-A., "Une nouvelle forme de théorie unitaire. Étude de la particule de spin 2", Ann. Phys. (Paris), 17, 158-207 (1942). also published by Masson, Paris 1942. (Cited on pages 104 and 166.)

[617] Tonnelat, M.-A., "Les phénomènes de gravitation, Étude des interactions entre la matière et la particule de spin 2", Ann. Phys. (Paris), 19, 396-445 (1944). 11e série. (Cited on pages 104 and 166.)

[618] Tonnelat, M.-A., "Structure générale d'une théorie unitaire des champs gravifique, électromagnétique et mésonique", C. R. Hebd. Seanc. Acad. Sci., 222, 1162-1164 (1946). [ARK]. (Cited on page 105.)

[619] Tonnelat, M.-A., "Théorie unitaire du champ physique. 1. Les tenseurs fondamentaux et la connexion affine", C. R. Hebd. Seanc. Acad. Sci., 228, 368-370 (1949). [ARK]. (Cited on page 106.)

[620] Tonnelat, M.-A., "Théorie unitaire du champ physique. 2. Cas d'une métrique symétrique", $C$. $R$. Hebd. Seanc. Acad. Sci., 228, 660-662 (1949). [ARK]. (Cited on page 106.) 
[621] Tonnelat, M.-A., "Théorie unitaire du champ physique. 3. Détermination des tenseurs fondamentaux", C. R. Hebd. Seanc. Acad. Sci., 228, 1846-1848 (1949). [ARK]. (Cited on page 106.)

[622] Tonnelat, M.-A., "Résolution des équations fondamentales d'une théorie unitaire purement affine", C. R. Hebd. Seanc. Acad. Sci., 230, 182-184 (1950). [ARK]. (Cited on pages 90, 107, and 111.)

[623] Tonnelat, M.-A., "Théorie unitaire affine. 2. Résolution rigoureuse de l'équation fondamentale", $C$. R. Hebd. Seanc. Acad. Sci., 231, 487-489 (1950). [ARK]. (Cited on pages 90 and 111.)

[624] Tonnelat, M.-A., "Compatibilité des équations de la théorie unitaire des champs", C. R. Hebd. Seanc. Acad. Sci., 232, 2407-2409 (1951). [ARK]. (Cited on page 112.)

[625] Tonnelat, M.-A., "Étude du système formé par la réunion de deux corpuscules de Dirac", J. Phys. Radium, 12, 516-520 (1951). [DOI]. (Cited on page 105.)

[626] Tonnelat, M.-A., "Les tentatives de rapprochement entre les constantes $\lambda$ (constante cosmologique) et $\mu_{0}$ (masse du photon)", J. Phys. Radium, 12, 829-832 (1951). [DOI]. (Cited on page 108.)

[627] Tonnelat, M.-A., "Théorie unitaire affine du champ physique", J. Phys. Radium, 12, 81-88 (1951). [DOI]. (Cited on pages 20, 72, 107, 108, 109, and 190.)

[628] Tonnelat, M.-A., "Compléments à la théorie unitaire des champs", J. Phys. Radium, 13, 177-185 (1952). (Cited on pages 107 and 108.)

[629] Tonnelat, M.-A., "Application de la solution générale des equations $g_{\mu \nu ; \rho}=0$ à la détermination d'une connexion affine particulière", C. R. Hebd. Seanc. Acad. Sci., 239, 231-233 (1954). [ARK]. (Cited on pages 90 and 98.)

[630] Tonnelat, M.-A., "Validité de la solution générale des equations d'Einstein $g_{\mu \nu ; \rho}=0$ dans le cas $\varphi=0 "$, C. R. Hebd. Seanc. Acad. Sci., 239, 1468-1470 (1954). [ARK]. (Cited on pages 90 and 112.)

[631] Tonnelat, M.-A., "La solution générale des équations d'Einstein $g_{+\nu ; \rho}=0$ ", J. Phys. Radium, 16, 21-38 (1955). (Cited on pages 112 and 113.)

[632] Tonnelat, M.-A., La théorie du champ unifié d'Einstein et quelques-uns de ses développements, (Gauthier-Villars, Paris, 1955). (Cited on pages 10, 12, 13, 15, 17, 23, 72, 98, 101, 102, 109, $110,111,115,121,124$, and 185.)

[633] Tonnelat, M.-A., "La solution générale des équations d'Einstein $g_{\mu_{+-} ; \rho}=0$ ", in Mercier, A. and Kervaire, M., eds., Fünfzig Jahre Relativitätstheorie - Cinquantenaire de la Théorie de la Relativité - Jubilee of Relativity Theory, Proceedings of the conference held in Bern, Switzerland, 11-16 July 1955, Helv. Phys. Acta Suppl., 29, pp. 192-197, (Birkhäuser, Basel, 1956). [DOI]. (Cited on page 112.)

[634] Tonnelat, M.-A., "Les équations approchées de la théorie du champ unifié d'Einstein-Schrödinger", Nuovo Cimento, 3, 902-920 (1956). [DOI]. (Cited on pages 113 and 150.)

[635] Tonnelat, M.-A., "Représentation de la matière en relativité générale et en théorie unitaire", Cah. Phys., 13, 1-11 (1958). (Cited on pages 115, 116, and 193.)

[636] Tonnelat, M.-A., "Solution générale des équations $g_{\mu \nu ; \rho}=0$. Expression de la connexion affine en fonction du tenseur fondamental $g_{\mu \nu}$ non dissocié", C. R. Hebd. Seanc. Acad. Sci., 246, 2227-2230 (1958). [ARK]. (Cited on pages 101 and 113.)

[637] Tonnelat, M.-A., "Sur la représentation des contributions matérielles et électromagnétiques dans une théorie purement géométrique", C. R. Hebd. Seanc. Acad. Sci., 251, 2892-2894 (1960). [ARK]. (Cited on pages 101, 116, and 119.) 
[638] Tonnelat, M.-A., "Théorie euclidienne du champ de gravitation. Énergie gravitationelle", C. R. Hebd. Seanc. Acad. Sci., 253, 2475-2477 (1961). [ARK]. (Cited on page 166.)

[639] Tonnelat, M.-A., "Inductions de gravitation", C. R. Hebd. Seanc. Acad. Sci., 254, 225-227 (1962). [ARK]. (Cited on page 168.)

[640] Tonnelat, M.-A., "Énergie gravitationelle et lois du mouvement dans une théorie linéaire et Minkowskienne du champ de gravitation", in Infeld, L., ed., Proceedings on Theory of Gravitation, conference in Warszawa and Jabłonna, 25-31 July, 1962, pp. 323-327, (Gauthier-Villars, Paris, 1964). (Cited on page 167.)

[641] Tonnelat, M.-A., Les théories unitaires de l'électromagnétisme et de la gravitation, Traité de physique théorique et de physique mathématique, 11, (Gauthier-Villars, Paris, 1965). (Cited on pages 10, 17, $101,110,115,116,119,120,129,166,170,172,174,185,187$, and 190.)

[642] Tonnelat, M.-A., Einstein's theory of unified fields, (Gordon and Breach, New York, London, Paris, 1966). With a preface by André Lichnerowicz. (Cited on pages 10, 109, and 111.)

[643] Tonnelat, M.-A., "Gravitational radiation and motion of a finite mass in an external field", in Treder, H.-J., ed., Entstehung, Entwicklung und Perspektiven der Einsteinschen Gravitationstheorie, Einstein Symposium Berlin, 2-5 November 1965, pp. 137-158, (Akademie-Verlag, Berlin, 1966). (Cited on page 169.)

[644] Tonnelat, M.-A., "Radiation gravitationelle et Mouvement des Sources", in Lichnerowicz, A. and Tonnelat, M.-A., eds., Fluides et champ gravitationnel en relativité générale, Paris, Collège de France, 19-23 Juin 1967, Colloques Internationaux du CNRS, 170, pp. 73-102, (Éditions du CNRS, Paris, 1969). (Cited on pages 166 and 169.)

[645] Tonnelat, M.-A., Histoire du principe de relativité, (Flammarion, Paris, 1971). (Cited on pages 10, 104, and 121.)

[646] Tonnelat, M.-A. and Bouche, L., "Quelques remarques le sur le schéma matière pure dans une théorie asymétrique du champ de gravitation pure", C. R. Hebd. Seanc. Acad. Sci., 250, 4289-4291 (1960). [ARK]. (Cited on page 155.)

[647] Tonnelat, M.-A. and Lederer, S., "Définition de l'impulsion-énergie gravitationelle dans une théorie euclidienne du champ de gravitation", C. R. Hebd. Seanc. Acad. Sci., 256, 371-373 (1963). [ARK]. (Cited on page 168.)

[648] Tonooka, K., "On three- and four-dimensional Finsler spaces with fundamental form $\sqrt[3]{a_{\alpha \beta \gamma} \chi^{\prime \alpha} \chi^{\prime \beta} \chi^{\prime \gamma}}$, , Tensor, New Ser., 9, 209-216 (1959). (Cited on page 175.)

[649] Treder, H.-J., "Der Materietensor in der unsymmetrischen Feldtheorie Einsteins", Wiss. Z. HumboldtUniv. Berlin, Math.-Naturw. R., 4(1), 9-10 (1955). (Cited on pages 116 and 118.)

[650] Treder, H.-J., "Stromladungsdefinition und elektrische Kraft in der einheitlichen Feldtheorie", Ann. Phys. (Leipzig), 19, 369-380 (1957). [DOI]. (Cited on pages 101 and 117.)

[651] Treder, H.-J., "Über eine Interpretation von Einsteins hermite-symmetrischer Feldtheorie", Tensor, New Ser., 23, 75-80 (1972). (Cited on page 102.)

[652] Udeschini, P., "Le equazioni di prima approssimazione nella nuova teoria relativistica unitaria di Einstein", Rend. Lincei, 9, 256-261 (1950). (Cited on page 162.)

[653] Udeschini, P., "Le equazioni di seconda approssimazione nella nuova teoria relativistica unitaria di Einstein. I", Rend. Lincei, Ser. VIII, 10, 21-24 (1951). (Cited on page 162.)

[654] Udeschini, P., "Le equazioni di seconda approssimazione nella nuova teoria relativistica unitaria di Einstein. II", Rend. Lincei, Ser. VIII, 10, 121-123 (1951). (Cited on page 162.) 
[655] Udeschini, P., "Sulle mutue azioni fra campo gravizionale e campo elettromagnetico", Rend. Lincei, Ser. VIII, 10, 390-394 (1951). (Cited on page 162.)

[656] Udeschini, P., "Spostamento delle righe spettrali per effeto di un campo magnetico elementare nella nuova teoria relativistica unitaria di Einstein", in Atti del Quarto congresso dell'Unione Matematica Italiana, Proceedings of the congress held in Taormina, $25-31$ October 1951, pp. 583-584, (Edizioni Cremonese, Roma, 1952). (Cited on page 163.)

[657] Udeschini, P., "Successiva linearizzazione delle ultime equazioni del campo unitario einsteiniano", Rend. Lincei, Ser. VIII, 15(3-4), 165-170 (1953). (Cited on page 162.)

[658] Udeschini, P., "Sviluppi dell'ultima teoria unitaria di Einstein", Rend. Sem. Mat. Fis. Milano, 27, 50-74 (1956). [DOI]. (Cited on page 162.)

[659] Udeschini, P., "Sopra una costante universale nella teoria unitaria di Einstein", Rend. Ist. Lombardo Accad. Sci. Lett., Sci. Mat. Fis. Chim. Geol., Ser. A, 97, 68-80 (1963). (Cited on page 163.)

[660] Udeschini, P., "On Einstein's Unified Field Theory", in Hoffman, B., ed., Perspectives in Geometry and Relativity: Essays in honor of Václav Hlavatý, pp. 436-447, (Indiana University Press, Bloomington; London, 1966). (Cited on page 163.)

[661] Utiyama, R., "Invariant theoretical interpretation of interactions", Phys. Rev., 101, 1597-1607 (1956). [DOI]. (Cited on page 156.)

[662] Utiyama, R. et al., "Report on 'Symposium on Gravity' Held at Research Institute for Fundamental Physics in April, 1962", Prog. Theor. Phys. Suppl., 25, 99-107 (1963). [DOI]. (Cited on pages 186 and 195.)

[663] Vaillant, J., "Sur les discontinuités du tenseur de courbure en théorie d'Einstein-Schrödinger", $C$. R. Hebd. Seanc. Acad. Sci., 253, 231-233 (1961). [ARK]. (Cited on page 129.)

[664] Vaillant, J., "Sur les discontinuités du tenseur de courbure en théorie d'Einstein-Schrödinger", $C$. R. Hebd. Seanc. Acad. Sci., 253, 1909-1911 (1961). [ARK]. (Cited on page 129.)

[665] Vaillant, J., "Sur les discontinuités du tenseur de courbure en théorie d'Einstein-Schrödinger", $C$. R. Hebd. Seanc. Acad. Sci., 254, 431-433 (1962). [ARK]. (Cited on page 130.)

[666] Vallée, R., Sur la représentations relativiste des fluides parfaits chargés, Ph.D. thesis, (Université de Paris, Paris, 1962). (Cited on page 140.)

[667] van Dongen, J., Einstein's Unification, (Cambridge University Press, Cambridge; New York, 2010). (Cited on pages 11 and 189.)

[668] Vanstone, J. R., "The General Static Spherically Symmetric solution of the 'Weak' Unified Field equations", Can. J. Math., 14, 568-576 (1962). [DOI]. (Cited on page 98.)

[669] Varga, O., "Über eine Klasse von Finslerschen Räumen, die die nichteuklidischen verallgemeinern", Comment. Math. Helv., 19, 367-380 (1946). [DOI]. (Cited on pages 175 and 176.)

[670] Veblen, O., "Formalism for conformal geometry", Proc. Natl. Acad. Sci. USA, 21, 168-173 (1935). [DOI]. (Cited on pages 28 and 178.)

[671] Veblen, O. and Hoffmann, B., "Projective relativity", Phys. Rev., 36, 810-822 (1930). [DOI]. (Cited on page 27.)

[672] Venini, C., "Integrazione delle equazioni di campo di seconda approssimazione dell'ultima teoria unitaria Einsteiniana", Rend. Ist. Lombardo Accad. Sci. Lett., Sci. Mat. Fis. Chim. Geol., Ser. A, 93, 731-742 (1959). (Cited on page 164.) 
[673] Venini, C., "Massa di un corpuscolo elettrizzato nella seconda approssimazione dell'ultima teoria unitaria Einsteiniana", Rend. Lincei, 27, 362-367 (1959). (Cited on page 164.)

[674] Venini, C., "Moto di dipoli elettrici nell'ultima teoria unitaria Einsteiniana", Rend. Lincei, 26, 490497 (1959). (Cited on page 164.)

[675] Venini, C., "Spostamento del perielio nell'orbita descritta da una particelle materiale elettrizzata nella prima approssimazione dell'ultima teoria unitaria einsteiniana", Rend. Lincei, 30, 728-733 (1961). (Cited on page 164.)

[676] Vierhaus, R. and vom Brocke, B., eds., Forschung im Spannungsfeld von Politik und Gesellschaft: Geschichte und Struktur der Kaiser-Wilhelm-/Max-Planck-Gesellschaft, (Deutsche Verlagsanstalt, Stuttgart, 1990). (Cited on page 179.)

[677] Vizgin, V. P., "Einstein, Hilbert, Weyl: Genesis des Programms der einheitlichen geometrischen Feldtheorien", NTM, 21, 23-33 (1984). (Cited on page 8.)

[678] Vizgin, V. P., Unified field theories in the first third of the 20th century, Science Networks. Historical Studies, 13, (Birkhäuser, Basel; Boston, 1994). [Google Books]. (Cited on page 10.)

[679] von Borzeszkowski, H.-H., Treder, H.-J. and Wahsner, R., Unitary field theory and prospects of generalized general relativity, MPIWG Preprint, 267, (Max Planck Institute for the History of Science, Berlin, 2004). (Cited on page 10.)

[680] von Weizsäcker, C. F., "Sur le problème d'une théorie unitaire des champs", in Louis de Broglie: physicien et penseur, pp. 135-143, (Éditions Albin Michel, Paris, 1953). (Cited on page 96.)

[681] Vrănceanu, G., "Sur une théorie unitaire non-holonome des champs", J. Phys. Radium, 7, 514-526 (1936). [DOI]. (Cited on page 34.)

[682] Vrănceanu, G., "Anhang”, in Vorlesungen über Differentialgeometrie, Vol. 2, pp. 382-385, (Akademie Verlag, Berlin, 1961). In deutscher Sprache bearb. und hrsg. von M. Pinl. (Cited on page 17.)

[683] Wald, R. M., General Relativity, (University of Chicago Press, Chicago, 1984). [ADS], [Google Books]. (Cited on page 14.)

[684] Wald, R. M., "Spin-two fields and general covariance", Phys. Rev. D, 33, 3613-3625 (1986). [DOI], [ADS]. (Cited on page 168.)

[685] Weinberg, S., Dreams of a Final Theory, (Pantheon Books, New York, 1992). (Cited on page 196.)

[686] Weinberg, S., "The Making of the Standard Model", in 't Hooft, G., ed., 50 Years of Yang-Mills Theory, pp. 99-117, (World Scientific, Singapore; Hackensack, NJ, 2005). [DOI]. (Cited on page 9.)

[687] Wentzel, G., "Zur Frage der Äquivalenz von Lichtquanten und Korpuskelpaaren", Z. Phys., 92, 337-358 (1934). [DOI]. (Cited on page 8.)

[688] Weyl, H., "Gravitation und Elektrizität", Sitzungsber. Preuss. Akad. Wiss., 1918(XXVI), 465-478 (1918). with "Nachtrag" of Einstein on p. 478 and "Erwiderung des Verfassers" on pp. 478-480 (1918). (Cited on page 9.)

[689] Weyl, H., Raum, Zeit, Materie, (Springer, Berlin, 1921), 4th edition. (Cited on page 53.)

[690] Weyl, H., "Zur Infinitesimalgeometrie: Einordnung der projektiven und konformen Auffassung", Nachr. Koenigl. Gesellsch. Wiss. Goettingen, Math.-Phys. Kl., 1921, 99-112 (1921). (Cited on page 16.)

[691] Weyl, H., Mathematische Analyse des Raumproblems, (Springer, Berlin, 1923). (Cited on page 16.)

[692] Weyl, H., "Elektron und Gravitation. I.", Z. Phys., 56, 330-352 (1929). [DOI]. (Cited on pages 9 and 155.) 
[693] Weyl, H., "Relativity Theory as a Stimulus in Mathematical Research", in Eisenhart, L. P., ed., Theory of Relativity in Contemporary Science, Papers read at the Celebration of the Seventieth Birthday of Professor Albert Einstein in Princeton, March 19, 1949, Proc. Amer. Phil. Soc., 93, pp. 535-541, (American Philosophical Society, Philadelphia, 1949). [Google Books]. (Cited on page 78.)

[694] Weyssenhoff, J., "Further contributions to the dynamics of spin-fluids and spin-particles", Acta Phys. Pol., 9, 26-33 (1947). (Cited on page 154.)

[695] Weyssenhoff, J. and Raabe, A., "Relativistic dynamics of spin-fluids and spin-particles", Acta Phys. Pol., 9, 7-18 (1947). (Cited on page 154.)

[696] Wheeler, J. A., "Geons", Phys. Rev., 97, 511-536 (1955). [DOI]. (Cited on page 193.)

[697] Wheeler, J. A., "Wie steht es heute mit Einsteins Idee, alles als Geometrie zu verstehen?", in Treder, H.-J., ed., Entstehung, Entwicklung und Perspektiven der Einsteinschen Gravitationstheorie, Einstein-Symposium Berlin, 2-5 November 1965, pp. 14-25, (Akademie-Verlag, Berlin, 1966). (Cited on page 186.)

[698] Will, C. M., Theory and Experiment in Gravitational Physics, (Cambridge University Press, Cambridge; New York, 1981), 2nd edition. (Cited on page 137.)

[699] Will, C. M., "The Confrontation between General Relativity and Experiment", Living Rev. Relativity, 9, lrr-2006-3 (2006). [DOI], [ADS], [arXiv:gr-qc/0510072]. URL (accessed 11 February 2014): http://www.livingreviews.org/lrr-2006-3. (Cited on page 137.)

[700] Winogradzki, J., "Sur la connexion des espaces affines", C. R. Hebd. Seanc. Acad. Sci., 232, 936-938 (1951). [ARK]. (Cited on page 122.)

[701] Winogradzki, J., "Contribution à la théorie des grandeurs physiques attachées aux particules de spin 1/2", Ann. Phys. (Paris), 8, 763-812 (1953). (Cited on page 122.)

[702] Winogradzki, J., "Sur les $\lambda$-transformations de la théorie unitaire d'Einstein-Schrödinger", C. $R$. Hebd. Seanc. Acad. Sci., 239, 1359-1361 (1954). [ARK]. (Cited on pages 20 and 122.)

[703] Winogradzki, J., "Le Groupe Relativiste de la Théorie Unitaire d'Einstein-Schrödinger", J. Phys. Radium, 16, 438-443 (1955). [DOI]. (Cited on pages 22, 24, 102, and 122.)

[704] Winogradzki, J., "Sur les 'identités de Bianchi' de la théorie unitaire d'Einstein-Schrödinger", $C$. $R$. Hebd. Seanc. Acad. Sci., 242, 74-76 (1956). [ARK]. (Cited on pages 88, 102, and 122.)

[705] Winogradzki, J., "Bookreport on 'M.-A. Tonnelat, La théorie du champ unifié d'Einstein et quelquesuns de ses développements', Paris 1955", J. Phys. Radium, 18, 523 (1957). (Cited on page 110.)

[706] Winogradzki, J., "Invariance et conservation en théorie des champs. Application de la théorie générale à l'étude d'un champ spinoriel", Cah. Phys., 13, 17-26 (1958). (Cited on page 122.)

[707] Woit, P., Not Even Wrong: The Failure of String Theory and the Continuing Challenge to Unify the Laws of Physics, (Vintage Press, London, 2007). (Cited on page 86.)

[708] Wrede, R. C., "“ $n$ ' dimensional considerations of basic principles A and B of the unified theory of relativity", Tensor, New Ser., 8, 95-122 (1958). (Cited on page 147.)

[709] Wyman, M., "Unified Field Theory", Can. J. Math., 2, 427-439 (1950). [DOI]. (Cited on pages 97 and 100.)

[710] Wyman, M. and Zassenhaus, H., "Zero Curvature Tensor in Einstein's Unified Field Theory", Phys. Rev., 110, 228-236 (1958). [DOI]. (Cited on page 99.)

[711] Xinh, N. X., "Le tenseur d'impulsion-énergie électromagnétique en présence de matière chargée, dans le cas des équations de liaison non linéaires de la théorie de Born-Infeld", C. R. Hebd. Seanc. Acad. Sci., 250, 468-470 (1960). [ARK]. (Cited on page 45.) 
[712] Yang, C. N. and Mills, R. L., "Conservation of isotopic spin and isotopic gauge invariance", Phys. Rev., 96, 191-195 (1954). [DOI]. (Cited on pages 9 and 156.)

[713] Yano, K., "Sur la nouvelle théorie unitaire de MM. Einstein et Bergmann", Proc. Imp. Acad. Japan, 14, 325-328 (1938). [DOI]. (Cited on page 34.)

[714] Yano, K. and Ohgane, M., "On Unified Field Theories", Ann. Math. (2), 55, 318-327 (1952). [DOI]. (Cited on page 34.)

[715] Yano, K. and Ohgane, M., "On six-dimensional unified field theories", Rend. Mat. Appl. (5), 13, 99-132 (1955). (Cited on page 139.)

[716] Yukawa, H., "On the Theory of Elementary Particles. I", Prog. Theor. Phys., 2(4), 209-215 (1947). [DOI]. (Cited on page 39.)

[717] Yukawa, H., "Models and Methods in the Meson Theory", Rev. Mod. Phys., 21, 474-479 (1949). [DOI]. (Cited on page 39.)

[718] Yukawa, H., "On the Radius of the Elementary Particle", Phys. Rev., 76, 300-301 (1949). [DOI]. (Cited on page 39.)

[719] Yukawa, H., "Quantum Theory of Non-Local Fields. Part I. Free Fields", Phys. Rev., 77, 219-226 (1950). [DOI]. (Cited on page 39.)

[720] Yukawa, H., "Structure and Mass Spectrum of Elementary Particles. I. General Considerations", Phys. Rev., 91, 415-416 (1953). [DOI]. (Cited on page 39.)

[721] Yukawa, H., "Structure and Mass Spectrum of Elementary Particles. II. Oscillator Model", Phys. Rev., 91, 416-417 (1953). [DOI]. (Cited on page 39.)

[722] Yukawa, H., "An attempt at a unified theory of elementary particles", in Imai, I., ed., Proceedings of the International Conference of Theoretical Physics 1953, Kyoto and Tokyo, September 1953, pp. 1-10, (Science Council of Japan, Tokyo, 1954). (Cited on page 39.)

[723] Zanella, A., "Successive linearizzazioni in una recente teoria relativistica unitaria", Rend. Ist. Lombardo Sci. Lett., Cl. Sci. Mat. Natur., III. Ser., 87, 575-592 (1954). (Cited on page 163.) 